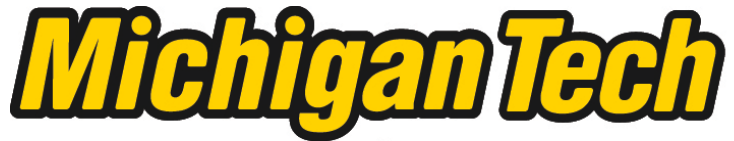 \\ Michigan Technological University Create the Future Digital Commons @ Michigan Tech
}

\section{Localized annealing of polysilicon microstructures by inductively heated ferromagnetic films}

Melissa L. Trombley

Michigan Technological University

Follow this and additional works at: https://digitalcommons.mtu.edu/etds

Part of the Electrical and Computer Engineering Commons

Copyright 2007 Melissa L. Trombley

\section{Recommended Citation}

Trombley, Melissa L., "Localized annealing of polysilicon microstructures by inductively heated ferromagnetic films", Dissertation, Michigan Technological University, 2007.

https://doi.org/10.37099/mtu.dc.etds/67

Follow this and additional works at: https://digitalcommons.mtu.edu/etds

Part of the Electrical and Computer Engineering Commons 


\title{
Localized Annealing of Polysilicon Microstructures by Inductively Heated Ferromagnetic Films
}

\author{
By: \\ MELISSA L. TROMBLEY
}

\begin{abstract}
A DISSERTATION
Submitted in partial fulfillment of the requirements

for the degree of

DOCTOR OF PHILOSOPHY

(Electrical Engineering)

MICHIGAN TECHNOLOGICAL UNIVERSITY
\end{abstract}


This dissertation, "Localized Annealing of Polysilicon Microstructures by Inductively Heated Ferromagnetic Films," is hereby approved in partial fulfillment of the requirements for the degree of DOCTOR OF PHILOSOPHY in the field of Electrical Engineering.

\section{DEPARTMENT or PROGRAM:}

Electrical Engineering

Signatures:

Dissertation Advisor

(Dr. Paul L. Bergstrom)

Committee (optional)

(Dr. Anand K. Kulkarni)

(Dr. Craig R. Friedrich)

(Dr. Bishnu P. Gogoi)

Department Chair

(Dr. Timothy J. Schulz)

Date 


\section{Acknowledgements}

The author would like to express sincere gratitude to all who helped to make this research possible and successful. The committee members were all invaluable resources in providing guidance and assistance when needed. Dr. Paul Bergstrom, the graduate advisor, was a marvelous teacher and friend who allowed tremendous flexibility in direction and scope of the project as well as and individual student ownership over the results. Dr. Anand Kulkarni (ECE), Craig Friedrich (ME-EM), and Bishnu Gogoi (Freescale Semiconductor) lent their wealth of experience to help focus the research goals to align as much as possible with the needs of the semiconductor industry while subject to the limitations (and advantages!) of the university environment.

Other wonderful faculty members (and hockey players!) during the nebulous journey through MTU included Dr. Timothy Schulz, Dr. Warren Perger, Dr. David Nitz, Dr. Peter Moran, Dr. Jeffrey Burl, Dr. Walter Milligan, and Dr. Sue BeskeDiehl. Sincere gratitude is also due to the amazing technical staff at MTU, including Bill Knudsen, Mike Chase, John Miller, Roland McKinstry, Scott Ackerman, Michelle Borkowski, Owen Mills, Edward Laitila, and Ruth Kramer.

Several members of the faculty technical staff at the University of Michigan's Solid State Electronics Laboratory and the University of Minnesota's Institute for Rock Magnetism were kind enough to donate their time and resources. Recognition and gratitude are owed to Dr. Kensall Wise, Dr. Khalil Najafi, Katharine Beach, Kimberly Appel, Timothy Brock, Mike Jackson, and Peter Solheid.

The student members of the Bergstrom, Friedrich, and other research groups were invaluable resources and friends in helping to see the research to fruition, especially as the final defense was near. The best of luck and success is wished to the following current and former students: Tom Wallner, Jin Zheng, Jianlin Liang, P. Santosh Karre, Hui Xia, Ben Arcand, Laura Walz, Raghav Vanga, Rodney Snow, Jack Grochowski, Andrew Gross, Michael Oisten, Brian Choponis, Paul Klustaitis, and many others.

Last, but certainly not least, are the friends and family members who provided support during these and other challenging time periods. Teddy Robert, you were always there even when you didn't know it; Dad and Marianne, Joanie Baloney, and the Trombley and Kitka families, you helped to make this all possible; Jacko and Jennifer Bukovich, you had better come visit; MTU Four-Wheelers and Chassell Bronco Club, you made college the amazing ten (ack!) years that they were. 


\begin{abstract}
The monolithic integration of dissimilar microsystems is often limited by conflicts in thermal budget. One of the most prevalent examples is the fabrication of active micro-electromechanical systems (MEMS), as structural films utilized for surface micromachining such as polysilicon typically require processing at temperatures unsuitable for microelectronic circuitry. A localized annealing process could provide for the post-deposition heat treatment of integrated structures without compromising active devices. This dissertation presents a new microfabrication technology based on the inductive heating of ferromagnetic films patterned to define regions for heat treatment. Support is provided through theory, finite-element modeling, and experimentation, concluding with the demonstration of inductive annealing on polysilicon inertial sensing structures. Though still in its infancy, the results confirm the technology to be a viable option for integrated MEMS as well as any microsystem fabrication process requiring a thermal gradient.
\end{abstract}




\section{Table of Contents}

Acknowledgements...................................................

Abstract.............................................................. ii

Table of Contents........................................................

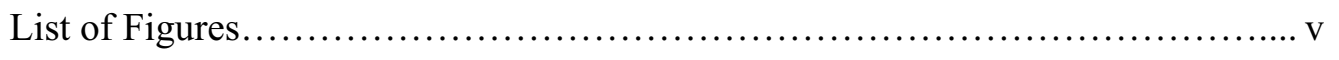

List of Tables.......................................................

Chapter 1: Localized Annealing in Microsystem Fabrication..................... 1

1.1: Overview of CMOS-MEMS Integration........................... 1

1.2: Integrated MEMS Technologies.................................. 4

1.3: Localized Annealing Methods...................................... 15

1.4: Other Applications.................................................

Chapter 2: Theory of Induction Heating.................................... 27

2.1: Electromagnetic Fundamentals...................................28

2.2: Selectivity through Material Properties.............................. 33

Chapter 3: Process Development through Simulation.......................... 49

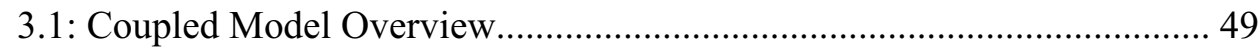

3.2: ANSYS Example Induction Heating Model.......................................... 51

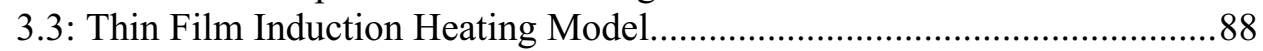

3.4: Thin Film Induction Heating Model with Thermal Isolation.................... 107

Chapter 4: Process Development through Experimentation..................... 118

4.1: Concept Verification............................................119

4.2: Inductive Annealing Test System................................. 128

4.3: Magnetic Properties of RF Sputtered Ferromagnetic Films.............. 142

4.4: Impact of Film Properties on Heat Generation......................... 152

Chapter 5: Annealing of Polysilicon Structures..................................................... 178

5.1: Blanket Polysilicon Films............................................ 178

5.2: Polysilicon Cantilever Beams.............................................................. 184

5.3: Polysilicon Accelerometer Structures................................................... 193

Chapter 6: Device Design and Fabrication..................................... 202

6.1: Design Considerations............................................203

6.2: Process Overview and Device Design................................210

6.3: Solid Model Generation..............................................220

6.4: Electrostatic Simulation...........................................224 
6.5: Mechanical Simulation............................................ 230

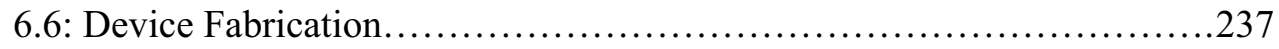

Chapter 7: Conclusion and Recommendations for Future Work.................. 248

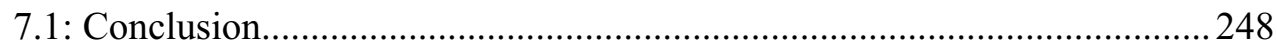

7.2: Recommendations for Future Work...................................................... 249

References............................................................ 252

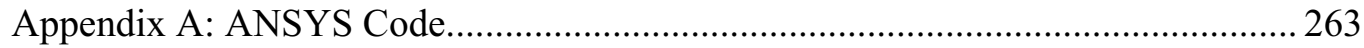

A.1: Simulation Code for ANSYS Example Induction Heating Model...........263

A.2: Material Properties for Thin Film Simulations........................................2268

A.3: Simulation Code for Thin Film Induction Heating Model...................... 275

A.4: Simulation Code for Thin Film Induction Heating Model with

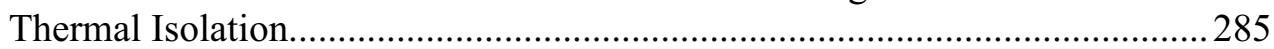

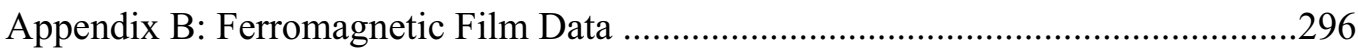

B.1: Nickel and Cobalt................................................296

B.2: Nickel-Iron Alloys........................................... 301

B.3: Iron-Cobalt Alloys.................................................. 307

B.4: Nickel-Iron-Cobalt Alloys....................................... 311

Appendix C: High Aspect Ratio Polysilicon Sensor Process............................... 314

C.1: Low-Temperature Deposition with Annealing....................... 314

C.2: High-Temperature Deposition without Annealing.......................3 318 


\section{List of Figures}

Figure 1.1: Illustrative structure describing the initial version of Cornell University's SCREAM Process [Zhang, et al., 1991]. 6

Figure 1.2: Cross-section of integrated pressure sensor fabricated from the University of Michigan Dissolved Wafer Process [Chavan, et al., 2000].................6

Figure 1.3: Illustration of LIGA processing [Ueno, et al., 1997]........................... 9

Figure 1.4: Illustration of the Carnegie Mellon CMOS-MEMS three-metal aluminum process [Zhang, et al., 1999]

Figure 1.5(a): Illustration of Sandia's $M^{3} E M S$ process [Allen, et al., 1998]. 9

Figure 1.5(b,c): Cross-section of Sandia rotary actuator (b) fabricated using SUMMIT-V process (c) consisting of five polysilicon layers with intermediate sacrificial oxides [Krygowski, et al., 1999]...

Figure 1.6: Illustration of Analog Devices Modular-MEMS process

[Palaniapan, et al., 2003].

Figure 1.7: Illustration of Bosch epi-poly process [Offenberg, et al., 1995].

Figure 2.1: Illustration of ferromagnetic domains [Eisberg, et al., 1985 (p.

502)].

Figure 2.2: Typical ferromagnetic hysteresis loops showing the effect of a magnetic field $H$ on the (a) magnetization $M$, and (b) magnetic flux density $B$ [Elliott, 1998 (p. 630)...

Figure 2.3: Magnetization curves for single-crystalline iron, nickel, and cobalt, [Honda, et al., 1926].

Figure 2.4: Illustration of the difference between (a) magnetically hard, and

(b) magnetically soft materials [Feynman, 1971 (pp. 37-10, 36-7)]....

Figure 2.5(a,b): Effect of temperature on the magnetization characteristics of

(a) nickel, and (b) FeZr alloys under different field conditions [Arajs, 1965;

Barandiaran, et al., 1994].

Figure 2.6: Illustration of electron alignment under (a) ferromagnetic, (b)

antiferromagnetic, and (c) ferrimagnetic exchange conditions [Eisberg, et al., 1985 (p. 503)].

Figure 3.1: Flow chart illustrating sequential coupled-solution modeling in

ANSYS. [ANSYS Coupled-Field Analysis Guide, Sec. 2.3] ................................50

Figure 3.2: Area plot of the ANSYS solid model....................................................5

Figure 3.3(a): Elemental plot of the meshed ANSYS solid model..........................56

Figure 3.3(b): Axisymmetric rotation of the meshed model................................... 56

Figure 3.4: Three-dimensional plot showing the magnitude of the z-

component of the magnetic vector potential across the coil interior.

Figure 3.5: Output plot generated by ANSYS example simulation code

showing the temperatures at the center (blue) and edge (purple) of the heated

billet.

Figure 3.6: Contour plot showing the electromagnetic field magnitudes along the y-direction, with a close up added of outer edge of heated billet.

Figure 3.7: (a) Two-dimensional contour plot, and (b) three-dimensional vector plot showing the y-component of the electromagnetic flux density, $B_{Y}$, for the ANSYS model.

Figure 3.8: Representation of the induced current densities, shown in (a) two dimensions, and (b) three dimensions.

Figure 3.9: Two-dimensional contour plot showing the $I^{2} R$ power generation within the heated structure of the ANSYS example model..... 
Figure 3.10: Contour plots shown in (a) two dimensions, and (b) three dimensions illustrating the final temperature distribution in the ANSYS model following the execution of the coupled simulation.

Figure 3.11(a): Graph showing the impact of frequency on the induced power density and total power dissipation.......

Figure 3.11(b): Relationship between induced power density and induced current density in for frequency values ranging from $50-450 \mathrm{kHz}$.

Figure 3.11(c): Curves showing the variation in temperature rise with frequency for the example ANSYS model.

Figure 3.12(a): Graph of the magnetic field strength and magnetic flux density with varying current levels applied to the magnetic coil.

Figure 3.12(b): Relationship between the impressed coil current and the induced current density in the inductively heated ANSYS structure.

Figure 3.12(c): Graph showing the maximum induced power density at the outer edge of the heated billet (blue) and total dissipated power in the billet (pink) with respect to the impressed coil current.

Figure 3.12(d): Variation in temperature rise in the heated structure with increasing current applied to the magnetic coil......

Figure 3.13(a): Variation in the magnetic field strength (blue) and the flux density at the outer edge of the heated billet (pink) with changes in magnetic permeability.

Figure 3.13(b): Graph of the total time-average power dissipation (pink) and the peak power density at the outer edge of the heated structure (blue) in the example model vs relative permeability

Figure 3.13(c): Illustration of the impact of the relative magnetic permeability on the rate of rise of the heated billet.

Figure 3.14(a): Graph showing the trends in flux density (blue) and skin depth (pink) in the heated billet of the ANSYS model vs temperature......

Figure 3.14(b): Variation in power dissipation and peak power density vs temperature

Figure 3.15(a): Impact of film thickness on induced power dissipation and peak power density values.

Figure 3.15(b): Impact of film radius on induced power dissipation and peak power density values 86

Figure 3.16(a): Area plot of the solid model with close-ups illustrating the layouts of the thin film regions. 90

Figure 3.16(b): Three-dimensional axisymmetric rotation of the thin film solid model showing only the substrate and film stack.

Figure 3.17: Meshed thin film model showing the relative mesh sizes for the different components.

Figure 3.18(a): Contour plot showing the y-component of the magnetic field strength that was produced by the solenoid coil.

Figure 3.18(b): Vector plot showing magnetic field strength and orientation..........94

Figure 3.19(a): Contour plot showing the magnitude of the x-component of the magnetic flux density, with a close-up on the NiFe19 film.

Figure 3.19(b): Contour plot showing the magnitude of the y-component of the magnetic flux density, with a close-up on the NiFe19 film. 95

Figure 3.20: Contour plot showing the induced current density within the thin film model. 96

Figure 3.21: Contour plot showing the relative power density dissipation in the 
thin film model.

Figure 3.22: Illustration of the points of measurement for plotting in the charts of simulated temperature with respect to time. 100

Figure 3.23(a): Graph of temperature, in Kelvin, vs time following a 10second run of the default, unconstrained thin film simulation.

Figure 3.23(b): Contour plot showing the final temperature distribution, in Kelvin, across the model after a 10 -second coupled simulation of the thin film model without temperature constraints on the substrate.

Figure 3.24(a): Graph of temperature $(\mathrm{K})$ vs time during a 6-second simulation of the thin film model with the back side of the substrate held at $300 \mathrm{~K}$

Figure 3.24(b): Contour plot showing the temperature distribution $(\mathrm{K})$ across the model following a 6-second simulation with the back side of the substrate held at $300 \mathrm{~K}$

Figure 3.25(a): Graph of temperature $(\mathrm{K})$ vs time during a 6-second simulation in which the outer edge of the substrate was held at $300 \mathrm{~K}$

Figure 3.25(b): Contour plot showing the temperature distribution $(\mathrm{K})$ in and around the $\mathrm{NiFe} 19$ film at simtime $=0.49 \mathrm{~s}$ with the outer edge of the substrate held at $300 \mathrm{~K}$.

Figure 3.25(c): Contour plot showing the temperature distribution $(\mathrm{K})$ in and around the NiFe19 film at simtime $=1.58 \mathrm{~s}$ with the outer edge of the substrate held at $300 \mathrm{~K}$. 105

Figure 3.25(d): Contour plot showing the temperature distribution $(\mathrm{K})$ across the model at simtime $=6 \mathrm{~s}$ with the outer edge of the substrate held at $300 \mathrm{~K}$..............106 Figure 3.26(a): Area plot of the isolation film solid model designed to enhance thermal isolation. 108

Figure 3.26(b): Three-dimensional rotational area plot showing the layout of the structure more clearly... 108

Figure 3.27: Element plot of the isolation film solid model showing the relative sizes of the divisions.

Figure 3.28: Illustration of the points of measurement for plotting in the charts of simulated temperature with respect to time for the isolation film model.

Figure 3.29(a): Graph of temperature (K) vs time during a 10s simulation of the isolation film solid model.

Figure 3.29(b): Contour plot showing the temperature distribution, in Kelvin, across the model following a 10 -second simulation with no thermal constraints placed on the substrate.

Figure 3.30(a): Graph of temperature $(\mathrm{K})$ vs time for a 6 -second simulation of the isolation film solid model.

Figure 3.30(b): Contour plot showing the temperature distribution, in Kelvin, across the isolation model following a 6-second simulation with the back side of the substrate held at $300 \mathrm{~K}$

Figure 3.31(a): Graph of temperature $(\mathrm{K})$ vs time for a 6 -second simulation of the isolation film solid model with the back side of the substrate held at $300 \mathrm{~K}$, a $20 \mu \mathrm{m} \mathrm{SiO}_{2}$ thickness, and the coil current increased by a factor of 10 .....

Figure 3.31(b): Contour plot showing the temperature distribution (K) across the isolation model following a 6-second simulation with the back of the substrate held at $300 \mathrm{~K}$, a $20 \mu \mathrm{m} \mathrm{SiO}_{2}$ thickness, and the coil current increased by a factor of 10 .

Figure 3.32: Graph showing the relationship between the current density 
applied to the magnetic coil and the resulting induced steady-state temperatures.

Figure 4.1: Cross-sectional diagram of polysilicon cantilever beams utilized in the second verification test...

Figure 4.2: Illustration of wafer partitioning into rectangular strips

approximately $1 \mathrm{~cm} \times 3 \mathrm{~cm}$ in size.

Figure 4.3: Images of inductively heated cobalt films showing (a) a lowtemperature anneal in progress in which heat generation was evident by the cobalt film discoloration and (b) the result of a high-temperature anneal

Figure 4.4: SEM images of cantilever beams from the (a) low-temperature and (b) high-temperature ends of the same test sample....

Figure 4.5: SEM images of the cantilever beam anchors illustrating a visible difference in the silicon grain structures between (a) the low-temperature and (b) the high-temperature areas of the sample.

Figure 4.6: Interferometric images of the beam anchor regions from the (a) low-temperature region and (b) high-temperature region again reflecting an improvement in surface texture as a result of inductive annealing.

Figure 4.7(a): X-Ray Diffraction output plot obtained from the low-

temperature region of a polysilicon beam sample.

Figure 4.7(b): X-Ray Diffraction output plot showing the considerable narrowing of the diffraction peaks that occurred as a result of heat treatment.

Figure 4.8: Full view of the BEAVIS test system showing the exterior layout........129

Figure 4.9: Interior of the BEAVIS vacuum chamber illustrating the manner in which the various components interacted.

Figure 4.10: Ameritherm Nova Star 3 induction heating system consisting of the (a) $3 \mathrm{~kW}$ self-tuning power supply and (b) remote heat station to which the coil was attached....

Figure 4.11: Means by which the substrate was scanned around the coil.

Figure 4.12: Alumina silicate (ceramic) substrate holder designed to secure the wafer vertically in front of the coil without placing restrictions on wafer diameter or heat sinking capability.

Figure 4.13: Components utilized for temperature measurement during induction heating.

Figure 4.14: Modified version of the BEAVIS test system for pyrometry calibration

Figure 4.15: Diagram of the Luxtron menu hierarchy.

Figure 4.16: Emissivity of polished silicon over temperature and wavelength of radiation with a phosphorous doping level of approximately $3 \mathrm{E} 14 \mathrm{~cm}^{-3}$ [Sato, 1967]

Figure 4.17: Illustration of the pyrometry calibration procedure.

Figure 4.18: Final results of pyrometry calibration showing a comparison of the thermocouple and pyrometry readings at various levels of the autotransformer.

Figure 4.19: $\mathrm{Ni}_{81} \mathrm{Fe}_{19}$ magnetization curves measured in the plane of the substrate

Figure 4.20(a): Relative permeability values for the three alloys of interest, normal to the substrate, extracted from the low-field VSM data...

Figure 4.20(b): Relative permeability values for the three alloys of interest, parallel to the substrate, extracted from the low-field VSM data. 151

Figure 4.21: Temperature scale provided by Thermographic Measurements for 
the MC490-10 multi-change thermally-sensitive paint [TMC, 2005].

Figure 4.22: Photograph showing a sample being heated using an early configuration of BEAVIS

Figure 4.23: Heated samples showing (a) variations in paint colors as a result of different induced temperatures, and (b) the result of allowing a sample to heat too high.

Figure 4.24: Illustration of the ferromagnetic ring concept for reducing the footprint of the inductive annealing process.

Figure 4.25(a): Initial square layout illustrating the dimensions utilized. 162

Figure 4.25(b): Modified square ring layout with interior corners rounded to better accommodate eddy current flow.

Figure 4.26: Plot of S1 FeCo data from Table 4.6 with error bars to compensate for the front-back temperature difference as well as to account for uncertainty in the pyrometry measurements $\left(+9^{\circ} \mathrm{C} /-1^{\circ} \mathrm{C}\right)$.

Figure 4.27: Illustration of $2.4 \mathrm{~cm}$-wide circular areas on $100 \mathrm{~mm}$ wafers, with (a) a solid interior and (b) a $1.2 \mathrm{~cm}$ inner diameter.....

Figure 4.28: Photograph showing the glow of a 7.50 $\mu \mathrm{m}$ hollow NiFe19 ring heated to approximately $600^{\circ} \mathrm{C}$, as measured by infrared pyrometry 168

Figure 4.29(a): Plot of temperature vs. coil power for both solid and hollow (as indicated) NiFe19 films at various thicknesses and heating conditions 170

Figure 4.29(b): Reduced data set from Figure 4.28(a) showing only values

from the stable temperature regions.

Figure 4.29(c): Linear trendline data for solid $\mathrm{FeCo}$ and $\mathrm{Au}$ circles of various thicknesses.

Figure 4.30: Pyrometry sensor data showing temperature drop from primary sensor at heated film region, at wafer center, to secondary sensor shifted $3.2 \mathrm{~cm}$ laterally (center-center spacing).

Figure 4.31: Illustration representing the heat flow to the two infrared sensors as a result of inductive coupling to a circular ring-shaped film centered around the rightmost sensor.

Figure 5.1: SEM images of $1 \mu \mathrm{m}$ LPCVD polysilicon surfaces (a) before, and (b) after a five-minute anneal at approximately $1000^{\circ} \mathrm{C}$ using a $2.5 \mu \mathrm{m}, 1 \mathrm{~cm}^{2}$ FeCo film deposited in a 400-550 Gauss magnetic field.

Figure 5.2: Interferometric images of polysilicon surface (a) before, and (b) after annealing showing a change in stress-induced curvature from highly compressive to moderately tensile.

Figure 5.3: SEM images showing cross-sections of $1.5 \mu \mathrm{m}$ sputtered

polysilicon film (a) before, and (b) after a ten-minute anneal at $1.5 \mathrm{~kW}$ using a $2.5 \mu \mathrm{m}, 4 \mathrm{~cm}^{2} \mathrm{FeCo}$ film.

Figure 5.4: Interferometric images of polysilicon surface (a) before, and (b) after annealing showing a change in stress-induced curvature from moderately compressive to nearly flat.....

Figure 5.5: Layout of the beam design used for structural annealing. 185

Figure 5.6: Photograph showing the appearance of the silicon substrate after the heating of a FeCo square ring.... 186

Figure 5.7: Photograph showing the inductive heating of a polysilicon beam cluster using a $2.4 \mathrm{~cm}$-wide hollow NiFe19 ring measuring $6.6 \mu \mathrm{m}$ in thickness........ 186 Figure 5.8(a): SEM image showing the initial surface morphology of the $3 \mu \mathrm{m}$ polysilicon beam pattern 188

Figure 5.8(b): SEM image taken from the center of the annealed polysilicon 
beam pattern 188

Figure 5.8(c): SEM image taken from a location mid-way between the center and edge of the annealed polysilicon beam pattern.

Figure 5.8(d): SEM image taken from the edge of the annealed polysilicon beam pattern.

Figure 5.9(a): X-Ray Diffraction output plot obtained from the as-deposited 3 $\mu \mathrm{m}$ LPCVD polysilicon which was formed at $625^{\circ} \mathrm{C}$ and patterned into cantilever beams.

Figure 5.9(b): X-Ray Diffraction output plot showing the impact of the 840$865^{\circ} \mathrm{C}$ anneal on the polysilicon grain structure.

Figure 5.10: Approximate layout utilized for the annealing of $3 \mu \mathrm{m}$ polysilicon sensing structures using a $7.1 \mu \mathrm{m}$ NiFe19 film patterned into square-shaped ring $2.4 \mathrm{~cm}$ in width with a $1.2 \mathrm{~cm}$ inner diameter.

Figure 5.11(a): SEM image showing the $3 \mu \mathrm{m}$ polysilicon surface taken from central region of the heat-treated set of inertial sensing structures

Figure 5.11(b): SEM image showing the polysilicon surface just inside of the thermal isolation trenches, at a distance of just under $4 \mathrm{~mm}$ from the center

Figure 5.11(c): SEM image showing the polyslicon film surface in the region between the thermal isolation trenches....

Figure 5.11(d): SEM image showing the polysilicon film surface in the region just outside of the thermal isolation trenches.

Figure 5.12: SEM image showing a visible gradient in the polysilicon texture over a $15 \mu \mathrm{m}$ lateral span.

Figure 5.13(a): X-Ray Diffraction output plot obtained from the as-deposited 3 $\mu \mathrm{m}$ LPCVD polysilicon, which was formed at $600^{\circ} \mathrm{C}$ and patterned to form inertial sensing structures.

Figure 5.13(b): X-Ray Diffraction output plot showing the impact of the 760$865^{\circ} \mathrm{C}$ anneal on the polysilicon grain structure.

Figure 6.1: SEM images of industrial inertial sensors illustrating the main features of (a) the Analog Devices ADXL150 50g lateral accelerometer [Samuels, 1996] and (b) the Motorola 50g z-axis accelerometer [Li, et al., 2001]... 202 Figure 6.2: Block diagram showing the primary components and transduction method of a typical capacitive linear accelerometer.

Figure 6.3: Beam-bending diagrams illustrating (a) the simple cantilever beam and (b) the double-clamped beam under a concentrated load, $F$, applied at $x=L \ldots . .204$ Figure 6.4: Schematic of a differential capacitor in which the output voltage $V_{0}$ is measured at the moving plate of the sensing structure and the input signals $+V_{R}$ and $-V_{R}$ are applied to each of the two fixed electrodes.

Figure 6.5(a): Silicon wafer (four-inch diameter, $0.25 \mathrm{~mm}$ thickness) with $1 \mu \mathrm{m}$ thermal silicon dioxide layer for minimal nitride stress and $0.25 \mu \mathrm{m}$ LPCVD silicon nitride foundation.

Figure 6.5(b): Device wafer following deposition and patterning of poly0 lower interconnect layer.

Figure 6.5(c): Device wafer following deposition and patterning of the poly0polyl dielectric.

Figure 6.5(d): Device wafer following deposition and patterning of the polyl structural layer.

Figure 6.5(e): Device wafer following deposition and patterning of the poly1metall dielectric.

Figure 6.5(f): Device wafer following deposition and patterning of the metal1 1 
upper interconnect layer.

Figure 6.5(g): Device wafer following the sacrificial etch for structural release..... 213

Figure 6.6(a): Full view of device layout on a $2 \times 2 \mathrm{~mm}$ die.

Figure 6.6(b): Right-hand side of the device showing connections to and anchoring of springs, structural electrodes and etch holes, stationary electrodes and anchors, self-test electrodes, ground plane, and upper and lower interconnects.

Figure 6.6(c): Close-up of sense electrode region showing dimensions of moving and stationary electrodes and surrounding gaps, vias, etch holes, and anti-stiction bumps.

Figure 6.7(a): Styles and sizes of folded-beam springs for each of the four sensor designs

Figure 6.7(b): Models of each of the four spring styles shown in Figure 6.6(d) using series and/or parallel combinations of single-beam springs.

Figure 6.7(c): Microsoft Excel spreadsheet showing the numerical details of each of the spring constant calculations

Figure 6.8: Fabrication process file utilized to construct the full solid model of the lateral accelerometer...

Figure 6.9(a): Solid model of the full accelerometer chip including the substrate and base dielectrics.

Figure 6.9(b): Close-up of the right-hand portion of the sensor surface showing details of the electrodes, both sensing and self-testing, as well as etch holes, a style-4 spring, and the upper interconnect layer.

Figure 6.9(c): Close-up of the right-hand portion of the sensor with the polyl, metall, and poly1-metall oxide layers removed, illustrating the poly0 pattern as well as the non-sacrificed portion of the poly0-polyl oxide layer.

Figure 6.10: Imported layout of the modified device in which the two green areas defined the portion to be utilized for solid model generation

Figure 6.11: Modified fabrication process used to produce the electrostatic model.

Figure 6.12(a): Meshed model of the capacitor banks utilized for the electrostatic model of the accelerometer.

Figure 6.12(b): Close-up of the left-hand electrode bank of the electrostatic device model illustrating details of the capacitive sub-structure and its mesh configuration

Figure 6.13(a): Voltages applied to the Center, Left, and Right conductor signals.

Figure 6.13(b-d): Definition of structural offset trajectory for the electrostatic simulation

Figure 6.14: Results of lateral accelerometer electrostatic simulation

Figure 6.15: Imported layout of structural region consisting only of the proof mass, moving electrodes, and springs.

Figure 6.16: Modified fabrication process file utilizing only the base layers, sacrificial layer, and poly1 structural layer.

Figure 6.17(a): Meshed model utilized for mechanical simulation.....

Figure 6.17(b): Close-up image of a spring region from the solid model showing the fine details of the mesh.

Figure 6.18(a): Re-attachment of the springs to the structure through linkage boundary conditions. 233

Figure 6.18(b): Surface boundary condition preventing motion of the four 
anchor patches in any direction.

Figure 6.18(c): Volumetric boundary conditions establishing two separate acceleration loads.

Figure 6.18(d,e): Acceleration loads applied separately but simultaneously to

the structure under the two Edit tabs in Figure 6.17(c).........................................2235

Figures 6.18(f-h): Definition of acceleration trajectory load MechBC1..................235

Figure 6.19: Mechanical simulation results for each of the four spring styles......... 236

Figure 6.20(a): Image of full device following patterning of the poly0-polyl

silicon dioxide layer.

Figure 6.20(b): Close-up of upper left-hand corner of device following poly0-

poly1 patterning.... 238

Figure 6.21: Image of epi-poly device layer following the polishing step and prior to patterning. 240

Figure 6.22(a): Image of sensor following patterning of the polyl device layer......241

Figure 6.22(b): Close-up image showing the lower left-hand corner of the poly1 structural layer following patterning....

Figure 6.23(a): Image showing the partial but incomplete trench refill

following deposition of the poly1-metal1 silicon dioxide layer.....

Figure 6.23(b): Close-up image of the lower left-hand corner of the device showing poly1-metall vias along the outer portion of the stationary electrodes.......244

Figure 6.24(a): SEM image of a completed, unreleased accelerometer.....

Figure 6.24(b): Close-up SEM image of the right-hand side of the device illustrating the incomplete refill of the structural trenches.

Figure B.1: Magnetic flux density plots for a RF sputtered nickel film illustrating properties both parallel and normal to the substrate at room temperature.

Figure B.2: Magnetization plots for the RF sputtered nickel film showing the effect of elevated temperature on its magnetic behavior in the direction normal to the substrate relative to the $25^{\circ} \mathrm{C}$ data.

Figure B.3: Magnetic flux density plots for an electroplated nickel film illustrating properties both parallel and normal to the substrate at room temperature.

Figure B.4: Magnetization plots for the electroplated nickel film showing the effect of elevated temperature on its magnetic behavior in the direction normal to the substrate relative to the $25^{\circ} \mathrm{C}$ data

Figure B.5: Magnetic flux density plots for a RF sputtered nickel film illustrating properties both parallel and normal to the substrate at room temperature.

Figure B.6: Magnetization plots for the in-situ magnetized RF sputtered nickel film showing the effect of elevated temperature on its magnetic behavior in the direction normal to the substrate relative to the $25^{\circ} \mathrm{C}$ data.

Figure B.7: Magnetic flux density plots for a RF sputtered cobalt film illustrating properties both parallel and normal to the substrate at room temperature.

Figure B.8: Magnetization plots for the RF sputtered cobalt film showing the effect of elevated temperature on its magnetic behavior in the direction normal to the substrate relative to the $25^{\circ} \mathrm{C}$ data

Figure B.9: Magnetic flux density plots for a RF sputtered cobalt film illustrating properties both parallel and normal to the substrate at room temperature. 
Figure B.10: Magnetization plots for the in-situ magnetized RF sputtered cobalt film showing the effect of elevated temperature on its magnetic behavior in the direction normal to the substrate relative to the $25^{\circ} \mathrm{C}$ data.

Figure B.11: Magnetic flux density plots for a $\mathrm{RF}$ sputtered $\mathrm{Ni}_{83} \mathrm{Fe}_{17}$ alloy illustrating properties both parallel and normal to the substrate at room temperature.

Figure B.12: Magnetization plots for the RF sputtered $\mathrm{Ni}_{83} \mathrm{Fe}_{17}$ alloy showing the effect of elevated temperature on its magnetic behavior in the direction normal to the substrate relative to that at $25^{\circ} \mathrm{C}$

Figure B.13: Magnetic flux density plots for a $\mathrm{RF}$ sputtered $\mathrm{Ni}_{81} \mathrm{Fe}_{19}$ alloy illustrating properties both parallel and normal to the substrate at room temperature.....

Figure B.14: Magnetization plots for the RF sputtered $\mathrm{Ni}_{81} \mathrm{Fe}_{19}$ alloy showing the effect of elevated temperature on its magnetic behavior in the direction normal to the substrate relative to that at $25^{\circ} \mathrm{C}$.

Figure B.15: Magnetic flux density plots for a $\mathrm{RF}$ sputtered $\mathrm{Ni}_{81} \mathrm{Fe}_{19}$ alloy illustrating properties both parallel and normal to the substrate at room temperature....

Figure B.16: Magnetization plots for the in-situ magnetized RF sputtered $\mathrm{Ni}_{81} \mathrm{Fe}_{19}$ alloy showing the effect of elevated temperature on its magnetic behavior in the direction normal to the substrate relative to that at $25^{\circ} \mathrm{C}$.....

Figure B.17: Magnetic flux density plots for a $\mathrm{RF}$ sputtered $\mathrm{Ni}_{45} \mathrm{Fe}_{55}$ alloy illustrating properties both parallel and normal to the substrate at room temperature.

Figure B.18: Magnetization plots for the RF sputtered $\mathrm{Ni}_{45} \mathrm{Fe}_{55}$ alloy showing the effect of elevated temperature on its magnetic behavior in the direction normal to the substrate relative to that at $25^{\circ} \mathrm{C}$....

Figure B.19: Magnetic flux density plots for a $\mathrm{RF}$ sputtered $\mathrm{Ni}_{45} \mathrm{Fe}_{55}$ alloy illustrating properties both parallel and normal to the substrate at room temperature.....

Figure B.20: Magnetization plots for the in-situ magnetized RF sputtered $\mathrm{Ni}_{45} \mathrm{Fe}_{55}$ alloy showing the effect of elevated temperature on its magnetic behavior in the direction normal to the substrate relative to that at $25^{\circ} \mathrm{C}$.

Figure B.21: Magnetization plots for the in-situ magnetized RF sputtered $\mathrm{Ni}_{45} \mathrm{Fe}_{55}$ alloy showing the effect of elevated temperature on its magnetic behavior in the direction parallel to the substrate relative to that at $25^{\circ} \mathrm{C}$

Figure B.22: Magnetic flux density plots for a RF sputtered $\mathrm{Fe}_{49} \mathrm{Co}_{50} \mathrm{~V}$ alloy illustrating properties both parallel and normal to the substrate at room Temperature.

Figure B.23: Magnetization plots for the RF sputtered $\mathrm{Fe}_{49} \mathrm{Co}_{50} \mathrm{~V}$ alloy showing the effect of elevated temperature on its magnetic behavior in the direction normal to the substrate relative to that at $25^{\circ} \mathrm{C}$.

Figure B.24: Magnetic flux density plots for a RF sputtered $\mathrm{Fe}_{49} \mathrm{Co}_{50} \mathrm{~V}$ alloy illustrating properties both parallel and normal to the substrate at room temperature.

Figure B.25: Magnetization plots for the magnetized RF sputtered $\mathrm{Fe}_{49} \mathrm{Co}_{50} \mathrm{~V}$ alloy showing the effect of elevated temperature on its magnetic behavior in the direction normal to the substrate relative to that at $25^{\circ} \mathrm{C}$. 308

Figure B.26: Magnetic flux density plots for a RF sputtered $\mathrm{Fe}_{49} \mathrm{Co}_{50} \mathrm{~V}$ alloy illustrating properties both parallel and normal to the substrate at room 
temperature

309

Figure B.27: Magnetization plots for the magnetized RF sputtered $\mathrm{Fe}_{49} \mathrm{Co}_{50} \mathrm{~V}$ alloy showing the effect of elevated temperature on its magnetic behavior in the direction normal to the substrate relative to that at $25^{\circ} \mathrm{C}$.

Figure B.28: Magnetization plots for a RF sputtered $\mathrm{Fe}_{49} \mathrm{Co}_{50} \mathrm{~V}$ alloy parallel and normal to the substrate.

Figure B.29: Magnetic flux density plots for a RF sputtered $\mathrm{Co}_{60}\left(\mathrm{Ni}_{81} \mathrm{Fe}_{19}\right)_{40}$ alloy illustrating properties both parallel and normal to the substrate at room temperature.

Figure B.30: Magnetization plots for the magnetized RF sputtered

$\mathrm{Co}_{60}\left(\mathrm{Ni}_{81} \mathrm{Fe}_{19}\right)_{40}$ alloy showing the effect of elevated temperature on its magnetic behavior in the direction normal to the substrate relative to that at $25^{\circ} \mathrm{C}$

Figure B.31: Magnetic flux density plots for a RF sputtered $\left(\mathrm{Ni}_{81} \mathrm{Fe}_{19}\right)_{70} \mathrm{Co}_{30}$ alloy illustrating properties both parallel and normal to the substrate at room temperature.

Figure B.32: Magnetization plots for the magnetized RF sputtered

$\left(\mathrm{Ni}_{81} \mathrm{Fe}_{19}\right)_{70} \mathrm{Co}_{30}$ alloy showing the effect of elevated temperature on its magnetic behavior in the direction normal to the substrate relative to that at $25^{\circ} \mathrm{C}$

Figure B.33: Magnetic flux density plots for a RF sputtered $\left(\mathrm{Fe}_{49} \mathrm{Co}_{50} \mathrm{~V}\right)_{70} \mathrm{Ni}_{30}$ alloy illustrating properties both parallel and normal to the substrate at room temperature.

Figure B.34: Magnetization plots for the magnetized RF sputtered $\left(\mathrm{Fe}_{49} \mathrm{Co}_{50} \mathrm{~V}\right)_{70} \mathrm{Ni}_{30}$ alloy showing the effect of elevated temperature on its magnetic behavior in the direction normal to the substrate relative to that at $25^{\circ} \mathrm{C}$ 


\section{List of Tables}

Table 1.1: Summary of Integrated CMOS-MEMS Technologies.............................16

Table 2.1: Inductive Heating Properties of Selected Materials................................44

Table 3.1: Element Types Utilized in the ANSYS Example Model........................52

Table 3.2: Variation of Frequency Values in the ANSYS Model............................68

Table 3.3: Theoretical and Simulated Frequency Trends for the ANSYS

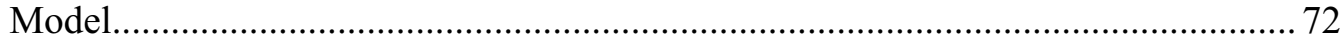

Table 3.4: Variation of Coil Current Values in the ANSYS Model........................ 73

Table 3.5: Theoretical and Simulated Coil Current Trends for the ANSYS

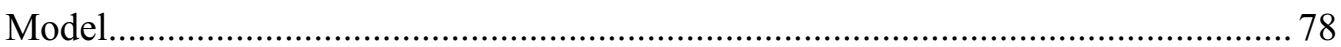

Table 3.6: Variation of Magnetic Permeability Values in the ANSYS Model......... 78

Table 3.7: Theoretical and Simulated Permeability Trends for the ANSYS

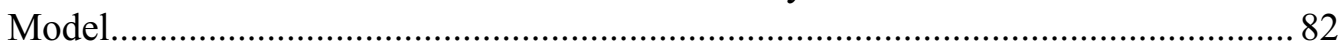

Table 3.8: Variation of Ambient Temperature Values in the ANSYS Model.......... 83

Table 3.9: Variation of Billet Dimensions in the ANSYS Model............................85

Table 3.10: Theoretical and Simulated Dimensional Trends for the ANSYS

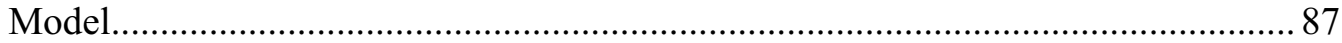

Table 3.11: Element Types Utilized in the Thin Film Model................................. 91

Table 3.12: Theoretical and Simulated Electromagnetic Quantities.........................98

Table 4.1: X-Ray Diffraction Data and Calculations for the Beam Anchors........... 126

Table 4.2: LSU/CAMD Nickel Electroplating Recipe...........................................143

Table 4.3: Summary of Ferromagnetic Film Properties..........................................146

Table 4.4: Electromagnetic Properties for Focus Materials....................................... 153

Table 4.5: Temperatures Induced in Initial FeCo Films........................................ 156

Table 4.6: Temperatures Induced in Inductively Heated Square "Rings"................ 164

Table 4.7: Density and Specific Heat for Selected Materials.................................. 172

Table 4.8: Trendline Slopes for 2cm Circular Film Data........................................172

Table 5.1: Stress Calculations for LPCVD Polysilicon Films................................. 181

Table 5.2: Stress Calculations for Sputtered Polysilicon Films................................ 184

Table 5.3: X-Ray Diffraction Data and Calculations for Polysilicon Beams........... 191

Table 5.4: X-Ray Diffraction Data and Calculations for Polysilicon Inertial

Sensing Structures. 


\section{Chapter 1: Localized Annealing in Microsystem Fabrication}

Technologies for localized heating have been applied to microsystem fabrication with moderate success, but limitations often exist with respect to flexibility in design and process, maximum temperature, and degree of localization. This chapter introduces the motivation for the process development presented in this document. Although numerous applications exist, the investigation is concentrated foremost on the annealing of polysilicon micromechanical structures for the purpose of monolithic integration with electronic circuitry, an industrially relevant problem that requires very high temperatures yet considerable localization and flexibility in order to minimize the impact to device development. A summary is first given of the typical challenges associated with integration in Section 1.1, followed by an analysis of several leading integrated or CMOS-compatible technologies from both industrial and academic facilities in Section 1.2. Section 1.3 presents alternate methods for localized heating and other types of selective processes for comparison with that to be presented in this dissertation, specifically with respect to the primary application. Finally, some additional applications that could benefit from these types of technologies are briefly discussed in Section 1.4 as opportunities for future research.

\section{1: Overview of CMOS-MEMS Integration}

As semiconductor devices continue to advance in capacity and complexity, one of the key objectives that researchers pursue is miniaturization. This includes not only enhancing the capability to produce smaller features but also developing means by which dissimilar components can be combined onto a single chip with minimal unused space. Design tradeoffs, contaminant materials, and conflicts in thermal budget can all introduce 
difficulty to the latter, and as a result the cost of integration often outweighs the spatial benefit. In the field of microelectromechanical systems (MEMS), monolithic fabrication is one of the most prevalent challenges in the development of active devices. Films deposited for surface micromachined structures typically require a high temperature deposition or a post-deposition anneal in order to relieve intrinsic stress and therefore prevent strain-induced curvature upon release. Conversely, electronic circuitry such as that used in complementary metal-oxide semiconductor (CMOS) or bipolar CMOS (BiCMOS) technologies is often incompatible with elevated temperatures. One of the most preferred microstructural materials is silicon, which poses no contamination risk and also has highly favorable and well-characterized mechanical and electrical properties. Of particular favor for industrial MEMS is fine-grained polycrystalline silicon, or polysilicon; smaller, more numerous grains (e.g., thickness/size 20 ) enhance isotropicity in mechanical and electrical properties [Srikar, et al., 2002; Painter, et al., 2003] and furthermore help to facilitate the manufacture of small-featured devices [Kahn, et al., 1996] with repeatable properties [Guckel, et al., 1988]. Material properties are highly dependent upon fabrication conditions, but polysilicon typically has a Young's modulus in the vicinity of 160GPa [Senturia, 2001 (pp. 193-6); Bustillo, et al., 1998], and resonant structures can be produced with measured quality factors exceeding 100,000 [Bustillo, et al., 1998]. Obtaining the desired morphology with low stress, preferably tensile in order to prevent structural buckling, requires deposition or annealing around or above $1000^{\circ} \mathrm{C}$ [French, et al., 1996; Guckel, et al., 1988]. Once fabricated, however, standard circuitry is typically assigned an upper limit of around $450^{\circ} \mathrm{C}$, above which aluminum- or copperbased interconnect layers begin to deteriorate in quality, exhibiting increasing resistance 
often accompanied by diffusion into the surrounding dielectrics [Sedky, et al., 2001; Franke, et al., 2003]. Furthermore, temperatures above those used in the formation of transistor junctions can result in further dopant diffusion, thus altering concentration profiles. As a result, the CMOS integration problem presents a formidable challenge that is important to resolve, particularly in industrial applications where efficient use of silicon area can yield a significant cost savings.

Integrated MEMS technologies are divided into three classifications based on the order in which components are fabricated: post-CMOS, MEMS-first, and interleaved. Post-CMOS processing has obvious thermal consequences as all high temperature MEMS procedures are performed after the temperature-sensitive electronics have been produced. Nevertheless, it is the preferred option of many device engineers for the simple reason that the more complex CMOS fabrication can be done at a dedicated foundry and passivated to protect the circuitry from relatively unclean MEMS processing. The opposite is a MEMS-first method, which solves the thermal issue of the former but has drawbacks related to contamination as the substrate is subject to additional handling and exposure to MEMS materials prior to CMOS processing. Measures taken to protect the circuitry may result in mechanical design limitations. Interleaved technologies attempt to reconcile some of the advantages and disadvantages of post-CMOS and MEMS-first processes through the selective fabrication of CMOS and MEMS devices and materials in an alternating fashion. This commonly entails performing initial dopant diffusions in the CMOS region, depositing structural layers in the MEMS region, completing the CMOS interconnects, and finally releasing the MEMS structures [Lewis, et al., 2003; Nunan, et al., 2000]. The potential exists to reduce processing steps by performing certain 
procedures simultaneously such as dopant activation and mechanical annealing. These types of technologies are often highly specific to a particular device and process, rendering design modification for subsequent generations less straightforward. As with MEMS-first, contamination concerns remain prevalent. Integrated micromechanical devices have been successfully mass-produced through technologies of all three types. Examples of each are provided with more detail in the next section.

\section{2: Integrated MEMS Technologies}

In the absence of a localized annealing method, CMOS-first technologies require all micromechanical fabrication procedures to be performed at relatively low temperatures or for very brief time durations. One solution to this problem is the use of bulk micromachined structures. Figure 1.1 shows a sample structure fabricated via Cornell University's initial Single Crystal Reactive Etching and Metallization (SCREAM) process. SCREAM technologies are based on structural formation using deep reactive ion etching (DRIE) followed by structural release through isotropic chemical etching. A silicon dioxide layer deposited by thermal oxidation and/or plasma-enhanced chemical vapor deposition (PECVD) protects the trench sidewalls from further etching during release. Thermal oxidation, if used, also helps to improve the sidewall quality. The authentic SCREAM technology requires masking steps both to define trench locations as well as to provide for electrode patterning [Zhang, et al., 1991]. A later version, called SCREAM-I, eliminates the latter by performing release etching prior to electrode deposition and allowing some metal to accumulate on the floor of the now-opened trenches [Shaw, et al., 1993]. This version has a trade-off, however, in that it increases the parasitic capacitance from device to substrate since the metallic surface can store 
more surface charge. Substantial modifications were made after the process was licensed for commercial application by EG\&G Sensors of Singapore (later acquired by Temic Automotive). The most significant drawback to the process, difficulty in isolating structures from the remainder of the substrate, is overcome through the use of silicon dioxide "buss-bars" in which additional trenches are formed prior to the structure near desired anchoring regions, undercut below and on three sides to eliminate contact to the bulk in all directions except that which connect to the structure, thermally oxidized, and refilled via low pressure chemical vapor deposition (LPCVD) [Sridhar, et al., 1998]. This modification doubles the complexity of the process but renders it much more practical for device fabrication. Etch rates and timings remain extremely critical, however, and design flexibility is somewhat limited. Another commercialized process utilizing the substrate as a structural material is the Dissolved Wafer Process (DWP) developed at the University of Michigan. Licensed by spin-off company Integrated Sensing Systems (ISSYS), the DWP involves the electrostatic bonding of silicon and glass (silicon dioxide) wafers for device encapsulation and the removal of the excess silicon. Several variations of the process have been published, including one in which the wafer is thinned to the desired device thickness through chemical-mechanical polishing (CMP), for example $120 \mu \mathrm{m}$ [Chae, et al., 2002], and the original in which a deep boron diffusion prior to bonding defines the shape of the structure and serves as an etch stop region in ethylene diamine pyrocatechol (EDP) solution [Gianchandani, et al., 1992; Chavan, et al., 2000]. Anodic bonding is commonly done at temperatures in the range of $300^{\circ} \mathrm{C}$ to $500^{\circ} \mathrm{C}$ with an applied voltage in the range of $800 \mathrm{~V}$ to $1000 \mathrm{~V}$ and therefore can be a CMOS-compatible procedure with respect to temperature [Gianchandani, et al., 1992; Juan, et al., 1996]. The 


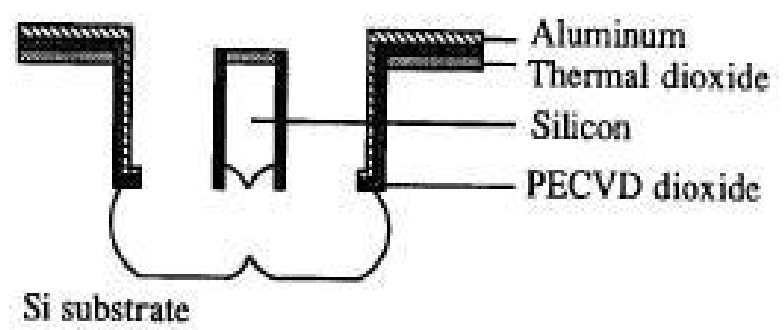

Figure 1.1: Illustrative structure describing the initial version of Cornell University's SCREAM Process [Zhang, et al., 1991 ${ }^{1}$ ].

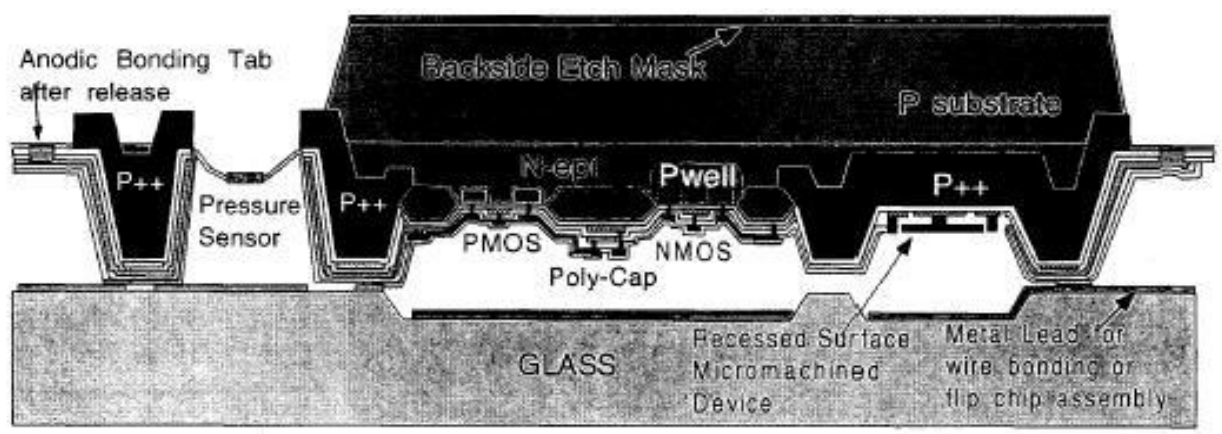

Figure 1.2: Cross-section of integrated pressure sensor fabricated from the University of Michigan Dissolved Wafer Process [Chavan, et al., 2000²].

boron diffusion step, however, utilizes temperatures in the vicinity of $1200^{\circ} \mathrm{C}$ and therefore technologies of the second type are classified as interleaved. Bonding pads and interconnects can be formed on either wafer, and metal islands are often used to provide for inter-wafer signal transport. The image in Figure 1.2 shows a cross-section of an integrated pressure sensor fabricated via an interleaved DWP. A heavily doped etch stop region protects the $2 \mathrm{P} / 2 \mathrm{M}$ BiCMOS region during device release [Chavan, et al., 2000]. Overall, the different DWP varieties offer a wide range of design and fabrication capabilities extending from the very simple to the fairly complex, and the thickness of the sensing structures is of virtually no limit. Viewpoints differ on the most desirable form of silicon for micromachining purposes. Single-crystalline silicon requires no heat treatment for reducing intrinsic stress and also exhibits higher electrical conductivity than

\footnotetext{
1 C1991 IEEE

2 C2000 IEEE
} 
polysilicon due to the absence of grain boundary scattering, but it lacks the mechanical isotropicity of fine-grained polysilicon. The impact of crystalline anisotropy on microstructural properties places boundaries on design capabilities and performance, particularly when applied to more complex devices such as gyroscopes or multi-axis sensors. For this reason, the vast majority of industrial inertial sensors are fabricated using polysilicon structures.

For CMOS-first surface micromachined devices, alternative structural materials to polysilicon remain under investigation. Considerable effort has been devoted to the study of polycrystalline germanium and/or silicon-germanium alloys due to the lower melting points and overall reduced thermal requirements of germanium with respect to silicon. Researchers at the University of California at Berkeley have demonstrated CMOScompatible processing of structures made from both n-type poly-germanium and p-type poly- $\mathrm{Si}_{0.35} \mathrm{Ge}_{0.65}$ with $\mathrm{SiO}_{2}$ and poly-Ge sacrificial layers, respectively [Franke, et al., 2003]. Ge and SiGe alloys have been reported to exhibit somewhat degraded mechanical properties with respect to pure silicon such as lower quality factor (Franke achieved maximum values of 30,000 for poly-Ge and 15,000 for poly-SiGe as compared to 80,000 or higher for poly-Si) and Young's Modulus (132 for poly-Ge as compared to 173 for poly-Si). Nevertheless, this is largely compensated for by excellent material and thermal compatibility $\left(937^{\circ} \mathrm{C}\right.$ melting point for $\mathrm{Ge}$ as compared to $1415^{\circ} \mathrm{C}$ for $\left.\mathrm{Si}\right)$ as well as opportunities for self-alignment due to the inability of germanium to nucleate on silicon dioxide [Li, et al., 1999]. Other research endeavors have focused on electrodeposited metallic structures fabricated through techniques such as sputter deposition or LIGA (LIthographie or lithography, Galvanoformung meaning electrodeposition, and 
Abformung meaning molding). A well-known sputter-based technology is that utilized by Texas Instruments (TI) to fabricate their Digital Micromirror Device (DMD), an integrated projection display [Van Kessel, et al., 1998]. This two-metal (2M) CMOS-first process consists of four aluminum layers, one electrical and three structural, with deepUV hardened photoresist used to form the air gaps necessary for motion. Each mirror measures $16 \mu \mathrm{m}$ square, and arrays ranging in size from $800 \times 600$ to $1280 \times 1024$ are manufactured. Largely developed in Germany, LIGA processing involves the formation of micromolds using thick photoresist or polyimide followed by an electroplating procedure, as shown in Figure 1.3. High aspect ratio metallic structures have been fabricated for purposes ranging from inertial sensors and microturbines to reusable forms for use in injection molding [Bacher, et al., 1995]. In the United States, significant research in metallic microstructures has primarily been concentrated at the University of Wisconsin at Madison, where their High Aspect Ratio Microsystems Technology (HARMST) process includes refinements such as high-energy photon bombardment for thicker photoresist structures and planarization to compensate for unequal electrodeposition rates in regions of different sizes [Guckel, 1998]. Electrodeposited metallic structures are advantageous for use in high aspect ratio devices in that they form rapidly at CMOS-compatible temperatures with relatively low stress. Plastic deformation is of concern for metallic structures, however, and devices are subject to fatigue over cyclic loading. A third class of integratable microstructural materials has been pioneered at Carnegie Mellon University in which structures are comprised by composites of silicon dioxide and one or more metallic layers. CMOS devices are fabricated in a foundry, typically using an Agilent three-metal process through the MOSIS service, with the 


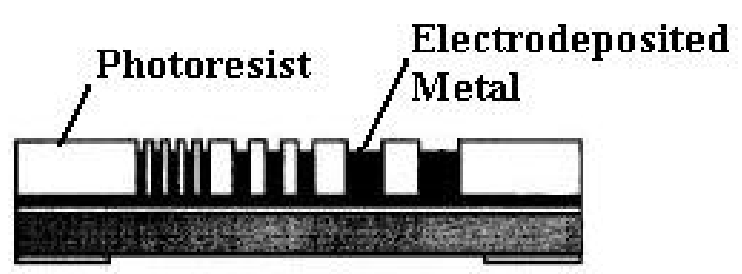

Figure 1.3: Illustration of LIGA processing showing the use of a thick photoresist (or other material) mold to define the shape of an electrodeposited metallic structure [Ueno, et al., $\left.1997^{1}\right]$.

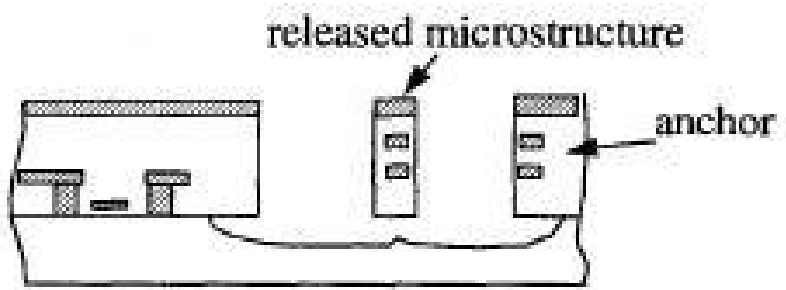

Figure 1.4: Illustration of the Carnegie Mellon CMOS-MEMS three-metal aluminum process demonstrating the use of the third metal layer to mask the shape of the microstructure [Zhang, et al., 1999 ${ }^{2}$ ].

silicon dioxide insulating layers filling the structural region and the metal mask set designed such that the interconnects lie within the structure as desired and the top metal layer provides a hard mask to define the shape of the device as shown in Figure 1.4. The structure is then released using an anisotropic trenching etch through the oxide followed by an isotropic release etch in the substrate. This process has the advantage of simplicity but the disadvantages of a lower Young's modulus than either polysilicon or polygermanium [Luo, et al., 2002; Franke, et al., 2003] and, more importantly, significant out-of-plane curling that varies with temperature due to non-uniformity in structural composition. The researchers compensate for this problem by attaching sensing structures to a rigid frame such that the curl of moving electrodes is parallel to the curl of thicker structures of lower stress, eliminating the need for an outer frame but introducing the trade-offs of increased moisture sensitivity and stiction susceptibility [Luo, et al., 2002].

\footnotetext{
1 (C)1997 IEEE

2 C1999 IEEE
} 
In summary, existing post-CMOS integration technologies tend to focus on the use of alternate materials to polysilicon. The range of trade-offs is vast, but in each case the process is specific to a particular material or class of materials and therefore flexibility in processing and, more particularly, technological upgrades, is restricted. The inductive annealing process introduced in Chapter 2 is applicable to nearly any type of device whether made of polysilicon, germanium, or otherwise, and can be applied in a wide range of configurations to suit different purposes, as will be shown.

Perhaps the most highly recognized MEMS-first technology is the Sandia Modular, Monolithic MEMS ( $\left.{ }^{3} E M S\right)$ process. Sandia approaches the aforementioned difficulties in MEMS-first CMOS fabrication by burying micromechanical structures in a depression as shown in Figure 1.5(a). This trench is formed using an anisotropic etchant to render a shape similar to that shown, with a depth slightly greater than the height of the structure. Beginning with a silicon nitride foundation, one or more layers of polysilicon are deposited with sacrificial silicon dioxide layers providing support and anchoring

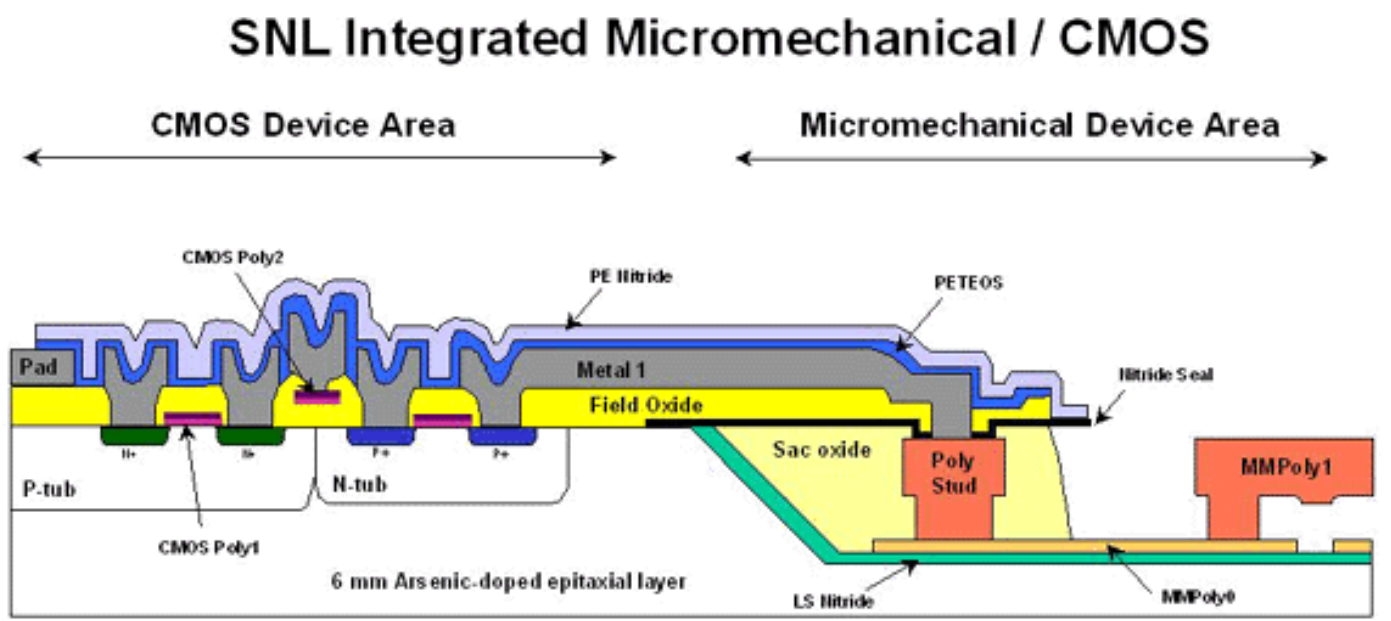

Figure 1.5(a): Illustration of Sandia's $M^{3} E M S$ process showing mechanical polysilicon structure formed within wafer recess with polysilicon stud(s) transporting signals to electronics at surface [Allen, et al., 1998 ${ }^{1}$.

\footnotetext{
1 C 1998 IEEE
} 


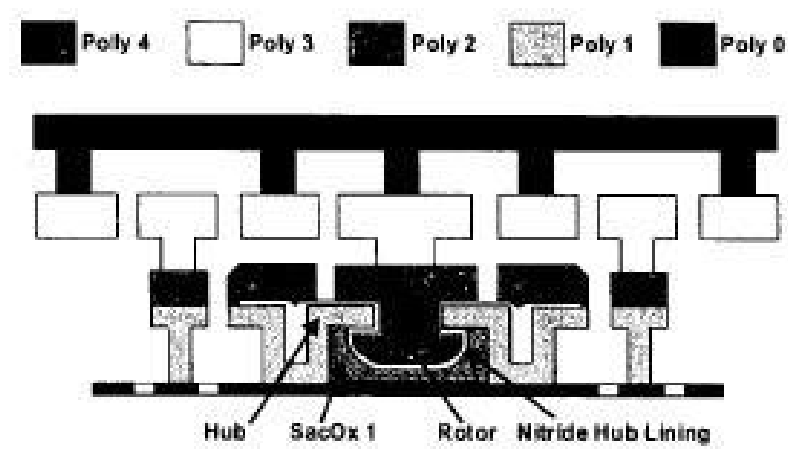

(b)

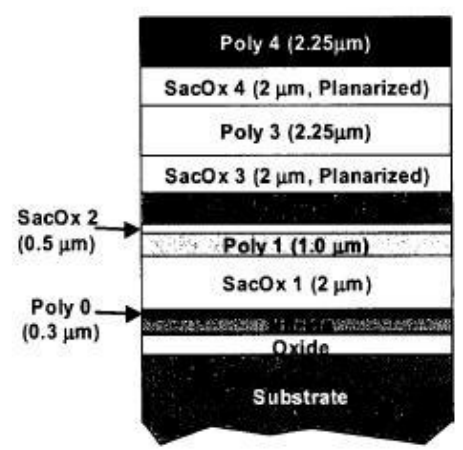

(c)

Figure 1.5(b,c): Cross-section of Sandia rotary actuator (b) fabricated using SUMMIT-V process (c) consisting of five polysilicon layers with intermediate sacrificial oxides [Krygowski, et al., $1999^{1}$ ].

locations. With a high degree of flexibility, $\mathrm{M}^{3} \mathrm{EMS}$ structures can vary from a singlelevel $6 \mu \mathrm{m}$ accelerometer as presented in Allen, et al., 1998, to a five-level rotary actuator like that of Figure 1.5(b) fabricated using the five-level Sandia Ultra-planar Multi-level MEMS Technology (SUMMiT-V) illustrated in Figure 1.5(c) [Krygowski, et al., 1999]. As the structures are formed, polysilicon studs are simultaneously built for the purpose of signal transport between mechanical and electrical devices. Just prior to sealing off the depression with a nitride layer, thermal annealing is done in order to ensure stability of the region during any high-temperature CMOS processing steps. Surface quality is of particular importance in order to keep CMOS fabrication unaffected, and CMP steps are used following certain oxide depositions, particularly those near the surface, so that the planarized wafers can be sent to a dedicated foundry. The micromechanical devices remain supported until after CMOS completion, at which point a masking step removes the nitride layer over the structure and a sacrificial etch releases it for operation. The $\mathrm{M}^{3} \mathrm{EMS}$ and SUMMIT technologies have a key advantage in that a wide range of polysilicon devices can be fabricated such as sensors, actuators, and gear trains, and as a

\footnotetext{
1 C)1999 IEEE
} 
result licensing agreements have been reached with various manufacturers, including Fairchild Semiconductor [Sandia, 2002], microsystem investment firm Ardesta [Ardesta, 2001], software developer Microcosm Technologies [Coventor, 2001], and Sandia's own spin-off company MEMX [MEMX, 2003]. A tedious approach to high aspect ratio processing, SUMMIT requires numerous, lengthy CMP procedures that are undesirable for industrial applications due to the added cost and reduced wafer throughput. This is exacerbated with increasing device thickness as additional metal layers are added. Furthermore, caution must be exercised in order to avoid contamination of the wafer surface, which may exclude certain materials from being used in MEMS devices and/or fabrication procedures. Despite these potential drawbacks, for the production of both simple and complex polysilicon structures the Sandia process is a respected technology.

As was mentioned in Section 1.1, interleaved technologies often exhibit a fabrication sequence in which transistor junctions are completed initially, followed by deposition and optional annealing of the structural film(s), circuitry metallization and passivation, and finally structural definition and release. The Analog Devices (ADI) Integrated MEMS (iMEMS) process utilizes exactly this format [Lewis, et al., 2003]. Producing perhaps the largest volume of inertial sensors for both automotive and consumer markets, ADI produces relatively thin $(2-4 \mu \mathrm{m})$ LPCVD polysilicon structures and maintains transistor junction areas sufficiently large so as not to be significantly impacted by structural deposition procedures. As with most interleaved technologies, the specific details used in the fabrication of each individual device are highly customized and therefore enhancements remain difficult to realize. Furthermore, the structural thickness limitation remains strict due to increasing intrinsic stress, and though ADI has 
directed considerable effort into optimizing the polysilicon deposition process [Nunan, et al., 2000], it has become apparent that iMEMS is being phased out. As device complexity continues to increase, ADI has begun presenting devices fabricated through various experimental technologies, most notably the Modular-MEMS process, illustrated in Figure 1.6. Shown in several different interleaved variations [Yasaitis, et al., 2003; Bhave, et al., 2003] as well as in a MEMS-first format [Palaniapan, et al., 2003; Kung, 1996], structural layers are deposited through one or more LPCVD polysilicon steps and removed from the substrate except for in the structural region. Device layers and the sacrificial layer at the surface are optionally planarized via CMP. The remainder of the wafer is covered with an epitaxial silicon layer, deposited such that the nucleation rate in the non-MEMS region is considerably higher, with a thickness at or slightly above the height of the structural module, and CMP planarization ensures a smooth, level surface. Circuitry is then fabricated in the high-quality non-structural regions, passivated, and finally the structure is defined if not so already and released. This new process ensures a clean surface for BiCMOS processing while simultaneously providing for its modularity, thus substantially increasing design flexibility. While structural thicknesses published have only reached $6 \mu \mathrm{m}$ thus far [Yasaitis, et al., 2003], removing the polysilicon from the remainder of the wafer helps to reduce the impact of film stress to the wafer. A second, also highly successful, genre of interleaved technologies is made up of the "epipoly" processes. Initially introduced for inertial sensor fabrication by the Robert Bosch Corporation [Offenberg, et al., 1995] and later developed in a similar form by ST Microelectronics [Galayko, et al., 2002], epipoly technologies make use of silicon epitaxy above a patterned silicon dioxide layer and polysilicon seed layer in order to 


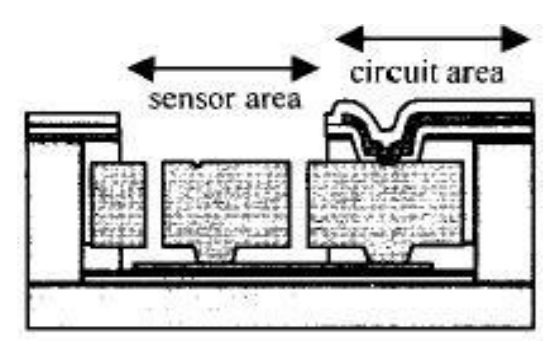

Figure 1.6: Illustration of Analog Devices Modular-MEMS process showing circuit area fabricated at surface of epitaxial MEMS encapsulation layer [Palaniapan, et al., $2003^{1}$ ].

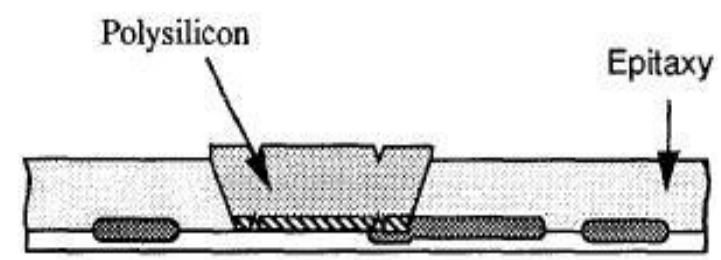

Figure 1.7: Illustration of Bosch epi-poly process demonstrating simultaneous, selective deposition of monocrystalline and polycrystalline silicon for use in transistor and transducer regions, respectively [Offenberg, et al., 1995²].

simultaneously form both monocrystalline and polycrystalline regions. As shown in Figure 1.7, the transducer polysilicon extends upward and outward from the seeded region. A buried diffusion layer provides for electrical connectivity to the structure as well as electrostatic biasing of other regions. Once the epitaxial deposition is complete, fabrication of the active devices takes place at the planarized surface of the singlecrystalline region. Following CMOS passivation, the polycrystalline region is trenched to define the shape of the structure, and finally the silicon dioxide layer is dissolved to release the device. Bosch utilizes its epipoly process to produce its entire line of inertial sensors with recent publications describing both linear [Reichenbach, et al., 2003] and rotational [Funk, et al., 1999] devices with published thicknesses reaching $11 \mu \mathrm{m}$. Structures are nearly free of intrinsic stress due to the high deposition temperatures, for example $1180^{\circ} \mathrm{C}$ was used to form the tri-axial linear accelerometer described in

1 (C)2003 IEEE

2 C1995 IEEE 
Reichenbach, et al., 2003, and deposition rates are on the order of $1.5 \mu \mathrm{m} /$ minute [Partridge, et al., 2001]. The resulting grain structure is extremely coarse, however, resulting in high surface roughness and low electrical conductivity despite in-situ doping, and as a result both CMP and doping procedures are required. Achieving uniform ex-situ doping of thick structures is difficult, however, and non-uniformity in the final dopant concentration has been reported to generate some compressive curvature, particularly for thicker structures. Tethers are designed such that compressive stress is converted to tensile in order to prevent buckling during device operation, but nevertheless a limit is placed on proof mass dimensionality [Offenberg, et al., 1995]. Bosch has worked closely with researchers at the Fraunhofer Institute and the University of Freiberg to characterize

and further refine the polysilicon quality [Lange, et al., 1996; Wagner, et al., 2003] and today has what is arguably the best technology in the industrial market.

The technologies evaluated in this section each exhibited unique strengths and weaknesses, summarized in Table 1.1, and it is likely that there exists no perfect integrated fabrication process. While university-based processes utilize a wide range of structural materials, the top-ranking manufacturers remain dedicated to polysilicon despite its thermal drawbacks. It is clear that a low-impact localized annealing method could be of considerable benefit to the microsystems industry with the capability to provide for modularity with optimum design flexibility.

\section{3: Selective Modification of Microsystem Properties}

Realization of the opportunities afforded by localized heating is not a unique conception. In recent years, several different methods to selectively manipulate the properties of microsystem devices and materials have been under investigation. The 


\begin{tabular}{|c|c|c|c|c|}
\hline \multicolumn{5}{|c|}{$\begin{array}{c}\text { TABLE } \mathbf{1 . 1} \\
\text { SUMMARY OF INTEGRATED CMOS-MEMS TECHNOLOGIES }\end{array}$} \\
\hline Technology & $\begin{array}{l}\text { Company/ } \\
\text { University }\end{array}$ & Type & Advantages & Disadvantages \\
\hline SCREAM & Cornell & Post-CMOS & $\begin{array}{l}\text { Simple, few masking } \\
\text { steps }\end{array}$ & $\begin{array}{l}\text { Bulk micromachined, } \\
\text { limited flexibility, } \\
\text { substrate isolation difficult }\end{array}$ \\
\hline DWP & U-Michigan & $\begin{array}{l}\text { Post-CMOS or } \\
\text { Interleaved }\end{array}$ & $\begin{array}{l}\text { Highest available } \\
\text { thicknesses }\end{array}$ & Bulk micromachined \\
\hline $\begin{array}{l}\text { Poly-Ge or } \\
\text { Poly-SiGe }\end{array}$ & UC-Berkeley & various & $\begin{array}{l}\text { Self-alignment, } \\
\text { lower temperatures } \\
\text { than Poly-Si }\end{array}$ & $\begin{array}{l}\text { Lower Young's Modulus } \\
\text { than Poly-Si, still requires } \\
\text { elevated temperatures }\end{array}$ \\
\hline DMD & $\begin{array}{l}\text { Texas } \\
\text { Instruments }\end{array}$ & Post-CMOS & $\begin{array}{l}\text { Low-stress } \\
\text { structures }\end{array}$ & $\begin{array}{l}\text { Plastic deformation and } \\
\text { fatigue concerns, aspect } \\
\text { ratio limitations }\end{array}$ \\
\hline HARMST & UW-Madison & Post-CMOS & $\begin{array}{l}\text { Low-stress } \\
\text { structures, high } \\
\text { aspect ratios }\end{array}$ & $\begin{array}{l}\text { Plastic deformation and } \\
\text { fatigue concerns }\end{array}$ \\
\hline $\begin{array}{l}\text { Composite } \\
\text { Laminates }\end{array}$ & Cornell & Post-CMOS & $\begin{array}{l}\text { Simple, uses CMOS } \\
\text { processing for film } \\
\text { depositoin }\end{array}$ & $\begin{array}{l}\text { Lowest Young's Modulus, } \\
\text { curvature problems severe } \\
\text { and difficult to correct }\end{array}$ \\
\hline $\begin{array}{l}\mathrm{M}^{3} \mathrm{EMS}+ \\
\text { SUMMIT }\end{array}$ & Sandia & MEMS-First & Flexible & $\begin{array}{l}\text { Numerous CMP steps, } \\
\text { aspect ratio limitations, } \\
\text { contamination concerns }\end{array}$ \\
\hline iMEMS & $\begin{array}{l}\text { Analog } \\
\text { Devices }\end{array}$ & Interleaved & $\begin{array}{l}\text { Highly optimized } \\
\text { polysilicon } \\
\text { processing }\end{array}$ & $\begin{array}{l}\text { Customized processes } \\
\text { difficult to upgrade, aspect } \\
\text { ratio limitations }\end{array}$ \\
\hline $\begin{array}{l}\text { Modular } \\
\text { MEMS }\end{array}$ & $\begin{array}{l}\text { Analog } \\
\text { Devices }\end{array}$ & $\begin{array}{l}\text { Interleaved or } \\
\text { MEMS-First }\end{array}$ & $\begin{array}{l}\text { Flexible, capable of } \\
\text { modularity }\end{array}$ & Aspect ratio limitations \\
\hline Epi-Poly & Bosch & Interleaved & $\begin{array}{l}\text { Low-stress HAR } \\
\text { structures, above- } \\
\text { average flexibility }\end{array}$ & $\begin{array}{l}\text { Coarse grains require extra } \\
\text { doping, causes issues with } \\
\text { uniformity and curvature }\end{array}$ \\
\hline
\end{tabular}

focused absorption or dissipation of energy has been reported for applications such as wafer bonding and polysilicon annealing. Like the technology presented in this document, some are based upon resistive heating with currents obtained either through direct contact or electromagnetic coupling to specific regions or materials. Others make use of high-energy laser beams, either in continuous or pulsed modes to single or multiple targets. A third class attempts to modify material properties through light alloying with specific materials in order to obtain the desired results with reduced thermal requirements. Research in these areas has primarily been done at academic facilities, and at the present time all methods either have limiting disadvantages or require further 
refinements that have kept them from being adopted by industry. Nevertheless, the continually increasing interest in resolving the CMOS-MEMS integration challenge reflects its industrial relevance.

Wafer-scale resistive heating methods have been successfully demonstrated with currents generated in either metals or semiconductors. The majority of published techniques of this type have been concentrated on wafer bonding applications. In 1999, researchers at the California Institute of Technology published a method by which silicon dies were bonded together through the microwave heating of thin gold films [Budraa, et al., 1999]. Test samples $5 \mathrm{~mm}$ in width were each prepared, prior to dicing, with $150 \AA$ thick chromium and $1200 \AA$ Á-thick gold films forming a $2 \mathrm{~mm}$-wide square ring above a $3 \mathrm{~mm}$-wide, $100 \mu \mathrm{m}$-deep recess. Two dies were stacked such that the gold regions were in contact, and a $2.45 \mathrm{GHz}$ electromagnetic field was applied at power levels of 100-300W

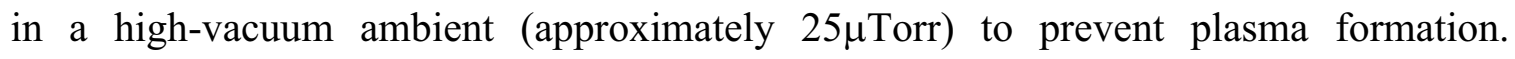
Temperature measurements were not provided, but leak tests on the results verified bond hermeticity. This research led to the founding of Altadena-based (California) Microwave Bonding Instruments (MBI) which continues to publish experimental and simulation results pertaining to the bonding of various types of substrates using microwave heating methods [Clendenin, et al., 2003; Budraa, et al., 2004]. A year after this study was published, researchers at the University of Michigan reported a fusion wafer bonding technique in which currents were applied through direct contact to line-shaped "microheaters" which also provided the bonding region on the device wafer [Cheng, et al., 2000]. Silicon-Pyrex fusion was achieved through the heating of $5 \mu \mathrm{m}$-wide, $1.1 \mu \mathrm{m}-$ thick polysilicon heaters fabricated on the silicon wafer to approximately $1300^{\circ} \mathrm{C}$ for five 
minutes with an applied pressure of $1 \mathrm{MPa}$. The quality of the resulting bond was further enhanced by the local softening of the glass, and SEM imaging verified that the two materials were completely merged. In the same publication, the technique was applied to silicon-gold eutectic bonding in which $5 \mu \mathrm{m}$-wide, $0.5 \mu \mathrm{m}$-thick gold resistors were heated to approximately $800^{\circ} \mathrm{C}$, again for five minutes and at $1 \mathrm{MPa}$, and once more the bond was shown to be thorough. This work was subsequently expanded upon at the University of California at Berkeley, where Pyrex-Pyrex and Pyrex-polycarbonate wafer bonding was performed with currents inductively applied to gold "bonding rings" [Cao, et al., 2002]. The rings varied in size from $1-1.2 \mathrm{~mm}$ in diameter, $6 \mu \mathrm{m}$ thick, and $100-200 \mu \mathrm{m}$ wide. Using a $10-15 \mathrm{MHz}$ power supply, temperatures in excess of $1000^{\circ} \mathrm{C}$ were induced with as little as $500 \mathrm{~W}$. Bonding was achieved is one minute or less, and again hermeticity was verified. The use of insulating wafers simplified the process requirements as the electrical resistivities of insulators prevent efficient inductive heating, and it is certain that the maintenance of localization would be much more difficult with semiconducting wafers as will be discussed further in the next chapter. At nearly the same time, researchers at the University of Wisconsin also began to publish results on wafer bonding [Thompson, et al., 2002] as well as dopant activation [Thompson, et al., 2001; Thompson, et al., 2003] using their electromagnetic induction heating (EMIH) technology. This research focused on electromagnetic coupling directly to silicon wafers, making use of the larger volume of the substrate to overcome the lower conductivity with respect to metals. Magnetic fields with frequencies of $13.56 \mathrm{MHz}, 2.45 \mathrm{GHz}$, and $30.5 \mathrm{GHz}$ were applied using a spiral coil for the former and resonant cavities for the latter two, and temperatures were measured with optical pyrometry. Direct silicon-silicon bonding 
without the use of an intermediate layer required a temperature of $1000^{\circ} \mathrm{C}$, and this was achieved at both $13.56 \mathrm{MHz}$ and $2.45 \mathrm{GHz}$ with power levels in the range of $900-1300 \mathrm{~W}$ [Thompson, et al., 2002]. Wafers of both $50 \mathrm{~mm}$ and $75 \mathrm{~mm}$ diameter were thoroughly bonded within five minutes, and knife-edge delamination testing confirmed that the wafer stacks shattered before separating. Due to electromagnetic skin effects, induced temperatures were at a maximum at the wafer edges, and in the case of the radio frequency (RF) bonding of $75 \mathrm{~mm}$ wafers a gradient was observed such that the temperature at the center was only approximately $780^{\circ} \mathrm{C}$. Bonding was nevertheless found to be complete, and the authors hypothesized that RF electric fields present at the bonding interface may have enhanced reaction kinetics such that the temperature requirement was reduced. The authors have similarly published results on the use of EMIH for dopant activation, making use of its high efficiency to demonstrate the utility of the rapid rate of temperature increase to achieve shallow dopant activation [Thompson, et al., 2003]. The next generation of active devices requires enhanced control over junction depths, and rapid drive-in processes such as rapid thermal annealing (RTA) are becoming necessary in order to achieve sufficient dopant activation with limited diffusion. The researchers utilized the process to perform "spike annealing," a $\sim$ zerosecond anneal in which the power was increased at a fixed rate until the target temperature was reached and then immediately removed. Using the $2.45 \mathrm{GHz}$ power supply, temperatures were ramped at a rate of $125^{\circ} \mathrm{C} / \mathrm{s}$ to $950^{\circ} \mathrm{C}$ and $1050^{\circ} \mathrm{C}$ in order to activate beam-line boron implants. The environmental oxygen content was reduced to $100 \mathrm{ppm}$ thorough nitrogen purging, thus limiting surface oxidation as well as oxygenenhanced diffusion. Under optimized conditions, "box-shaped" profiles, ideal for CMOS 
source/drain regions, were obtained with depths below $30 \mathrm{~nm}$ and resistivities of less than $600 \Omega /$ square, resulting in junctions suitable for $100 \mathrm{~nm}$ technologies. Resistive-based heating techniques offer many of the advantages of RTA processes with further benefits of increased efficiency and the capability for localization to specific regions or materials. Those based on electromagnetic induction are arguably more convenient as they do not require wafer probing to provide the means for energy generation. The adaptation of this relatively old manufacturing process to modern semiconductor fabrication continues to generate increasing interest and, pending further investigation, may become an enabling technology for new types of MEMS devices, integrated or otherwise.

The utilization of single- or pulsed-mode laser beams has been under investigation as an alternative to heat treatment for polysilicon thin films. The majority of research in this area has been directed toward thin-film transistor (TFT) applications such as active matrix liquid crystal displays (AMLCDs) for both the recrytallization of asdeposited amorphous silicon [Kuriyama, 1995] and dopant activation [Peng, et al., 2003]. Experimentation has shown laser-based methods to be highly successful in reducing thermal requirements, which not only facilitates monolithic integration with other circuitry but also enables the use of alternate substrates to quartz. The potential for the generation of stresses and stress gradients is high; shallow pulse depths and temperature gradients result in vertical non-uniformity, and the extremely rapid rate of cooling can exacerbate thermal expansion mismatch with the underlying layer(s) [Parr, et al., 2002]. Instruments of short wavelength and/or small pulse duration tend to be favored as they provide for maximum localizability, and consequently technological advancement of laser-based processes in microsystems fabrication is largely contingent upon the 
development of the laser technologies themselves. At the present time, considerable research is being devoted to the characterization of excimer laser processing as their resolution capability is suitable for microsystem applications [Holmes, 2001]. Excimer lasers emit radiation in the ultraviolet spectrum using a gas discharge. Their operation in the recrystallization of structural materials was recently reported by researchers at the University of California at Berkeley [Sedky, et al., 2004]. Using a $\mathrm{KrF}$ device with a $248 \mathrm{~nm}$ wavelength, $38 \mathrm{~ns}$ pulse width, and energy densities in the range of 120 $790 \mathrm{~mJ} / \mathrm{cm}^{2}$, relationships between various parameters of deposition and annealing were investigated for poly-SiGe films. TEM imaging showed the depth of energy penetration to be limited to approximately $0.8 \mu \mathrm{m}$, indicating the utility of the process in maintaining localization for CMOS compatibility but at the same time revealing a constraint that may prevent its promotion to thick films. The researchers partially addressed this by depositing SiGe bilayers in which the lower amorphous layer was subjected to a laserinduced recrystallization which subsequently altered the properties of the second layer. Through fine-tuning of laser energies as well as SiGe compositions and thicknesses, crystalline behavior was manipulated such that the stress gradients of the primary and secondary layers canceled to produce curvature-free structures. The thickest annealed films shown were cantilever beams consisting of a $1.4 \mu \mathrm{m}$-thick $\mathrm{Si}_{60} \mathrm{Ge}_{40}$ foundation deposited at $400^{\circ} \mathrm{C}$ and annealed at $320 \mathrm{~mJ} / \mathrm{cm}^{2}$ with a $1.4 \mu \mathrm{m}$-thick $\mathrm{Si}_{44} \mathrm{Ge}_{56}$ top layer. For the high aspect ratio structures targeted by industry, however, achieving gradient-free anneals will likely require a complex procedure consisting of multiple laser procedures during the film deposition process, though future research may prove otherwise. Methods to control the rate of recrystallization will likely be required as the rapid melting and 
solidification typically encountered with a laser-based anneal tend to result in a relatively coarse grain structure, desirable for TFT applications where conductivity is of high importance but less so for MEMS applications which benefit from the isotropicity of fine-grained structural materials. This has also been reported to generate voids in the grain structure [Peng, et al., 2003]. Thus there are several stress-related concerns affiliated with laser-based annealing. It is a relatively unexplored field, however, and should not be ruled out as a contender for the next revolutionary technology in the MEMS industry.

A third class of technologies, also largely developed for the TFT industry, takes advantage of silicon interactions with specific metals to lower the temperature requirements for recrystallization. Depending on the mode of operation, these processing techniques are referred to as metal-induced crystallization (MIC) or metal-induced lateral crystallization (MILC). The most commonly used reagent is nickel, and it has been shown that at moderately elevated temperatures (typically at or slightly above $500^{\circ} \mathrm{C}$ ) the $\mathrm{NiSi}_{2}$ particles from the interface are able to diffuse through the as-deposited amorphous silicon, both vertically and laterally, breaking bonds along the way. At these moderately elevated temperatures, the broken bonds can be made to crystallize into an ordered lattice using the single-crystalline substrate as a seed [Kawazu, et al., 1990; Hayzelden, et al., 1993]. Maximum silicon film thicknesses, therefore, are dependent upon the reagent diffusivity at a given annealing temperature. Patterning of the metallic films allows for selectivity. Although the lateral rate of diffusion must be taken into consideration for the characterization of resolution capabilities, the significant reduction in thermal requirements renders this field of technologies very appealing. Researchers at the Hong 
Kong University of Science and Technology have suggested the adaptation of MILC to integrated sensor fabrication [Wang, et al., 2000-1; Wang, et al., 2001]. Publications demonstrate pressure sensors consisting of high-quality polysilicon piezoresistors with diaphragms made of a low temperature oxide (LTO) and $\mathrm{Si}_{\mathrm{x}} \mathrm{N}_{\mathrm{y}}$ film stack. The $400 \mathrm{~nm}$ thick silicon resistors are deposited in amorphous form via LPCVD at $550^{\circ} \mathrm{C}$ and are furnace annealed at $500-550^{\circ} \mathrm{C}$ using a $5 \mathrm{~nm}$-thick nickel film. To achieve results comparable with their benchmark $620^{\circ} \mathrm{C}$ LPCVD polysilicon, however, the authors recommend following up with a brief high-temperature anneal, e.g. $1000^{\circ} \mathrm{C}$ for 30 minutes. Other MIC/MILC variations in the literature include the use of pulsed RTA to expedite crystallization times [Leung, et al., 2001] and a hybrid process in which an excimer laser enhances annealing results [Murley, et al., 2001]. While metal-induced processes still require slightly higher temperatures than are recommended for post-CMOS fabrication with aluminum interconnects, changing metals or even light alloying may enable full integration. The resulting films are prone to anisotropic crystallinity as the grains tend to be long and narrow, extending toward the metal-silicon interface. This affects both mechanical and electrical properties, with the former being of particular concern for high aspect ratio MEMS structures. Uniform annealing of thick films has not been shown to date and would likely require either significantly elevated temperatures to enhance diffusion or possibly composite structures consisting of thin metal layers (e.g., $5 \mathrm{~nm}$ of nickel) spaced vertically throughout the structural layer (e.g., every $1 \mu \mathrm{m}$ ). Annealed films do contain a small impurity concentration, though it is thought that the vast majority of metal atoms remain electrically inactive [Wang, et al., 2000-2]. The effect of gradients in both concentration and annealing quality on structural curvature will 
require investigation. The ability to achieve localization through very simple and inexpensive means, however, is a clear advantage over the other techniques discussed.

The three different classifications of localization technologies examined in this section all exhibit the potential to solve the CMOS integration problem. Each imparts a unique set of challenges that must be overcome, but the processes are still in their infancy and will almost certainly advance with time and effort. The technology to be presented in this document is of the first type, is induction-based, and attempts to maximize localization through the use of specific materials for maximum magnetic coupling. It too requires further optimization before becoming suitable for industrial manufacturing, but the initial results show tremendous promise.

\section{4: Other Applications}

The benefits of selective processing techniques remain at the present time largely unexplored in the microsystems industry. While structural annealing is the primary focus of the investigation under review, localization may be of use under any circumstance in which high-temperature processing is necessary [Bergstrom, et al., 2004]. For example, thin films that are in the process of being deposited or grown by either physical vapor deposition (PVD) or chemical vapor deposition (CVD) means could be locally annealed in-situ. Thermal treatment during the deposition or growth would enable local alteration of many material properties including mechanical stress, stress gradients, optical transmission, electrical conduction, and many other effects that are well documented in the literature. Also, deposition rates for CVD and thermally-driven processes, such as $\mathrm{SiO}_{2}$ growth from silicon, could be significantly altered (less so for PVD) by applying heat to certain regions while a reactive gas load is applied to the system, thus selectively 
enhancing the rate of reaction. Researchers at the University of California at Berkeley have recently published results from a related technique in which resistive heat generated through directly applied currents is used to locally enhance the growth rate of PECVD polysilicon [Joachim, et al., 2003]. Similar capabilities exist for chemical-based etching processes such as Reactive Ion Etching (RIE) in which elevated temperatures could be used to selectively enhance reaction rates, possibly enabling film profiles not possible otherwise. New types of dopant profiles may also become available through selective control of diffusion rates; this may be of use in the development of active devices as well as for processes such as the Dissolved Wafer Process that make use of dopant-based etch stops. Ferromagnetic films deposited for Magnetic Random Access Memory (MRAM) often require a magnetic anneal, a low-temperature anneal in the presence of a constant magnetic field, in order to establish the uniaxial anisotropy required for digital data storage capability [Jun, et al., 2004]. CMOS-compatible temperature elevation could be achieved through inductive heating, either using a time-varying field separate from the constant poling field or a single field with both direct and alternating components, or through other localized means [Bergstrom, et al., 2007]. Finally, packaging techniques including wafer bonding could be done using methods such as inductively/resistively heated films or inductively/resistively melted solder. Some initial research in this area was discussed in Section 1.3. These examples are by no means exhaustive, but they demonstrate a portion of the wide range of opportunities afforded by selective processing technologies. It is likely that in the future such methods will not only enhance existing devices and fabrication methods as suggested here but also enable the creation of completely new types of systems and applications. 


\section{Conclusion}

This chapter presented the motivation for research into a localized microstructural annealing technique. Existing methods utilized for monolithic CMOS integration were examined as well as various techniques presently under investigation for the selective modification of targeted materials and/or regions of a device. Opportunities for improvement were evident, specifically with respect to conflicting thermal budgets of dissimilar systems. The remainder of this dissertation presents the development of a new microfabrication process based on the induction heating of thin ferromagnetic films for the purpose of facilitating the modular integration of MEMS structures with CMOS circuitry. While actual CMOS devices were not used, localization is shown via the presence of annealed and unannealed devices in close vicinity on a single substrate, with the unannealed devices understood to represent an active region. Chapter 2 provides a more focused discussion on the direction chosen for this research and the theoretical advantages expected as a result of intentional differences in material properties. This is followed with the demonstration and characterization of the capabilities of the inductive annealing process through both finite-element modeling (Chapter 3 ) and experimentation (Chapters 4 and 5). Finally, Chapter 6 presents the initial development of a high aspect ratio accelerometer fabrication technology, with a full device designed and simulated, in hopes of eventually incorporating inductive annealing into the process. The overall objectives of this dissertation were to provide convincing evidence that the process could provide an industrially acceptable solution to the CMOS-MEMS integration problem and to lay the foundation for its eventual incorporation into the fabrication of functional, integrated devices. 


\section{Chapter 2: Theory of Induction Heating}

Induction heating is a technique commonly utilized in metallurgical industries to modify the properties of materials, particularly iron-based metals. An alternative to ambient heating, it has the advantage of allowing the localized generation of thermal energy in specific regions of a device through differences in the properties of the constituent materials and manipulation of the behavior of the applied magnetic field. For this reason, induction heating has several potential applications in microelectronic and microsystem fabrication, though its use has traditionally been limited to crystal growing [Ciszek, 1985] and susceptor heating [Rafferty, et al., 2002] until recently, as was discussed in Section 1.3. The approach under investigation takes advantage of the higher magnetic permeabilities of ferromagnetics with respect to typical microsystem materials in order to enhance process compatibility among monolithically integrated microsystems with conflicting thermal budgets. The selective heating of regions on a wafer can provide substantial flexibility in the design and fabrication of each individual system as well as reduce the overall size of the integrated device. This chapter discusses the theory and requirements behind this technology, beginning with justification through basic electromagnetic principles in Section 2.1 and continuing with opportunities for localization through differences in material properties in Section 2.2. Section 2.3 concludes the chapter with the proposal of the inductive annealing technology to be presented in this dissertation, with a discussion of the anticipated advantages and limitations as well as considerations for achieving the desired heat and localization results for the annealing of polysilicon microstructures in a CMOS-compatible environment. 


\section{1: Electromagnetic Fundamentals}

Induction heating is the combined result of two primary behaviors, eddy current heating and hysteresis heating. Eddy current heating typically receives greater mention and in most applications is the more significant factor [Tudbury, 1960 (pp. 1-12-1-13); Rudnev, et al., 1997 (p. 782)]. Heat generation is a strong function of frequency, magnetic permability, and electrical conductivity, and is exhibited in all materials to different degrees. Hysteresis heating, on the other hand, is limited to the ferromagnetic set of materials and depends on the nature of their resistance to re-magnetization. This section presents the fundamentals of eddy current heating; hysteresis will be clarified in the next section.

When an object is located within a time-varying magnetic field, an electric field of the same frequency is induced according to Faraday's law [Rudnev, et al., 1997 (p. 780)],

$$
\nabla \times E=-\frac{\partial B}{\partial t}
$$

where $E$ denotes the induced electric field $(\mathrm{V} / \mathrm{m})$ and $B$ is the magnetic flux density $(\mathrm{T})$ which is proportional to the magnetic field strength $H(\mathrm{~A} / \mathrm{m})$ by the material's permeability, $\mu(\mathrm{H} / \mathrm{m})$ as per the magnetic constitutive relation. The electric field gives rise to eddy currents that propagate in circular paths perpendicular to the applied magnetic field, and resistive losses lead to heat generation. The region of maximum current density occurs at the outer edge of the material as a result of Lenz's law which states that the induced currents generate secondary magnetic fields that act to oppose the primary, preventing a runaway condition and consequently causing the current magnitude to decay exponentially toward the center as [Rudnev, et al., 1997 (p. 796)] 


$$
J_{r}=J_{0} e^{-r / \delta}
$$

Equation (2.2) defines the electromagnetic skin effect and reflects the magnitude of the current density $J_{r}\left(\mathrm{~A} / \mathrm{m}^{2}\right)$ at a distance $r(\mathrm{~m})$ from the edge for a given peak current density $J_{0}$. This indicates that $\exp ^{-1}=63.2 \%$ of the eddy current magnitude lies within the first skin depth, and therefore $\exp ^{-2}=86.4 \%$ of the power dissipation. The skin depth, $\delta$, for a good electrical conductor simplifies to [Balanis, 1989 (pp. 149-151)]

$$
\delta=(\pi \sigma \mu f)^{-1 / 2}
$$

Materials that provide efficient energy coupling (i.e., higher electrical conductivity and magnetic permeability) tend to result in a narrower skin depth because the larger induced currents oppose the incident field more effectively, but a limit is reached at which the skin depth becomes so narrow that the effective resistance is high. Frequency selection thus plays an important role in process efficiency. Shen, et al., derived an expression for the heat generation in a hollow cylinder subject to a magnetic field applied parallel to its axis, thus producing eddy currents in the $\Phi$-direction. The time-average induced power dissipation in the cylinder was shown to be approximately [Shen, et al., 1995]

$$
P_{I N D}=(\pi a t d \sigma) \frac{\left(\pi f \mu H_{0}^{2}\right)^{2}}{1+(\pi f \mu \sigma a d)^{2}}
$$

where the dimensions $d, a$, and $t$ corresponded to the wall thickness, radius, and thickness of the cylinder (or film) in meters, the material properties $\mu$ and $\sigma$ corresponded to its magnetic permeability $(\mathrm{H} / \mathrm{m})$ and electrical conductivity $(1 / \Omega-\mathrm{m})$, and $H_{0}$ was the applied magnetic field strength. For analysis, the wall thickness of the cylinder will be assumed to be equal to the skin depth, as it is in this outermost "shell" that the majority of the power generation takes place. Rearranging and simplifying the variables in (2.4) and 
adding the subscript $F$ to indicate a film property gives the simplified form

$$
P_{I N D}=\frac{1.16 \pi a^{3} t_{F} H_{0}^{2}}{\sigma_{F} \delta_{F}\left(a_{F}^{2}+\delta_{F}^{2}\right)}
$$

where the scaling factor 1.16 has been added to account for the portion of the power dissipation not generated inside the first skin depth. The equation reflects a two-part piecewise behavior, depending on the relationship between the skin depth and the radius of the heated cylinder. Dividing the equation into separate portions for $a_{F}>>\delta_{F}$ and $a_{F}<<\delta_{F}$, the final result was

$$
P_{I N D}=\left\{\begin{array}{cc}
\frac{1.16 \pi H_{0}^{2} t_{F}}{\sigma_{F}}\left(\frac{a_{F}}{\delta_{F}}\right) & a_{F}>>\delta_{F} \\
\frac{1.16 \pi H_{0}^{2} t_{F}}{\sigma_{F}}\left(\frac{a_{F}}{\delta_{F}}\right)^{3} & a_{F}<<\delta_{F}
\end{array} .\right.
$$

The two modes reflected the importance of the relationship between the radial dimension of the heated device and its skin depth, which was dependent on its electromagnetic properties and the frequency of the applied magnetic field. Heat generation always benefits from increased frequency and permeability, but the effect of increasing the electrical conductivity depends upon the mode in which the induction heating process is taking place. Limitations can also arise as a result of the nature of the device and the desired outcome of the process. Raising the frequency too high can lead to appreciable heating in non-targeted materials, reducing process selectivity. The literature suggests that for a given material the frequency is typically chosen so as to obtain an $a_{F} / \delta_{F}$ ratio of two to four [Tudbury, 1960 (p. 1-71)]. Further frequency increase produces relatively little benefit in the target material compared to that in the others as it pushes them up the steeper slope of (2.6b), but, nevertheless, higher frequencies may be necessary if high 
temperature is required from a small volume as is the case for the primary application described in this dissertation, polysilicon annealing.

The output power dissipated in an inductively heated material is not directly measurable, but the average power delivered by the induction power supply is typically displayed on the unit. Therefore, relationships were examined between the supply level and the induced temperature, the latter of which was available via infrared pyrometry as will be discussed in Chapter 4. The power delivered to the inductive coil is linearly related to that generated by the power supply, or

$$
P_{S} \propto P_{C},
$$

as the resonant tank circuit establishing the field frequency contains only linear components (see Section 4.2). Considering the coil-film system to be a form of transformer, the power applied to the coil is also expected to be proportional to that dissipated in the heated material. This can be shown empirically via the equation for the power stored in an inductor [Irwin, 1996 (p 262)],

$$
P_{C}=L i \frac{d i}{d t},
$$

where $L(\mathrm{H})$ is the inductance of the coil, $i(\mathrm{~A})$ is the time-varying current through the coil, and $d i / d t(\mathrm{~A} / \mathrm{s})$ is its rate of change. Assuming the current to be sinusoidal, as was the case for this research [Irwin, 1996 (p. 458)],

$$
i(t)=I \cos (w t),
$$

and therefore,

$$
P_{C} \propto I^{2} .
$$


The magnetic field induced in a solenoid is assumed to be uniform, if unperturbed, and oriented parallel to its central axis. Its value is approximately [Nave, 2005-5]

$$
H_{0}=\frac{N_{C}}{l_{C}} I
$$

where $N_{C}$ is the number of coil turns and $l_{C}$ is the physical length of the coil. Substituting (2.9) into (2.8) gives the result

$$
P_{C} \propto H_{0}^{2} .
$$

Finally, since the power dissipation is also proportional to the square of $H_{0}$,

$$
P_{C} \propto P_{I N D} .
$$

The power dissipation is also proportional to the ideal change in absolute temperature, which follows the behavior [Nave, 2005-4,6]

$$
P_{I N D}=\frac{E}{t}=\frac{m_{F} s_{F} \Delta T}{t}
$$

where the dissipated power is the quotient of the thermal energy, $E(\mathrm{~J})$, and time duration, $t(\mathrm{~s})$, with the former equal to the product of the mass, $m_{F}(\mathrm{~kg})$, specific heat, $s_{F}(\mathrm{~J} / \mathrm{K})$, and temperature rise, $\Delta T(\mathrm{~K})$. Assuming that the dimensions of the heated material are sufficiently large such that (2.6a) is valid,

$$
\frac{\pi H_{0}^{2} t_{F}}{\sigma_{F}}\left(\frac{a_{F}}{\delta_{F}}\right)=\frac{m_{F} s_{F} \Delta T}{t} .
$$

Solving for $\Delta T$,

$$
\begin{aligned}
\Delta T & =\frac{\pi t_{F} t}{m_{F} s_{F} \sigma_{F}}\left(\frac{a_{F}}{\delta_{F}}\right) H_{0}^{2} \\
& =\frac{\pi t_{F} t}{m_{F} s_{F} \sigma_{F}}\left(\frac{a_{F}}{\delta_{F}}\right)\left[K_{P S} P_{S}\right],
\end{aligned}
$$


and converting from film mass to volume via density,

$$
\Delta T=\frac{t K_{P S}}{\rho_{F} a_{F} s_{F} \sigma_{F} \delta_{F}} P_{S},
$$

where $K_{P S}$ is a constant of proportionality that relates the applied magnetic field to the supply power and will be determined experimentally in Section 4.4. The actual temperature induced is expected to deviate somewhat from the theoretical value as energy is transported away from the heated film via thermal conduction, convection, and radiation. These effects also help to provide for the establishment of a steady-state temperature for a given input power, provided that a thermal runaway condition is not encountered (see Chapter 4). Considerable effort was devoted throughout the course of this research toward the minimization of conduction across the wafer and convection to the ambient; radiation was necessary for temperature measurement. Specific considerations for the inductive heating of thin films such as temperature measurement and the relationships that govern the efficiency of heat generation are discussed in Section 2.3.

\section{2: Selectivity through Material Properties}

Any material that conducts an electric current is capable of being inductively heated. As suggested by the equations of the previous section, however, some materials interact with magnetic fields more strongly than others. The magnetic behavior of a material is foremost determined by its uncompensated electron moments, primarily spin and secondarily orbital. As electrons accumulate in the valence band of an atom or molecule, it is energetically favorable according to Hund's Rule for them to assume states with parallel spins until all such states are filled, at which point they begin to fill states of 
the opposite spin, forming spin-up and spin-down pairs. Diamagnetic materials such as silicon and silicon dioxide are those with no unpaired electrons, and their behavior in the presence of a magnetic field is determined by orbital moments which rotate antiparallel as a result of Lenz's Law, resulting in a small negative susceptibility and weak repulsion [Dobson, 2000; Eisberg, et al., 1985 (p. 493)]. Unpaired electrons, on the other hand, give a material an uncompensated spin moment whose magnitude overrides that of orbital moments and instead attempts to align parallel to an applied field. Materials classified as paramagnetic have at least one unpaired electron per atom or molecule and exhibit a small positive susceptibility. The degree of alignment can be estimated using Curie's Law, [Eisberg, et al., 1985 (pp. 493-497)]

$$
M=C \frac{H}{T},
$$

where $M$ is the net magnetization $(\mathrm{A} / \mathrm{m}), H$ is the field strength $(\mathrm{A} / \mathrm{m}), T$ is the absolute temperature $(\mathrm{K})$, and $C$ is the material-specific Curie constant. The approximate magnetic susceptibility, $\chi$, of a material is given by the ratio $C / T$ and is related to the relative permeability, $\mu_{r}$, through the relation

$$
\chi=\mu_{r}-1
$$

As the field strength increases, the torque imparted upon the spin moments causes them to align more strongly. Equation (2.15) is only valid to the first order and does not reflect high-field limitations, nor does it remain accurate at very low temperatures. The predicted thermal behavior also breaks down in the case of metals as conduction band electrons are not affiliated with a particular atom and aligning forces are dominated by random thermal motion, resulting in lower susceptibilities as well as lower sensitivity to moderate 
temperature changes. Paramagnetics fail to retain magnetic alignment upon field removal as thermal agitation immediately randomizes dipole orientation.

The approach under investigation for localized annealing takes advantage of the superior magnetic properties of ferromagnetic materials. Of the presently known elements, ferromagnetic behavior is exhibited in iron, nickel, cobalt, and certain crystal forms of several rare earth metals at very low temperatures [Eisberg, et al., 1985 (p. 497); Wohlfarth, 1980 (pp. 188-189)]. Ferromagnetism is a consequence of paramagnetism under specific electron exchange conditions. It has been found that a system of indistinguishable fermions, the class of particles under which electrons fall, exhibits a wavefunction that is antisymmetric, a condition from which the Pauli exclusion principle is derived [Shankar, 1994 (pp. 263-265); Eisberg, et al., 1985 (p. 308)]. This entails that electrons from a multi-electron atom or an interacting set of atoms must have either a symmetric spatial function with antisymmetric spin, or an antisymmetric spatial function with symmetric spin. In the example of a two-electron system, the former and latter variations render solutions in one and three possible forms, respectively, which are referred to as the singlet and triplet states [Shankar, 1994 (pp. 403-405); Eisberg, et al., 1985 (pp. 310-314)]. It can be shown that electrons in a triplet state (or a similar state in the case of a many-electron system), having an antisymmetric spatial component, exhibit nearly zero probability of having similar spatial coordinates, meaning that they exist further apart, though of course not sufficiently distant to become non-interacting. Singletstate electrons, however, have an additive-form spatial function that reflects a doubled likelihood of being found very close together. This phenomenon is not related to Coulomb interaction or any other classical event and is referred to as an exchange force 
[Eisberg, et al., 1985 (p. 316)]. A symmetric spin function indicates parallel moment alignment, which is contrary to the standard antiparallel magnetic alignment classically anticipated. The term exchange energy denotes the energy difference in a system that results from exchange forces. Ferro- and antiferromagnetism are obtained from symmetric and antisymmetric exchange forces, respectively. Ferromagnetism occurs when atomic spacing and radii cause inter-atom electrons to interact such that those unpaired align in a parallel fashion. Figure 2.1 illustrates the concept of ferromagnetic domains in which a system is comprised of regions over which all magnetic moments are aligned in the same direction. In the absence of an applied field, the moment sum across all domains results in zero net magnetization. As the figure shows, however, applying a magnetic field of increasing strength causes quasi-parallel domains to become dominant, growing in size and reversing weaker domains until eventually saturation is reached. Strong field reinforcement in ferromagnetics results in larger susceptibility values than for paramagnetics. This magnetization is largely retained upon field removal due to the irreversibility of domain wall motion, provided that the material is not subject to a strong mechanical force or an elevated temperature [Eisberg, et al., 1985 (pp. 497-502)].
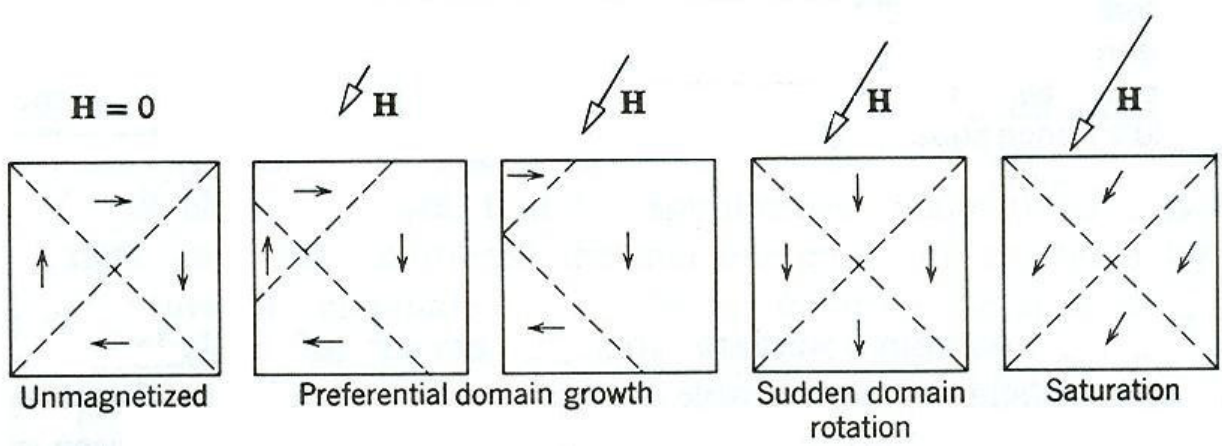

Figure 2.1: Illustration of ferromagnetic domains showing the result of increasing magnetic field strength. Quasi-parallel domains become dominant and grow in size while moments rotate toward saturation [Eisberg, et al., 1985 (p. 502) ${ }^{1}$ ].

\footnotetext{
${ }^{1}$ Reprinted with permission from John Wiley \& Sons, Inc.
} 
Furthermore, a change or reversal in field direction reveals the reluctance of the electron moments and domain walls to move accordingly, a behavior referred to as magnetic hysteresis. Figure 2.2 illustrates typical hysteresis loops for a ferromagnetic material. As shown in Figure 2.2(a), the magnetization, $M$, initially increases rapidly with increasing magnetic field, $H$, until the material begins to approach saturation, at which point the plot levels off. The flux density, $B$, is related to the magnetization through the relationship indicated in Figure 2.2(b). Its region of greatest slope occurs in the unsaturated region of the curve, but even beyond saturation it continues to increase at a reduced rate as $\mu_{0} H$, the free-space relation. The plots also illustrate key terms that define a ferromagnet's behavior. The coercivity or coercive force, $H_{C}$, is the reverse field required to return a saturated material to zero flux density $(B=0)$. This is related to but different from the intrinsic coercive force, $H_{C l}$, which is the reverse field required to de-magnetize a material $(M=0)$. Similarly, the remanent magnetization, $M_{r}$, and remanent induction or

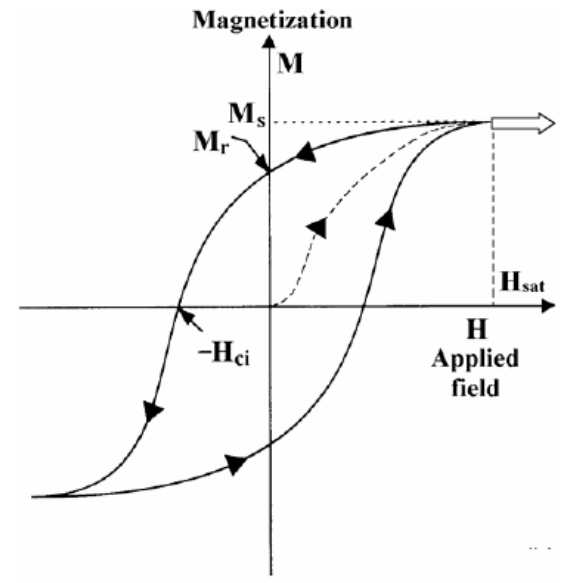

(a)

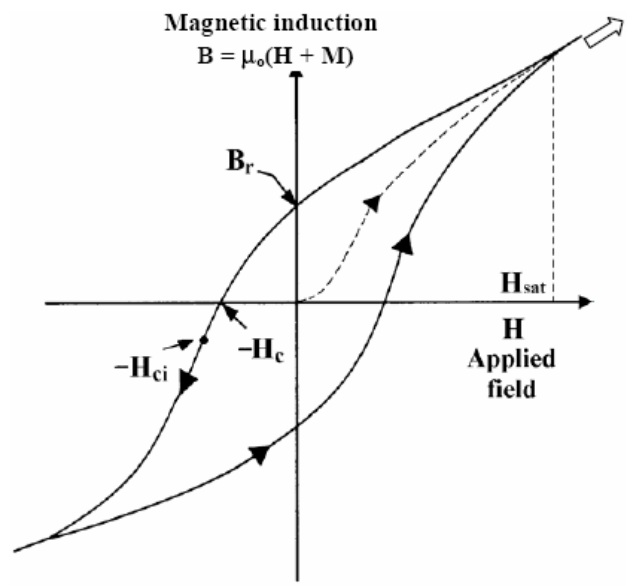

(b)

Figure 2.2: Typical ferromagnetic hysteresis loops showing the effect of a magnetic field $H$ on the (a) magnetization $M$, and (b) flux density $B$. Both variables initially increase in magnitude with magnetic field, but the magnetization eventually reaches a saturation level while the flux density continues to increase as $\mu_{0} H$ [Elliott, 1998 (p. 630) ${ }^{1}$.

\footnotetext{
${ }^{1}$ Reprinted with permission from John Wiley \& Sons, Inc.
} 

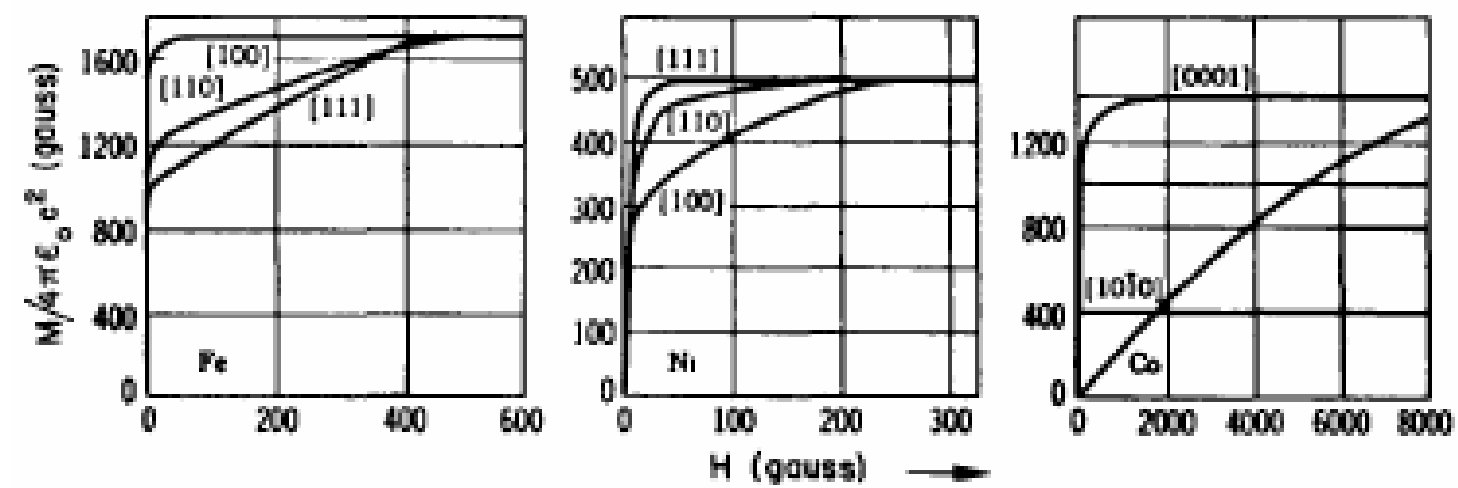

Figure 2.3: Magnetization curves for single-crystalline iron, nickel, and cobalt, illustrating the magnetocrystalline anisotropy that occurs along different crystal planes. As the field strength increases in magnitude, the easy axis direction is the first to reach saturation, corresponding to the highest permeability values [Honda, 1926].

remanence, $B_{r}$, are the respective magnetization and induction that remain once the field is removed. Finally, $H_{S a t}$ is the field at which saturation is reached, and $M_{s}$ is the saturation magnetization level. The actual shape of a material's hysteresis loop depends upon its specific properties such as crystal structure, atomic and electronic spacing, and electronic interaction behavior. Certain crystal planes as well as material formation parameters can yield varying degrees of anisotropy and the establishment of magnetic easy and hard axes as illustrated in Figure 2.3. The magnetic easy axis is the direction along which the least amount of magnetic energy is required to reach saturation, whereas the hard axis is that which requires the strongest field is required to reach saturation. The figure shows the magnetization behavior for iron, nickel, and cobalt in their standard room-temperature crystalline structures (body-centered cubic, face-centered cubic, and hexagonal close packed, respectively) [Honda, 1926]. Since the slope of the B-H curve is by definition the magnetic permeability, $\mu_{r}$ is maximized in the easy axis direction. The physical dimensions of a ferromagnet and its crystals can also give rise to anisotropy. A magnetized material exhibits magnetic charges at its surface that give rise to an opposing 
field. Structures or grains that are narrow will have a relatively stronger demagnetizing field than those that are wide. Thus if a material or its grains have different dimensions in different directions (as is usually the case for a thin film), a shape anisotropy will occur that encourages easy axis formation along the direction of greatest size. This may or may not be consistent with the magnetocrystalline anisotropy defined previously, and in many cases one phenomenon supersedes the other. Furthermore, thermal variations in electronic interactions can result in transitions from one mode to the other [IRM, 2005]. The area enclosed by a hysteresis loop, most specifically its width, is another significant factor in determining the applications for which a ferromagnet is best suited. A material with a narrow hysteresis loop is referred to as a soft magnet, indicating rapid switching and relatively easy reversal. Magnetically soft materials are used in the manufacture of high-speed devices such as transformers and electromagnets. A hard magnet, on the other hand, exhibits a larger coercive force and therefore retains its magnetization more

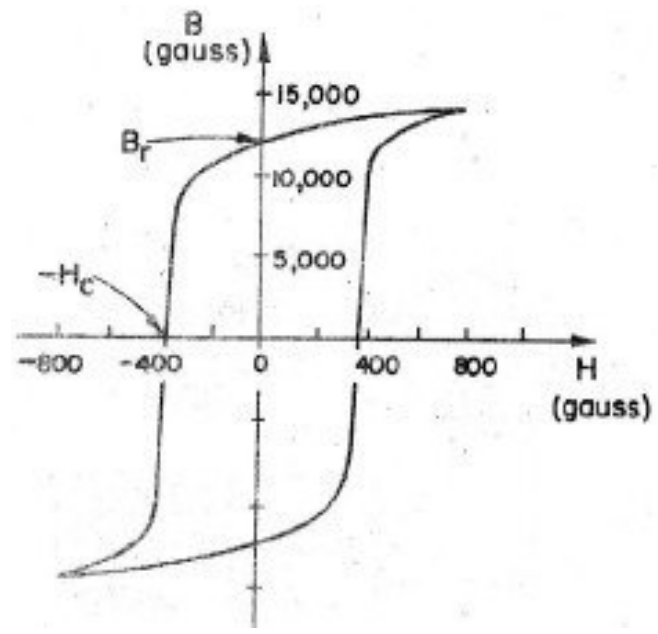

(a)

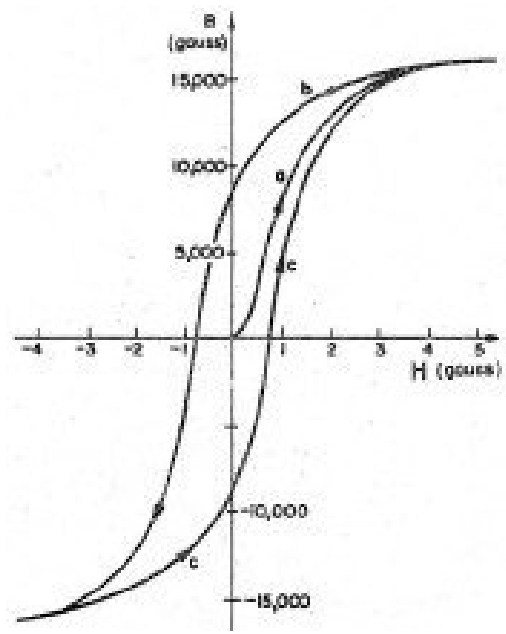

(b)

Figure 2.4: Illustration of the difference between (a) magnetically hard, and (b) magnetically soft materials. A hard magnet has a wide hysteresis loop and retains an applied magnetization more strongly than a soft magnet, which has a narrow hysteresis loop [Feynman, et al., 1989 (pp. 37-10, 36-7) ${ }^{1}$ ].

\footnotetext{
${ }^{1}$ Reprinted with permission from the California Institute of Technology.
} 
effectively. Permanent magnets are made from magnetically hard materials. The concepts of magnetic hardness and softness are illustrated in Figure 2.4. The image in Figure 2.4(a) shows a hysteresis loop for Alnico $V$, an alloy consisting of iron (51\%), cobalt (24\%), nickel (14\%), aluminum (8\%), and copper (3\%), which is considered a hard magnet. Figure 2.4(b) shows a hysteresis loop for soft iron, a class of low-carbon steel materials, all of which form soft magnets [Feynman, et al., 1989]. A squareness ratio can be defined as the ratio between the zero-field remanence, $B_{r}$, and the saturation magnetization, $H_{\text {Sat }}$. For memory and logic applications, a squareness ratio close to unity is desirable as it yields an optimal combination of logic state stability and rapid switching [Soohoo, 1985 (p. 243)].

Similar to paramagnets, magnetic alignment in a ferromagnet generally degrades with temperature as is shown in the examples of Figure 2.5. Increasing random thermal motion ultimately produces a condition at which electronic interaction breaks down. Each ferromagnetic material exhibits a characteristic temperature, the Curie point, above which ferromagnetic behavior ceases in favor of simple paramagnetism with susceptibility again following Equation (2.15) with a temperature offset. The figure demonstrates that the effect of increased temperature is largely dependent upon the applied field strength. At moderate fields, elevated temperature tends to have relatively minimal impact until slightly below the Curie temperature, at which point magnetic properties degrade rapidly. Under low fields, however, temperature can actually enhance electronic interaction up to a certain point, but nevertheless the Curie limit remains unchanged. Material composition and purity, crystal phases and transformations, and external forces such as pressure can also influence thermal magnetic behavior. The Curie points for iron, nickel, and cobalt, 


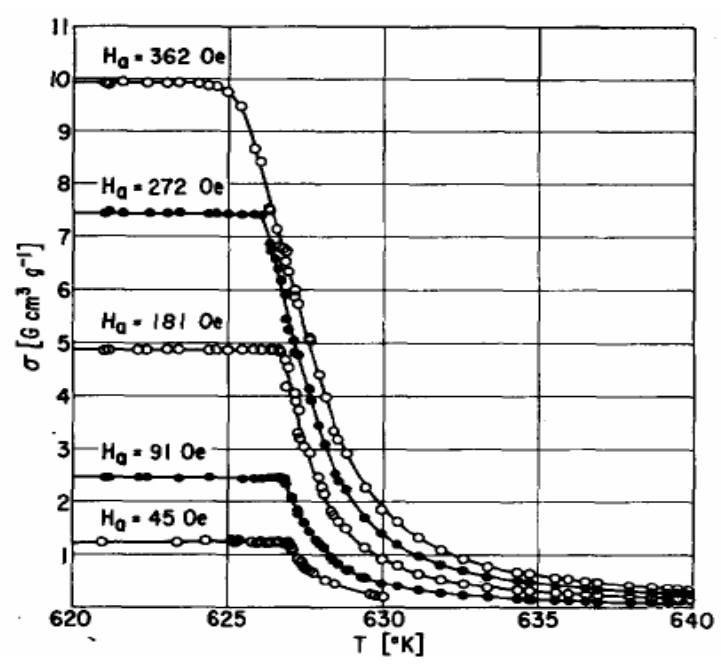

(a)

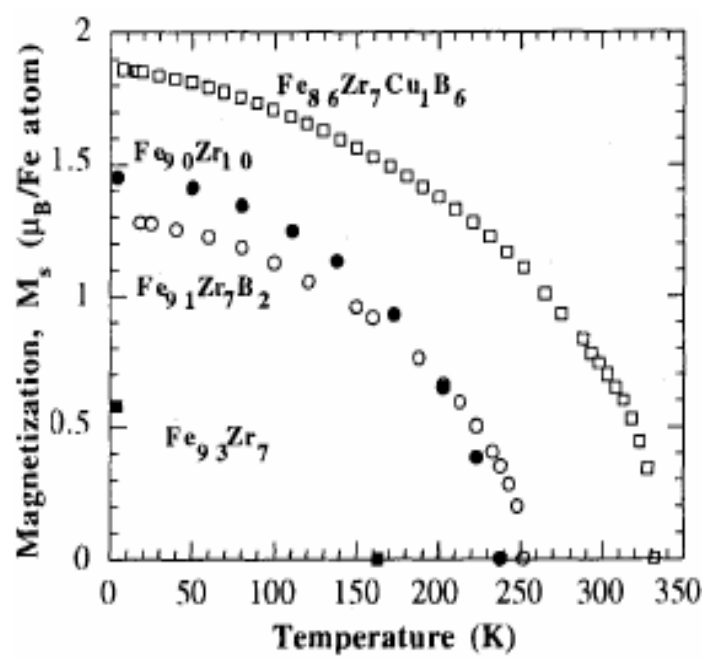

(b)

Figure 2.5: Effect of temperature on the magnetization characteristics of (a) nickel, and (b) FeZr alloys under different field conditions. The images illustrate the sharp decrease in susceptibility that occurs near the Curie temperature for all ferromagnetic materials [Arajs, 1965' ; Barandiaran, et al., 1994²].

are $1034 \mathrm{~K}\left(761^{\circ} \mathrm{C}\right), 631 \mathrm{~K}\left(358^{\circ} \mathrm{C}\right)$, and $1400 \mathrm{~K}\left(1127^{\circ} \mathrm{C}\right)$, respectively [Eisberg, et al., 1985 (p. 500)].

Closely related to ferromagnetism are antiferromagnetism and ferrimagnetism. The former can be observed in several rare earth metals and compounds such as $\mathrm{MnO}_{2}$ in which antiparallel alignment completely or almost completely cancels any net magnetic moment for the system. The latter can be observed in certain composites consisting of multiple ferromagnetics or antiferromagnetics in which an antiferromagnetic-like crystal forms such that the weaker set of moments only partially cancels the stronger, resulting in an overall magnetization similar to a ferromagnet but with slightly different electromagnetic behavior. These two forms of magnetism also reduce to paramagnetism above a critical temperature. Figure 2.6 summarizes the three types of magnetic behaviors

\footnotetext{
${ }^{1}$ Reprinted with permission from the American Institute of Physics. Arajs, S., "Paramagnetic Behavior of Nickel just Above the Ferromagnetic Curie Temperature," Journal of Applied Physics 36. C1965 American Institute of Physics.

2 C1994 IEEE
} 


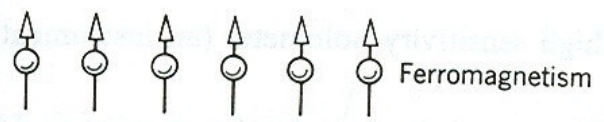

(a)

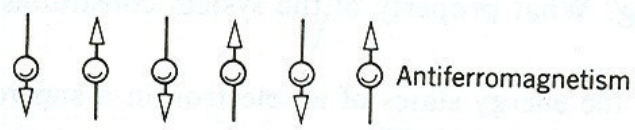

(b)

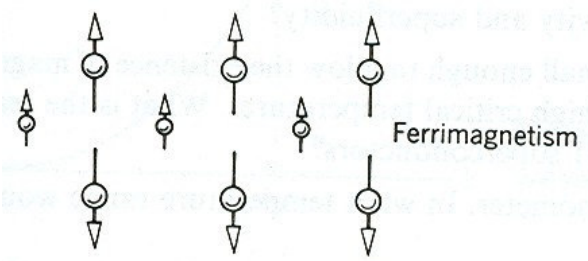

(c)

Figure 2.6: Illustration of electron alignment under (a) ferromagnetic, (b) antiferromagnetic, and (c) ferrimagnetic exchange conditions. Ferromagnetism occurs in systems with symmetric spin functions whereas antiferromagnetism and ferrimagnetism are results of antisymmetric spin functions [Eisberg, et al., 1985 (p. 503) ${ }^{1}$ ].

derived from exchange interactions [Eisberg, et al., 1985 (p. 503)].

Hysteresis heating was mentioned in the beginning of Section 2.1 as one of the two primary factors contributing to an induction heating process. It is a result of the nonzero energy required to re-magnetize a ferromagnetic material upon a change in fieldstrength and/or direction. Under an alternating external field, the volumetric energy consumed per cycle is equal to the area enclosed by the hysteresis loop and is given by Warburg's law as [Bozorth, 1993 (pp. 507-508)]

$$
E=\oint H d B
$$

where the energy $E$ is in Joules $/ \mathrm{m}^{3}$. Power dissipation increases with both coercive force and saturation level, and therefore is generally largest for magnetically hard materials. The vast majority is converted into thermal energy, which can become non-negligible at high frequencies. As was mentioned previously, for most induction applications hysteresis heating is assumed less prevalent than eddy current heating and has been

\footnotetext{
${ }^{1}$ Reprinted with permission from John Wiley \& Sons, Inc.
} 
neglected in the quantitative comparison of the inductive heating capabilities of different materials presented shortly. It also is excluded from the Finite Element Model (FEM) of Chapter 3 as hysteresis computation is beyond the capabilities of the ANSYS software utilized for this work. Nevertheless, it adds to the inductive heating efficiency of ferromagnetic materials and thus further increases their superiority over nonferromagnetics in terms of thermal energy generation.

Equations (2.3) and (2.6) reflect the capability of an induction heating process to take advantage of differences in electromagnetic material properties in order to obtain heating selectivity. Power absorption due to eddy currents increases with permeability and load size, while the effect of conductivity depends on the region in which the process is operating. A simple comparison of the heating efficiencies of several common microsystem materials and ferromagnetics for a given field strength is presented in Table 2.1. The effect of temperature on material properties, including Curie temperature, is neglected and the material properties shown are room temperature values. The dimensions used for radius, $a$, and thickness, $t$, are $0.5 \mathrm{~cm}$ and $1 \mu \mathrm{m}$, respectively. The transition frequency, $f_{T R r}$, is defined as that at which the power dissipation begins to follow Equation (2.6b) instead of (2.6a), meaning that the effect of frequency on heating efficiency becomes enhanced and the process optimization can degrade quickly. The skin depth, $\delta$, and power density, $P / V$, calculations of the rightmost two columns were all done at a frequency of $375 \mathrm{kHz}$ (the approximate frequency utilized during the majority of experimentation) using equations (2.3) and (2.6), respectively. The power dissipation values confirm that the ferromagnetic metals, which constitute the last six rows, have the capacity to produce thermal energy more efficiently than each of the microsystem 


\begin{tabular}{|c|c|c|c|c|c|}
\hline \multicolumn{6}{|c|}{$\begin{array}{c}\text { TABLE } \mathbf{2 . 1} \\
\text { INDUCTIVE HEATING PROPERTIES OF SELECTED MATERIALS }\end{array}$} \\
\hline Material & $\mu_{\mathrm{r}}$ at $25 \mathrm{C}$ & $\begin{array}{c}\sigma \text { at } 25 \mathrm{C} \\
\Omega^{-1} \mu \mathrm{m}^{-1}\end{array}$ & $\begin{array}{c}\mathrm{F}_{\mathrm{TR}} \\
\mathrm{Hz}\end{array}$ & $\begin{array}{c}\delta \text { at } \\
375 \mathrm{kHz} \\
\mu \mathrm{m}\end{array}$ & $\begin{array}{c}\mathrm{P} / \mathrm{V} \text { at } \\
375 \mathrm{kHz} \\
2 \mathrm{kA} / \mathrm{m} \\
\mathrm{W} / \mathrm{m}^{3}\end{array}$ \\
\hline Aluminum & $1.0000165^{1}$ & $41.4^{2}$ & $2.45 \mathrm{E} 2$ & $1.28 \mathrm{E} 2$ & $1.51 \mathrm{E} 7$ \\
\hline Copper & $0.99999454^{1}$ & $64.8^{2}$ & $1.56 \mathrm{E} 2$ & $1.02 \mathrm{E} 2$ & 1.21E7 \\
\hline Germanium, intrinsic & $0.9999884^{1}$ & $2.13 \mathrm{E}-6^{3}$ & $4.76 \mathrm{E} 9$ & $5.63 \mathrm{E} 5$ & $5.26 \mathrm{E}-6$ \\
\hline Germanium, $10^{15} \mathrm{~cm}^{-3}$ & $0.9999884^{1}$ & $5.00 \mathrm{E}-5^{4}$ & 2.03E8 & $1.16 \mathrm{E} 5$ & $2.55 E-5$ \\
\hline Germanium, $10^{21} \mathrm{~cm}^{-3}$ & $0.9999884^{1}$ & $1^{4}$ & $1.01 \mathrm{E} 4$ & $8.22 \mathrm{E} 2$ & $9.73 \mathrm{E} 7$ \\
\hline Gold & $0.999972^{1}$ & $48.8^{2}$ & $2.08 \mathrm{E} 2$ & $1.18 \mathrm{E} 2$ & $1.39 \mathrm{E} 7$ \\
\hline Molybdenum & $1.000072^{1}$ & $20.6^{2}$ & $4.92 \mathrm{E} 2$ & $1.81 \mathrm{E} 2$ & 2.14E7 \\
\hline Palladium & $1.00054^{1}$ & $10.2^{2}$ & $9.93 \mathrm{E} 2$ & $2.57 \mathrm{E} 2$ & 3.05E7 \\
\hline Platinum & $1.000193^{1}$ & $10.4^{2}$ & $9.74 \mathrm{E} 2$ & $2.55 \mathrm{E} 2$ & $3.02 \mathrm{E} 7$ \\
\hline Silicon, intrinsic & $0.99999688^{1}$ & $4.35 \mathrm{E}-10^{3}$ & $2.33 \mathrm{E} 13$ & $3.94 \mathrm{E} 7$ & $7.51 \mathrm{E}-8$ \\
\hline Silicon, $10^{15} \mathrm{~cm}^{-3}$ & $0.99999688^{1}$ & $2.5 \mathrm{E}-5^{4}$ & $4.05 \mathrm{E} 8$ & $1.64 \mathrm{E} 5$ & $1.80 \mathrm{E}-5$ \\
\hline Silicon, $10^{21} \mathrm{~cm}^{-3}$ & $0.99999688^{1}$ & 14 & $1.01 \mathrm{E} 4$ & $8.22 \mathrm{E} 2$ & $9.73 \mathrm{E} 7$ \\
\hline Tantalum & $1.000154^{1}$ & $8.20^{2}$ & $1.24 \mathrm{E} 3$ & $2.87 \mathrm{E} 2$ & $3.40 \mathrm{E} 7$ \\
\hline Titanium & $1.000151^{1}$ & $2.56^{2}$ & $3.96 \mathrm{E} 3$ & $5.14 \mathrm{E} 2$ & $6.08 \mathrm{E} 7$ \\
\hline Tungsten & $1.000053^{1}$ & $20.7^{2}$ & $4.89 \mathrm{E} 2$ & $1.81 \mathrm{E} 2$ & 2.14E7 \\
\hline Cobalt, $99 \%$ & $250 \max ^{5}$ & $17.9^{2}$ & 2.26 & $1.23 \mathrm{E} 1$ & 3.64E8 \\
\hline Iron, $99 \%$ & $6,000 \max ^{6}$ & $11.7^{2}$ & $1.44 \mathrm{E}-1$ & 3.10 & 2.20E9 \\
\hline Iron, $99.9 \%$ & $350,000 \max ^{6}$ & $11.7^{2}$ & $2.47 \mathrm{E}-3$ & $4.06 \mathrm{E}-1$ & $1.68 \mathrm{E} 10$ \\
\hline Nickel, 99\% & $600 \max ^{5}$ & $16.2^{2}$ & 1.04 & 8.34 & $5.92 \mathrm{E} 8$ \\
\hline 78 Permalloy & $100,000 \max ^{7}$ & $6.3^{7}$ & $1.61 \mathrm{E}-2$ & 1.04 & $1.23 \mathrm{E} 10$ \\
\hline Supermalloy & $800,000 \max ^{7}$ & $1.7^{7}$ & $7.45 \mathrm{E}-3$ & $7.05 \mathrm{E}-1$ & $6.68 \mathrm{E} 10$ \\
\hline
\end{tabular}

materials, both semiconducting and metallic, as a result of their larger magnetic permeabilities. This power difference can be enhanced by maximizing the volume of the heated ferromagnetic film compared to that of the microsystem metals.

The data presented in Table 2.1 is highly idealized, and in actuality the effect of factors such as temperature, composition, and frequency on electromagnetic properties can be quite significant. Electrically, metallic resistivities decrease with temperature

${ }^{1}$ CRC, 1999 (pp. 4-131 - 4-138).

${ }^{2}$ CRC, 1999 (pp. 12-45 - 12-47).

${ }^{3}$ Sze, 1981 (pp. 850-851).

${ }^{4}$ Sze, 1981 (pp. 32-33).

${ }^{5}$ Balanis, 1989 (p. 55).

${ }^{6}$ CRC, 1999 (pp. 12-117 - 12-125).

${ }^{7}$ CRC, 1989 (p. E-128). 
while those of doped semiconductors increase. As was mentioned previously, magnetic susceptibilities are also impacted by temperature and ferromagnetic values may increase or decrease prior to rapid descent near the Curie points. Ferromagnetics can be inductively heated above their Curie temperatures, but the energy absorption efficiency drops considerably. The result is that more input power is required to continue raising the temperature, which is worsened by the simultaneous elimination of hysteresis heating. This places a limit on the temperature differential realizable within a composite sample, as the additional power required to heat a paramagnetized ferromagnetic can result in the generation of appreciable heating in the normal paramagnetics. The relationship between magnetic susceptibility, Curie temperature, and film dimensionality is important to the design of related devices and processes and is the reason that the experimental data presented in Chapter 4 utilizes films relatively large in area that often contain high concentrations of cobalt. The purity and crystal structure of a material also play a role in determining its magnetic behavior. The permeability values shown in Table 2.1 are theoretical maxima, meaning that they represent the maximum slopes on magnetization curves from crystals of high purity and relatively low strain. This signifies the criticality of the method of formation and/or annealing. Finally, the frequency of the applied field can lead to magnetic degradation if it is sufficiently high that lag in electron moment response becomes significant. This behavior again depends on the structural properties of a given material, and while some studies have shown a considerable decrease in permeability in the $10 \mathrm{kHz}$ range as a result of interaction with thermal oxide films, it is generally assumed that permeabilities remain reasonably unchanged until beyond 100MHz [Bozorth, 1993 (pp. 798-803)]. 


\section{Conclusion}

The electromagnetic theory behind induction heating was presented in this chapter. Through differences in material properties and process parameters, specific regions of a composite structure can be targeted for concentrated thermal energy generation. The simulations and experiments presented in the remainder of this dissertation are based on these principles and were used to develop a means by which microstructures can be locally annealed ex-situ without adversely affecting nearby active devices. The inductive heating of a thin film region is a new application of a wellcharacterized metallurgical technology. The dimensions and fabrication methods of the heated materials, however, present several challenges not typically encountered in traditional induction heat-treating processes. Since the ferromagnetic films are formed through a physical deposition method, typically RF sputtering, their magnetic permeabilities are inferior to those of the corresponding bulk materials. The relatively small dimensions of the heated films influence the heat generation capability in multiple ways. Equations (2.6) indicate that the power absorbed is largely dependent upon both the film thickness and area, but the overall relationship between the volume and area is not immediately obvious. The volume of film directly affects its ability to absorb magnetic energy, and larger volumes allow the magnetic field to pass through more material. The area of the film, referring to the plane within which the eddy currents propagate, interacts with the skin depth and places a limitation on the efficiency of the process. From discussions that occurred during a May 2001 meeting at Inductoheat with induction heating professionals Valery Rudnev, Raymond Cook, and other experienced engineers, it was learned that the small dimensions of an inductively heated film could be 
partially overcome by applying the magnetic fields perpendicular to the wafer, thus inducing the eddy currents to flow within a plane parallel to the surface. This allows the user to take advantage of the relatively large lateral dimensions of the film, typically on the millimeter scale, thus reducing the impact of the micron-scale thickness on the process. The frequency required to ensure that 3-4 skin depths occur within the surface dimensions is considerably reduced, increasing process selectivity while simultaneously decreasing the effect of field frequency on ferromagnetic properties. Test results have shown that it provides for the efficient heating of very thin films $(\sim 2 \mu \mathrm{m})$ in the $100 \mathrm{kHz}$ range, allowing for the use of standard induction heating equipment. A drawback to this approach, however, is that thin film permeabilities are typically much lower in the perpendicular direction due to the reduced number of magnetic dipoles available for alignment reinforcement, and hysteresis measurements often resemble the sample hardaxis diagram of Figure 2.4(a). Deposition of one or more surface films to adjust stress/strain parameters and/or a post-deposition measure such as magnetic annealing can help to improve these properties [Soohoo, 1965 (pp. 89-90)], though in general the inplane magnetic properties are still expected to be superior.

Perhaps the most difficult challenge that the technology development must overcome is the discrepancy between the volume of the heated film and that of the substrate. While silicon is not considered an induction-efficient material, its higher volume with respect to a thin film could result in significant eddy current heating nevertheless, particularly in the case of heavily doped wafers. This was observed during experimentation as is discussed in Chapter 4. Thus, wafers of low doping are preferred for this process, a potentially significant drawback since industrial manufacturers tend to 
prefer heavily doped wafers for CMOS processing to alleviate parasite latch-up conditions [Deferm, et al., 1988]. This limitation can be alleviated in part or in full through optimization of the ferromagnetic film deposition process in order to realize optimum magnetic properties. A second concern brought about by silicon wafers is the relatively high thermal conductivity. This means that thermal energy conducted into the wafer will rapidly spread laterally, reducing process localization. Barriers to heat transport are therefore an important part of a wafer-based localized heating technique. They further serve to increase process efficiency as they help to confine the annealing energy to the region within which it is needed. 


\section{Chapter 3: Process Development through Simulation}

Modeling an induction heating process presents many challenges. Solutions employ both Maxwell's Equations and principles of heat transport. Material properties play a large role in determining induction heating efficiency, and many of those properties exhibit strong temperature dependence. Secondary items such as convection and radiation can be significant, as can the impact of magnetic hysteresis. This chapter presents the modeling work done using the ANSYS finite element modeling software to support the proof-of-concept of the inductive annealing process. Two different models are discussed, the first being a relatively simple induction heating simulation formed via code obtained from an ANSYS example, and the second being a more customized representation of the heating of a thin film in a vacuum ambient. The results shown are highly consistent with the theoretical analysis as pertaining to electromagnetics, but induced temperature values remain difficult to predict. Furthermore, the modeling of thin films presents obstacles with respect to element aspect ratio and memory allocation. The limitations of the model and suggestions for future revisions are discussed at the end of the chapter.

\section{1: Coupled Model Overview}

An induction heating simulation requires modeling both electromagnetic and thermal behaviors. ANSYS provides two different methods to obtain coupled solutions: the direct method in which a single coupled-field element provides multiple degrees of freedom so as to perform a simultaneous solution, and the sequential method in which individual models are constructed for each system and applied sequentially with the output(s) of each iteration providing input(s) for the next [ANSYS Coupled-Field 
Analysis Guide, Sec 1.2]. An induction heating problem compels the latter method due to the complex means by which the system response evolves, with its electromagnetic behavior changing as the temperature increases, thus requiring multiple iterations of the electromagnetic and thermal solutions [Ibid., Sec 2.1]. Each sub-model is separately optimized and stored in a physics environment, which contains all information related to element definitions, material properties, boundary conditions, solution options, and results [Ibid., Sec 2.2]. The mesh is common to both environments and switches between the two sets of element types as each environment is utilized. Figure 3.1 illustrates the sequential method of coupled-solution modeling. The physical model is built and meshed

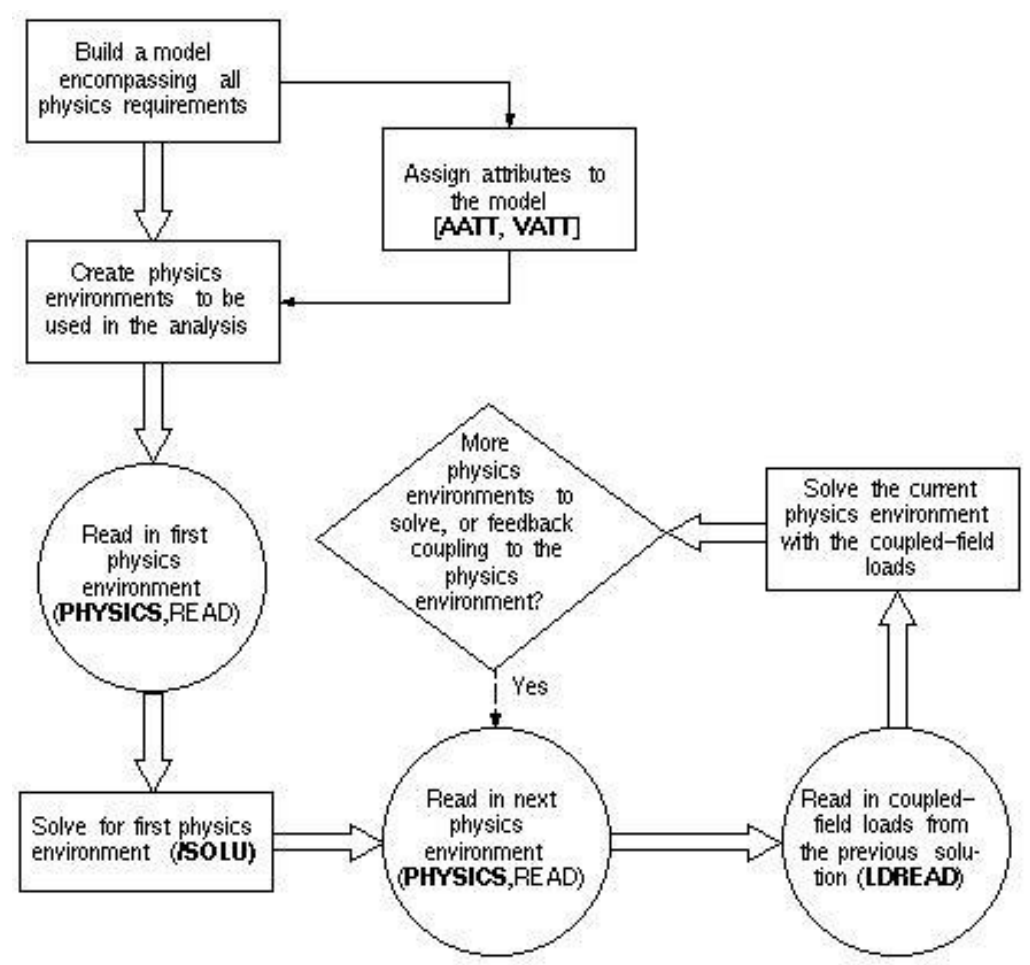

Figure 3.1: Flow chart illustrating sequential coupled-solution modeling in ANSYS. The model is first constructed with both physics environments created. The solution algorithm then consists of a loop in which the environments are individually called, with the coupled loads from the previous sub-solution applied as boundary conditions, until the user's criteria for completion have been satisfied [ANSYS Coupled-Field Analysis Guide, $\left.\operatorname{Sec} 2 \cdot 3^{1}\right]$.

\footnotetext{
${ }^{1}$ Image courtesy of ANSYS, Inc.
} 
using the element types of the first physics environment, with the condition that the elements must be seamlessly convertible to those of the other environment (eg, from 8 noded quadrilateral magnetic planar element to 8-noded quadrilateral thermal planar element), and each individual physics environment is written to its own file with all of the required information to perform the sub-solutions [Ibid., Sec. 2.3-2.4]. In the case of induction heating, the first solver provides for the generation of magnetic fields and flux densities, induced current densities, and resistive power loss densities based on an AC current in a magnetic coil. The second solver then converts the matrix of induced Joule heat values into one of temperatures, and it models heat propagation through the device via thermal conduction and transfer into the ambient via convection and radiation. The user specifies the amount of time that the thermal model simulates before re-evaluation of the electromagnetic fields, i.e., switching to the electromagnetic model and back, is required. This process repeats for the desired number of cycles. Execution of the simulation can be done either manually via the ANSYS graphical user interface, or with an input file containing commands from the ANSYS instruction set. The latter method is typically preferred, as otherwise the repeated conversion between the two environments becomes rather tedious. The two models presented in the next two sections are both codebased. The full code for each is given in Appendix A, with Sections 3.2 and 3.3 dedicated to their explanations and presentation of results.

\section{2: ANSYS Example Induction Heating Model}

The ANSYS Coupled-Field Analysis Guide is available on the Internet through multiple sources including the ANSYS website, the address for which can be obtained from the References chapter. ANSYS provided several example models to illustrate the 


\begin{tabular}{llll}
\hline \hline \multicolumn{4}{c}{ TABLE 3.1 } \\
\multicolumn{1}{c}{ ElEMENT TYPES UTILIZED IN THE ANSYS EXAMPLE MODEL } \\
\hline Description & Element & Electromagnetic & Thermal \\
& Number & Element Type & Element Type \\
\hline Structure & 1 & Plane13 & Plane55 \\
Coil and Air & 2 & Plane13 & Null0 \\
Structure Surface & 3 & Null0 & Surf151 \\
\hline \hline
\end{tabular}

use of physics environments, one of which was an induction heating model [Ibid., Sec. 2.10]. The code provided by ANSYS is duplicated in Appendix A.1, with additional comments provided for clarity. In essence, the code constructed the model, set up the two physics environments, and then executed the simulation. During the building of the model, the designer(s) primarily defaulted to the electromagnetic element types and material properties, and then converted to their thermal counterparts once the electromagnetic physics environment had been defined. The model began with the typical first step of defining the element types, as this was required before meshing. The three element pairs utilized are summarized in Table 3.1. The heated structure, or billet, utilized the Plane13 and Plane55 element types. These are both two-dimensional, fournoded planar elements that allow for axisymmetry, meaning that they can be utilized to form a three-dimensional model by rotating the two-dimensional model about the y-axis. This model, along with that presented in the next section, was an axisymmetric model as specified in the element type declarations. Plane13 provides for coupled-field solid elements that can be utilized for magnetic, electrical, thermal, and/or structural modeling but with limited degree-of-freedom combinations. Induced temperatures from Joule heating can be computed, but only at the post-processing stage, and thus transient thermal modeling is not enabled [ANSYS Element Reference, Element Library: Plane13]. Plane55, on the other hand, is strictly for thermal modeling and provides for thermal 
conduction from one element to the next [Ibid., Element Library: Plane55]. The inductive coil and surrounding air had similar electromagnetic requirements to the heated structure, referring to the capacity for current conduction (impressed or induced) and magnetic field continuity, and thus also utilized Plane13 for the electromagnetic model. These components were not relevant to the thermal model, however, and thus were converted to the Nullo, or non-simulated, element type in order to conserve memory and thus increase solver velocity [ANSYS Commands Reference, Command Dictionary: ET]. The third element type was formed only at the outer surface of the heated structure in order to enable the modeling of thermal radiation. Not utilized in the electromagnetic model, this was designated as a Surf151 element in the thermal model. A Surf151 element is overlaid onto the outer edge of a two-dimensional element. It can be considered a one-dimensional element but is in actuality two-dimensional because it utilizes a single, remote node called a space node to collect the radiated energy in the far field [ANSYS Element Reference, Element Library: Surf151].

The next portion of the code provided variable definitions for both models. Parameters for structural dimensions (both), harmonic frequency and minimum skin depth (electromagnetic), transient time-stepping (thermal), and physics environment timing (both) were established. The axisymmetric structures were defined through a series of radii establishing distances from the $\mathrm{y}=$ axis, with an overall thickness dimension applied to the entire model. The calculation of the minimum skin depth established the minimal mesh dimension, as accurate simulation requires a minimum of 1-2 elements per skin depth [ANSYS Electromagnetic Field Analysis Guide, Section 3.5.1]. The value tinc set the time duration of each thermal simulation, and the value ftime set the total 
simulation time; thus, their ratio determined the number of iterations of the simulation loop.

The model then defined the material properties for the electromagnetic simulation, specifically, the magnetic permeability and electrical resistivity values for the heated structure (material 2), magnetic coil (material 3), and surrounding air (material 1). Both properties for the billet were temperature-dependent, with the permeability decreasing and the resistivity increasing with increasing temperature. The other materials did not require temperature dependence since they were not included in the thermal simulation.

The structures were next constructed and meshed. Using the previously-defined radius variables, the billet, air gap between billet and coil, coil, and outer air region were built in the form of rectangles. Axisymmetric rotation during simulation later converted these into concentric rings; thus the model became a cylindrical billet surrounded by a single concentric coil loop, illustrated in Figure 3.2. Mesh sizing was done via keypoint selection. The mesh size in the heated structure was modulated from $1 / 2$ of the minimum skin depth, calculated previously, at the outer edge to 40 skin depths at the inner edge (center of the the area attributes were established, associating each area number with the appropriate material number and element type. Once the two-dimensional solid meshing was complete, the space node was added at the origin and the single Surf151 radiation element was added to the outer edge element of the billet. The layout of the mesh is illustrated in two dimensions in Figure 3.3(a) and in three dimensions in Figure 3.3(b). The images show the linearly-graded mesh from the center to the edge of the heated structure. This provided for a sufficiently fine grid at the outer edge of the billet, where 


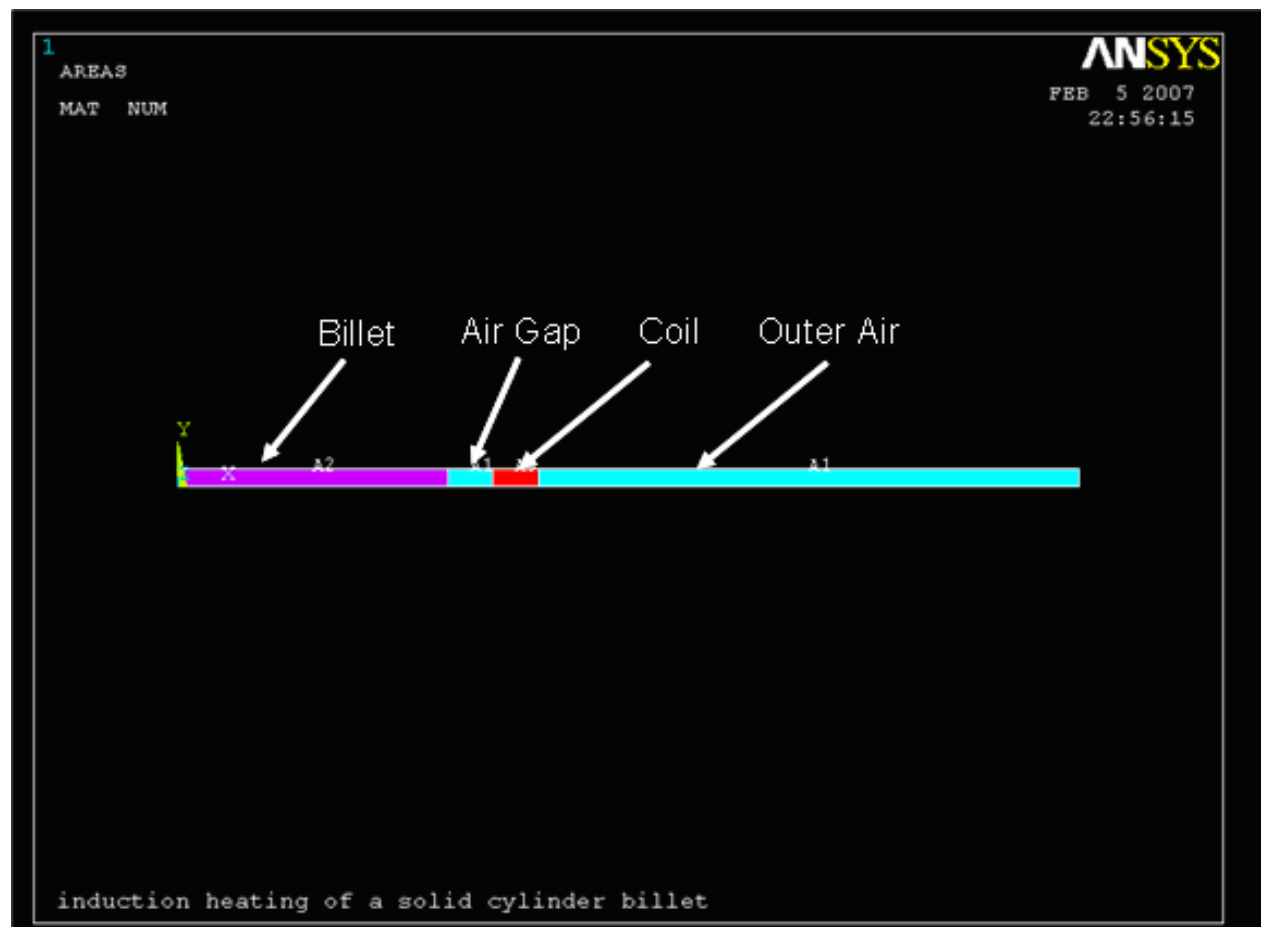

Figure 3.2: Area plot of the ANSYS solid model illustrating the simulation of the four modeled regions as two-dimensional rectangles. The horizontal dimensions of the rectangles were defined in terms of radii from the center of rotation, or y-axis. The solver then rotated the model about the center to create the cylindrical billet and coil loop.

the majority of heat generation takes place according to the skin effect (see Section 2.1), while conserving memory in the relatively uninteresting interior portion. With the model, material properties, and mesh fully defined, the electromagnetic physics environment was only missing its loads, boundary conditions, and solution options. The single load required was the current density through the coil cross-section, which was set to $15 \mathrm{E} 6 \mathrm{~A} / \mathrm{m}^{2}$ for a total current of $37.5 \mathrm{~A}$. The flux-normal boundary condition was applied to the $y$-axis, meaning that the z-component of the magnetic vector potential was set to zero on these nodes. This reflected the nature of the magnetic vector potential in the interior of a solenoid or current loop. The magnetic vector potential, $A\left(\mathrm{~T}^{*} \mathrm{~m}\right)$, is defined in terms of the magnetic field, $H(\mathrm{~A} / \mathrm{m})$, as [Balanis, 1989 (p. 256)] 


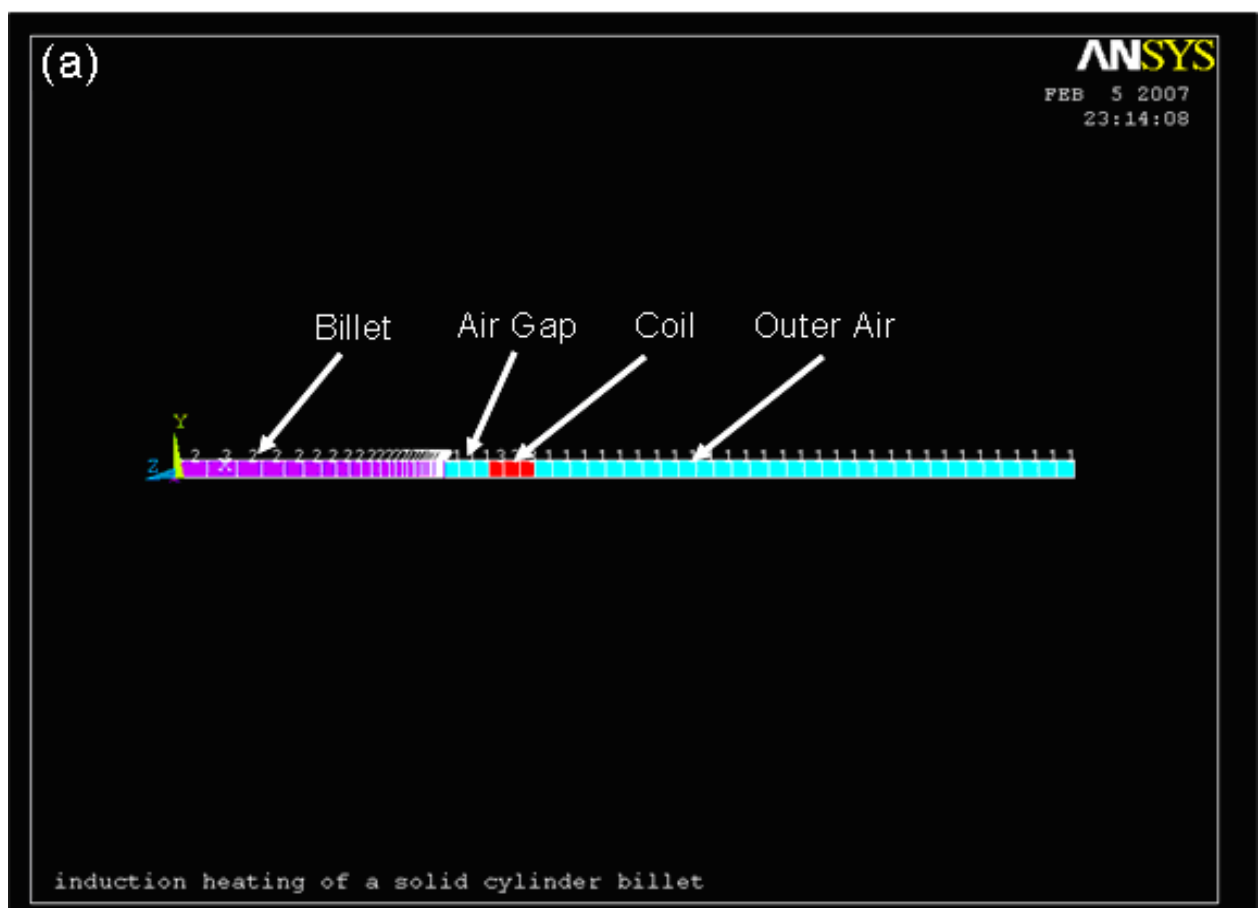

Figure 3.3(a): Elemental plot of the meshed ANSYS solid model. The billet was meshed such that the element size coincided with half the minimum required skin depth at the outer edge and 40 times the minimum skin depth at the inner edge. The remainder of the mesh was given elements $1 \mathrm{~mm}$ in height and width.

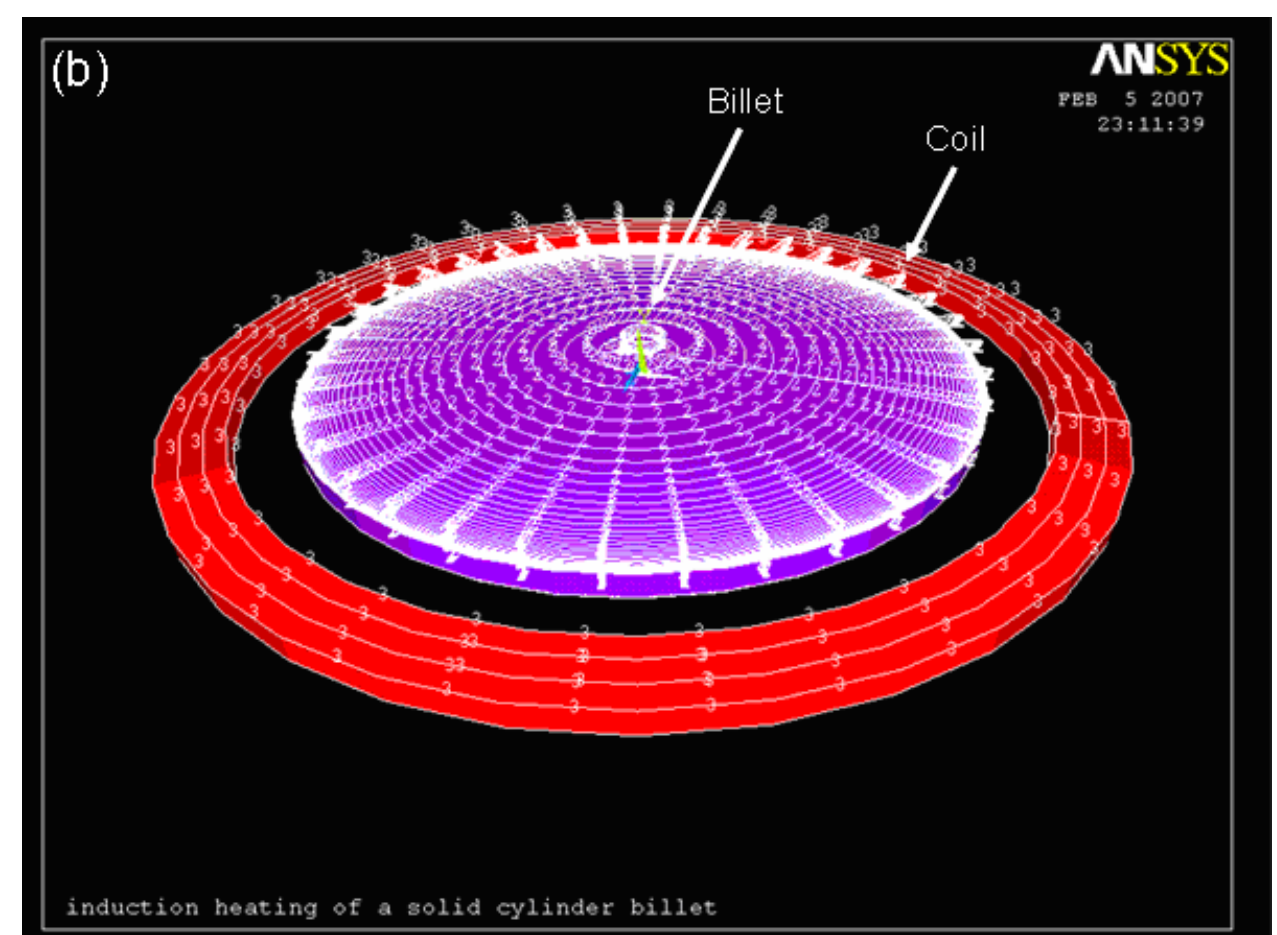

Figure 3.3(b): Axisymmetric rotation of the meshed model, showing only the billet and coil. The model provided for the simulation of a cylinder slice; the temperature gradient from center to edge was assumed to be uniform throughout the billet thickness. 


$$
H=\frac{1}{\mu} \Delta x A
$$

From Section 2.1, $H$ was understood to be uniform through the cross-section of the coil, whether a solenoid or a single loop, and oriented along the vertical direction (parallel to the y-axis). This signified that $A$ had a uniform curl and thus a circular shape. Solving for $H_{Y}$ in cylindrical coordinates gives [Balanis, 1989 (p. 266)]

$$
H_{Y}=\frac{1}{\mu \rho}\left[\frac{\partial}{\partial \rho}\left(\rho A_{\phi}\right)-\frac{\partial A}{\partial \phi}\right] .
$$

Since $A$ was circular, its variation with $\phi$ was zero and the second interval can be neglected. Substituting the magnetic field through a current loop into $H_{Y}$ [Nave, 2005-3],

$$
H=\frac{I}{2 R}
$$

where $R(\mathrm{~m})$ is the coil radius, the equation becomes

$$
\frac{I}{2 R}=\frac{1}{\mu \rho}\left[\frac{\partial}{\partial \rho}\left(\rho A_{\phi}\right)\right] .
$$

Integration with respect to the radial dimension, $\rho$, gives

$$
A_{\phi}=\frac{\mu I}{4 R} \rho
$$

where $\mu(\mathrm{H} / \mathrm{m})$ is the magnetic permeability of the medium, $I$ (A) is the current through the solenoid, $R(\mathrm{~m})$ is the radius of the solenoid, and $\rho(\mathrm{m})$ is the distance from its center. The equation indicates that the magnetic potential circulated about the axis of symmetry (y-axis), i.e., pointed in the z-direction on the $\mathrm{x}-\mathrm{y}$ plane (normal to the page in the default two-dimensional view) and increased linearly with distance from the axis. Setting $A_{Z}$ to zero at the y-axis was thus a natural condition as the radius term was equal to zero; 


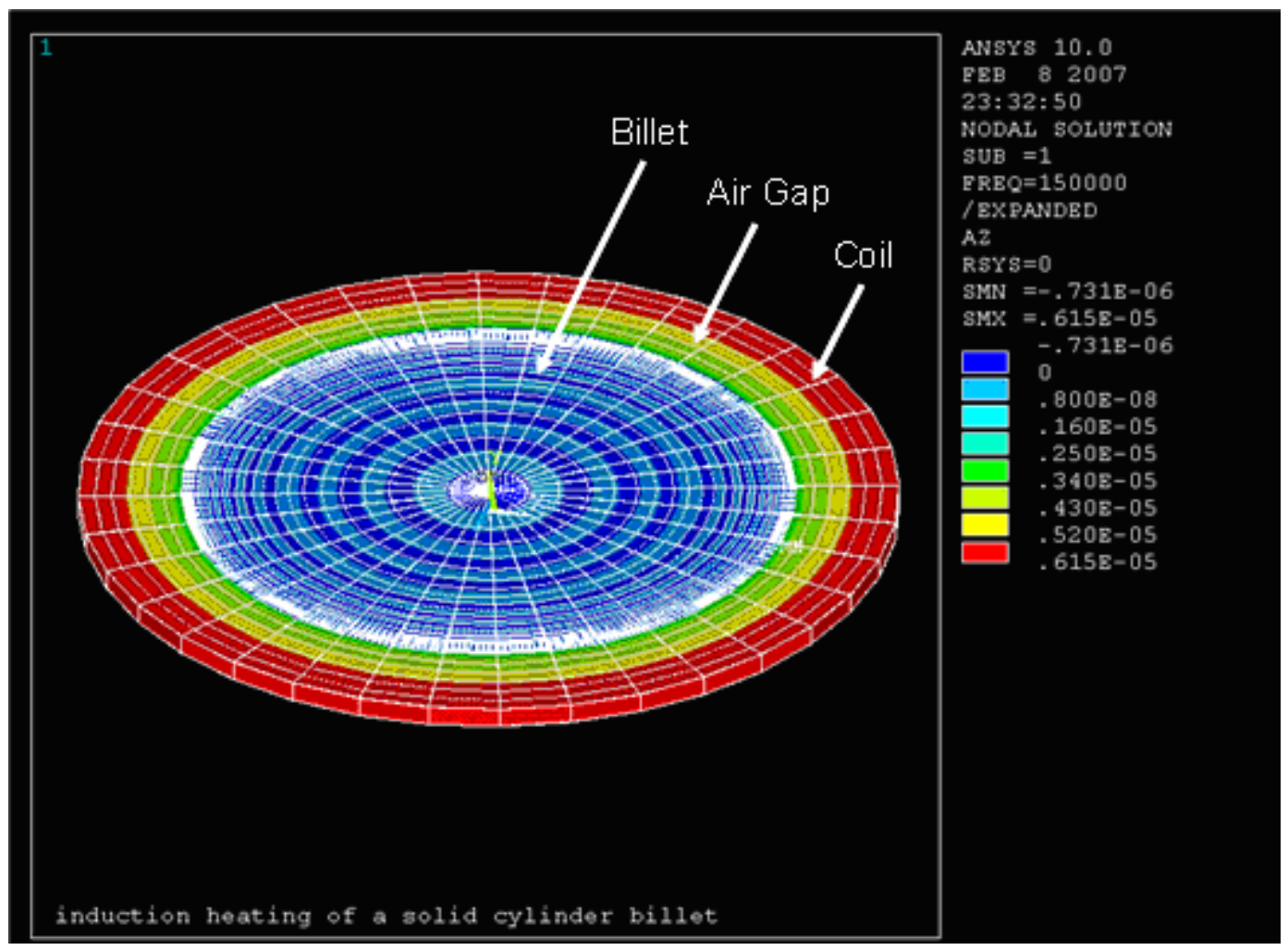

Figure 3.4: Three-dimensional plot showing the magnitude of the z-component of the magnetic vector potential across the coil interior. The magnitude followed the predicted linear behavior in the air gap but was quickly reduced to approximately zero in the billet due to field cancellation from the skin effect. ANSYS used $A_{Z}$ as the primary degree of freedom from which the remainder of the magnetic behavior was derived [ANSYS Element Reference, Element Library: Plane13].

furthermore, the line of symmetry provided no return path for the potential loop. Figure

3.4 illustrates the magnitude of the z-component (or $\phi$-component) of the magnetic vector potential as computed by ANSYS during the first iteration of the electromagnetic solution. Note that the magnitude in the air gap appeared to show a linear decrease toward the center. The cancelling fields generated within the billet, however, disrupted the linearity in $\rho$ and furthermore produced an odd-looking result in which adjacent elements alternated between positive and negative decaying values, apparently the means by which ANSYS resolved the behavior of the magnetic material in an alternating magnetic field. The simulated values in the air gap were within an order of magnitude 
from theory; Equation (3.5) predicted an $A_{Z}$ value of $1.01 \mathrm{E}-5$ at the edge joining the billet and air gap, whereas ANSYS computed approximately 3E-6, which was lower by a factor of 3.37. Differences were attributed to non-ideality in field uniformity as compared with the assumptions made in the theoretical analysis, exacerbated by close proximity to the coil. With the boundary conditions thus fully defined, the code set up the solution as a harmonic analysis with a frequency of $150 \mathrm{kHz}$, and lastly stored the electromagnetic physics environment for later use in the coupled solution.

The simulation code resumed with the conversion of the model from harmonic electromagnetic to transient thermal. The element types were first converted to their thermal counterparts. Next, the material properties relevant to the thermal simulation were defined, those being the thermal conductivity, enthalpy, and surface emissivity for the heated structure. The enthalpy values reflected the initial heat energy stored in the material. Enthalpy changes with temperature according to [Chang, 1994 (pp. 211-215)]

$$
\Delta H=m s \Delta T .
$$

where $m$ refers to the mass $(\mathrm{kg})$ of the heated sample, $s\left(\mathrm{~J} / \mathrm{kg}^{\circ} \mathrm{C}\right)$ is its specific heat, and $\Delta T\left({ }^{\circ} \mathrm{C}\right)$ is its change in temperature. The conductivity and enthalpy were both assigned temperature dependence, with the former decreasing and the latter increasing with temperature. Finally, the initial conditions and solution options were established. The analysis type was defined, the temperature scale was set to Celsius, the far-field temperature was set to $25^{\circ} \mathrm{C}$, and the range of time steps was provided. This information completed the requirements for the thermal model, and the thermal physics environment was stored. 
With both physics environments completed, the solution was executed via a doloop. The number of iterations was dependent upon the ratio of ftime, the total time of simulation, and tinc, the time duration of each thermal simulation. Beginning with the electromagnetic model, an if-else statement placed a uniform temperature of $100^{\circ} \mathrm{C}$ on the entire model for the first solution, and utilized the final temperature matrix from the previous thermal simulation for each subsequent solution, read in via the ldread command. Once the electromagnetic solution was completed, the model switched to the thermal environment. The ldread command was again utilized, this time for the purpose

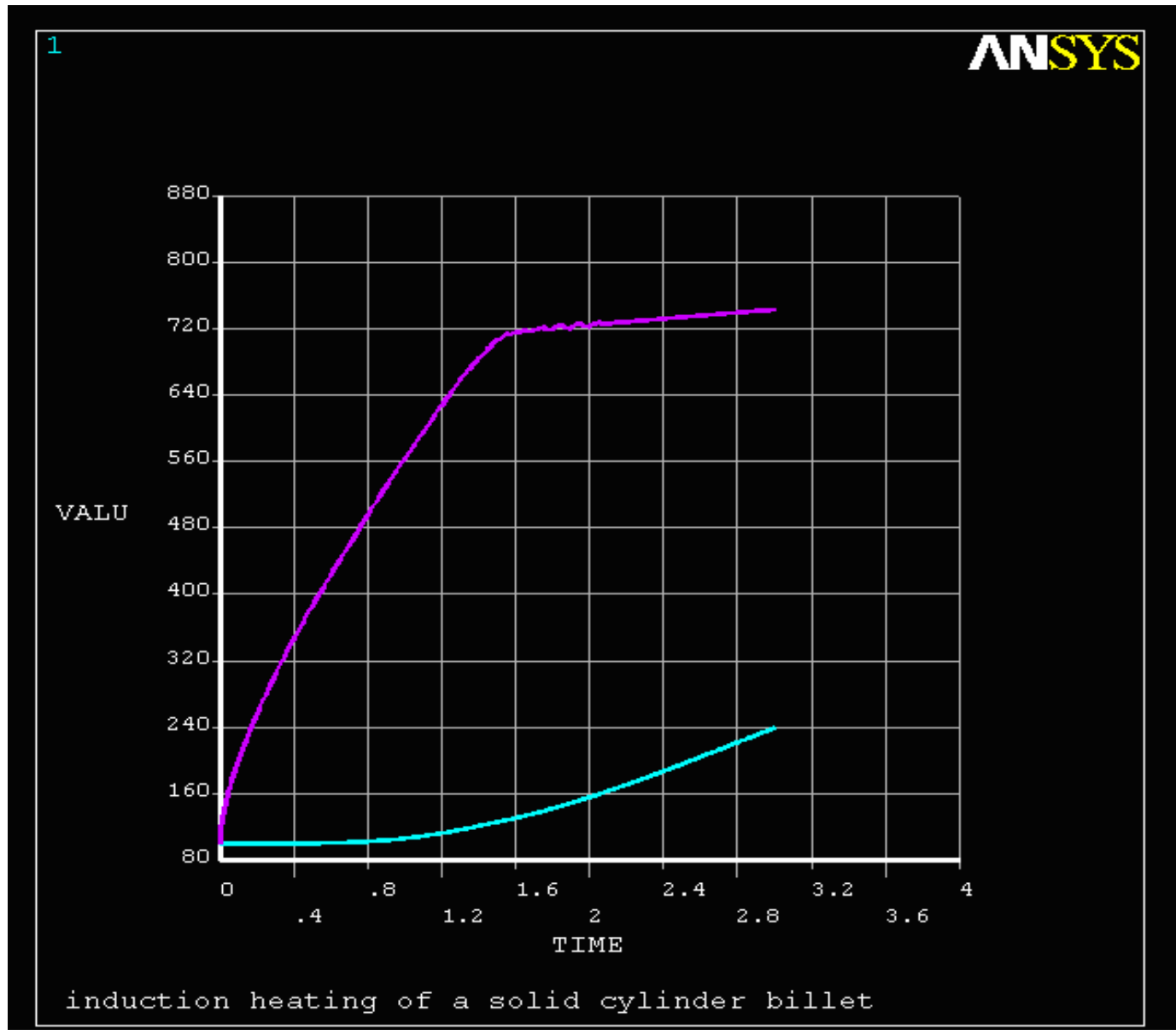

Figure 3.5: Output plot generated by ANSYS example simulation code showing the temperatures, in Celsius degrees, at the center (blue) and edge (purple) of the heated billet. The vast majority of heat generation took place at the outer edge, due the skin effect, whereas the gradual heat rise at the center was due to thermal conduction. 
of reading in the most recent matrix of Joule heat values, and the thermal solution began/resumed execution until the time duration specified, time, had been reached. The final section of the code was focused on the display of the temperature data from the center and outer edge of the heated billet. Figure 3.5 shows the output plot generated upon the conclusion of the simulation. As expected, the outer edge (purple) heated much more quickly than the center (blue). The skin effect caused the vast majority of heat generation to take place at the edge, as expected, and the gradual temperature rise at the center was brought about by thermal conduction. This gradient can be manipulated by adjusting the power input to the coil, thus controlling the rate of rise at the edge, depending on the desired outcome of the heat treatment procedure.

Figures 3.6-3.10 illustrate some of the underlying behaviors within the simulation relevant to the theoretical analysis of Chapter 2 . Those obtained from the electromagnetic simulation were computed using an ambient temperature of $100^{\circ} \mathrm{C}$. Figure 3.6 (a) shows the y-component of the magnetic field strength, $H_{Y}$, along the two-dimensional crosssection. The field strength within the free space region was nearly uniform at approximately $38 \mathrm{kA} / \mathrm{m}$. Removing the billet from the model verified relatively good field uniformity $(33 \mathrm{kA} / \mathrm{m}<H<38 \mathrm{kA} / \mathrm{m})$ within the entire region encircled by the coil. The close-up illustrated the deterioration of the field strength within the billet region, caused by the cancelling fields created by the induced eddy currents. Like the $A_{Z}$ plot in Figure 3.4 , the interior of the billet showed alternating regions of weak positive and negative values; this pattern was repeated in the flux densities and current densities as shown in the subsequent figures. The two-dimensional flux density plot in Figure 3.7(a) repeated the same basic pattern as the magnetic field strength. Multiplying the $38 \mathrm{kA} / \mathrm{m}$ field 


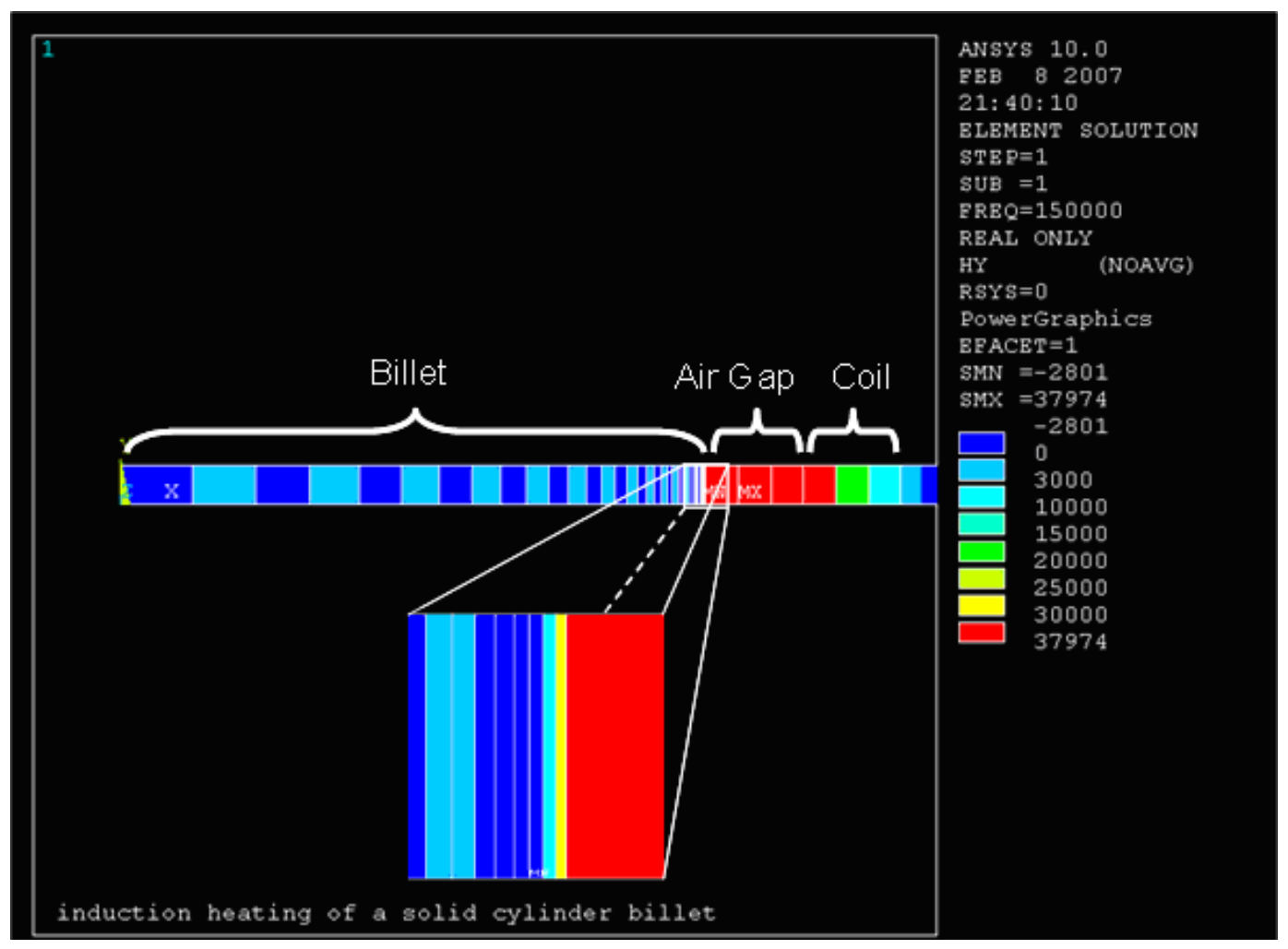

Figure 3.6: Contour plot showing the electromagnetic field magnitudes along the $y-$ direction, with a close up added of outer edge of heated billet. The magnetic field strength was approximately $38 \mathrm{kA} / \mathrm{m}$ in free space, and deteriorated sharply after the first skin depth (two elements) inside the billet.

strength by the free-space permeability, $4 \pi \mathrm{E}-7$, gave an expected free-space flux density of $0.048 \mathrm{~T}$ which was in agreement with the contour range in the "air gap" region shown in the figure. The ratio between the maximum flux density value, which occurred at the outer edge of the heated billet, and the free-space value was 147.3 , reflecting the approximate magnetic permeability of the structure (actually 194.5 at $100^{\circ} \mathrm{C}$ ). The threedimensional vector plot in Figure 3.7(b) helps to show the nature of the flux densities for the system as it was interpreted by the electromagnetic solver. The different flux magnitudes were illustrated by both the color and height variations of the vectors, which were displayed at each node. The relative flux density values, combined with differences 

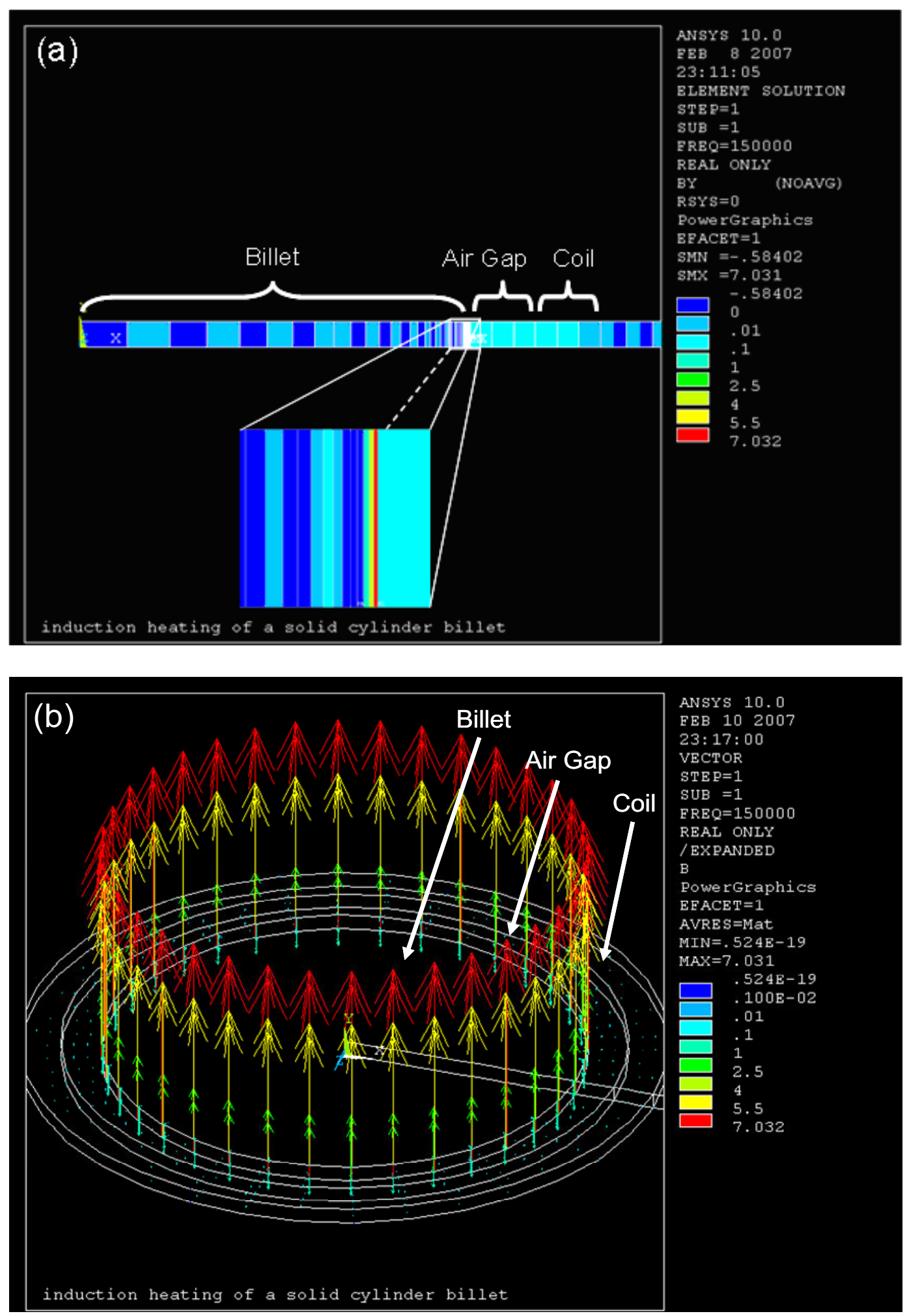

Figure 3.7: (a) Two-dimensional contour plot, and (b) three-dimensional vector plot showing the y-component of the electromagnetic flux density, $B_{Y}$, for the ANSYS model The close-up of the outer edge of the billet in (a) demonstrated the greater extent to which the magnetic material was polarized by the field. The color and height of the vectors in (b) further illustrated the relative flux density magnitudes at the different nodes. 

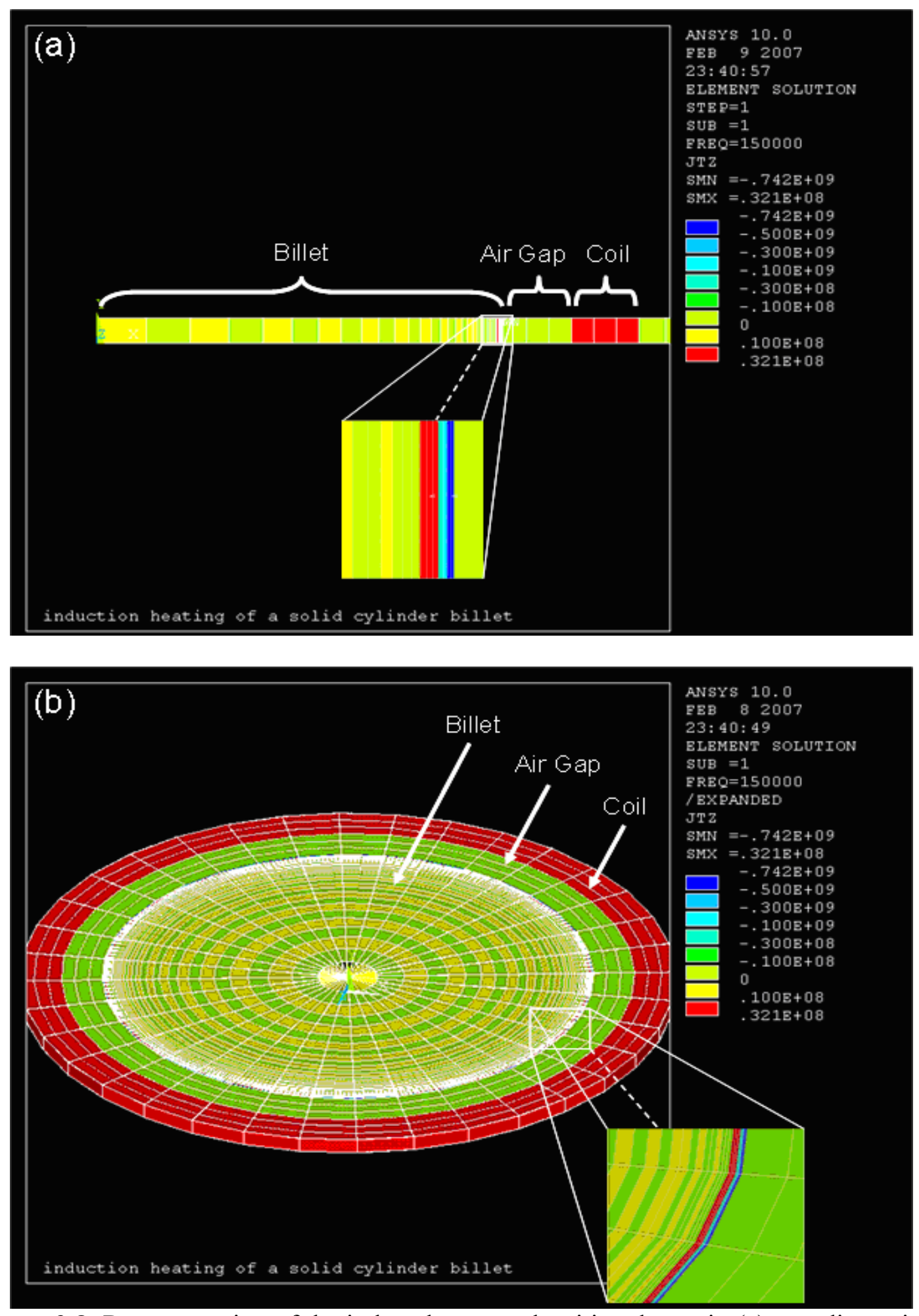

Figure 3.8: Representation of the induced current densities shown in (a) two dimensions, and (b) three dimensions. The values reflected both the impressed current density, applied to the coil, and the induced current density, generated in both the billet and surrounding air. Both the impressed and induced (eddy) currents propagated in a circular path about the axis of symmetry. 
in electrical conductivity among the materials, were converted into a gradient of current density values as shown in Figures 3.8(a,b). The two-dimensional representation in (a) again described a similar behavior as the corresponding field and flux density plots, with the peak values occurring at the outer edge of the structure as expected and quickly decaying toward the center. Note that the current density at the outer edge was higher in magnitude than that at the coil. Recognizing the impact of differences in element sizes and their capability to offset density calculations, the actual current through the narrowest billet elements was manually calculated to be approximately half (17.2A) of that through the coil $(37.5 \mathrm{~A})$. The relative impact on adjacent elements within the billet was negligible. Within the billet, the current density decayed to approximately $40 \%$ of its

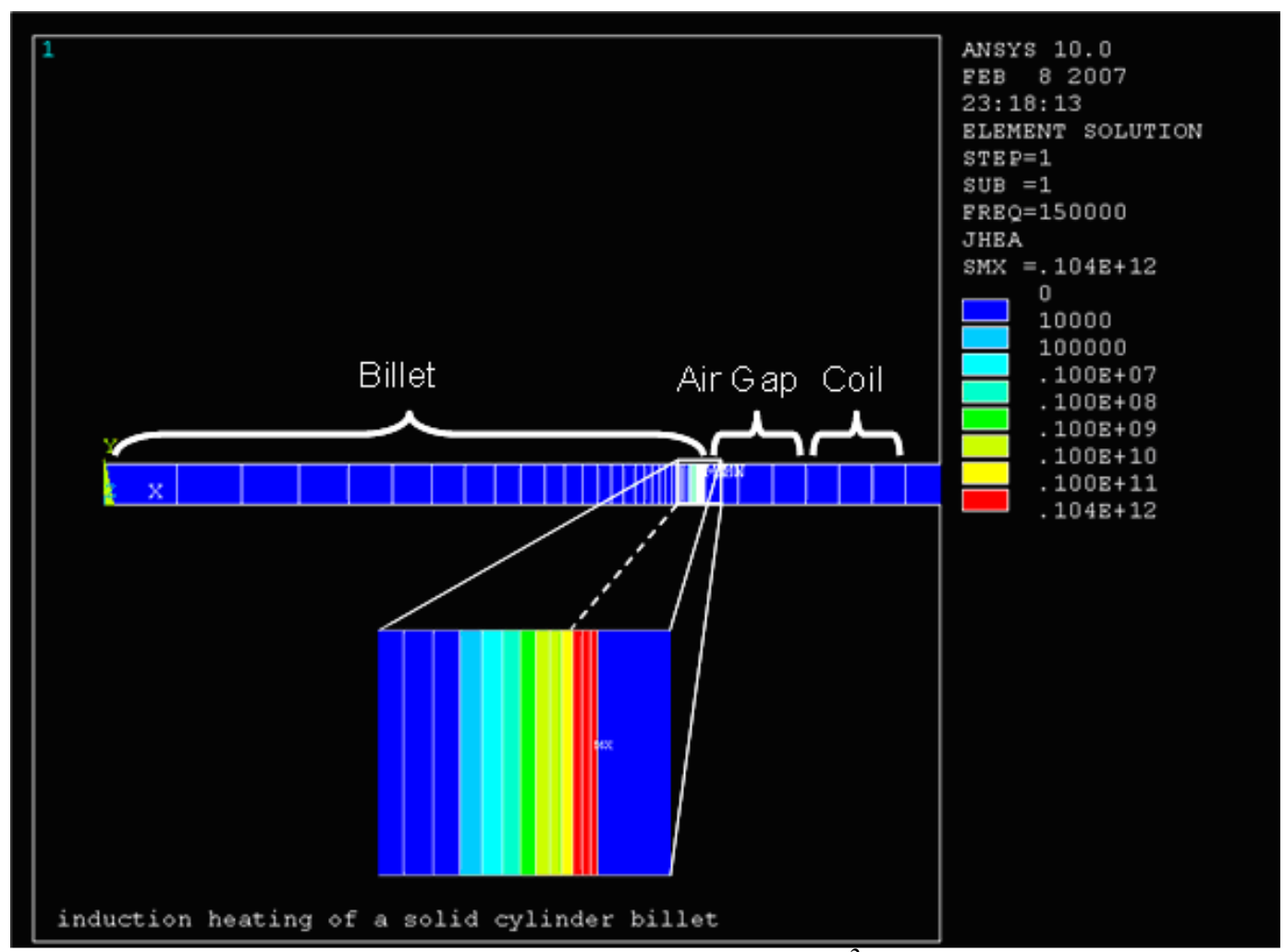

Figure 3.9: Two-dimensional contour plot showing the $I^{2} R$ power generation within the heated structure of the ANSYS example model. The power density magnitude decayed from that at the outer edge by approximately an order of magnitude in 1.5 skin depths, and by two orders of magnitude for each skin depth thereafter. 
peak value after one skin depth (two elements), consistent with the skin effect equation which predicted a drop of $63 \%$. The outermost current rings propagated in a direction opposite that of the coil due to the requirement of field cancellation as was discussed in Section 2.1. The ANSYS result was strange, however, in that although the current magnitudes decayed toward the center as expected, they switched direction after the first four elements (two skin depths), thus initiating the oscillatory pattern as exhibited in the other electromagnetic variables. Figure 3.9 shows the power density generated via $I^{2} R$ Joule heating. The quantitative analysis that follows will show that the power density within the billet was proportional to the square of the current density by a factor nearly identical to its resistivity. With a predicted decay rate of twice that of the current density $\left(\exp ^{-2}\right)$, the results were once again consistent with theory in that the power density magnitude dropped by an order of magnitude after 1.5 skin depths ( 3 elements) whereas theory predicted a $95 \%$ reduction at that location. The Joule heat values were the final piece of the electromagnetic puzzle; they were input directly into the thermal model to generate temperature values. Figures 3.10(a,b) show the final temperature distribution obtained after executing the full simulation provided by ANSYS. The results are consistent with the temperature-vs-time plot in Figure 3.5, in which the temperature ranged from a maximum of $743^{\circ} \mathrm{C}$ at the outer edge to a minimum of approximately $240^{\circ} \mathrm{C}$ at the center. Increasing the simulation time further showed that the peak temperature failed to increase beyond $750^{\circ} \mathrm{C}$, whereas the minimum temperature continued to slowly rise due to thermal conduction. Equalization was not attainable due to limitations in computer memory. 

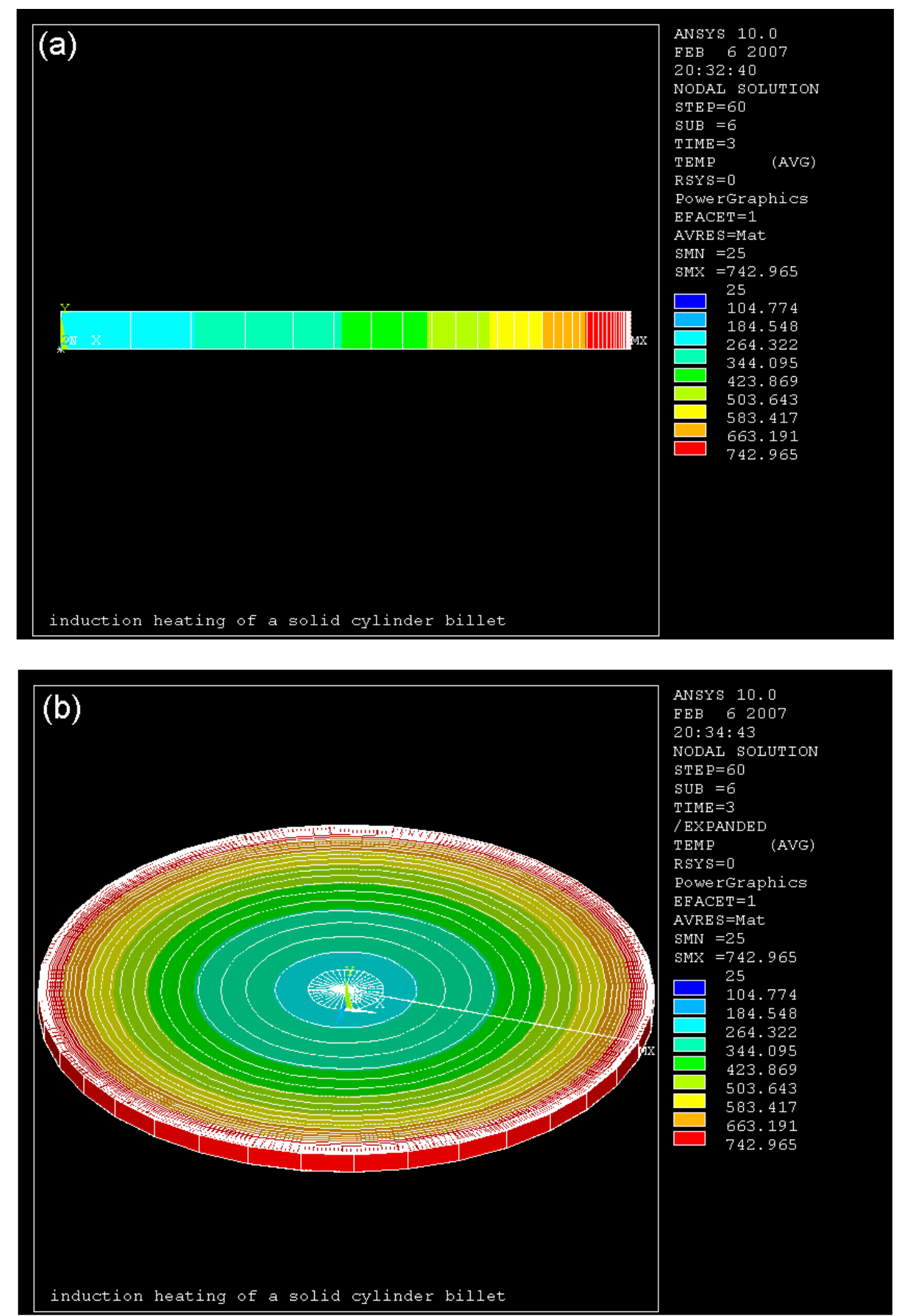

Figure 3.10: Contour plots shown in (a) two dimensions, and (b) three dimensions illustrating the final temperature distribution in the ANSYS model following the execution of the coupled simulation. After three seconds of heating, the temperature values ranged from $743^{\circ} \mathrm{C}$ at the outer edge to approximately $240^{\circ} \mathrm{C}$ at the center. 
In order to compare the results of the simulation with the theorized relationships of Section 2.1, variations of the model were executed in which selected adjustments were made to the field frequency, coil current density, magnetic permeability values, billet temperature, and billet dimensions. All purely electromagnetic models were executed assuming a uniform temperature of $100^{\circ} \mathrm{C}$ unless otherwise indicated, which was the starting temperature of the coupled simulation, and only one variable was altered at a time. Table 3.2 summarizes the results of varying the harmonic frequency from 50$450 \mathrm{kHz}$. The coil current density remained fixed at $1.5 \mathrm{E} 7 \mathrm{~A} / \mathrm{m}^{2}$ (or $37.5 \mathrm{~A}$ ) and all material properties and structural dimensions left at default. The magnetic field strength and magnetic flux density were unaffected by the changes in frequency, despite the change in element sizes within the billet due to the different SkinD calculations, thus confirming that the mesh was not a limiting factor in the simulation results. Figure 3.11(a) shows a graph of the power dissipation values with respect to frequency, where the blue curve represented the peak power density as measured at the outer edge of the billet, and the pink curve represented the time-average power dissipation in the full structure. Equation (2.6a) predicted that the total power dissipation would vary inversely with the skin depth, which in turn varies inversely with the square root of frequency.

\begin{tabular}{llllll}
\hline \hline \multicolumn{5}{c}{ TABLE 3.2 } \\
\hline $\begin{array}{l}\text { Frequency } \\
(\mathrm{kHz})\end{array}$ & $\begin{array}{l}\text { Peak Current } \\
\begin{array}{l}\text { Density } \\
\left(\mathrm{A} / \mathrm{m}^{2}\right)\end{array}\end{array}$ & $\begin{array}{l}\text { Peak Power } \\
\text { Density } \\
\left(\mathrm{W} / \mathrm{m}^{3}\right)\end{array}$ & $\begin{array}{l}\text { Total } \\
\text { Power }^{*}(\mathrm{~W})\end{array}$ & $\begin{array}{l}\text { Temp at } \\
\mathrm{T}=3 \mathrm{~s}\left({ }^{\circ} \mathrm{C}\right)\end{array}$ & $\begin{array}{l}\text { Time to } \\
700^{\circ} \mathrm{C}(\mathrm{s})\end{array}$ \\
\hline 50 & $4.25 \mathrm{E} 8$ & $3.39 \mathrm{E} 10$ & 209.8 & 614.1 & 3.8 \\
100 & $6.04 \mathrm{E} 8$ & $6.86 \mathrm{E} 10$ & 297.3 & 727.7 & 2.1 \\
150 & $7.42 \mathrm{E} 8$ & $1.04 \mathrm{E} 11$ & 364.4 & 743 & 1.45 \\
300 & $1.05 \mathrm{E} 9$ & $2.06 \mathrm{E} 11$ & 516 & 757.3 & 0.8 \\
450 & $1.29 \mathrm{E} 9$ & $3.10 \mathrm{E} 11$ & 632.5 & 765 & 0.55 \\
\hline \hline
\end{tabular}

* Electromagnetic model was run assuming a uniform temperature of $100^{\circ} \mathrm{C}$. 
Solving the equation in terms of frequency, the total power dissipation, $P(\mathrm{~W})$, was expected to behave as

$$
P=\frac{1.16 H_{0}^{2} a t \pi^{1.5} \sqrt{\mu}}{\sqrt{\sigma}} \sqrt{f}
$$

The power density values reported by ANSYS were given in terms of $\mathrm{W} / \mathrm{m}^{3}$, reflecting the three-dimensionality of the axisymmetric simulation. The as-drawn two-dimensional elements were converted to volumetric entities through rotation about the y-axis, giving each element a total size of $(2 \pi \mathrm{a})$ *height*width. The widths of the elements (xdimension) within the billet were proportional to the maximum skin depth, with the width of the smallest element (the location of maximum power density) equal to $0.5 \delta$, and the heights were all equal to $0.001 \mathrm{~m}$. The power density, $P_{D}$, was predicted to vary as

$$
P_{D}=\frac{0.63 P}{V_{E}}=\frac{0.63 P}{(0.5 \delta t)(2 \pi a)}=0.63\left[1.16 H_{0}^{2} \pi \mu\right] f .
$$

where the factor 0.63 was added by recognizing that the power decayed as $\exp ^{-2 r / \delta}$ and thus approximately $63 \%$, or $1-\exp ^{-\left(2^{*} 0.58 / \delta\right)}$, of the power dissipation occurred in this outermost element. Microsoft Excel was utilized to fit a power regression to each of the two curves, and the resulting trends were confirmed to vary with $f^{d / 2}$ and $f^{l}$, respectively. To analyze the coefficients, the values utilized by the simulation for material properties and structural dimensions were entered, along with the $38 \mathrm{kA} / \mathrm{m}$ field strength obtained from Figure 3.6. The results, shown in Table 3.3, were in close proximity to the simulated values, with offsets of 1.20 and 1.27 , respectively. The data was also utilized to examine the relationship between induced power density and induced current density. The graphed data is given in Figure 3.11(b). Since both terms were density values, their relationship was unaffected by element sizing. Fitting a power curve to the data, the variables were 


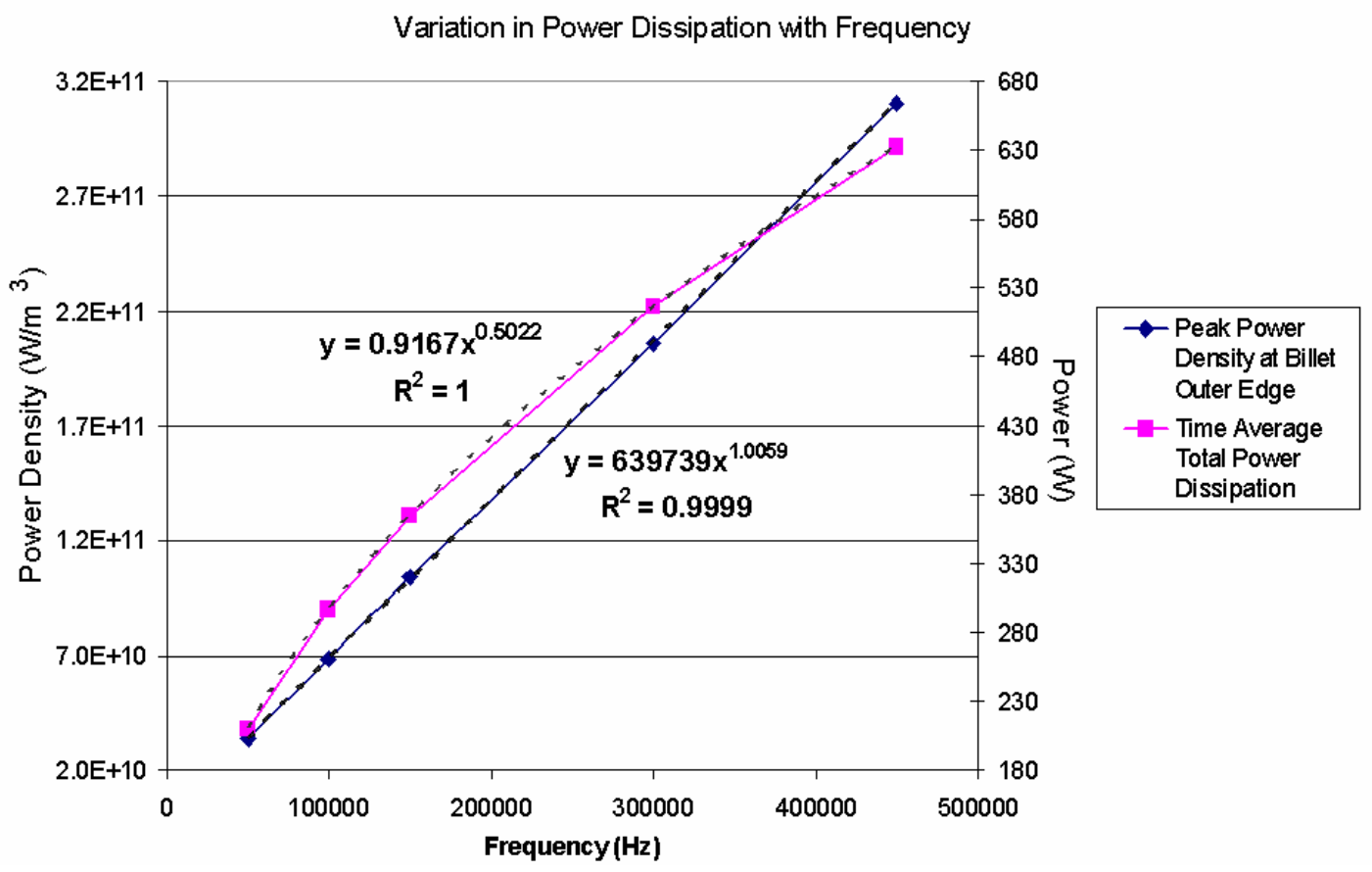

Figure 3.11(a): Graph showing the impact of frequency on the induced power density (blue) and total power dissipation (pink). The total power varied with the square root of frequency as predicted by Equation (2.6a). The power density exhibited a linear behavior due to the simultaneous change in element size.

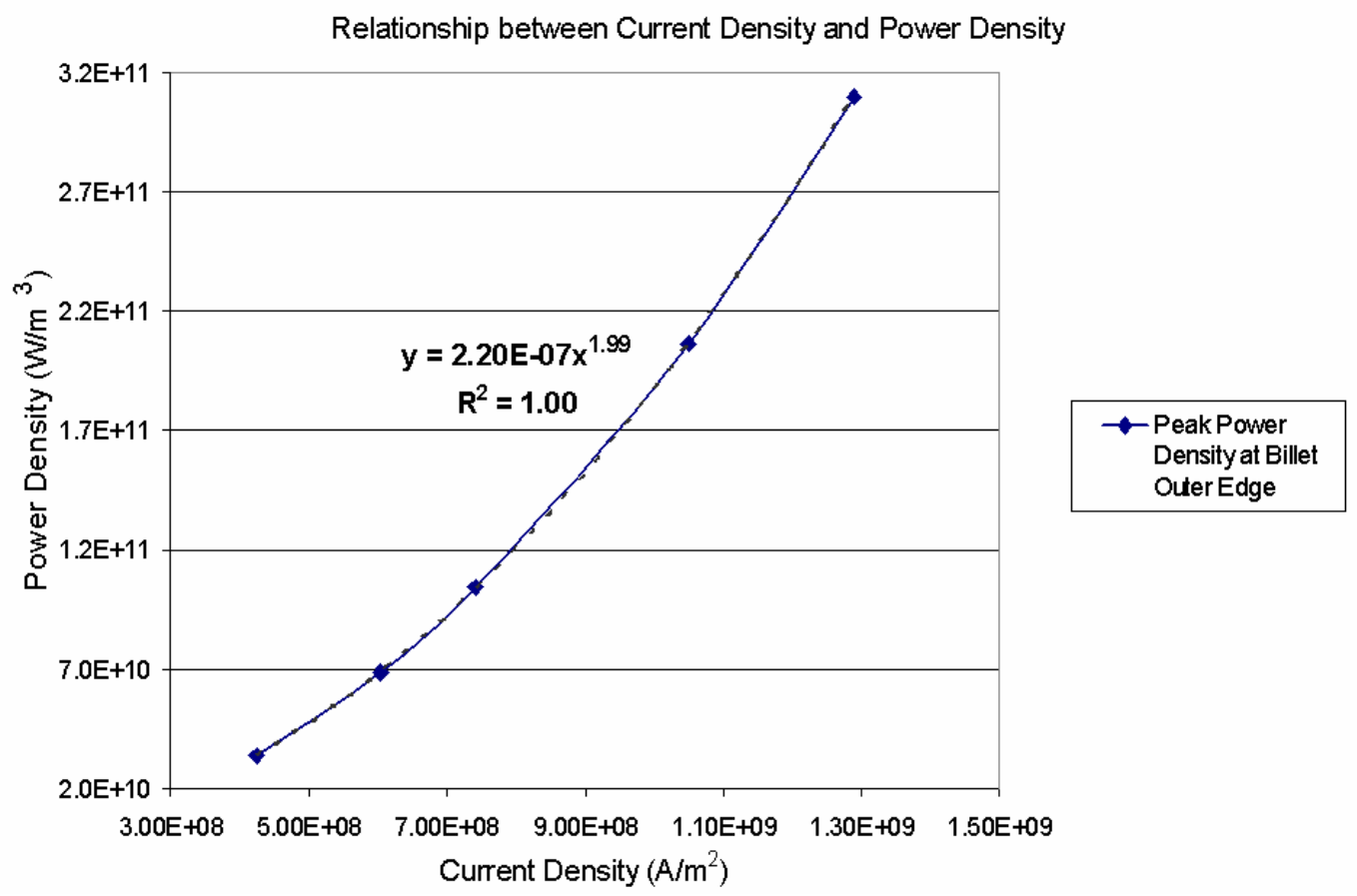

Figure 3.11(b): Relationship between induced power density and induced current density for frequency values ranging from $50-450 \mathrm{kHz}$. The power density varied as the square of the current density, with the constant of proportionality approximately equal to the resistivity of the heated structure $\left(\rho=2.54 \mathrm{E}-7 \Omega-\mathrm{m}\right.$ at $\left.100^{\circ} \mathrm{C}\right)$. 


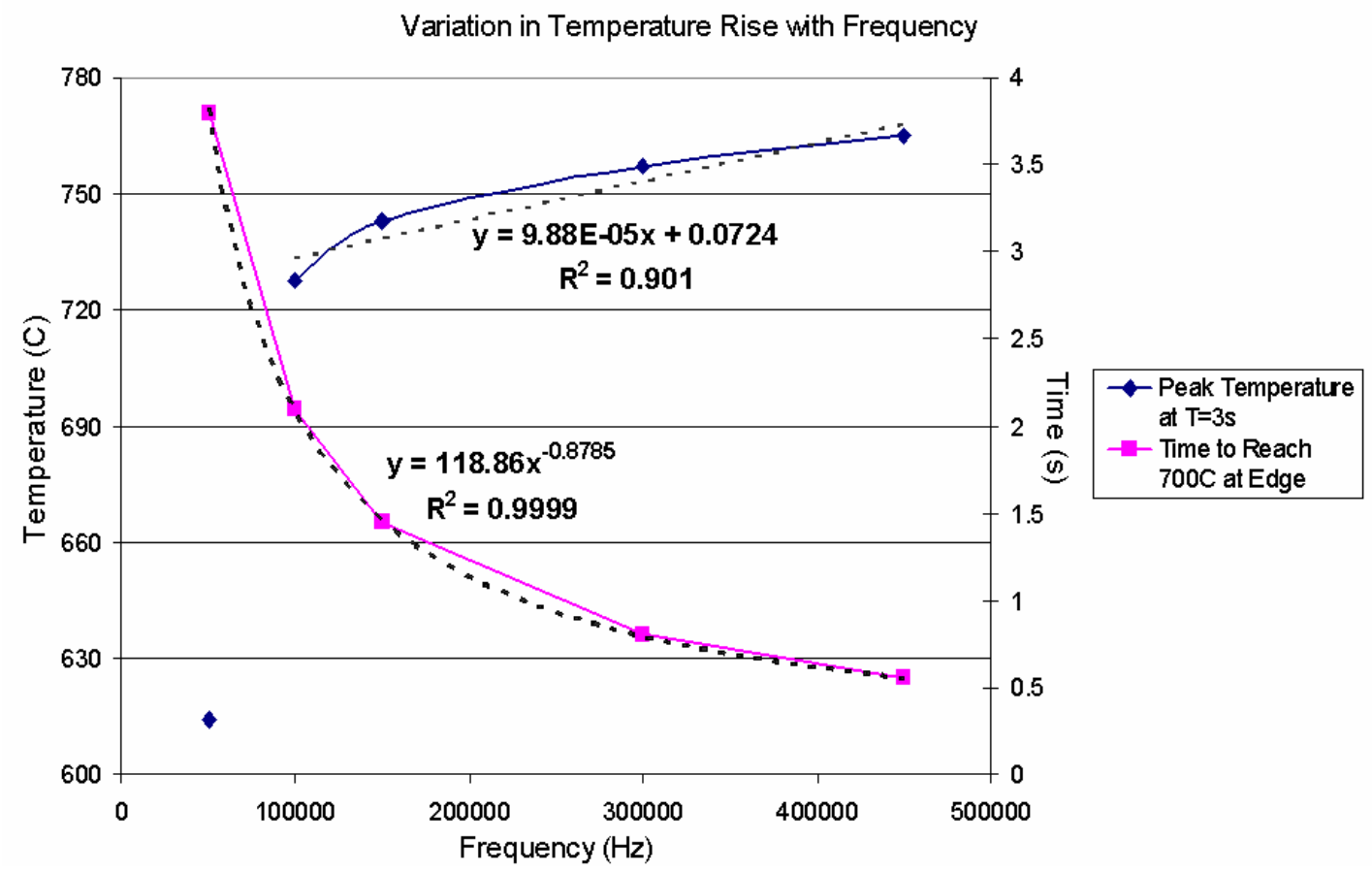

Figure 3.11(c): Curves showing the variation in temperature rise with frequency for the example ANSYS model. The temperature after a three-second simulation (blue) exhibited a transition temperature at around $700^{\circ} \mathrm{C}$, which was believed to be due to the Curie point. The time requirement to reach $700^{\circ} \mathrm{C}$ decreased (pink) had an exponent in close proximity to the theoretical value.

confirmed to exhibit a squared relationship. Furthermore, the proportionality constant (2.20E-7) was almost identical to the resistivity of the steel billet $(2.54 \mathrm{E}-7 \Omega-\mathrm{m})$, thus confirming the $I^{2} R$ expectation. Lastly, the impact of field frequency on the results of the full coupled model was studied, with the results described in Figure 3.11(c). The blue curve shows the final temperature value after the three-second simulation, and the pink curve shows the time required to reach an edge temperature of $700^{\circ} \mathrm{C}$. Based on Equation (2.14) and the trending of the power density (which the transient thermal model utilized to compute the temperature values) with the first power of the frequency, the anticipated behavior was expected to follow

$$
P_{D} \propto f \propto \frac{m s \Delta T}{t}
$$


Thus, for a constant time (i.e., 3 seconds) the temperature rise was expected to be linear with $f$, and for a constant temperature rise (i.e., $\Delta \mathrm{T}=700-100^{\circ} \mathrm{C}$ ), the time requirement was expected to vary inversely with $f$. As the figure shows, the temperature rise exhibited somewhat of a piecewise-linear result. This leveling off was likely caused by the sharp drop in permeability at approximately $720^{\circ} \mathrm{C}$ due to the billet having reached its Curie point; a similar transition was observed in this range for the other analyzed parameters. Within the relatively narrow temperature range, however, the trend showed good linearity. The time produced an exponent of -0.8789 , relatively close to the -1.0 expectation. The magnitudes of the coefficients were not analyzed, as the density and specific heat of the billet would have been required to compare them with Equation (2.14). The complete data set for the frequency analysis is summarized in Table 3.3. Overall, the induction heating trends followed the theoretical behavior with frequency very well, with all evaluated trends within $32 \%$ of the theoretical prediction. Thus, the analysis of Chapter 2 was well supported.

\begin{tabular}{|c|c|c|c|c|c|}
\hline \multicolumn{6}{|c|}{$\begin{array}{c}\text { TABLE } \mathbf{3 . 3} \\
\text { AND SIMULATED FREOUENCY TRENDS F }\end{array}$} \\
\hline Variable & $\begin{array}{l}\text { Theoretical } \\
\text { Exponent }\end{array}$ & $\begin{array}{l}\text { Simulated } \\
\text { Exponent }\end{array}$ & $\begin{array}{l}\text { Theoretical } \\
\text { Coefficient }\end{array}$ & $\begin{array}{l}\text { Simulated } \\
\text { Coefficient }\end{array}$ & $\begin{array}{l}\text { Coefficient } \\
\text { Offset }\end{array}$ \\
\hline $\begin{array}{l}\text { Total Power vs } \\
\text { Frequency }\end{array}$ & 0.5 & 0.502 & 1.10 & 0.917 & 1.20 \\
\hline $\begin{array}{l}\text { Peak Power } \\
\text { Density vs } \\
\text { Frequency }\end{array}$ & 1 & 1.01 & $8.10 \mathrm{E} 5$ & $6.40 \mathrm{E} 5$ & 1.27 \\
\hline $\begin{array}{l}\text { Peak Power } \\
\text { Density vs Peak } \\
\text { Current Density }\end{array}$ & 2 & 1.99 & $2.54 \mathrm{E}-7$ & $2.20 \mathrm{E}-7$ & 1.15 \\
\hline $\begin{array}{l}\text { Peak } \\
\text { Temperature vs } \\
\text { Frequency }\end{array}$ & 1 & 1.00 & $\mathrm{~N} / \mathrm{A}$ & $9.88 \mathrm{E}-5$ & N/A \\
\hline $\begin{array}{l}700 \mathrm{C} \text { Time vs } \\
\text { Frequency }\end{array}$ & -1 & -0.879 & N/A & 119 & N/A \\
\hline
\end{tabular}




\begin{tabular}{|c|c|c|c|c|c|c|c|}
\hline \multicolumn{8}{|c|}{ Table 3.4} \\
\hline $\begin{array}{l}\text { Coil } \\
\text { Current } \\
\text { (A) }\end{array}$ & $\begin{array}{l}\text { Field } \\
\text { Strength } \\
(\mathrm{A} / \mathrm{m})\end{array}$ & $\begin{array}{l}\text { Peak Flux } \\
\text { Density } \\
\text { (T) }\end{array}$ & $\begin{array}{l}\text { Peak } \\
\text { Current } \\
\text { Density } \\
\left(\mathrm{A} / \mathrm{m}^{2}\right)\end{array}$ & $\begin{array}{l}\text { Peak } \\
\text { Power } \\
\text { Density } \\
\left(\mathrm{W} / \mathrm{m}^{3}\right)\end{array}$ & $\begin{array}{l}\text { Total } \\
\text { Power } \\
(\mathrm{W})\end{array}$ & $\begin{array}{l}\text { Temp } \\
\text { at } \\
\mathrm{T}=3 \mathrm{~s} \\
\left({ }^{\circ} \mathrm{C}\right)\end{array}$ & $\begin{array}{l}\text { Time to } \\
700^{\circ} \mathrm{C} \\
\text { (s) }\end{array}$ \\
\hline 18.75 & 18946 & 3.516 & $3.71 \mathrm{E} 8$ & $2.95 \mathrm{E} 10$ & 91.1 & 145.2 & 12 \\
\hline 37.50 & 37892 & 7.031 & 7.42E8 & $1.04 \mathrm{E} 11$ & 364.4 & 743 & 1.45 \\
\hline 75.00 & 75784 & 14.062 & $1.48 \mathrm{E} 9$ & $4.14 \mathrm{E} 11$ & 1547.6 & 805.2 & 0.15 \\
\hline 112.50 & 113676 & 21.093 & $2.23 \mathrm{E} 9$ & $9.32 \mathrm{E} 11$ & 3279.7 & 1108 & 0.05 \\
\hline 150.00 & 151568 & 28.124 & $2.97 \mathrm{E} 9$ & $1.66 \mathrm{E} 12$ & 5830.5 & 1696 & 0.02 \\
\hline
\end{tabular}

* Electromagnetic model was run assuming a uniform temperature of $100^{\circ} \mathrm{C}$.

The second set of variations focused on the current applied to the magnetic coil. The current densities were varied from $7.5 \mathrm{E} 6 \mathrm{~A} / \mathrm{m}^{2}$ to $60 \mathrm{E} 6 \mathrm{~A} / \mathrm{m}^{2}$, which equated to a range of $18.75-150 \mathrm{~A}$. The harmonic frequency and material properties were standardized at their default values. Table 3.4 summarizes the numerical results. In this case, the magnetic field strength and flux density values were affected, which was anticipated as per Equation (3.3). Figures 3.12(a) illustrates their simulated trends, with power curves again fitted by Microsoft Excel. Both the field and flux density exhibited a high degree of linearity with coil current, consistent with the equation. The anticipated coefficients for $H_{Y}$ and $B_{Y}$ were

$$
\begin{aligned}
& H_{Y} \rightarrow \frac{1}{2 R} \\
& B_{Y} \rightarrow \frac{\mu}{2 R},
\end{aligned}
$$

where $R(\mathrm{~m})$ represented the radius of the coil and $\mu(\mathrm{H} / \mathrm{m})$ was the magnetic permeability of the heated billet. Substituting the values gave theoretical coefficients of 28.6 and $6.98 \mathrm{E}-3$, respectively, which were offset by factors of 34.5 and 26.9 from the simulated coefficients. Investigation into the means by which ANSYS computed the 
magnetic field revealed that the magnitude was completely independent of the coil radius, and instead followed Equation (2.11), the equation for a solenoid coil, with 1 turn and a total length of $0.001 \mathrm{~m}$ (the thickness of the ring), an unexpected result as the magnetic vector potential followed the equation for a single loop much more closely. The theoretical coefficients were thus changed to

$$
\begin{aligned}
H_{Y} & \rightarrow \frac{N_{C}}{\ell_{C}} \\
B_{Y} & \rightarrow \frac{\mu N_{C}}{\ell_{C}}
\end{aligned}
$$

where $N_{C} / l_{C}$ evaluated to exactly 1000 . The new coefficients yielded values of 1000 and 0.244 , respectively, both of which were very close to the simulated coefficients. The theoretical and simulated values for the exponents and coefficients are summarized in

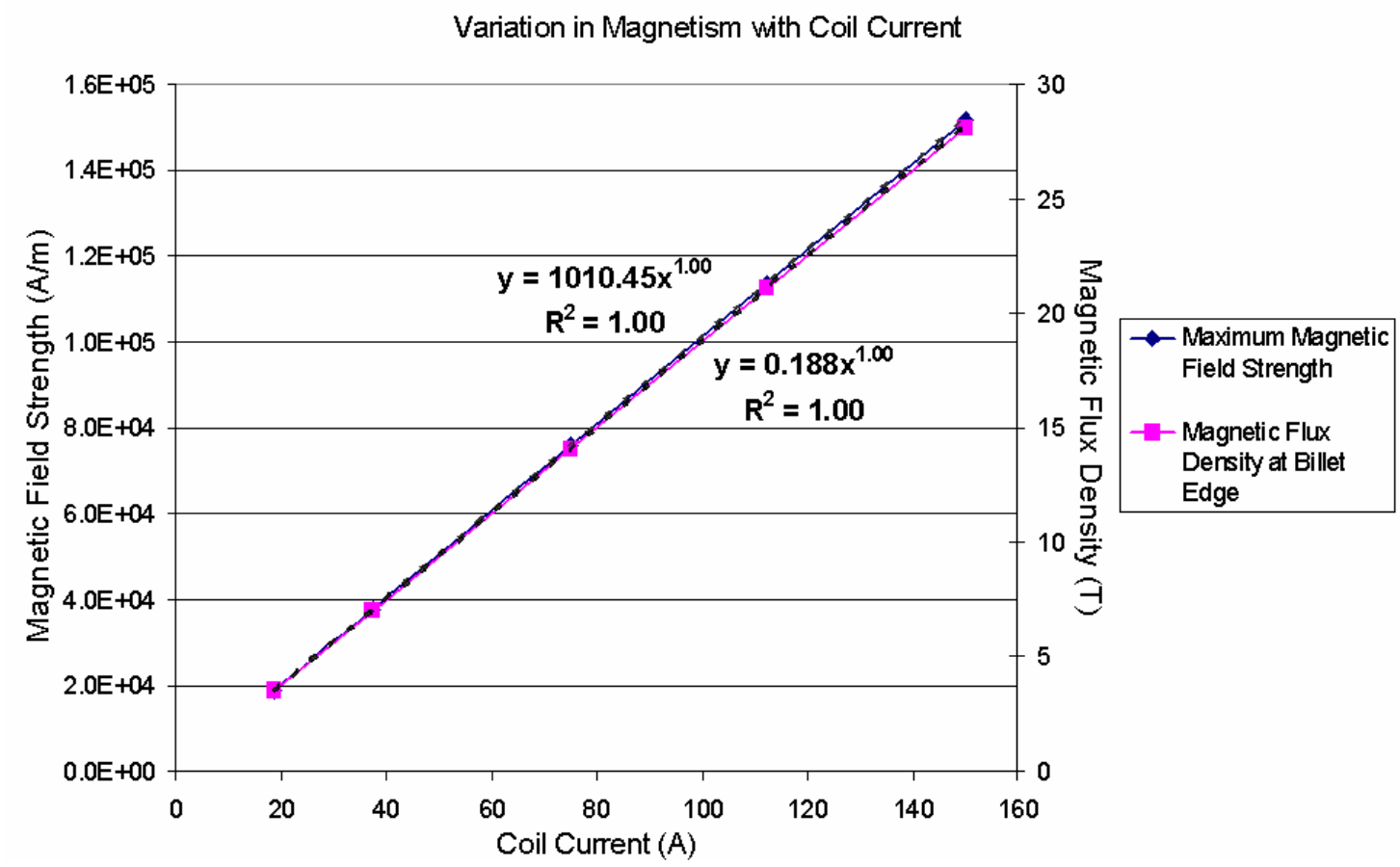

Figure 3.12(a): Graph of the magnetic field strength (blue) and magnetic flux density (pink) with varying current levels applied to the magnetic coil. Both dependent variables exhibited a linear relationship with coil current as anticipated, and their coefficients were well matched to the expected magnetic field in a solenoid. 
Table 3.5. Figures 3.12(b,c) summarize the effect of coil current on the induced current and power values within the heated structure. The anticipated relationships were again derived from Equation (2.6a), combined with the skin depth relation (Equation (2.3)) and the formula for the field induced in a solenoid (Equation (2.10)). The anticipated behavior of the total power dissipation with respect to coil current, $I_{C}(\mathrm{~A})$, was derived as

$$
P=\frac{1.16 \pi a N^{2}}{\sigma \delta t} I_{C}^{2},
$$

The relation for the peak power density was again obtained by dividing by the volume of the outermost billet element and scaling by 0.63 to account for skin depth, resulting in

$$
P_{D}=0.63 \frac{1.16 N^{2}}{\sigma \delta^{2} t^{2}} I_{C}^{2} .
$$

Lastly, $P_{D}$ was replaced with $J^{2} \rho$ as per the analysis of Figure 3.11(b), giving the result

$$
J=\frac{N \sqrt{0.63 * 1.16}}{\delta t} I_{C}
$$

The power regressions fit to the curves in Figures 3.12(b,c) confirmed that the power and power density both varied with the square of the coil current whereas the induced current density varied linearly with $I_{C}$. Substituting the values utilized to build the simulation yielded the coefficients shown in Table 3.5. All results were once again highly consistent with the simulation, with a maximum offset of 1.15 from the simulated value. Finally, the rate of temperature rise with increasing coil current is illustrated in Figure 3.12(d), where the temperature at the edge of the billet after three seconds is shown in blue and the time to reach an edge temperature of $700^{\circ} \mathrm{C}$ is shown in pink. Modifying Equation (3.8) using the squared relationship between coil current and power density gave the result

$$
P_{D} \propto I_{C}^{2} \propto \frac{m s \Delta T}{t}
$$




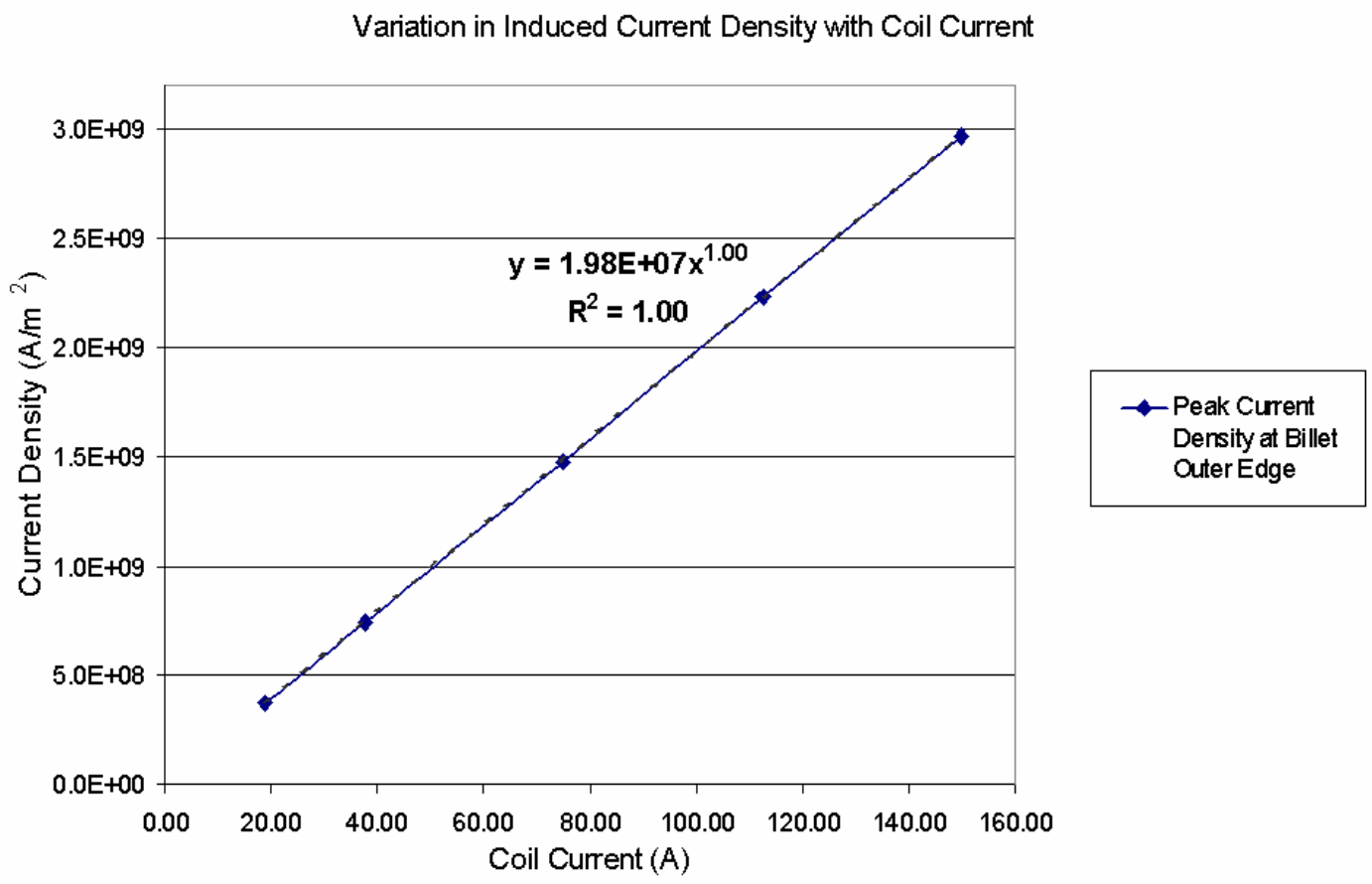

Figure 3.12(b): Relationship between the impressed coil current and the induced current density in the inductively heated ANSYS model. The two variables exhibited a linear relationship as predicted by Equation (3.11a) with a well-matched coefficient. Thus the coil-billet system could be considered a quasi-transformer.

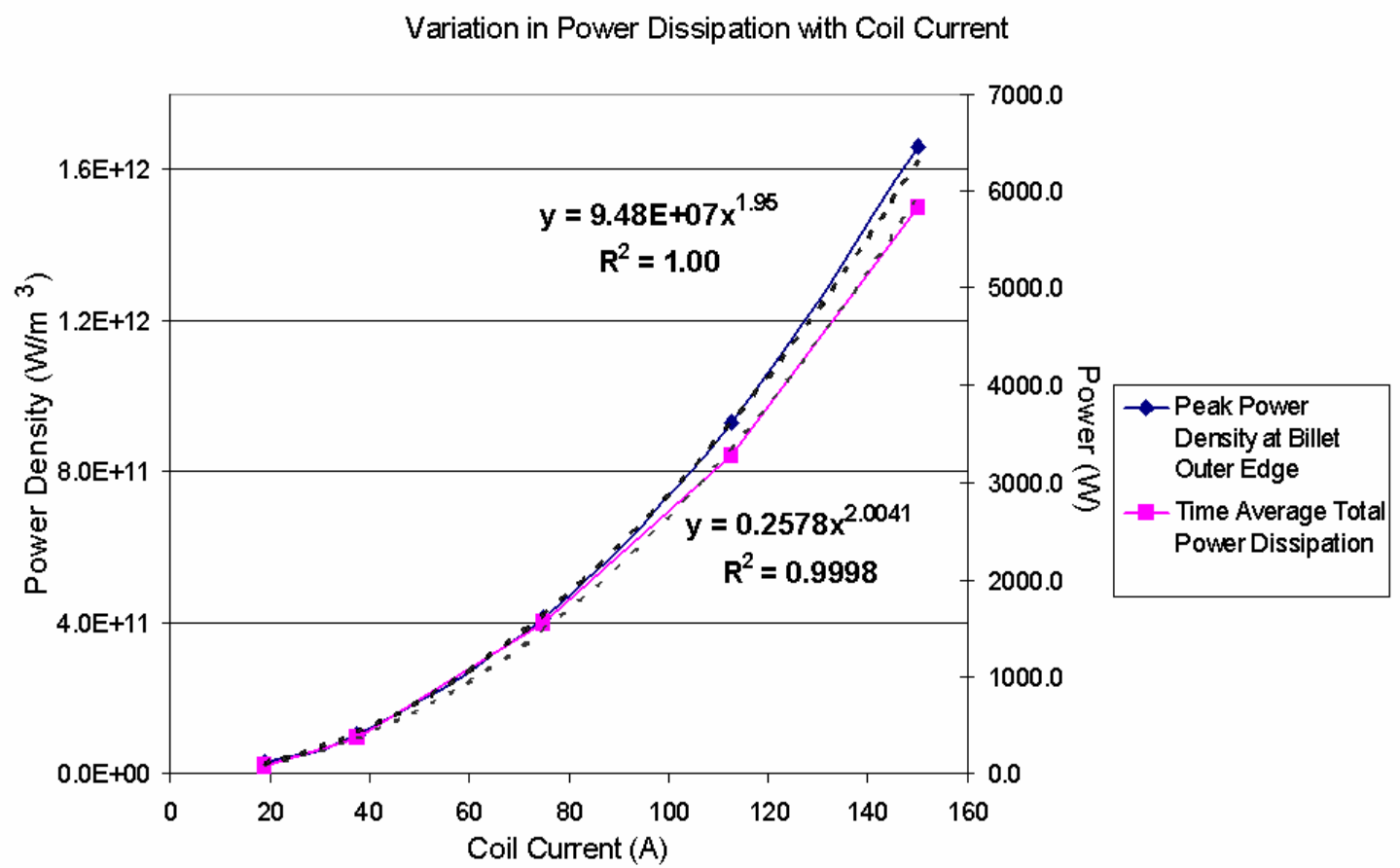

Figure 3.12(c): Graph showing the maximum induced power density at the outer edge of the heated billet (blue) and total dissipated power in the billet (pink) with respect to the impressed coil current. Both quantities varied with the square of the coil current, consistent with the theoretical prediction. 


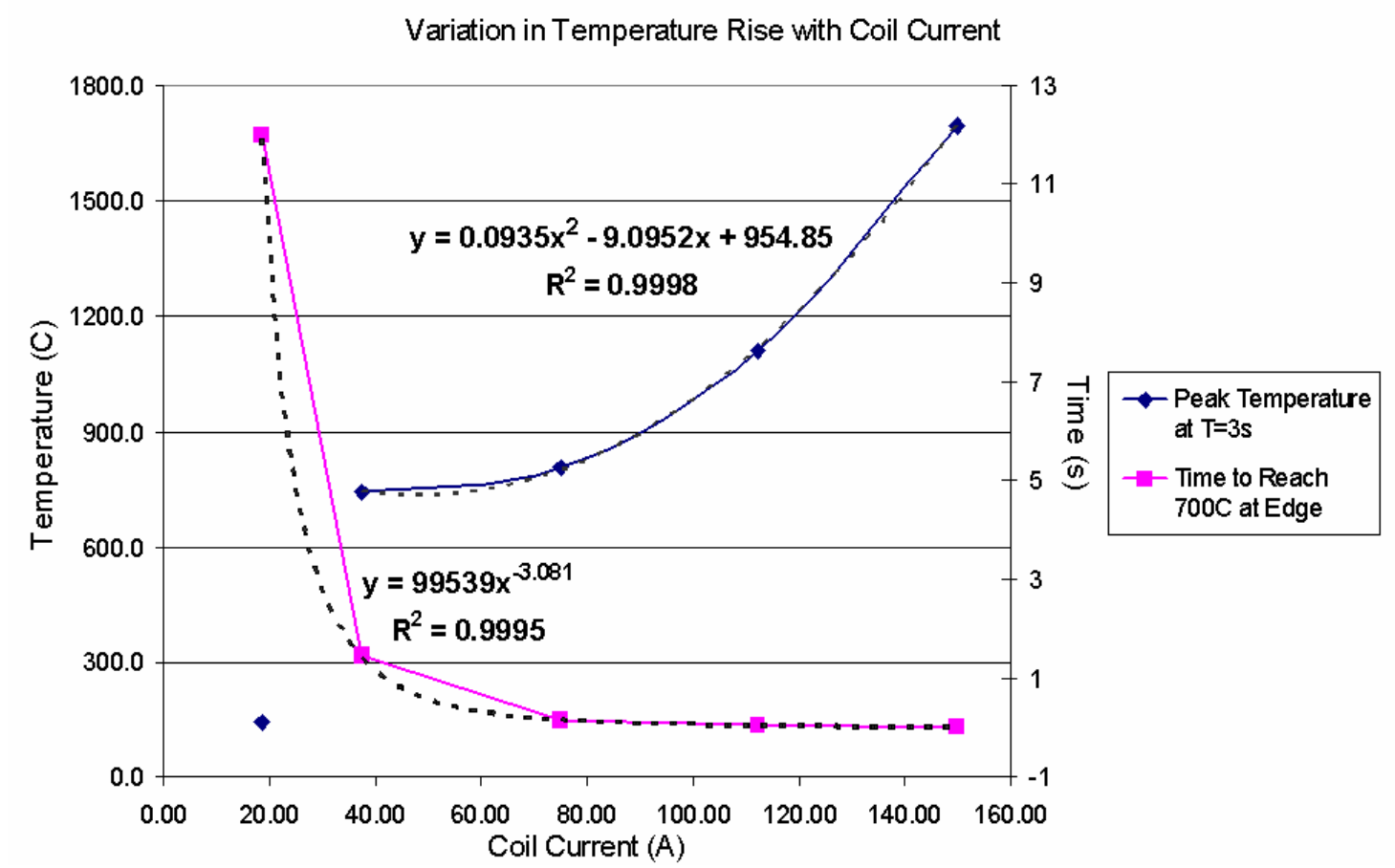

Figure 3.12(d): Variation in temperature rise in the heated structure with increasing current applied to the magnetic coil. The peak temperature in the billet three seconds into the simulation (blue) exhibited a parabolic shape at temperatures above $700^{\circ} \mathrm{C}$. The time required to reach $700^{\circ} \mathrm{C}$ (pink) decayed as approximately $I_{C}{ }^{-3}$.

and thus the temperature and time curves were anticipated to vary with $I_{C}{ }^{2}$ and $I_{C}{ }^{-2}$, respectively. The graph shows that once again the peak temperatures followed the predicted shape for points above $700^{\circ} \mathrm{C}$, with both linear and constant offsets, but exhibited a transition below this temperature, believed to be due to the $\sim 720^{\circ} \mathrm{C}$ Curie temperature of the heated billet. The time requirement for the temperature to rise to $700^{\circ} \mathrm{C}$ was found to vary inversely with the cube of the coil current rather than its square as predicted, not an exact match with theory but within reason. Again, the evaluation of the theoretical coefficients and comparison with simulation was beyond the scope of this work. The theoretical electromagnetic coefficients, however, were once again shown to be highly accurate. 
TABLE 3.5

THEORETICAL AND SimUlated COIL CURRENT TRENDS FOR THE ANSYS MODEL

\begin{tabular}{llllll}
\hline Variable & $\begin{array}{l}\text { Theoretical } \\
\text { Exponent }\end{array}$ & $\begin{array}{l}\text { Simulated } \\
\text { Exponent }\end{array}$ & $\begin{array}{l}\text { Theoretical } \\
\text { Coefficient }\end{array}$ & $\begin{array}{l}\text { Simulated } \\
\text { Coefficient }\end{array}$ & $\begin{array}{l}\text { Coefficient } \\
\text { Offset }\end{array}$ \\
\hline $\begin{array}{l}\text { Magnetic Field } \\
\text { vs Current }\end{array}$ & 1 & 1.00 & 1000 & 1010 & 0.990 \\
$\begin{array}{l}\text { Flux Density } \\
\text { vs Current }\end{array}$ & 1 & 1.00 & 0.244 & 0.188 & 1.23 \\
$\begin{array}{l}\text { Current } \\
\text { Density vs }\end{array}$ & 1 & 1.00 & $1.82 \mathrm{E} 7$ & $1.98 \mathrm{E} 7$ & 0.919 \\
$\begin{array}{l}\text { Current } \\
\text { Total Power vs }\end{array}$ & 2 & 2.00 & 0.296 & 0.258 & 1.15 \\
$\begin{array}{l}\text { Current } \\
\text { Peak Power }\end{array}$ & 2 & 1.95 & $8.42 \mathrm{E} 7$ & $9.48 \mathrm{E} 7$ & 0.888 \\
$\begin{array}{l}\text { Density vs } \\
\text { Current }\end{array}$ & & & & & \\
$\begin{array}{l}\text { Peak Temp vs } \\
\text { Current }\end{array}$ & 2 & 2 & N/A & $9.35 \mathrm{E}-2$ & N/A \\
$\begin{array}{l}700 C \text { Time vs } \\
\text { Current }\end{array}$ & -2 & -3.08 & N/A & $99.5 \mathrm{E} 3$ & N/A \\
\hline
\end{tabular}

A key component of the thin film inductive annealing process was the manipulation of relative permeability values among the constituent materials of a microsystem device. Figures 3.13(a-c) illustrate the behavior of the variables of interest with respect to changes in magnetic permeability, where the temperature-dependent magnetic permeability values in the billet were scaled from one-fourth their original value to double, equating to $100^{\circ} \mathrm{C}$ relative permeability values of $48.6-389$. The

\section{TABLE 3.6}

Variation of Magnetic Permeability Values In the ANSYS Model

\begin{tabular}{lllllll}
\hline $\begin{array}{l}\text { Relative } \\
\text { Permeability } \\
\text { Factor }\end{array}$ & $\begin{array}{l}\text { Peak Flux } \\
\text { Density } \\
(\mathrm{T})\end{array}$ & $\begin{array}{l}\text { Peak } \\
\text { Current } \\
\begin{array}{l}\text { Density } \\
\left(\mathrm{A} / \mathrm{m}^{2}\right)\end{array}\end{array}$ & $\begin{array}{l}\text { Peak } \\
\text { Power } \\
\text { Density } \\
\left(\mathrm{W} / \mathrm{m}^{3}\right)\end{array}$ & $\begin{array}{l}\text { Total } \\
\text { Power }^{*} \\
(\mathrm{~W})\end{array}$ & $\begin{array}{l}\text { Temp at } \\
\mathrm{T}=3 \mathrm{~s}\left({ }^{\circ} \mathrm{C}\right)\end{array}$ & $\begin{array}{l}\text { Time to } \\
700^{\circ} \mathrm{C}(\mathrm{s})\end{array}$ \\
\hline 0.25 & 2.018 & $3.90 \mathrm{E} 8$ & $3.19 \mathrm{E} 10$ & 179.6 & 190.6 & 4.8 \\
0.5 & 3.816 & $5.41 \mathrm{E} 8$ & $5.84 \mathrm{E} 10$ & 255.3 & 718 & 2.7 \\
1 & 7.031 & $7.42 \mathrm{E} 8$ & $1.04 \mathrm{E} 11$ & 364.4 & 743 & 1.45 \\
2 & 13.97 & $1.05 \mathrm{E} 9$ & $2.06 \mathrm{E} 11$ & 516.3 & 757.4 & 0.75 \\
\hline \hline
\end{tabular}

* Electromagnetic model was run assuming a uniform temperature of $100^{\circ} \mathrm{C}$. 


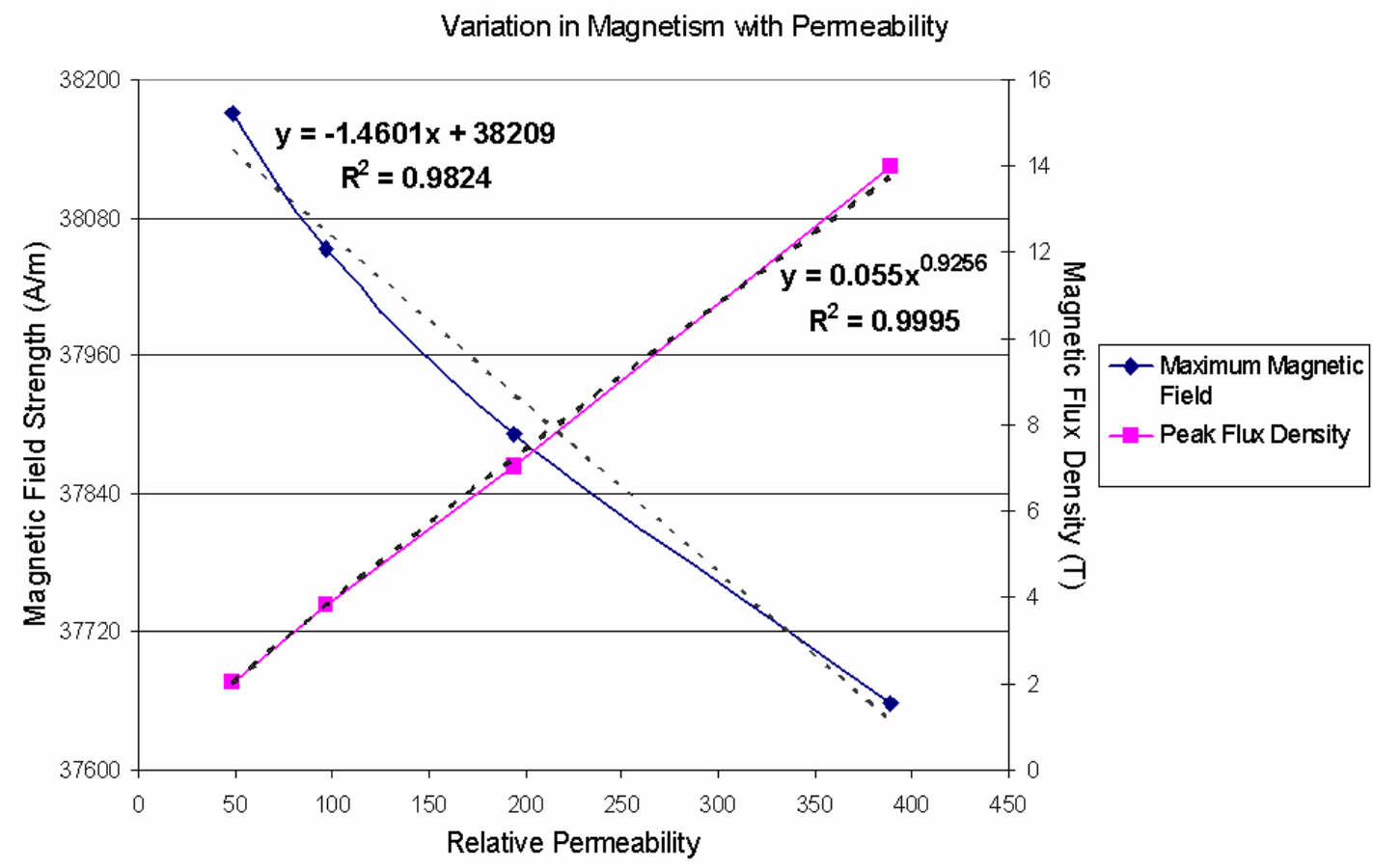

Figure 3.13(a): Variation in the magnetic field strength (blue) and the flux density at the outer edge of the heated billet (pink) with changes in magnetic permeability. The field strength showed little change, ranging from $37.7-38.2 \mathrm{~A} / \mathrm{m}$ over the $8 \mathrm{X}$ permeability range utilized. The flux density varied approximately linearly as was expected.

frequency and coil current density were standardized at their default values, and the electrical conductivity was not modified. The data utilized to generate the graphs is provided in Table 3.6. The impact on the free-space magnetic field, shown in Figure 3.13(a), was expected to be minimal if any. The slight decrease observed in field strength with increasing permeability was likely caused by the increased power consumption in the structure with respect to the free space ambient. The magnetic flux density, on the other hand, was expected to vary linearly with permeability as per the constitutive relation [Rudnev, et al., 1997 (p. 780)]

$$
B=\mu H=\left[\mu_{0} H\right] \mu_{R}
$$

The curves were plotted with respect to the relative magnetic permeability, $\mu_{R}$, and thus a coefficient of $\mu_{0} H=0.0478$ was expected. The simulated result, shown in Table 3.7 , was 
approximately a factor of 1.15 higher and thus showed good agreement. The induced power and power density trends were expected to behave very similarly to those encountered for changes in harmonic frequency, as the frequency and permeability both impacted the skin depth (and thus power dissipation) in the same manner. Exchanging the variables $\mu$ and $f$ in Equations (3.7) gave

$$
\begin{gathered}
P=\frac{1.16 H_{0}^{2} a t \pi^{1.5} \sqrt{f} \sqrt{\mu_{0}}}{\sqrt{\sigma}} \sqrt{\mu_{R}} \\
P_{D}=\frac{0.63 P}{V_{E}}=\frac{0.63 P}{(0.5 \delta t)(2 \pi a)}=0.63\left[1.16 H_{0}^{2} \pi f \mu_{0}\right] \mu_{R} .
\end{gathered}
$$

which evaluated to 30.6 and 4.06E8, respectively. Comparing these values with those obtained from Figure 3.13(b), the theoretical coefficients that were computed fell within a factor of 0.63 of the simulated values. The temperature trends were also expected to exhibit similar behaviors to those observed with respect to frequency, or

$$
P_{D} \propto \mu_{R} \propto \frac{m s \Delta T}{t} .
$$

Thus the permeability was predicted to vary linearly with temperature rise and inversely with time duration. The behaviors in Figure 3.13(c) exhibited almost identical patterns to those encountered in Figure 3.11(c), with the three-second temperature having good linearity in the region above $\sim 700^{\circ} \mathrm{C}$ but falling off at lower temperatures, and the $700^{\circ} \mathrm{C}$ time requirement having an exponent relatively close to the predicted value. Overall, the results continued to help strengthen the link between theory and simulation, justifying the focus of the process development which was to utilize differences in magnetic permeability to establish a controllable temperature gradient on the surface of a semiconducting wafer. 


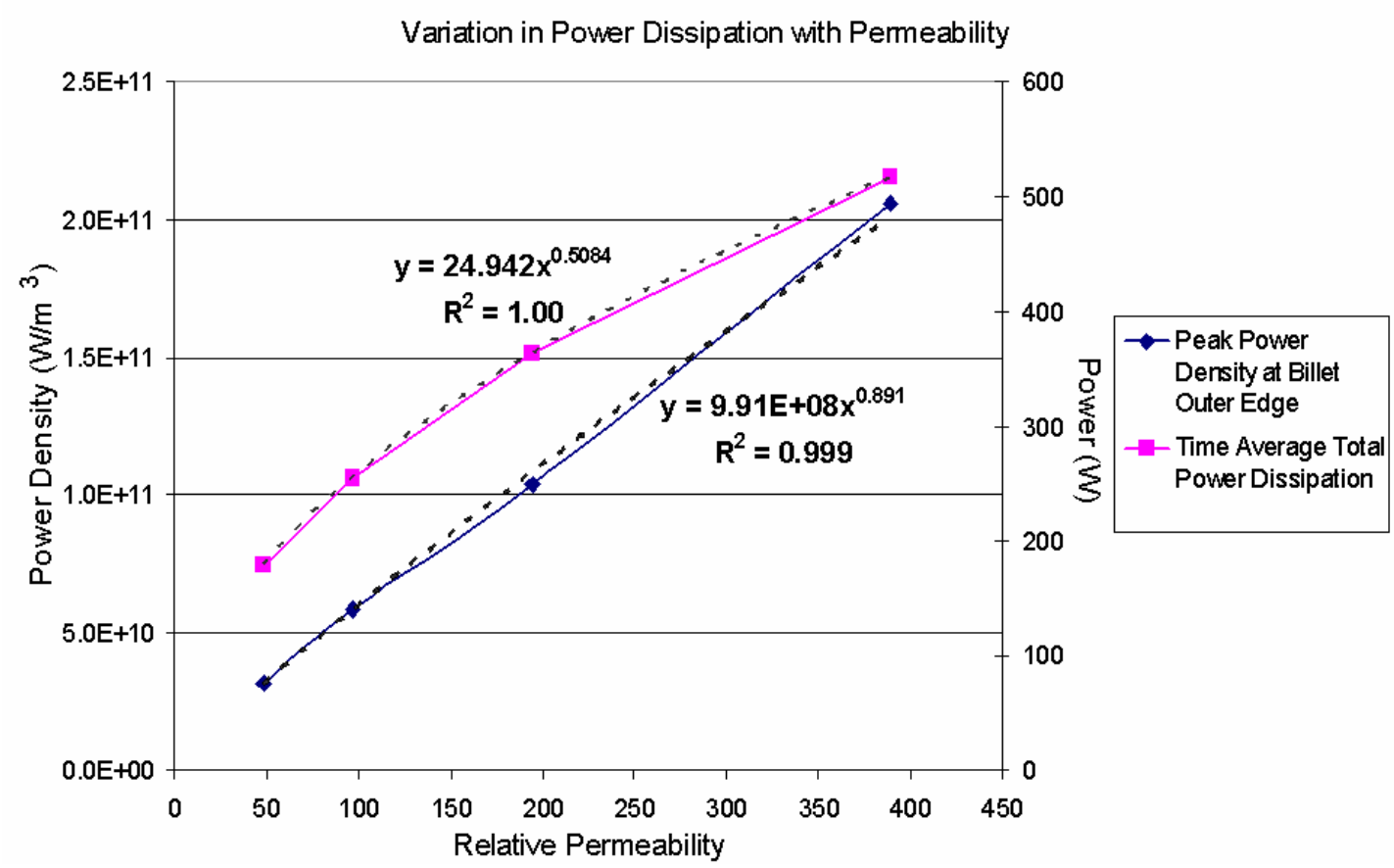

Figure 3.13(b): Graph of the total time-average power dissipation (pink) and the peak power density at the outer edge of the heated structure (blue) in the ANSYS model vs relative permeability. Theoretical trends were very similar to those computed for frequency variation and were once again confirmed to be accurate.

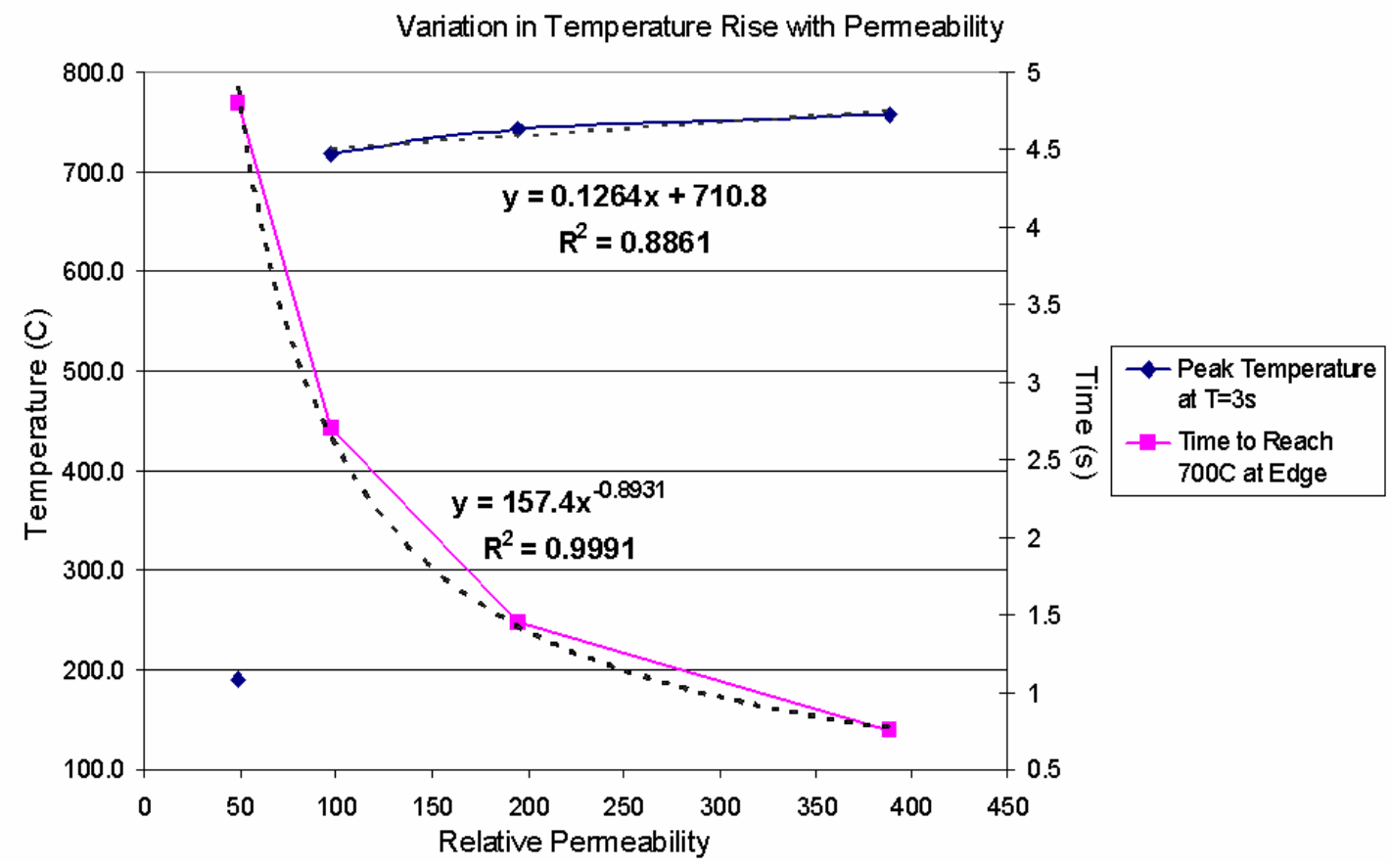

Figure 3.13(c): Illustration of the impact of the relative magnetic permeability on the rate of rise of the heated billet. The trend in temperature after a 3-second simulation (blue) was again shown exhibit a transition around $700^{\circ} \mathrm{C}$, with ideal theoretical behavior only followed above the transition point. The trend in time duration to reach $700^{\circ} \mathrm{C}$ (pink) exhibited approximately the anticipated profile. 


\begin{tabular}{|c|c|c|c|c|c|}
\hline \multicolumn{6}{|c|}{ TABLE 3.7} \\
\hline Variable & $\begin{array}{l}\text { Theoretical } \\
\text { Exponent }\end{array}$ & $\begin{array}{l}\text { Simulated } \\
\text { Exponent }\end{array}$ & $\begin{array}{l}\text { Theoretical } \\
\text { Coefficient }\end{array}$ & $\begin{array}{l}\text { Simulated } \\
\text { Coefficient }\end{array}$ & $\begin{array}{l}\text { Coefficient } \\
\text { Offset }\end{array}$ \\
\hline $\begin{array}{l}\text { Magnetic Field } \\
\text { vs Permeability }\end{array}$ & 0 & 1 & 0 & -1.46 & $\mathrm{~N} / \mathrm{A}$ \\
\hline $\begin{array}{l}\text { Flux Density vs } \\
\text { Permeability }\end{array}$ & 1 & 1.00 & 4.78E2 & $5.50 \mathrm{E} 2$ & 0.869 \\
\hline $\begin{array}{l}\text { Total Power vs } \\
\text { Permeability }\end{array}$ & 0.5 & 0.508 & 30.6 & 24.9 & 1.23 \\
\hline $\begin{array}{l}\text { Peak Power } \\
\text { Density vs }\end{array}$ & 1 & 0.891 & $6.25 \mathrm{E} 8$ & $9.91 \mathrm{E} 8$ & 0.631 \\
\hline $\begin{array}{l}\text { Permeability } \\
\text { Peak } \\
\text { Temperature vs }\end{array}$ & 1 & 1 & N/A & 0.126 & N/A \\
\hline $\begin{array}{l}\text { Permeability } \\
700 \mathrm{C} \text { Time vs } \\
\text { Permeability }\end{array}$ & -1 & -0.893 & N/A & 157 & N/A \\
\hline
\end{tabular}

All of the previous graphs of electromagnetic behaviors were obtained assuming a uniform $100^{\circ} \mathrm{C}$ ambient, which was the starting temperature of the coupled solution. To illustrate the changing properties with temperature, the steady-state electromagnetic solutions were obtained under temperatures from $100-900^{\circ} \mathrm{C}$. Table 3.8 summarizes the data used to generate the plots in Figures 3.14(a,b). Figure 3.14(a) illustrates the decrease in edge flux density (blue) due to decreasing magnetic permeability, the relationship

\begin{tabular}{lllll}
\hline \hline \multicolumn{5}{c}{ TABLE 3.8 } \\
\multicolumn{5}{c}{ VARIATION OF AMBIENT TEMPERATURE VALUES IN THE ANSYS ModEL } \\
\hline $\begin{array}{l}\text { Ambient } \\
\begin{array}{l}\text { Temperature } \\
\left({ }^{\circ} \mathrm{C}\right)\end{array}\end{array}$ & $\begin{array}{l}\text { Skin Depth } \\
(\mathrm{m})\end{array}$ & $\begin{array}{l}\text { Peak Flux } \\
\text { Density }(\mathrm{T})\end{array}$ & $\begin{array}{l}\text { Peak Power } \\
\text { Density } \\
\left(\mathrm{W} / \mathrm{m}^{3}\right)\end{array}$ & $\begin{array}{l}\text { Total Power } \\
(\mathrm{W})\end{array}$ \\
\hline 100 & $4.70 \mathrm{E}-5$ & 7.031 & $1.04 \mathrm{E} 11$ & 364.4 \\
300 & $6.37 \mathrm{E}-5$ & 7.045 & $1.07 \mathrm{E} 11$ & 456.1 \\
500 & $8.39 \mathrm{E}-5$ & 6.425 & $1.01 \mathrm{E} 11$ & 519.5 \\
700 & $1.26 \mathrm{E}-4$ & 4.31 & $6.97 \mathrm{E} 10$ & 496.8 \\
750 & $4.12 \mathrm{E}-4$ & 0.4709 & $7.98 \mathrm{E} 09$ & 163.8 \\
800 & $1.35 \mathrm{E}-3$ & 0.04822 & $7.83 \mathrm{E} 08$ & 50.61 \\
900 & $1.40 \mathrm{E}-3$ & 0.04822 & $7.83 \mathrm{E} 08$ & 52.39 \\
\hline \hline
\end{tabular}


between which was shown to be linear in Equation (3.12). The skin depth (pink), on the other hand, depended upon both the magnetic permeability and the electrical conductivity, both of which decreased with increasing temperature. The impact of the Curie temperature at approximately $720^{\circ} \mathrm{C}$ was clearly visible and carried over into the dependent behaviors of power dissipation and peak power density, shown in Figure 3.14(b). The total time-average power dissipation for the billet showed an initial increase in magnitude with temperature. This was because the gradual decrease in magnetic permeability and its skin depth impact was more than compensated for by the stronger decrease in electrical conductivity (see Equation (3.7a)). Once the Curie point was reached, however, the permeability fell sharply and the power dissipation followed. Since the peak power density at the outer edge was completely independent of conductivity as

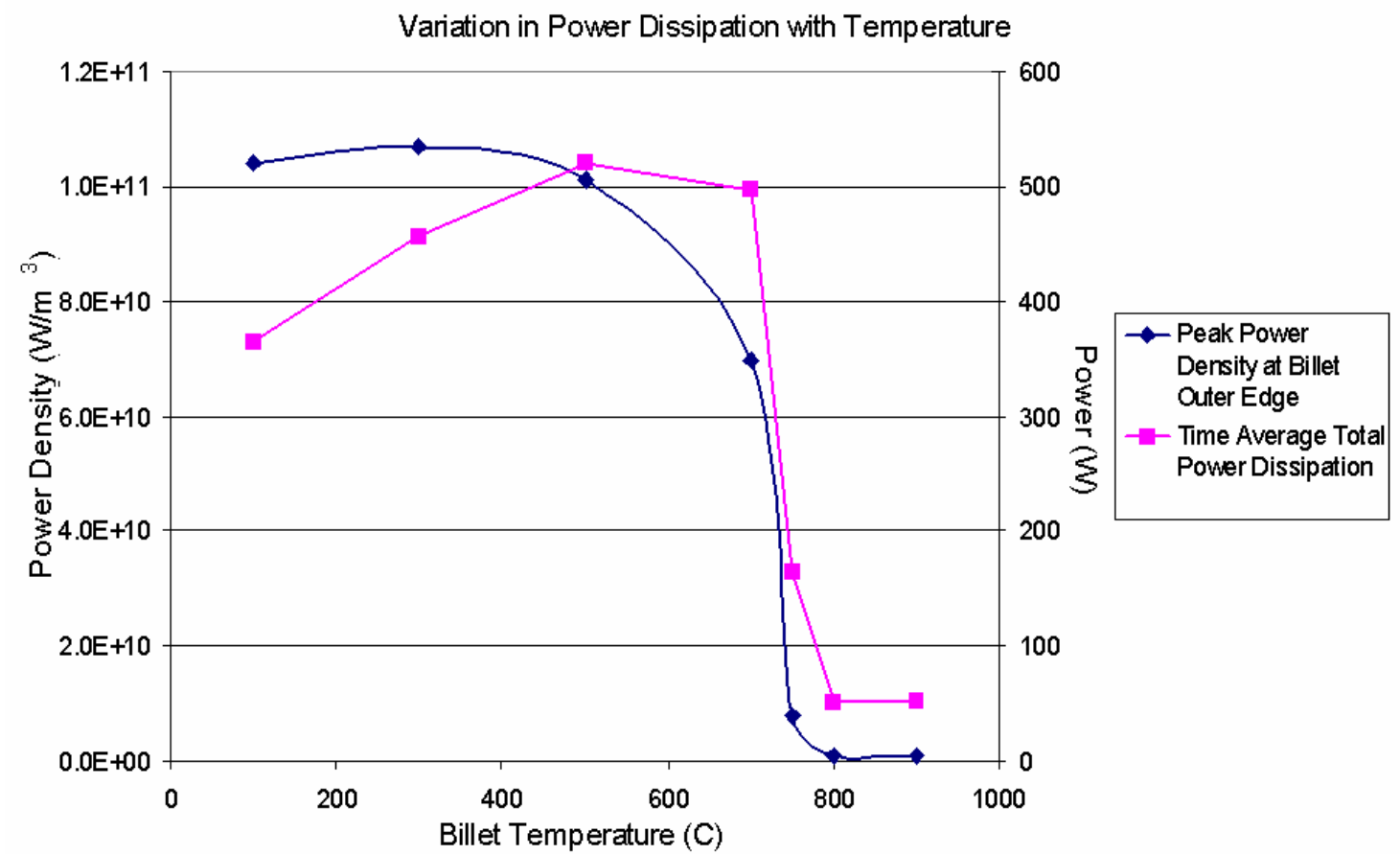

Figure 3.14(a): Graph showing the trends in flux density (blue) and skin depth (pink) in the heated billet of the ANSYS model vs temperature. The flux density decreased with increasing temperature due to the reduced magnetic permeability, and the skin depth simultaneously increased (enhanced further by the decreasing electrical conductivity). 


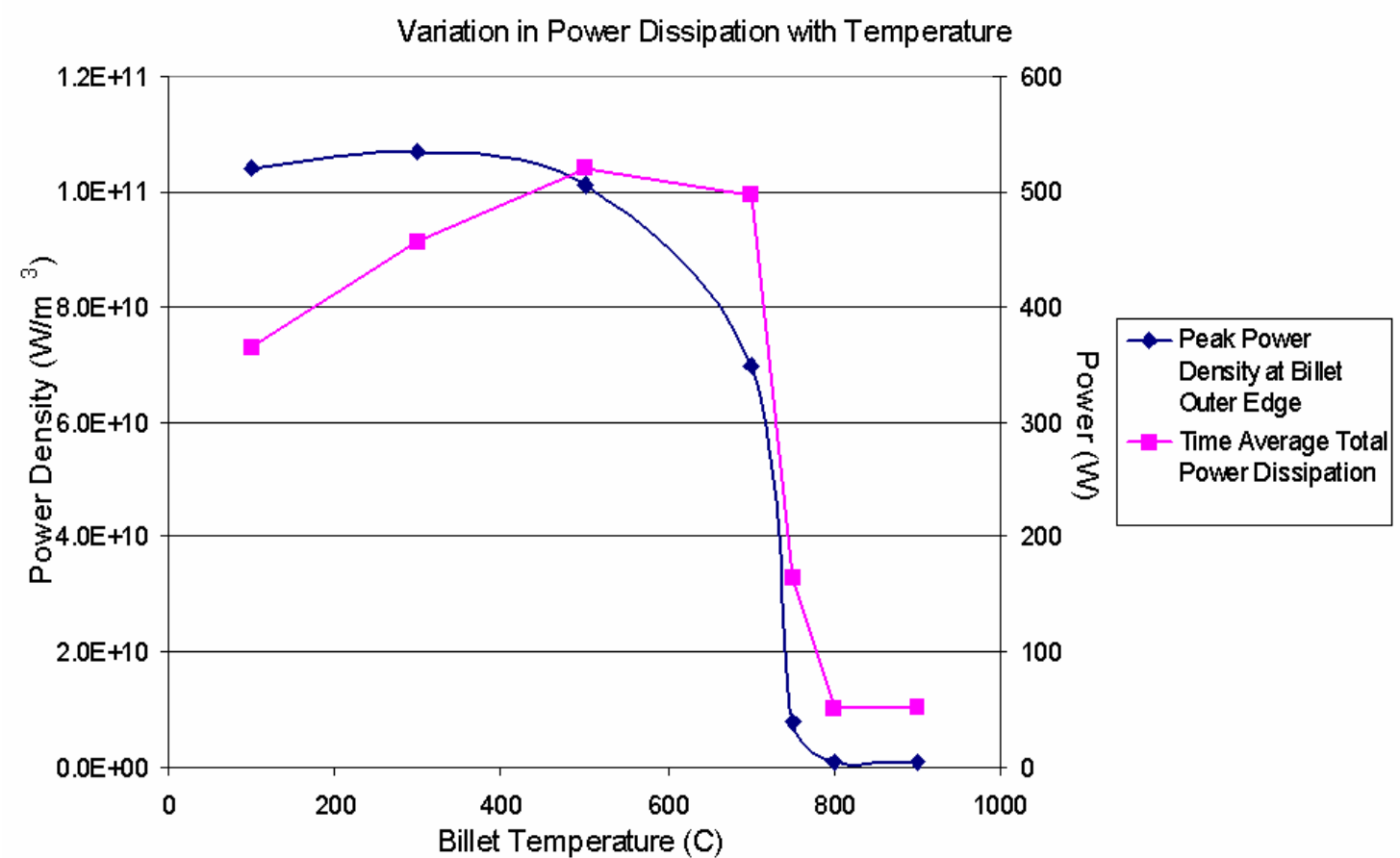

Figure 3.14(b): Variation in power dissipation and peak power density vs temperature. The power dissipation (pink) initially rose due to the decreasing electrical conductivity but then quickly dropped when the skin depth rose sharply at the Curie temperature. The power density (blue) showed a very small initial rise with temperature but began to fall much earlier due to the simultaneous increase in element size.

can be seen from Equation (3.7b), a permeability-based trend was produced almost identical to that of the magnetic flux density. Quantitative analysis of these behaviors is beyond the scope of this analysis and primarily reflected the unique material properties of the constituent material in the billet. However, the graphs are helpful in understanding the transitional nature of the three-second temperature trends shown in Figures 3.11(c), 3.12(d), and 3.13(c).

Lastly, and perhaps most importantly, the scaling of the model to dimensions more typical of a thin film was examined. Equation (2.6a) suggested that the total power dissipation should vary linearly in both the thickness and radius of the billet. Substituting the default values utilized by the model, including the $38 \mathrm{kA} / \mathrm{m}$ field strength, gave the anticipated relationships 


$$
P=[2.46 E 7] t a
$$

and

$$
P_{D}=\frac{0.63 P}{(0.5 \delta t)(2 \pi a)}=[1.06 E 11]
$$

Equation (3.15b) indicates that the power dissipation density was expected to be independent of both the billet thickness and radius due to the cancelling effect of the element volume, and thus a constant value of 1.06E11 was forecast. Table 3.9 shows the data obtained by individually varying the billet thickness and radius values from $5 \mu \mathrm{m}$ $1 \mathrm{~mm}$ and $0.5 \mathrm{~cm}-1.5 \mathrm{~cm}$, respectively, where the peak values in each range were those utilized in the default simulation. The values are plotted versus their respective dependent variables in Figures 3.15(a,b). The trends in total power dissipation both exhibited the linearity predicted by ( $3.15 \mathrm{a})$. To analyze the coefficients, $a=0.015$ was substituted into the thickness-based trend, and $t=0.001$ was substituted into the radius-based trend. The calculated values, shown in Table 3.10, were within a factor of 0.967 of simulation. The

\begin{tabular}{|c|c|c|c|c|c|}
\hline \multicolumn{6}{|c|}{$\begin{array}{c}\text { TABLE } 3.9 \\
\text { VARIATION OF BILLET DIMENSIONS IN THE ANSYS MODEL }\end{array}$} \\
\hline$\frac{\frac{0}{2}}{\frac{\pi}{\pi}}$ & Value & $\begin{array}{l}\text { Peak } \\
\text { Power } \\
\text { Density }^{*} \\
\left(\mathrm{~W} / \mathrm{m}^{3}\right)\end{array}$ & $\begin{array}{l}\text { Total } \\
\text { Power } \\
(\mathrm{W})\end{array}$ & $\begin{array}{l}\text { Temp at } \\
\mathrm{T}=3 \mathrm{~s}\left({ }^{\circ} \mathrm{C}\right)\end{array}$ & $\begin{array}{l}\text { Time to } \\
700^{\circ} \mathrm{C}(\mathrm{s})\end{array}$ \\
\hline \multirow{4}{*}{ 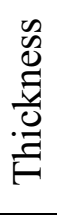 } & $5 \mu \mathrm{m}$ & $1.04 \mathrm{E} 11$ & 1.82 & 743 & 1.45 \\
\hline & $10 \mu \mathrm{m}$ & $1.04 \mathrm{E} 11$ & 3.64 & 743 & 1.45 \\
\hline & $100 \mu \mathrm{m}$ & $1.04 \mathrm{E} 11$ & 36.4 & 743 & 1.45 \\
\hline & $1 \mathrm{~mm}$ & $1.04 \mathrm{E} 11$ & 364.4 & 743 & 1.45 \\
\hline \multirow{3}{*}{ 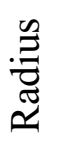 } & $0.5 \mathrm{~cm}$ & $1.03 \mathrm{E} 12$ & 121.1 & 773 & 0.9 \\
\hline & $1.0 \mathrm{~cm}$ & $1.03 \mathrm{E} 12$ & 242.4 & 754 & 1.3 \\
\hline & $1.5 \mathrm{~cm}$ & $1.04 \mathrm{E} 11$ & 364.4 & 743 & 1.45 \\
\hline
\end{tabular}
trends in power density were indeed constant values as predicted, with an offset factor of 


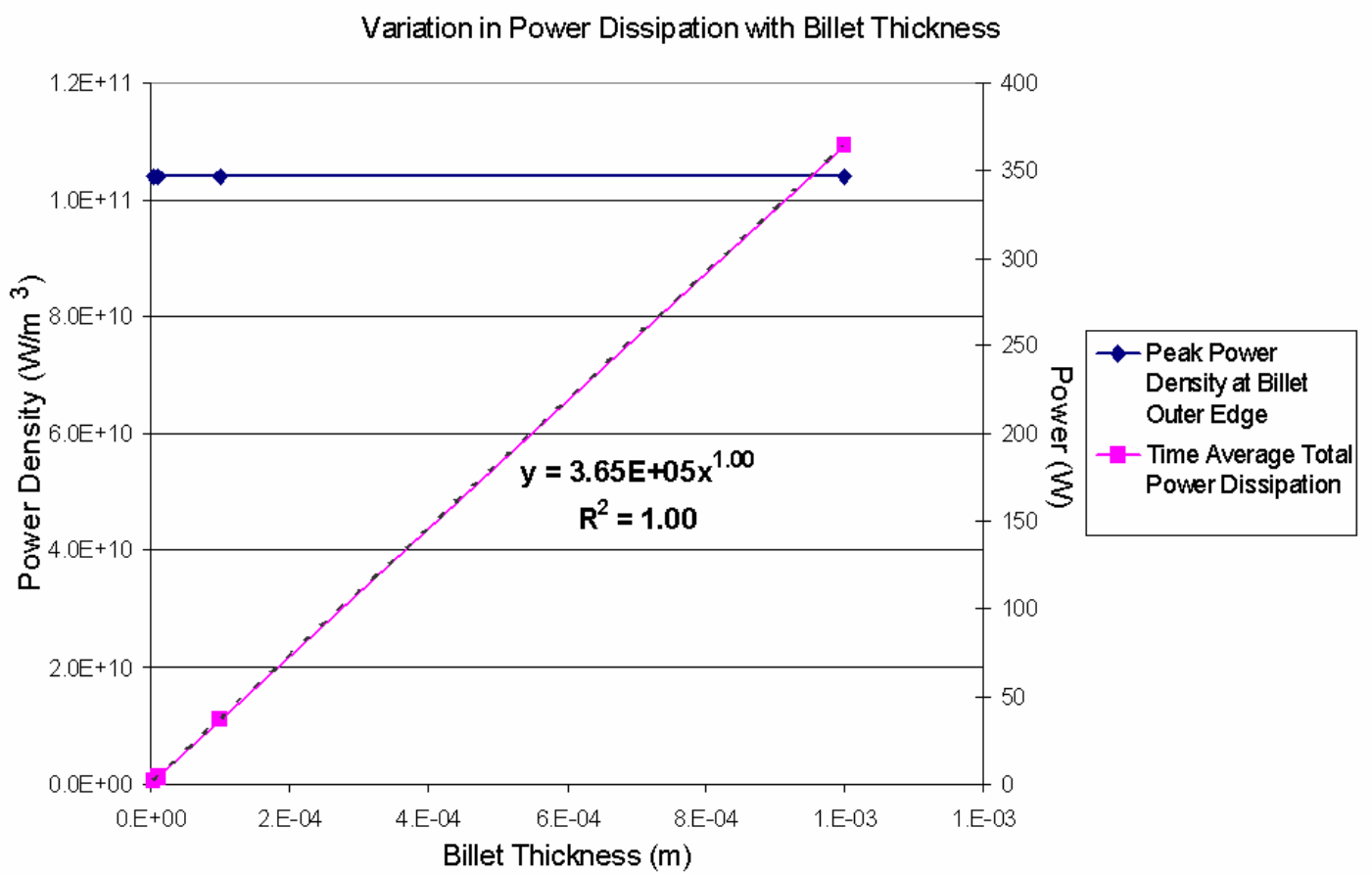

Figure 3.15(a): Impact of film thickness on induced power dissipation (pink) and peak power density (blue) values. The power dissipation varied linearly with thickness as predicted by Equation (3.15a), whereas the peak power density was independent of thickness as predicted by Equation (3.15b).

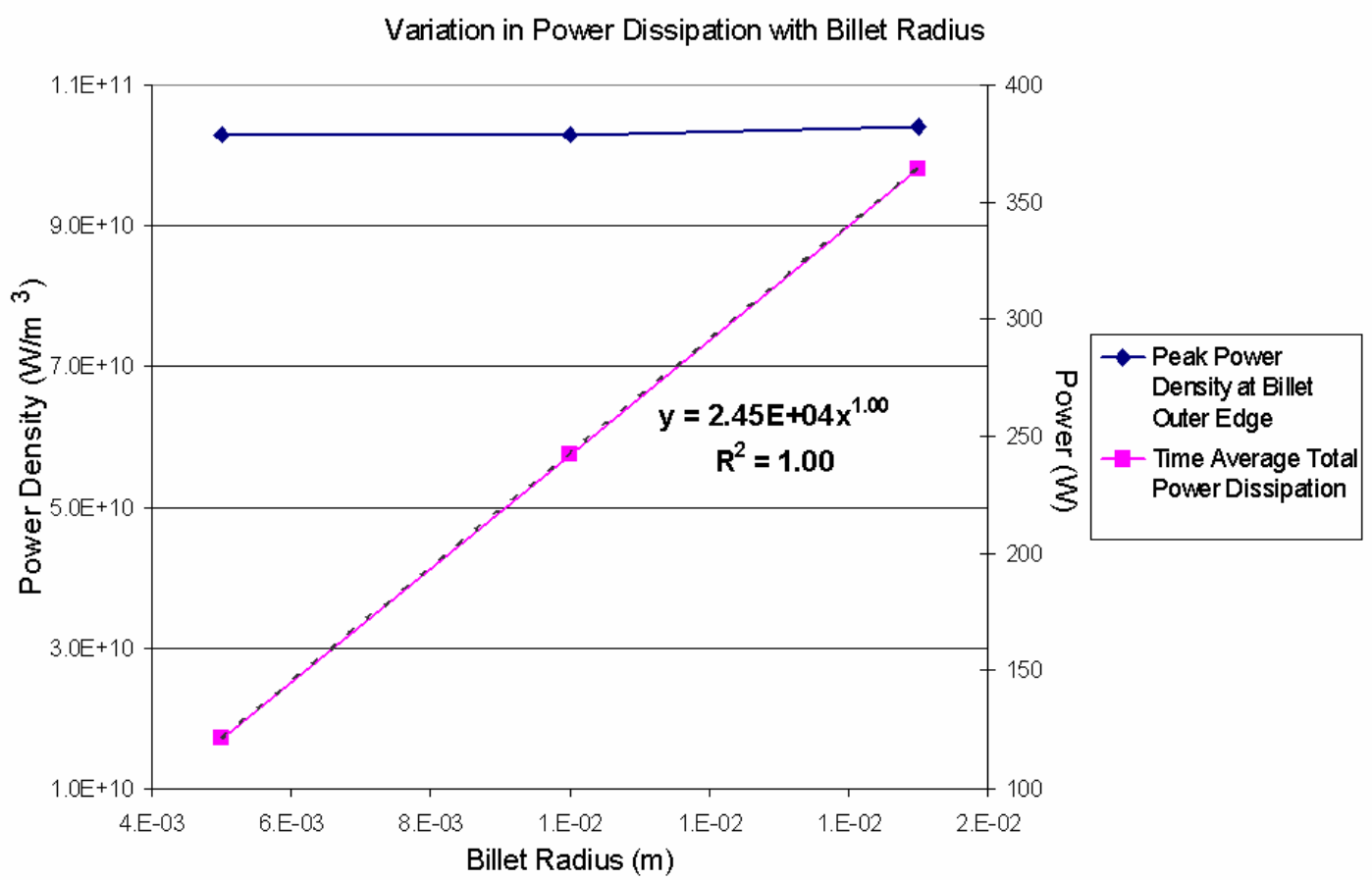

Figure 3.15(b): Impact of film radius on induced power dissipation (pink) and peak power density (blue) values. The power dissipation varied linearly with thickness as expected, but the power density showed negligible variation. This unexpected result was caused by the conversion between two and three dimensions. 
TABLE 3.10

THEORETICAL AND SimUlated DimENSIONAL TRENDS FOR THE ANSYS MODEL

\begin{tabular}{llllll}
\hline Variable & $\begin{array}{l}\text { Theoretical } \\
\text { Exponent }\end{array}$ & $\begin{array}{l}\text { Simulated } \\
\text { Exponent }\end{array}$ & $\begin{array}{l}\text { Theoretical } \\
\text { Coefficient }\end{array}$ & $\begin{array}{l}\text { Simulated } \\
\text { Coefficient }\end{array}$ & $\begin{array}{l}\text { Coefficient } \\
\text { Offset }\end{array}$ \\
\hline $\begin{array}{l}\text { Total Power vs } \\
\text { Thickness }\end{array}$ & 1 & 1 & $3.68 \mathrm{E} 5$ & $3.65 \mathrm{E} 5$ & 1.01 \\
$\begin{array}{l}\text { Peak Power } \\
\begin{array}{l}\text { Density vs } \\
\text { Thickness }\end{array}\end{array}$ & 0 & 0 & $1.06 \mathrm{E} 11$ & $1.04 \mathrm{E} 11$ & 1.02 \\
$\begin{array}{l}\text { Total Power vs } \\
\text { Radius }\end{array}$ & 1 & 1 & $2.46 \mathrm{E} 4$ & $2.45 \mathrm{E} 4$ & 0.967 \\
$\begin{array}{l}\text { Peak Power } \\
\begin{array}{l}\text { Density vs } \\
\text { Radius }\end{array}\end{array}$ & 0 & 0 & $1.06 \mathrm{E} 11$ & $1.04 \mathrm{E} 11$ & 1.02 \\
\hline \hline
\end{tabular}

1.02 between theoretical and simulated values. Attempts were made to scale the radius values smaller than $5 \mathrm{~mm}$, but the model quickly broke down and was unrepairable with changes in element size. At a $1 \mathrm{~mm}$ radius, for example, the magnetic field strength in free space, which should not have been impacted, more than doubled in magnitude whereas the flux density induced in the billet simultaneously deteriorated. It was evident that a billet radius of 20 skin depths or less was not possible to simulate with the existing model. This was not a concern, however, as the dimensions utilized in experimentation were typically on the order of $1-2 \mathrm{~cm}$.

In summary, the example ANSYS coupled-solver induction heating model was highly valuable in justifying the theoretical analysis of Chapter 2. The electromagnetic trends were all confirmed to a high degree of accuracy. This provided a basis from which the experimental results could be anticipated and/or explained. The model had limitations, however, for fully describing the envisioned inductive annealing process in that it only accounted for thermal conduction within a single material. Thus a more detailed structure was needed in order to assess permeability and temperature gradients 
across multiple materials (eg, a film stack) as well as radiant energy loss from the relatively larger horizontal surface into the ambient. The model was also found to "blow up" under certain circumstances, such as can be seen in the data for the higher current values in Table 3.4. The blow-up was characterized by a rapid, uncontrolled rise in temperature. A similar effect was often encountered during experimentation, as will be shown in Chapter 4, but it was difficult to determine the ability of the model to predict the thermal runaway condition as there were many other contributing factors (such as the lack of an appreciable ambient into which thermal energy could spread). The customized thin film model presented in the next section also exhibited the blow-up condition under certain circumstances when the energy levels were raised too high.

\section{3: Thin Film Induction Heating Model}

The ANSYS example induction heating model presented in the previous section provided justification for the theoretical equations of Chapter 2. Missing, however, was the simulation of a composite structure so as to illustrate the capability for selective heating and engineered temperature gradients. The thin film induction heating models presented in this section and the next attempted to resolve this through the establishment of thin film stacks above a silicon substrate. The simulation code for the first thin film model is provided in Appendix A.3. The instructions flow in a similar arrangement to those utilized in the ANSYS model. The first portion defined the axisymmetric layout of the components, which was done almost entirely via connected keypoints. The only exception was the magnetic coil, which was drawn as a hollow circle with dimensions attempting to replicate those of the solenoid used during experimentation. Note that only one turn of the coil was simulated in order to conserve simulation time and memory; the 
190A coil current was multiplied by three to produce the same magnetic field. The solid model is pictured in Figure 3.16(a,b). The film stack formed three concentric circles about the y-axis, where the innermost was a $3 \mu \mathrm{m}$-thick polysilicon film extending to a $0.45 \mathrm{~cm}$ radial dimension $(9 \mathrm{~mm}$ total diameter), the middle ring was a $7.3 \mathrm{~mm}$-thick NiFe19 film extending from $0.6-1.2 \mathrm{~cm}$, and the outermost ring was another $3 \mu \mathrm{m}$-thick polysilicon film extending from $1.35-1.80 \mathrm{~cm}$. These were all patterned above a $500 \mu \mathrm{m}-$ thick silicon substrate, with a $1 \mu \mathrm{m}$-thick silicon dioxide base layer. The dimensions were chosen to coincide with the experiment presented in Section 5.2, in which a cluster of cantilever beams measuring 9x9mm was enclosed by a hollow NiFe19 ring with a $2.4 \mathrm{~cm}$ outer diameter and a $1.2 \mathrm{~cm}$ inner diameter. The ferromagnetic film was heated to approximately $840^{\circ} \mathrm{C}$, and the resulting temperature gradient produced varying polysilicon morphologies at different distances from the ferromagnet.

The next section of the code was dedicated to the fragmentation of the model into a mesh suitable for simulation. Meshing was particularly challenging for a model of this type. Thin films form areas with high aspect ratios, meaning that their height/width (or width/height) ratios are significantly above unity. High aspect ratio elements, however, can lead to inaccuracy in the solution [ANSYS Modeling and Meshing Guide, Sec 7.5.7]. ANSYS issues a warning when it encounters an aspect ratio above 20. At this point, the solution may still be accurate, but the likelihood of a problem becomes increasingly likely beyond this point. Thus achieving an accurate solution for the thin film model required a relatively large number of elements. Larger element numbers translated directly into a larger model size, however, and thus the computational requirements as well as the simulation time were impacted. Furthermore, an additional constraint on 


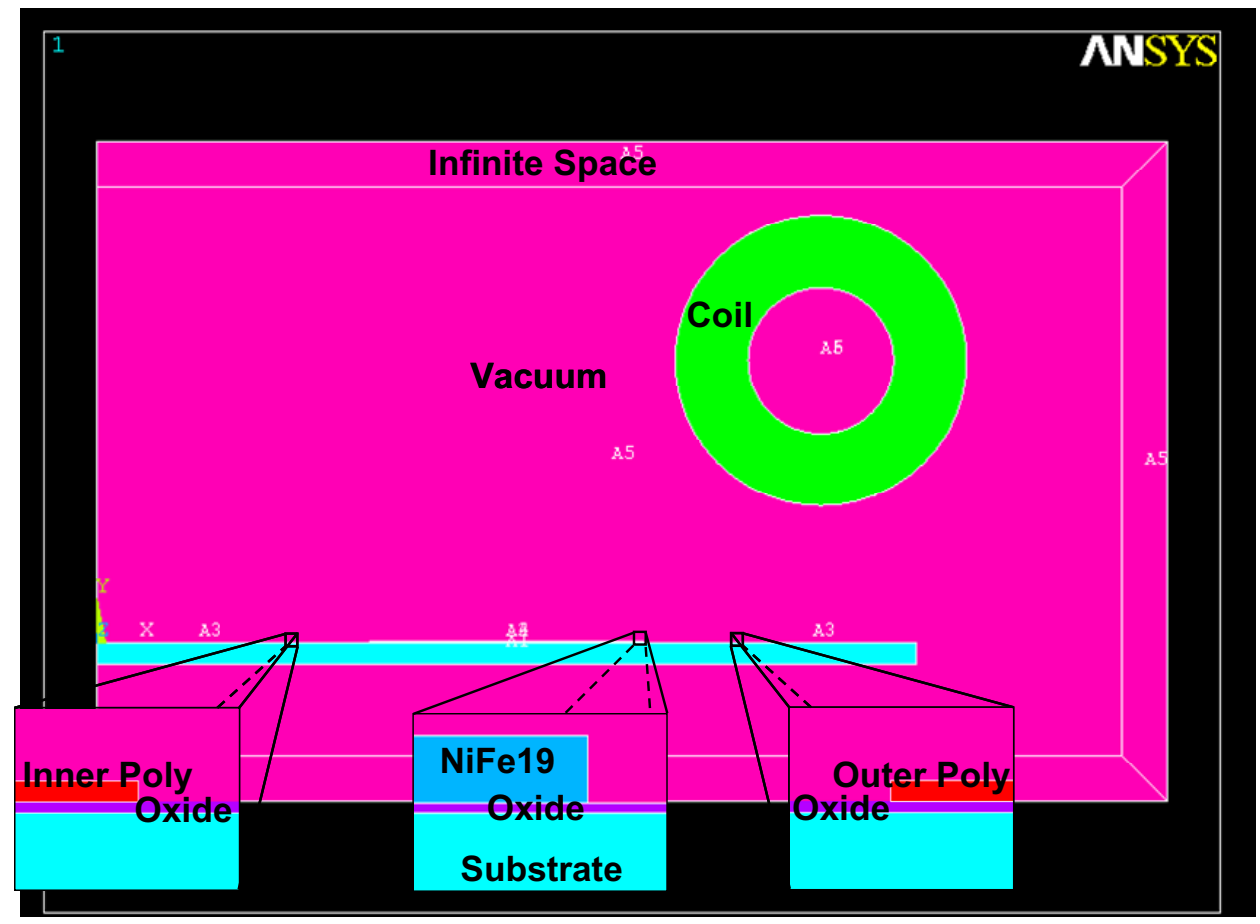

Figure 3.16(a): Area plot of the solid model with close-ups illustrating the layouts of the thin film regions. An axisymmetric model, the film layout formed three concentric rings above the oxide-covered substrate; the inner and outer rings formed polysilicon devices, and the middle ring was a heated NiFe19 film.

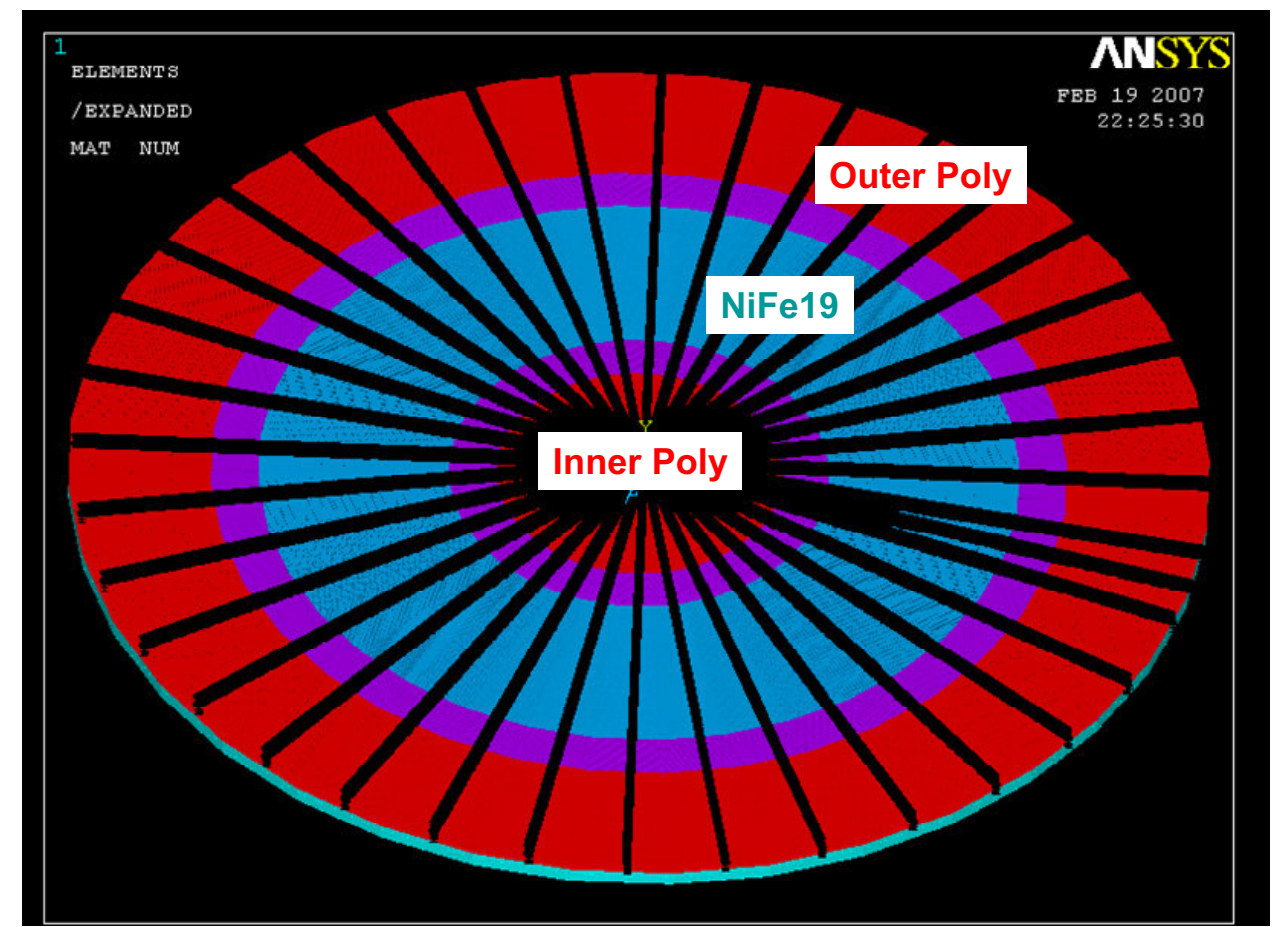

Figure 3.16(b): Three-dimensional axisymmetric rotation of the thin film solid model showing only the substrate and film stack. The inner and outer poly rings (red) enclosed the inductively heated $\mathrm{NiFe} 19$ ring (blue) from which they received thermal energy. 
model size was present due to the use of ANSYS' University Package which enabled the use of all simulation packages with a strict limitation on the node count. The original development of the model was hindered by a 16,000-node limit, which made the present model impossible to simulate. The recent upgrade to a 120,000-node package was the key enabling factor that rendered the model development successful. The meshing section of the code began with the electromagnetic element type definitions as illustrated in Table 3.11. Plane53 and Plane77 were the 8-noded equivalent versions of the 4-noded Plane13 and Plane55 elements utilized in the ANSYS example model. The other key difference was the addition of a row of infinite-surface elements (Infin110) around the outer edge of the model. This provided for an expansion of the free space region, thus allowing the model to seem larger than it was as-drawn. The material properties, defined in external files, were read in via the mpread command. The property values that were utilized are provided in Appendix A.2, along with their individual sources. The electromagnetic properties for the NiFe19 film were obtained from actual measurements of the RFsputtered thin film properties. The ambient was modeled after a 50mTorr vacuum, which was the ambient under which the majority of the experiments were performed. The mesh was generated on each area individually, beginning with the thin films and ending with the infinite ambient areas. The element size within the NiFe19 film was fixed at $4 \mu \mathrm{m}$,

\begin{tabular}{llll}
\hline \hline \multicolumn{4}{c}{ TABLE 3.11 } \\
\multicolumn{1}{c}{ ELEMENT TYPES UtILIZED IN THE THIN FILM MODEL } \\
\hline Description & Element & Electromagnetic & Thermal \\
& Number & Element Type & Element Type \\
\hline Structure & 1 & Plane53 & Plane77 \\
Coil and Air & 2 & Plane53 & Null0 \\
Outer Edge & 3 & Infin110 & Infin110 \\
NiFe19 Surface & 4 & Nul10 & Surf151 \\
\hline \hline
\end{tabular}




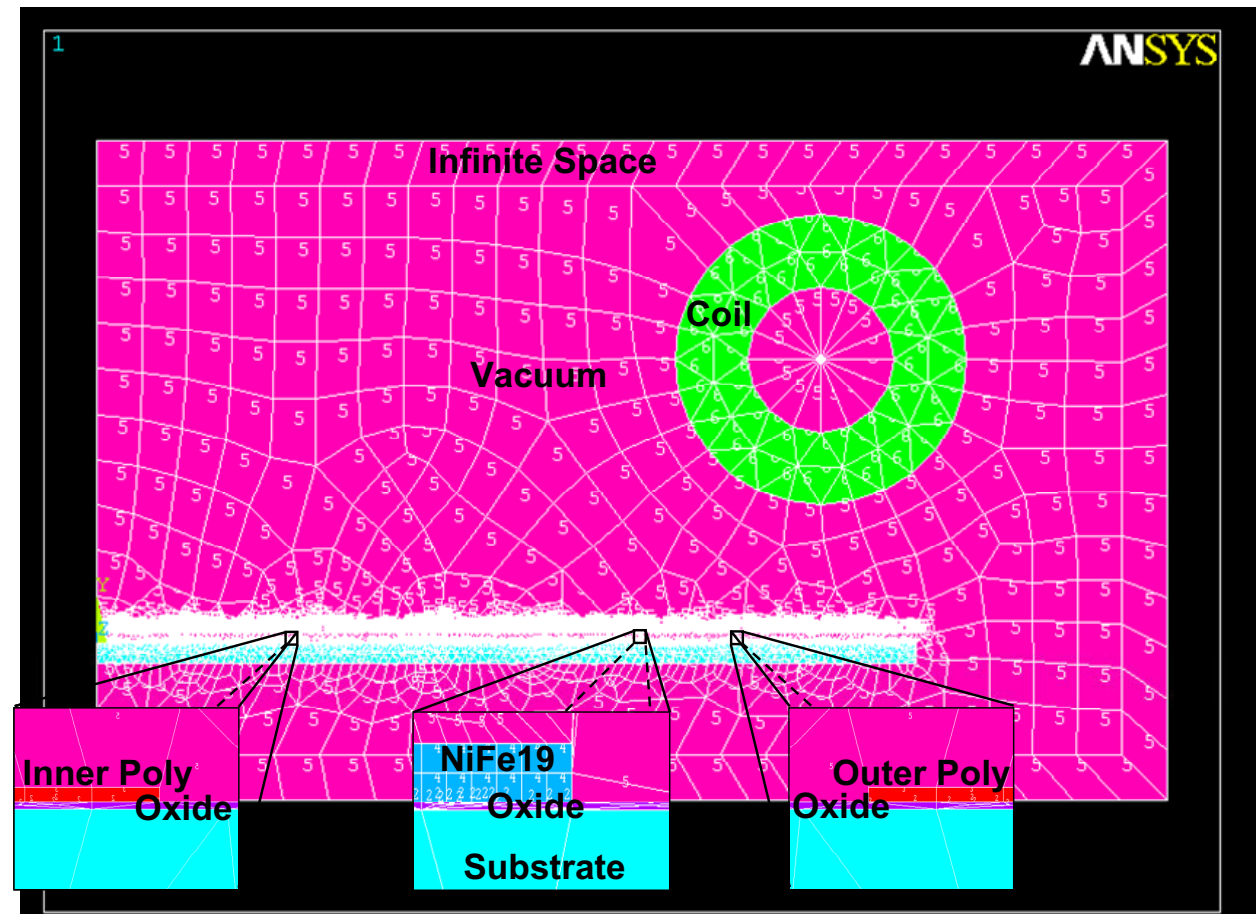

Figure 3.17: Meshed thin film model showing the relative mesh sizes for the different components. The NiFe19 film was meshed to $4 \mu \mathrm{m}$ divisions throughout the entire structure, which provided more than sufficient resolution for the $94.9 \mu \mathrm{m}$ skin depth.

provided more than sufficient resolution within the calculated $94.9 \mu \mathrm{m}$ skin depth. The meshed model is shown in Figure 3.17, where the three close-up images illustrate the relative mesh sizes for the different components. Lastly, the Surf151 line elements were generated on the top surface of the NiFe19 film. The esurf command was utilized to simultaneously generated the mesh and assigned the space node.

The loads and boundary conditions were essentially the same as those utilized in the ANSYS example model. The unique load to the entire model was once again the current density applied to the coil, which in this case was computed to deliver $3 * 190=570$ A through the $24.1 \mathrm{~mm}^{2}$ cross-section. The flux-normal boundary condition was also applied as before, meaning that the z-component of the magnetic vector potential was set to zero along the y-axis. The use of infinite surface elements around the 
outer edge was a new feature, however; this required an additional boundary condition to designate the external infinite edge. A third condition was optionally utilized to fix the temperature behind or at the edge of the substrate, to simulate the effect of substrate temperature regulation. Finally, the harmonic frequency was defined at $375 \mathrm{kHz}$, which was the frequency at which nearly all experimentation was done, and the electromagnetic physics environment was stored.

The electromagnetic simulation was tested before proceeding with the creation of the thermal physics environment. The plots in Figures 3.18-3.21 present the results specific to the room-temperature electromagnetic solution. The magnetic field produced by the coil was oriented along the y-direction through the center of the coil. The magnitude of $H_{Y}$ is shown in Figure 3.18(a). From Equation (2.11), the theoretical value for a three-turn coil with $190 \mathrm{~A}$ (or a one-turn coil with $570 \mathrm{~A}$ ) was $18.0 \mathrm{kA} / \mathrm{m}$., which was consistent with the region of the plot internal to the coil. Closer to the substrate, the field strength was reduced slightly to $\sim 14 \mathrm{kA} / \mathrm{m}$ due to the distance from the source. The effect of distance is illustrated more clearly in the vector plot of Figure 3.18(b). The heated structure was subject to a weakened field with some off-axis curvature in the x-direction, the latter of which increased considerably with distance from the central axis. The field strength in the vicinity of the substrate translated to a free-space magnetic flux density of 0.0176T, which was in agreement with the free-space regions of the contour plots of Figures 3.19(a,b). The flux density behaviors within the ferromagnetic film were far less predictable than those encountered in the ANSYS example model, and were most likely caused by a combination of field curvature and anisotropy in the magnetic permeability. The measured relative permeability values of the NiFe19 films were 48.3 in the plane of 


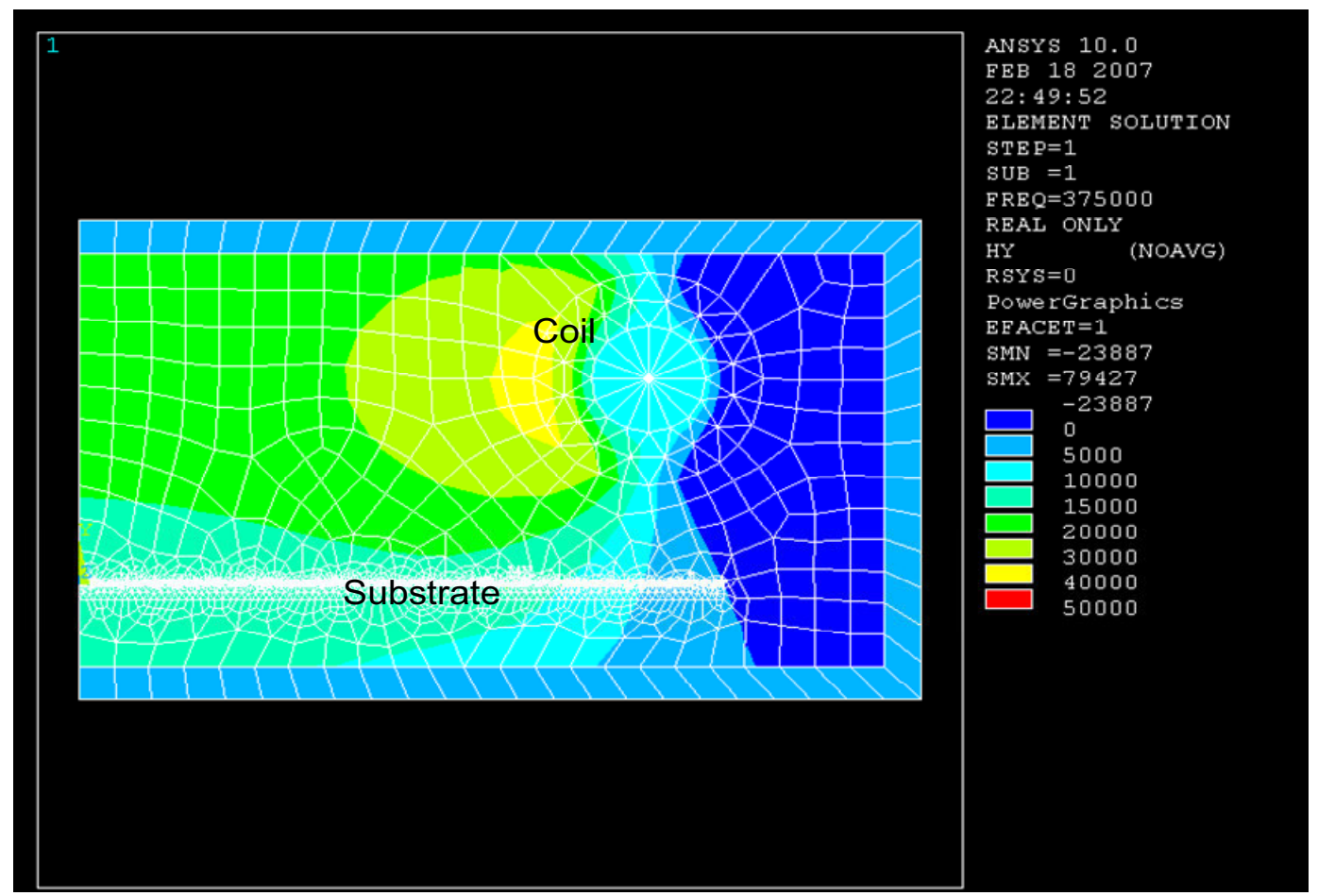

Figure 3.18(a): Contour plot showing the y-component of the magnetic field strength that was produced by the solenoid coil. The magnitude in the interior of the coil was consistent with the theoretical prediction of $18.0 \mathrm{kA} / \mathrm{m}$.

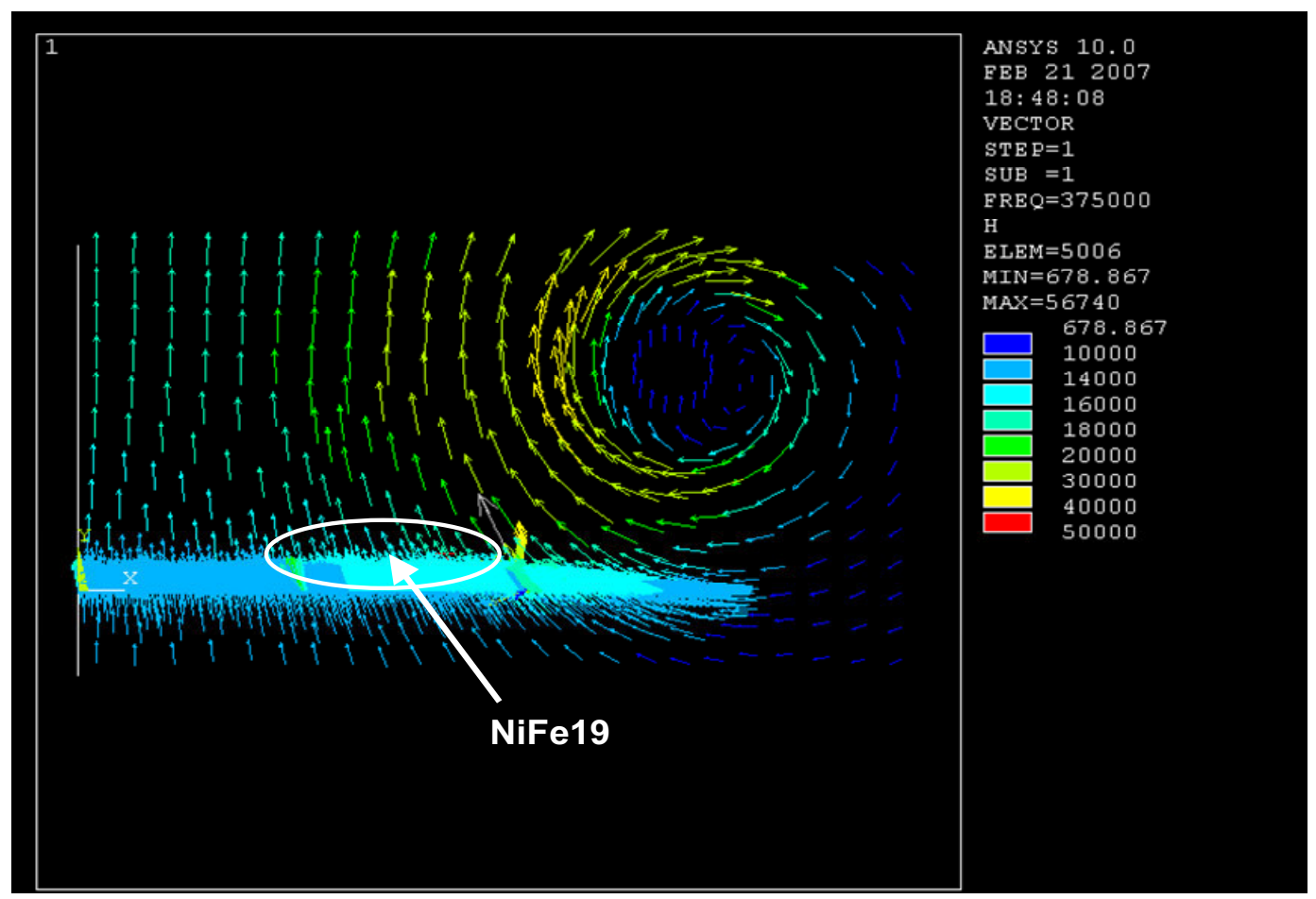

Figure 3.18(b): Vector plot showing magnetic field strength and orientation. The field passing through the NiFe19 film had a magnitude of $\sim 14 \mathrm{kA} / \mathrm{m}$ and had an appreciable component in the $\mathrm{x}$-direction. 


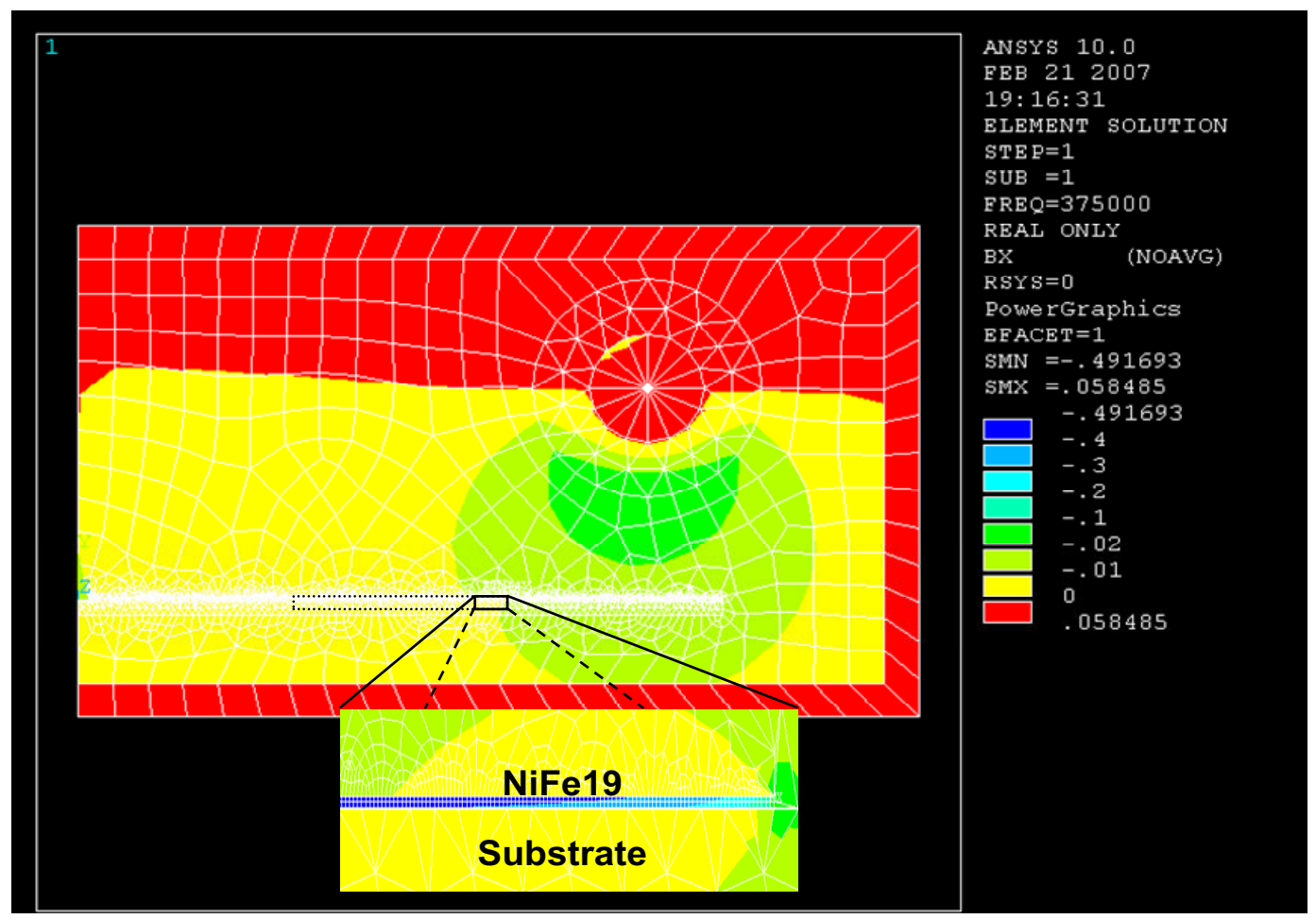

Figure 3.19(a): Contour plot showing the magnitude of the $\mathrm{x}$-component of the magnetic flux density, with a close-up on the NiFe19 film. The curvature of the magnetic field, combined with the anisotropic permeability, rendered $B_{X}$ the dominant component.

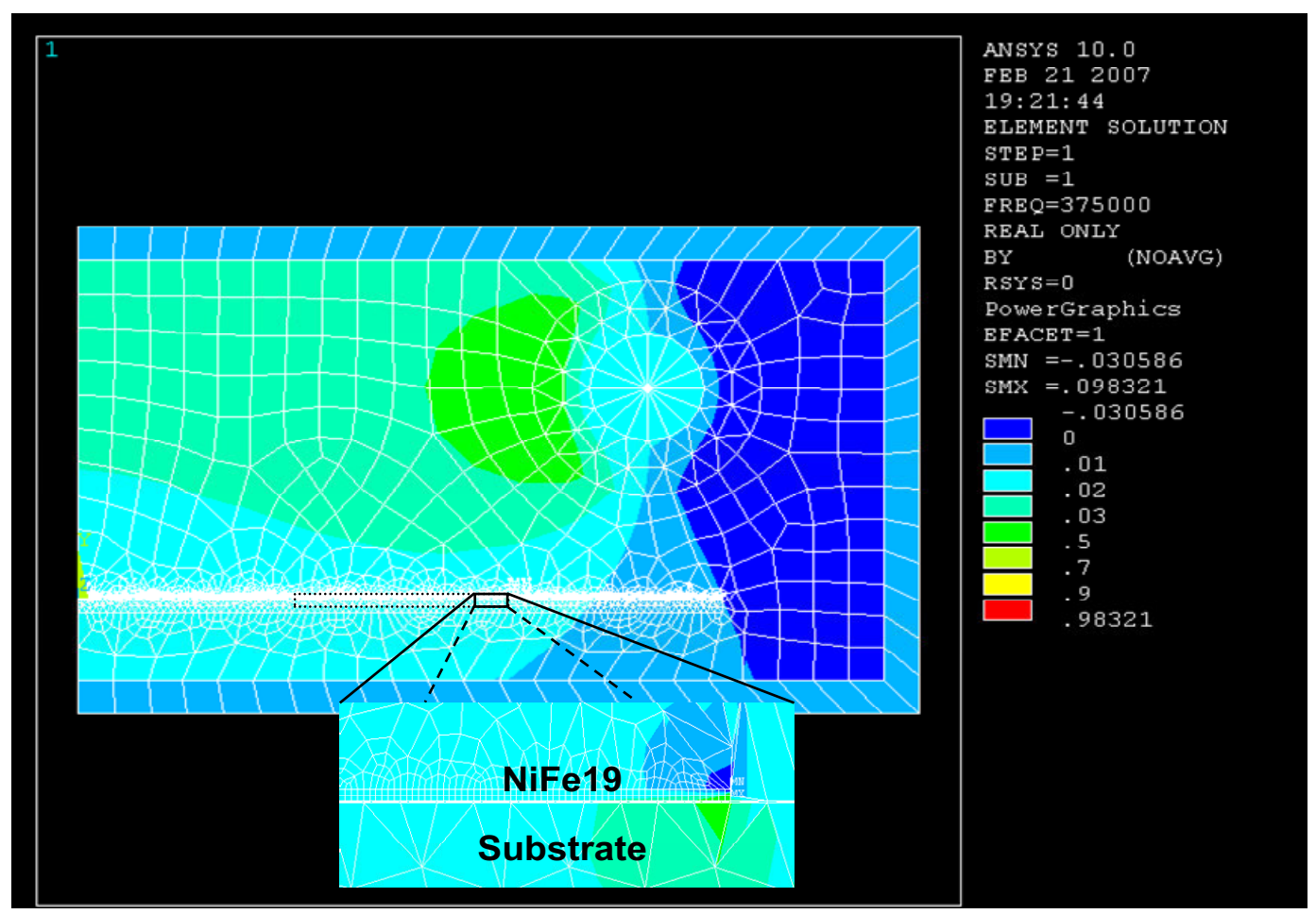

Figure 3.19(b): Contour plot showing the magnitude of the y-component of the magnetic flux density, with a close-up on the NiFe19 film. Theory predicted a $B_{Y}$ value of $0.277 \mathrm{~T}$ for the given field strength, but the actual value was roughly equal to that of free space. 
the film $\left.\rho_{X}=\rho_{Z}\right)$ and 15.8 in the normal direction $\left(\rho_{Y}\right)$. The measurement of these values is discussed in detail in Section 4.3. As the figures show, the flux density values produced in the simulated film had much stronger components in the x-direction than in the $y$ direction, where the value of $B_{Y}$ was essentially that of free space other than an anomaly at the interface. The peak value in $\mathrm{B}_{\mathrm{X}}$ was $0.492 \mathrm{~T}$, which corresponded to an effective permeability of 28.0. This was approximately equal to the mean of the two permeability values, $32.3\left(\rho_{X Y}\right)$, suggesting that the rotated alignment of the ferromagnetic domains was driven by the vector summation. Figure 3.20 shows the contour plot for $J_{Z}$, the current density in the z-direction (or $\Phi$-direction). $J_{X}$ and $J_{Y}$ were not valid degrees of freedom in the two-dimensional model due to the unspecified element depth, but it was believed that the out-of-plane eddy currents were significant. The in-plane induced

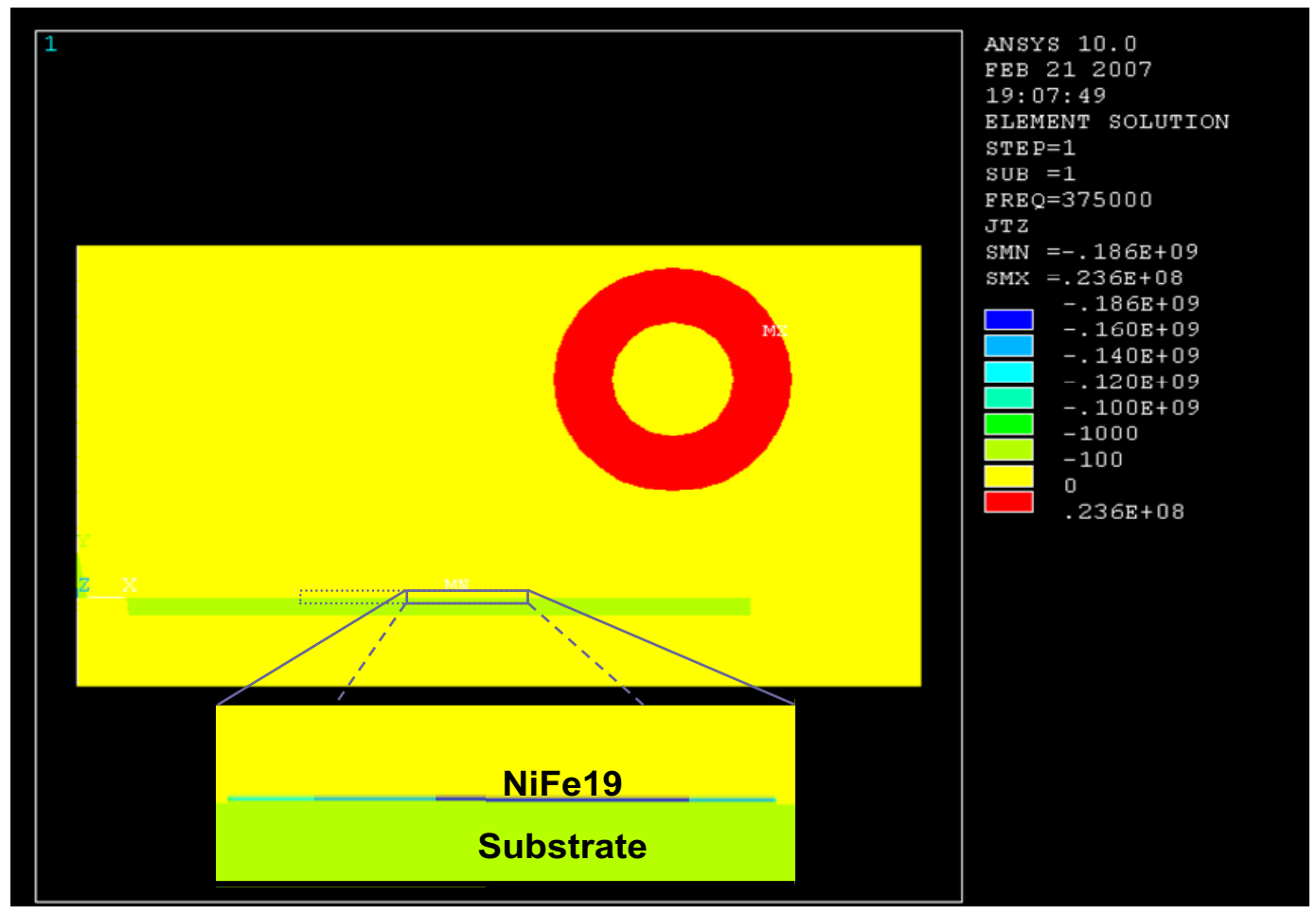

Figure 3.20: Contour plot showing the induced current density within the thin film model. The values in the NiFe19 film reached a peak magnitude of $1.86 \mathrm{E} 8 \mathrm{~A} / \mathrm{m}^{2}$, whereas the peak magnitude in the substrate was below $1000 \mathrm{~A} / \mathrm{m}^{2}$. 


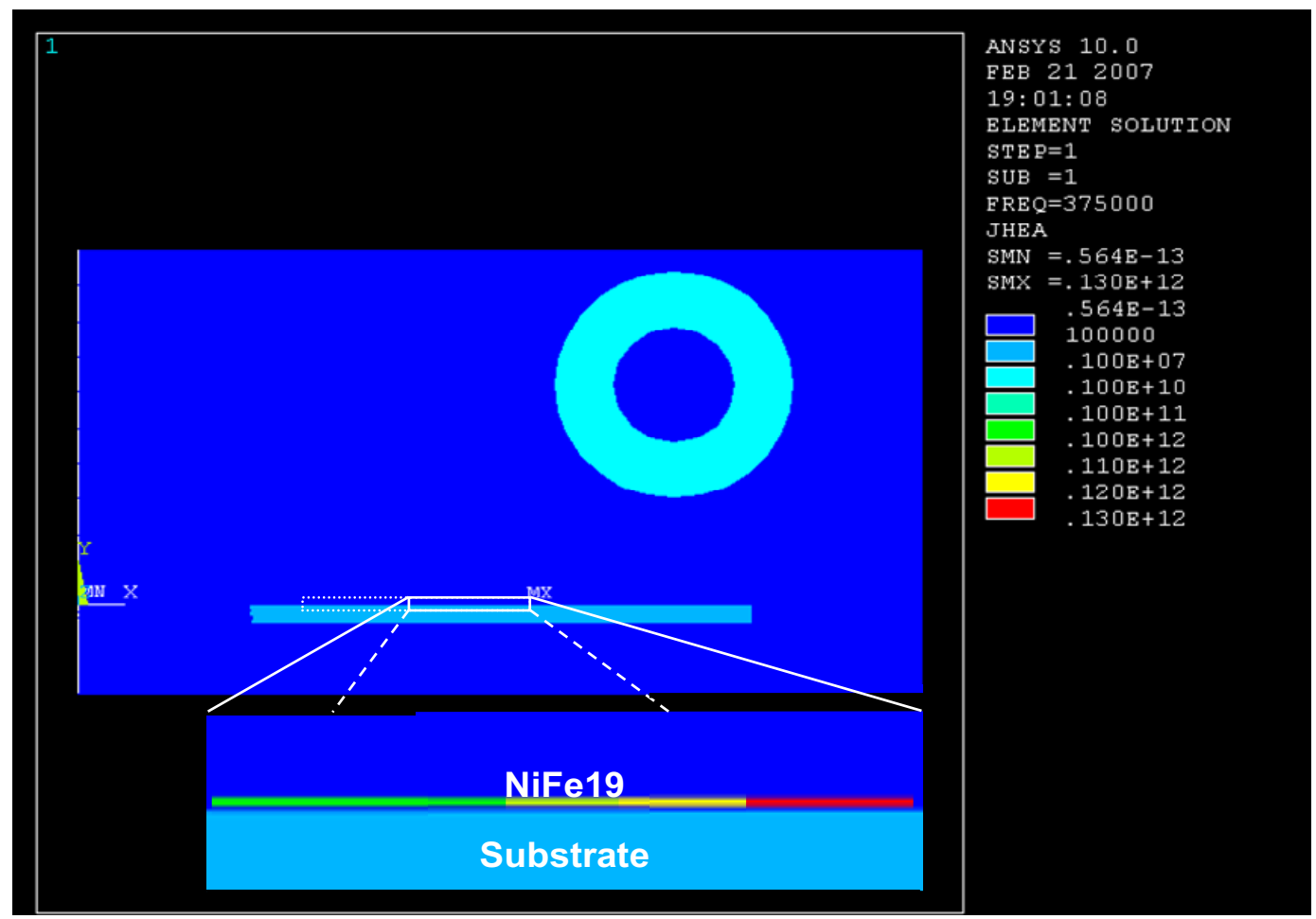

Figure 3.21: Contour plot showing the relative power density dissipation in the thin film model. The ferromagnetic film reached a peak density value of $1.30 \mathrm{E} 11 \mathrm{~W} / \mathrm{m}^{3}$, which was at least six orders of magnitude higher than that in the substrate.

currents were once again opposite the direction of the applied current, with the current density in the ferromagnetic film reaching a peak magnitude of $1.86 \mathrm{E} 8 \mathrm{~A} / \mathrm{m}^{2}$, the majority of the substrate falling within the $100-1000 \mathrm{~A} / \mathrm{m}^{2}$ contour, and the remainder of the model failing to exceed $100 \mathrm{~A} / \mathrm{m}^{2}$ (and was likely much lower). Note that the peak magnitudes of both $J_{Z}$ and $B_{X}$ did not occur exactly at the outer edge; this was believed to be a result of the non-uniformity in the magnetic field. Finally, the power density contour plot is shown in Figure 3.21. The NiFe19 film once again demonstrated considerable superiority over the other materials, including the substrate. Comparison against theoretical values was not straightforward, as the effective permeability value was uncertain. Table 3.12 shows a comparison of the calculated values using Equation (2.6a) to obtain the theoretical power, factoring out the element sizes to obtain the density, and 


\begin{tabular}{lllll}
\hline \hline \multicolumn{5}{c}{ TABLE 3.12 } \\
\multicolumn{1}{c}{ TheORETICAL AND Simulated ElECTROMAGNETIC QuANTITIES } \\
\hline Variable & Simulated & Calculated & Calculated & Calculated \\
& Value & using $\rho_{\mathrm{X}}$ & using $\rho_{\mathrm{Y}}$ & using $\rho_{\mathrm{XY}}$ \\
\hline Power $(\mathrm{W})$ & 204.4 & 0.242 & 0.139 & 0.197 \\
$\begin{array}{l}\text { Power Density } \\
\left(\mathrm{W} / \mathrm{m}^{3}\right)\end{array}$ & $1.30 \mathrm{E} 11$ & $2.05 \mathrm{E} 10$ & $6.68 \mathrm{E} 9$ & $1.36 \mathrm{E} 10$ \\
$\begin{array}{l}\text { Current } \\
\text { Density }\left(\mathrm{A} / \mathrm{m}^{2}\right)\end{array}$ & $1.86 \mathrm{E} 8$ & $3.12 \mathrm{E} 8$ & $1.78 \mathrm{E} 8$ & $2.54 \mathrm{E} 8$ \\
\hline \hline
\end{tabular}

dividing by the resistivity to obtain the square of the current density (which was shown in the previous section to be procedurally correct). The table shows that the theoretical power dissipation values were all offset by three orders of magnitude from the total simulated value, which was computed by ANSYS using the powerh command. The power density values were considerably closer, with only one order of magnitude between them. Converting these to current density values, however, yielded results that were surprisingly close to the $J_{Z}$ obtained from Figure 3.20 , with $\rho_{Y}$ (which was 15.8) producing a theoretical value equal to 0.96 times the simulated value. The significance of these results was not entirely clear, but the close correlation of $\rho_{Y}$ with the $J_{Z}$ value suggested that the in-plane current density still followed the anticipated behavior but that the total power distribution was significantly affected by the unknown out-of-plane currents. Note that ANSYS was not capable of modeling hysteresis heating, and electric fields were not included as a degree of freedom, so all power dissipation should have been through eddy currents. Thus the theoretical model was only partially upheld in this more realistic scenario.

With the electromagnetic physics environment written and tested, the model generation continued with the establishment of the thermal physics environment. The conversion was essentially the same as was done in the ANSYS example model; the 
element types were switched over to their thermal counterparts, and the boundary conditions specific to the thermal model were applied. The outermost edges of the infinite surface elements were once again designated as infinite boundaries and were assigned a constant $300 \mathrm{~K}$ temperature. The optional temperature constraints on the back side and outer edge of the substrate were also available. Finally, the automated time-stepping options were defined to range from $10 \mathrm{~ms}$ to $1 \mathrm{~ns}$, with a $1 \mu$ s starting point, and the thermal physics environment was stored.

The execution of the solution was modeled after the code from the ANSYS example model, in which a repeating do-loop sequentially performed the electromagnetic simulation, followed by the thermal simulation, for the specified number of iterations. One key difference, however, was that it was split into two separate loops, where the first was responsible for the simulation start-up and executed until simtime $=1 \mathrm{sec}$ with increments of $50 \mathrm{~ms}$ between electromagnetic re-evaluations, and the second drove the simulation to completion with $0.5 \mathrm{~s}$ increments and the maximum time step of the thermal environment increased to $0.1 \mathrm{~s}$. This allowed the model to maintain accuracy during the critical formation stage but reduced the simulation time by several hours. At the conclusion of the simulation, specific nodes were selected (via the keypoints to which they were attached) for temperature evaluation. The plvar command was utilized to generate plots of the inner and outer edges of the ferromagnetic film and the nearest edges of the two polysilicon films. The locations of these sites are illustrated in Figure 3.22. They were intended to illustrate the relative rates of temperature rise and the degree of thermal localization achieved by the structure. As the results will show, however, localization was limited by the high thermal conductivity of the substrate. 


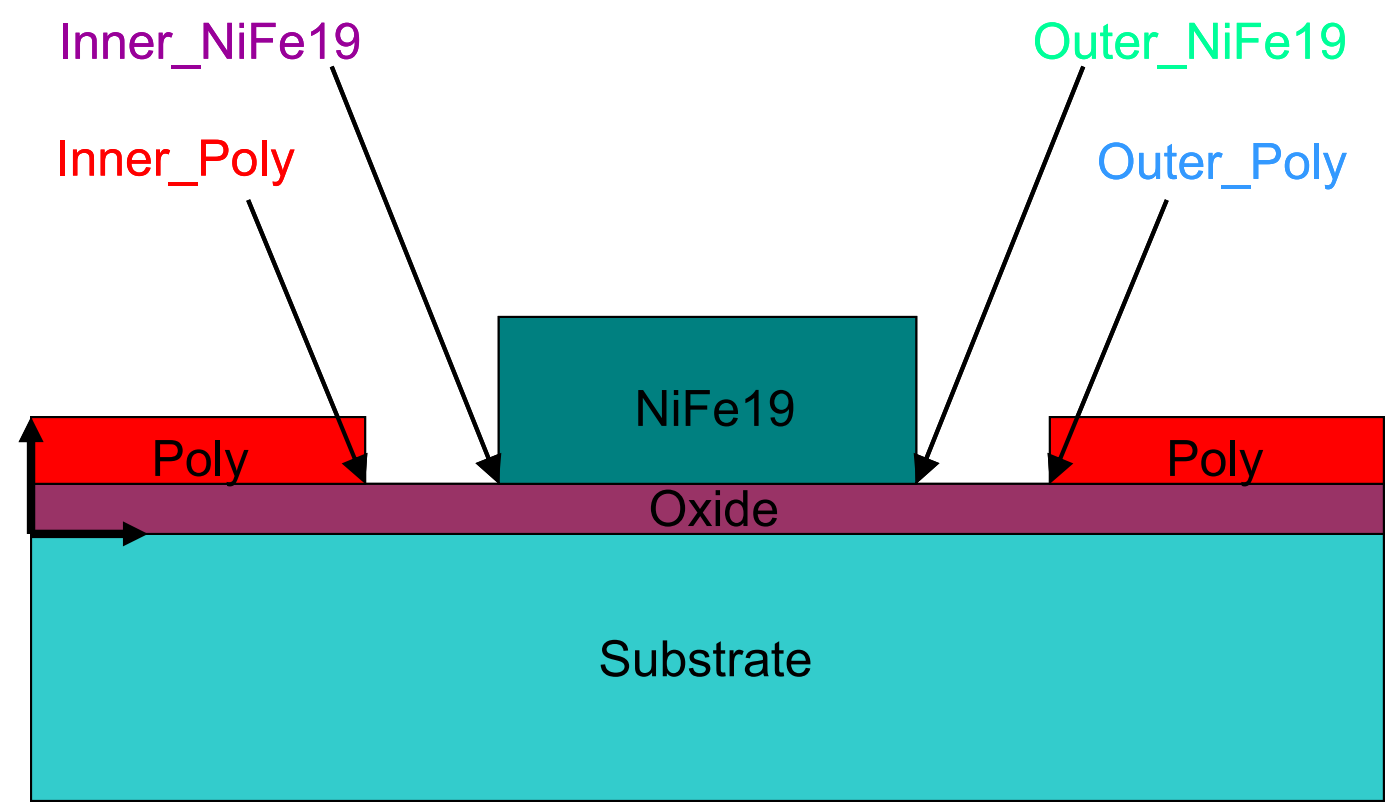

Figure 3.22: Illustration of the points of measurement for plotting in the charts of simulated temperature with respect to time. The four locations were intended to help illustrate the degree of localization obtained within the film stack.

The simulation was run under three different conditions: with no temperature constraints, with the back side of substrate constrained to $300 \mathrm{~K}$, and with the edge of substrate constained to $300 \mathrm{~K}$. The results of the unconstrained simulation are presented in Figures 3.23(a,b). The temperature plot in Figure 3.23(a) shows an initial temperature rise at approximately $230 \mathrm{~K} / \mathrm{s}$ that slowed down to $40 \mathrm{~K} / \mathrm{s}$ upon reaching the $\sim 650 \mathrm{~K}$ Curie temperature of the NiFe19 film. The final temperature after 10 s peaked at $1008 \mathrm{~K}$, or $735^{\circ} \mathrm{C}$, with the maximum value occurring near the horizontal center of the film as indicated in Figure 3.23(b). The plots reflected a minimal degree of localization due to the high thermal conductivity of the silicon substrate. With temperatures of approximately $975 \mathrm{~K}$ at the center and $955 \mathrm{~K}$ at the edge, the temperature gradients evaluated to

$$
\nabla T_{\text {Inner }}=\frac{1000-975 \mathrm{~K}}{0.6 \mathrm{~cm}}=41.7 \mathrm{~K} / \mathrm{cm}
$$




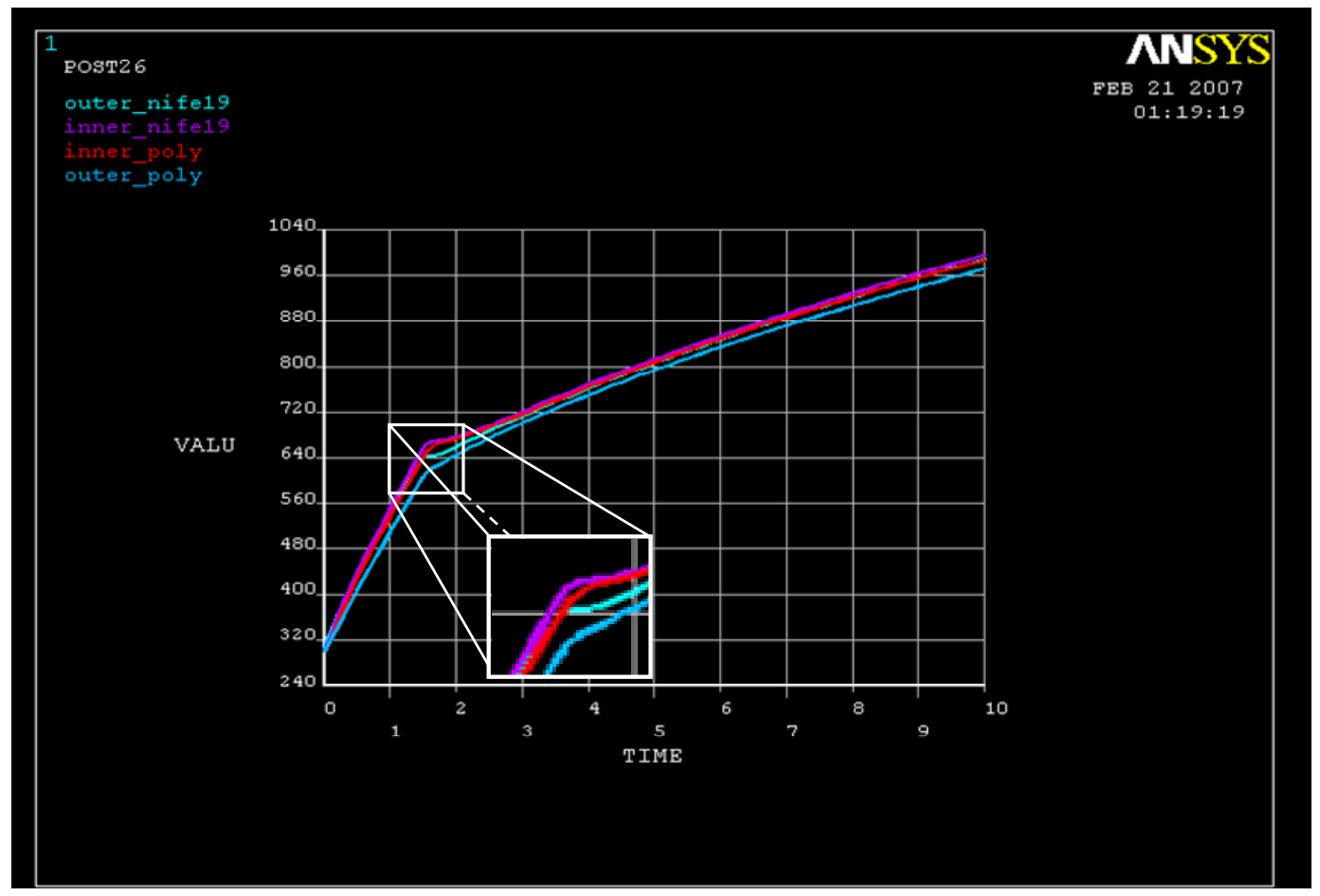

Figure 3.23(a): Graph of temperature, in Kelvin, vs time following a 10-second run of the default, unconstrained thin film simulation. The rate of temperature rise slowed down as the ferromagnet reached its Curie temperature, which was at approximately $650 \mathrm{~K}$.

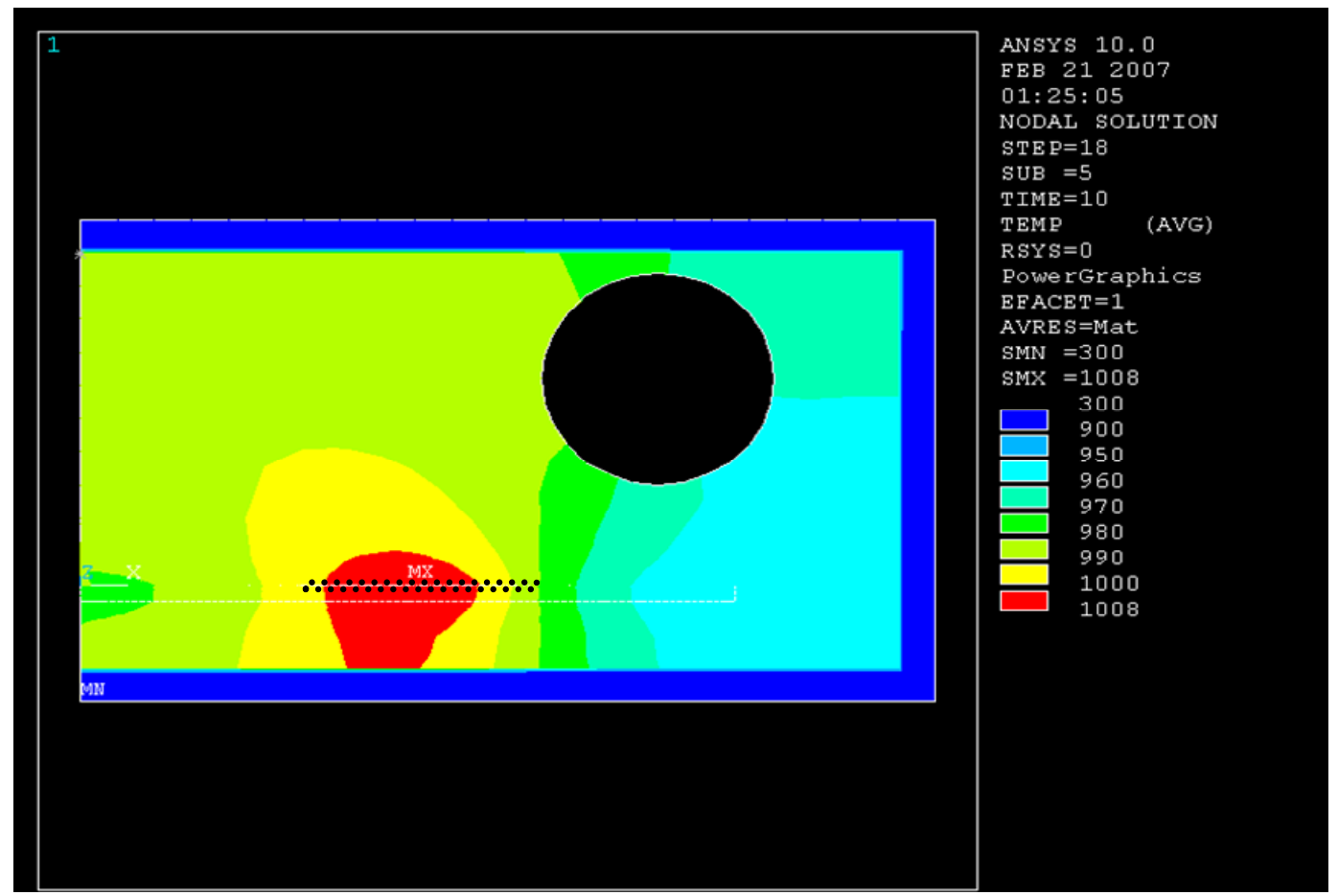

Figure 3.23(b): Contour plot showing the final temperature distribution, in Kelvin, across the model after a 10-second coupled simulation of the thin film model without temperature constraints on the substrate. The high thermal conductivity of the silicon substrate prevented the creation of an appreciable temperature gradient. 
and

$$
\nabla T_{\text {Outer }}=\frac{1000-955 \mathrm{~K}}{0.775 \mathrm{~cm}}=58.1 \mathrm{~K} / \mathrm{cm}
$$

Thus there was considerable room for improvement, and the importance of preventing the thermal energy from being drawn into the substrate was clear. Figures 3.24(a,b) illustrate the effect of regulating the temperature at the back side of the substrate to $300 \mathrm{~K}$, presumably through a heat sink. As the chart of temperature vs time in Figure 3.24(a) shows, all four temperatures almost immediately stabilized to their permanent values, with the polysilicon regions fixed at $300 \mathrm{~K}$ and the outer and inner NiFe19 regions at $302 \mathrm{~K}$ and $300.6 \mathrm{~K}$, respectively. This corresponded to gradients of

$$
\nabla T_{\text {Outer }}=\frac{302-300 \mathrm{~K}}{0.15 \mathrm{~cm}}=13.33 \mathrm{~K} / \mathrm{cm}
$$

and

$$
\nabla T_{\text {Inner }}=\frac{300.6-300 \mathrm{~K}}{0.15 \mathrm{~cm}}=4.00 \mathrm{~K} / \mathrm{cm}
$$

Thus regulating the substrate temperature helped to drive the process to a steady-state but created an excessive temperature draw on the ferromagnetic power source. This same effect was observed during experimentation, in which placing a heat sink behind the substrate prevented any measurable temperature changes from being induced. Finally, Figures 3.25(a-d) show the results of an attempted middle ground through the regulation of the temperature at the edge of the substrate. This configuration was representative of the wafer stage designed by MTU undergraduates for the custom inductive heating test system described in the next chapter when utilized without a heat sink. The primary difference was that the smaller sample size utilized in the simulation rendered the point of contact closer to the center than in reality. The temperature-vs-time plot in Figure 3.25(a) was quite unique; the four temperatures initially rose quite rapidly, but just as they began 


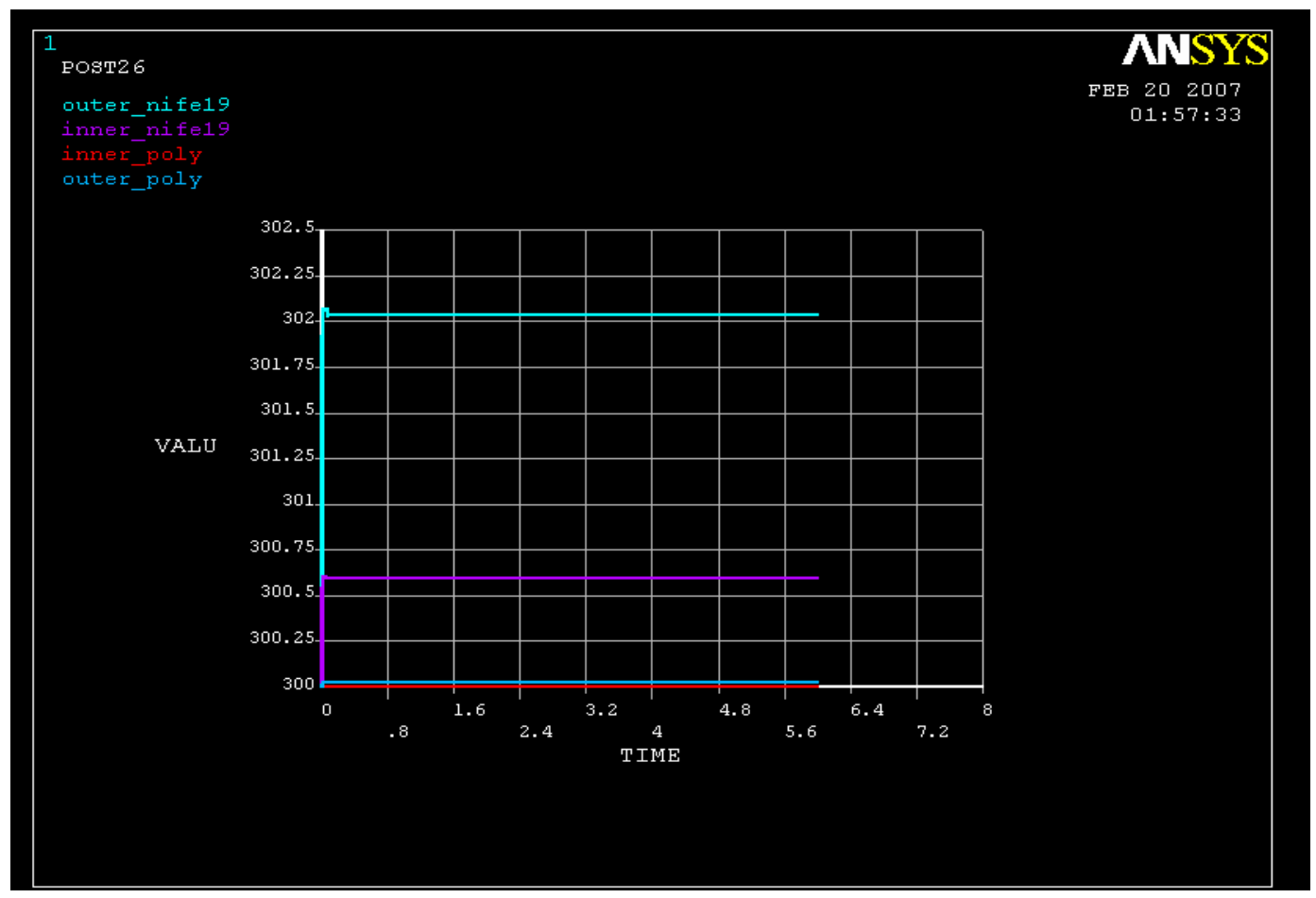

Figure 3.24(a): Graph of temperature (K) vs time during a 6-second simulation of the thin film model with the back side of the substrate held at $300 \mathrm{~K}$. The result was a severe limitation on the induced temperatures due to the heat sinking of the entire structure.

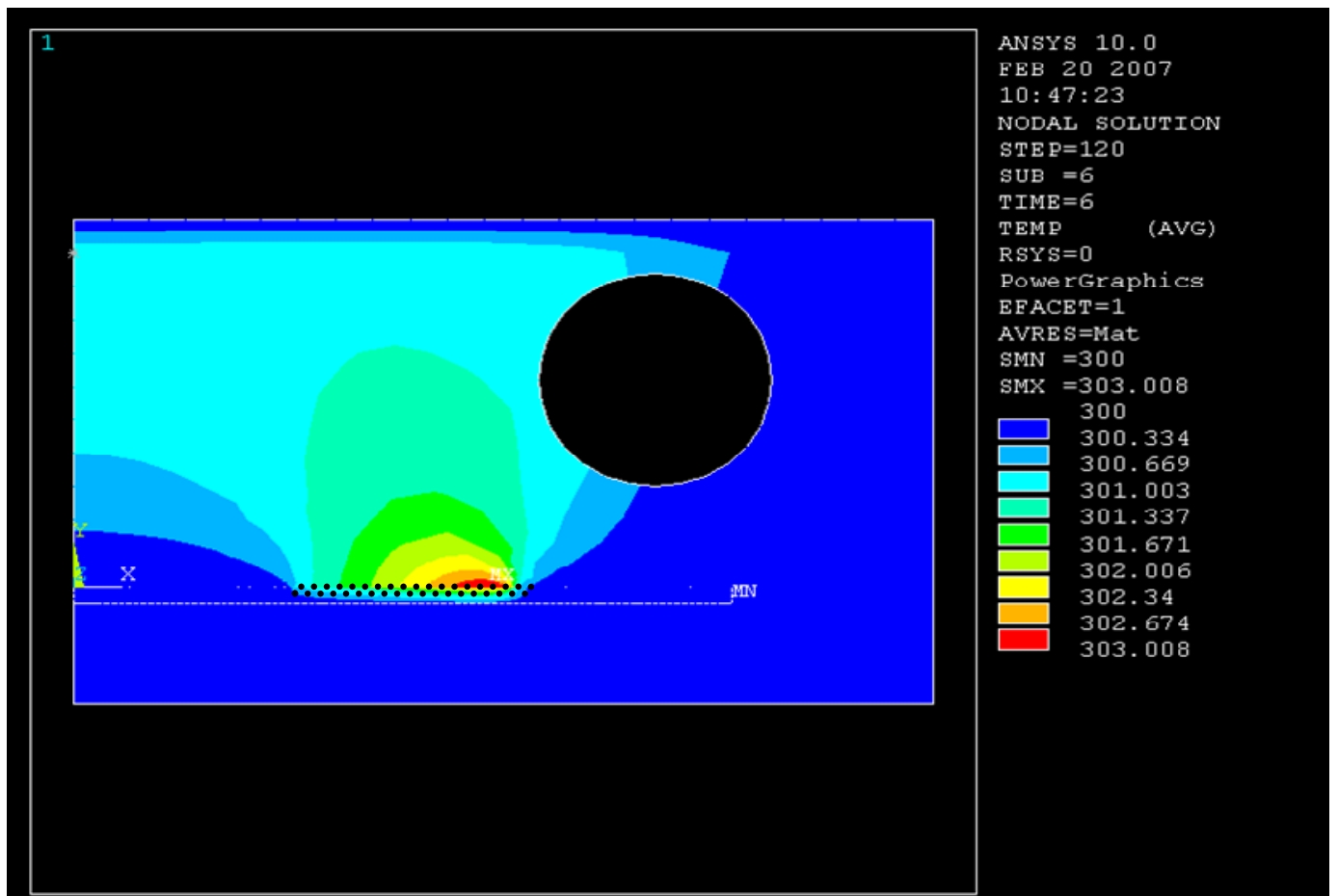

Figure 3.24(b): Contour plot showing the temperature distribution (K) across the model following a 6 -second simulation with the back side of the substrate held at $300 \mathrm{~K}$. The thermal energy was prevented from spreading laterally, but the attainable temperature rise was severely reduced. 


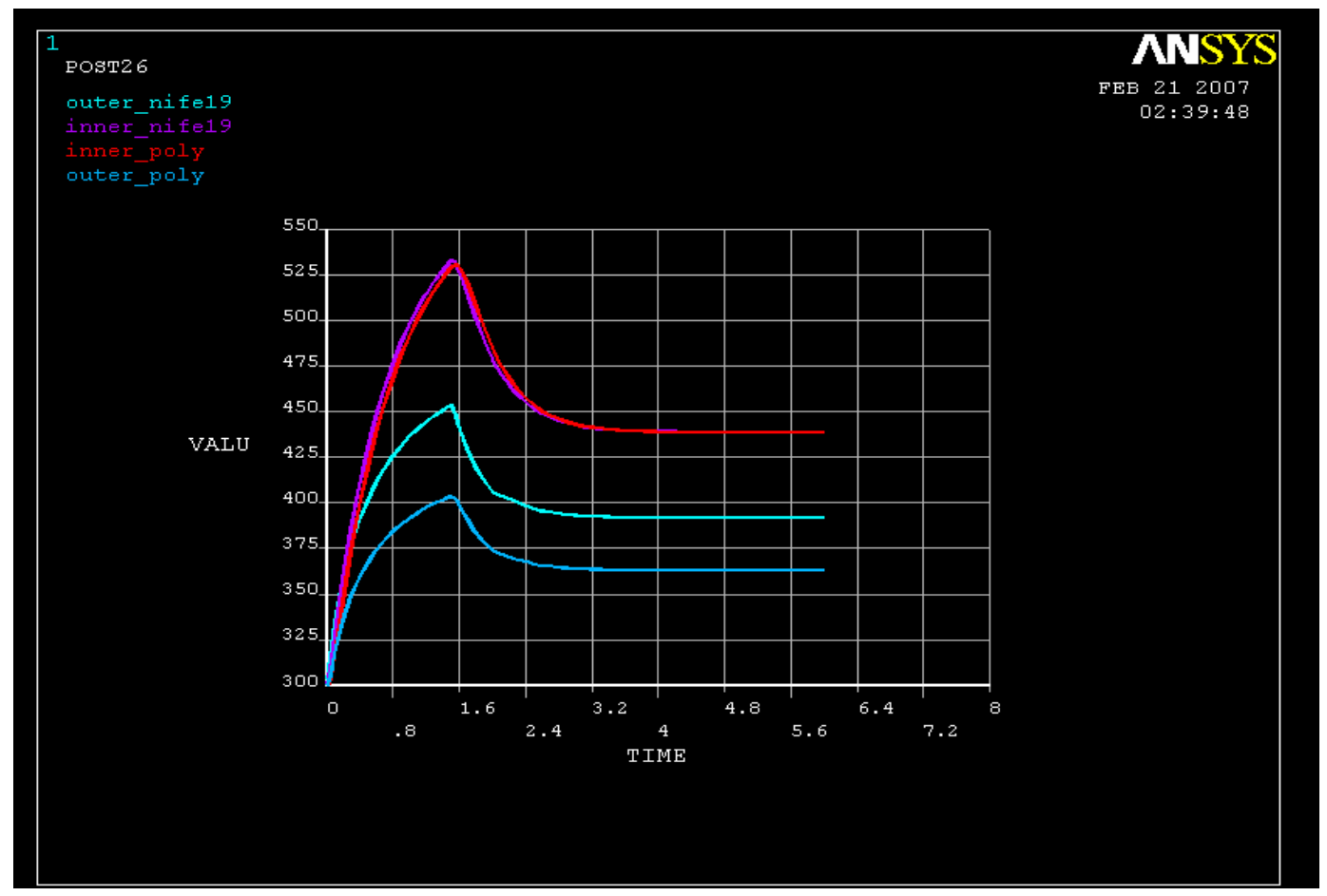

Figure 3.25(a): Graph of temperature (K) vs time during a 6-second simulation in which the outer edge of the substrate was held at $300 \mathrm{~K}$. The temperatures reached higher steady-state values than they did with the back side of the substrate fixed, but they were unable to overcome the lateral temperature gradient that was set up across the substrate.

to stabilize, they each suddenly dropped back down and to approximately $10-15 \%$ below their peak values. To understand this phenomenon, contour plots at various time snapshots were obtained. Figure 3.25(b) shows a close-up on the ferromagnetic film at simtime $=0.492 \mathrm{~s}$. The figure shows that heat generation initiated in a manner typical for the model, with the temperature rise initiating from the approximate center of the NiFe19. The maximum value at this time was $440 \mathrm{~K}$, approximately equal to that of the final distribution. The temperatures for each of the four curves reached their peaks at around $1.58 \mathrm{~s}$; Figure $3.25(\mathrm{c})$ shows that by this time, the source of heat generation had shifted toward the far inside portion of the ferromagnet. At this point, the curves began sharply trending downward, retaining the same qualitative distribution but decreasing in magnitude as shown in Figure 3.25(d). The unusual behavior was thus the result of two 


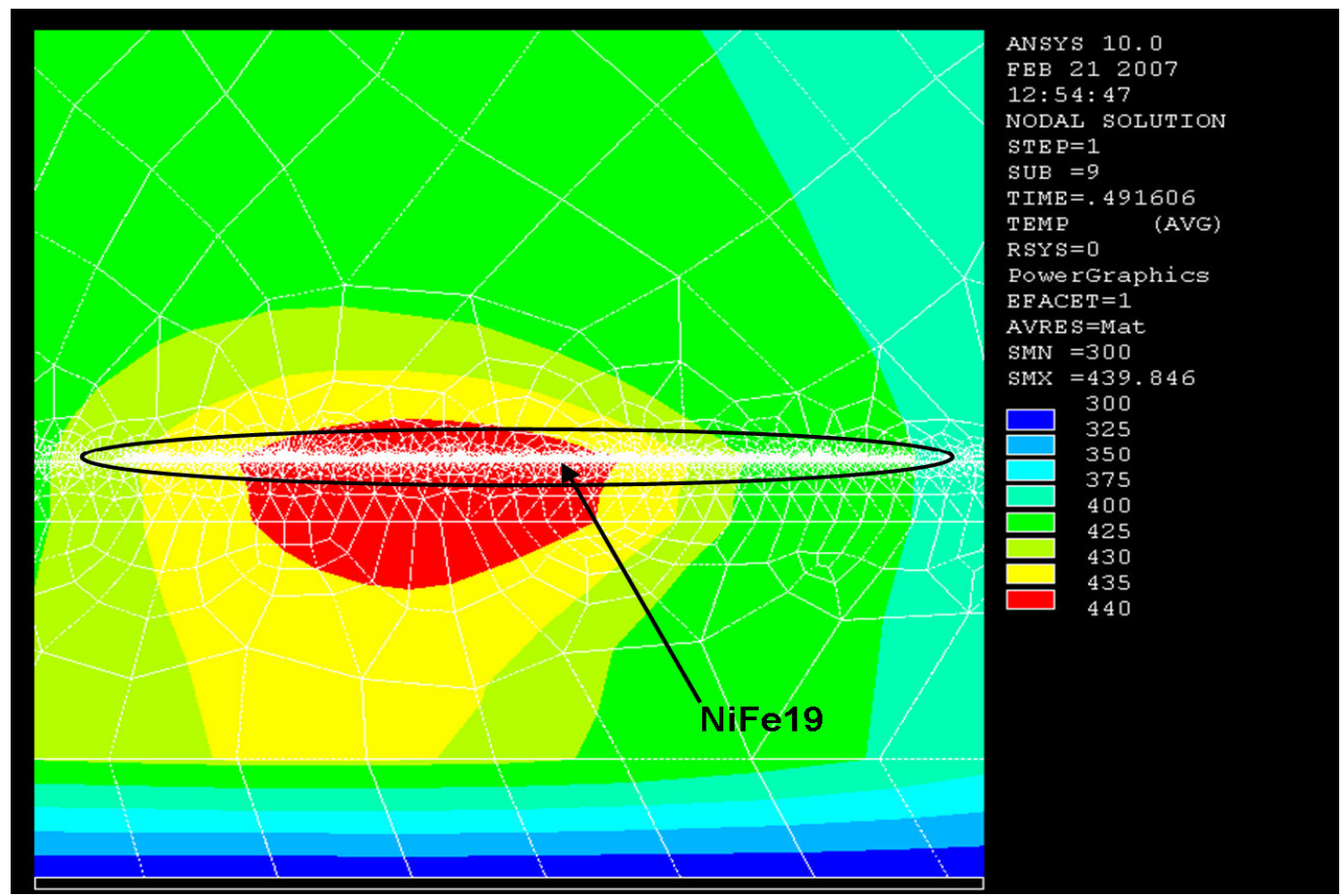

Figure 3.25(b): Contour plot showing the temperature distribution $(\mathrm{K})$ in and around the $\mathrm{NiFe} 19$ film at simtime $=0.49 \mathrm{~s}$ with the outer edge of the substrate held at $300 \mathrm{~K}$. The early temperature distribution bore close resemblance to that of the unperturbed model.

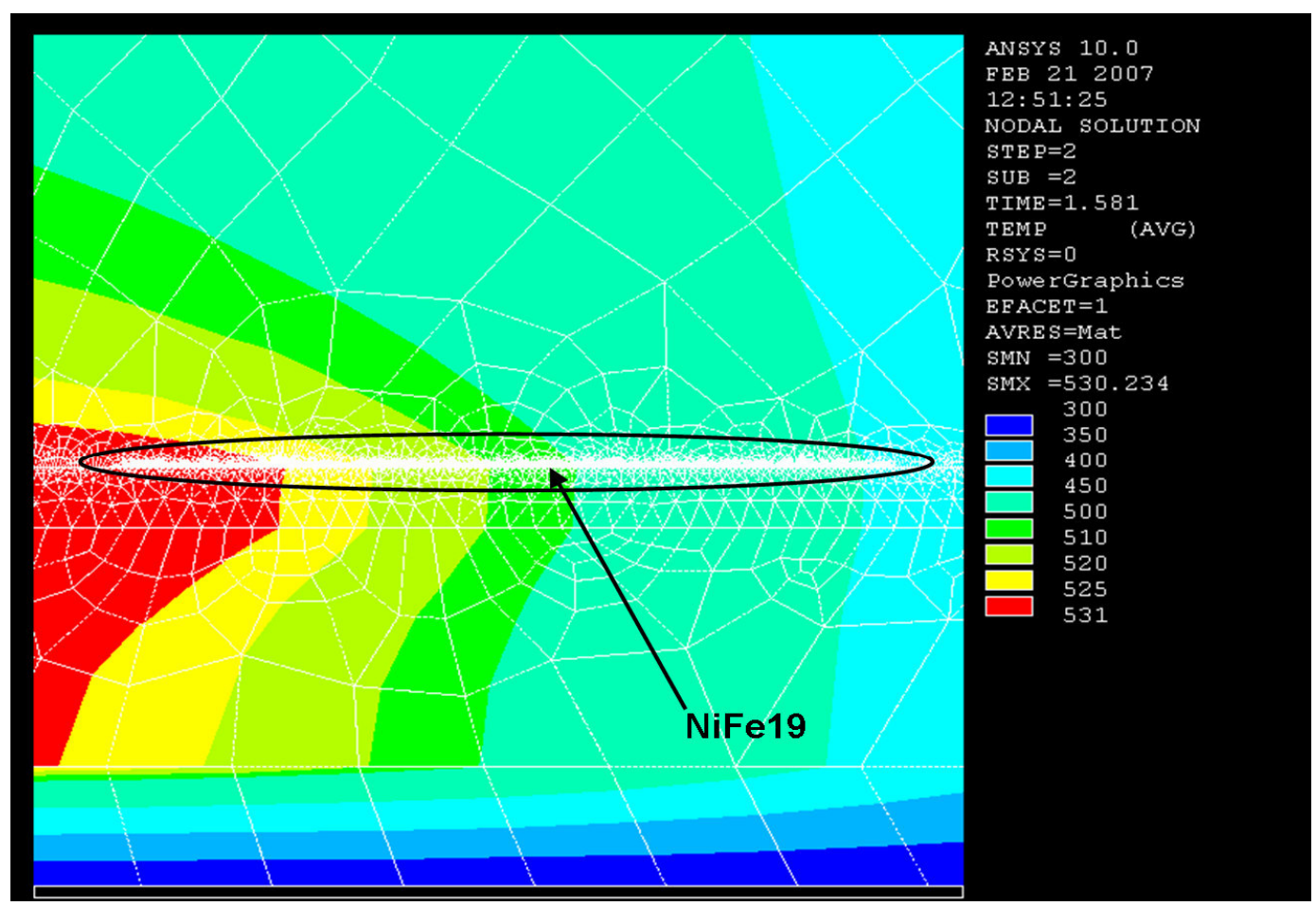

Figure 3.25(c): Contour plot showing the temperature distribution $(\mathrm{K})$ in and around the $\mathrm{NiFe} 19$ film at simtime $=1.58 \mathrm{~s}$ with the outer edge of the substrate held at $300 \mathrm{~K}$. This was the time at which all temperature values peaked, and by this point the heat source had shifted to the inside portion of the NiFe19 ring. 


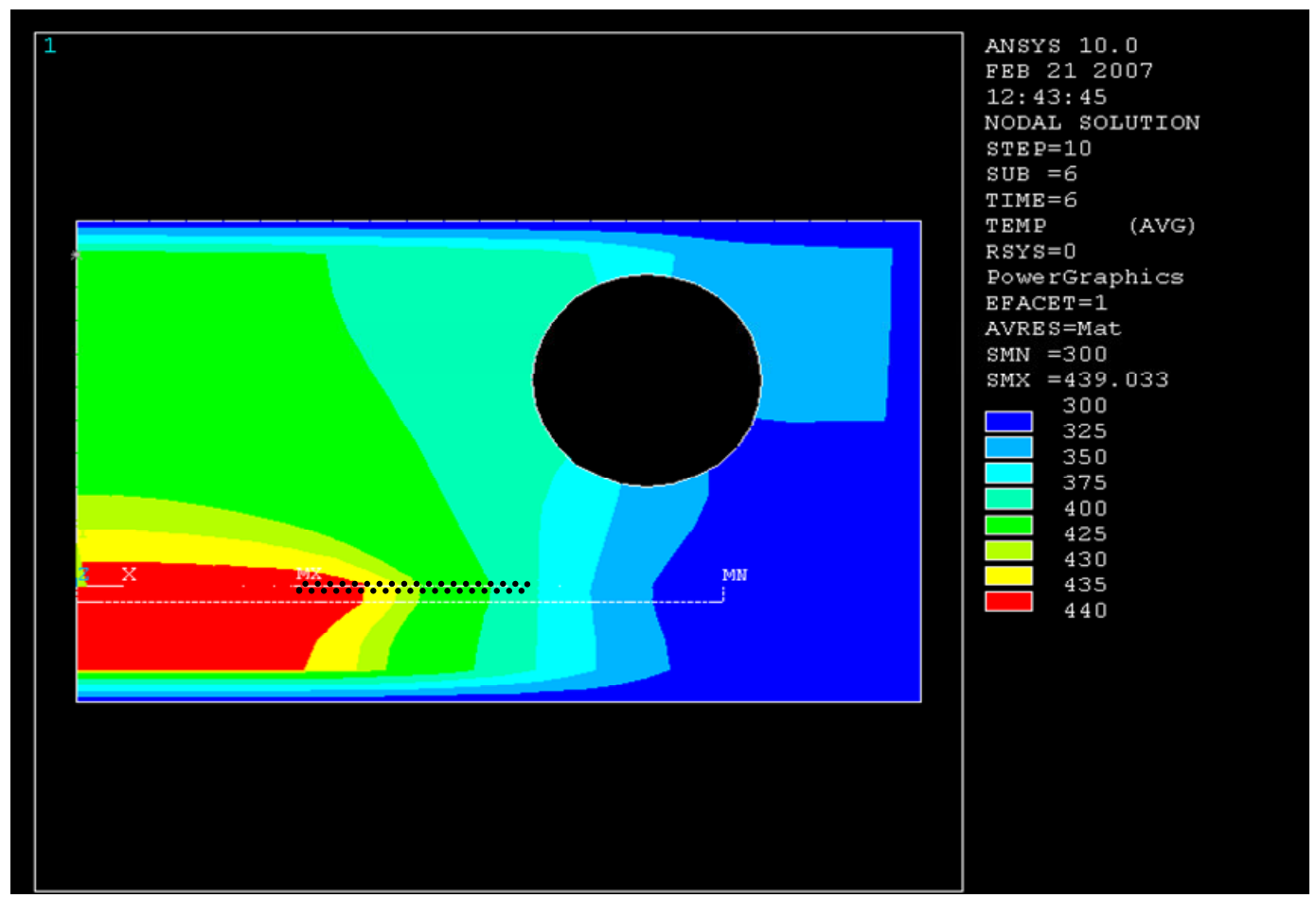

Figure 3.25(d): Contour plot showing the temperature distribution $(\mathrm{K})$ across the model at simtime $=6 \mathrm{~s}$ with the outer edge of the substrate held at $300 \mathrm{~K}$. The contours indicate that the temperature distribution retained the same shape as that shown in Figure 3.25(c).

competing phenomena, similar in nature to that encountered when the back side of the substrate was regulated but with a different final outcome. At first, the inductively heated film and the $300 \mathrm{~K}$ heat sink operated as separate sources. Once their ranges collided, however, the heat sink became dominant as it was capable of sinking an infinite amount of energy. The end result was that the $300 \mathrm{~K}$ heat sink dominated the behavior in the outer portion of the structure, while the film retained control over the interior (note that the location of maximum temperature was still within the NiFe19 film and thus the temperature at the origin was slightly lower). The temperature gradients computed to

$$
\nabla T_{\text {Outer }}=\frac{390-363 \mathrm{~K}}{0.15 \mathrm{~cm}}=180 \mathrm{~K} / \mathrm{cm}
$$

and 


$$
\nabla T_{\text {Inner }}=\frac{438-438 K}{0.15 \mathrm{~cm}} \approx 0 \mathrm{~K} / \mathrm{cm}
$$

Once again, similar effects were observed during experimentation when the substrate was pinned at the edges. The strong lateral gradient that was established rendered a configuration of this type unusable for device application, however, as there would be considerable non-uniformity among devices. The persistent trade-off between process control and annealing temperature was often re-optimized as experimentation led to improved designs in the test samples and in the test system itself. The model presented in the next section shows various attempts at improving on the results of the original thin film model through thermal isolation, i.e., selective placement and dimensions of silicon dioxide layers.

\section{4: Thin Film Induction Heating Model with Thermal Isolation}

Resolving the thermal isolation challenge was a critical factor in achieving the desired temperature gradients suitable for high-temperature annealing in the vicinity of temperature-sensitive CMOS components, and thereby realizing a successful technology. The model presented in this section drew from the results of the original thin film model presented in the previous section. The simulation code, presented in Appendix A.4, was essentially the same as that in Appendix A.3, which was described in detail previously. The only difference was the film stack in the heated structure. For this device, the $7.1 \mu \mathrm{m}$ ferromagnetic film was patterned directly above a $3 \mu \mathrm{m}$-thick blanket polysilicon layer. The intent was to vary the thickness of the buried oxide in order to prevent the majority of the thermal energy from reaching the substrate, and to instead utilize the annealed polysilicon layer to provide the necessary thermal conduction within itself. Thermal 


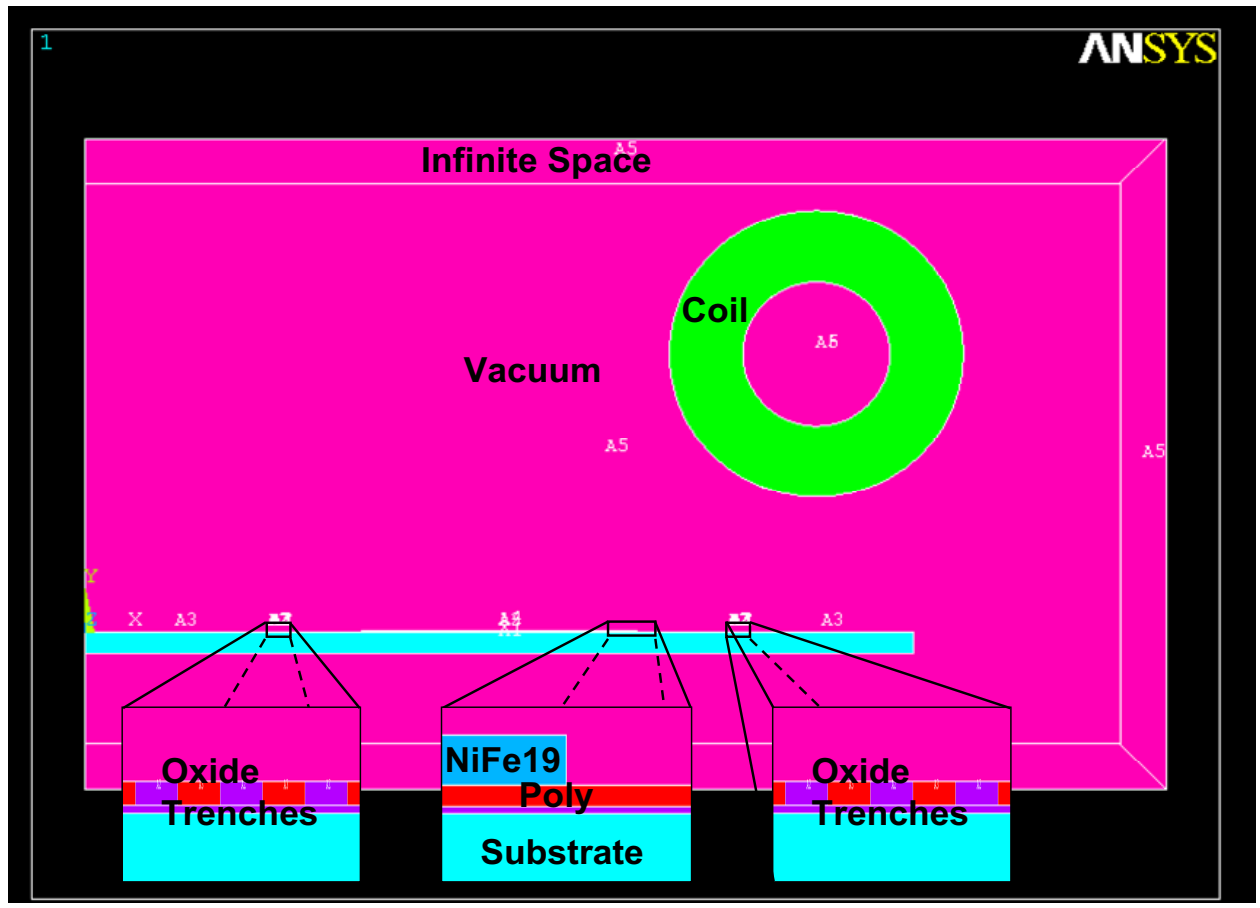

Figure 3.26(a): Area plot of the isolation film solid model designed to enhance thermal isolation. Blanket $\mathrm{SiO}_{2}$ and polysilicon films were formed above the substrate, and a $\mathrm{NiFe} 19$ film was patterned directly above the polysilicon. Trenches in the polysilicon on each side of the ferromagnet were intended to provide lateral isolation.

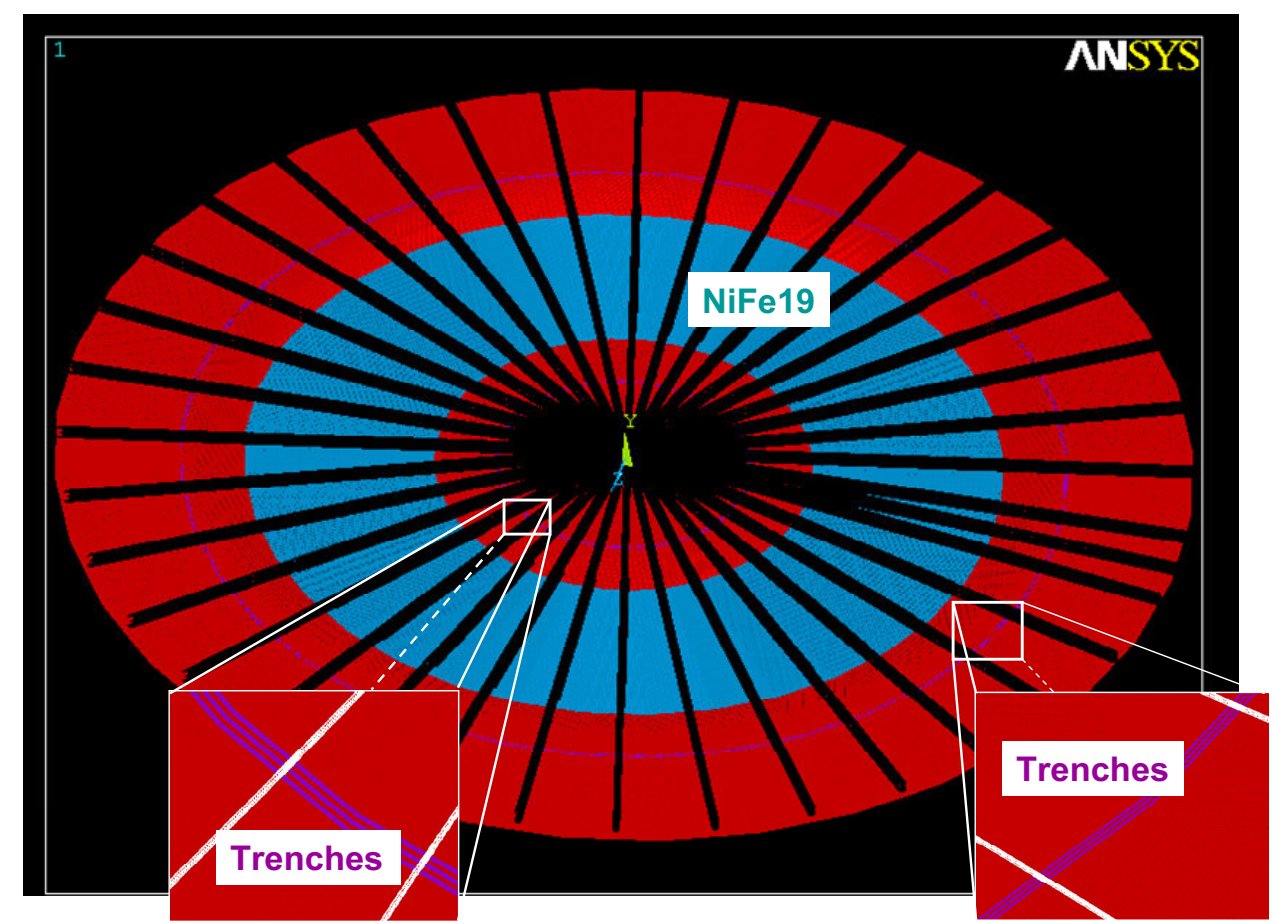

Figure 3.26(b): Three-dimensional rotational area plot showing the layout of the structure more clearly. The NiFe19 ring was of the same dimensions as those of the original thin film model. Three rows of trench rings were formed both inside and outside of the heated film. 


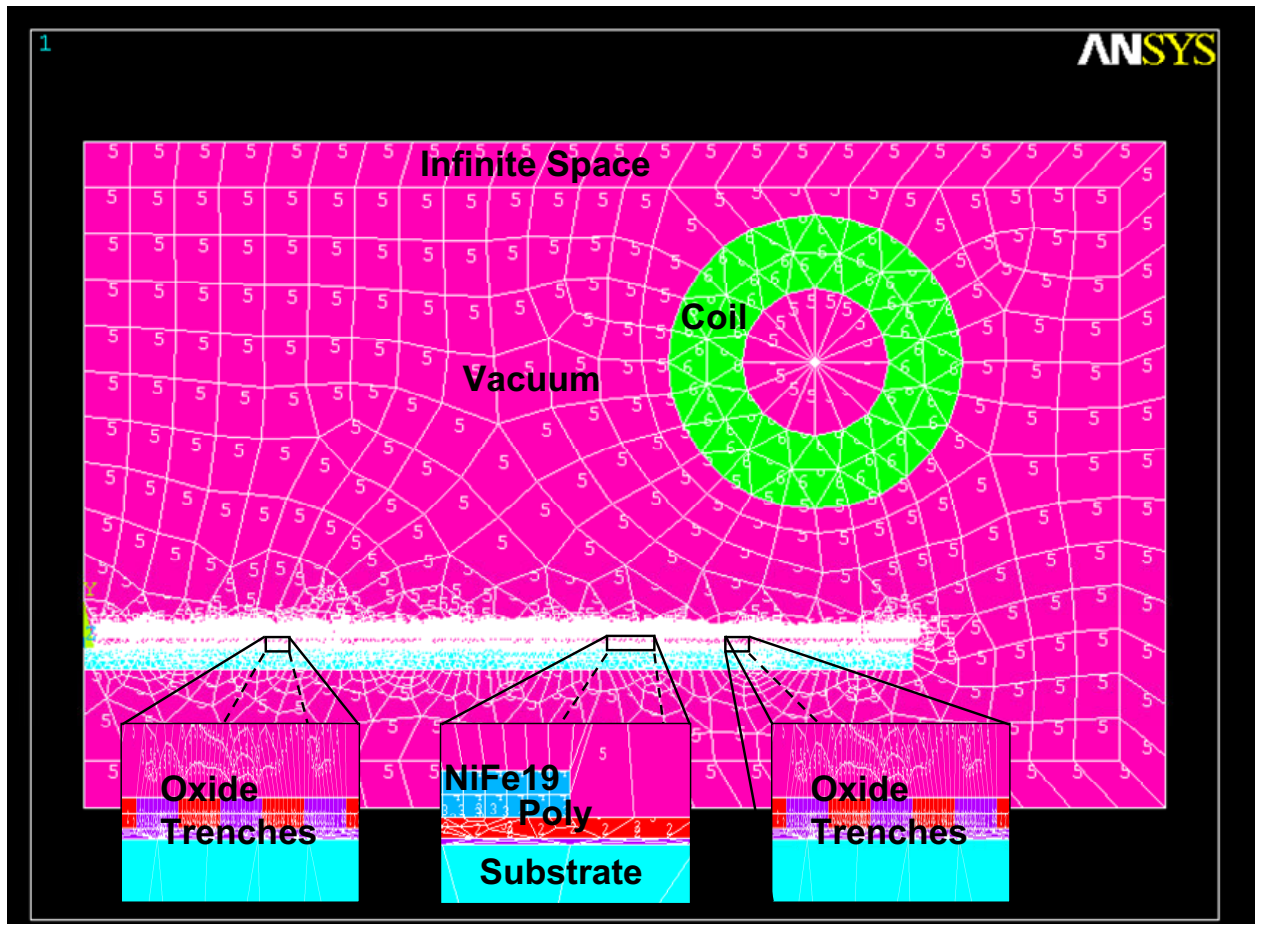

Figure 3.27: Element plot of the isolation film solid model showing the relative sizes of the divisions. Mesh dimensions were the same as those utilized in the original thin film model, and ranged from $4 \mu \mathrm{m}$ in the $\mathrm{NiFe} 19$ film to $2 \mathrm{~mm}$ in the infinite vacuum elements.

isolation trenches were etched into the polysilicon in order to provide lateral containment. The solid model is shown in Figure 3.26(a), with close-ups showing the NiFe19 film in the center and the two sets of oxide-filled isolation trenches on each side. The threedimensional plot in Figure 3.26(b) helps to more clearly illustrate the layout of the structure. The NiFe19 film formed a ring about the y-axis that extended from $0.6-1.2 \mathrm{~cm}$ in radius, the same as was utilized in the original thin film model. Its thickness was reduced slightly in order to coincide with the experimental results presented in Section 5.3. On both sides of the ferromagnetic ring were three rows of oxide-filled trenches, each of which measured $20 \mu \mathrm{m}$ in width with $20 \mu \mathrm{m}$ spaces between them. Since the heated film was essentially unchanged, the electromagnetic properties presented in the previous section were still applicable, and thus only the temperature results from the 


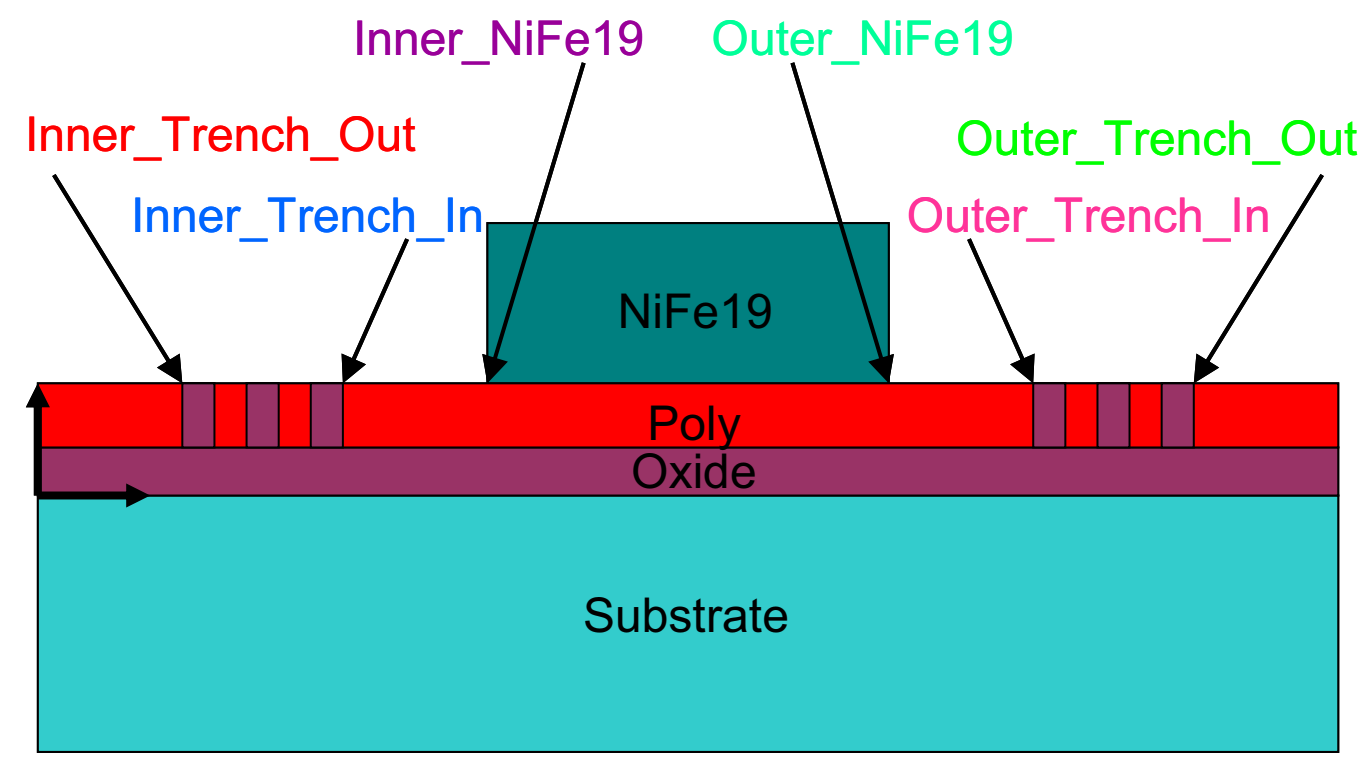

Figure 3.28: Illustration of the points of measurement for plotting in the charts of simulated temperature with respect to time for the isolation film model. The six locations were intended to help illustrate the degree of localization obtained within the film stack

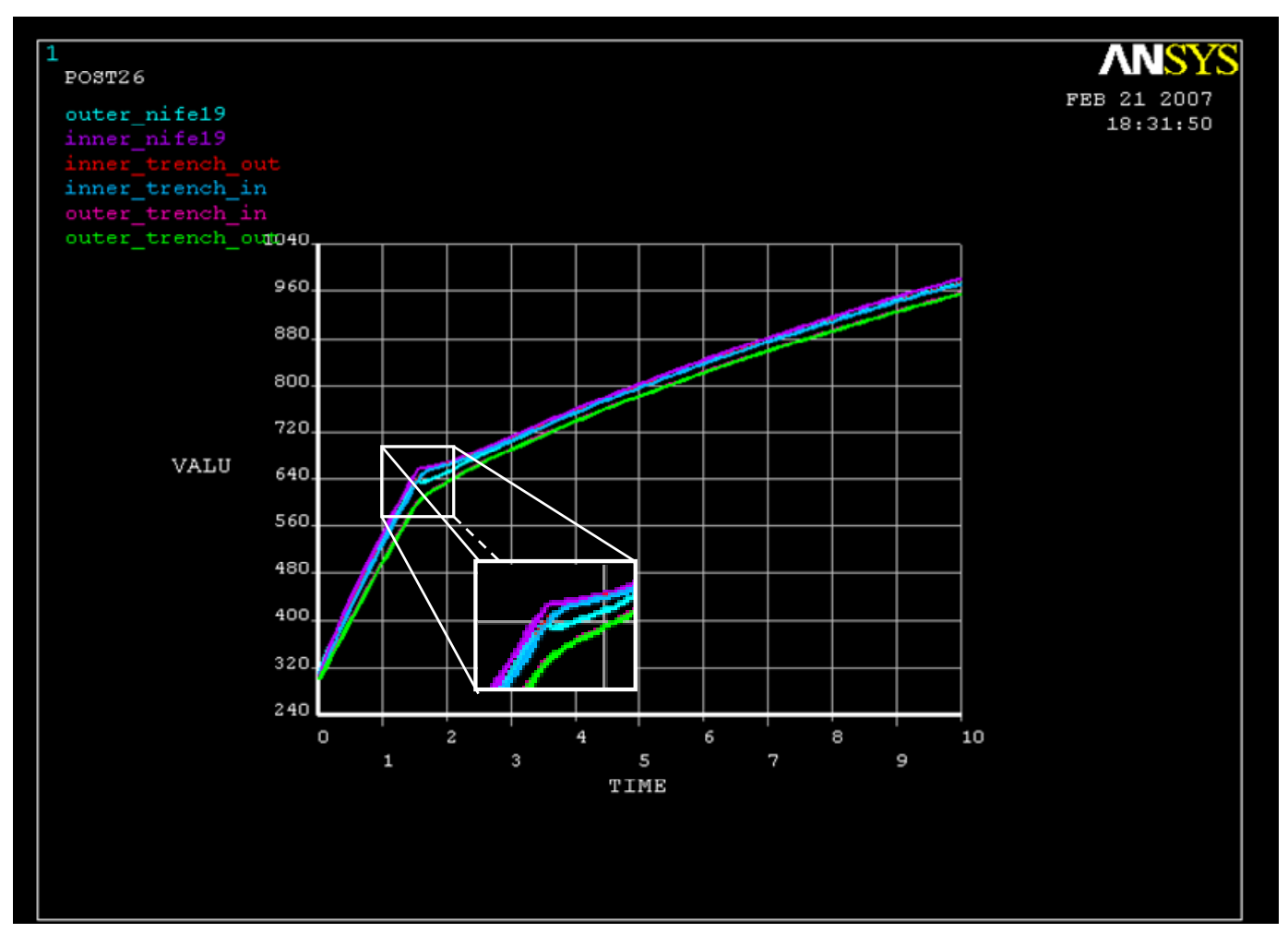

Figure 3.29(a): Graph of temperature $(\mathrm{K})$ vs time during a 10s simulation of the isolation film solid model. The plot was nearly identical to that from the original thin film model, shown in Figure 3.23(a), as the high thermal conductivity of the substrate once again caused the model to be "flooded" with thermal energy. 


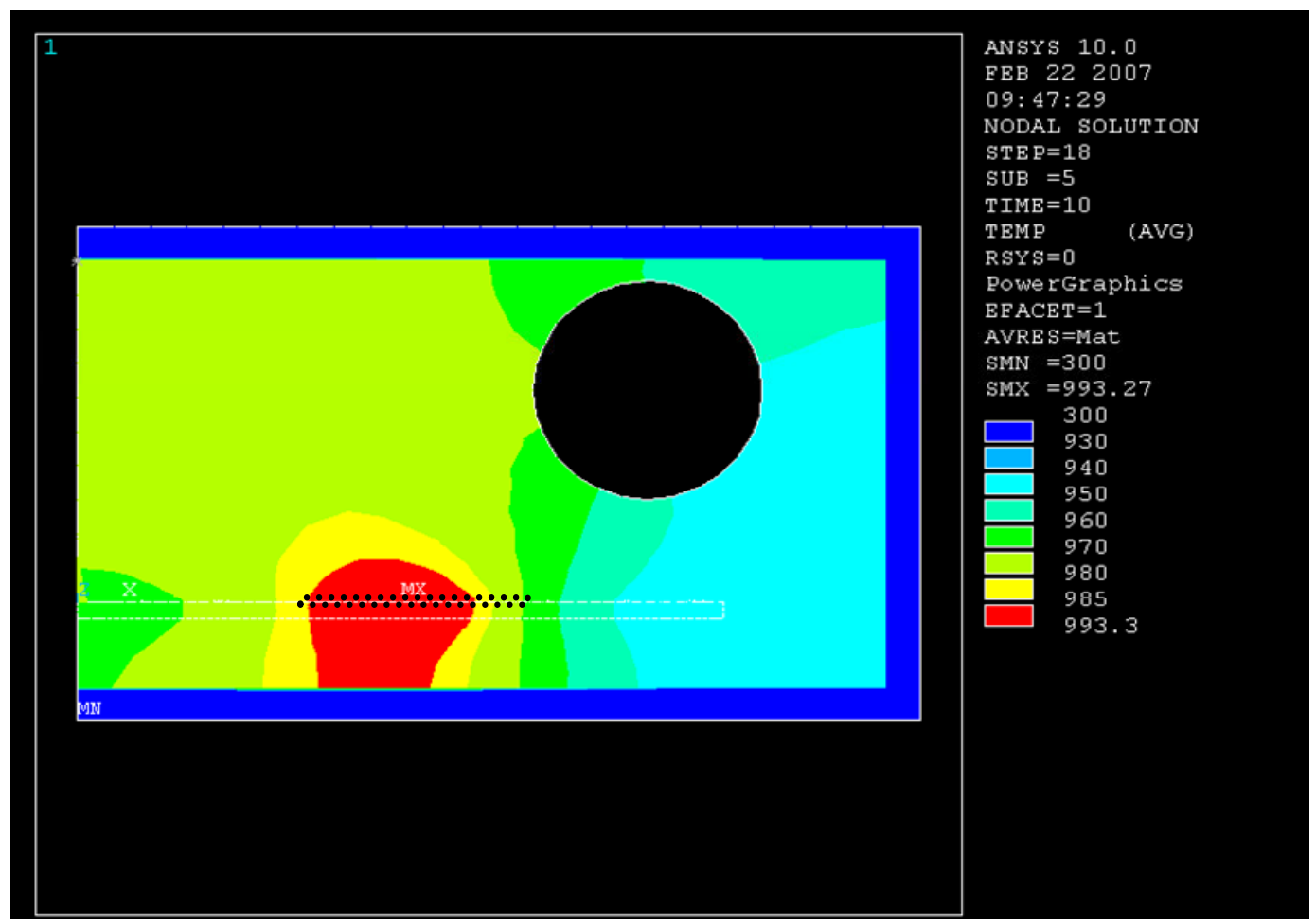

Figure 3.29(b): Contour plot showing the temperature distribution, in Kelvin, across the model following a 10 -second simulation with no thermal constraints placed on the substrate. The results were nearly identical to those from Figure 3.23(b).

coupled solution will be presented. The points of temperature measurement for this model are shown in Figure 3.28. Figures 3.29(a,b) show the results of the simulation using only the default $1 \mu \mathrm{m}$-thick base silicon dioxide layer with no temperature constraints on the substrate. The resulting temperature trends and distribution showed no appreciable difference from those obtained in the original model, as the simulation was once again controlled by the high thermal conductivity of the silicon substrate. Pinning the back side or outer edge of the substrate also produced identical results to the original model, indicating that it was still dominating the thermal gradient.

To remove the contribution of the substrate to thermal conduction, the base $\mathrm{SiO}_{2}$ film was increased in thickness to $20 \mu \mathrm{m}$. While this excessive thickness was rather unrealistic for real-world applications, the intent was to simulate the effect of a more 
effective thermal barrier such as an oxide/nitride/oxide film stack or other highly insulating material. Of the three configurations (unregulated, fixed substrate back side, and fixed substrate edge), the most interesting results were obtained with the back side of the substrate fixed at $300 \mathrm{~K}$. Figures $3.30(\mathrm{a}, \mathrm{b})$ show the results obtained without any additional modifications. Although the improvement over the non-isolation counterpart of Figures 3.24(a,b) still left much to be desired, the magnitude of the temperature differential from ferromagnetic film to substrate climbed from $3 \mathrm{~K}$ to $14 \mathrm{~K}$. Thus the substrate was still drawing considerable thermal energy out of the heated film, but it was theorized that this could be overcome by finding a balance between magnetic field energy and $\mathrm{SiO}_{2}$ thickness. The latter was immaterial to the power requirement as it provided a vertical temperature differential but not an energy sink, so analysis focused on varying the current density within the magnetic coil. In practice, increasing the field strength can be done through increased power (thus requiring a larger power supply) or through coil optimization (increased turns and/or decreased length - but note that higher resistance decreases the output current). The default current density was $2.36 \mathrm{E} 7 \mathrm{~A} / \mathrm{m}^{2}$, which corresponded to a total current of 570A (equivalent to 190A through a three-turn coil). The result of increasing the current density by a factor of 10 , to $2.36 \mathrm{E} 8 \mathrm{~A} / \mathrm{m}^{2}$, is illustrated in Figures 3.31(a,b). The temperature curves in Figure 3.31(a) exhibited an initial instability, producing a square-wave trend. This behavior was encountered with all of the simulations that used the $300 \mathrm{~K}$ back side boundary condition, more so with increasing coil current, but the results eventually converged toward final steady-state values. The contour plot from simtime $=6 \mathrm{~s}$, shown in Figure 3.31(b), demonstrated good thermal isolation between the NiFe19 film and the substrate. The temperatures were assumed to 


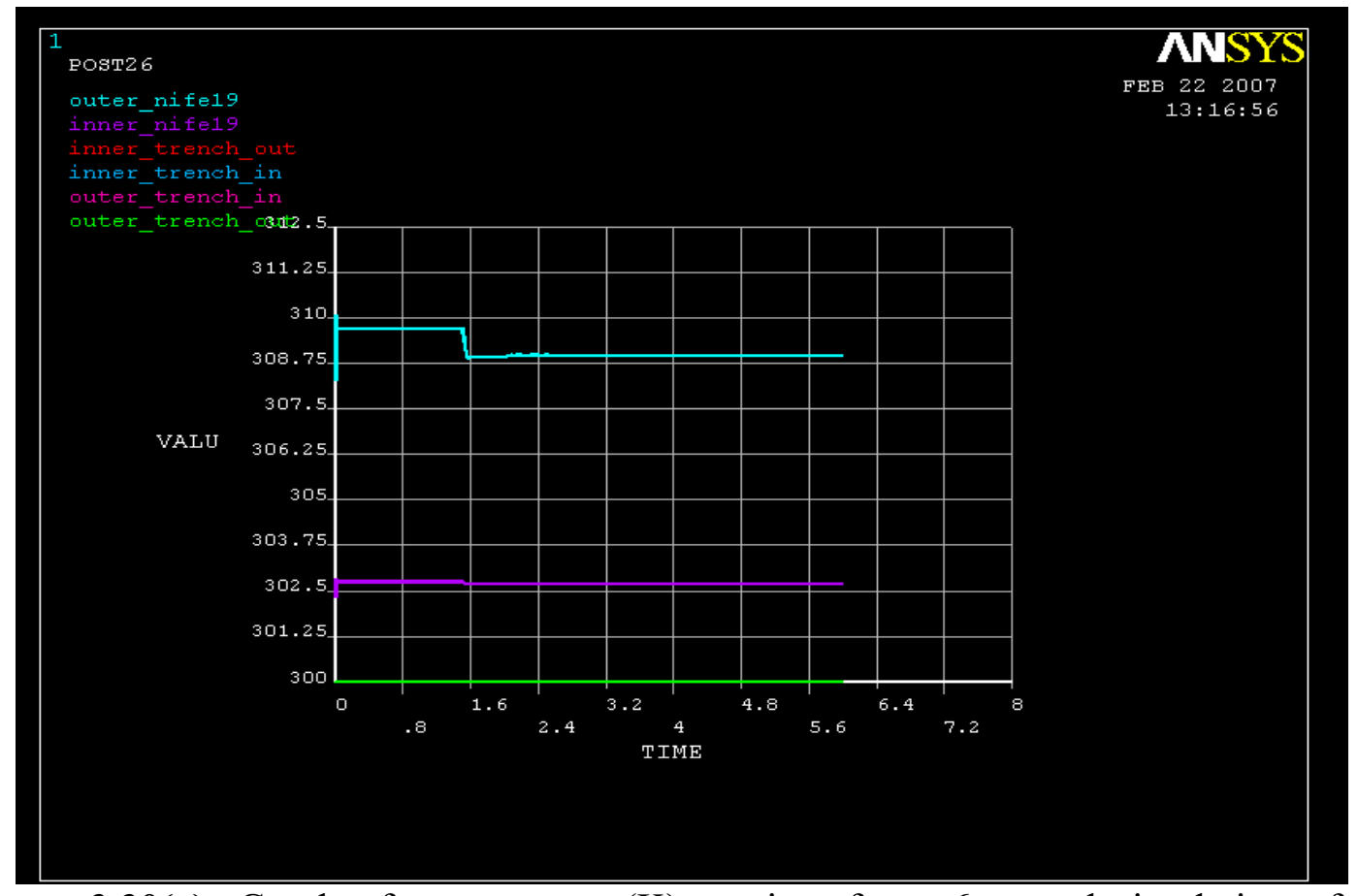

Figure 3.30(a): Graph of temperature $(\mathrm{K})$ vs time for a 6-second simulation of the isolation film solid model with the back side of the substrate held at $300 \mathrm{~K}$ and the $\mathrm{SiO}_{2}$ thickness increased to $20 \mu \mathrm{m}$. Although the temperature gradients were small, the relative improvement over the original simulation suggested that the direction might be fruitful.

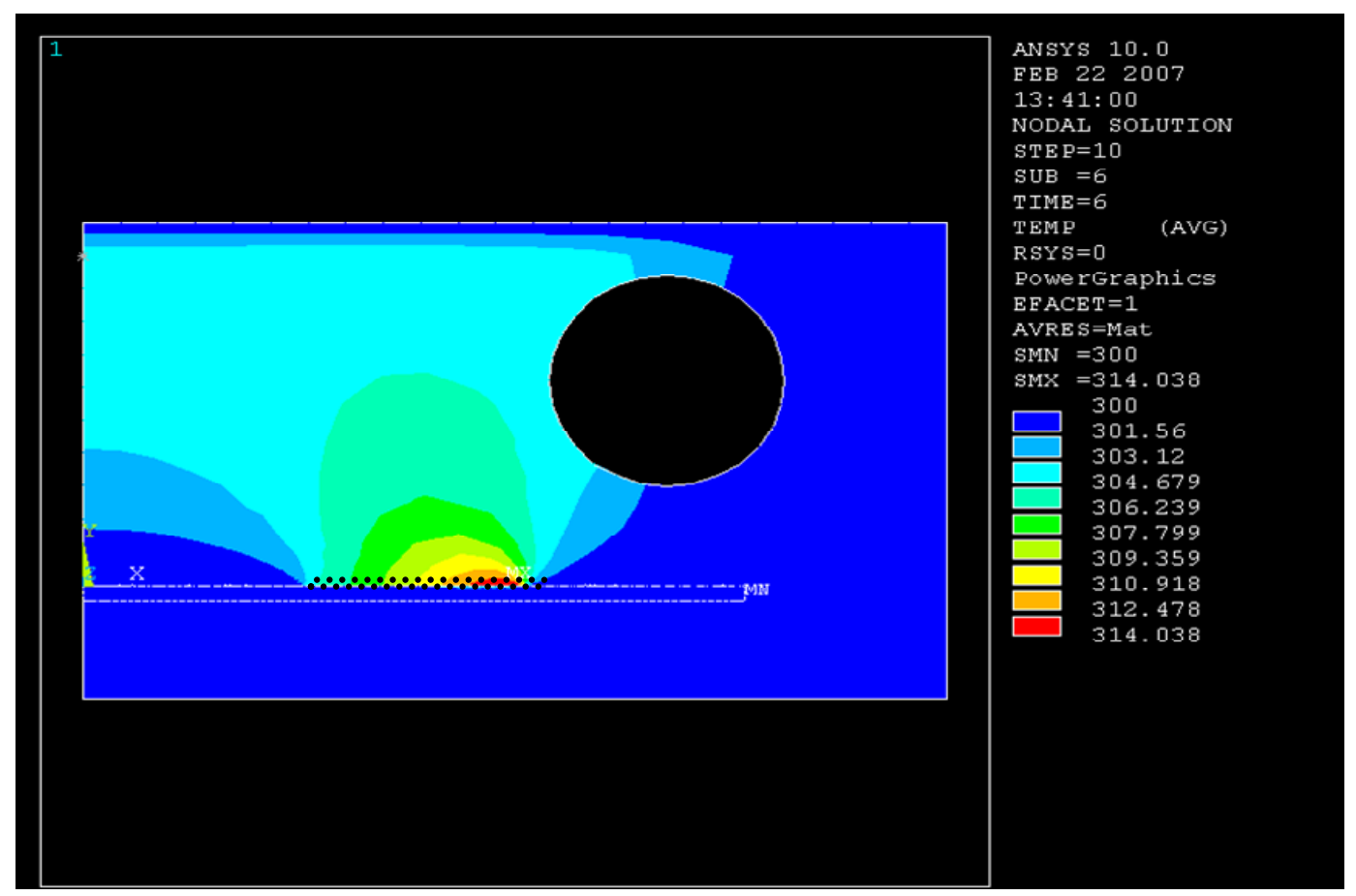

Figure 3.30(b): Contour plot showing the temperature distribution, in Kelvin, across the isolation model following a 6-second simulation with the back side of the substrate held at $300 \mathrm{~K}$ and the base oxide thickness increased to $20 \mu \mathrm{m}$. The thicker base oxide layer shielded nearly all of the thermal energy from the substrate. 


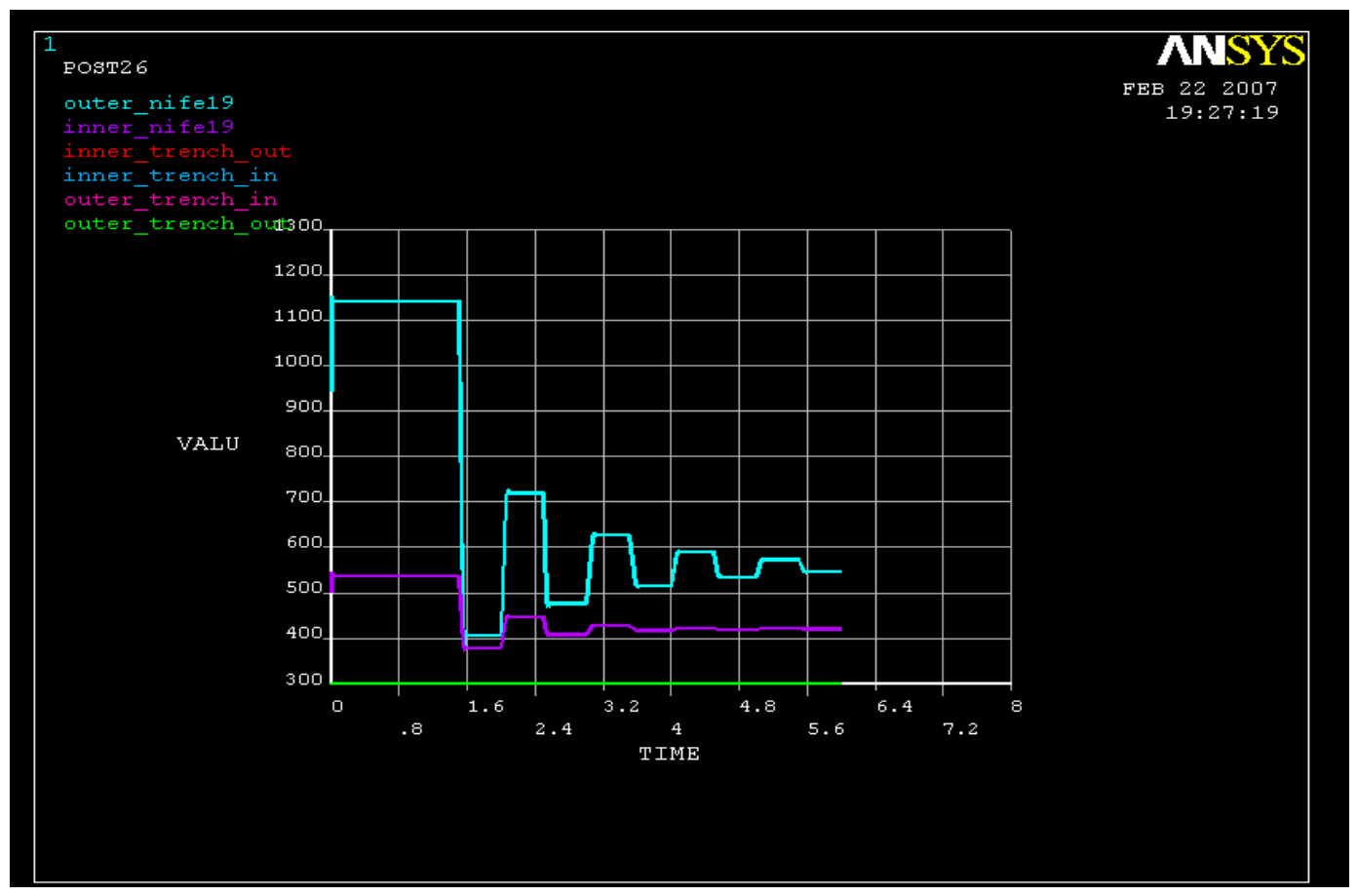

Figure 3.31(a): Graph of temperature $(\mathrm{K})$ vs time for a 6-second simulation of the isolation film solid model with the back side of the substrate held at $300 \mathrm{~K}$, a $20 \mu \mathrm{m} \mathrm{SiO}_{2}$ thickness, and the coil current increased by a factor of 10 . The solution exhibited some instability but was converging toward a $250 \mathrm{~K}$ differential between film and substrate.

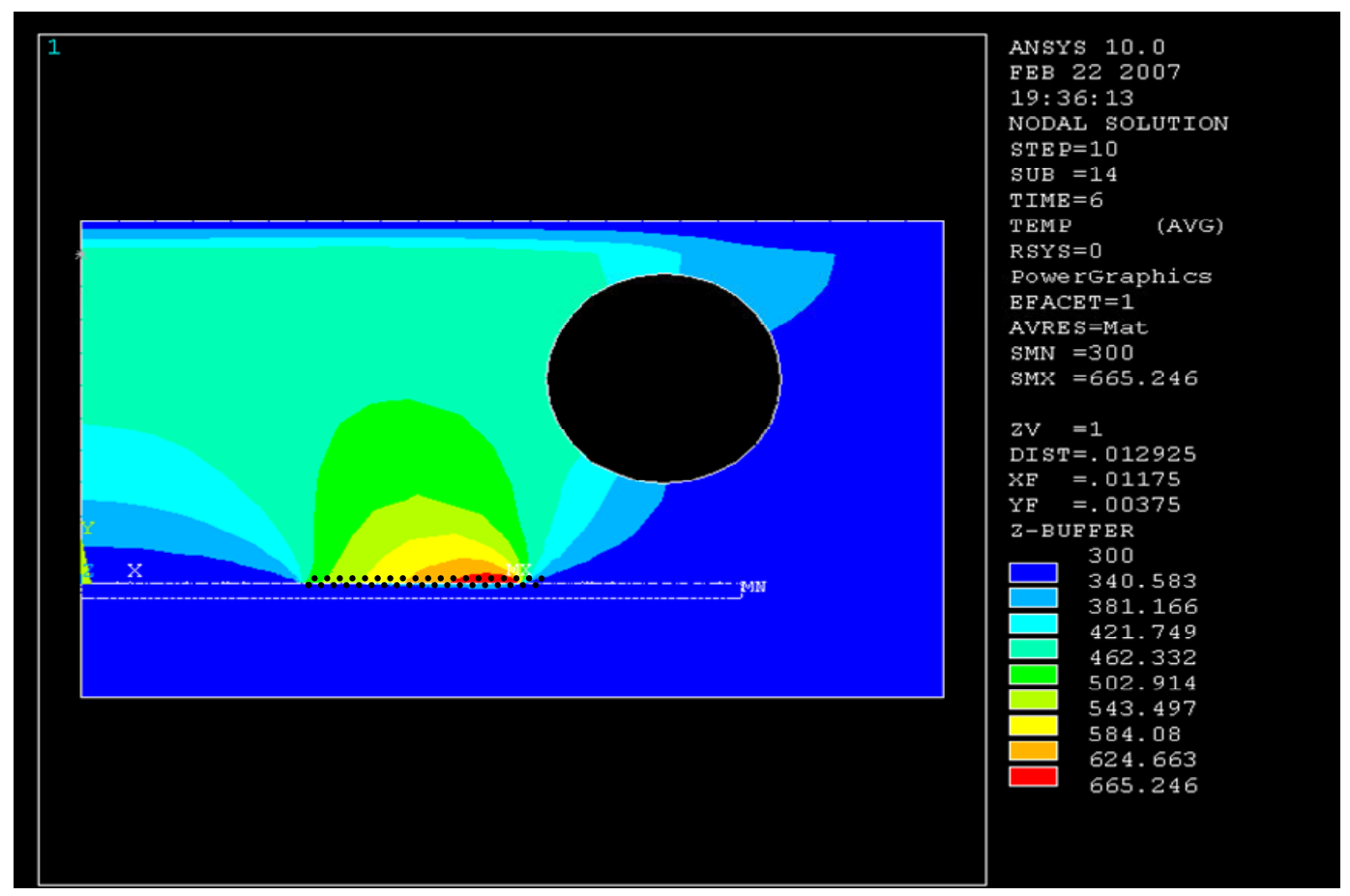

Figure 3.31(b): Contour plot showing the temperature distribution (K) across the isolation model following a 6-second simulation with the back of the substrate held at $300 \mathrm{~K}$, a $20 \mu \mathrm{m} \mathrm{SiO} \mathrm{Si}_{2}$ thickness, and the coil current increased by a factor of 10 . Nearly all of the energy was contained in the NiFe19 film, which had a peak temperature of $665 \mathrm{~K}$. 
have reached their approximate steady-state values at the end of the 6-second simulation. With a steady-state maximum temperature of $665 \mathrm{~K}$, the simulation results demonstrated the capability to maintain a stable differential of $365 \mathrm{~K}$ from film to substrate. The drawback, however, was that the lateral energy spread through the polysilicon film was minimal, and in fact insufficient energy reached the trench regions to enable the testing of their effectiveness. Despite having the same high thermal conductivity as the bulk silicon, the polysilicon film was relatively thin and apparently did not provide a good conduction path. Thus, another trade-off was revealed, suggesting the need for further design revision. Possible solutions include the use of thicker polysilicon films, enhancing the lateral thermal conduction using an additional layer, or patterning the inductively heated film directly above the polysilicon region to be annealed. Further adjustments to the coil

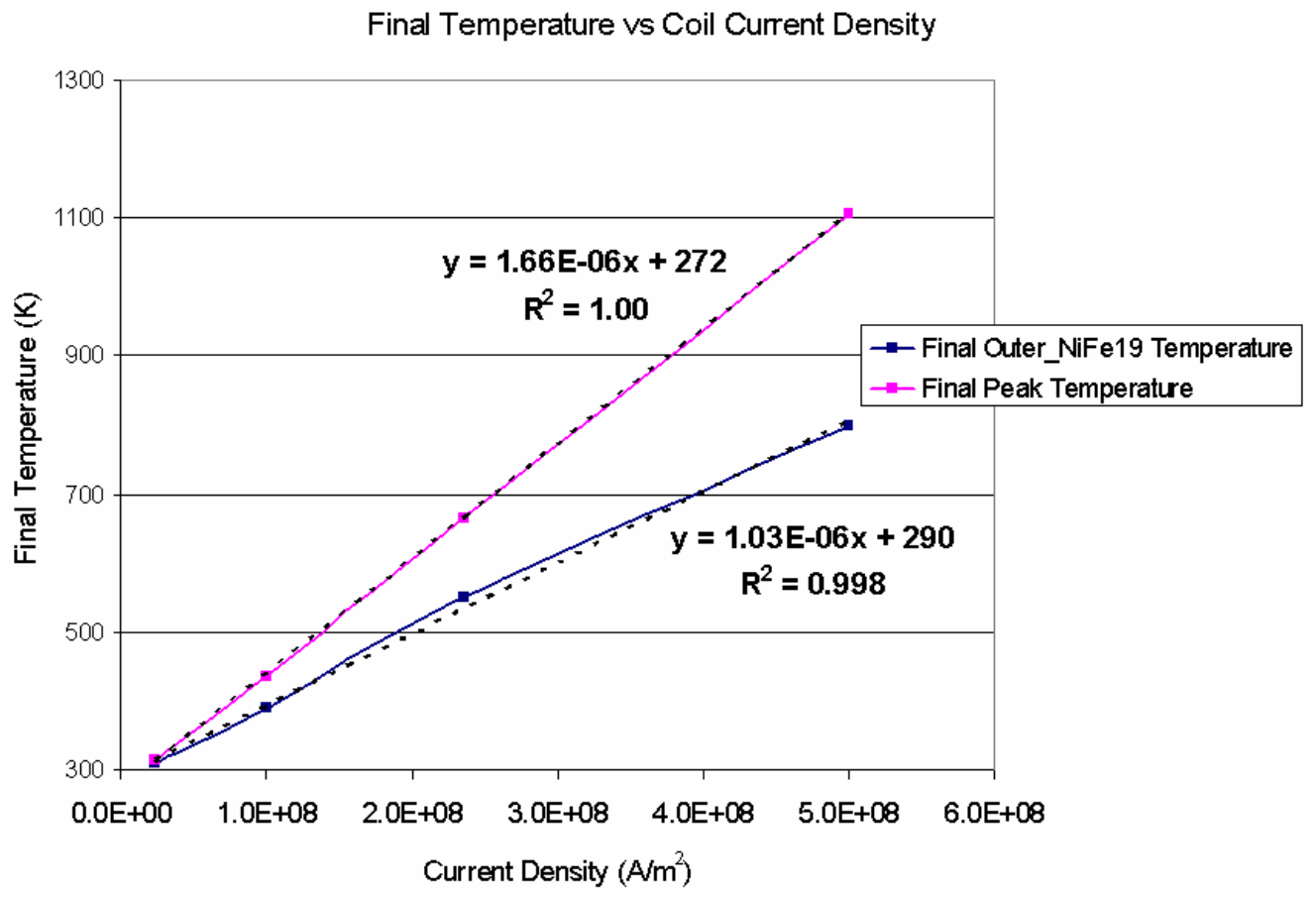

Figure 3.32: Graph showing the relationship between the current density applied to the magnetic coil and the resulting induced steady-state temperatures. The temperature at the outer edge of the NiFe19 film (blue) and the maximum temperature within the film (pink) both varied linearly with current and thus were linear in magnetic field strength. 
current yielded the same qualitative results, with the only difference being the numerical values. Figure 3.32 shows a graph of the induced temperatures with respect to the applied current density. Both the temperature at the outer edge of the NiFe19 film and the maximum steady-state temperature within the film were linear with current (and thus magnetic field strength). This was inconsistent with the theoretical prediction as well as the results of the ANSYS example simulation, both of which indicated the temperature values should vary with the square of the coil current. Closer examination of the impact on the various dependent parameters within the electromagnetic simulation showed that the power and power density values both varied with $J^{2}$ as expected, so the discrepancy fell within the thermal model. The increase in directions available for the induced thermal energy to flow was the likely cause. The two linear trends suggested that an average temperature within the NiFe19 film (and thus the polysilicon below) of $1000^{\circ} \mathrm{C}$ could be

reached using a current density of approximately $8 \mathrm{E} 8 \mathrm{~A} / \mathrm{m}^{2}$, which corresponded to a magnetic field strength of $1.79 \mathrm{E} 6 \mathrm{~A} / \mathrm{m}$. This was a factor of 99.7 above the present $1.8 \mathrm{E} 4$ $\mathrm{A} / \mathrm{m}$ field strength. Available power supplies from Ameritherm with the same frequency range reach up to $12 \mathrm{~kW}$ in output power, a factor of four increase over that available from the Nova Star 3 utilized for experimentation [Ameritherm, 2007]. Thus the remaining factor of $\sim 25$ would have to come from optimization of the structural layout and magnetic coil.

\section{Conclusion}

This chapter presented the generation and results of three different finite-element models designed to simulate induction heating processes. The first model, developed by ANSYS, was highly idealized in that the heated structure was of relatively large 
dimensions and was located completely in the interior of the solenoid, thus producing a magnetic field entirely oriented along the y-axis. Nevertheless, it provided a critical link to the theoretical equations of Chapter 2, with most simulated electromagnetic parameters falling within a factor of 1.27 of their theoretical counterparts, and it also provided a baseline from which the more complex thin film models were established. Development of the thin film models was focused on enhancing the controllable thermal energy gradients between the regions that were intended for heating and those that were not. Progress was made using silicon dioxide films as thermal barriers, but considerable room for improvement remained. The most successful results utilized unrealistically thick thermal barrier films and extremely high coil current levels, which suggested that further enhancements in process efficiency and energy containment were needed. Research into alternate barrier film materials and/or stacks will be a key component of the second generation of the process development. This will increase the thermal gradient capabilities, and thus the loss of energy to the surroundings. Combined with improved ferromagnetic film properties, success in this area will help to drive the success of the technology. 


\section{Chapter 4: Experimental Results}

While the theoretical and computational analyses of the inductive annealing process seemed to suggest feasibility, it was only through experimentation that its practicality could be evaluated. Unexpected challenges were certain to arise, and several were indeed encountered during the early phase of testing. Much of the middle phase was spent examining methods to overcome these challenges, some of which resulted only in minor process changes while others placed limitations on device conditions. The possible solutions are by no means exhausted, however, and it is likely that continued research with fresh ideas could help to lift the constraints that presently exist. This chapter presents the highlights of the technology evolution to date. It begins with the initial proof-of-concept testing in Section 4.1. Based on the results of these and subsequent studies, a custom test system was assembled at MTU specifically targeting the efficient induction heating of thin ferromagnetic films. Section 4.2 details the construction and relevant features of the Bergstrom Electromagnetic Annealing Vacuum Induction System (BEAVIS). Process optimization also required a study into the magnetic properties of the various ferromagnetic films available for deposition with the Perkin Elmer 2400-8J sputtering system. The results of these measurements are presented in Section 4.3. Proceeding with three selected alloys, Section 4.4 examines the important relationships between film dimensions and heat generation, utilizing the various challenges encountered to drive the evolution of the inductive annealing process and corresponding designs. Overall, the data showed a continual trend toward a fully functional technology in which the CMOS integration problem could be overcome with minimal impact to 
device design. While this has yet to be fully proven, it was clear that induction heating was a worthwhile direction for further research.

\section{1: Concept Verification}

Initial experimentation was performed at the Lepel induction heating test facility in Edgewood, New York, where various power supplies were available with frequencies ranging from tens of $\mathrm{kHz}$ to several $\mathrm{MHz}$. The first test samples used to demonstrate the application of induction heating to ferromagnetic films consisted of $1 \mu \mathrm{m}$-thick nickel films evaporated onto silicon wafers, masked in situ to yield circles approximately $2 \mathrm{~cm}$ in diameter. The films yielded rapid, thorough heating to approximately $400^{\circ} \mathrm{C}$ at frequencies of a few MHz. Temperature measurement was done using a handheld infrared pyrometry gun. This confirmed that a magnetic field applied to a thin film could result in a measurable temperature elevation, but the research goals required $1000^{\circ} \mathrm{C}$ for polysilicon annealing. To determine whether this range of temperatures could be reached, a second set of samples was prepared containing rows of polysilicon cantilever beams above a 4" silicon substrate. The beams were made of undoped LPCVD polysilicon deposited at $625^{\circ} \mathrm{C}$, producing films of high stress and stress gradient that, without annealing, would exhibit significant out-of-plane curvature [French, 2002]. The beams were patterned as indicated in Figure 4.1. Cobalt was selected to serve as the inductively heated film because its Curie temperature allows efficient heating at and above $1000^{\circ} \mathrm{C}$ and therefore could provide the thermal energy needed to initiate grain regrowth in polysilicon. To prevent the cobalt from coming into contact with the silicon, which would result in the formation of cobalt silicides, a 100nm PECVD oxide film was deposited over the entire wafer, as the literature indicates it to be an effective barrier to 


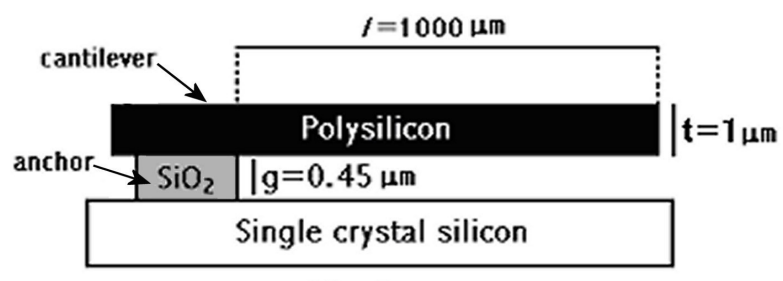

side view

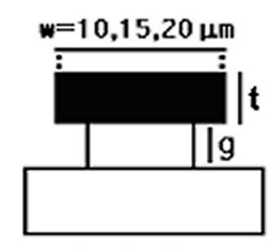

back view

Figure 4.1: Cross-sectional diagram of polysilicon cantilever beams utilized in the second verification test. A timed etch of the underlying sacrificial silicon dioxide layer provided for beam anchoring ${ }^{1}$.

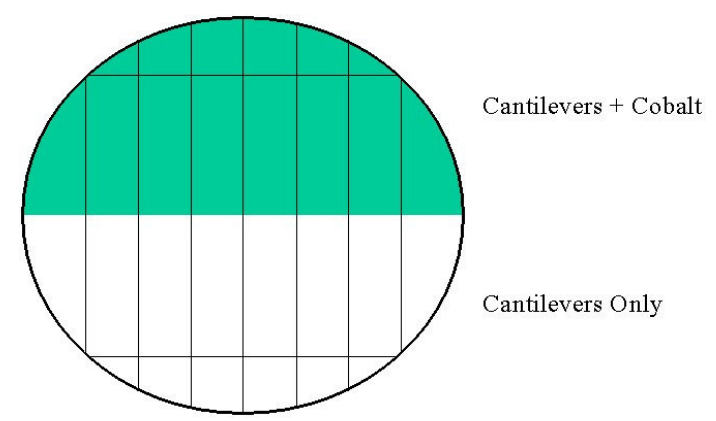

Figure 4.2: Illustration of wafer partitioning into rectangular strips approximately $1 \mathrm{~cm} \mathrm{x}$ $3 \mathrm{~cm}$ in size. Half of each sample was covered with a $100 \mathrm{~nm}$ cobalt film so as to produce adjacent annealed and unannealed regions.

cobalt diffusion [Detavernier, et al., 2000]. A 100nm-thick cobalt film was evaporated onto one side of the wafer using a shadow mask. Finally, the wafer was partitioned into rectangular strips such that half of each strip was covered with cobalt and half was bare as is illustrated in Figure 4.2. Returning to Lepel, solenoidal coils were chosen so as to subject the samples to a strong, relatively uniform magnetic field with a dominant component in the $z$-direction. As was discussed in Section 2.3, the samples were oriented such that the eddy currents were induced in the $r-f$ plane in order to reduce the dependence of the required frequency on the film thickness. Figure 4.3(a) shows a lowtemperature anneal in progress, evident by the discoloration spreading through the cobalt film, with the frequency and transmitted power at approximately $4.95 \mathrm{MHz}$ and $2 \mathrm{~kW}$, respectively. Thermally-sensitive paint indicated that the temperature had reached around

\footnotetext{
${ }^{1}$ Courtesy of Bishnu Gogoi.
} 


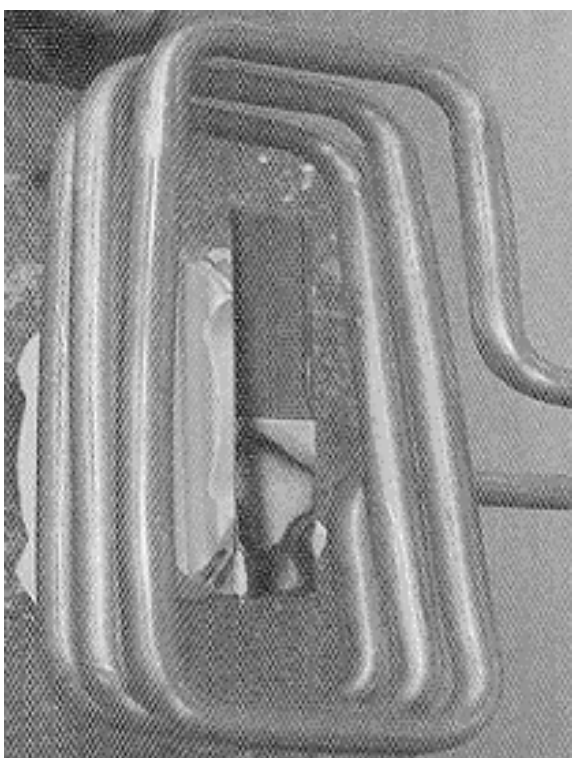

(a)

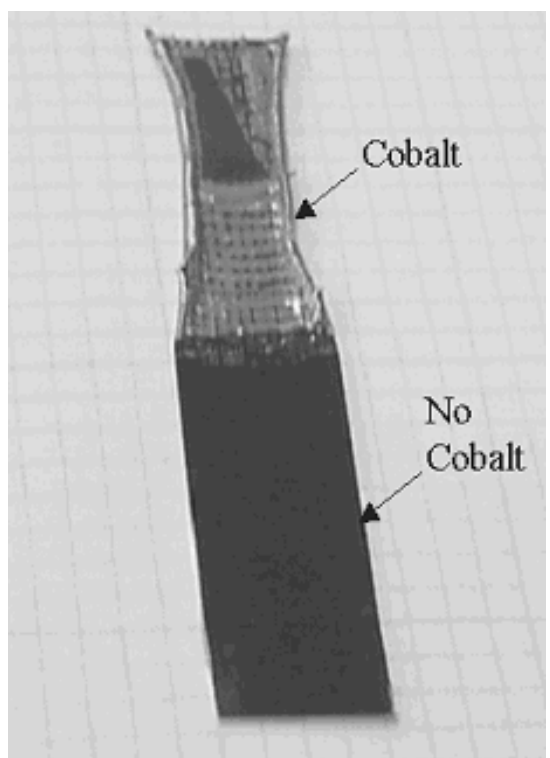

(b)

Figure 4.3: Images of inductively heated cobalt films showing (a) a low-temperature anneal in progress in which heat generation was evident by the cobalt film discoloration and (b) the result of a high-temperature anneal in which the cobalt-covered side of the sample had deformed. All testing was done in ambient conditions.

$400^{\circ} \mathrm{C}$. Figure $4.3(\mathrm{~b})$ shows the outcome of increasing the coil power beyond $2 \mathrm{~kW}$, at which point the cobalt-covered side of the sample began to glow brightly and the process lost stability, reaching temperatures in excess of the silicon melting point, $1410^{\circ} \mathrm{C}$, almost instantaneously. The unchanged shape of the low-temperature side of the sample verified that a thermal gradient had successfully been obtained. It was hypothesized that the loss of stability occurred as a result of the increasing generation of electronic carriers in the substrate under the cobalt which enabled the silicon to inductively heat more efficiently, leading to a thermal runaway condition. The considerable difficulty encountered in controlling the temperature beyond $400-500^{\circ} \mathrm{C}$ lends support to this theory. This suggested that wafer doping was a critical factor and that additional measures might be required such as heat-sinking the substrate or introducing additional thermal isolation in the vicinity of the microstructural areas. The doping level of the substrates used for this 
experimentation was not characterized.

In spite of the deformation that occurred at the edges of the high-temperature end of the samples, the structures located in their interior were left undamaged. After removing the cobalt in a piranha $\left(50 \mathrm{vol} \% \mathrm{H}_{2} \mathrm{SO}_{4}, 50 \mathrm{vol} \% \mathrm{H}_{2} \mathrm{O}_{2}\right)$ solution, the oxide layers above and below the beams were etched in 5:1 buffered hydrofluoric acid (BHF) to expose and release them. The samples were soaked in isopropyl alcohol, methanol, and then pentane following the sacrificial etch, but problems with stiction were still encountered as a result of the cantilever length. Nevertheless, the SEM images in Figure 4.4 illustrate a clear difference between structures on opposite sides of the sample from Figure 4.3(b). The initial curvature due to the intrinsic stress gradient was effectively eliminated, and manually lifting the ends of the heat-treated beams from the substrate confirmed them to be flat. The impact of the anneal on the polysilicon surface texture is illustrated in the SEM images of Figure 4.5, where the change from a relatively coarse appearance to near uniformity suggests that significant regrowth had taken place. Both sets of SEM images were obtained using a JEOL 6400 system. This change in surface texture was quantified using a ADE Phase Shift white light interferometric microscope (IFM). Figure 4.6 shows the digitized profiles of the anchor regions, again showing a

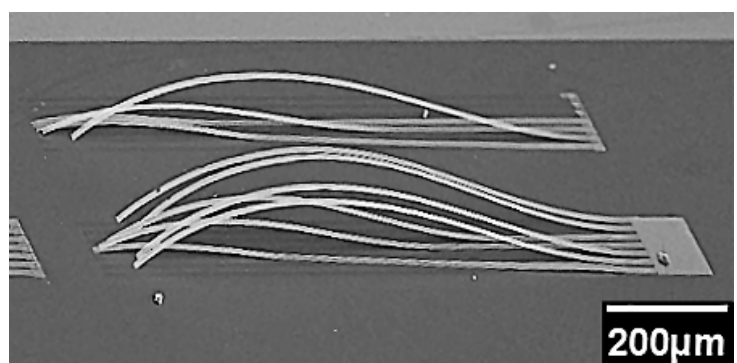

(a)

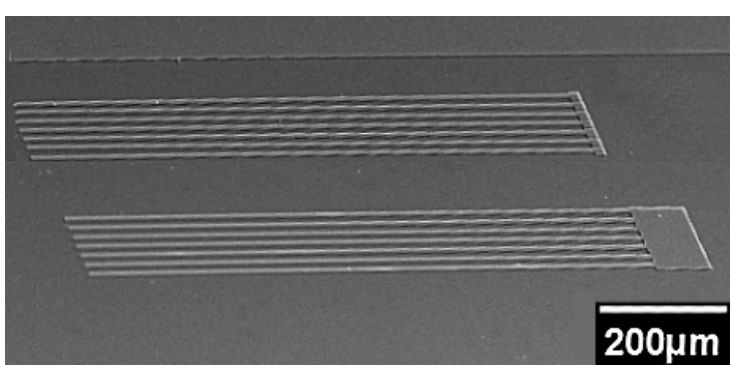

(b)

Figure 4.4: SEM images of cantilever beams from the (a) low-temperature and (b) hightemperature ends of the same test sample. A significant reduction in the intrinsic stress gradient was evident as a result of the inductive anneal. 


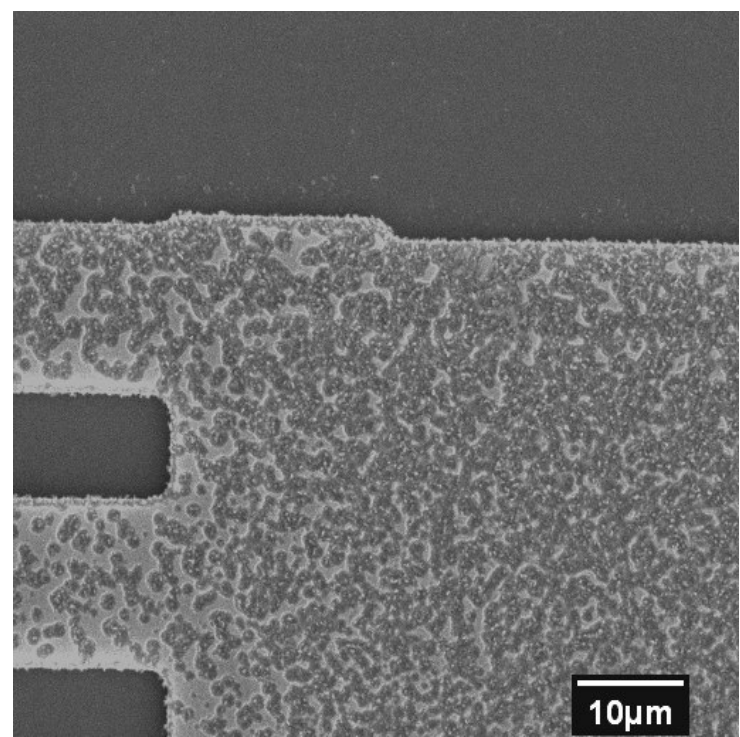

(a)

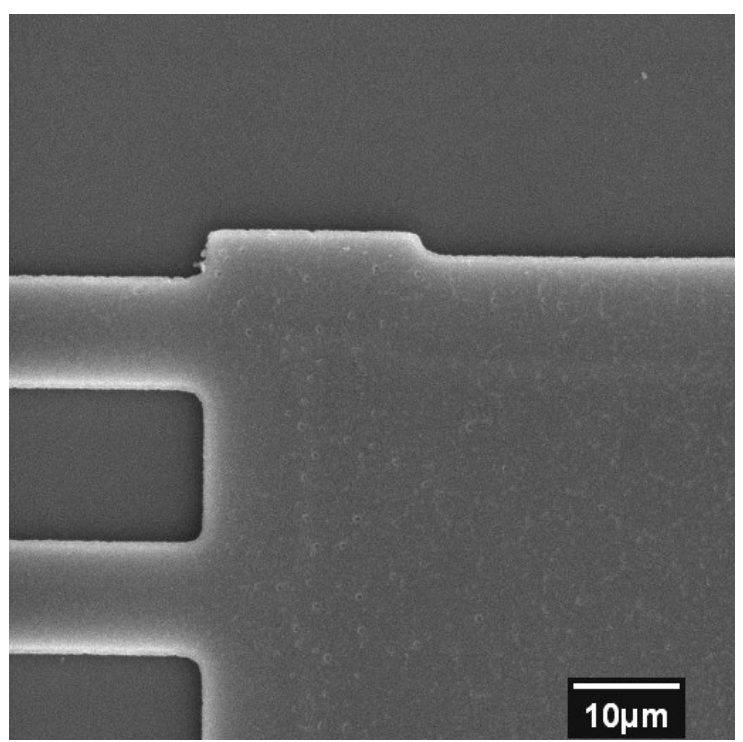

(b)

Figure 4.5: SEM images of the cantilever beam anchors illustrating a visible difference in the silicon grain structures between (a) the low-temperature and (b) the hightemperature areas of the sample.

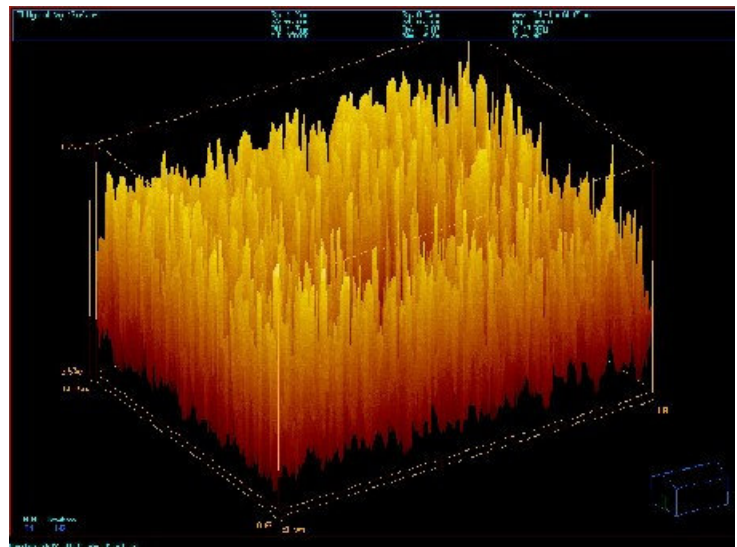

(a)

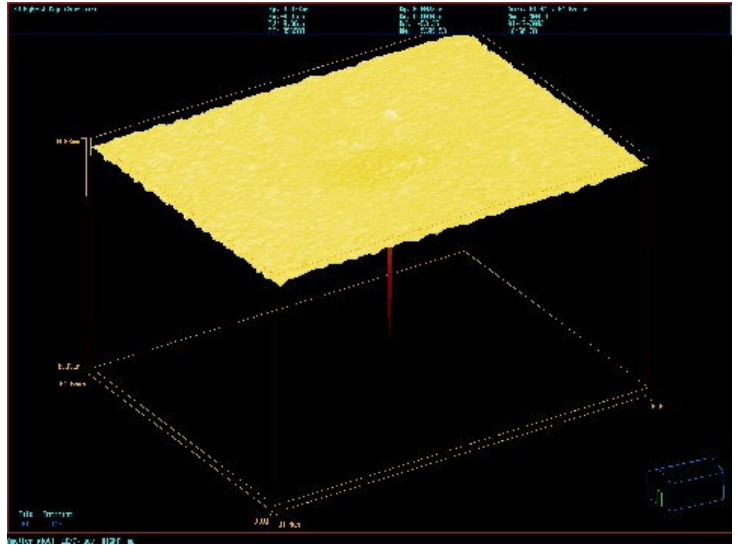

(b)

Figure 4.6: Interferometric images of the beam anchor regions from the (a) lowtemperature region and (b) high-temperature region again reflecting an improvement in surface texture as a result of inductive annealing. The microscope interface software indicated that roughness values had been reduced by nearly two orders of magnitude.

smoothed texture following heat treatment, and the IFM interface software determined that the RMS $\left(\mathrm{R}_{\mathrm{q}}\right)$ and average $\left(\mathrm{R}_{\mathrm{a}}\right)$ roughness values had decreased from $0.37 \mu \mathrm{m}$ and $0.31 \mu \mathrm{m}$, respectively, to $0.0072 \mu \mathrm{m}$ and $0.0034 \mu \mathrm{m}$, a factor of nearly two orders of magnitude. Confirmation of changes in the grain structure and size was obtained through 
XRD analysis using a Scintag XDS2000 Powder Diffractometer. Utilizing reference data provided by the National Bureau of Standards [NBS, 2001], scans were done for the three most prevalent angle-orientation pairs for polysilicon: $\left\langle 111>\right.$ at $\left.28.4429^{\circ},<220\right\rangle$ at $47.3036^{\circ}$, and $<311>$ at $56.1221^{\circ}$. Note that the angles given are actually equal to twice the diffraction angle. The full-width, half-maximum (FWHM) values generated for each of the three peaks, given in degrees, were then utilized to compute the average crystallite sizes according to the Scherrer formula,

$$
\text { Size }=\frac{0.9 \lambda}{B \cos \theta}
$$

where $\lambda(\mathrm{nm})$ is the $\mathrm{x}$-ray wavelength, $B$ (radians) is the FWHM value, or breadth, of the selected peak, and $\theta$ (radians) is the diffraction angle for that peak [Cullity, 1956 (p 262)]. The proportionality constant is referred to as the shape factor or Scherrer constant and is commonly assigned the value of 0.9 based on the assumption of spherical crystallites. Crystallites and grains are not synonymous; crystallites exist within grains and are bounded by items such as grain boundaries, dislocations, and other various imperfections. Thus the average crystallite size provided a minimum value for the average grain size, with their proximity dependent upon material quality. Before calculations could be done, however, it was first necessary to make an adjustment to the FWHM values in order to account for any error introduced by the system. This was done using a characterized polysilicon reference sample known to be of large crystallite size. Based on recommendations made by Edward Laitila of the MTU Materials Science and Engineering Department, the offset adjustments were made as follows:

$$
B_{1}=B_{F W H M}-B_{R E F}
$$




$$
\begin{gathered}
B_{2}=\sqrt{B_{F W H M}^{2}-B_{R E F}^{2}} \\
B_{A D J}=\sqrt{B_{1} B_{2}}
\end{gathered}
$$

where $\mathrm{B}_{\mathrm{FWHM}}$ is the as-measured FWHM value, $\mathrm{B}_{\mathrm{REF}}$ is the FWHM value of the corresponding peak from the reference data, and $\mathrm{B}_{\mathrm{ADJ}}$ is the final, adjusted value to be utilized in Equation (4.1). The diffraction data was also utilized to compute the average lattice strain and stress as indicated by the difference between the measured diffraction angle from the ideal, relaxed value. The formula for lattice strain can be derived from Bragg's law,

$$
n \lambda=2 d \sin \theta
$$

where $n$ is a integer, reflecting the requirement for an integral number of wavelengths between x-rays reflected from a sample in order to achieve constructive interference and thus maximum signal, and $d(\mathrm{~m})$ is the inter-atomic spacing [Nave-1, 2005]. Solving for $d$ and substituting into the characteristic equation for strain, $\varepsilon(\mathrm{m} / \mathrm{m}$, or unitless) [REF],

$$
\varepsilon=\frac{d-d_{0}}{d_{0}}
$$

the lattice strain can be related to the diffraction angle as

$$
\varepsilon=\frac{\sin \theta_{0}-\sin \theta}{\sin \theta}
$$

Strain values can then be converted to stresses via multiplication by the Young's Modulus; a value of $160 \mathrm{GPa}$ was used for all stress calculations as it represented a typical polysilicon Modulus [Senturia, 2001 (p. 196)]. Table 4.1 summarizes the data from XRD scans on the reference polysilicon sample and the annealed and unannealed test samples as well as the results of the calculations. The output waveforms generated by the 
diffractometer are given in Figures 4.7(a) and 4.7(b). The data and plots show that annealing rendered all three peaks considerably narrower, consistent with increased grain

\begin{tabular}{|c|c|c|c|c|c|c|}
\hline \multicolumn{6}{|c|}{ TABLE 4.1 } & X-RAY DIFFRACTION DATA AND CALCULATIONS FOR BEAM ANCHORS \\
\hline$\theta$ & $\begin{array}{l}2 \theta, \\
\text { degrees }\end{array}$ & $\begin{array}{l}\mathrm{B}_{\mathrm{FWHM}}, \\
\text { radians }\end{array}$ & $\begin{array}{l}\mathrm{B}_{\mathrm{ADJ}}, \\
\text { radians }\end{array}$ & $\begin{array}{l}\text { Scherrer Cry. } \\
\text { Size, nm }\end{array}$ & $\begin{array}{l}\text { Strain, } \\
\mathrm{mm} / \mathrm{m}\end{array}$ & $\begin{array}{l}\text { Stress, } \\
\mathrm{MPa}\end{array}$ \\
\hline \multirow{3}{*}{ 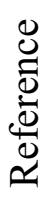 } & 28.4479 & 0.001290 & - & - & - & - \\
\hline & 47.3050 & 0.001417 & - & - & - & - \\
\hline & 56.1273 & 0.001370 & - & - & - & - \\
\hline \multirow{3}{*}{ 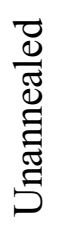 } & 28.5607 & 0.002878 & 0.002021 & 70.78 & -3.619 & -579.0 \\
\hline & 47.4676 & 0.004136 & 0.003251 & 46.59 & -2.607 & -417.2 \\
\hline & 56.2768 & 0.003887 & 0.003026 & 51.97 & -1.745 & -279.2 \\
\hline \multirow{3}{*}{ 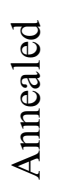 } & 28.5212 & 0.001587 & 0.000524 & 273.3 & -2.355 & -376.8 \\
\hline & 47.4054 & 0.002180 & 0.001124 & 134.7 & -1.613 & -258.0 \\
\hline & 56.2074 & 0.001977 & 0.000931 & 168.9 & -0.9366 & -149.9 \\
\hline
\end{tabular}

Polysilicon XRD Data, Low Temperature Region

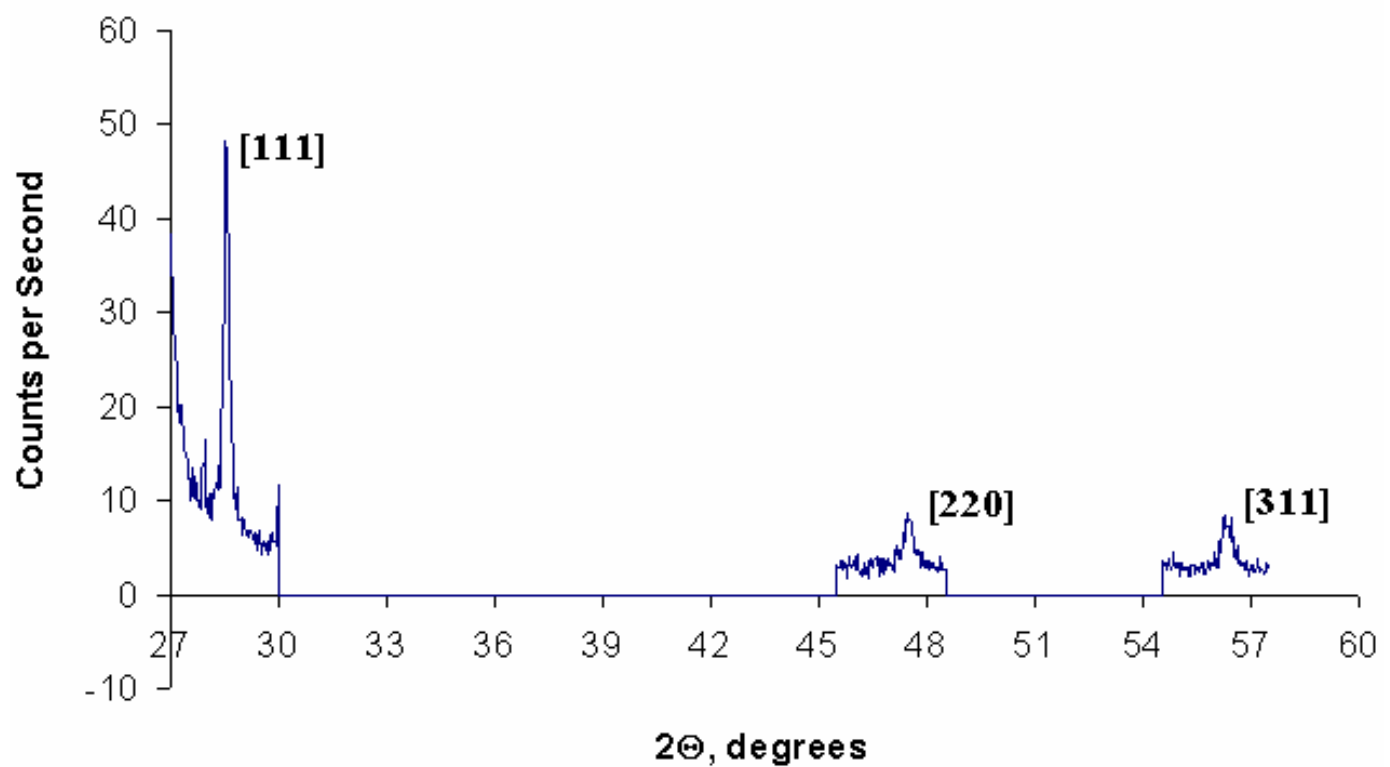

Figure 4.7(a): X-Ray Diffraction output plot obtained from the low-temperature region of a polysilicon beam sample. The plot illustrates the shapes and locations of the three dominant diffraction peaks, those being the [111], [220], and [311] orientations. 


\section{Polysilicon XRD Data, High Temperature Region}

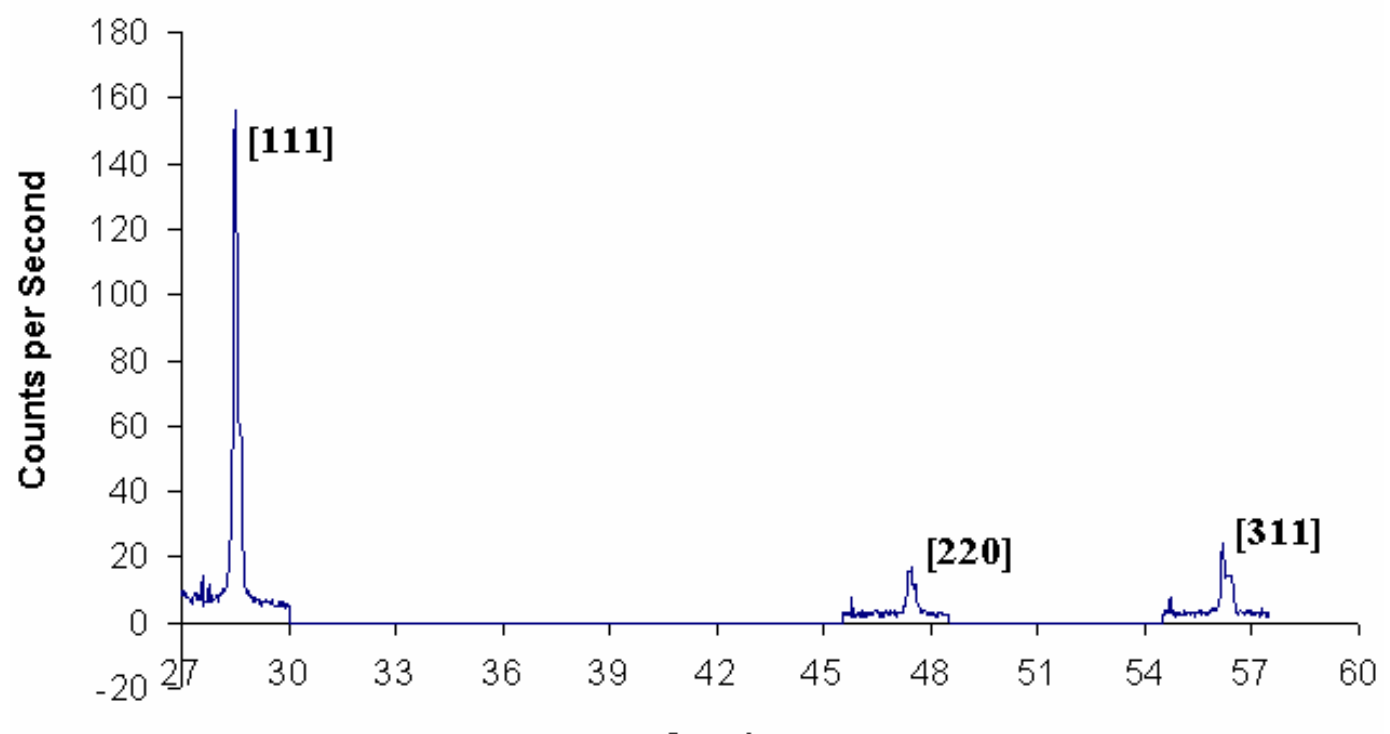

$2 \Theta$, degrees

Figure 4.7(b): X-Ray Diffraction output plot showing the considerable narrowing of the diffraction peaks that occurred as a result of heat treatment. Note that the vertical scale was increased by a factor of three.

sizes. Averaging the calculated crystallite sizes for the three measured orientations yielded final values of $56.45 \mathrm{~nm}$ and $192.3 \mathrm{~nm}$ for the as-deposited and heated structures, respectively. Stress and strain values also showed a measurable change. The average lattice strain dropped from $2.657 \mathrm{~mm} / \mathrm{m}$ to $1.635 \mathrm{~mm} / \mathrm{m}$, a reduction of $38.5 \%$, and stress was reduced from an average of $425.1 \mathrm{MPa}$ compressive to $261.6 \mathrm{MPa}$ compressive. Although these results indicated that anneal was not yet complete, with the polysilicon still exhibiting considerable compressive stress, they confirmed that the process had in fact produced a very significant change within a one-second time duration. Thus it had been shown that temperatures sufficient for polysilicon annealing could be reached through inductive coupling to ferromagnetic films and that the capability existed for thermal localization based on strategic film placement. The first of several challenges had been revealed, however, and process control would continue to impose difficulties 
throughout the course of this research. The end result would be a limit placed upon the doping level of the substrate, though it was anticipated that continued research into improving ferromagnetic film properties would alleviate this requirement as maximizing coupling would help to prevent the silicon from becoming induction efficient. It was furthermore concluded that the process would benefit considerably from heating in a vacuum ambient, as the high ratio of surface area to thickness resulted in substantial convective losses. A moderate vacuum level would help to retain thermal energy as well as prevent film oxidation at high temperatures. Thus began the construction of a custom test system at MTU focused on the optimization of the induction heating of a thin film on a semiconducting wafer.

\section{2: Inductive Annealing Test System}

The BEAVIS test system was installed in Room 312 of the Mining and Materials Building at MTU. Its construction began in the Fall of 2002 with the donation of a Norton Research Corporation (NRC) 3117 thermal evaporation system from the MTU Biomedical Engineering Department and the loan of an Ameritherm Nova Star 3 induction heating system from Dr. Walter Milligan of the Materials Science and Engineering Department. The evaporation hardware was removed from the system and the pump stack was upgraded with newer mechanical and diffusion pumps, both manufactured by Varian. MDC pneumatic solenoids were installed for the foreline and roughing valves, and a toggle valve was plumbed into a compressed nitrogen line for

chamber venting. The manual high-vacuum gate valve was left in place. Pressure transduction utilizes a NRC 563-P ionization gauge at the diffusion pump and Type 0531 
thermocouple gauges on the chamber and foreline, with values displayed on a GranvillePhillips 330 ion gauge controller and an MKS Type 286 controller, respectively.

Several attempts were made before the induction coil was successfully installed within the vacuum chamber. The original intent was to place the Ameritherm remote heat station outside of the chamber and transport the inductive signal inside to the coil through custom feedthroughs consisting of hollow copper tubing to allow for cooling water passage. It was learned, however, that the excessive length of copper tubing, along with the many unavoidable bends, resulted in power losses that considerably reduced the heating capability of the coil. In the end, the heat station was disassembled and modified for mounting inside the chamber in order to minimize the distance to the coil.

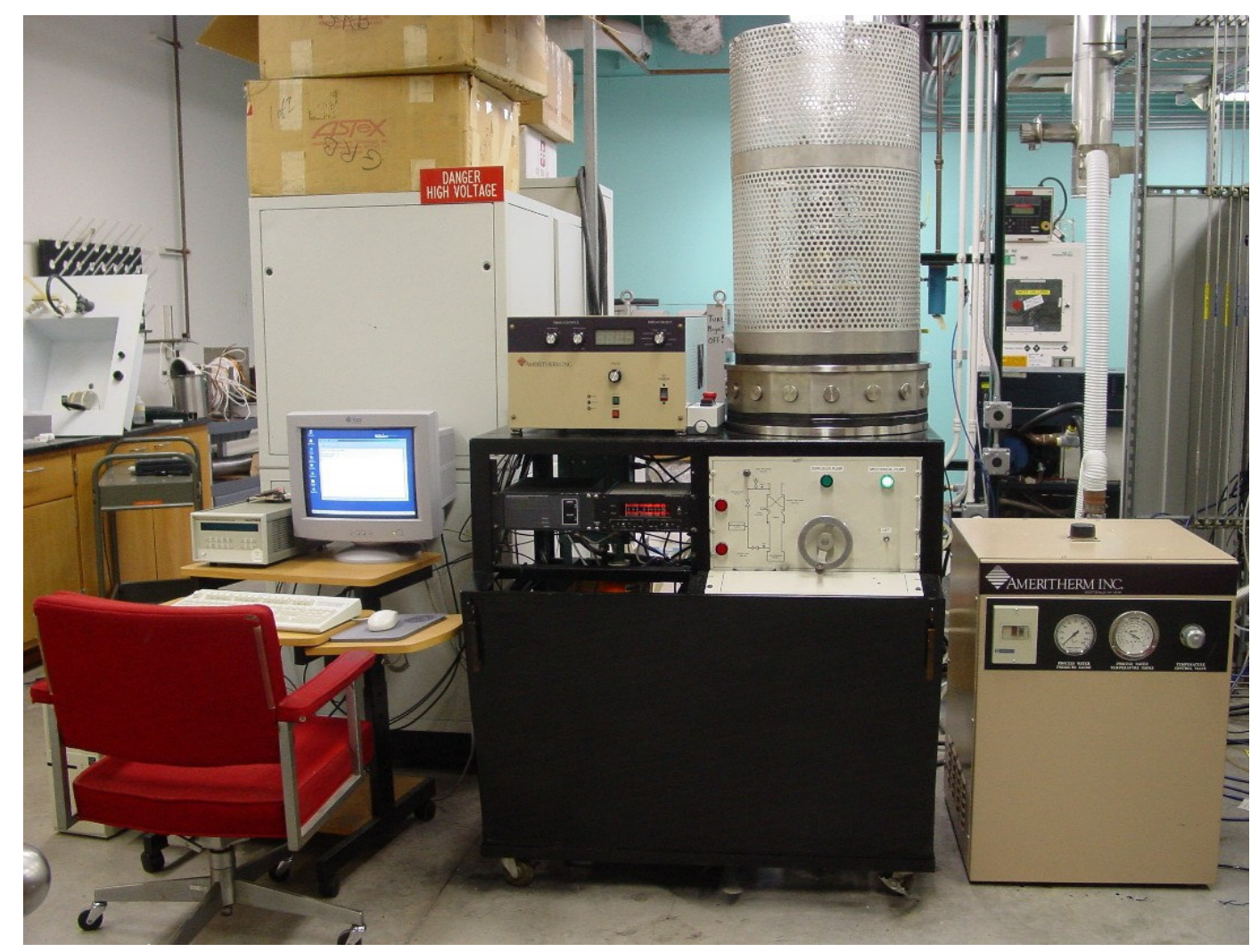

Figure 4.8: Full view of the BEAVIS test system showing the exterior layout. The system consisted of an induction power supply that operated a remote heat station within a vacuum chamber. It was constructed for the purpose of optimizing the induction heating of thin ferromagnetic films. 
Instrumentation utilized to support the inductive heating process included a heat exchanger to provide cooling water for the power supply, heat station, and coil, a stepperpowered stage to allow for wafer scanning, and infrared pyrometry for temperature measurement. The system and its significant components are pictured in Figures 4.8-13. Figure 4.8 shows the complete system. To the right of the main vacuum system was the Ameritherm heat exchanger; to the left was a small desk with a computer, stepper controller, and pyrometer. The interior of the chamber is shown in Figure 4.9. It consisted of two effective zones; the front portion contained the coil and its supporting hardware, and the back portion contained the equipment associated more directly with the wafer. The components relevant to the induction aspect of the system are shown in Figure 4.10.

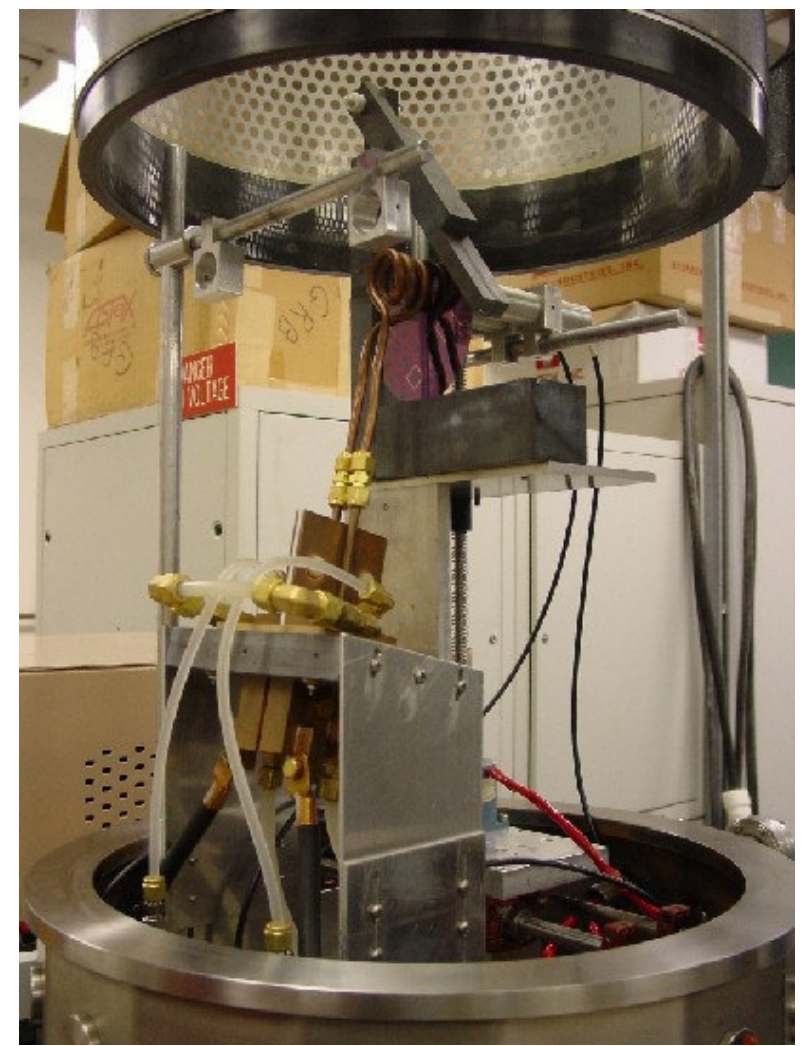

Figure 4.9: Interior of the BEAVIS vacuum chamber illustrating the manner in which the various components interacted. The front half of the chamber contained the induction coil and associated hardware, while the back half contained the motorized stage, substrate holder, and infrared sensors. 


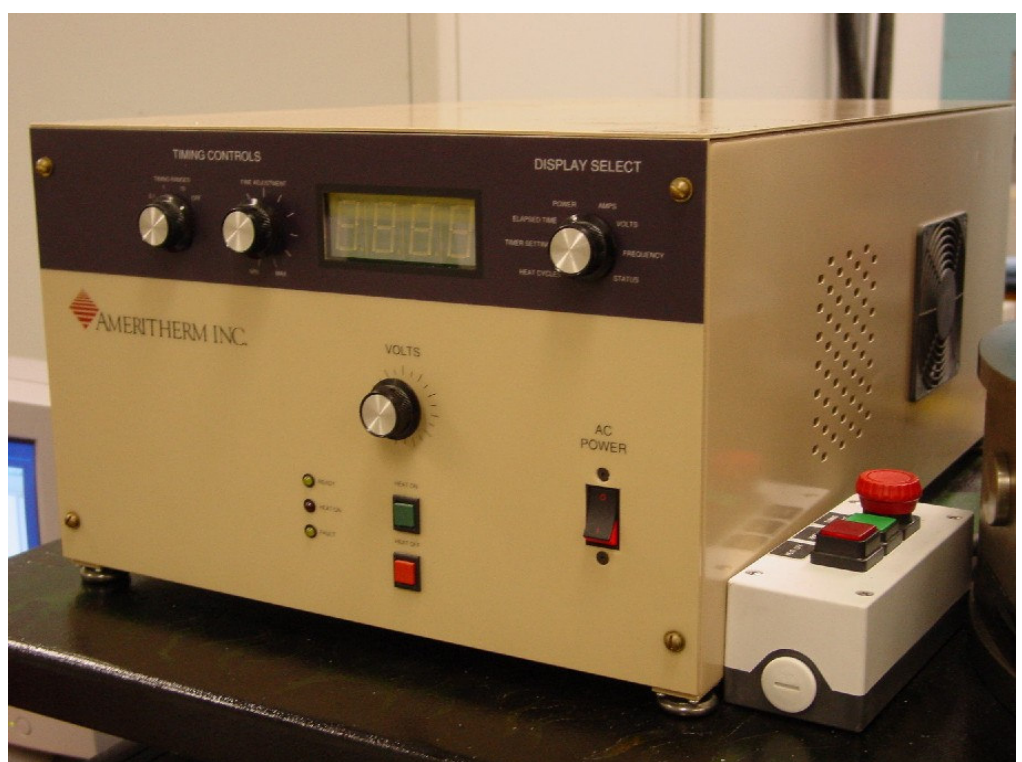

(a)

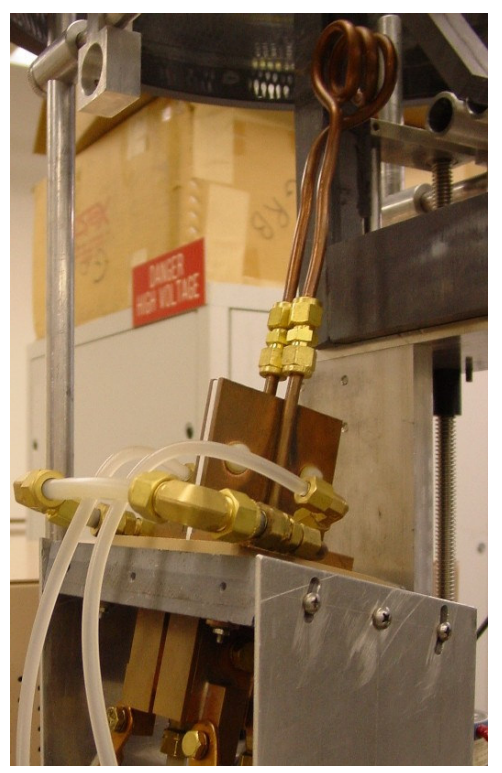

(b)

Figure 4.10: Ameritherm Nova Star 3 induction heating system consisting of the (a) $3 \mathrm{~kW}$ self-tuning power supply and (b) remote heat station to which the coil was attached. The heat station was partially disassembled and mounted to an adjustable stand within the vacuum chamber.

Figure 4.10(a) shows the Nova Star 3 power supply, which had a 3kW maximum output rating (though only $2 \mathrm{~kW}$ was usually obtained) and self-tuning capability within the 50$450 \mathrm{kHz}$ frequency range based on coil inductance and heat station capacitance; these two components were connected in parallel and made up a resonant tank circuit, a typical configuration for induction heating systems as it allows for the generation of large coil current values without requiring substantial supply currents and also minimizes the distance over which these currents must travel [Celem, 2005]. The heat station with its custom mounting stand is shown in Figure 4.10(b). It required two separate cooling water loops; one cooled the station itself while a second passed through the hollow copper coil. $\mathrm{AC}$ power was connected to each side of the station, with the two signals separated by a dielectric plate. Thus the heat station utilized six vacuum feedthroughs in total. The coil utilized throughout the course of this research was a three-turn solenoid measuring 1.25 " 


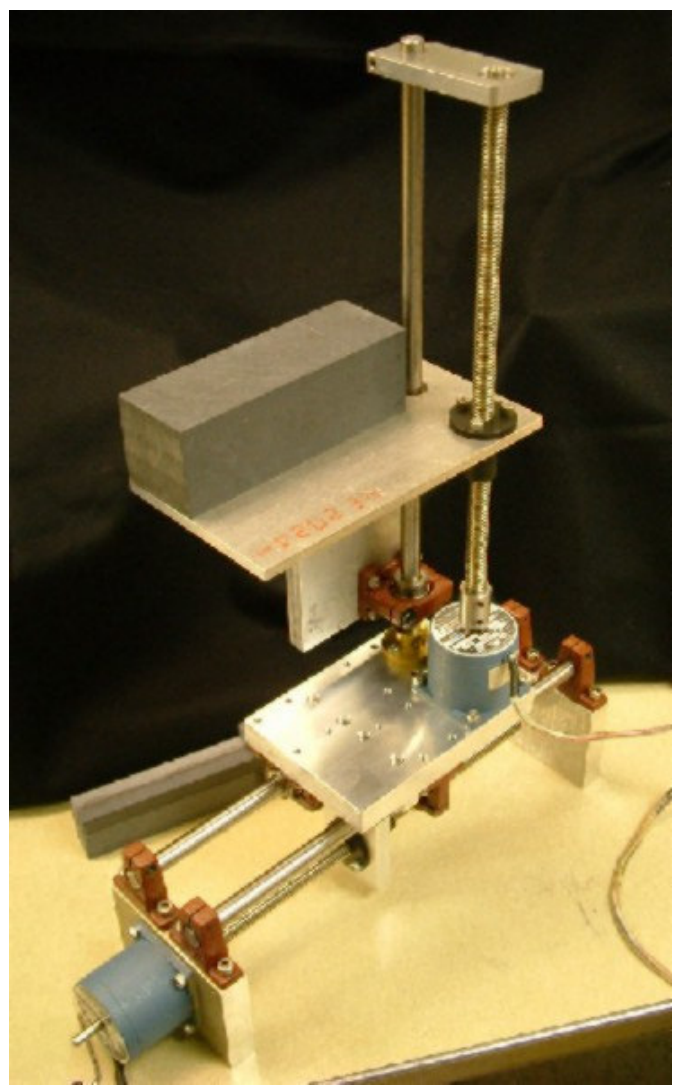

(a)

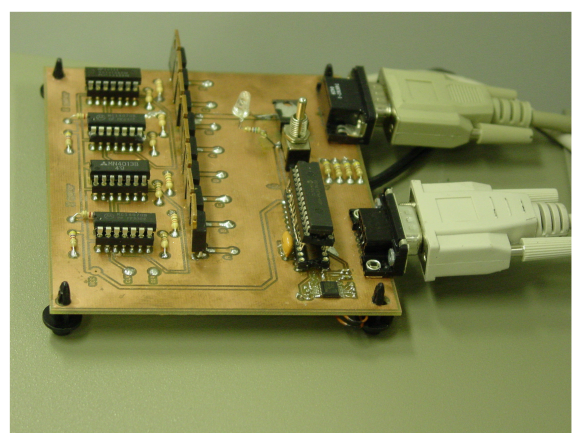

(b)

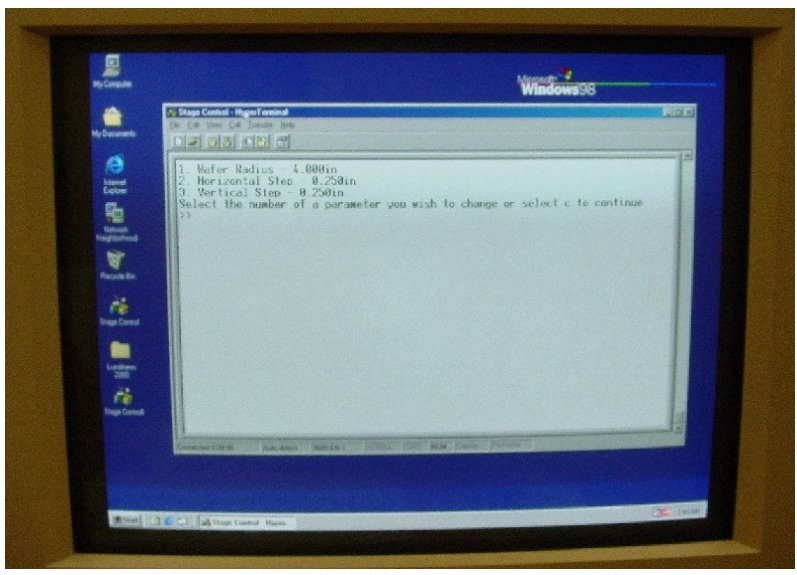

(c)

Figure 4.11: Means by which the substrate was scanned around the coil. The wafer stood vertically on the (a) stepper-powered stage, which allowed for precise horizontal and vertical motion through signals generated by the (b) control circuitry. A computer program, shown in part in (c), provided the user interface.

in length with a 1" inner diameter. While the coil remained in place, the wafer was scanned horizontally and vertically around it by means of a motorized stage, shown in Figure 4.11(a), which utilized two stepper motors operated by the control circuit in Figure 4.11(b). The board contained a Microchip Technology PIC16F873A programmable integrated controller (PIC) which was uploaded with coding that converted instructions from the user, given through a serial communications program such as Hyperterminal as shown in Figure 4.11(c), into the alternating series of outputs required to turn the steppers in the chosen direction. The stage was designed to allow for the complete scanning of wafers up to 8 " in diameter. Its top surface provided for the 


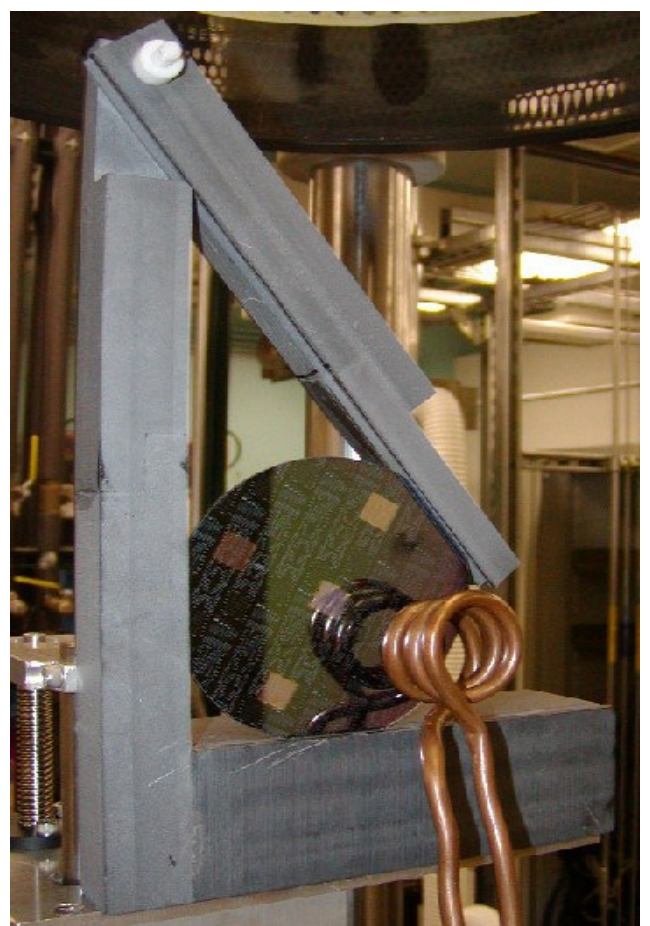

Figure 4.12: Alumina silicate (ceramic) substrate holder designed to secure the wafer vertically in front of the coil without placing restrictions on wafer diameter or heat sinking capability. Shown here is a 4" silicon wafer with $1 \mathrm{~cm}^{2}$ FeCo films in an attempt to heat groups of polysilicon test structures.

securing of the substrate holder, shown in Figure 4.12 with a 4" silicon wafer. Made of alumina silicate, a machineable ceramic, so as not to allow efficient inductive coupling, the substrate holder was triangular in shape with an adjustable arm that rotated on a pivot so as to also enable the heating of various wafer sizes. The substrate rested in a tapered channel so that a heat sink could be placed behind it if desired with a gravity-enhanced contact force. Finally, the monitoring of the temperatures being generated in the targeted and non-targeted regions of the substrate was an essential capability if thorough polysilicon heating was to be achieved without risking damage to the wafer as was encountered previously. This was accomplished using infrared pyrometry. Two light sensors, or collimators, were purchased from Multimode Fiber Optics and custom fiber optic feedthroughs were obtained through JT Ingram Sales and Marketing. All optics 


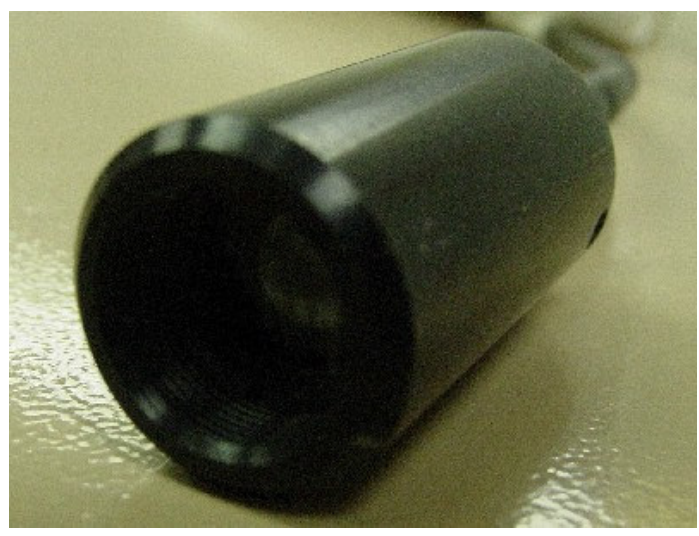

(a)

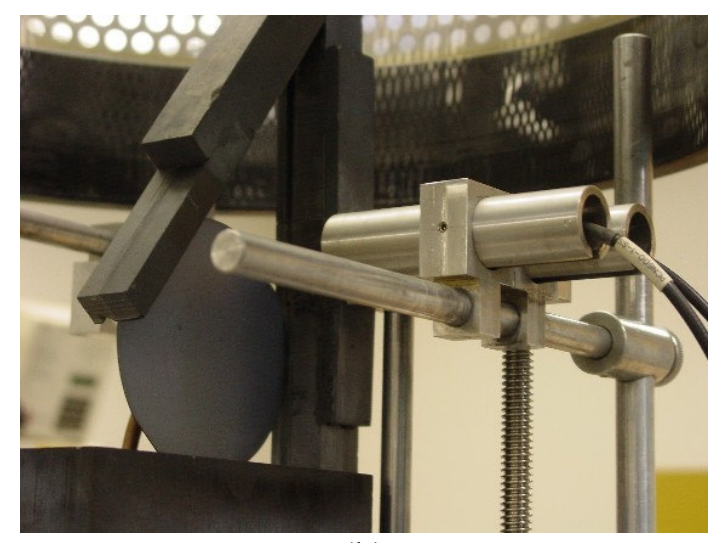

(b)

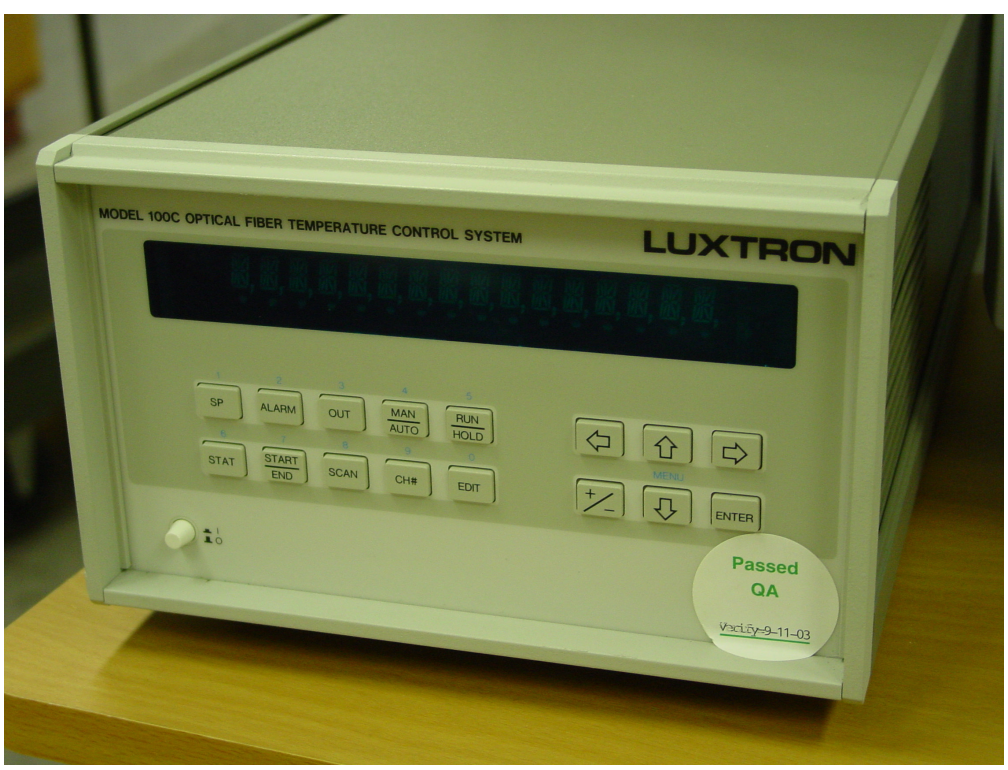

(c)

Figure 4.13: Components utilized for temperature measurement during induction heating. The (a) light sensors were mounted to an adjustable support bar located behind the wafer as shown in (b). Fiber cables directed the optical signal to the (c) Luxtron pyrometer which sequentially displayed the corresponding temperature values.

utilized on the system had a $600 \mu \mathrm{m}$ core and were intended to function within the 750 $1000 \mathrm{~nm}$ wavelength range typical of infrared pyrometry with a 0.22 numerical aperture (NA). Pictured in Figure 4.13(a), the model LC-10 sensors allowed for focal point adjustment, enabling control over the spot size from which they collected thermal radiation. The spot size could be made smaller than $1 \mathrm{~mm}$ if desired; it was set at approximately $1 \mathrm{~mm}$ on each sensor for the fixed distance to the wafer. The pyrometry 
sensors were mounted on a cantilevered bar as shown in Figure 4.13(b). Vertical bars located both in front of and behind the wafer provided tremendous flexibility in the securing of instrumentation components such as these through the various adjustments available in the number and placement of the secondary horizontal beams as well as the individual mounts specific to each device. The sensors were held in place with aluminum tubes that could slide forward or backward within the mounts and were located approximately halfway back within the tubes in order to help to shield them from external light sources. Together with the baseplate feedthroughs, optical fiber cable, also purchased from MultiMode, transmitted the light signals collected by the sensors to a Luxtron 100C Optical Fiber Temperature Control System, pictured in Figure 4.13(c). The Luxtron system read up to four channels and sequentially displayed the calibrated output of each activated signal. Its output temperature range was valid from $325-1900^{\circ} \mathrm{C}$, indicating that it read $325^{\circ} \mathrm{C}$ at room temperature, and it had the capability for serial port communications, though this feature wasn't utilized.

Calibration of the temperature readout was a formidable challenge, consuming nearly an entire semester, in large part due to the failure of the original pyrometer which was subsequently replaced with the Luxtron. The test system was partially reconfigured in order to make room for two Model 5305-5 tungsten filament heat lamps manufactured by Research Inc. Since the lamps required both alternating power and cooling water, the coil was removed so that its power and water inputs could be utilized. The lamps were installed in front of the wafer using the same bar-beam apparatus as the pyrometry sensors. Their power level was modulated using a Staco Type 3PN1010 variable autotransformer rated for $140 \mathrm{~V}$ and up to $10 \mathrm{~A}$. Once the setup was complete, a 
SensArray 1501 Series 4" silicon wafer instrumented with a K-type thermocouple was placed in the wafer holder and connected through a thermocouple feedthrough to a Honeywell DC3003 readout display. Calibration was done under vacuum in order to protect the thermocouple, as it was only rated for $500^{\circ} \mathrm{C}$ under ambient conditions. The modified version of the test system is illustrated in Figure 4.14. A square ceramic sheet measuring approximately $6 \times 6$ " was utilized to both absorb the radiant light with minimal reflection as well as to help prevent it from reaching the sensors as it greatly affected their reading. Since even a small reflection was found to influence the output, aluminum foil was utilized to further suppress the light. The features of the Luxtron pyrometer relevant to calibration were found through the main menu, which was accessed using the

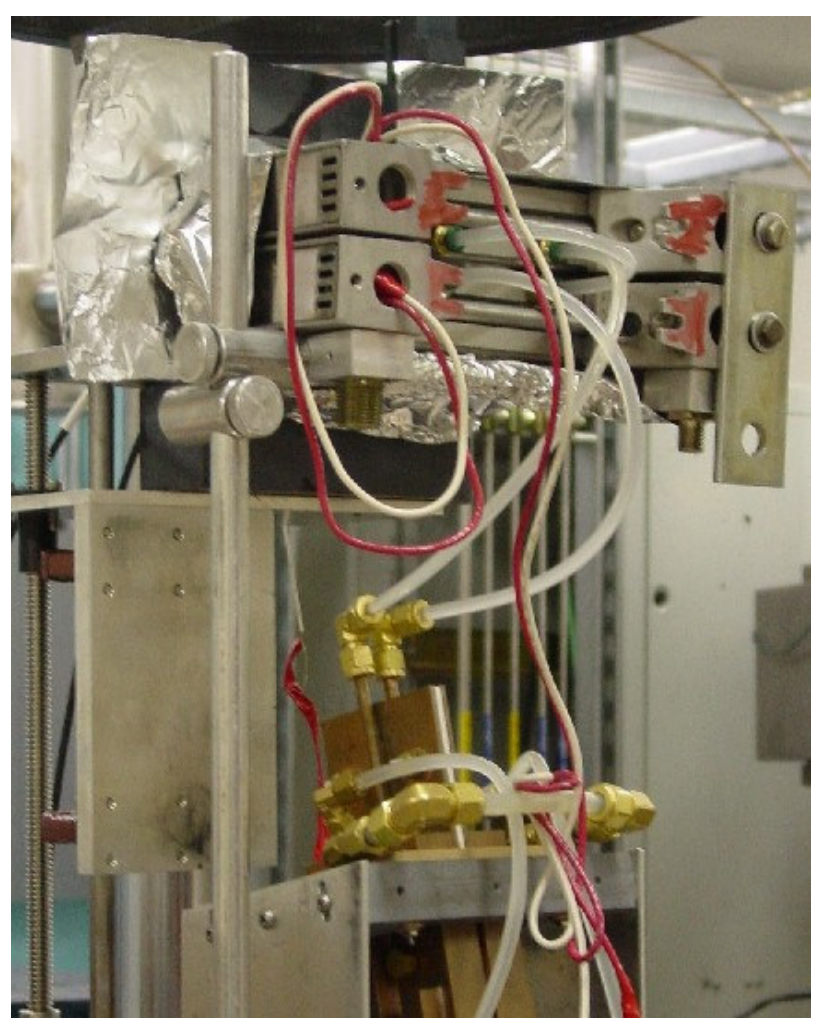

Figure 4.14: Modified version of the BEAVIS test system for pyrometry calibration. Heat lamps provide radiant energy on a square ceramic sheet which then transfers this energy to the thermocouple wafer behind it. Optical sensors measure the wafer temperature and are calibrated at the Luxtron pyrometer such that the thermocouple and pyrometry measurements correspond. 


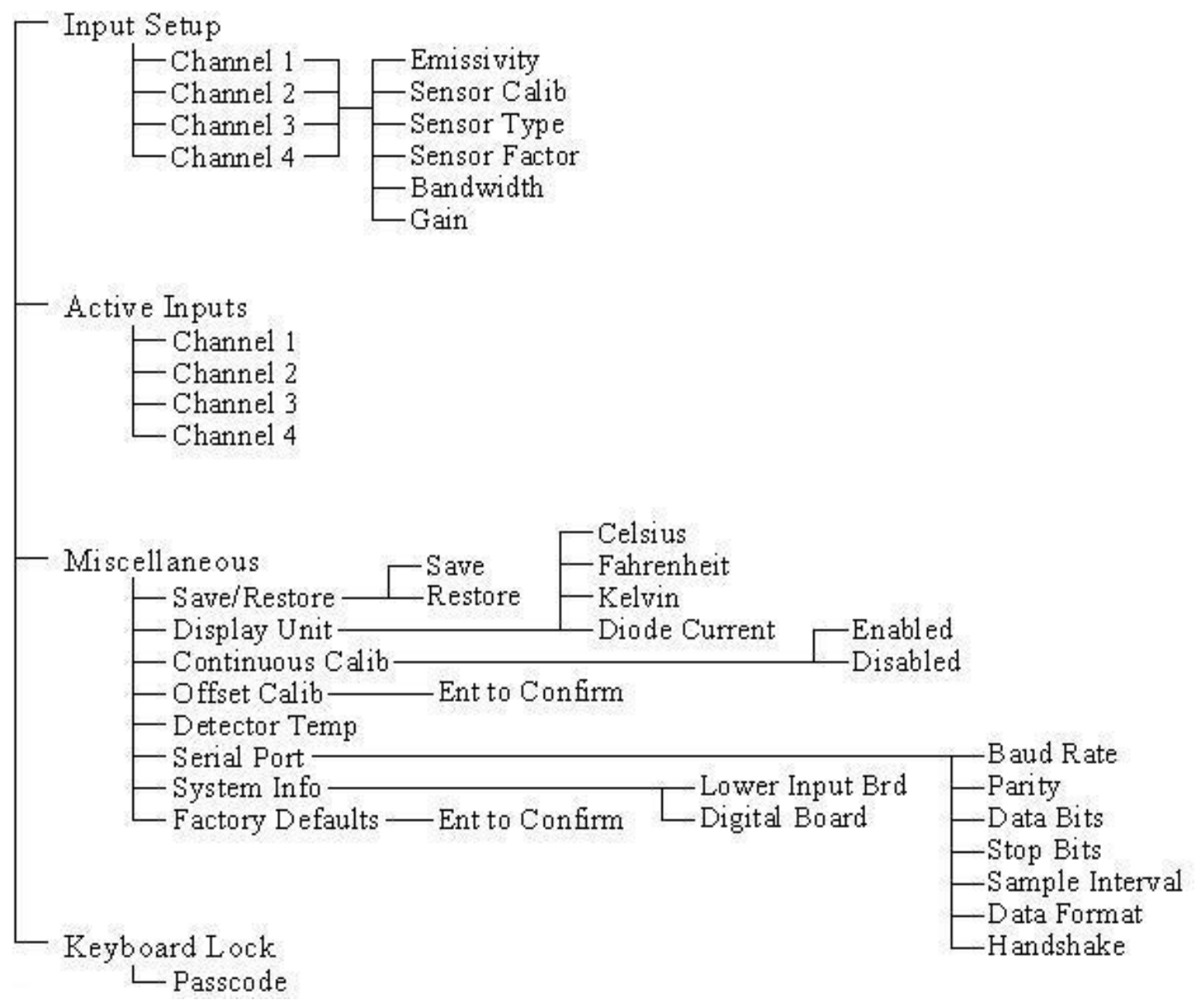

Figure 4.15: Diagram of the Luxtron menu hierarchy. The up/down arrows on the box moved between levels and the left/right arrows scrolled through the various options within a level. Calibration was done through the Input Setup menu using the Emissivity, Sensor Calib, and Sensor Factor options.

arrows located on the lower right-hand side of the face. The menu hierarchy for the system is shown in Figure 4.15. The up/down arrows were used to move between levels, while the right/left arrows scrolled through the particular options within the selected level. The main options of interest were Emissivity, Sensor Calib(ration), and Sensor Factor located under the Input Setup heading. These values could be programmed independently of each other for each of the four channels. Emissivity is an indication of the degree to which a substance radiates or absorbs thermal energy. It ranges from 0-1 and varies with factors such as material composition, crystallinity, surface finish, 
temperature, and radiation wavelength. The influence it has on pyrometry accuracy can be understood from the Stefan-Boltzmann law,

$$
q_{R A D}=\sigma_{S} \varepsilon\left(T_{S}^{4}-T_{A}^{4}\right),
$$

where $q_{R A D}\left(\mathrm{~W} / \mathrm{m}^{2}\right)$ is the radiated heated flux, $k_{B}\left(5.67 \mathrm{E}-8 \mathrm{~W} / \mathrm{m}^{2} \mathrm{~K}^{4}\right)$ is the StefanBoltzmann constant, $e_{S}$ is the emissivity, $T_{S}(\mathrm{~K})$ is the surface temperature, and $T_{A}(\mathrm{~K})$ is the ambient temperature [Rudnev, et al., 1997 (p. 777)]. It is nearly impossible to obtain a highly accurate emissivity value for a surface from the literature, as variations occur from manufacturer to manufacturer and can even be significant at different points on a single wafer. Fortunately, the effect of emissivity error is greatly minimized by the fact that the temperature is raised to the fourth power in Equation (4.6); thus when the pyrometer attempts to convert from the input Q value to an actual temperature, the offset portion is reduced to its fourth root. For example, if surface with an emissivity of 0.7 is at a temperature of $800^{\circ} \mathrm{C}$ but the pyrometer believes it to be 0.6 , a $14 \%$ offset, it would compute approximately $831^{\circ} \mathrm{C}$, a $3.9 \%$ offset. For the purposes of this experimentation, a $31{ }^{\circ} \mathrm{C}$ error would not cause significant problems, though for many other processes it would. Initial emissivity values were obtained from Sato who generated data on the emissivity of a polished silicon surface [Sato, 1967]. Sato's famous graph is shown in Figure 4.16 and indicates the silicon emissivity to be fairly consistent with respect to temperature in the $750-1000 \mathrm{~nm}$ wavelength range at around 0.65 . It is important to note that this data was for a relatively thick sample $(2 \mathrm{~mm})$ that was polished on both sides, and consequently some adjustments were required. A second offset term encountered in pyrometry is the sensor factor, which is an indication of the efficiency with which the various items in the optical circuit transmit light [Advanced Energy, 2002]. Energy is lost 


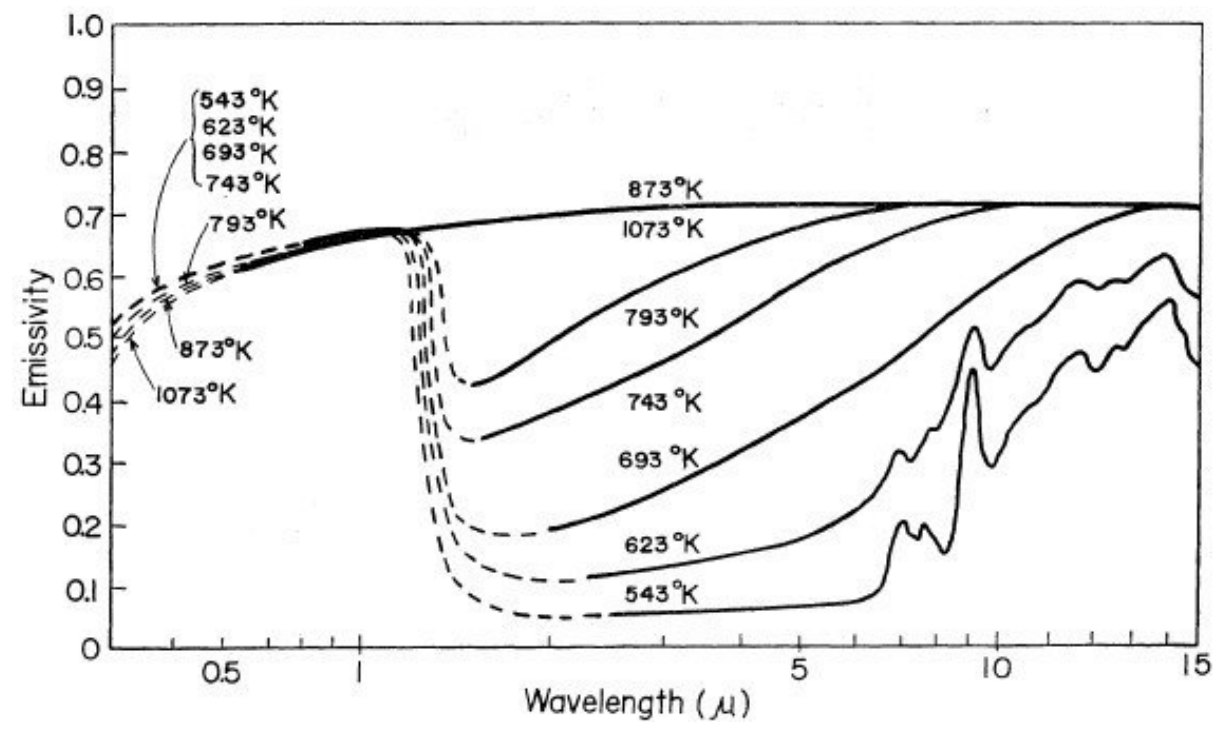

Figure 4.16: Emissivity of polished silicon over temperature and wavelength of radiation with a phosphorous doping level of approximately $3 \mathrm{E} 14 \mathrm{~cm}^{-3}$. The data indicates a nearly constant value in the wavelength range of interest for infrared pyrometry [Sato, $1967^{1}$ ].

at each junction, throughout the cables, and even at the sensor itself. The Luxtron system had the capability to automatically determine the sensor factors using the Sensor Calib feature which allowed the user to input a reference temperature provided that its value was known. Based on the emissivity and the previous sensor factor value, a new factor would be calculated such that the temperature reading matched the user-supplied value. Thus, the calibration was performed over several iterations in which the heat lamps were increased to full intensity, producing a thermocouple reading of approximately $550^{\circ} \mathrm{C}$, and the exact measurement was supplied to the pyrometer for each of the two channels in use. As the temperature was gradually ramped down, the degree to which the readings began to deviate from the thermocouple was noted and the emissivity value was adjusted accordingly. The means by which the two sets of readings was observed is illustrated in Figure 4.17 , in which the temperature was at its maximum value and the pyrometry readings had just been adjusted. Eventually, an emissivity value of 0.63 was settled upon

\footnotetext{
${ }^{1}$ Image reprinted with permission from the Japanese Journal of Applied Physics.
} 


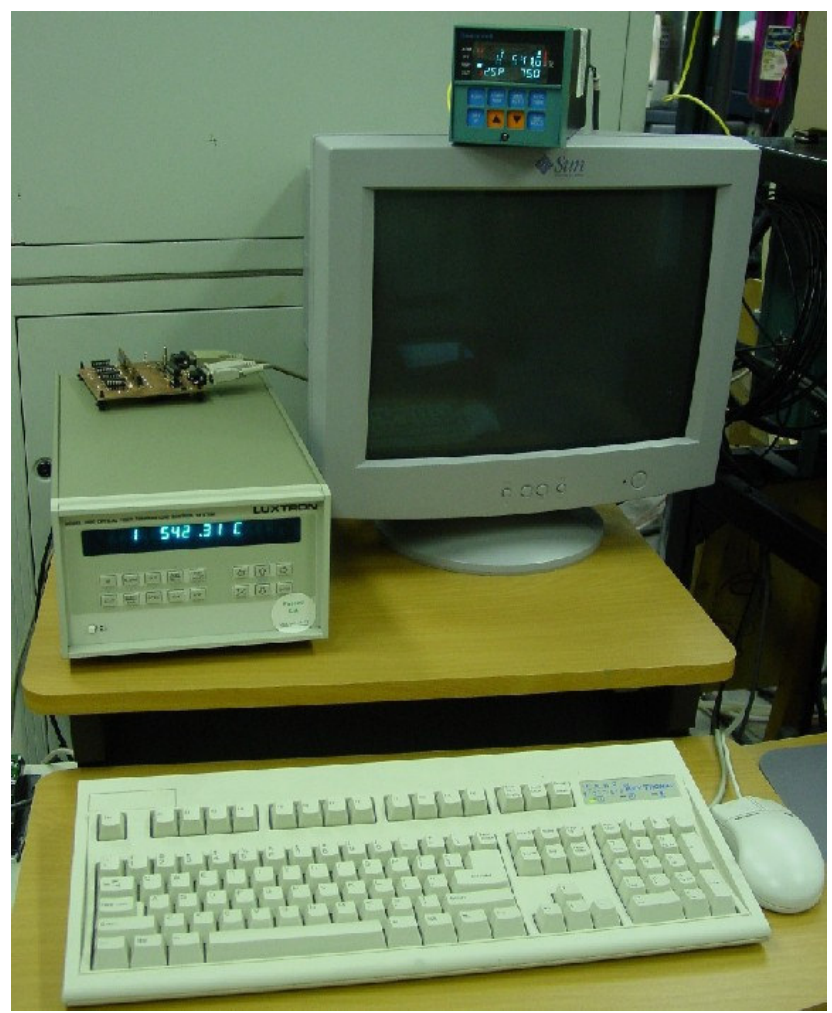

Figure 4.17: Illustration of the pyrometry calibration procedure in which the thermocouple readout was displayed on the green-colored system above the computer monitor while the pyrometry readout was displayed on the tan-colored system to the left of the monitor. The pyrometer had just been supplied with the known reference temperature.

with the resulting sensor factors for Channel 1 and Channel 2 determined to be 1.882 and 1.579 , respectively. Figure 4.18 contains a plot of the thermocouple reading and the two pyrometry readings over the calibration range utilized, $325-550^{\circ} \mathrm{C}$. The results showed an error of less than $10^{\circ} \mathrm{C}$ over the $225^{\circ} \mathrm{C}$ span. While it would have been preferred to extend the upper temperature limit, pyrometry inherently increases in accuracy at higher temperatures as it is becomes less susceptible to interference from background radiation [Luxtron, 2004] and therefore it was concluded that the measurements should be acceptable over the desired range of polysilicon annealing temperatures.

Accurate temperature monitoring was the final goal to be fulfilled for the first generation of the BEAVIS test system, reaching completion in August of 2005 and 


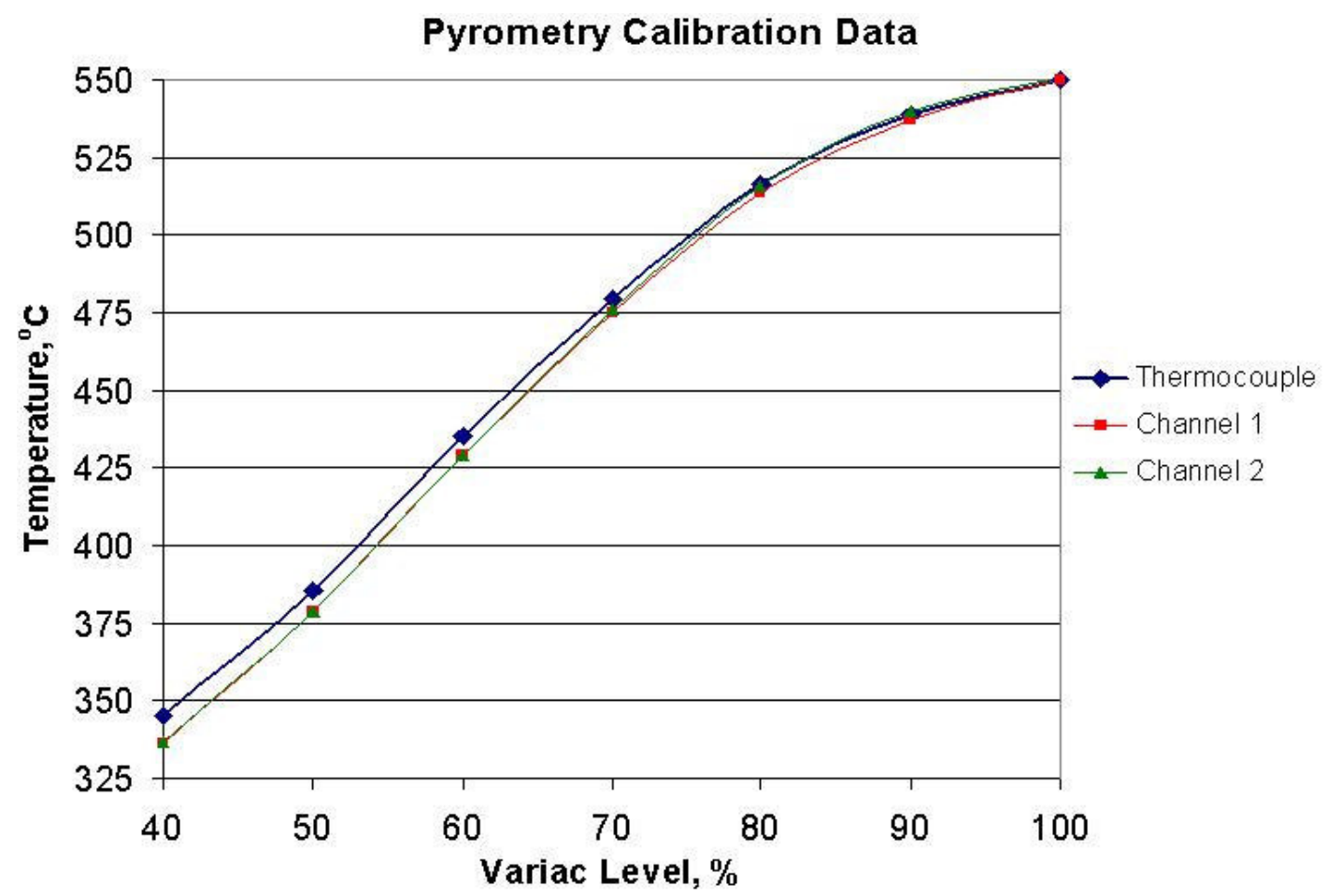

Figure 4.18: Final results of pyrometry calibration showing a comparison of the thermocouple and pyrometry readings at various levels of the autotransformer. The pyrometry had a maximum error of $-9^{\circ} \mathrm{C}$ to $+1{ }^{\circ} \mathrm{C}$ over the full range of calibration.

concluding nearly three years of design, assembly, and modification. The author would like to express sincere gratitude to the many students and staff members who contributed to this effort. MTU research associates Michael Chase, Roland McKinstry, and John Miller, graduate student Thomas Wallner, and undergraduates Brian Choponis, Andrew Gross, Paul Klustaitis, Scott Mollard, Michael Oisten, Robert Simon, and Kevin Zeits assisted in various aspects of the system design and hardware installation. The experiments that were carried out during and after construction verified that the majority of the original objectives with respect to efficient induction heating had been achieved. The capability for further improvement still remained, with second-generation purchases likely including a larger power supply and a thermal imaging camera. Nevertheless, polysilicon annealing was demonstrated on the system as is shown later in this chapter. 


\section{3: Magnetic Properties of RF Sputtered Ferromagnetic Films}

Ferromagnetic films deposited at MTU for induction annealing on the BEAVIS test system were primarily formed on a Perkin-Elmer 2400-8J RF sputtering system. The tool was acquired with four ferromagnetic targets: $\mathrm{Ni}, \mathrm{Ni}_{83} \mathrm{Fe}_{17}, \mathrm{Ni}_{81} \mathrm{Fe}_{19}, \mathrm{Fe}_{49} \mathrm{Co}_{50} \mathrm{~V}$; two additional targets, $\mathrm{Co}$ and $\mathrm{Ni}_{45} \mathrm{Fe}_{55}$, were subsequently purchased. This assortment presented several alternatives, and consequently an investigation was done into the magnetic qualities of these materials in order to choose the best film for the process. Of particular interest were the permeability in the direction normal to the substrate and the ability to retain ferromagnetism at high temperatures. Referring to Figure 2.4, shown in Section 2.2 and obtained from Honda, et al., properties such as the saturation magnetization level, $M_{S A T}$, and the applied field required to reach saturation, $H_{S A T}$, could be anticipated for the pure elements while linear combinations were used to predict the approximate behavior of their alloys. The plots suggested that materials high in nickel and iron would saturate very quickly $\left(H_{S A T-N i} \sim 100 \mathrm{Oe}\right.$ and $\left.H_{S A T-F e} \sim 200 \mathrm{Oe}\right)$, while those high in cobalt would saturate relatively slowly $\left(H_{S A T-C o} \sim 4000\right.$ Oe). Saturation levels were expected to be highest for films high in iron and cobalt $\left(M_{S A T-F e} \sim 1700 \mathrm{G}\right.$ and $\left.M_{S A T-C o} \sim 1500 \mathrm{G}\right)$, and lowest for those high in nickel $\left(M_{S A T-N i} \sim 500 \mathrm{G}\right)$. Examining the Curie temperature values given in Eisberg, et al., 1985 (p. 500), materials high in nickel should lose their ferromagnetic qualities at the lowest temperatures $\left(T_{C-N i}=358^{\circ} \mathrm{C}\right)$, those high in iron at moderate temperatures $\left(T_{C-F e}=761^{\circ} \mathrm{C}\right)$, and those high in cobalt at relatively high temperatures $\left(T_{C-C o}=1127^{\circ} \mathrm{C}\right)$ [Honda, et al., 1926]. These initial parameters were all of qualitative value, but thin film properties are quite different from those of bulk materials and thus actual measurements were vital to process optimization. 
The sputtered films were assumed to be mostly amorphous, and this was certain to have an impact on the degree to which magnetic dipoles interacted. In Raeburn, et al., 1978, a study was done in which amorphous and polycrystalline samples of iron were analyzed; it was found the saturation magnetization values were typically much lower for the amorphous samples, with a difference of as much as $50 \%$. Much of the difference was likely a result of the lower density exhibited by an amorphous material; it was discussed in Chapter 2 that inter-atomic spacing is one of the critical factors in the determination of ferromagnetic properties. Raeburn, et al., suggested that possible explanations could include a change in the distribution of exchange interactions, a change in the density of states, and even an introduction of some antiferromagnetic coupling.

Thin film magnetic measurements were obtained using a Princeton Measurements Vibrating Sample Magnetometer (VSM) at the University of Minnesota's Institute for Rock Magnetism (IRM). This system allows for the characterization of a magnetic material's hysteresis curve under ambient temperatures between $10 \mathrm{~K}$ and $1025 \mathrm{~K}\left(-261^{\circ} \mathrm{C}\right.$ and $752^{\circ} \mathrm{C}$ ) under applied fields of up to $1.8 \mathrm{~T}$ in magnitude [Solheid, et al., 1995 (pp. 1, 6)]. Data was obtained for each of the various sputtered films as well some additional sputtered alloys fabricated by alternating between two targets for specific time durations within a five-minute duty cycle. An electroplated nickel film was also produced for

\begin{tabular}{ll}
\hline \hline \multicolumn{1}{c}{ TSU/CAMD NICKEL ELECTROPLATING RECIPE } \\
\hline Chemical & Grams per liter of $\mathrm{H}_{2} \mathrm{O}$ \\
\hline $\mathrm{NiSO}_{4}$ & 300 \\
$\mathrm{NiCl}_{2}$ & 45 \\
$\mathrm{H}_{3} \mathrm{BO}_{4}$ & 45 \\
Sodium Saccharin & 8 \\
Sodium Lauryl Sulfate & 0.5 \\
Coumarin & 0.5 \\
\hline \hline
\end{tabular}


comparison purposes, deposited according to the Louisiana State University (LSU) Center for Advanced Microstructures and Devices (CAMD) recipe indicated in Table 4.2. Many of the samples were formed in the presence of an applied magnetic field from one or more permanent magnets. Manufactured by Magnetic Component Engineering (MCE), each magnet measured 4" square with normal field strength in the range of approximately 200-300 Gauss. It was anticipated that the magnet(s) would help to improve the magnetic quality of the films in the normal orientation by encouraging the adatoms to nucleate in a manner that aligned with the applied field, thus reducing the free energy. In addition, a few samples were subject to the photolithography process, as factors such as photoresist bake temperatures and photoresist-imparted stresses could potentially have an impact on magnetic properties. Half of these then underwent a two-hour magnetic anneal at $300^{\circ} \mathrm{C}$ in a nitrogen-ambient furnace using one of the same MCE magnets in order to determine whether this might be a useful method to improve properties beyond those of the asdeposited films. All films were deposited above a silicon substrate $500 \mu \mathrm{m}$ in thickness with a $1 \mu \mathrm{m}$ thermally-grown silicon dioxide layer preventing silicon-metal interaction. Table 4.2 summarizes the conditions by which each sample was prepared and the results that were obtained. The entire data collection is presented graphically in Appendix B. The VSM generated raw data as the magnetic moment of the material in $\mathrm{A}^{*} \mathrm{~m}^{2}$. Normalizing by the film's volume provided the magnetization values in $\mathrm{A} / \mathrm{m}$. Flux density values were then obtained through the relation

$$
B=\mu_{0}(H+M)
$$

where the flux density, $B$ (Tesla), is proportional to the sum of the applied field, $H(\mathrm{~A} / \mathrm{m})$, and the magnetization, $M(\mathrm{~A} / \mathrm{m})$, by the permeability of free space, $\mu_{0}(\mathrm{H} / \mathrm{m})$ [Balanis, 
1989 (p. 54)]. The appendix contains plots of both the flux density and magnetization for each sample, with individual analyses provided in the figure captions. The initial magnetization curves exhibited a significant linear component (i.e., it appeared that the films failed to saturate) as a result of the non-negligible contribution of the silicon substrate. A linear equation was formed for each sample based upon the average slope in the saturation regions, with a y-intercept at the origin, and this was utilized to remove the non-ferromagnetic offset from the data. The outcome of this modification is illustrated in Figure 4.19; the $\mathrm{Ni}_{81} \mathrm{Fe}_{19}$ curve was flattened at the saturation regions and was otherwise minimally affected. This modified data was used to provide the values for Table 4.3. If saturation was obtained for a given sample and orientation, the saturation level was found by rounding the magnetization values to three significant figures and determining the

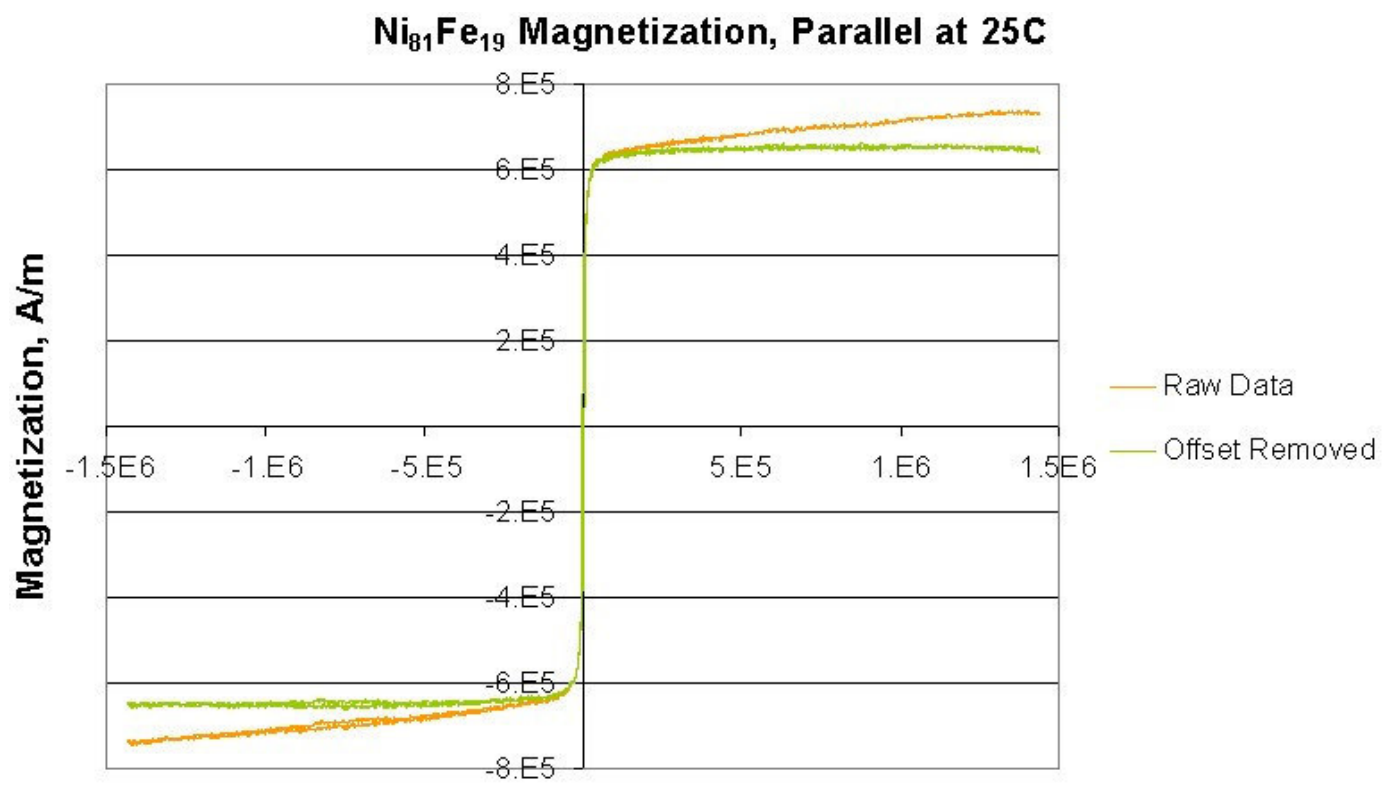

Applied Field, A/m

Figure 4.19: $\mathrm{Ni}_{81} \mathrm{Fe}_{19}$ magnetization curves measured in the plane of the substrate. The first curve shows the magnetization calculated directly from the magnetic moment data. The non-zero slope in the saturation regions was a result of the non-ferromagnetic materials present in the sample, namely the substrate. The second curve shows the effect of subtracting the linear component from the data such that only the ferromagnetic film was characterized. 


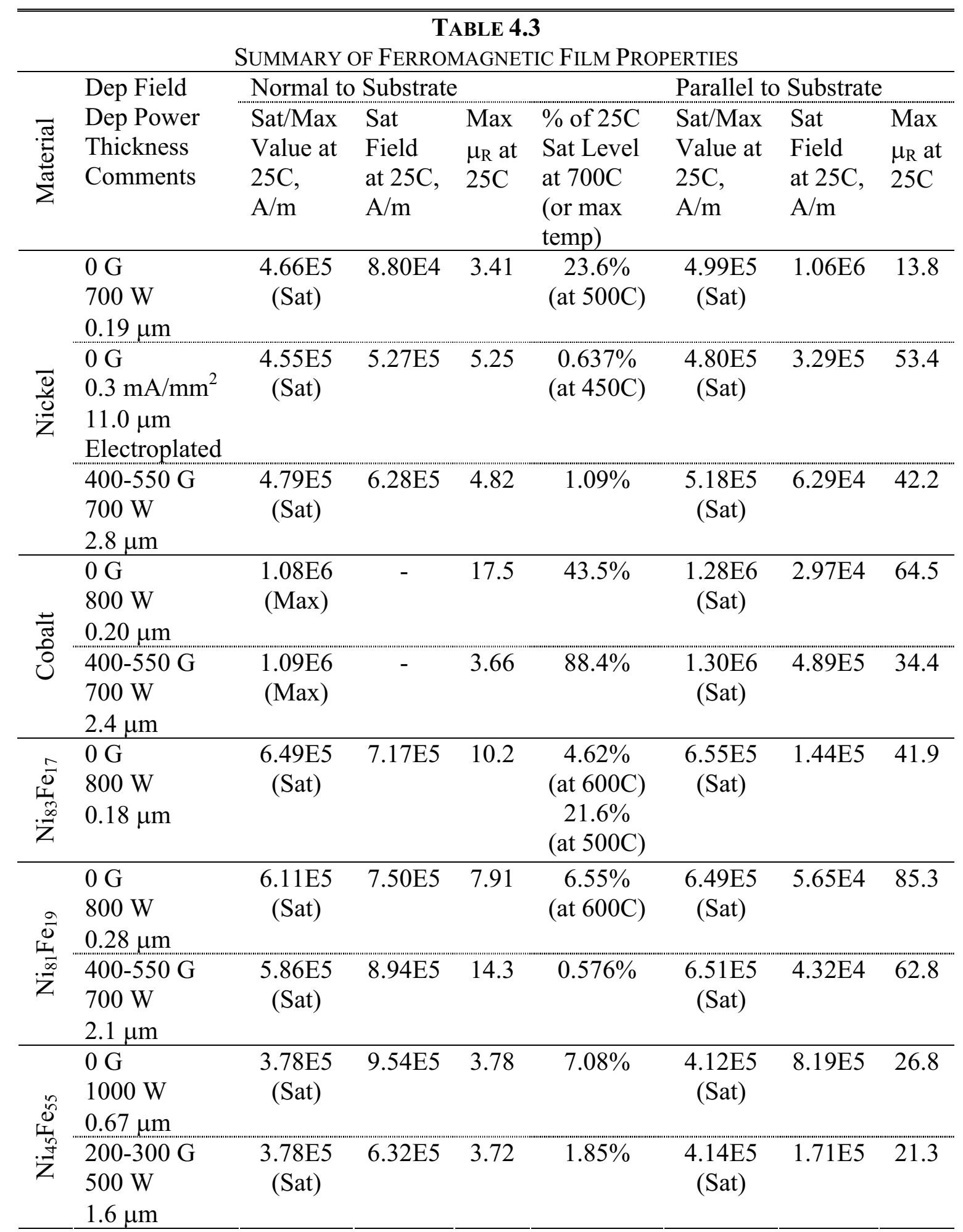




\begin{tabular}{|c|c|c|c|c|c|c|c|c|}
\hline \multirow{6}{*}{ 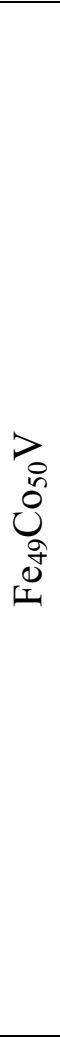 } & $\begin{array}{l}0 \mathrm{G} \\
850 \mathrm{~W} \\
0.20 \mu \mathrm{m}\end{array}$ & $\begin{array}{c}1.11 \mathrm{E} 6 \\
\text { (Max) }\end{array}$ & - & 6.56 & $2.76 \%$ & $\begin{array}{c}1.26 \mathrm{E} 6 \\
\text { (Sat) }\end{array}$ & $1.58 \mathrm{E} 5$ & 29.7 \\
\hline & $\begin{array}{l}200-300 \mathrm{G} \\
700 \mathrm{~W} \\
1.7 \mu \mathrm{m}\end{array}$ & $\begin{array}{c}9.76 \mathrm{E} 5 \\
(\mathrm{Max})\end{array}$ & - & 6.57 & $96.9 \%$ & $\begin{array}{c}\text { 1.27E6 } \\
\text { (Sat) }\end{array}$ & $5.13 \mathrm{E} 4$ & 106 \\
\hline & $\begin{array}{l}400-550 \mathrm{G} \\
700 \mathrm{~W} \\
3.0 \mu \mathrm{m}\end{array}$ & $\begin{array}{l}9.43 \mathrm{E} 5 \\
(\mathrm{Max})\end{array}$ & - & 1.83 & $99.3 \%$ & $\begin{array}{c}1.26 \mathrm{E} 6 \\
\text { (Sat) }\end{array}$ & $3.52 \mathrm{E} 4$ & 112 \\
\hline & $\begin{array}{l}200-300 \mathrm{G} \\
300 \mathrm{~W} \\
0.40 \mu \mathrm{m}\end{array}$ & $\begin{array}{c}1.10 \mathrm{E} 6 \\
\text { (Sat) }\end{array}$ & $8.03 \mathrm{E} 5$ & 8.84 & - & $\begin{array}{c}1.17 \mathrm{E} 6 \\
\text { (Sat) }\end{array}$ & $2.02 \mathrm{E} 5$ & 59.2 \\
\hline & $\begin{array}{l}200-300 \mathrm{G} \\
300 \mathrm{~W} \\
0.40 \mu \mathrm{m} \\
\text { Litho }\end{array}$ & $\begin{array}{c}1.15 \mathrm{E} 6 \\
\text { (Sat) }\end{array}$ & 7.94E5 & 9.45 & - & $\begin{array}{c}1.22 \mathrm{E} 6 \\
\text { (Sat) }\end{array}$ & 4.29E5 & 56.6 \\
\hline & $\begin{array}{l}200-300 \mathrm{G} \\
300 \mathrm{~W} \\
0.40 \mu \mathrm{m} \\
\text { Litho, } \\
\text { Anneal }\end{array}$ & $\begin{array}{c}1.24 \mathrm{E} 6 \\
\text { (Sat) }\end{array}$ & $9.54 \mathrm{E} 5$ & 4.05 & - & $\begin{array}{c}\text { 1.28E6 } \\
\text { (Sat) }\end{array}$ & $2.54 \mathrm{E} 5$ & 44.0 \\
\hline 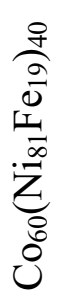 & $\begin{array}{l}400-550 \mathrm{G} \\
700 \mathrm{~W} \\
3.1 \mu \mathrm{m} \\
\text { Alternate } \\
\text { targets } \\
(3: 00,2: 00)\end{array}$ & $\begin{array}{c}8.08 \mathrm{E} 5 \\
(\mathrm{Max})\end{array}$ & - & 3.08 & $70.5 \%$ & $\begin{array}{c}\text { 8.71E5 } \\
\text { (Sat) }\end{array}$ & $8.70 \mathrm{E} 4$ & 62.1 \\
\hline 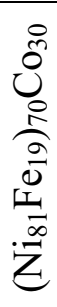 & $\begin{array}{l}400-550 \mathrm{G} \\
700 \mathrm{~W} \\
2.8 \mu \mathrm{m} \\
\text { Alternate } \\
\text { targets } \\
(3: 30,1: 30)\end{array}$ & $\begin{array}{c}9.66 \mathrm{E} 5 \\
(\mathrm{Max})\end{array}$ & - & 4.84 & $40.3 \%$ & $\begin{array}{c}1.01 \mathrm{E} 6 \\
\text { (Sat) }\end{array}$ & $2.33 \mathrm{E} 4$ & 95.9 \\
\hline 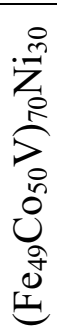 & $\begin{array}{l}400-550 \mathrm{G} \\
700 \mathrm{~W} \\
3.0 \mu \mathrm{m} \\
\text { Alternate } \\
\text { targets } \\
(3: 30,1: 30)\end{array}$ & $\begin{array}{c}1.08 \mathrm{E} 6 \\
(\mathrm{Max})\end{array}$ & - & 4.51 & $26.7 \%$ & $\begin{array}{c}1.44 \mathrm{E} 6 \\
\text { (Sat) }\end{array}$ & $3.93 \mathrm{E} 4$ & 124 \\
\hline
\end{tabular}


overall mode; this eliminated the impact of random data fluctuations. The saturation field was then the applied field at which the magnetization reached $95 \%$ of the computed saturation level. For samples that did not reach saturation, the maximum overall magnetization was examined in lieu of a saturation value. In either case, permeabilities were computed at each data point by calculating the slope of the flux density $(B-H)$ curve using three points before and after; the table shows the maximum value for each sample and orientation. Finally, the effect of elevated temperature on the magnetization normal to the substrate (the direction of interest for this research) was quantified with the ratio of the saturation/maximum level at maximum temperature, usually $700^{\circ} \mathrm{C}$, to that at $25^{\circ} \mathrm{C}$.

Overall, the data reflected several interesting trends. All samples reached saturation when magnetized in the plane of the substrate, but many did not saturate in the normal direction. The effect of the applied magnetic field normal to the substrate during film formation was mixed, particularly at relatively high deposition power levels. Some samples exhibited permeabilities that increased in the normal direction but decreased in the in-plane direction, some exhibited the reverse behavior, others increased in both directions, while still others decreased in both. In general, however, the in-situ magnetized samples tended to saturate or approach saturation more rapidly than their non-magnetized counterparts. Examining the data set for $\mathrm{Fe}_{49} \mathrm{Co}_{50} \mathrm{~V}$, the most thoroughly analyzed material, reducing the deposition power apparently enabled the in-situ magnetic field to more strongly impact the process of film formation; the permeabilities in the normal direction were higher for the $300 \mathrm{~W}$ samples than for those deposited at $700 \mathrm{~W}$, and the magnetization curves reached saturation whereas at $700 \mathrm{~W}$ they did not. The data also seemed to suggest that both photolithography and magnetic annealing had minimal 
impact on the film properties, and if anything the magnetic anneal reduced the film quality, likely due to film oxidation from residual oxygen in the nitrogen supply or possibly even nitridation.

Comparing experiment with theory, the saturation levels were typically very close to those predicted by the Honda figures, though not unexpectedly the ternary alloys differed considerably as their interactions are more complex and the alloys were not fully uniform in composition. The conversion from either Oersteds or Gauss to $\mathrm{A} / \mathrm{m}$ entails multiplication by $1000 / 4 \pi$ (approximately 80 ), but differences between the cgs and MKS systems of units introduce an additional factor of $4 \pi$ between the cgs magnetization, $I$, and its MKS counterpart, $M$, resulting in an overall conversion factor of 1000 [Reed, et al., 1983; Bozorth, 1993 (pp. 2-5)]). For example, Honda predicted nickel to saturate at around $500 \mathrm{G}$, or approximately $5 \mathrm{E} 5 \mathrm{~A} / \mathrm{m}$. The values obtained from the three nickel samples ranged from $4.55 \mathrm{E} 5$ to $5.18 \mathrm{E} 5$, including both the parallel and normal orientations. It was anticipated that higher fields would be required in order to reach saturation due to the amorphous crystal structure of the films. In actuality, saturation fields showed a large degree of variation, ranging from slightly lower than that shown for the equivalent bulk material to up to two orders of magnitude higher, and defendable conclusions were difficult to make in this regard. The thermal properties of the samples, on the other hand, were in excellent agreement with expectations. The films comprising nickel as the primary alloy were the quickest to degrade in ferromagnetic quality. Cobalt and the iron-cobalt alloys, on the other hand, retained their properties very well, usually showing little to no reduction in saturation level or permeability over the full temperature range. Perhaps the most interesting behavior was that exhibited by the $\mathrm{Ni}_{45} \mathrm{Fe}_{55}$ alloy. As 
the temperatures were increased, the saturation levels initially fell rapidly, more so than for the other NiFe alloys despite having a much higher iron content. By $300^{\circ} \mathrm{C}$, the samples were nearly paramagnetic in nature, but then at $400^{\circ} \mathrm{C}$ a sudden increase in magnetization took place. Beyond this temperature, the values decreased as normal. This behavior repeated for multiple samples, with and without in-situ magnetization, and in both orientations. This suggests that a phase transformation may have been taking place in the $\mathrm{Ni}_{45} \mathrm{Fe}_{55}$ films, perhaps increasing their crystallinity. The next section will show that this particular alloy did not inductively heat as well as was expected. A magnetic anneal in which this phase change takes place might help to improve its capabilities.

This initial investigation into the magnetic properties of the RF sputtered ferromagnetic films produced significant information about their behavior. While engineering led to improvements in ferromagnetic quality normal to the substrate, it nevertheless remained true that the permeability values were far below expectation and further research in this area would be imperative to the success of the inductive annealing technology. The $\mathrm{Ni}_{81} \mathrm{Fe}_{19}$ films appeared to have the best permeability values and were expected to be the most likely to heat well since nickel has been found to yield films of high quality (low stress). The $\mathrm{Fe}_{49} \mathrm{Co}_{50} \mathrm{~V}$ films, on the other hand, exhibited slightly reduced permeabilities but retained them to much higher temperatures. Subsequent research concentrated primarily on these two materials, henceforth referred to as NiFe19 and $\mathrm{FeCo}$, respectively, with a lesser investigation into the heating capabilities of the $\mathrm{Ni}_{45} \mathrm{Fe}_{55}$ (henceforth referred to as NiFe55) alloy which was still believed to have high potential. Examining these three materials more closely in the magnetic field range typical of that output by BEAVIS (see Section 3.2), linear regressions were fit for each 
temperature value and low-field permeabilities were extracted from their slopes. The variation in permeability over temperature for the three alloys is shown in the plots of Figures $4.20(\mathrm{a}, \mathrm{b})$. Normal to the substrate, NiFe19 exhibited an initial high permeability

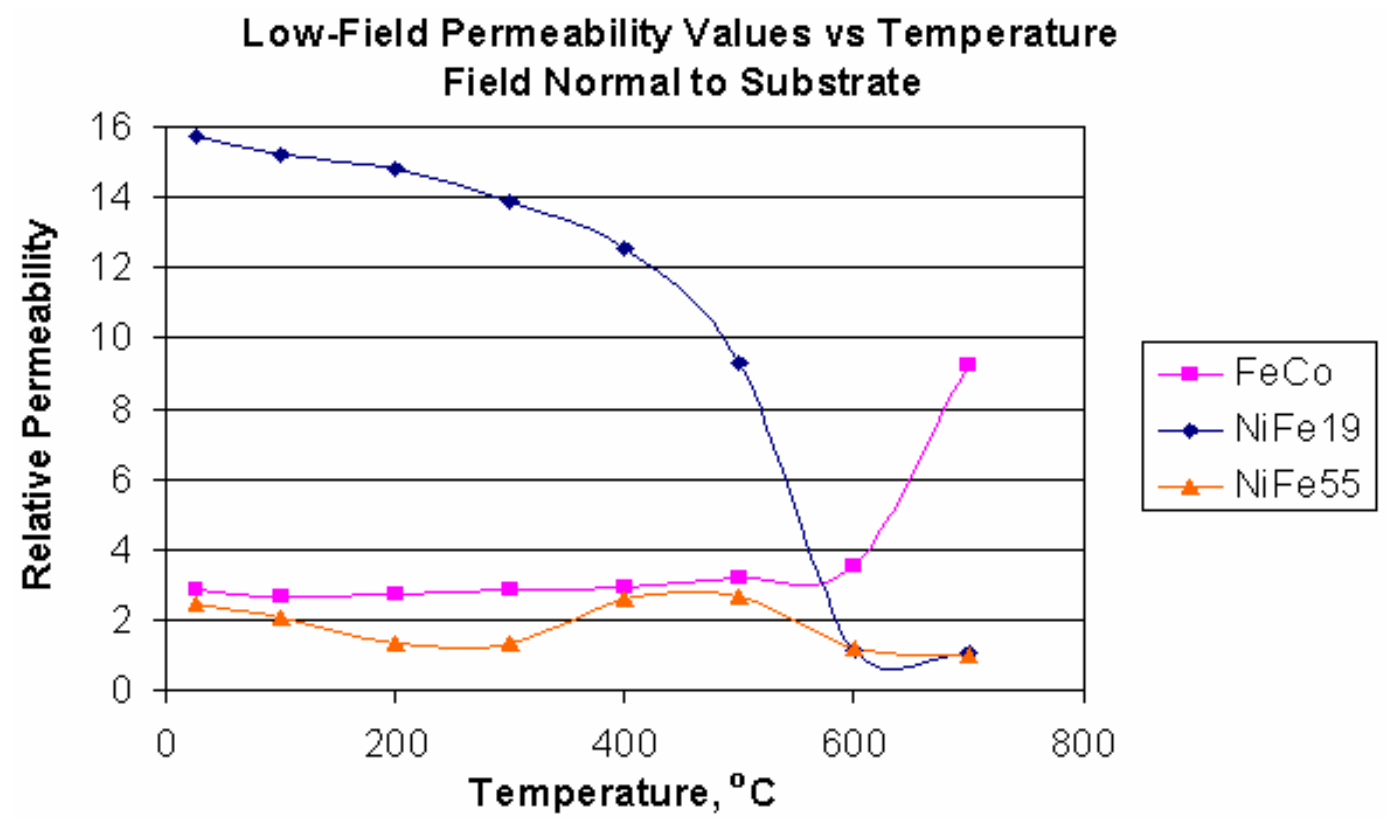

Figure 4.20(a): Relative permeability values for the three alloys of interest, normal to the substrate, extracted from the low-field VSM data. At each temperature, a linear regression was fit to the $B$-vs- $H$ curve using only the values for which $|H|<1 \mathrm{E} 4 \mathrm{~A} / \mathrm{m}$.

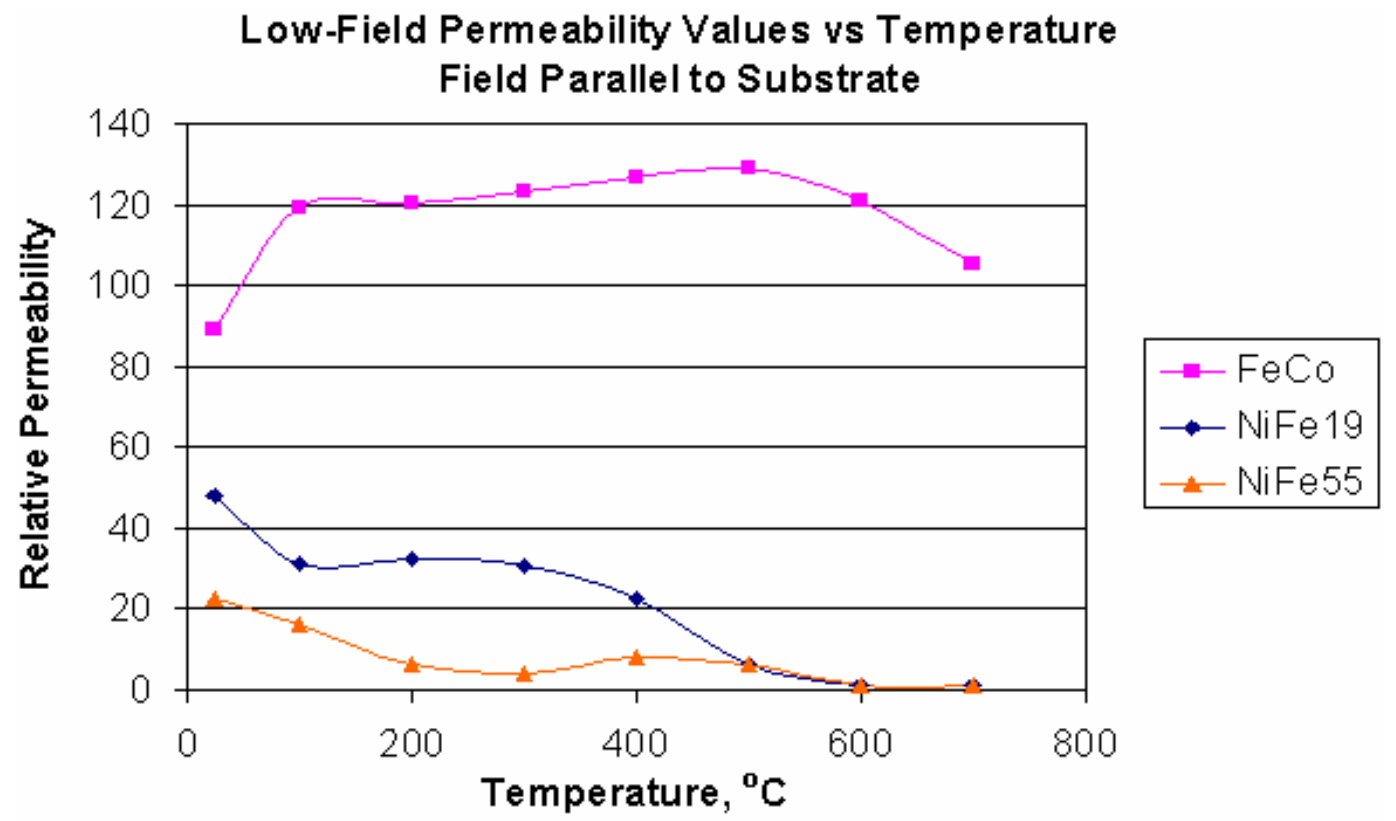

Figure 4.20(b): Relative permeability values for the three alloys of interest, parallel to the substrate, extracted from the low-field VSM data. 
that gradually decreased with temperature up to approximately $400^{\circ} \mathrm{C}$ and then began to fall more rapidly. $\mathrm{FeCo}$, on the other hand, slowly increased in permeability up to $600^{\circ} \mathrm{C}$ with a sharp rise taking place at $700^{\circ} \mathrm{C}$ that suggested another possible phase transformation. In the plane of the substrate, $\mathrm{FeCo}$ maintained a relatively high permeability throughout the entire temperature range whereas $\mathrm{NiFe} 19$ started at a moderate level that again began to drop rapidly after $400^{\circ} \mathrm{C}$. The low-field data for the NiFe55 alloy again showed surprisingly low permeability values with a phase transformation again occurring at around $400^{\circ} \mathrm{C}$ in both directions. In summary, the data showed that $\mathrm{NiFe} 19$ and $\mathrm{FeCo}$ were both promising materials for use in the inductive annealing process; the former exhibited the best magnetic properties in the lower half of the $25-1000^{\circ} \mathrm{C}$ temperature range whereas the latter exhibited the best properties in the upper half. The next two sections demonstrate polysilicon annealing with both alloys and compare their actual heating capabilities.

\section{4: Impact of Film Properties on Heat Generation}

The relationship between the spatial dimensions of an object and its ability to convert energy from magnetic to thermal is reflected in Equations (2.5) of Section 2.1. The equations indicate that power generation increases linearly with the thickness of the sample, but thicker films have a drawback in that they tend to exhibit higher intrinsic stress which reduces conductivity and furthermore increases the likelihood of delamination. Like frequency, the effect of area is piecewise; the power generation initially increases as the third power of the radius until a quasi-saturation is reached, at which point the relationship becomes linear. The dimension at which this transition occurs is approximately equal to the skin depth, $\delta$. Typically, induction heating processes 
are designed such that the dimensions of the sample through which eddy currents propagate are several times larger than the skin depth (see Section 2.1), but process parameters such as low frequency or poor electromagnetic properties can render the reverse situation, in which case these dimensions become especially important. As was discussed in Section 4.1, however, larger surface areas can enhance power loss mechanisms such as convection and radiation. Thus the relationship between film area and overall volume is important to have thoroughly characterized. This section focuses on two alloys in particular, FeCo and NiFe19. The NiFe55 alloy was also studied to a small degree in order to determine whether the phase change discussed in the previous section could be of benefit. Table 4.4 summarizes the measured electromagnetic properties of these alloys and shows the calculated skin depth dimensions for each at $375 \mathrm{kHz}$, the approximate frequency at which testing was done $(+/-5 \mathrm{kHz})$. This is the frequency selected by the Ameritherm self-tuning circuitry for the coil that was utilized, a three-turn, 1" (inner diameter) coil made of 1/4" (outer diameter) copper tubing, with a $0.66 \mu \mathrm{F}$ tank capacitor. The permeabilities shown in the table are the maxima from the low-field values and were utilized to generate the plots in Figure 4.20(a). Resistivity measurements were obtained using an A\&M Fell Model A four-point probe system. Skin depths were calculated using Equation (2.3). The skin depth values suggest that the

\begin{tabular}{|c|c|c|c|}
\hline \multicolumn{4}{|c|}{ TABLE 4.4} \\
\hline Alloy & $\begin{array}{l}\text { Max. Low-Field } \\
\text { Permeability }\end{array}$ & $\begin{array}{l}\text { 25C Conductivity, } \\
\Omega^{-1} \mu \mathrm{m}^{-1}\end{array}$ & $\begin{array}{l}\text { Skin Depth at } \\
375 \mathrm{kHz}, \mu \mathrm{m}\end{array}$ \\
\hline FeCo & 9.23 & 3.30 & 148.9 \\
\hline NiFe19 & 15.8 & 5.92 & 84.98 \\
\hline $\mathrm{NiFe} 55$ & 2.63 & 2.52 & 319.3 \\
\hline $\mathrm{Au}$ & 1 & 18.1 & 193.2 \\
\hline $\mathrm{Cr}$ & 1 & 1.24 & 738.1 \\
\hline
\end{tabular}


NiFe19 alloy should heat with the highest efficiency, at least at room temperature. However, the high-temperature VSM data in the previous section indicated that it would also be the quickest to lose its heating efficiency as temperatures increase. For this reason, it was hypothesized that the FeCo films would yield the best overall results.

Infrared pyrometry was not yet available during the initial phase of characterization, and consequently thermally-sensitive paint was utilized to approximate induced temperatures. Manufactured by Thermographic Measurements Corporation (TMC), the MC490-10 multi-change paint has a temperature range of $490-1250^{\circ} \mathrm{C}$ [Thermographic Measurements, 2005]. The color strip shown in Figure 4.21 illustrates the manner in which temperatures were determined. Finer calibration was done on-site by heating a series of painted samples to the temperatures indicated in the figure. Each sample consisted of a $1 \mathrm{~cm}^{2}$ section of a diced silicon wafer, and the paint "dots" were approximately $1 / 8$ " in diameter. Heating took place in a Barnstead/Thermolyne FA17381 box furnace, and samples were held at temperature for 15 minutes in order to ensure full color change as the manufacturer specifies a ten-minute minimum. Matching each result to a location on the color strip was relatively straightforward, as the temperature to which the sample had been heated was already known. This was somewhat subjective

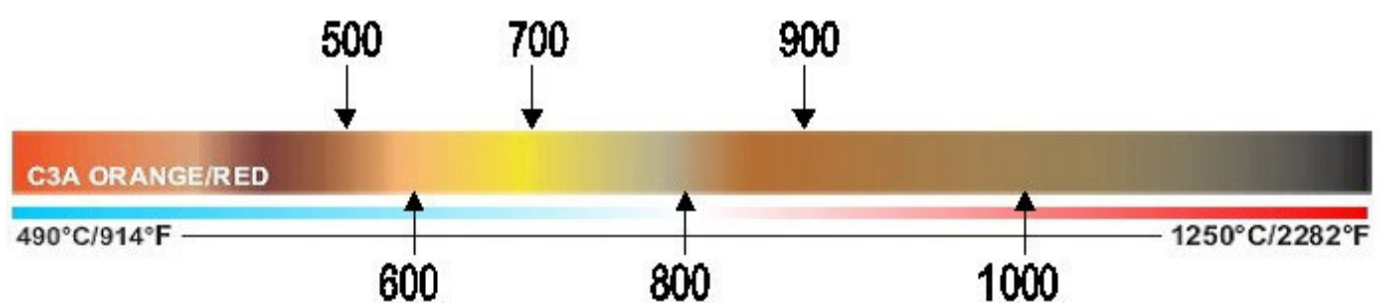

Figure 4.21: Temperature scale provided by Thermographic Measurements for the MC490-10 multi-change thermally-sensitive paint. The color strip was calibrated by heating painted samples for 15 minutes each at temperatures from $500-1000^{\circ} \mathrm{C}$, in $100^{\circ} \mathrm{C}$ intervals [Thermographic Measurements, $2005^{1}$ ].

\footnotetext{
${ }^{1}$ Image and information provided courtesy of Thermographic Measurements Ltd.
} 
with an unknown temperature, however, as the color strip contained multiple sections of brown/orange shades. Thus, the measurement of induced temperature values yielded only estimates and required some knowledge of the approximate result (based on observations such as film discoloration and/or peeling, intensity of glowing, etc). Nevertheless, important qualitative information was obtained from these early experiments.

The first samples to be successfully heated on the BEAVIS test system were FeCo films deposited under an applied magnetic field in the range of 400-500 Gauss. Silicon substrates were prepared with a thermal silicon dioxide layer $(\sim 1 \mu \mathrm{m})$, polysilicon films of various thicknesses deposited via either LPCVD or RF sputtering, and finally covered with a thin $(\sim 100 \mathrm{~nm})$ sputtered silicon dioxide layer. The wafers were then diced into $2 \times 2 \mathrm{~cm}$ sections. FeCo films of different thickness were deposited and either left as blanket $4 \mathrm{~cm}^{2}$ films or patterned into smaller areas via shadow masking during deposition or photolithography after deposition. With a minimum skin depth of $148.9 \mu \mathrm{m}$, it was anticipated from the theoretical analysis that films having lateral dimensions of $1 \mathrm{~mm}$ or higher would heat with reasonably high efficiency, but the relatively low power available from the applied magnetic field turned out to be insufficient to generate appreciable heating in regions smaller than $1 \mathrm{x} 1 \mathrm{~cm}$ in size. Table 4.5 summarizes the temperature values obtained under full inductive power $(\sim 2.1 \mathrm{~kW})$ for $1 \mathrm{~cm}^{2}$ and $4 \mathrm{~cm}^{2}$ films. The data showed several interesting trends. Comparing the results of the $1 \mathrm{~cm}^{2}$ samples patterned via photolithography with those patterned via shadow masking, the latter was observed to perform much better. It was initially hypothesized that the photolithography process might be imparting some sort of deteriorating effect on the films' magnetic properties as a result of perhaps the various baking procedures or even photoresist-induced stresses. It 


\begin{tabular}{lccccc}
\hline \hline \multicolumn{5}{c}{ TABLE 4.5 } \\
& TEMPERATURES INDUCED IN INITIAL FECO FILMS & \\
\hline Sputtering & Deposition & Approximate & Film & Patterning & Approximate \\
Power/Voltage & Time & Thickness & Area & Method & Temperature \\
\hline $400 \mathrm{~W} / 335 \mathrm{~V}$ & 0.5 hour & $0.3 \mu \mathrm{m}$ & $1 \times 1 \mathrm{~cm}$ & Lithography & $<490^{\circ} \mathrm{C}$ \\
$400 \mathrm{~W} / 335 \mathrm{~V}$ & 1 hour & $0.6 \mu \mathrm{m}$ & $1 \times 1 \mathrm{~cm}$ & Lithography & $<490^{\circ} \mathrm{C}$ \\
$400 \mathrm{~W} / 335 \mathrm{~V}$ & 2 hours & $1.2 \mu \mathrm{m}$ & $1 \times 1 \mathrm{~cm}$ & Lithography & $500^{\circ} \mathrm{C}$ \\
$400 \mathrm{~W} / 335 \mathrm{~V}$ & 4 hours & $2.5 \mu \mathrm{m}$ & $1 \times 1 \mathrm{~cm}$ & Lithography & $525^{\circ} \mathrm{C}$ \\
\hline $600 \mathrm{~W} / 475 \mathrm{~V}$ & 0.5 hour & $0.6 \mu \mathrm{m}$ & $1 \times 1 \mathrm{~cm}$ & Lithography & $<490^{\circ} \mathrm{C}$ \\
$600 \mathrm{~W} / 475 \mathrm{~V}$ & 1 hour & $1.1 \mu \mathrm{m}$ & $1 \times 1 \mathrm{~cm}$ & Lithography & $500^{\circ} \mathrm{C}$ \\
$600 \mathrm{~W} / 475 \mathrm{~V}$ & 2 hours & $2.2 \mu \mathrm{m}$ & $1 \times 1 \mathrm{~cm}$ & Lithography & $525^{\circ} \mathrm{C}$ \\
$600 \mathrm{~W} / 475 \mathrm{~V}$ & 4 hours & $4.4 \mu \mathrm{m}$ & $1 \times 1 \mathrm{~cm}$ & Lithography & $550^{\circ} \mathrm{C}$ \\
\hline $400 \mathrm{~W} / 335 \mathrm{~V}$ & 0.5 hour & $0.3 \mu \mathrm{m}$ & $1 \times 1 \mathrm{~cm}$ & Shadow & $500^{\circ} \mathrm{C}$ \\
$400 \mathrm{~W} / 335 \mathrm{~V}$ & 1 hour & $0.6 \mu \mathrm{m}$ & $1 \times 1 \mathrm{~cm}$ & Shadow & $550^{\circ} \mathrm{C}$ \\
$400 \mathrm{~W} / 335 \mathrm{~V}$ & 2 hours & $1.2 \mu \mathrm{m}$ & $1 \times 1 \mathrm{~cm}$ & Shadow & $800^{\circ} \mathrm{C}$ \\
$400 \mathrm{~W} / 335 \mathrm{~V}$ & 4 hours & $2.5 \mu \mathrm{m}$ & $1 \times 1 \mathrm{~cm}$ & Shadow & $1000^{\circ} \mathrm{C}$ \\
\hline $400 \mathrm{~W} / 335 \mathrm{~V}$ & 0.5 hour & $0.3 \mu \mathrm{m}$ & $2 \times 2 \mathrm{~cm}$ & N/A & $500^{\circ} \mathrm{C}$ \\
$400 \mathrm{~W} / 335 \mathrm{~V}$ & 1 hour & $0.6 \mu \mathrm{m}$ & $2 \times 2 \mathrm{~cm}$ & N/A & $525^{\circ} \mathrm{C}$ \\
$400 \mathrm{~W} / 335 \mathrm{~V}$ & 2 hours & $1.2 \mu \mathrm{m}$ & $2 \times 2 \mathrm{~cm}$ & N/A & $1000^{\circ} \mathrm{C}$ \\
$400 \mathrm{~W} / 335 \mathrm{~V}$ & 4 hours & $2.5 \mu \mathrm{m}$ & $2 \times 2 \mathrm{~cm}$ & N/A & $>1400^{\circ} \mathrm{C}$ \\
\hline \hline
\end{tabular}

was shown in Section 4.3, however, that the VSM measurements did not support this theory. At the time, the significance of the substrate doping level was not yet recognized, and most early experimentation was done using samples of unknown doping. Based on observations from subsequent research, however, it became apparent that the substrates used for that particular set of tests were of much higher doping than those used for the other three sets, which all came from the same source. The $0.3 \mu \mathrm{m}$ FeCo film likely contributed very little to the initial $500^{\circ} \mathrm{C}$ temperature, but as the film thickness was increased, the film/substrate combination became progressively more effective, eventually reaching around $1000^{\circ} \mathrm{C}$ with a film thickness of approximately $1.2 \mu \mathrm{m}$. Examining the two sets of $1 \mathrm{~cm}^{2}$ photolithographically-patterned films in which the second set was deposited at a higher power than the first, the data showed that the increased film thicknesses exhibited by the higher-power films were for the most part 
negated by the reduction in film quality (decreased normal permeability due to increased stress). These temperature measurements were in much better agreement with data obtained later on substrates known to be of low doping concentrations (typically 10-20 $\Omega$-cm p-type silicon, equivalent to around $1 \mathrm{E} 15 \mathrm{~cm}^{-3}$ [Sze, 1981 (p 32)]). For the $4 \mathrm{~cm}^{2}$ samples, even the thinnest films yielded color changes in the thermal paint. The photograph in Figure 4.22 shows the heating of the $2.5 \mu \mathrm{m}$ film. The sample remained stable up to approximately $1.5 \mathrm{~kW}$, after which it quickly entered the same thermal runaway state as was encountered previously. The resulting deformed shape is shown in Figure 4.23(b) and illustrates the relative temperature distribution across the sample at the instant of melting; the locations of highest temperature appear to be at the edges of the sample, which is consistent with the skin effect concept. Figure 4.23(a) shows the back sides of three of the other heated samples, with the various paint colors reflecting

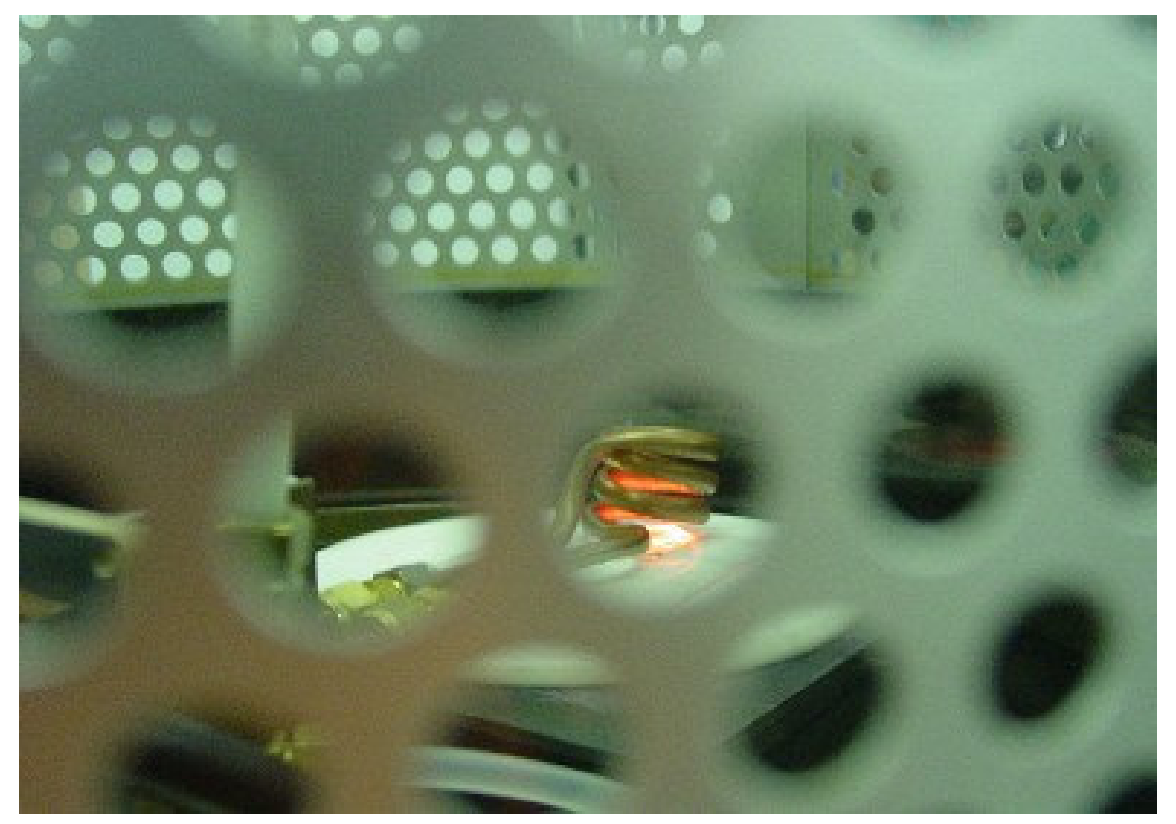

Figure 4.22: Photograph showing a sample being heated using an early configuration of BEAVIS. This sample measured $4 \mathrm{~cm}^{2}$ in area and $2.5 \mu \mathrm{m}$ in thickness. It was being heated at approximately $1.5 \mathrm{~kW}$ to around $1000^{\circ} \mathrm{C}$. Glowing was typically first observed at around $600^{\circ} \mathrm{C}$ and grew in intensity with increasing temperature. 


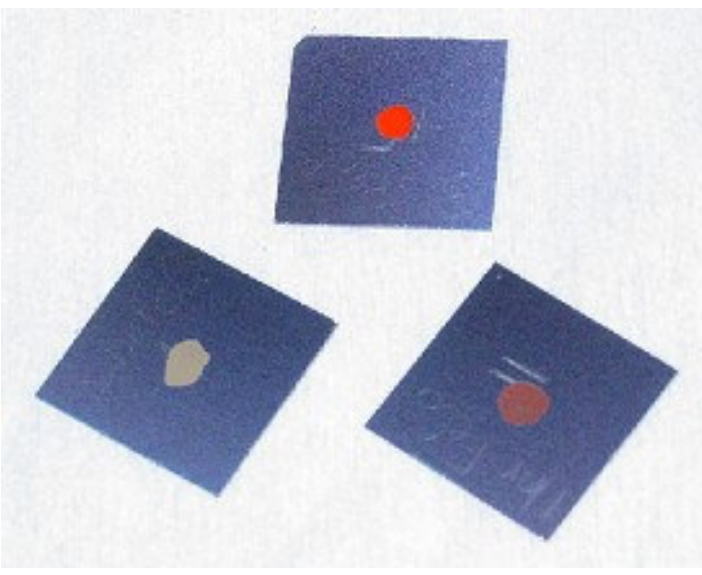

(a)

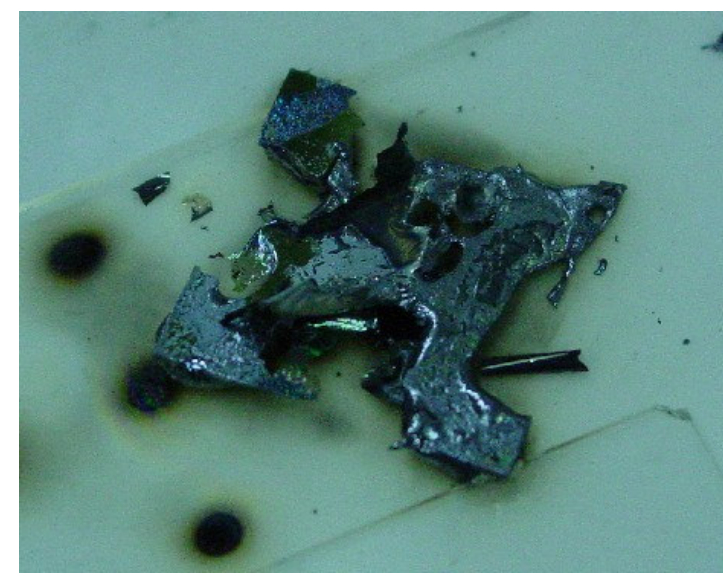

(b)

Figure 4.23: Heated samples showing (a) variations in paint colors as a result of different induced temperatures, and (b) the result of allowing a sample to heat too high. The shape of the melted sample reflected the nature of eddy current heating; the regions of highest temperature occurred at the edges of the sample, where the current density was the highest, while the center and corners remained relatively cool.

different induced temperatures. Although the data indicated an unexpectedly high level of difficulty in miniaturizing the footprint of the technology, temperatures sufficient for polysilicon annealing were nevertheless induced in a controllable manner. SEM and interferometric images from these films are provided in Section 5.1. Miniaturization is a key factor, however, in determining the potential success of any microsystem technology. The approach to improvement was twofold: examining methods by which the normal magnetic properties could be improved, and reconsidering an alternative approach to the ferromagnetic film layout. For the former, attempts were made at magnetic annealing, a process by which a sample is heated in the presence of a magnetic field in order to condition the ferromagnetic domains in the intended direction. The latter led to the development of the ferromagnetic ring concept, a design that took advantage of the fact that the majority of the heat generated during induction heating was originating at the edges of the film and thus the center portion was unnecessary. 
A standard procedure in the processing of ferromagnetic films, magnetic annealing is a means by which magnetic properties can be optimized for the desired application. The literature provides numerous examples of magnetic annealing for the conditioning of domains in both the in-plane [Mizutani, et al., 2000; Garcia, et al., 2002; Vas'ko, et al., 2002; Chen, et al., 2005] and normal [Garcia, et al., 2002; Zhang, et al., 2004] orientations. In Mizutani, et al., 2000, $0.8 \mu \mathrm{m} \quad \mathrm{Co}_{56} \mathrm{Ni}_{14} \mathrm{Fe}_{30}$ alloys were electroplated under an 800e magnetic field applied parallel to the substrate, establishing an in-plane easy axis, and annealed in a vacuum ambient at temperatures ranging from $150-400^{\circ} \mathrm{C}$ with a $100 \mathrm{Oe}$ in-plane field applied either parallel or perpendicular to the easy axis. Annealing parallel to the easy axis tended to improve the magnetic properties in that direction. The effect of annealing perpendicular to the easy axis was dependent upon both the temperature and the duration of the procedure, but it was shown that the easy axis direction could be changed from the as-deposited orientation. This occurred more quickly at higher temperatures, but even at the lowest temperatures the axis change was observed when sufficient time duration was allowed (around 2 hours). Nevertheless, the anisotropy constant, or relative "easiness", associated with the new easy axis was always found to be inferior to that measured along the original easy axis prior to annealing. The authors hypothesized that this was due to a remnant component of the initial anisotropy [Mizutani, et al., 2000]. Examples of perpendicular magnetic annealing are provided in Garcia, et al., and Zhang, et al. In the former, $(\mathrm{Pt}-\mathrm{Co})_{\mathrm{n}}-\mathrm{FeMn}$ multilayers were deposited to a thickness on the order of tens of microns via magnetron sputtering, which was found to impart a stray normal field of up to $40 \mathrm{Oe}$, and annealed at $180^{\circ} \mathrm{C}$ in a vacuum ambient with a 2000 Oe field applied either in-plane or normal to the film. It was found that the Pt- 
Co interface tended to favor a perpendicular easy axis, whereas the Co-FeMn interface favored an in-plane easy axis, and thus the final results were highly dependent upon the combined effects of annealing properties, the individual film thicknesses, and the total number of multilayers [Garcia, et al., 2002]. In Zhang, et al., film stacks consisting of $\mathrm{Fe}_{60} \mathrm{Pt}_{40}-\mathrm{MgO}$ layers were prepared via $\mathrm{RF}$ sputtering with each thickness on the order of a few nanometers. The purpose of the $\mathrm{MgO}$ was to limit the interaction between the $\mathrm{Fe}_{60} \mathrm{Pt}_{40}$ grains in order to reduce switching noise as well as to condition the grain texture. The samples were annealed in a vacuum ambient at $500-600^{\circ} \mathrm{C}$ under a perpendicularly applied field. While the perpendicular magnetic properties tended to improve with annealing temperature, once again the individual film thicknesses played a significant role in determining the quality of the final results. The authors believed that this was primarily an effect of the build-up of film stress [Zhang, et al., 2004]. Thus increasing the film thickness can introduce a trade-off; more magnetic dipoles are available to interact with the applied magnetic field, but lattice strain may reduce their individual abilities to respond. For the inductive annealing process, an attempt was made to incorporate magnetic annealing in order to compensate for the relatively low quality of the asdeposited films. Annealing was done both in vacuum and in a nitrogen ambient at temperatures up to $250^{\circ} \mathrm{C}$ and $300^{\circ} \mathrm{C}$, respectively, based upon the limitations of the two different furnaces. Some of these results are presented in Table 4.6 with the data from the heating of "square rings" of different sizes and materials. Consistent with the VSM results from Section 4.3, the data showed that the magnetic anneals failed to improve the transverse film properties and in fact usually reduced them. This is one of the suggested areas of further research to investigate during the next phase of technology optimization. 
In spite of efforts made to engineer the ferromagnetic films to realize the full potential of the inductive annealing technology, characterization data showed that this would not likely be possible with the existing fabrication capabilities. In order to reduce the process footprint below the $1 \mathrm{~cm}^{2}$ goal, a new design concept was envisioned that resulted in a change from solid films to hollow rings. As was discussed in Chapter 2, the result of the skin effect was that the vast majority of heat generation took place at the outer edges of the inductively heated films. Therefore, it was hypothesized that the ferromagnetic films could be hollowed with minimal impact to the induced temperatures; this was confirmed experimentally as will be shown. The ferromagnetic ring concept allowed for the use of large "effective areas" with much less material required. Its

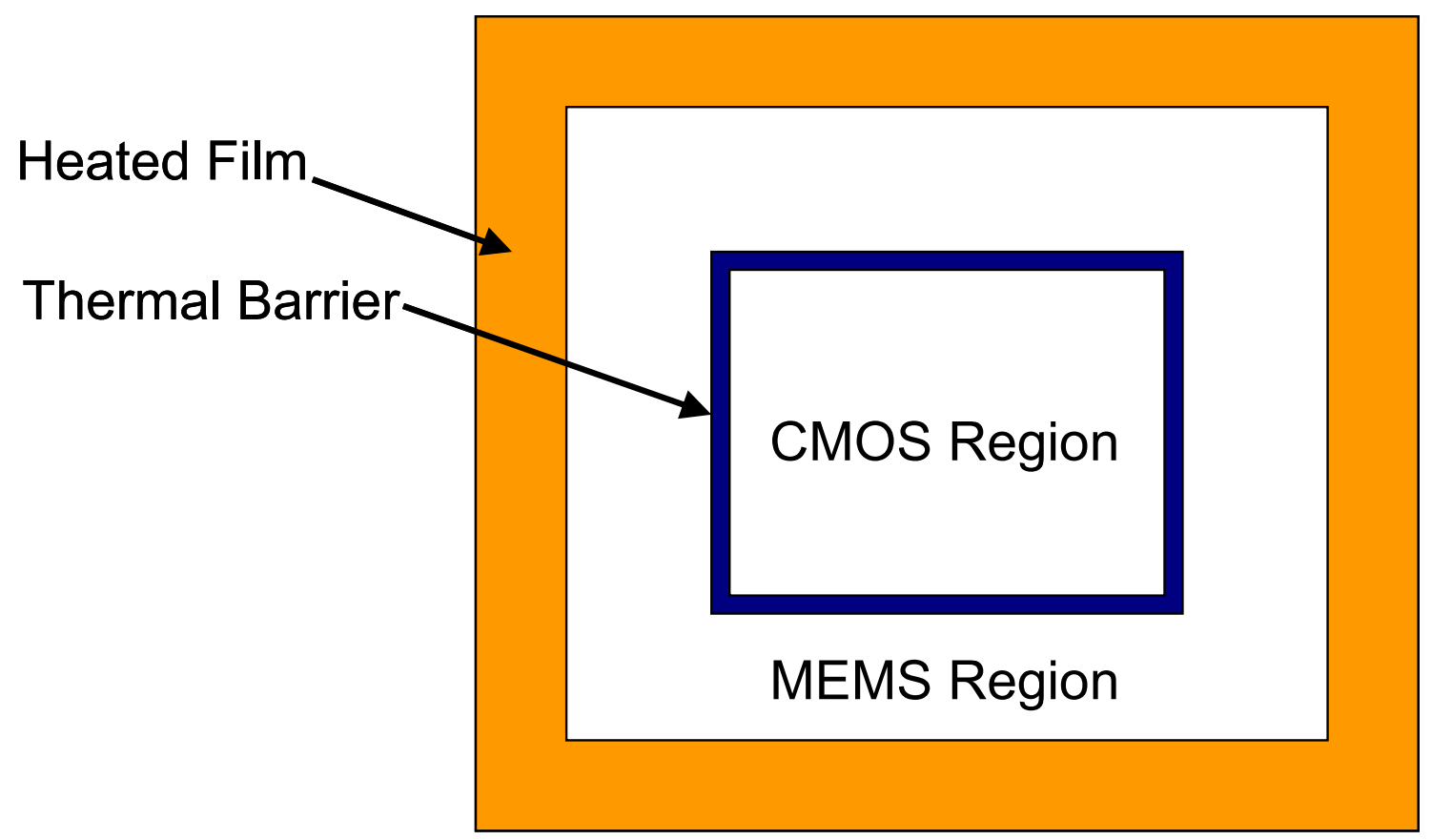

Figure 4.24: Illustration of the ferromagnetic ring concept for reducing the footprint of the inductive annealing process. The ferromagnetic film is patterned in the shape of a hollow ring, either round or square, such that multiple devices are enclosed. With the help of a thermal isolation region, the induced thermal energy is confined to the outer devices while the inner devices remain relatively cool. These devices represent chips containing CMOS circuitry. 
intended usage is illustrated in Figure 4.24, which shows the combined use of heated rings with a thermal isolation region between the CMOS and MEMS areas. The remainder of the experimentation presented in this section made use of both round- and square-shaped ferromagnetic rings.

The first sets of ferromagnetic rings to be heated were deposited to various thicknesses in the $1-7 \mu \mathrm{m}$ range with areas patterned as shown in Figure 4.25(a). For analysis, these four squares were designated S1, S2, S3, and S4 with the labels beginning at the upper right-hand corner and continuing in a counter-clockwise fashion. After initial results indicated that induced temperatures still remained lower than desired, a design

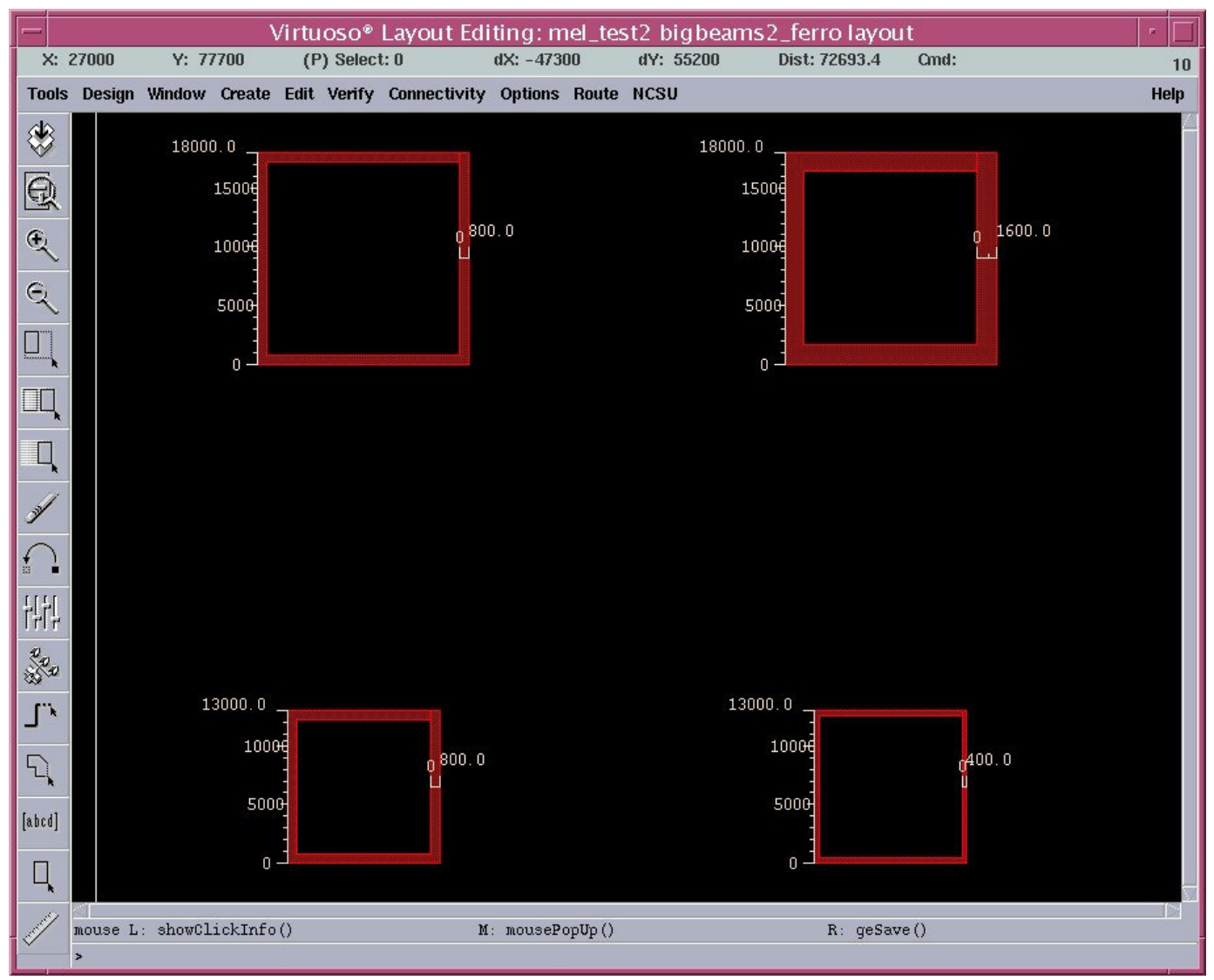

Figure 4.25(a): Initial square layout illustrating the dimensions utilized. Two different outer diameters were used, $1.3 \mathrm{~cm}$ and $1.8 \mathrm{~cm}$, with different sidewall widths in order to vary the resistance to eddy current flow. 


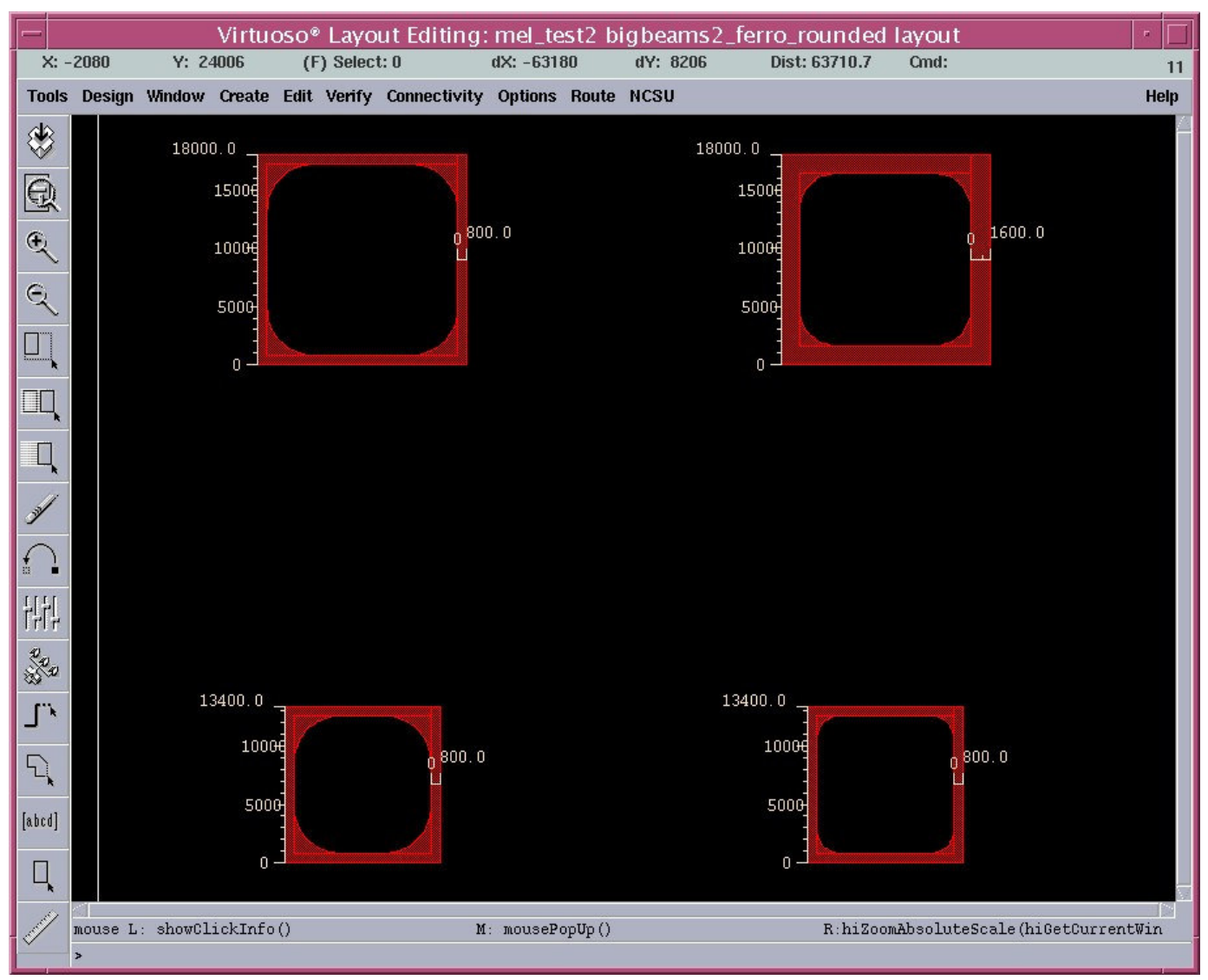

Figure 4.25(b): Modified square ring layout with interior corners rounded to better accommodate eddy current flow. Note that some dimensions have been increased slightly from those of the initial design in order to attempt to obtain higher temperatures.

change was made to the pattern in which the interior corners of the squares were rounded in order to attempt to improve eddy current flow; this modified pattern is shown in Figure 4.25(b). It should be noted that some of the dimensions were increased as well, specifically the diameter of the two smaller squares (S3 and S4) and the sidewall width of S4. The induced temperatures, measured using pyrometry, are presented in Table 4.6. The data shows results for the three alloys of interest (FeCo, NiFe19, and NiFe55), though the investigation continued to focus primarily on FeCo with considerable effort made to enhance its heating capabilities through magnetic annealing. The films were deposited onto full 4" silicon wafers boron-doped to $10-20 \Omega$-cm with $1 \mu \mathrm{m}$ of thermally- 
grown silicon dioxide. These high-resistivity substrates were selected in order to minimize the silicon interaction with the magnetic field. The "yes" and "no" information provided under the "Magnetic Dep., Anneal" column indicates whether a magnetic field was utilized during film deposition and whether a magnetic anneal was performed after

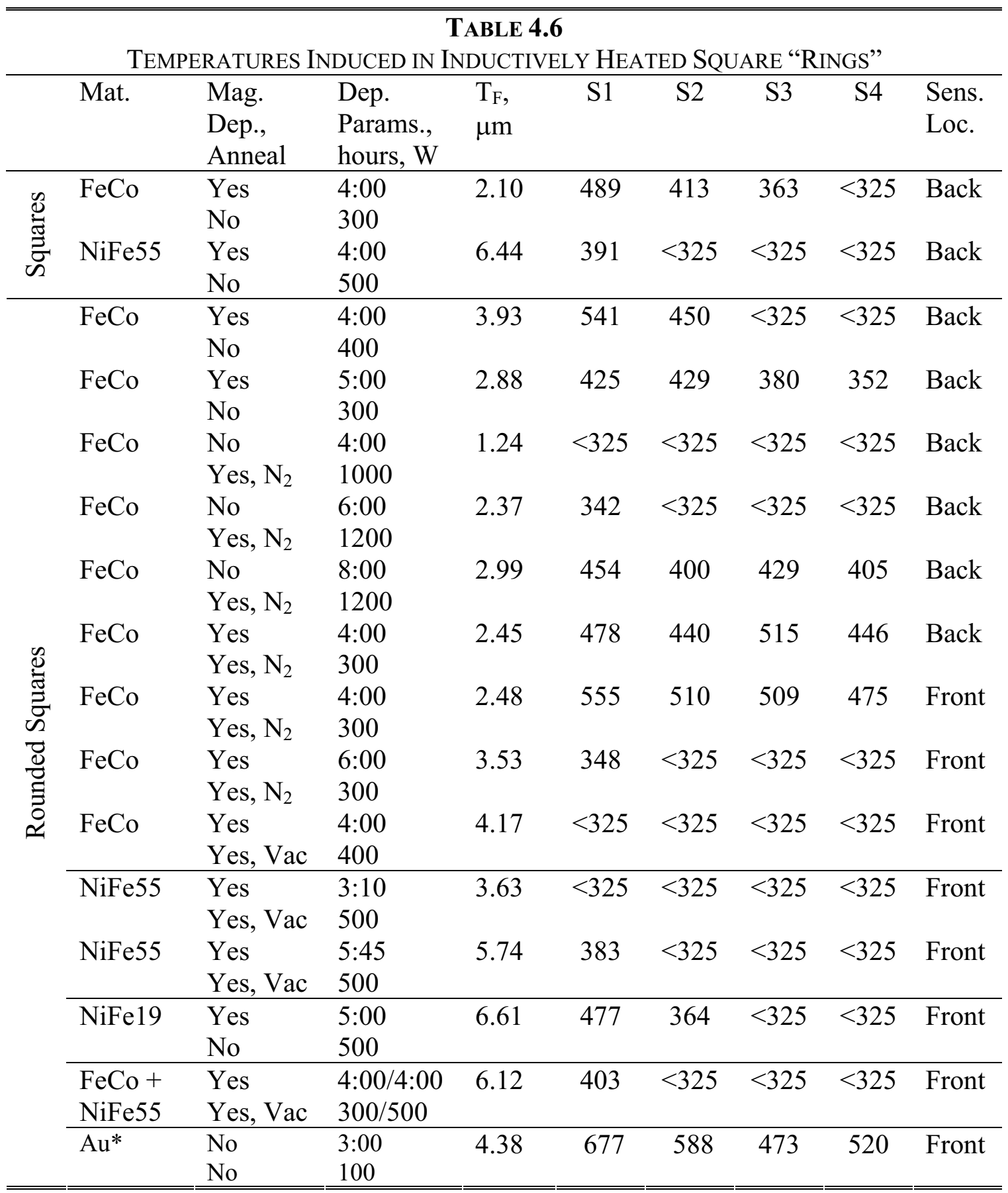




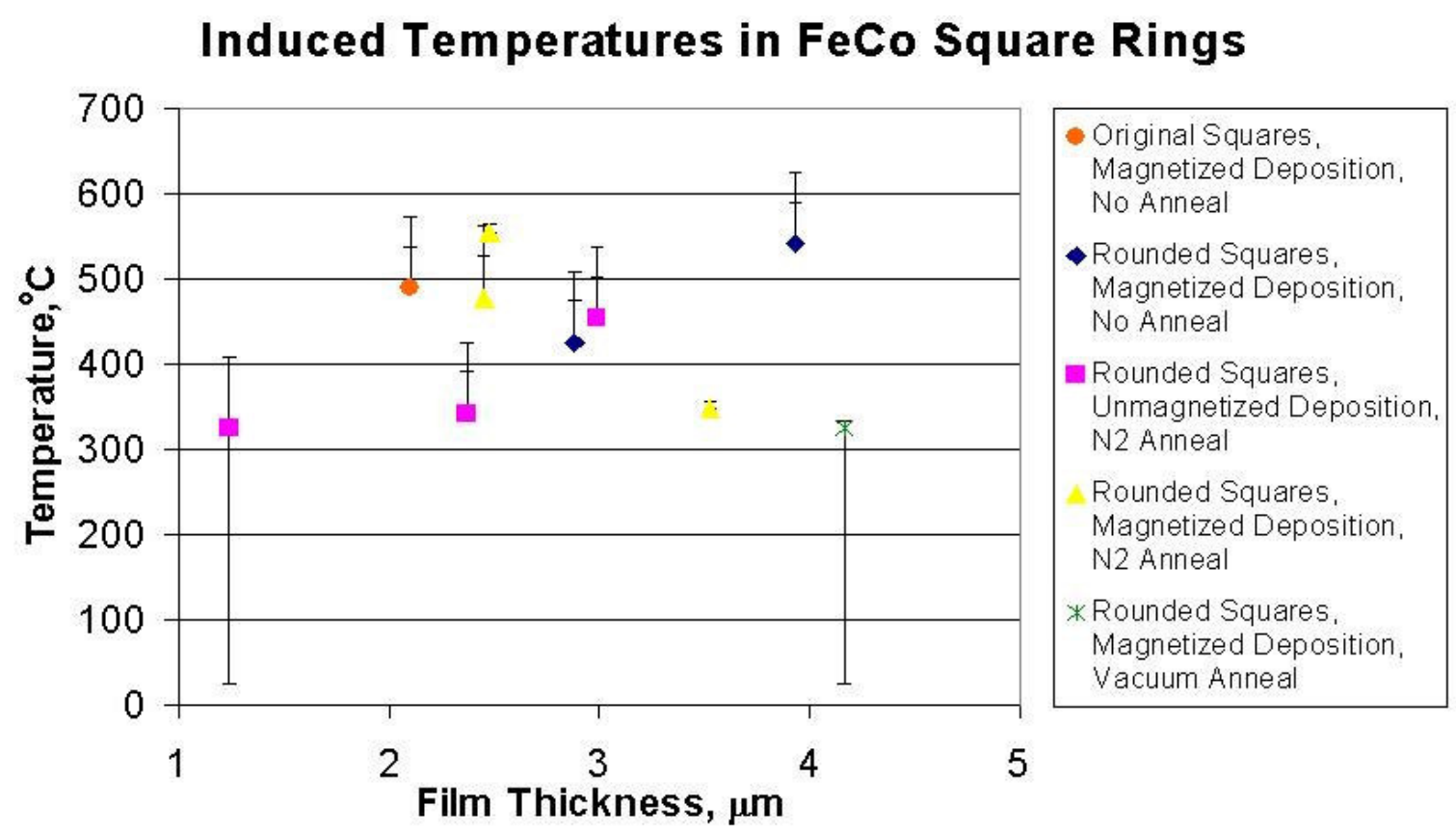

Figure 4.26: Plot of S1 FeCo data from Table 4.6 with error bars to compensate for the front-back temperature difference as well as to account for uncertainty in the pyrometry measurements $\left(+9^{\circ} \mathrm{C} /-1^{\circ} \mathrm{C}\right)$.

patterning, respectively. If applicable, the type of anneal is also indicated (nitrogen or vacuum ambient). Lastly, the location of the pyrometry sensors is indicated (front or back) with respect to the wafer surface; measurements were initially taken from the back sides of the wafers so as to ensure consistency that would not be affected by surface features, including the ferromagnetic films themselves. It was later found that differences in surface features did not significantly alter the pyrometry readings and thus the sensors were moved to face the front sides of the wafers so that the actual induced temperatures would be known. The front-back temperature difference was observed to vary within the $50-75^{\circ} \mathrm{C}$ range for the simple film stack. This difference is compensated for via error bars in Figure 4.26, in which the FeCo temperature results at the largest square, S1, are plotted with respect to film thickness. The bars also account for the uncertainty in pyrometry measurements $\left(+9^{\circ} \mathrm{C} /-1^{\circ} \mathrm{C}\right.$ for $\mathrm{T}>325^{\circ} \mathrm{C}$, or $25^{\circ} \mathrm{C} \leq \mathrm{T} \leq 325^{\circ} \mathrm{C}$ for $\left.\mathrm{T} \leq 325^{\circ} \mathrm{C}\right)$ as was 
indicated in Section 4.2. Overall, the data reflected the complexity of the relationship between inductive heating capability and film thickness. For the samples with thinner ferromagnetic films $(t<3 \mu \mathrm{m})$, the induced temperatures tended to vary approximately linearly with thickness, in good agreement with Equation (2.6a). The best results occurred under both a magnetized deposition and a post-pattern magnetic anneal (nitrogen ambient), and it was hypothesized that a higher annealing temperature might yield further improvement. The thicker samples, however, followed a different trend; unannealed samples continued to follow the same linear slope, but those subjected to magnetic annealing failed to generate an appreciable temperature rise. Furthermore, nearly all samples that underwent magnetic annealing in a vacuum ambient, regardless of composition, performed rather poorly. The root cause of this behavior was not investigated in further detail but was believed to be primarily an effect of increased film stress with a possible contribution from film oxidation. The multitude of interrelationships involved in magnetic annealing was discussed previously and the design of an optimized magnetic annealing process presents a formidable challenge of its own, particularly under the thermal budget limitations imposed by a post-CMOS fabrication technology. Continued research in this area may be of high importance to the improvement of thin film permeabilities and thus the overall inductive annealing process. These S1 trends were consistent for the S2, S3, and S4 squares as well, with the temperature magnitudes tending to decrease with decreasing surface area. Comparing the behaviors of the different ferromagnetic materials, the FeCo films yielded the best performance for a given film thickness. Their weakness, however, was in their high intrinsic stresses, observed through both discoloration (cloudiness) and delamination 
problems, which placed an upper limit on the film thickness range. On the other hand, films absent of cobalt were able to be deposited to higher thicknesses. This can be understood in part by examining mineral hardness values for iron, nickel, and cobalt, which are equal to 4.0, 4.0, and 5.0, respectively, using Mohs' scale of mineral hardness. This relative scale ranges from 1.0 to 10.0 without units, where the maximum value is assigned to diamond, and is approximately logarithmic, meaning that each integral increment reflects an increase in hardness by a factor of ten [Winter, 2006]. The hardness of a given material is one of many factors that determine the resulting intrinsic stress. This relationship was examined in Davies, et al., and attributed to higher yield stress which reduced the migration of nucleates during deposition [Davies, et al., 2004]. Gold, with a mineral hardness of 2.5 , tended to produce films of very high quality having measured resistivity values very close to those of the bulk material. This was believed to be the reason that the gold benchmark samples generated the best overall results, despite being non-magnetic. The significance of this outcome was to confirm the need for improved ferromagnetic film quality. As was concluded in Section 4.3, the measured magnetic properties were far below expectation. The additional impacts of resistivity and film stress further degraded the inductive heating capabilities of the ferromagnetic alloys that were utilized. The ability to optimize these relationships will determine the success or failure of the inductive annealing technology.

With limited success from the inductively heated square rings, the remaining experimentation utilized thick $(\sim 6 \mu \mathrm{m})$ circular films of relatively large surface area $(2 \mathrm{~cm}$ outer diameter) in order to better characterize the process at higher temperatures and later achieve polysilicon annealing on more complex structures. The two wafers in Figure 4.27 
illustrate the two patterns that were used; the wafer in (a) formed a solid circle with a $2.4 \mathrm{~cm}$ outer diameter while that in (b) was hollow with a $1.2 \mathrm{~cm}$ inner diameter. Based on the aforementioned results, research focus shifted to the NiFe19 alloy as its films exhibited lower intrinsic stresses and thus could be deposited to greater thicknesses than

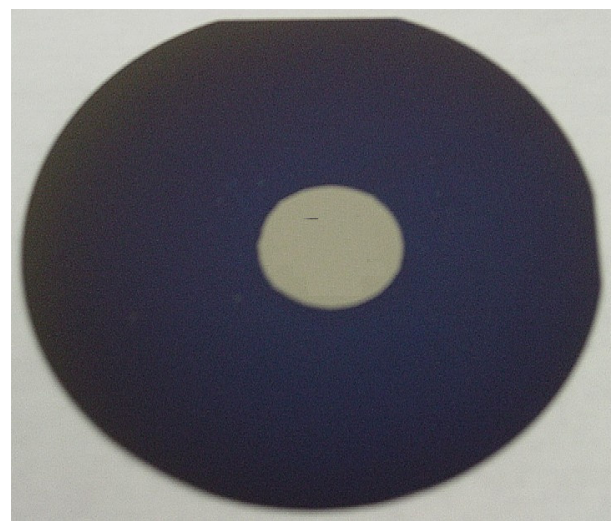

(a)

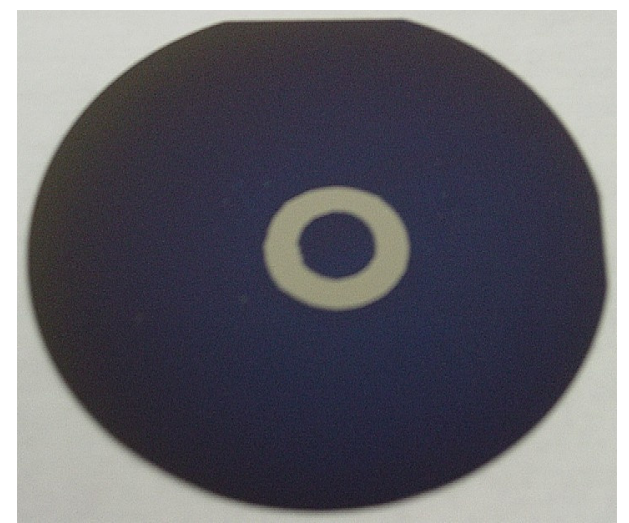

(b)

Figure 4.27: Illustration of $2.4 \mathrm{~cm}$-wide circular areas on $100 \mathrm{~mm}$ wafers, with (a) a solid interior and (b) a $1.2 \mathrm{~cm}$ inner diameter. The rings shown were patterned from NiFe19 films approximately $6 \mu \mathrm{m}$ in thickness.

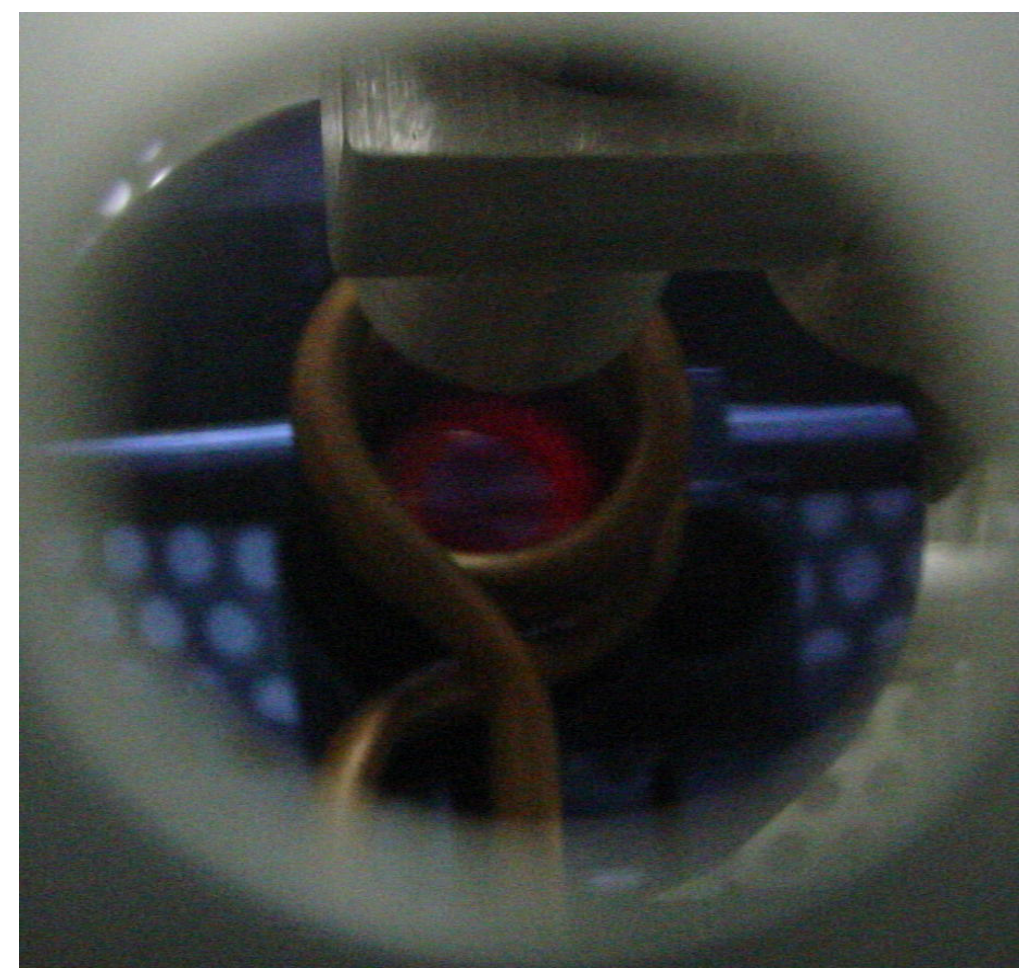

Figure 4.28: Photograph showing the glow of a $7.50 \mu \mathrm{m}$ hollow NiFe19 ring heated to approximately $600^{\circ} \mathrm{C}$, as measured by infrared pyrometry. 
FeCo as well as heat to higher temperatures without delaminating. The photograph in Figure 4.28 shows the heating of a hollow NiFe19 ring and illustrates the faint glowing that was typically observed at around $600^{\circ} \mathrm{C}$. For each sample, the coil power was gradually increased and the induced temperature was allowed to stabilize at each test point, which typically required around ten seconds. Figure 4.29(a) shows the temperature-vs-power data for five different NiFe19 samples. The legend indicates the measured film thicknesses, whether the films were patterned in solid or hollow circles, and any special processing conditions, if applicable. The first two samples utilized solid NiFe19 circles of different thicknesses whereas the last three utilized hollow rings heated under different circumstances; Sample3 utilized the default process, Sample 4 was heated using a reduced coil frequency of $272 \mathrm{kHz}$ via substitution of the $0.66 \mu \mathrm{F}$ tank capacitor for its $1.25 \mu \mathrm{F}$ counterpart, and Sample 5 examined the effect of a ceramic heat sink pressed against the back side of the wafer. The trends indicate that the temperature-power relationships were all approximately linear within the stable operating regions, i.e., the temperatures at which thermal runaway did not occur. Thermal runaway was typically initiated at approximately $800^{\circ} \mathrm{C}$, but the thinnest film went unstable at only around $600^{\circ} \mathrm{C}$. This supported the theory that optimizing the heating efficiency of the ferromagnetic film (e.g., with increased thickness) would help to prevent the runaway condition from occurring. To quantitatively analyze the trends in the stable regions, the data series were truncated to remove the points of instablility and linear equations were assigned as shown in Figure 4.29(b). For each line, the computed equation and correlation coefficient, $R^{2}$, are provided. The $R^{2}$ values were all 0.97 or higher, reflecting a high degree of linearity which was consistent with the temperature-vs-power 


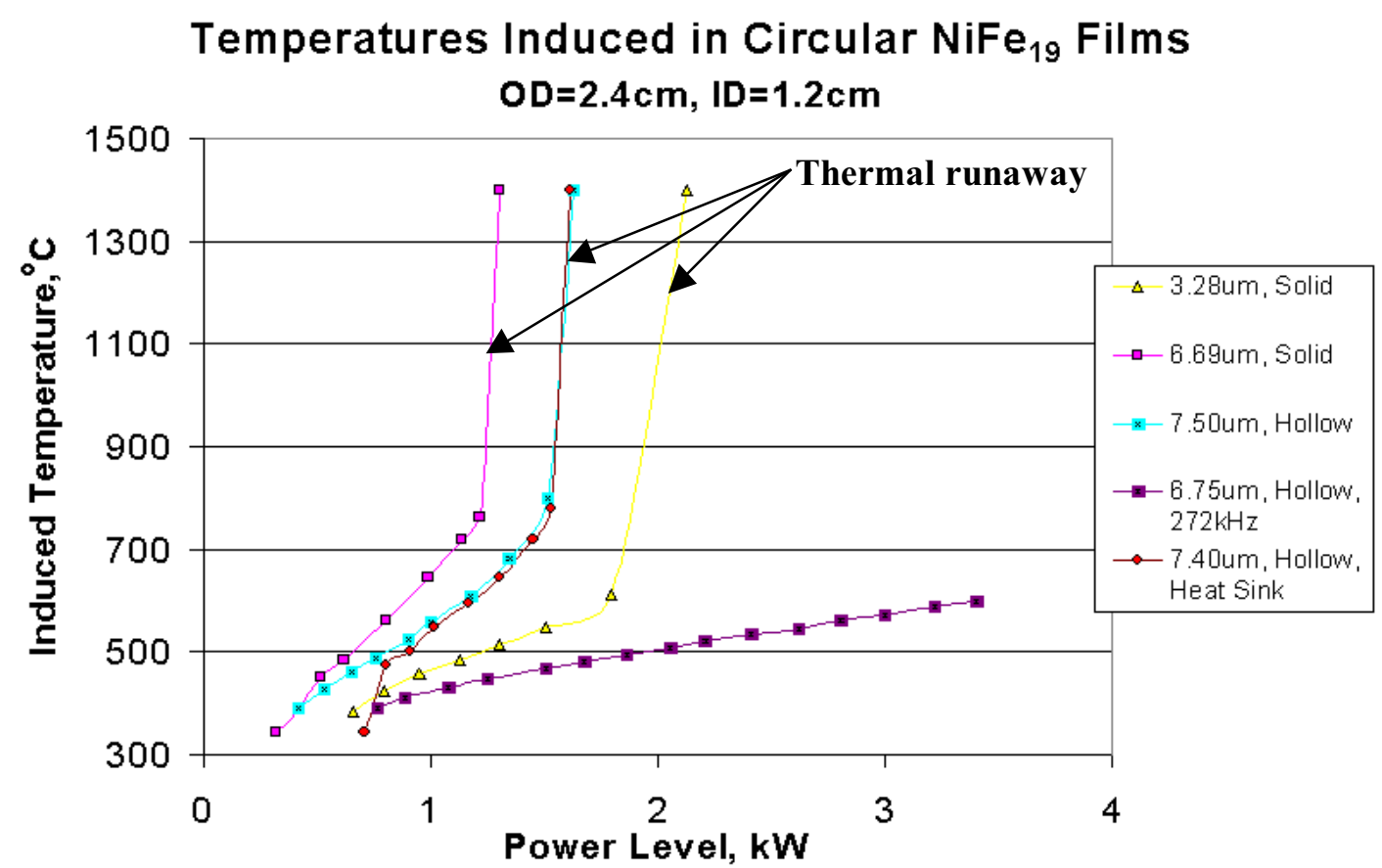

Figure 4.29(a): Plot of temperature vs. coil power for both solid and hollow (as indicated) $2.4 \mathrm{~cm} \mathrm{NiFe} 19$ films at various thicknesses and heating conditions. Stability was retained to approximately $800^{\circ} \mathrm{C}$, an improvement over that from the proof-ofconcept testing, with nearly linear behavior in the stable region.

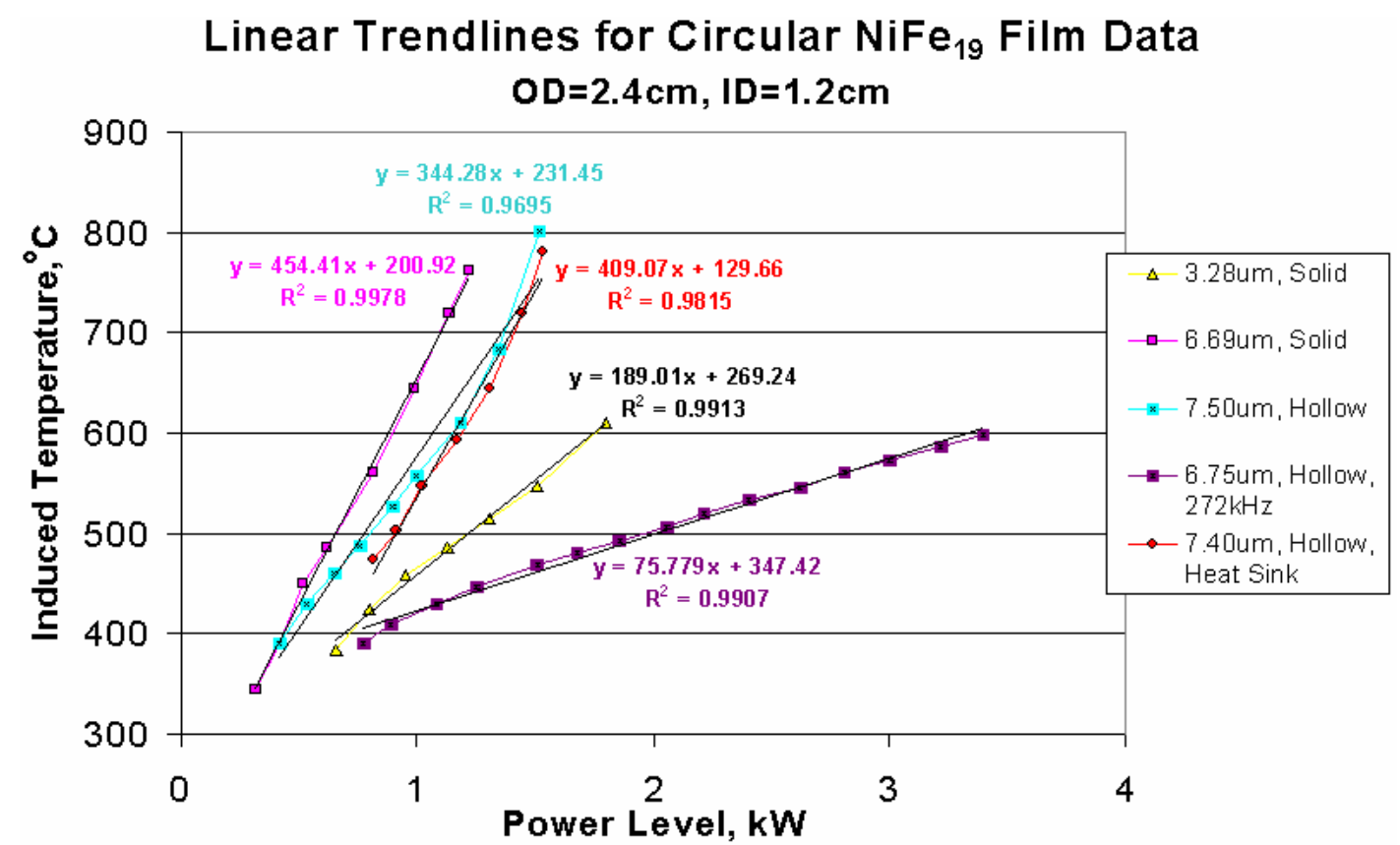

Figure 4.29(b): Reduced data set from Figure 4.28(a) showing only values from the stable temperature regions. Linear trendlines for each curve were obtained via Microsoft Excel, with the slope providing an indication of the energy conversion efficiency from magnetic to thermal. 


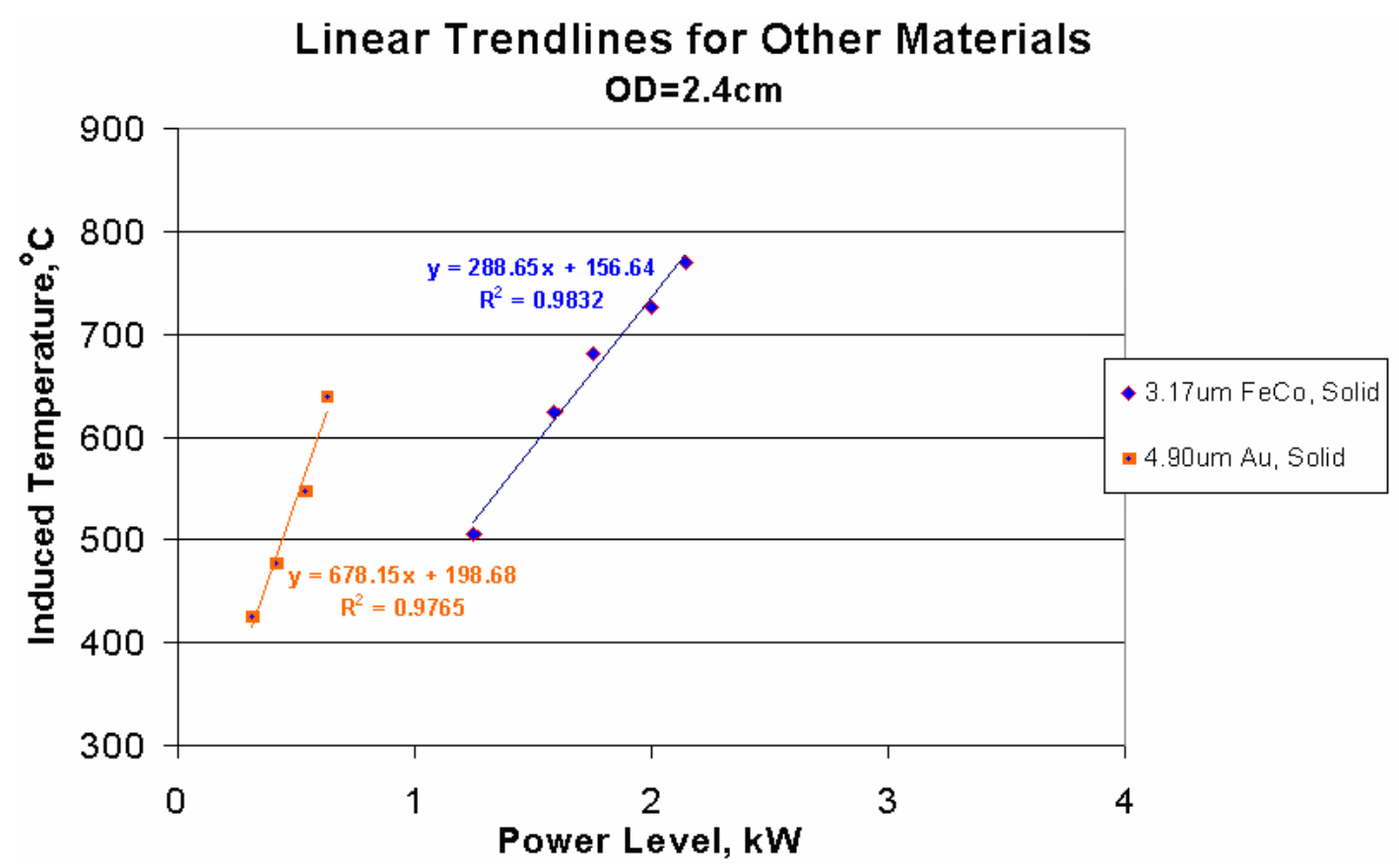

Figure 4.29(c): Linear trendline data for solid $\mathrm{FeCo}$ and $\mathrm{Au}$ circles of various thicknesses. The data series for $\mathrm{FeCo}$ and $\mathrm{Au}$ have been truncated to show only their stable regions.

relationship derived in Equation (2.15c) and subsequently confirmed in the simulations of Chapter 3. To compare the theoretical and experimental slope values, the proportionality constant $\mathrm{K}_{\mathrm{PS}}$ was first required. This value reflected the relationship between the supply power and resulting magnetic field. Measurements of the coil voltage via oscilloscope indicated that at full power $(2 \mathrm{~kW})$ the coil current was approximately $190 \mathrm{~A}$ in amplitude. Equation (2.11) indicates that a current of this strength with the three-turn, 1.25in-long solenoidal coil utilized should produce a magnetic field of $18.0 \mathrm{kA} / \mathrm{m}$. Therefore, the constant $K_{P S}$ was equal to $1.61 \mathrm{E} 5 \Omega^{-1} \mathrm{~m}^{-2}$. Theoretical power and slope values were then calculated using the electromagnetic properties from Table 4.4, the density and specific heat values from Table 4.7 (note that alloy values were obtained via interpolation), and a heating time of $10 \mathrm{~s}$ (the approximate time required for temperatures to reach their new values following a power increase). The theoretical and experimental results are 


\begin{tabular}{lll}
\hline \hline & \multicolumn{2}{c}{ TABle 4.7 } \\
\multicolumn{2}{c}{ Density AND SPECIFIC HEAT FOR SElECTED MATERIALS } \\
\hline Material & Density $^{1}\left(\mathrm{~kg} / \mathrm{m}^{3}\right)$ & Specific Heat $^{2}\left(\mathrm{~J} / \mathrm{kg}^{*} \mathrm{~K}\right)$ \\
\hline $\mathrm{Ni}$ & 8908 & 440 \\
$\mathrm{Fe}$ & 7874 & 440 \\
$\mathrm{Co}$ & 8900 & 420 \\
$\mathrm{NiFe} 19$ & 8712 & 440 \\
$\mathrm{FeCo}$ & 8387 & 430 \\
$\mathrm{Au}$ & 19300 & 128 \\
\hline \hline
\end{tabular}

summarized in Table 4.8, together with the data from the comparison films in Figure 4.29(c). For the most part, the experimental slope values were greater than their theoretical predictions, but all were within an order of magnitude except for the Au film. Offsets were attributed to differences in thin film properties from the bulk material values that were utilized, particularly the density. Comparing the two solid NiFe19 films $(3.28 \mu \mathrm{m}$ and $6.69 \mu \mathrm{m})$, theory predicted both samples would generate the same power density within the skin depth, $\delta$, and that therefore both would have the same temperature-generation characteristics due to the increased amount of film material to heat, thus negating the thickness advantage. The experimental slope values, however,

\begin{tabular}{|c|c|c|c|}
\hline \multicolumn{4}{|c|}{ TABLE 4.8} \\
\hline Sample Description & $\begin{array}{l}\text { Theoretical } \\
\text { Power Density } \\
\text { within } \delta, \mathrm{W} / \mathrm{m}^{3}\end{array}$ & $\begin{array}{l}\text { Theoretical } \\
\text { Slope, }{ }^{\circ} \mathrm{C} / \mathrm{kW}\end{array}$ & $\begin{array}{l}\text { Experimental } \\
\text { Slope, }{ }^{\circ} \mathrm{C} / \mathrm{kW}\end{array}$ \\
\hline $3.28 \mu \mathrm{m}$ NiFe 19, Solid & $3.78 \mathrm{E} 9$ & 69.58 & 189.01 \\
\hline $6.69 \mu \mathrm{m}$ NiFe19, Solid & $3.78 \mathrm{E} 9$ & 69.58 & 454.41 \\
\hline 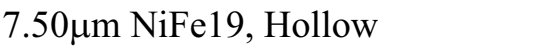 & $3.78 \mathrm{E} 9$ & 69.58 & 344.28 \\
\hline $6.75 \mu \mathrm{m} \mathrm{NiFe} 19$, Hollow, $272 \mathrm{kHz}$ & 2.74E9 & 59.26 & 75.779 \\
\hline $7.40 \mu \mathrm{m}$ NiFe 19 , Hollow, Heat Sink & $3.78 \mathrm{E} 9$ & $834.9^{3}$ & 409.07 \\
\hline $3.17 \mu \mathrm{m}$ FeCo. Solid & 2.21E9 & 75.70 & 288.65 \\
\hline $4.90 \mu \mathrm{m} \mathrm{Au}$, Solid & $2.39 \mathrm{E} 8$ & 15.53 & 678.15 \\
\hline
\end{tabular}

${ }^{1}$ CRC, 1989 (pp. B-68 - B-146)

${ }^{2}$ CRC, 1989 (pp. D-180 - D-181)

${ }^{3} 2 \mathrm{~min}$ stabilization time utilized for calculations, rather than the typical $10 \mathrm{~s}$ 
suggested that the thinner sample was affected considerably more by surface loss mechanisms, an expected result as its volume/surface-area ratio was halved. Furthermore, the difference between the solid and hollow films was found to be more significant than expected, with the hollow film encountering a $24.3 \%$ drop in temperature generation efficiency. The root cause for this difference was unclear, but may have been related to the additional film stress created during patterning which often resulted in film delamination. The reduced-frequency slope yielded a very close match with theory. This dramatic change most likely indicated a secondary effect within the power supply that affected the coil current values for a given power output and thus producing a different $K_{P S}$ value. This theory was supported in that the lower frequency enabled the power supply to reach a $3.5 \mathrm{~kW}$ output rather than the normal $2 \mathrm{~kW}$ maximum. Finally, the impact of heat sinking the substrate was to significantly reduce the rate of temperature rise. Given sufficient time for stabilization (typically $\sim 2$ minutes between data points rather than the typical $\sim 10$ seconds), however, the final temperatures followed nearly the same trend as its default-process counterpart (Sample 3). Thus the heat sink acted as a buffer and otherwise did not have a significant effect on the temperature distribution. Its increased heating time greatly impacted the theoretical slope, but the result was only separated from the experiment value by approximately a factor of two.

Table 4.8 also presented results from the heating of alternate film materials. The FeCo slope was predicted to exceed that of the NiFe19 film of similar thickness by $6.12^{\circ} \mathrm{C} / \mathrm{kW}$, or $8.80 \%$, while in actuality it surpassed NiFe19 by $99.6^{\circ} \mathrm{C} / \mathrm{kW}$, or $52.7 \%$. The benefit of increasing heating efficiency was often greater than that predicted by theory, as secondary effects like absorption of magnetic energy by the substrate were not 
taken into account in the equations. As was mentioned previously, though, stress-induced thickness limitations for the FeCo films rendered this slope an approximate upper limit. The most significant discrepancy between theory and experiment was encountered in the results from the Au sample, whose measured slope far exceeded the theoretical value by a factor of 43.7. For reasons already discussed, the high film quality of the sputtered $\mathrm{Au}$ films inevitably enabled them to surpass all of the present ferromagnetics in initial heat generation capacity. The relatively early loss of stability, however, suggested that it was easier to be overpowered by the silicon substrate. Nevertheless, the importance of emphasizing future research into magnetic film properties was clear.

While the degree of heating localization has not received a high degree of attention in this chapter, it was in fact one of the most important considerations in the technology as it provided an indication of whether the final goal of annealing polysilicon microstructures in the vicinity of CMOS circuitry could be realistically achieved. The data plotted in Figure 4.30 shows the difference in temperature measurements between the sensor pointed directly the heated circular film (Sensor1), which was centered within the wafer, and a second sensor (Sensor2) which was spaced $3.2 \mathrm{~cm}$ away center-center (the closest span available due to the radius of the sensors and their supports). As was discussed in Section 4.2, the focal lengths of the sensors were mechanically adjusted to measure the light intensity within a $1 \mathrm{~mm}$ spot size. The traces all show that the temperature difference increased as the overall temperature increased, indicating that the thermal energy generation was surpassing the capabilities of thermal conduction to a progressively greater extent. Of interest was the fact that two distinct trends were followed. The two samples that generated the upper trend both utilized solid films that 


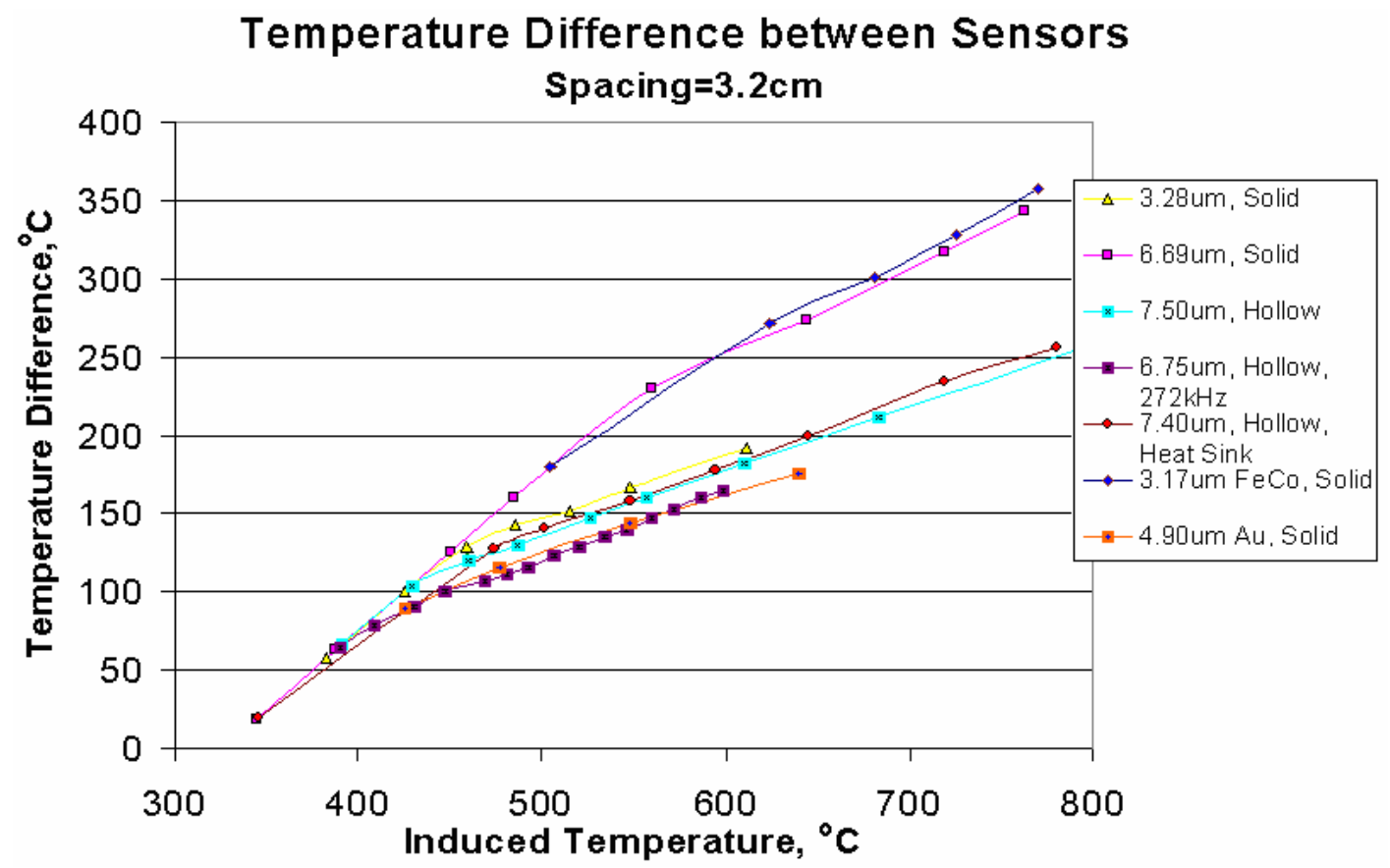

Figure 4.30: Pyrometry sensor data showing temperature drop from primary sensor at heated film region, at wafer center, to secondary sensor shifted $3.2 \mathrm{~cm}$ laterally (centercenter spacing), plotted with respect to the primary (induced) temperature. Temperature differences increased with increasing temperature, indicating that power generation was occurring more rapidly than thermal conduction.

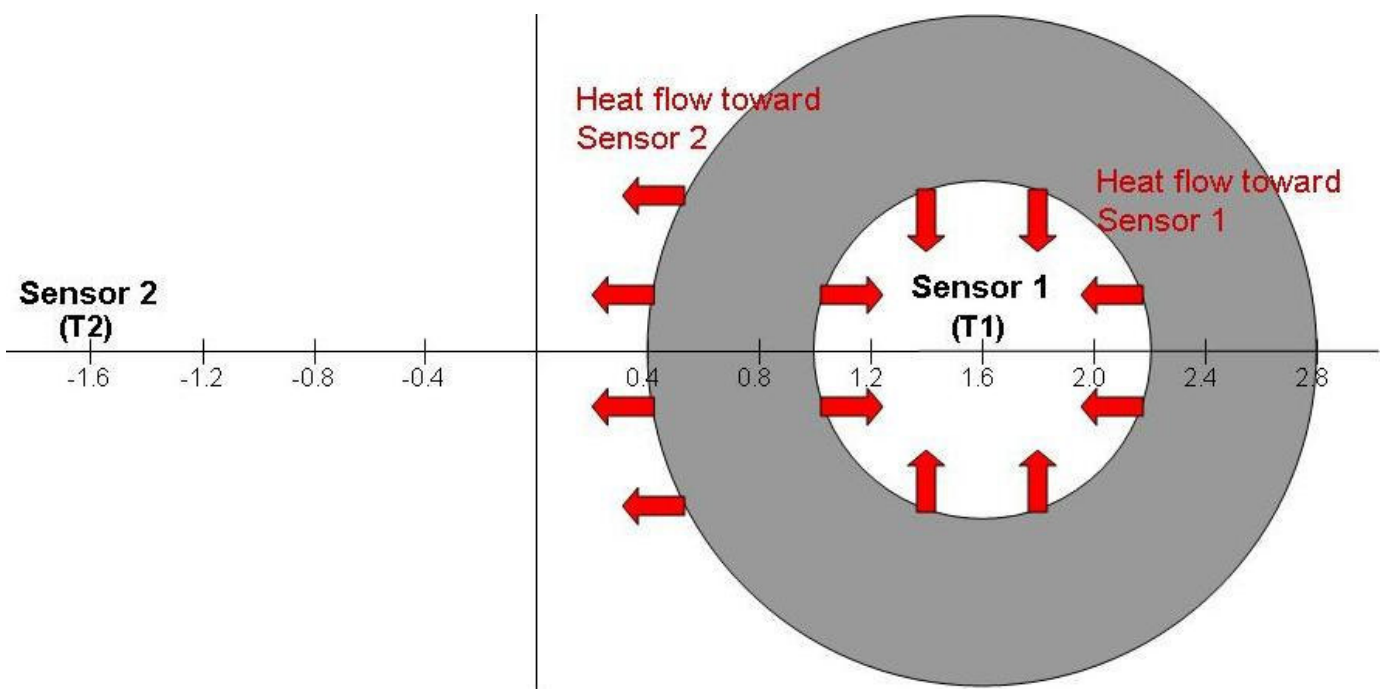

Figure 4.31: Representation of the relative placement of the two pyrometry sensors with respect to the inductively heated ferromagnetic films. Assuming a uniform temperature within the metal-covered region, the entire temperature differential occurred over a distance of $2 \mathrm{~cm}$. 
produced relatively efficient power generation as compared to the others, thus maximizing joule heating segregation against the substrate. Assuming that the temperature within the ferromagnetic film was relatively uniform due to internal thermal conduction, the entire $300-350^{\circ} \mathrm{C}$ temperature drop occurred across a $2 \mathrm{~cm}$ distance as illustrated in Figure 4.31. This corresponded to a gradient of $150-175^{\circ} \mathrm{C} / \mathrm{cm}$ (or K/cm). Estimation of the gradient inside the ring was more challenging. It was recognized that the configuration corresponded somewhat to that of the thin film solid model of Section 3.3 in which the outer edge of the substrate was regulated to $300 \mathrm{~K}$ (via the wafer chuck), though the wafer radius in the simulation was artificially thinned to $1.8 \mathrm{~cm}$. The simulated gradient internal to the hollow film was essentially zero, however, which contradicted the visual observation of a fainter (or non-existent) glow at the center as can be seen in the photograph of Figure 4.28. The three-dimensional system was examined in terms of cylindrical surfaces of varying radii. The heat flow outward from the film to the second sensor experienced a reduction in intensity due to the spread from a $1.2 \mathrm{~cm}$ radius to a $3.2 \mathrm{~cm}$ radius, or

$$
I_{\text {Sensor } 2}=I_{\text {Film }} \frac{2 \pi \rho_{\text {Film }}}{2 \pi \rho_{\text {Sensor } 2}}=0.375 I_{\text {Film }}
$$

The heat flow inward, however, was all directed toward the center. Therefore, the FilmSensor2 temperature gradient was assumed to be $1 / 0.375=2.67$ times larger than the FilmSensor1 gradient. Neglecting radiation and heat sinking effects, the internal gradient was estimated to be $56.3-65.6^{\circ} \mathrm{C} / \mathrm{cm}$ and the temperature at Sensorl was therefore approximately $33.8-39.4^{\circ} \mathrm{C}$ below that of a hollow film. The gradient at the center of a solid film was likely somewhat smaller due to heat conduction within the metal. This data, together with the results of the thin film simulations, pointed out a fundamental 
problem with the use of the wafer chuck without a heat sink. The pinning of the temperature at the wafer edge established a lateral gradient that would most certainly impact device-to-device uniformity in a real application. Modeling suggested that the solution was to regulate the back side of the wafer, thus impacting all devices equally, and find a way to increase the strength of the magnetic field.

\section{Conclusion}

This chapter presented the evolution to date of the inductive annealing process. Connections were made between theory and experimentation, with the calculated trends in temperature rise with respect to supply power turning out within an order of magnitude of experiment. Being the first generation of the technology, its development was far from complete. Nevertheless, many of the challenges revealed were overcome through the ongoing engineering of the test system, magnetic film properties, and the layout of the heat-treated system. Improvements in heating efficiency helped to clarify and alleviate the thermal runaway issue, which was especially important for wafer-scale processing. Further progress will require considerable improvement in film properties, both electromagnetic and structural (stress and delamination). Film deposition techniques and magnetic annealing are key focus areas. The next chapter shows the application of $\mathrm{NiFe}_{19}$

films to the heating of actual polysilicon films, both blanket and patterned. Variations in film morphology demonstrated the generation of controllable temperature gradients. 


\section{Chapter 5: Annealing of Polysilicon Structures}

The ultimate goal of this research project was to utilize induction heating to demonstrate the annealing of polysilicon microstructures. The work presented in the previous chapter provided the means by which to do so; the work presented in the present chapter demonstrates the capability to reach the desired result. Beginning with blanket polysilicon films in Section 5.1, both a visible change in morphology and a measured reduction in curvature are shown. Section 5.2 illustrates annealing applied to cantilever beam structures using inductively heated NiFe19 hollow rings. Finally, Section 5.3 presents the most successful results obtained to date in which the inductive annealing process was applied to lateral accelerometer structures with gradient enhancement provided by thermal isolation trenches. In both of the latter two sections, pre- and postanneal structures were contrasted using both Scanning Electron Microscope (SEM) and X-Ray Diffraction (XRD) analyses, confirming the results both qualitatively and quantitatively. The annealing results came very close to reaching the goals as defined in Chapter 1 but were limited by the controllability and efficiency factors discussed previously. The suggested methods for reaching those goals are summarized in Chapter 7.

\section{1: Blanket Polysilicon Films}

The development of the inductive annealing process and its dedicated test system has concentrated on the ultimate goal of optimizing the localized annealing of polysilicon microstructures. This section presents some of the pre- and post-processing images and data from polysilicon films, beginning with unpatterend blanket layers, continuing with simple beam structures, and finally ending with more complex accelerometer structures. The results contain both SEM and IFM surface images as well as x-ray diffraction 
analyses, and they demonstrate changes in surface texture, changes in film stress, and the establishment of measurable temperature gradients. Section 4.4 showed that despite considerable improvements in process efficiency, thermal runaway concerns continued to place an upper limit of $800^{\circ} \mathrm{C}$ on the process temperature for full wafers. This was far less of a problem for diced samples, however, as less silicon was available to create a runaway condition. Thus the images and data presented in this section show the results of annealing at $1000^{\circ} \mathrm{C}$ for diced samples and at $800^{\circ} \mathrm{C}$ for full-wafer samples. Despite being slightly below the target temperature, clear differences were observable in the polysilicon morphology and diffractometry of the full-wafer samples. The results were consistent with those reported in the literature for lower-temperature anneals.

The earliest samples to be successfully annealed on BEAVIS were obtained from diced wafers and heated to around the $1000^{\circ} \mathrm{C}$ target. The SEM images in Figure 5.1 show the surface texture of $1 \mu \mathrm{m}$ LPCVD polysilicon films before and after annealing for five minutes at approximately $1000^{\circ} \mathrm{C}$, measured via thermal paint. Annealing utilized a $2.5 \mu \mathrm{m}$-thick FeCo film deposited on a $1 \mathrm{~cm}^{2}$ sample of relatively high doping

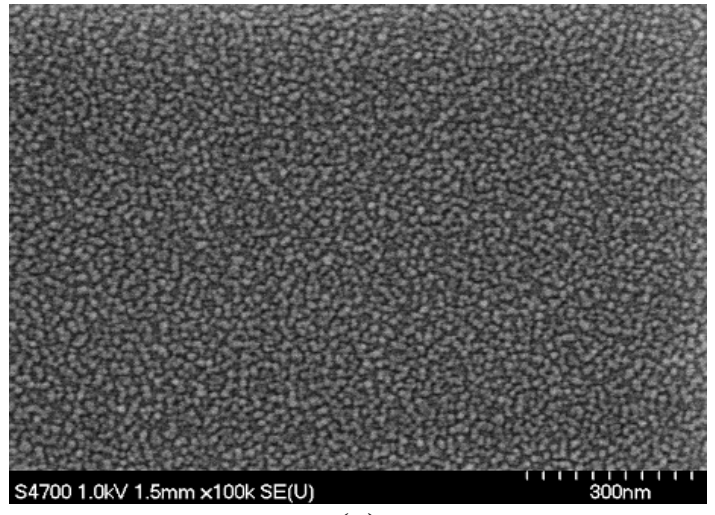

(a)

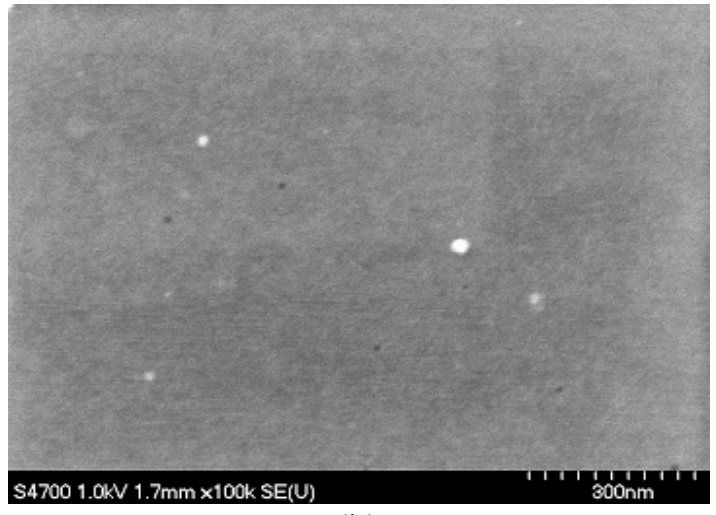

(b)

Figure 5.1: SEM images of $1 \mu \mathrm{m}$ LPCVD polysilicon surfaces (a) before, and (b) after a five-minute anneal at approximately $1000^{\circ} \mathrm{C}$ using a $2.5 \mu \mathrm{m}, 1 \mathrm{~cm}^{2} \mathrm{FeCo}$ film deposited in a 400-550 Gauss magnetic field. The initial morphology showed approximately $20 \mathrm{~nm}$ grains very clearly whereas the texture of the post-anneal sample was barely visible. 


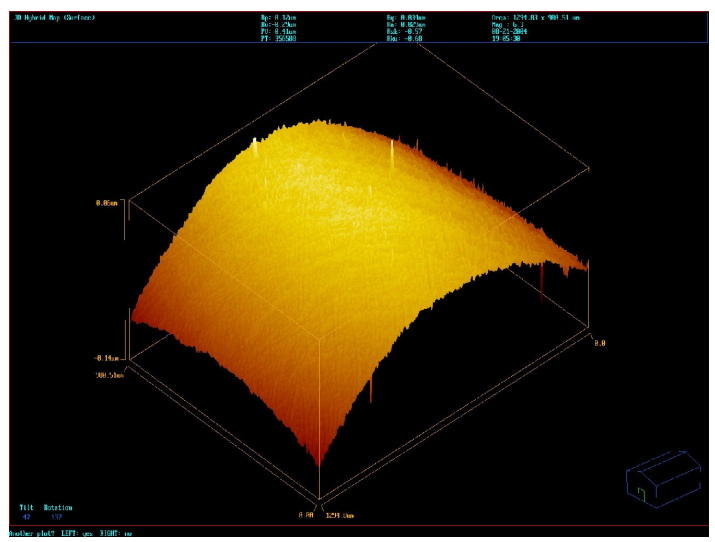

(a)

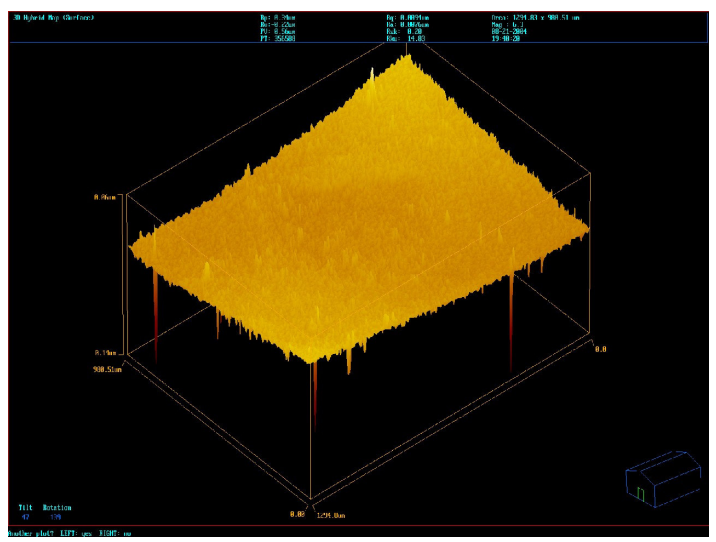

(b)

Figure 5.2: Interferometric images of polysilicon surface (a) before, and (b) after annealing showing a change in stress-induced curvature from highly compressive to moderately tensile. Surface roughness measurements indicated a reduction in magnitude from $0.031 \mu \mathrm{m}$ to $0.0094 \mu \mathrm{m}$ RMS and $0.029 \mu \mathrm{m}$ to $0.0076 \mu \mathrm{m}$ average.

concentration as compared to that preferred for full-wafer processing $\left(<1 \mathrm{E} 15 \mathrm{~cm}^{-3}\right)$. The resulting film morphology no longer exhibited the well-defined grains visible in 5.1(a), instead appearing almost homogeneous on the micron scale. Figure 5.2 shows interferometric surface scans of the same two films. The initial curvature reflected the compressive stress typically associated with as-deposited polysilicon films, whereas the final curvature indicated a mild tensile stress. Approximated stress values were obtained from these figures through Stoney's Equation,

$$
\sigma_{F}=\frac{E_{S}}{1-v_{S}} \frac{t_{S}^{2}}{6 R t_{F}}
$$

where the stress, $\sigma(\mathrm{MPa})$, is a function of the Young's Modulus, $E_{S}(\mathrm{~Pa})$, Poisson's ratio, $v$, and thickness, $t_{S}(\mathrm{~m})$, of the substrate, the thickness, $t_{F}(\mathrm{~m})$, of the film, and the radius of curvature, $R(\mathrm{~m})$, of the substrate-film combination [Chen, et al., 2002]. The radius of curvature can be calculated from the horizontal and vertical dimensions of the IFM plots using the relation 


$$
R=\frac{l^{2}}{2 b}
$$

where $l(\mathrm{~m})$ represents the chord length of the sector, or lateral dimension, and $b(\mathrm{~m})$ represents the bow of the wafer, or vertical dimension [Chen, et al., 2002]. The Stoney Equation assumes a uniform stress throughout the film of interest, and thus does not account for stress gradients. This reduced form of the original equation also assumes that the film thickness was much smaller than that of the substrate. Lastly, the assumption is made that the substrate was absent of curvature prior to film deposition. This second assumption was unverifiable, as the wafers from which these samples were generated were obtained with films already present. They also had a $1 \mu \mathrm{m}$ LPCVD silicon dioxide between the substrate and polysilicon film; this film was also on the back side of the wafer, however, and was not believed to have contributed significantly to the overall curvature. Thus some error was present in the calculated stress magnitudes, but they were believed to be in the appropriate range. Table 5.1 summarizes the details and results of the calculations for this particular set of samples. The $l$ (horizontal) values were identical for both samples and were calculated using the geometric mean of the lateral dimensions indicated in the headings $(1294.83 \mathrm{~mm} \times 980.51 \mathrm{~mm})$. The $b$ dimensions were estimated from the figures using the vertical scale. This yielded radius of curvature values of $4.88 \mathrm{~m}$ and $15.9 \mathrm{~m}$ for the unannealed and annealed samples, respectively. These were then

\begin{tabular}{lll}
\hline \hline \multicolumn{3}{c}{ TABLE 5.1 } \\
\multicolumn{2}{c}{ STRESS CALCULATIONS FOR LPCVD POLYSILICON FILMS } \\
\hline Value & Unannealed & Annealed \\
\hline $1, \mathrm{~mm}$ & 1.13 & 1.13 \\
$\mathrm{~b}, \mu \mathrm{m}$ & -0.13 & 0.04 \\
$\mathrm{R}, \mathrm{m}$ & -4.88 & 15.9 \\
$\sigma, \mathrm{MPa}$ & -1542 & 474 \\
\hline \hline
\end{tabular}


substituted into Equation (4.8) and, using a Young's Modulus of 130GPa and a Poisson's Ratio of 0.278 as per measurements done by Franca, et al., for $<100>$ silicon, the stress values were obtained [Franca, et al., 2004]. They confirmed quantitatively that a significant reduction in the magnitude of the intrinsic stress occurred as a result of inductive heat treatment, and that furthermore the type of stress was changed from compressive to tensile. Identical samples heated for different time durations showed a negligible morphology change for heating durations at or below 2 minutes.

A similar analysis was done using sputter-deposited silicon, prepared at MTU on the Perkin Elmer 2400-8J RF sputtering system. Films were deposited to a thickness of $1.5 \mu \mathrm{m}$ in three $0.5 \mu \mathrm{m}$ intervals (chamber remained at vacuum) in order to allow the system time to disperse any heat buildup, with a $1 \mu \mathrm{m}$ silicon dioxide layer providing an interface between the film and the substrate. Substrates were diced into $2 \times 2 \mathrm{~cm}$ samples, and annealing was done using a $2.5 \mu \mathrm{m}$ FeCo film heated to approximately $1000^{\circ} \mathrm{C}$. The cross-sectional SEM images in Figure 5.3 compare the initial film morphology with that following a ten-minute anneal. The initial film showed almost no discernable texture, indicative of the amorphous crystalline structure present. After annealing, however, a significant change had taken place, with $10-20 \mathrm{~nm}$ grains beginning to take shape. This restructuring and simultaneous densification resulted in the formation of voids within the film as can be observed in the figure. The image shows the lowest of the three $0.5 \mu \mathrm{m}$ segments in the film, with the interface to the $\mathrm{SiO}_{2}$ layer visible in the lower right-hand corner, and discontinuous interface to the second polysilicon film segment in the upper left-hand corner. This discontinuity was likely the result of poor vacuum and took place at both poly-poly interfaces, but nevertheless the morphology looked identical in all three 


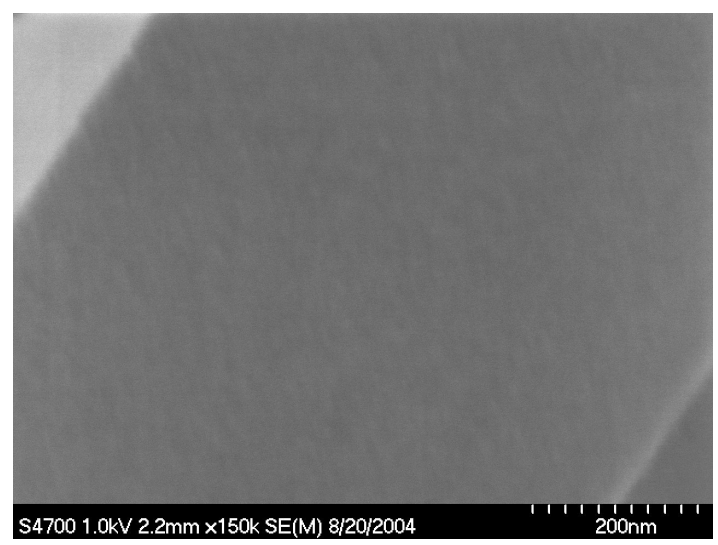

(a)

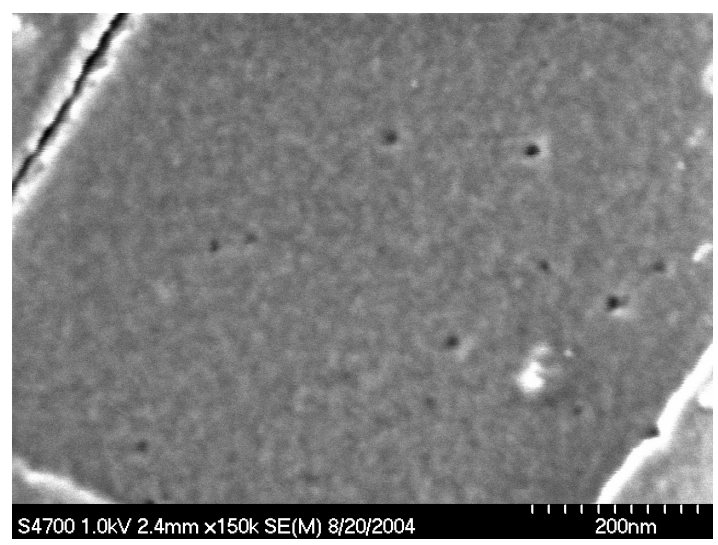

(b)

Figure 5.3: SEM images showing cross-sections of $1.5 \mu \mathrm{m}$ sputtered polysilicon film (a) before, and (b) after a ten-minute anneal at $1.5 \mathrm{~kW}$ using a $2.5 \mu \mathrm{m}, 4 \mathrm{~cm}^{2} \mathrm{FeCo}$ film. The initially amorphous film was beginning to show the presence of grains as well as voids in the lattice as the relatively low-density amorphous material assumed increased order.

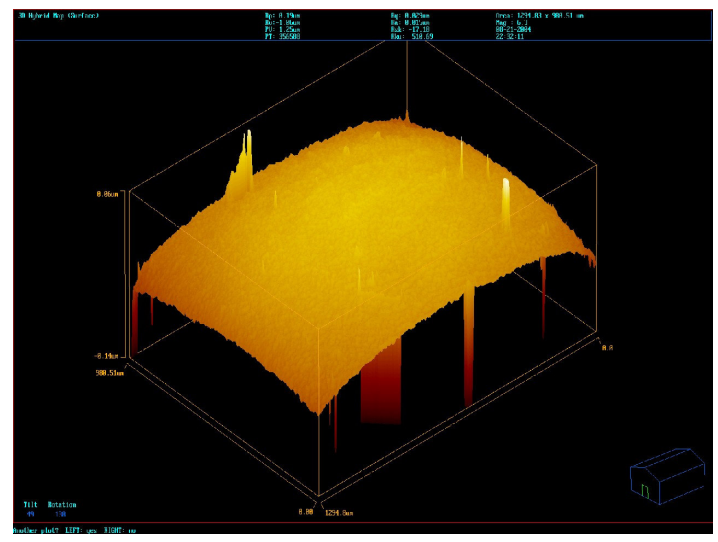

(a)

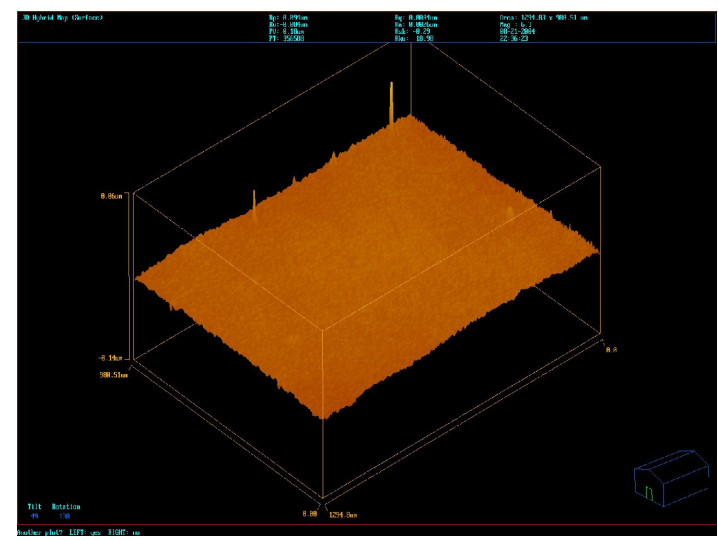

(b)

Figure 5.4: Interferometric images of polysilicon surface (a) before, and (b) after annealing showing a change in stress-induced curvature from moderately compressive to nearly flat. Surface roughness measurements indicated a reduction in magnitude from $0.029 \mu \mathrm{m}$ to $0.0031 \mu \mathrm{m}$ RMS and $0.015 \mu \mathrm{m}$ to $0.0026 \mu \mathrm{m}$ average.

segments as the gap was only on the order of 10nm. IFM images are presented in Figure 5.4 and again illustrate a change in curvature, but unlike the LPCVD sample shown previously the film stress remained compressive in nature. The details of the stress calculations are shown in Table 5.2 and were done using the same equations, assumptions, and material constants as were used for the LPCVD samples shown previously. The radius of curvature values for the unannealed and annealed samples were 


\begin{tabular}{|c|c|c|}
\hline \multicolumn{3}{|c|}{ TABLE 5.2} \\
\hline STRESS & NSS FOR SPUT & OLYSILICON FILMS \\
\hline Value & Unannealed & Annealed \\
\hline $1, \mathrm{~mm}$ & 1.13 & 1.13 \\
\hline $\mathrm{b}, \mu \mathrm{m}$ & -0.10 & -0.01 \\
\hline $\mathrm{R}, \mathrm{m}$ & -6.35 & -63.5 \\
\hline$\sigma, \mathrm{MPa}$ & -395 & -39.5 \\
\hline
\end{tabular}

estimated at $6.35 \mathrm{~m}$ and $63.5 \mathrm{~m}$, respectively, and the resulting stresses were $395 \mathrm{MPa}$ and 39.5MPa, both compressive. Although the resulting film was of lower density than the LPCVD polysilicon film and not tensile as is preferred for MEMS devices (see Section 1.1), its low-temperature deposition made it a potential precursor to the inductive annealing process. Tensile stress was likely achievable with alternate annealing times and/or temperatures. Alternate possibilities included PECVD polysilicon and cathodicarc-based polysilicon alloys.

\section{2: Polysilicon Cantilever Beams}

Graduating from blanket films to patterned structures was greatly facilitated by the ferromagnetic ring concept (see Section 4.4), as it enabled the use of larger heated films and reduced the thermal stress imparted upon the devices. The first structures to be utilized were simple beams patterned from a $3 \mu \mathrm{m}$-thick CVD polysilicon layer grown at $625^{\circ} \mathrm{C}$ via $\mathrm{SiH}_{4}$ decomposition in a TMX9K 4304 diffusion furnace. A $1 \mu \mathrm{m}$ thermal $\mathrm{SiO}_{2}$ film provided a base layer. The layout for each beam cluster is shown in Figure 5.5. It consisted of eight variations of the same basic design, which incorporated five singleclamped cantilever beams, one each of two styles of folded beams, and five doubleclamped beams. This pattern was repeated four times horizontally in order to vary beam lengths $(500 \mu \mathrm{m}, 1000 \mu \mathrm{m}, 2000 \mu \mathrm{m}$, and $3000 \mu \mathrm{m})$ and twice vertically in order to vary 


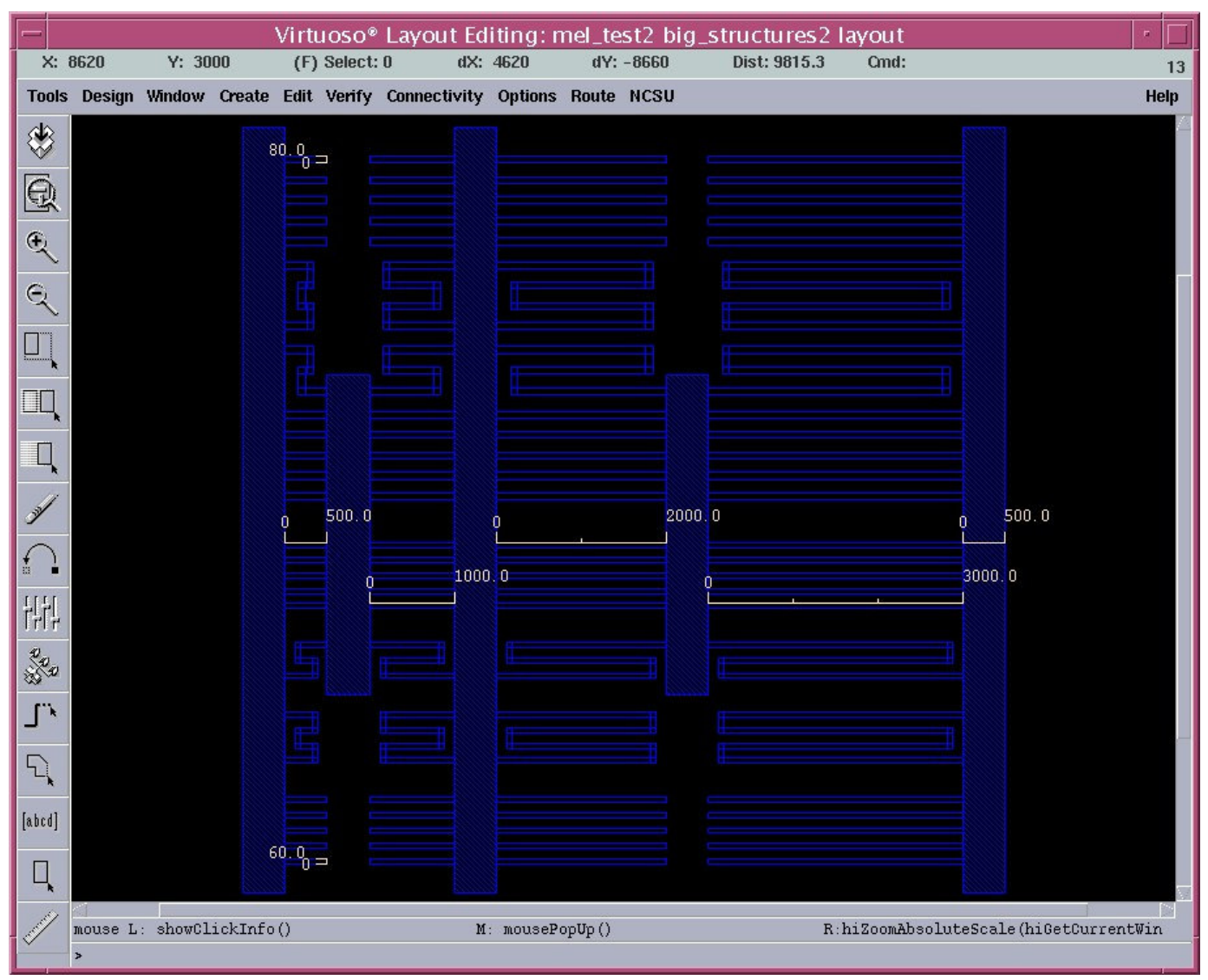

Figure 5.5: Layout of the beam design used for structural annealing. The pattern consisted of cantilever, double-clamped, and folded beams with widths of $60 \mu \mathrm{m}$ (top half) and $80 \mu \mathrm{m}$ (bottom half) and lengths of $500 \mu \mathrm{m}, 1000 \mu \mathrm{m}, 2000 \mu \mathrm{m}$, and $3000 \mu \mathrm{m}$.

beam widths $(60 \mu \mathrm{m}$ and $80 \mu \mathrm{m})$. Large vertical strips $500 \mu \mathrm{m}$ in width provided for anchor regions in the event that the beams were to be released. Each beam cluster measured $9 \mathrm{~mm}$ on each side, and they were patterned into a grid as can be seen in Figures 5.6 and 5.7. Heating was done using either round or square-shaped ferromagnetic rings formed around a single cluster. Figure 5.6 illustrates a common effect that often took place during the cool-down phase of an anneal. The square-shaped ring pictured around the beam cluster was not the actual FeCo pattern utilized to perform the heat treatment but was instead the footprint left behind when the highly-stressed ferromagnetic film delaminated from the substrate. Annealing temperatures as low as $500^{\circ} \mathrm{C}$ were sufficient 


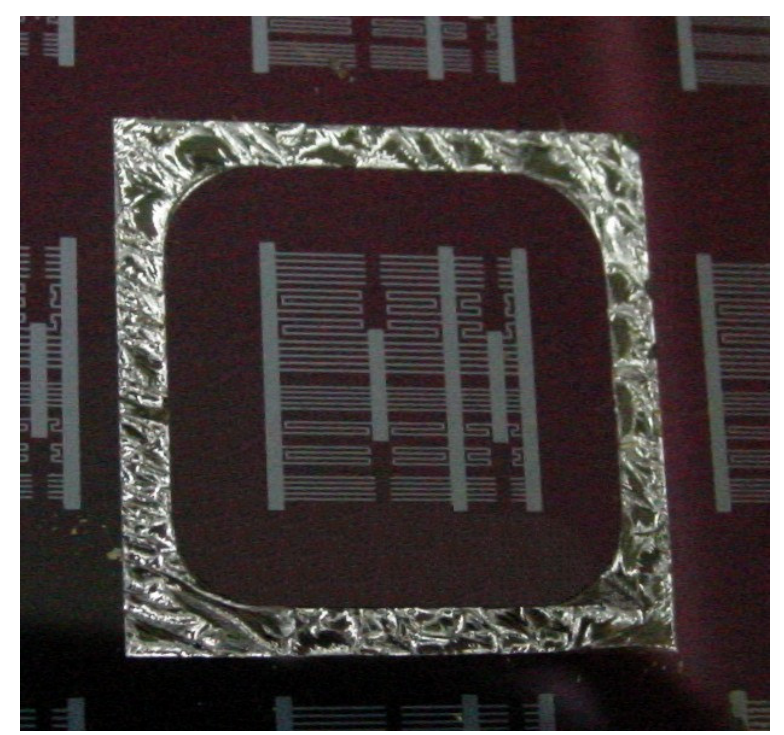

Figure 5.6: Photograph showing the appearance of the silicon substrate after the heating of a FeCo square ring. Pyrometry focused at the center of the pattern indicated a temperature of approximately $500^{\circ} \mathrm{C}$. The film peeled during cool-down, removing the underlying $\mathrm{SiO}_{2}$ as well as portions of the substrate.

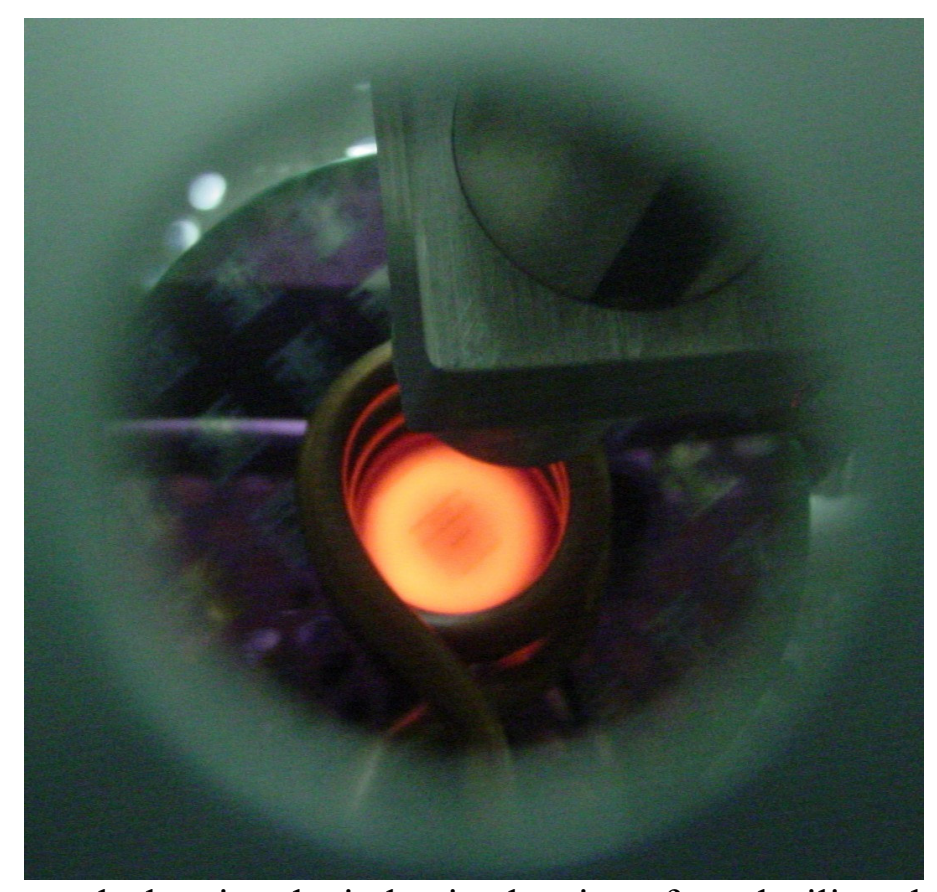

Figure 5.7: Photograph showing the inductive heating of a polysilicon beam cluster using a $2.4 \mathrm{~cm}$-wide hollow NiFe19 ring measuring $6.6 \mu \mathrm{m}$ in thickness. The temperature was approximately $800^{\circ} \mathrm{C}$ as indicated by infrared pyrometry.

to induce significant thermal expansion mismatch stresses in both FeCo and NiFe19. Adhesion problems were not encountered during the warm-up phase, as the rate could be 
precisely regulated through power supply manipulation, but despite efforts to reduce the rate of chamber venting, delamination during cool-down remained an ongoing challenge. This was primarily due to the fact that little or no cooling took place while the samples remained under vacuum and thus it was difficult to reduce the impact of the thermal shock and turbulence created upon the introduction of nitrogen gas. The delaminating force was sufficient to also remove the underlying silicon dioxide film as well as pieces of the substrate. Among the recommendations for future research in Chapter 7 is the development of a controlled cool-down procedure. Figure 5.7 illustrates the bright glowing observed around the beams during heat treatment. The glow intensity typically began at around $600^{\circ} \mathrm{C}$ and increased with temperature, becoming almost white in color during thermal runaway. Figures 5.8(a-d) present SEM images from the heat treatment of polysilicon beams via inductive coupling to a $7.3 \mu \mathrm{m} \mathrm{NiFe} 19$ ring patterned like that in Figure 4.27(b). Annealing was done for ten minutes, and pyrometry indicated a temperature of $840^{\circ} \mathrm{C}$ at the center of the ring. As was discussed in the previous section, temperatures were limited to the $800^{\circ} \mathrm{C}$ vicinity for this type of film geometry in order to avoid initiating a runaway condition. While below the $1000^{\circ} \mathrm{C}$ target stated in Section 1.1, the literature indicates temperatures in this lower range to be nevertheless sufficient to produce a measurable change in polysilicon morphology [French, 2002; Guckel, et al., 1988-2; Suarez, et al., 1992]. Post-anneal properties can be somewhat unpredictable, however, as the moderate thermal energy level often activates only a subset of the restructuring mechanisms. This can result in conflicting behaviors for the various grain orientations, including size reduction [Campo, et al., 1995] and significant increases in stress [French, et al., 1996]. These behaviors were observed in the lower-temperature 


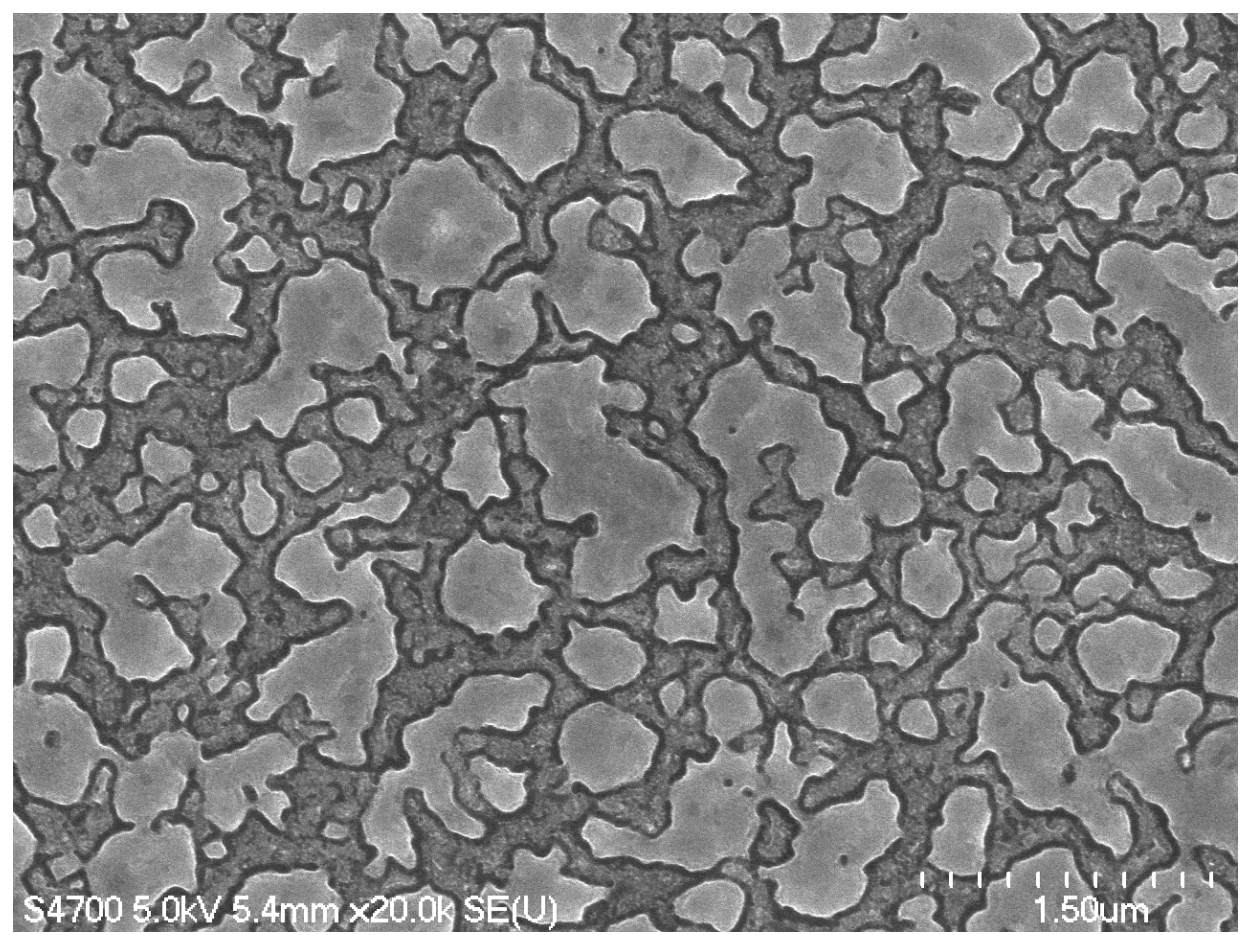

Figure 5.8(a): SEM image showing the initial surface morphology of the $3 \mu \mathrm{m}$ polysilicon beam pattern. All thick polysilicon films were mechanically polished before patterning in order to reduce the surface roughness and thus improve the quality of the subsequent films utilized for annealing.

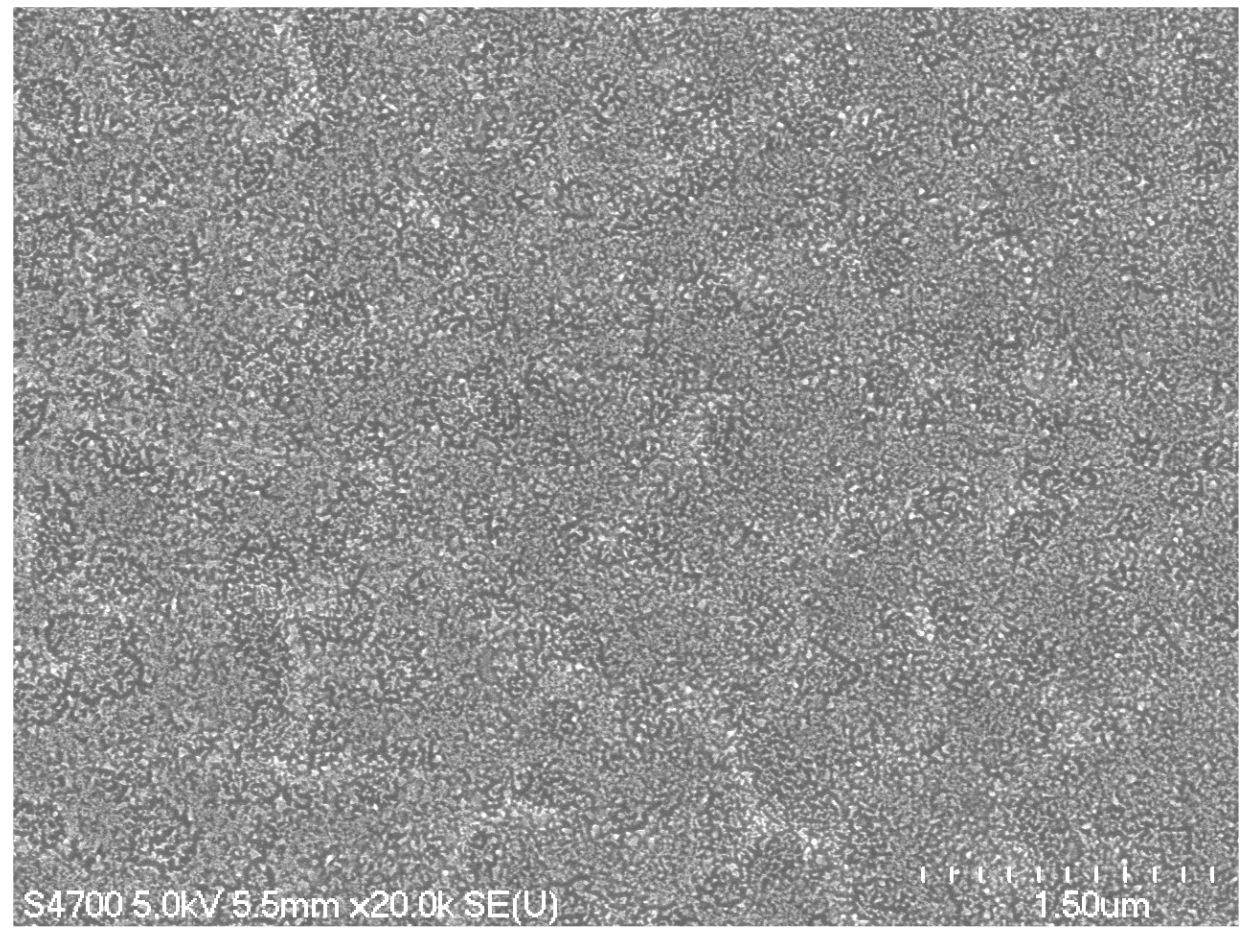

Figure 5.8(b): SEM image taken from the center of the annealed polysilicon beam pattern. The texture reflected the initiation of film restructuring, with numerous voids and little morphology definition. The temperature measured at this location was $840^{\circ} \mathrm{C}$. 


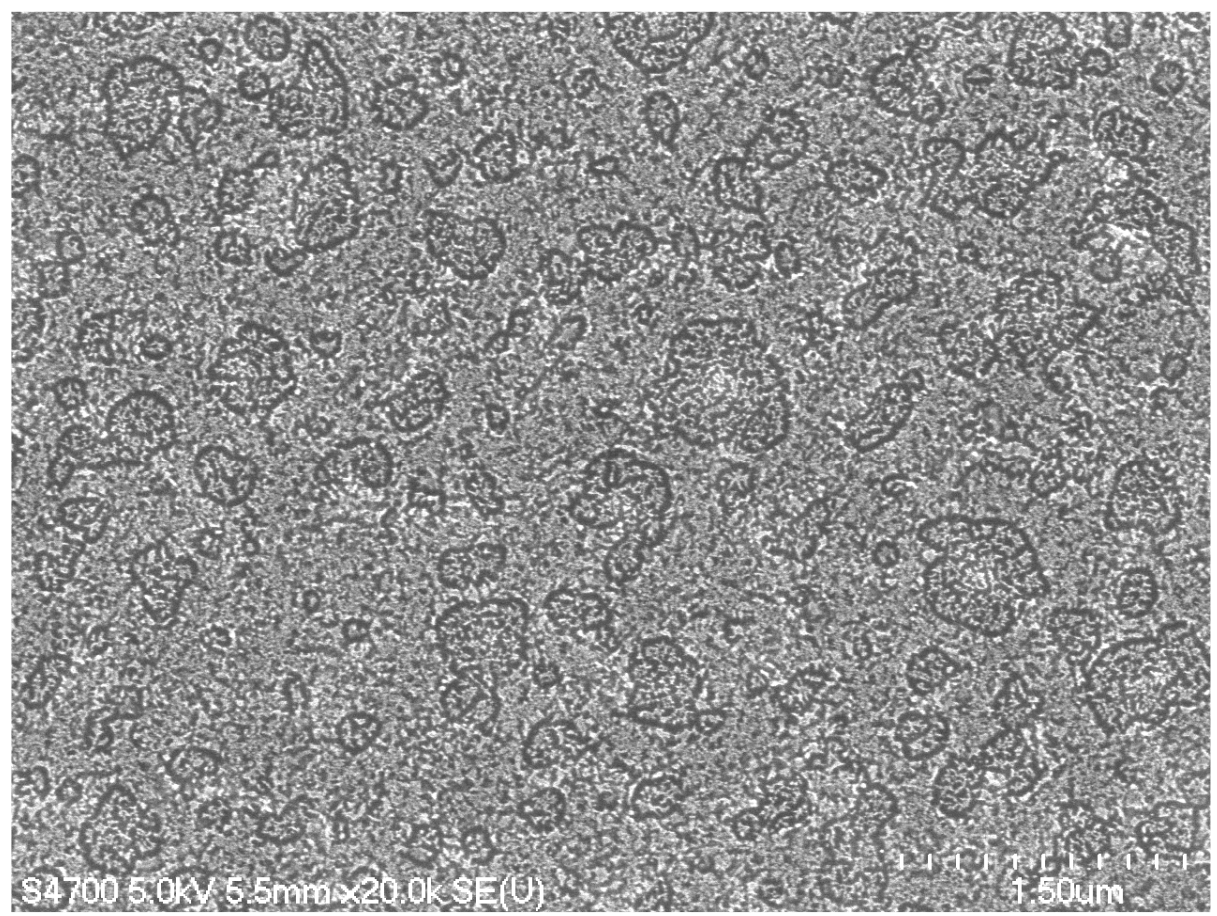

Figure 5.8(c): SEM image taken from a location mid-way between the center and edge of the annealed polysilicon beam pattern. The film morphology showed increased definition as compared to that in (b), with regions of higher density beginning to take shape, as a result of a higher annealing temperature. The temperature was estimated to be $853^{\circ} \mathrm{C}$.

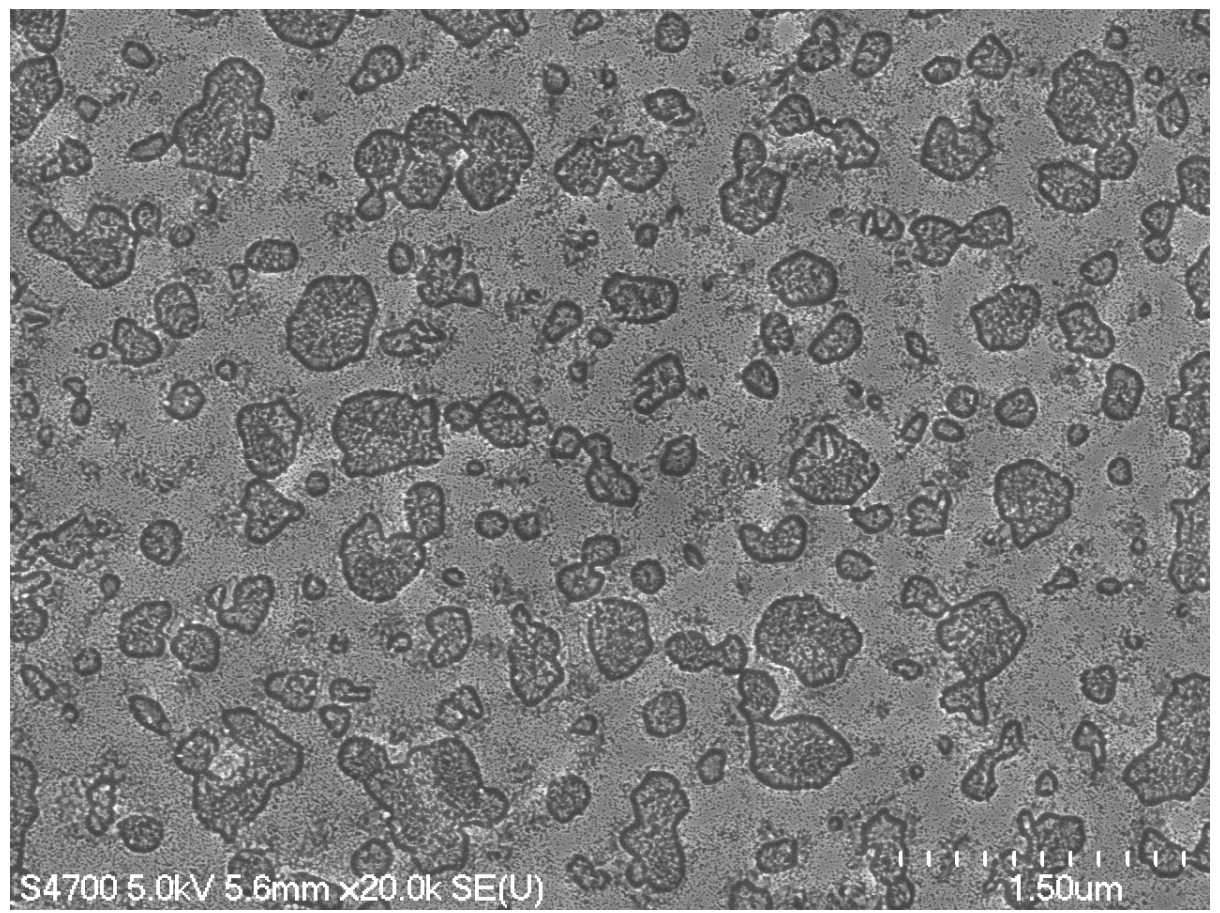

Figure 5.8(d): SEM image taken from the edge of the annealed polysilicon beam pattern. Proximity to the NiFe19 rings rendered this the region of maximum temperature. The texture reflected the furthest progress of the anneal, with the film consisting of lowdensity islands engulfed by a densified lattice. The estimated temperature was $865^{\circ} \mathrm{C}$. 
anneal data, as will be shown. Figure 5.8(a) illustrates the unannealed polysilicon surface. Its unusual appearance can be attributed to the fact that the relatively thick film was mechanically polished prior to patterning. The remaining three figures show the polysilicon surface texture at different locations within the annealed beam cluster, illustrating the horizontal temperature gradient within the anneal region. The image in Figure 5.8(b) was taken from near the center of the pattern and thus illustrates the region of lowest temperature, which was at approximately $840^{\circ} \mathrm{C}$ as the pyrometry sensor was centered with respect to the coil and focused to a $1 \mathrm{~mm}$ spot size. The image illustrated the beginnings of film restructuring. The surface had changed considerably, appearing more uniform as though the film was attempting to repair itself from stresses induced during polishing. Morphology lacked clear definition, however, with numerous voids distributed at random throughout the lattice. Moving outward to a location midway between the center and edge of the beam pattern, Figure 5.8(c) demonstrates the impact of a slightly higher annealing temperature. Voids in the lattice were starting to be forced together as a result of enhanced film recrystallization and densification. Finally, Figure 5.8(d) shows the polysilicon region of maximum annealing temperature, located at the outer edge of the pattern. The film morphology at this location exhibited superior restructuring as compared to that shown in (b) and (c), with the "islands" of low density crystallites surrounded by an increasingly densified lattice. Thus the inductive annealing process had been utilized to produce a significant temperature gradient across a polysilicon device and, consequently, a visible morphology gradient. The magnitude of this gradient was estimated using the measured temperature differential data from Figure 4.30. Analysis of the figure for a hollow NiFe19 ring $\sim 7 \mu \mathrm{m}$ in thickness heated to $800^{\circ} \mathrm{C}$ 
yielded temperature gradient estimates of $150^{\circ} \mathrm{C} / \mathrm{cm}$ in the region outside of the heated ring and $56.3^{\circ} \mathrm{C} / \mathrm{cm}$ inside the ring. Applying linear interpolation, the approximate temperature values for the center, middle $(r=0.225 \mathrm{~cm})$, and edge $(r=0.45 \mathrm{~cm})$ regions of the beam cluster were $840^{\circ} \mathrm{C}, 853^{\circ} \mathrm{C}$, and $865^{\circ} \mathrm{C}$, respectively. This $25^{\circ} \mathrm{C}$ differential in the center-to-edge annealing temperature had a significant impact on the appearance of the polysilicon surface, which suggests that the impact on the performance of a released sensor would also have been significant.

The impact of the various levels of partial annealing was exemplified in the XRay Diffraction (XRD) data, which is summarized in Table 5.3 (see Section 4.1 for a description of the headings and calculation methods). Figures 5.9(a,b) show the pre- and post-anneal output waveforms. Unlike the clear results of the thorough anneal in the proof-of-concept test, the data from this partial anneal reflected an earlier stage of recrystallization. The computed Scherrer crystallite sizes indicated appreciable grain

\begin{tabular}{|c|c|c|c|c|c|c|}
\hline \multicolumn{7}{|c|}{$\begin{array}{ll}\text { TABLE } 5.3 \\
\text { X-RAY DIFFRACTION DATA AND CALCULAT }\end{array}$} \\
\hline$\theta$ & $\begin{array}{l}2 \theta, \\
\text { degrees }\end{array}$ & $\begin{array}{l}\mathrm{B}_{\mathrm{FWHM}}, \\
\text { radians }\end{array}$ & $\begin{array}{l}\mathrm{B}_{\mathrm{ADJ}}, \\
\text { radians }\end{array}$ & $\begin{array}{l}\text { Scherrer Cry. } \\
\text { Size, } \mathrm{nm}\end{array}$ & $\begin{array}{l}\text { Strain, } \\
\mathrm{mm} / \mathrm{m}\end{array}$ & $\begin{array}{l}\text { Stress, } \\
\mathrm{MPa}\end{array}$ \\
\hline \multirow{3}{*}{ 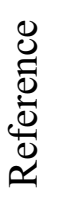 } & 28.4479 & 0.001290 & - & - & - & - \\
\hline & 47.3050 & 0.001417 & - & - & - & - \\
\hline & 56.1273 & 0.001370 & - & - & - & - \\
\hline \multirow{3}{*}{ 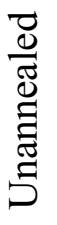 } & 28.5130 & 0.009159 & 0.008448 & 16.93 & -2.092 & -334.7 \\
\hline & 47.4519 & 0.002164 & 0.001105 & 137.1 & -2.357 & -377.1 \\
\hline & - & - & - & - & - & - \\
\hline \multirow{4}{*}{ 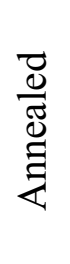 } & 28.5174 & 0.002157 & 0.001225 & 116.8 & -2.233 & -357.3 \\
\hline & 47.4562 & 0.002190 & 0.001136 & 133.3 & -2.425 & -388.1 \\
\hline & 55.4999 & 0.001906 & 0.000843 & 185.9 & 7.466 & 1195 \\
\hline & 56.2502 & 0.002644 & 0.001697 & 91.94 & -1.436 & -229.7 \\
\hline
\end{tabular}




\section{Polysilicon Beam XRD Data, Unannealed Sample}

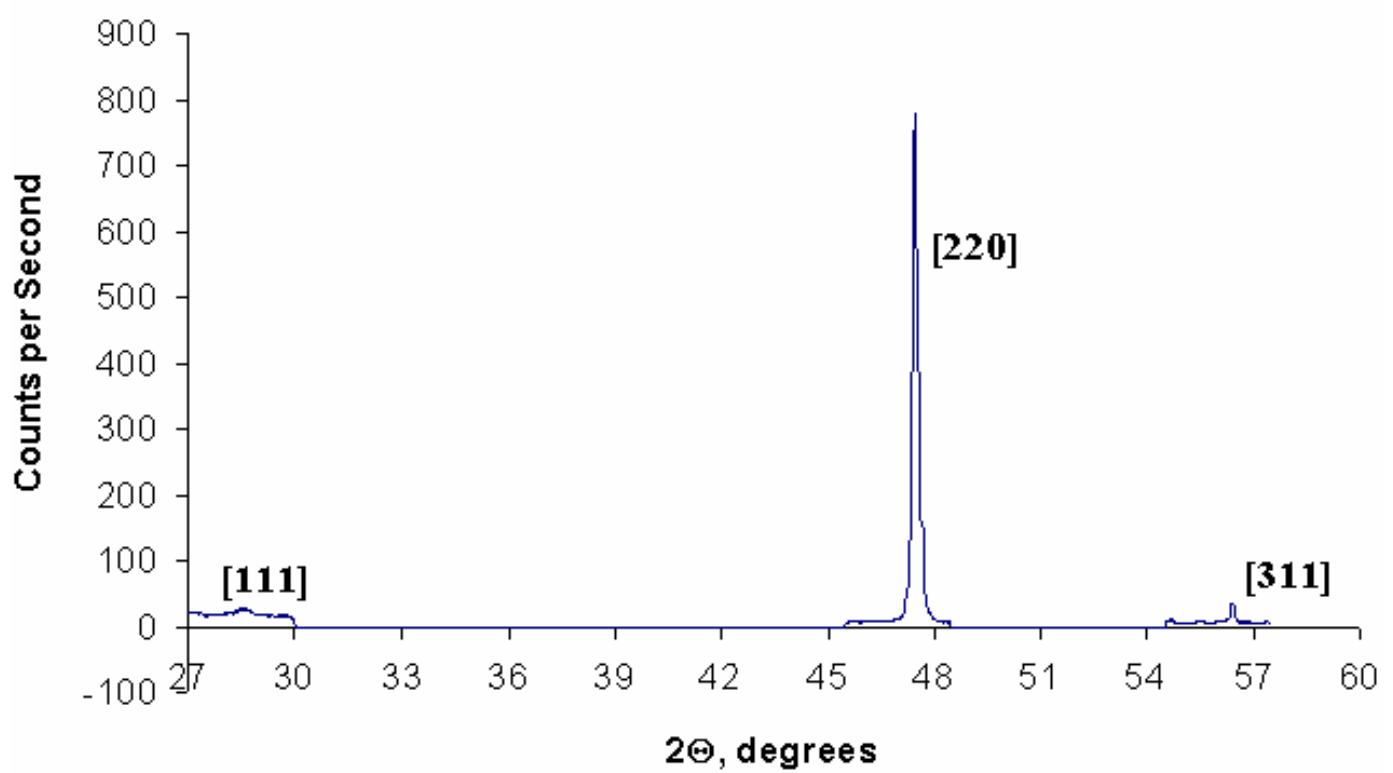

Figure 5.9(a): X-Ray Diffraction output plot obtained from the as-deposited $3 \mu \mathrm{m}$ LPCVD polysilicon which was formed at $625^{\circ} \mathrm{C}$ and patterned into cantilever beams. The three dominant grain orientations were [111], [220], and [311], though the diffractometer did not produce a measurement for the [311] peak. Note that these were the same orientations examined in polysilicon films of Section 4.1 .

Polysilicon Beam XRD Data, Annealed Sample

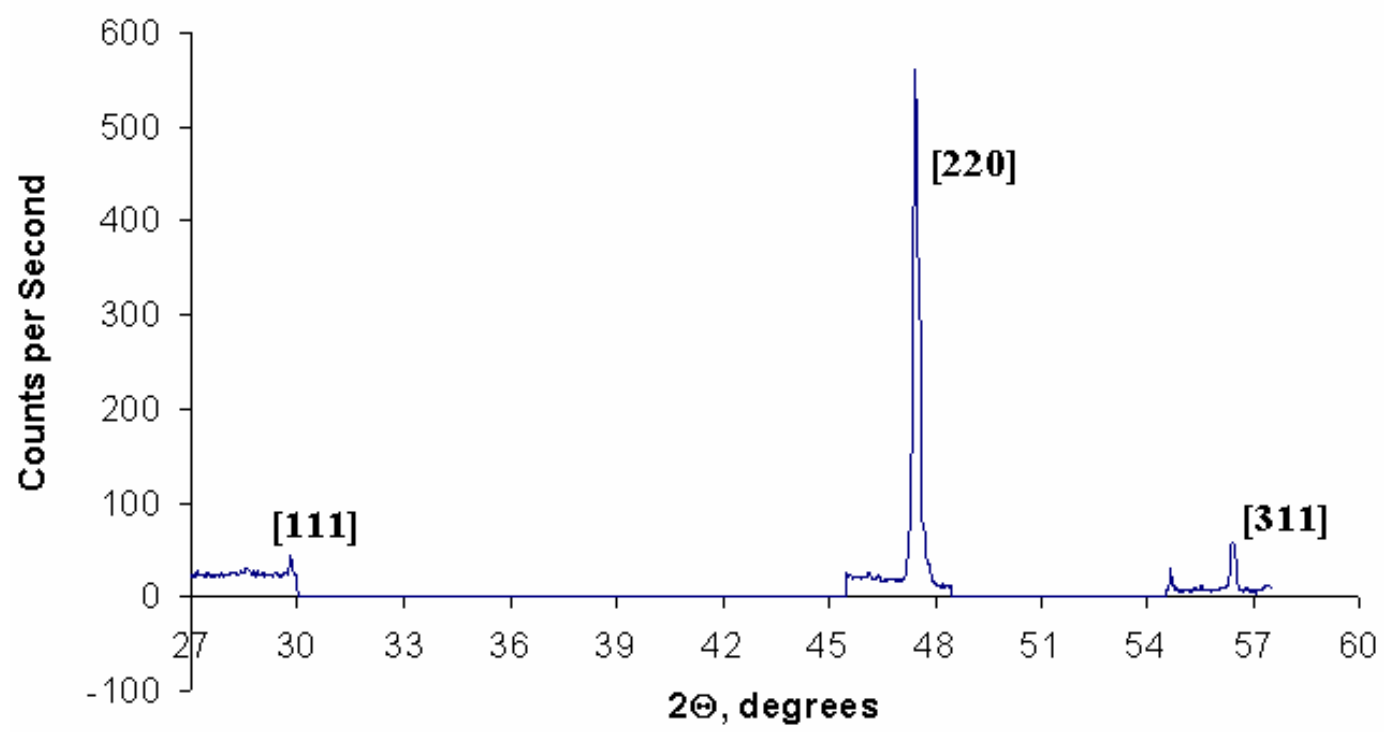

Figure 5.9(b): X-Ray Diffraction output plot showing the impact of the $840-865^{\circ} \mathrm{C}$ anneal on the polysilicon grain structure. The [111] and [311] grains grew considerably, whereas the [220] grains were actually found to have been slightly reduced in size. Stress and strain values were rendered higher due to the incomplete anneal. 
growth along the [111] and [311] axes but a small reduction in size in the [220] direction. The [311] grains, which were undetectable in the unannealed sample, appeared at two distinct diffraction angles, one of which was computed to have tensile stress while the other was computed to have compressive stress. Furthermore, the film stresses along the [111] and [220] directions both became slightly more compressive. The implication of the results was that grain growth had been initiated, but with insufficient energy to achieve complete reversal of the less-dominant orientations. Nevertheless, a measurable difference in the polysilicon was demonstrated via both qualitative changes in morphology and quantitative changes in grain structure.

\section{3: Polysilicon Accelerometer Structures}

The ultimate goal of the research project was to demonstrate the application of the inductive annealing process to polysilicon devices. Processing difficulties prevented this from taking place on complete, functional inertial sensors as was originally intended (see Chapter 6), but some success was achieved with basic accelerometer structures. The layout of the sensor is shown in Figure 6.6(a); refer to Section 6.2 for a description of the structural design. The sensing structures were patterned into a $3 \mu \mathrm{m}$-thick LPCVD polysilicon film deposited at $600^{\circ} \mathrm{C}$ via $\mathrm{SiH}_{4}$ decomposition in a Tempress 6400 furnace and mechanically polished to reduce surface roughness. Figure 5.10 illustrates the relative placement of the heated film with respect to the sensors. The square-ring-shaped NiFe19 film encircled a $6 \times 6$ grid of $2 \times 2 \mathrm{~mm}$ devices, and was patterned via a shadow mask to have a $1.2 \mathrm{~cm}$ inner diameter and a $2.4 \mathrm{~cm}$ outer diameter. Note that this outer diameter was considerably larger than that illustrated in the figure. Thermal isolation 


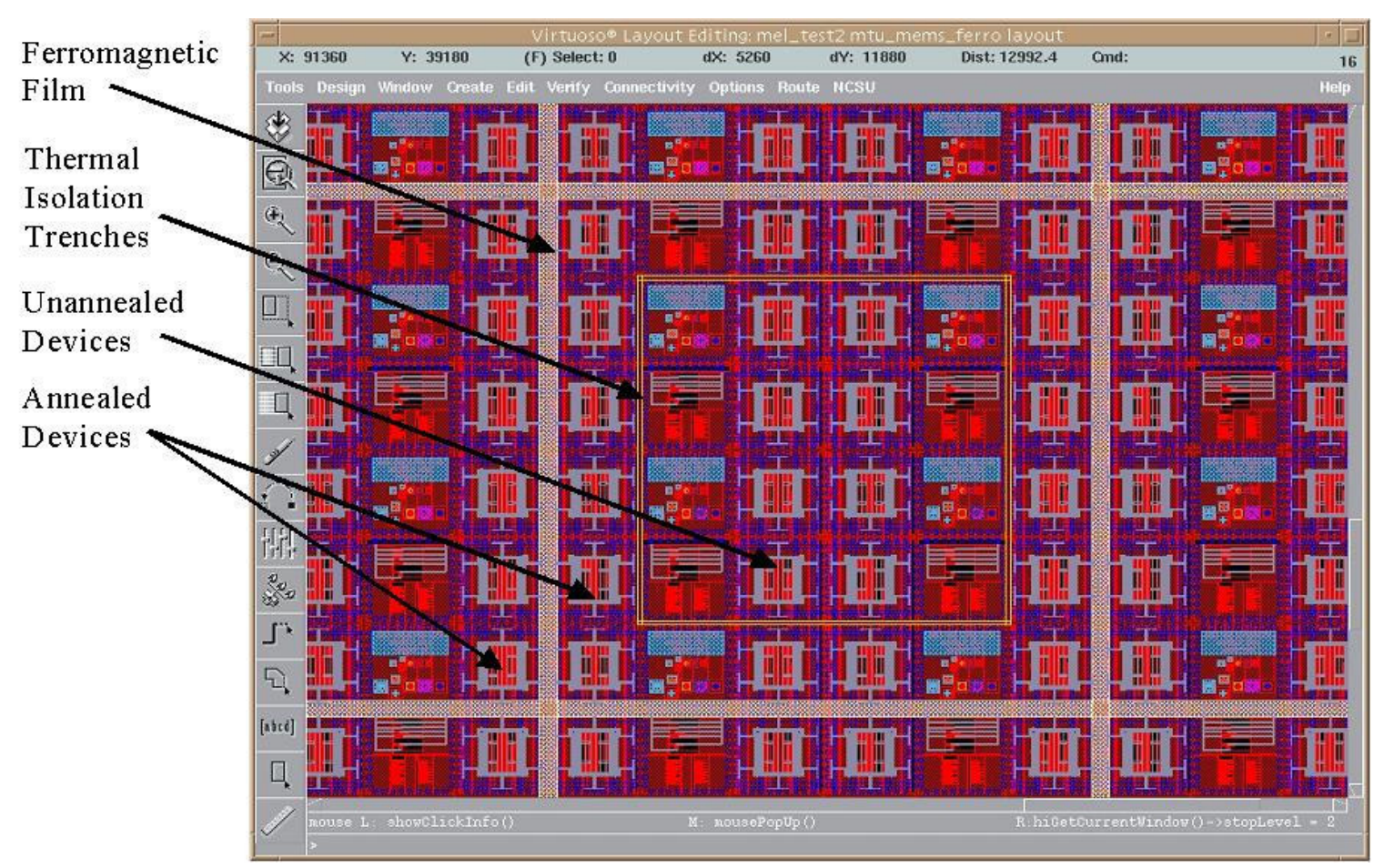

Figure 5.10: Approximate layout utilized for the annealing of $3 \mu \mathrm{m}$ polysilicon sensing structures using a $7.1 \mu \mathrm{m} \mathrm{NiFe} 19$ film patterned into square-shaped ring $2.4 \mathrm{~cm}$ in width with a $1.2 \mathrm{~cm}$ inner diameter. Two rows of trenches were etched through the polysilicon in order to help shield the devices that were not intended for heat treatment.

trenches were utilized to divide the high-temperature and low-temperature regions in the interior of the heated film, similar to the method described in the thin film isolation model of Section 3.3. In this case, the trenches were patterned to form two rows $40 \mu \mathrm{m}$ in width with a $20 \mu \mathrm{m}$ space between them. The NiFe19 film was heated at full power for ten minutes. Pyrometry indicated a temperature of $760^{\circ} \mathrm{C}$ at the center. This temperature was considerably lower than the $840^{\circ} \mathrm{C}$ reached during the cantilever beam experiment, though the induced temperatures in the heated NiFe19 rings were likely similar in magnitude due to their similar dimensions. Like the results presented in Section 5.2, this relatively low temperature rendered the annealing incomplete. The lower polysilicon deposition temperature of $600^{\circ} \mathrm{C}$ helped to produce a measurable difference nonetheless. The induced temperature gradient inside the inner diameter the heated NiFe19 ring was 
assumed to be the same as that computed in Section 4.4 and applied previously in Section 5.2, that being $56.3^{\circ} \mathrm{C} / \mathrm{cm}$. The impact of the thermal trenches was impossible to determine directly and was therefore estimated by assuming that the temperature induced by the film was indeed $865^{\circ} \mathrm{C}$, the estimated temperature in the NiFe19 ring of Section 5.2. These assumptions yielded temperature values of $783^{\circ} \mathrm{C}$ and $854^{\circ} \mathrm{C}$ on the inside and outside of the trenches, respectively. The temperature difference across the $20 \mu \mathrm{m}$ intertrench polysilicon was relatively small at only $0.113^{\circ} \mathrm{C}$, and therefore it was concluded that each of the two trenches reduced the surface temperature by around $35.6^{\circ} \mathrm{C}$.

Figures 5.11(a-d) present SEM images of the post-anneal surface morphology obtained at various locations within the interior of the heated ring. All images utilized the same magnification. Figure 5.11(a) shows the appearance of the polysilicon near the center of the heated region, which had lowest annealing temperature. The initial appearance exhibited large, continuous sections with dimensions ranging from $\sim 100$ $400 \mathrm{~nm}$. The surface morphology slightly inside of the trenches is shown in Figure 5.11(b). The image shows a trend toward increased fineness of the features, though up close they still looked quite similar to those of Figure 5.11(a). Examining the polysilicon region between the two trenches, Figure 5.11(c) shows an interesting blend of coarse and fine in which some of the larger, continuous regions were still visible but the areas between them were taking on an increased granular appearance. The surface as a whole was becoming increasingly three-dimensional. Finally, the image in Figure 5.11(d) shows the morphology slightly outside of the trench region. The film had become completely granular in appearance, with feature sizes averaging around $20 \mu \mathrm{m}$. The collection of images demonstrated a behavior similar that that of the annealed beam clusters in Section 


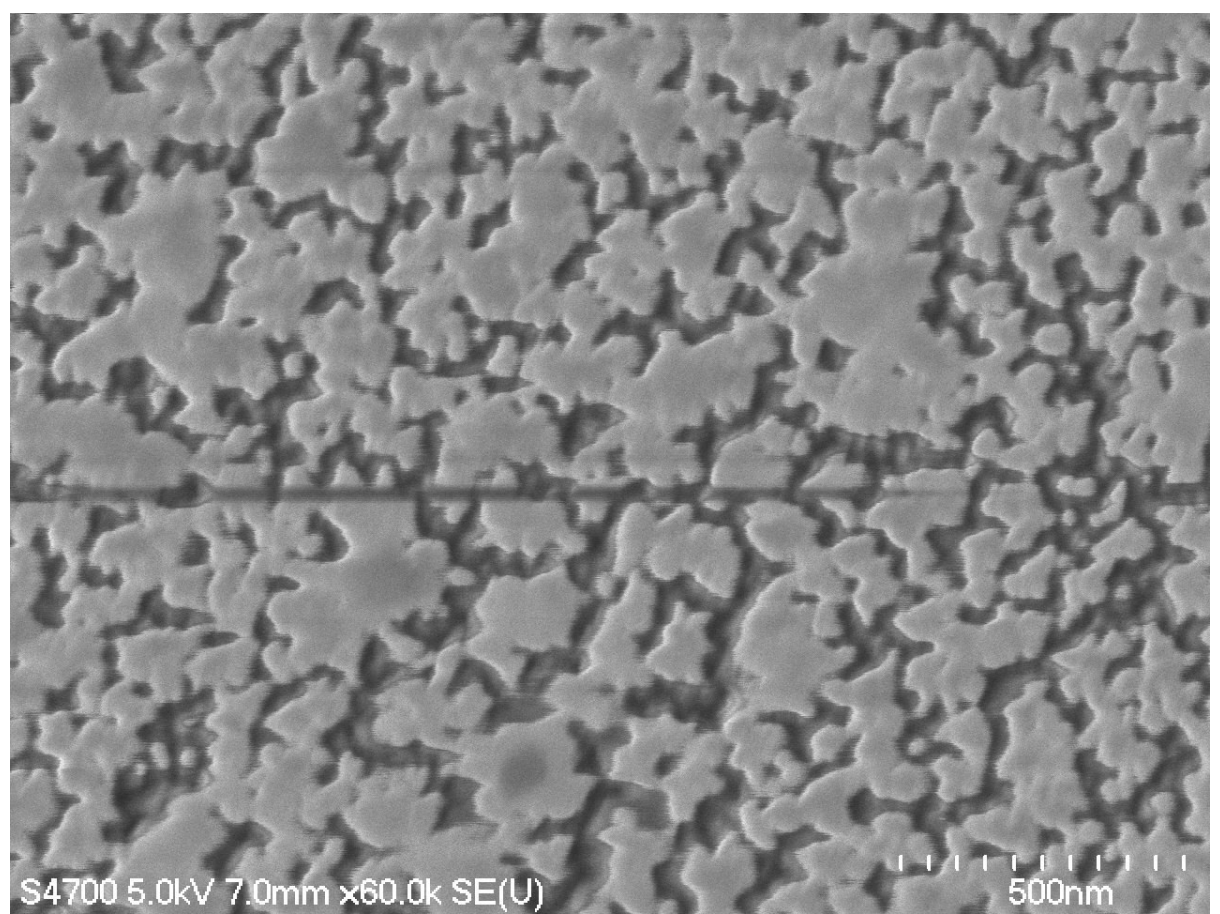

Figure 5.11(a): SEM image showing the $3 \mu \mathrm{m}$ polysilicon surface taken from central region of the heat-treated set of inertial sensing structures. This was the area of lowest temperature, measured via pyrometry to be $760^{\circ} \mathrm{C}$. Note that the films were mechanically polished prior to heat treatment.

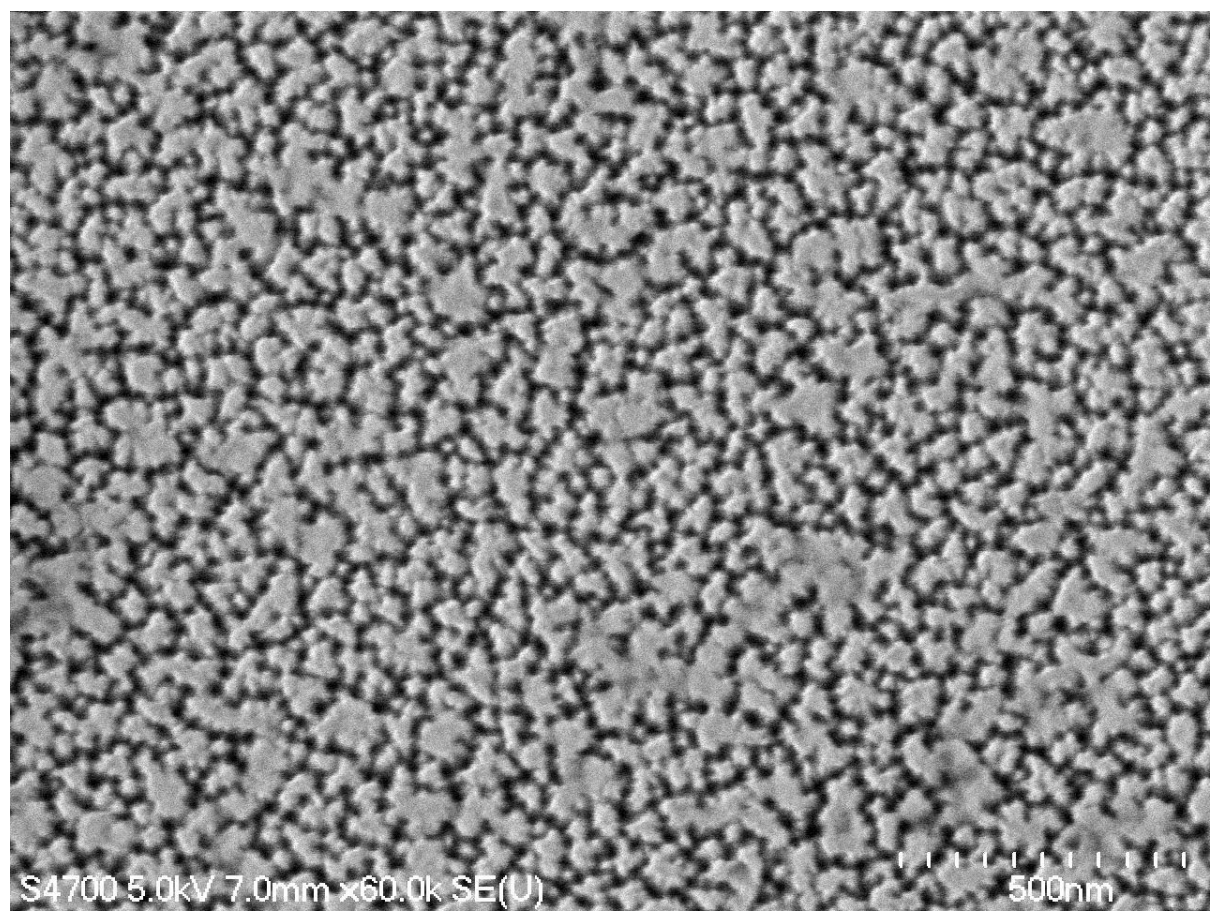

Figure 5.11(b): SEM image showing the polysilicon surface just inside of the thermal isolation trenches, at a distance of just under $4 \mathrm{~mm}$ from the center. The temperature at this location was estimated to be $783^{\circ} \mathrm{C}$. The film surface looked very similar to that of (a) except with relatively finer features. 


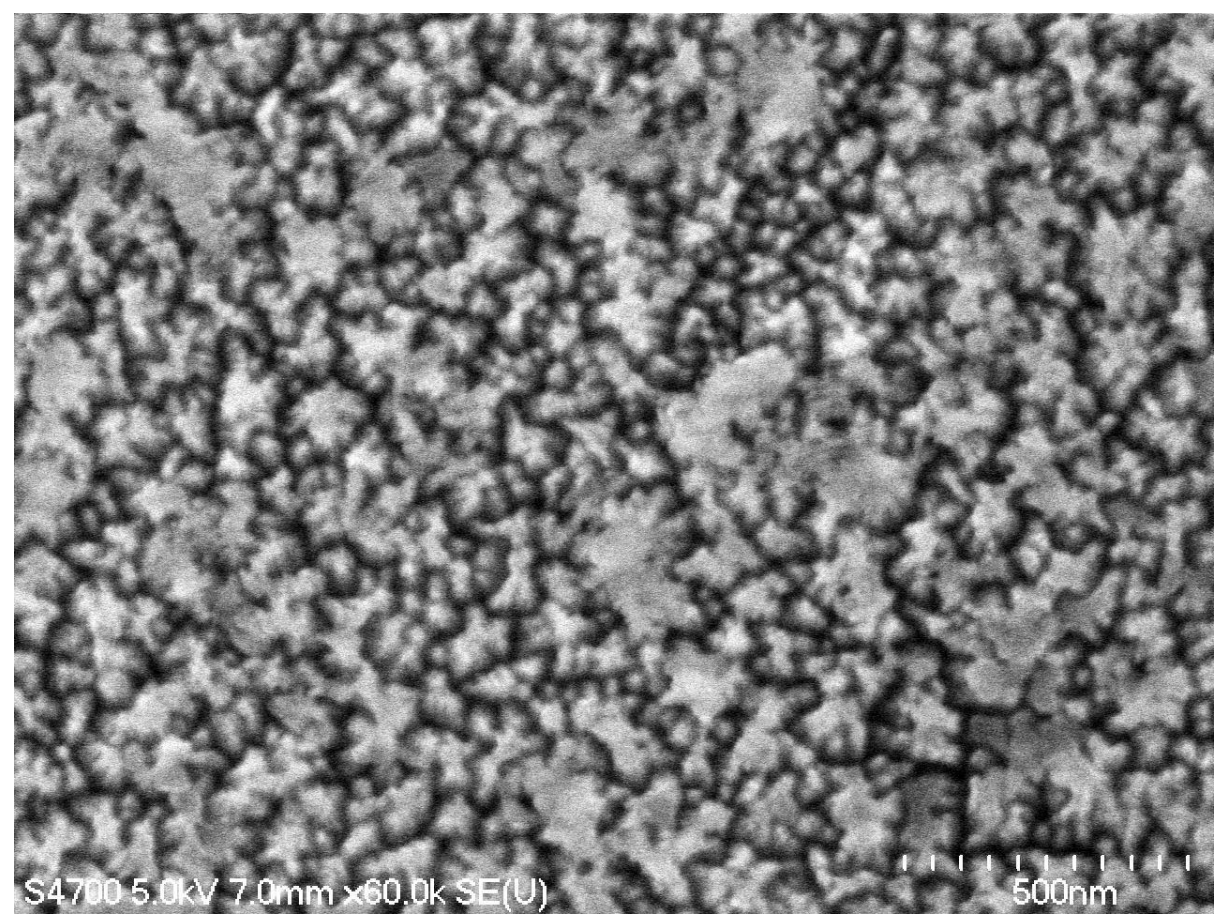

Figure 5.11(c): SEM image showing the polyslicon film surface in the region between the thermal isolation trenches. The temperature at this location was estimated to be $818^{\circ} \mathrm{C}$. The surface exhibited a mixture of small, granular features like those in (d) and larger, continuous features like those from (a) and (b).

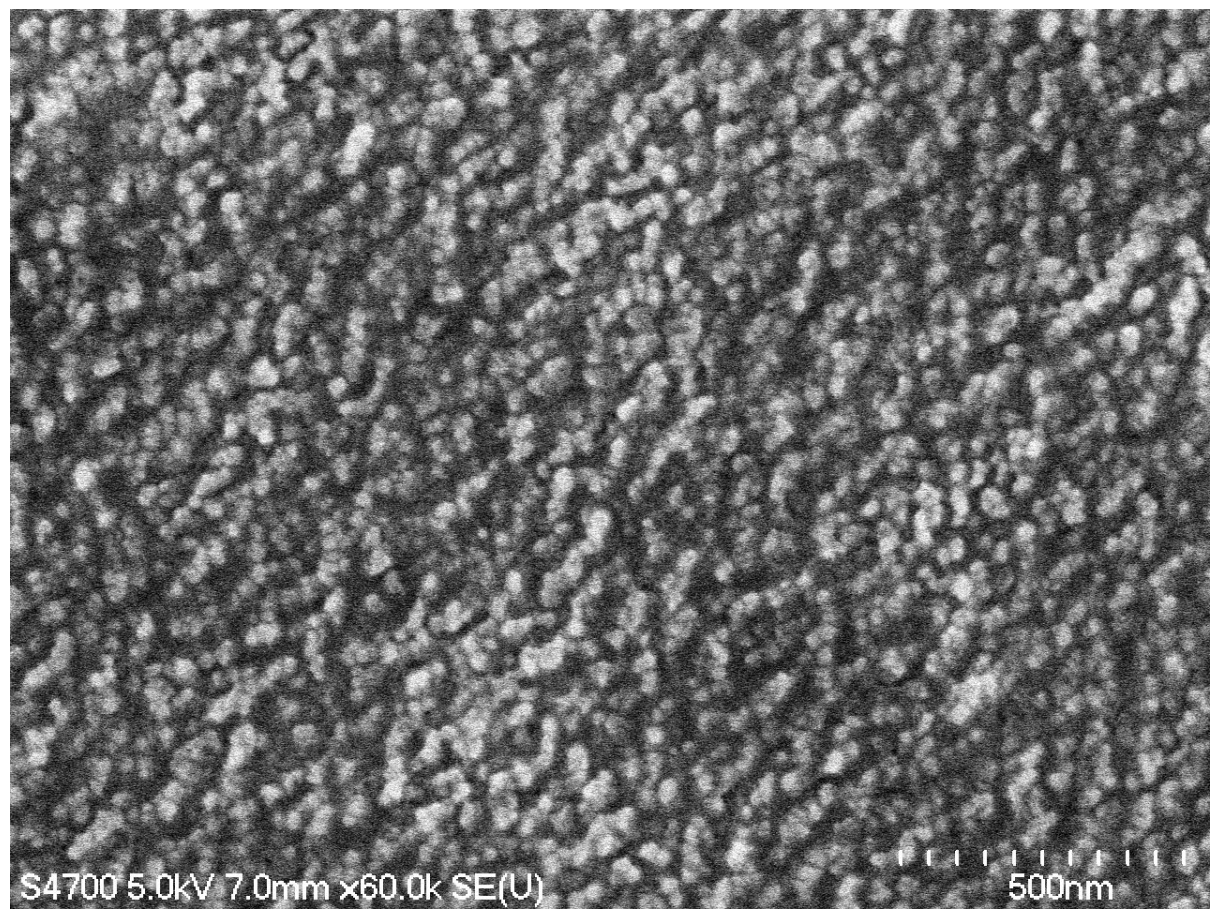

Figure 5.11(d): SEM image showing the polysilicon film surface in the region just outside of the thermal isolation trenches. The morphology had entirely taken on a fine, granular appearance, with features approximately $20 \mathrm{~nm}$ in size. The temperature at this location was estimated to be $854^{\circ} \mathrm{C}$. 
5.2. The two films were deposited at two different temperatures, and the as-deposited properties of LPCVD polysilicon are documented in the literature as being quite different in the $560-600^{\circ} \mathrm{C}$ range (ellipsoidal grains) from the $600-700^{\circ} \mathrm{C}$ range (columnar grains) [Heuer, 2000]. Thus the resulting morphology trends were expected to be quite different. The change in morphology across the $600^{\circ} \mathrm{C}$ film was sufficiently pronounced that a gradient in texture was visible over a lateral span of $\sim 15 \mu \mathrm{m}$ as is shown in Figure 5.12. The image was captured from just slightly inside the trench region, at a location near that from which Figure 5.11(b) was obtained. The temperature of heat treatment in that region had been estimated at $783^{\circ} \mathrm{C}$, and the corner-corner differential calculated to

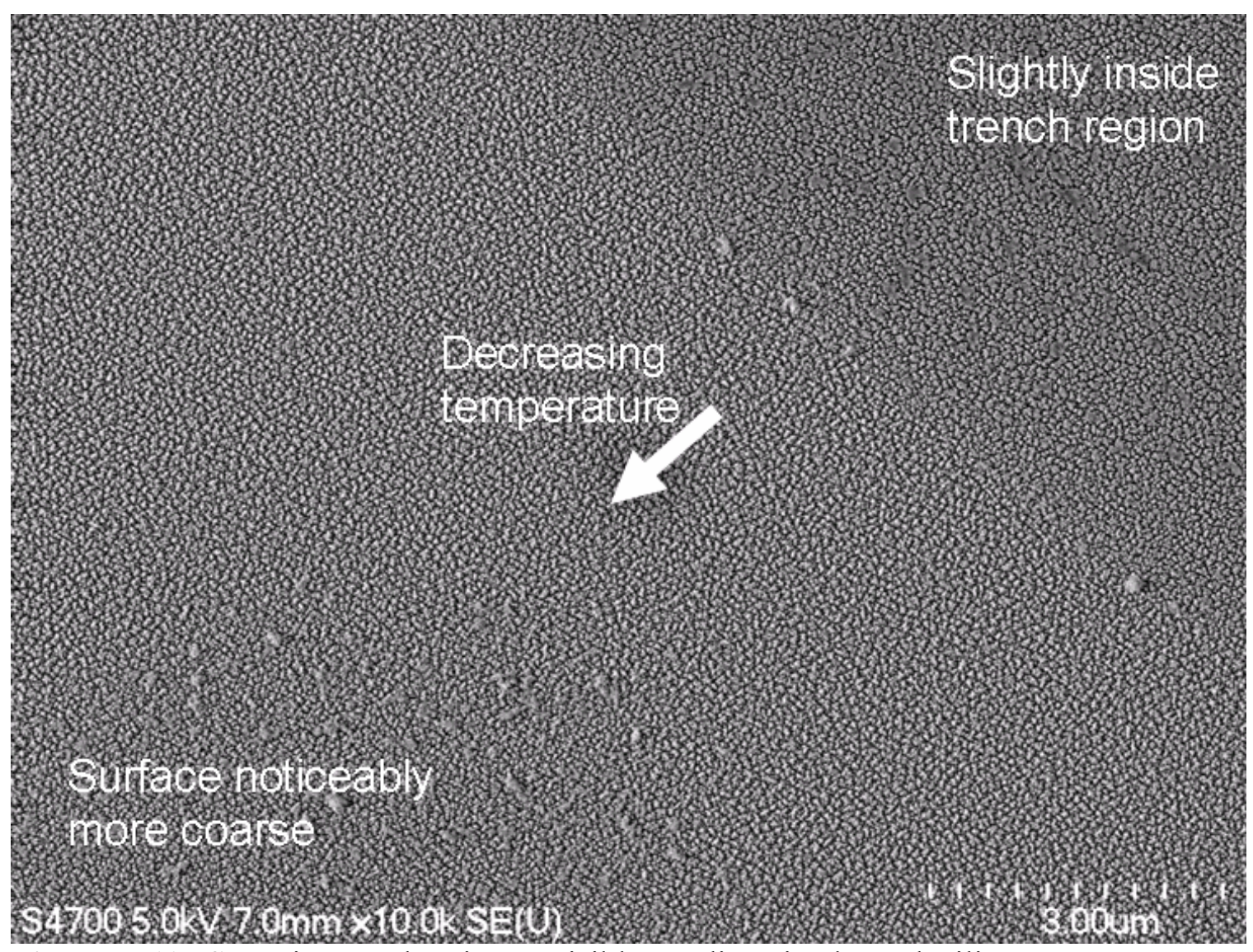

Figure 5.12: SEM image showing a visible gradient in the polysilicon texture over a $15 \mu \mathrm{m}$ lateral span. The upper right-hand corner corresponded to a location just slightly inside the trench region, which was estimated to have an annealing temperature of $783^{\circ} \mathrm{C}$. The temperature differential from corner to corner was approximately $0.1{ }^{\circ} \mathrm{C}$ 
approximately $0.1{ }^{\circ} \mathrm{C}$. As the figure illustrates, the surface texture was relatively fine at the upper right-hand corner, which was in closest proximity to the innermost trench. The lower left-hand corner, on the other hand, showed noticeably larger islands. Furthermore, the image showed multiple boundary lines parallel to the trenches, i.e., normal to the direction of heat flow. These were presumed to represent different activation energies.

Similar to the results presented in Section 5.2, quantitative comparison of initial and annealed samples via X-Ray Diffraction produced mixed results. The samples were analyzed along the same three crystallographic axes, those being [111], [220], and [311]. The values and their calculations are summarized in Table 5.4, with the diffraction waveforms given in Figures 5.13(a,b). As was the case for the analysis in the previous section, the analyzed sample contained the full interior region of the NiFe19 film and thus contained a spectrum of annealing results. The annealed polysilicon exhibited an increase in crystallite size along the [220] and [311] orientations, but showed a slight

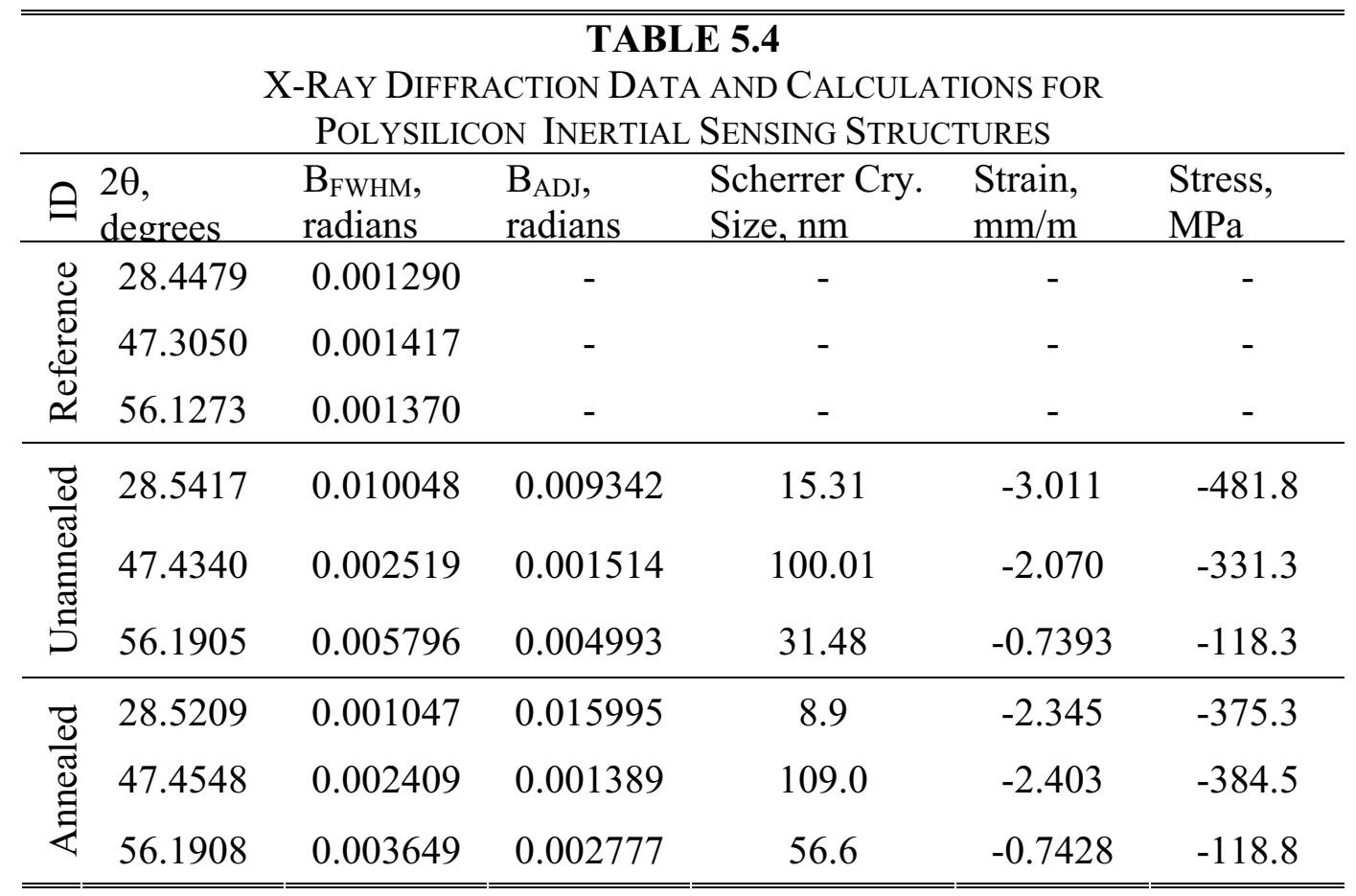




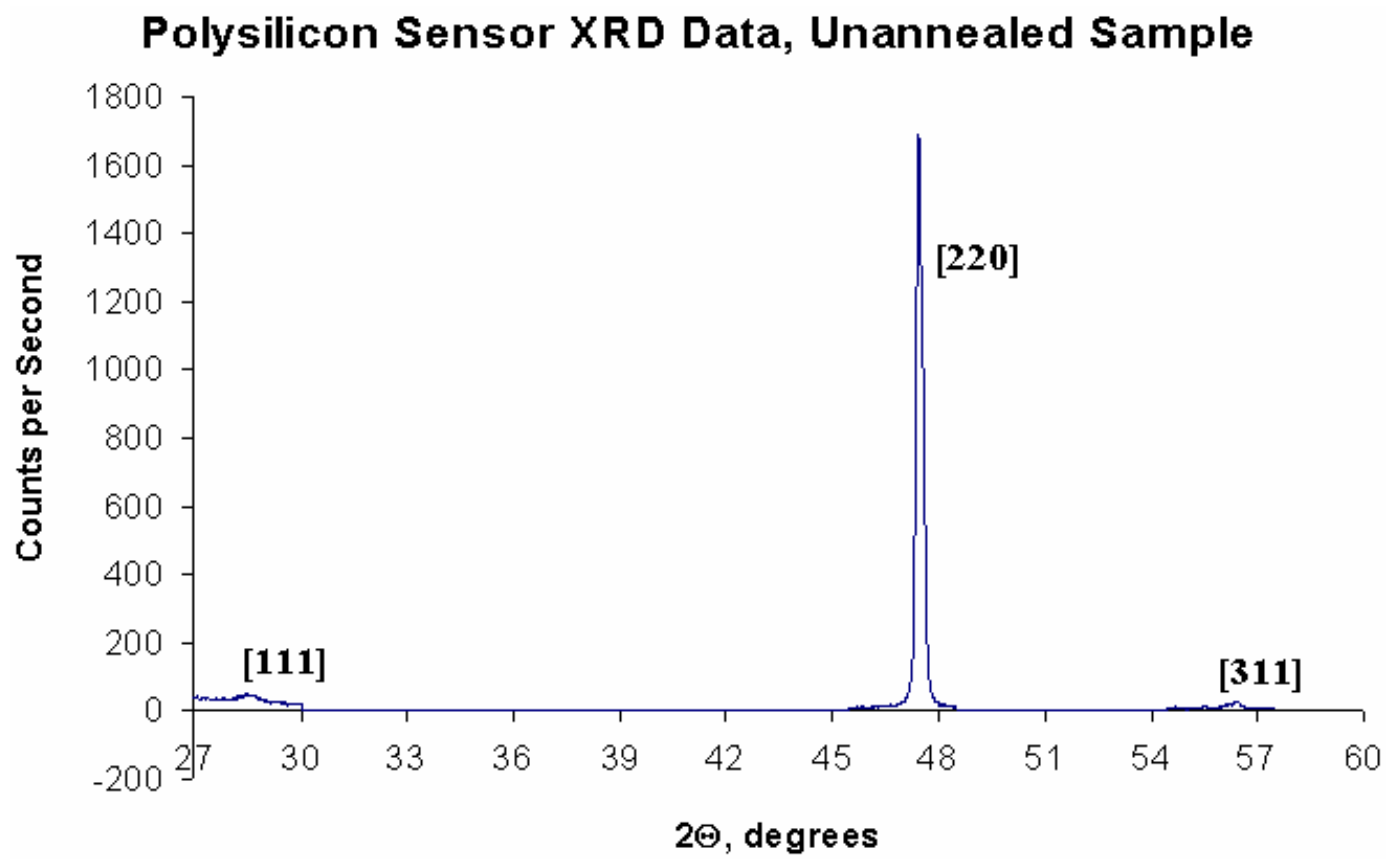

Figure 5.13(a): X-Ray Diffraction output plot obtained from the as-deposited $3 \mu \mathrm{m}$ LPCVD polysilicon, which was formed at $600^{\circ} \mathrm{C}$ and patterned to form inertial sensing structures. The three dominant grain orientations were [111], [220], and [311] as in the previous samples.

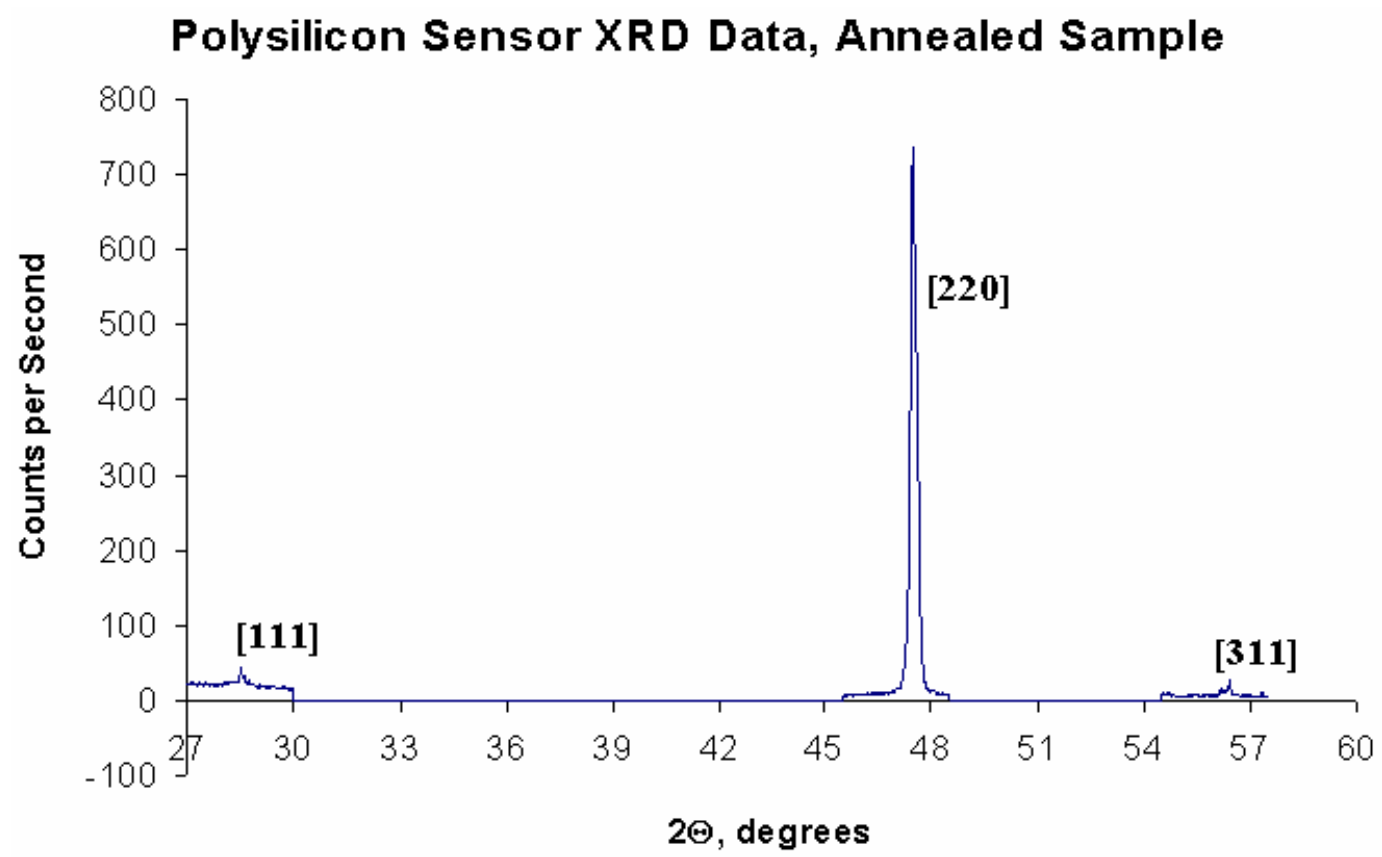

Figure 5.13(b): X-Ray Diffraction output plot showing the impact of the $760-865^{\circ} \mathrm{C}$ anneal on the polysilicon grain structure. Like the cantilever beam polysilicon of Section 5.2, the [111] and [311] crystallites increased in size while those in [220] were made slightly smaller. The stress in the [111] grains became less compressive, but that in [220] increased somewhat and that in [311] showed a negligible change. 
decrease along the [111] orientation. On the other hand, the calculated stress in the [111] grains became $22.2 \%$ less compressive, but that in [220] actually showed a $16.3 \%$ increase while that in [311] registered a negligible change. The partial anneal appeared to have created a scenario in which the [220] grains were dominant but unable to grow significantly due to having insufficient energy to cause reversal in the other grains, thus becoming even more compressively stressed. The key result, however, was the repeated creation of a controlled morphology change on a polysilicon structure. The expectation was that with further research, methods for improvement would be revealed.

\section{Conclusion}

This chapter presented the application of the process development work of Chapters 3 and 4 to the annealing of polysilicon films and microstructures. Induced temperatures sufficient for annealing were measured using infrared pyrometry and demonstrated through changes in film curvature, appearance of surface texture, and crystallite size and stress. Further optimization of the technology will help to increase the range of sustainable annealing temperatures. Emphasis must be placed on increased coupling to the ferromagnetic films and decreased thermal conduction to the silicon substrate. Furthermore, increasing the annealing uniformity within the polysilicon is also of high importance. Forming the ferromagnetic film directly above the structure, with a thin diffusion barrier such as $\mathrm{SiO}_{2}$ between them, would provide the ideal situation but would also require that the thermally-induced stresses in the metal be reduced significantly so as not to cause device tear-out. 


\section{Chapter 6: Device Design and Fabrication}

The application of a new technology to the production of fully functional micromechanical devices presents a more complex set of challenges than those typically encountered with basic test structures. Whether or not obstacles can be overcome without compromising flexibility in process or design will establish its actual utility. This chapter presents the first iteration in the development of a CMOS-compatible high aspect ratio (HAR) device technology based on the inductive annealing process. The lateral accelerometer was selected for the purpose of technology demonstration. This class of sensors is of high importance to the microsystems industry and has applications ranging from automotive safety to consumer electronics. While individual designs are quite unique, lateral accelerometers generally resemble that of Figure 6.1(a) in which a series of electrodes are attached to either side of a moving seismic mass, whereas vertical or zaxis accelerometers are often similar to Figure 6.1(b) in which the movable electrode is formed from lower surface of the seismic mass. The chapter begins with the presentation

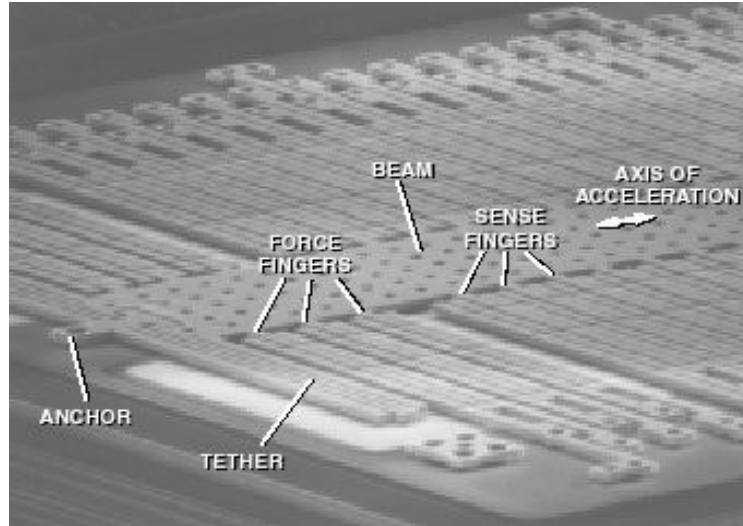

(a)

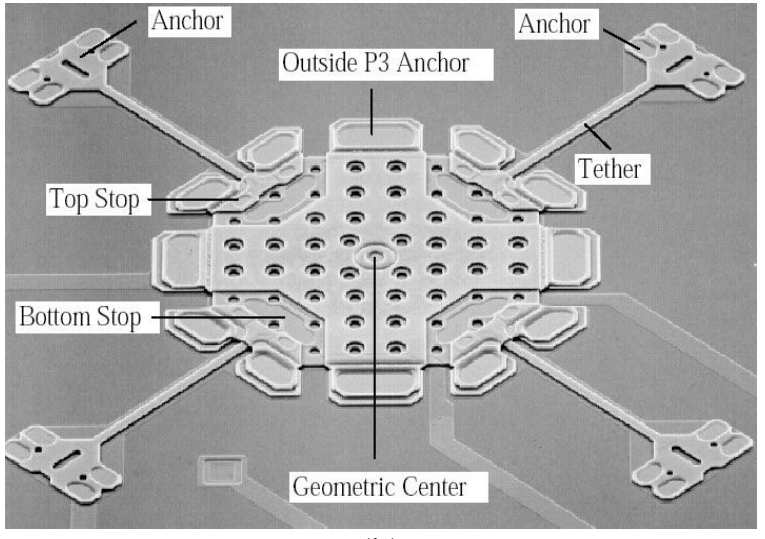

(b)

Figure 6.1: SEM images of industrial inertial sensors illustrating the main features of (a) the Analog Devices ADXL150 50g lateral accelerometer [Samuels, 1996 ${ }^{1}$ ] and (b) the Motorola 50g z-axis accelerometer [Li, et al., $2001^{2}$ ].

${ }^{1}$ Courtesy of Analog Devices, Inc.

2 C2001 IEEE 
of the definitions and equations relevant to device design in Section 6.1. Section 6.2 details the design process including the selection of the layers to be utilized and their targeted thicknesses, the specification of design criteria, and the accelerometer layout. The next three sections are devoted to the finite element modeling of the device using the Coventorware software, beginning with an overview of the model generation process (Section 6.3) and then examining the details of the independent electrical (Section 6.4) and mechanical (Section 6.5) simulations. Finally, images and measurements from the actual fabrication and device characterization are given in Section 6.6. Although the intent had been to utilize the sensor as a means by which to demonstrate the application of the inductive annealing technology on a full device, processing challenges were encountered that ultimately rendered the devices non-functional. These challenges are elaborated in the sixth section, along with recommendations for improvement.

\section{1: Design Considerations}

The detection of a change in velocity, whether linear or rotational, entails the conversion of a mechanical force into an electrical signal. The block diagram in Figure 6.2 illustrates the basic operation of a capacitive accelerometer, a linear inertial sensor that transduces motion by means of a variable capacitance. Accelerometers consist of a movable proof mass anchored to a substrate through a flexible suspension, with viscous damping often provided through a pressurized ambient. The inherent inertia of the mass causes it to resist an applied acceleration (or deceleration) according to Newton's second law [Elwenspoek, et al., 2001 (p. 133)],

$$
F_{A}=m a,
$$


where the inertial force, $F_{A}(\mathrm{~N})$, increases with the magnitude of the mass, $m(\mathrm{~kg})$, of the structure upon which the acceleration, $a\left(\mathrm{~m} / \mathrm{s}^{2}\right)$, is applied. The result is that the structure shifts laterally with respect to the substrate. Its range of motion is determined by the rigidity of the suspension according to Hooke's law [Senturia, 2001 (p. 112)],

$$
F_{S}=-k_{Z} \Delta z
$$

in which the spring constant, $k_{Z}(\mathrm{~N} / \mathrm{m})$, determines the strength of the restoring force, $F_{S}$ $(\mathrm{N})$, produced for a given deflection, $\Delta z(\mathrm{~m})$. The springs, or tethers, utilized in micromachined accelerometers typically consist of combinations of simple beams like those in Figure 6.1. Values for their factors are approximated through standard beambending equations, with the two most relevant forms derived from the cantilever beam,

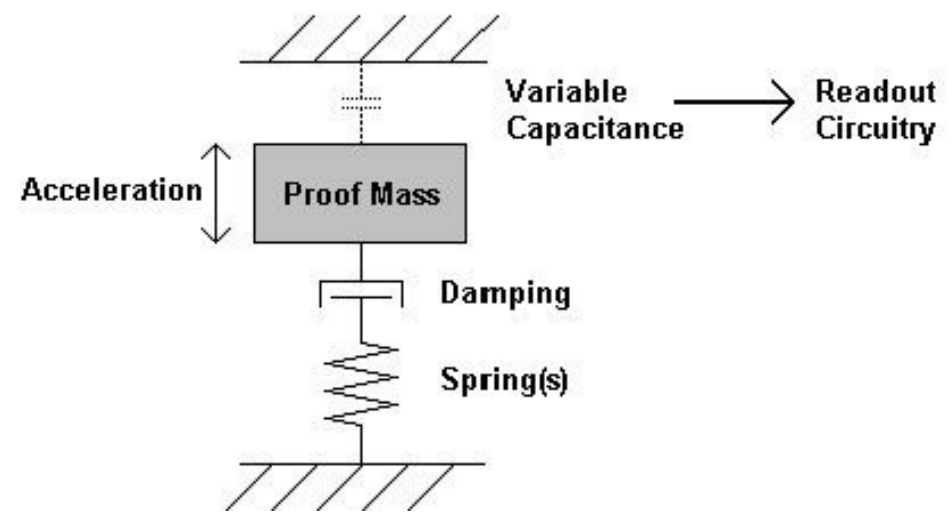

Figure 6.2: Block diagram showing the primary components and transduction method of a typical capacitive linear accelerometer. An applied acceleration causes a shift in a movable proof mass with respect to the substrate; the change in capacitance between mobile and stationary electrodes is detected by customized readout circuitry.

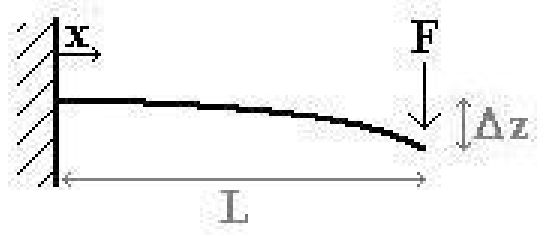

(a)

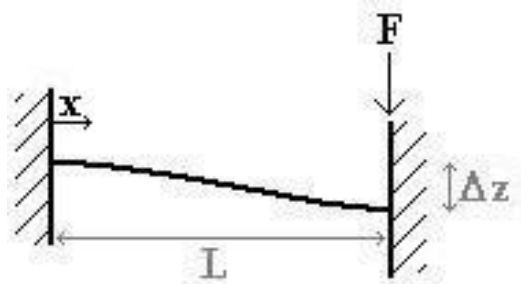

(b)

Figure 6.3: Beam-bending diagrams illustrating (a) the simple cantilever beam and (b) the double-clamped beam under a concentrated load, $F$, applied at $x=L$. 
shown in Figure 6.3(a), and the double-clamped beam, shown in Figure 6.3(b). Equations for the tip deflection under a concentrated load can be found using the Euler-Bernoulli equation [Eibeck, et al., 2000; Senturia, 2001 (p. 215)] for the bending of thin beams,

$$
\frac{d^{2} z}{d x^{2}}=\frac{M}{E I},
$$

where the second derivative of the deflection, $\Delta z(\mathrm{~m})$, with respect to the distance from the support, $x(\mathrm{~m})$, is a function of the internal moment at $x, M\left(\mathrm{~N}^{*} \mathrm{~m}\right)$, the Young's Modulus of the constituent material, $E\left(\mathrm{~N} / \mathrm{m}^{2}\right.$ or $\left.\mathrm{Pa}\right)$, and the area moment of inertia of the beam cross-section, $I\left(\mathrm{~m}^{4}\right)$. Free-body diagrams for the cases of Figures 6.3(a) and 5.3(b) yield the respective bending moments

$$
M=F(L-x) \quad M=F(L-x)+M_{L}
$$

in which the term $M_{L}$ in the latter equation refers to the reaction moment at the moving support, and the boundary conditions for each scenario are

$$
\begin{array}{ll}
y(0)=0 & y(0)=0 \\
\left.\frac{d y}{d x}\right|_{x=0}=0 & \left.\frac{d y}{d x}\right|_{x=0}=0 . \\
\left.\frac{d y}{d x}\right|_{x=L}=0
\end{array}
$$

Solving the differential equation produces the following results for the deflection at $x=L$ :

$$
\Delta z=\frac{L^{3}}{3 E I} F \quad \Delta z=\frac{L^{3}}{12 E I} F .
$$

The area moment of inertia of a rectangular cross-section about the central axis is given by [Spiegel, et al., 1994 (p. 133)]

$$
I=\frac{W H^{3}}{12},
$$


where $W$ and $H$ represent the width (y-dimension) and height (z-dimension) of the beam, respectively. Substituting (5.7) into (5.6a,b) and solving for the spring constant $k_{Z}$ using (5.2) gives the final result:

$$
k_{Z}=\frac{E W H^{3}}{4 L^{3}} \quad k_{Z}=\frac{E W H^{3}}{L^{3}} .
$$

Note that $(5.8 \mathrm{a}, \mathrm{b})$ refers to the spring constant for a beam bending in the vertical $(\mathrm{z})$ direction. In the case of a lateral accelerometer, deflection occurs in the horizontal (y) direction and therefore the terms $W$ and $H$ must be exchanged in order to calculate $k_{Y}$. Springs such as those shown in Figure 6.1 simultaneously derive behaviors from each of the two different beam-bending models. Numerical results are typically closer to those of the double-clamped beam due to the fact that the "free" ends are somewhat constrained by either a mostly-rigid proof mass or the semi-rigid bends that attach additional beams to make up a folded spring. Once approximate values for the individual beams have been obtained, complex spring designs can be characterized through series and/or parallel combinations of single-beam springs. It should be understood that the results are suitable for initial design purposes only as it is difficult to estimate the effects of elasticity in spring folds and the proof mass, and consequently simulation through finite element modeling or other means is strongly recommended.

Several important aspects of device behavior are directly affected by the values of $m$ and $k$ that result from the chosen design, beginning with the range of accelerations over which it operates. The sensitivity of the device is defined as its deflection for a given acceleration, or [Gad-el-Hak, 2002 (p. 24-6)]

$$
S=\frac{\Delta y}{a} .
$$


Under "quasi-static" conditions, the device responds to the input stimulus with minimal delay, indicating relatively low damping and a sufficiently large resonant frequency so as to minimize interference with sensor operation [Senturia, 2001 (p. 499)]. This yields the relationship

$$
F_{A}=F_{S}
$$

and therefore

$$
S=\frac{m}{k} .
$$

The differential equation modeling the sensor gives the resonant frequency as

$$
\omega_{0}=\sqrt{\frac{k}{m}} .
$$

which can also be written in the form

$$
\omega_{0}=\sqrt{\frac{a}{\Delta y}} .
$$

Therefore, it is the ratio between $k$ and $m$ and not their actual values that defines these primary characteristics of device operation. Additional parameters such as quality factor and noise performance also rely on these two values as well as the coefficient of viscous damping. This term is utilized to obtain a specific transient behavior. Sensors are typically designed such that they operate as close to critical damping as possible with moderate overdamping in order to avoid any overshoot or ringing that can increase the time required for stabilization and potentially cause false triggering. A thorough analysis of damping coefficients and sensor transients is given in The MEMS Handbook [Gad-elHak, 2002 (pp. 24-7 - 24-12]. Viscous damping is not a critical factor for the device design under review and therefore will not be discussed further. 
At the present time, capacitance-based sensing is the method of choice for industrial sensor manufacturing. Many of these devices are utilized in automotive applications and are therefore subject to a wide range of thermal ambient conditions. Capacitive sensors are relatively immune to temperature changes, especially when compared with other methods such as piezoresistive, piezoelectric, electron tunneling, etc, and are furthermore inherently simple. Their means of operation makes use of movable and stationary electrodes, with the former attached to the proof mass and the latter fixed to the substrate (though electrically isolated). The distance between the two sets of electrodes, $d(\mathrm{~m})$, changes as the structure moves, which affects the overall capacitance as approximately [Senturia, 2001 (p 126)]

$$
C=\frac{\varepsilon A}{d}
$$

where the capacitance, $C(\mathrm{~F})$, depends also on the permittivity (or dielectric constant) of the gas within the gap, $\varepsilon(\mathrm{F} / \mathrm{m})$, and the electrode surface area, $A(\mathrm{~m})$, on either side of the gap. This is the standard equation for a parallel-plate capacitor, which neglects the contribution of fringing fields at the perimeter. The calculation of an accurate fringing capacitance tends to be somewhat difficult, and various complex equations exist with varying degrees of accuracy under different conditions [Leus, et al., 2004]. A simple estimate can be obtained, however, by adding the gap distance to that of the electrodes, thus giving effective length and width values that are each larger by a factor of $2 d$ [Calvert, 2003]. Lateral accelerometers typically incorporate numerous electrodes for both the moving and stationary signals, with the individual components connected together to act as one large capacitor in order to maximize sensing resolution. A subset of the capacitive classification of devices utilizes differential capacitive sensing in which 


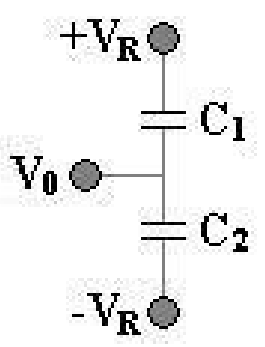

Figure 6.4: Schematic of a differential capacitor in which the output voltage $\mathrm{V}_{0}$ is measured at the moving plate of the sensing structure and the input signals $+V_{R}$ and $-V_{R}$ are applied to each of the two fixed electrodes.

two sets of stationary electrodes are fabricated, with one fixed electrode on each side of every moving electrode. The basic configuration of such a device is illustrated in Figure 6.4. The moving structure provides the center plate from which the output voltage $V_{0}$ is measured, and the two stationary electrodes on opposite sides of the proof mass form capacitors $C_{1}$ and $C_{2}$ to which a reference voltage $+V_{R}$ and $-V_{R}$ are applied. The advantage of such a design can be seen from the characteristic equation for a differential capacitor [Senturia, 2001 (p. 502)],

$$
V_{0}=\frac{C_{1}-C_{2}}{C_{1}+C_{2}} V_{R}=\frac{d_{2}-d_{1}}{d_{2}+d_{1}} V_{R}
$$

in which the simplification is made using the assumption that the surface area values for $C_{1}$ and $C_{2}$ are the same. At the unstressed position of the structure, the values of $d_{1}$ and $d_{2}$ are identical and the output voltage is zero. As the structure moves and the values of $d_{1}$ and $d_{2}$ change, one of the two capacitances will increase while the other decreases, but the sum $d_{2}+d_{1}$ always remains consistent. Therefore, the output voltage is a scaled fraction of the reference voltage, linear in $d_{2}-d_{1}$, with the sign reflecting the direction of the shift. This is the approach that was selected for the sensor presented in this chapter. Specific parameters and dimensions are detailed in the next section. 
A final consideration for device design is the means by which it will be tested. Confirmation of proper performance over the chosen acceleration range as well as robustness under cyclic loading, thermal stresses, and impact shock requires sensors to undergo a wide range of validation procedures. Providing each device with self-test capability enables a rapid check without requiring substantial time or instrumentation, allowing many faulty sensors to be quickly discarded. Self-testing of a lateral inertial sensor is typically done by incorporating a dedicated set of stationary electrodes. The application of a DC voltage generates an electrostatic force between the capacitive plates that causes the structure to deflect. Equilibrium is reached when the counter-force of the springs balances the capacitive force, or [Senturia, 2001 (pp. 132-137)]

$$
k \Delta y=\frac{1}{2 d} C V^{2} .
$$

The shift can then be detected using the sense electrodes in order to verify that the output capacitance change is within the proper range. Nearly all devices are provided with some type of self-testing means. Although passing self-test doesn't guarantee functionality, it does give evidence of proper formation of the structure and interconnects, spring constant values, and thorough release without stiction.

\section{2: Process Development and Device Design}

The design of a device begins with the definition of a fabrication process. While the details of the process are subject to change as the device is tested and refined, the initial version serves as a starting point in which parameters are chosen such as proof mass thickness and number of interconnect layers. The MTU high aspect ratio (HAR) accelerometer process revolves around a $20 \mu \mathrm{m}$-thick polysilicon structure with a $2 \mu \mathrm{m}$ 
minimum feature size. The fabrication sequence is illustrated in Figures 6.5(a-g). It begins with 4" silicon wafers purchased with $1 \mu \mathrm{m}$ of thermally grown silicon dioxide on the front and back sides. A silicon nitride layer, targeted at $0.25 \mu \mathrm{m}$, provides a base layer for the device as the oxide would not withstand the final release etch. The wafer crosssection at this point in the fabrication is shown in Figure 6.5(a). Signal transport below the structure utilizes a $0.5 \mu \mathrm{m}$ heavily doped polysilicon interconnect layer (poly0), which also is used to fabricate a sub-structural ground plane to electrostatically bias the region as is illustrated in Figure 6.5(b). This is followed with a $2 \mu \mathrm{m}$-thick poly0-poly1 silicon dioxide inter-layer dielectric, shown in Figure 6.5(c), whose patterning not only defines vias for electrical signals but also the structural anchor points. The heavily doped $20 \mu \mathrm{m}$ polysilicon structural layer (poly1) is shown in Figure 6.5(d) and is used to form the sensing structure. It also provides for the transfer of signals between the upper and lower interconnects. The $2 \mu \mathrm{m}$ minimum feature size assigned to the process is primarily applied to this layer and dictates the sizes and shapes of features such as springs, etch holes through the structure to facilitate device release, and the gap between sensing electrodes. A second $2 \mu \mathrm{m}$ silicon dioxide layer, poly1-metall, refills the trenches in the patterned poly1 layer and defines via locations for the upper interconnect layer as shown in Figure 6.5(e). It is at this point that the inductive annealing process takes place if desired, consisting of the deposition, patterning, heating, and removal of a ferromagnetic film such as $\mathrm{FeCo}$ or $\mathrm{NiFe}_{19}$. Finally, a $0.5 \mu \mathrm{m}$ aluminum film (metal1), shown in Figure 6.5(f), is deposited and patterned to form the upper interconnect layer which is comprised of the connections to the individual sensing electrodes as well as the peripheral bonding 
pads. Device release is accomplished through the timed sacrificial etch of the poly1metall and poly0-poly1 oxides. The etch must be sufficiently long so as to ensure release

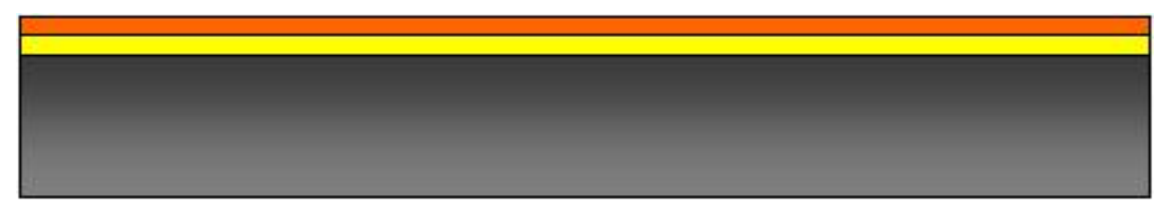

Figure 6.5(a): Silicon wafer (four-inch diameter, $0.25 \mathrm{~mm}$ thickness) with $1 \mu \mathrm{m}$ thermal silicon dioxide layer for minimal nitride stress and $0.25 \mu \mathrm{m}$ LPCVD silicon nitride foundation.

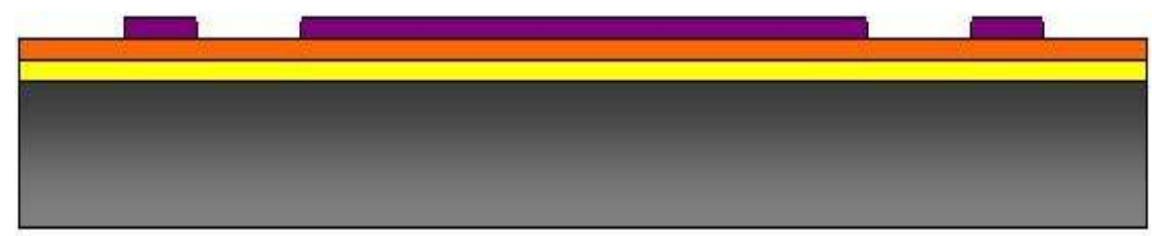

Figure 6.5(b): Device wafer following deposition and patterning of poly0 lower interconnect layer. Outer portions represent electrical traces while the center region reflects the ground plane below the structure.

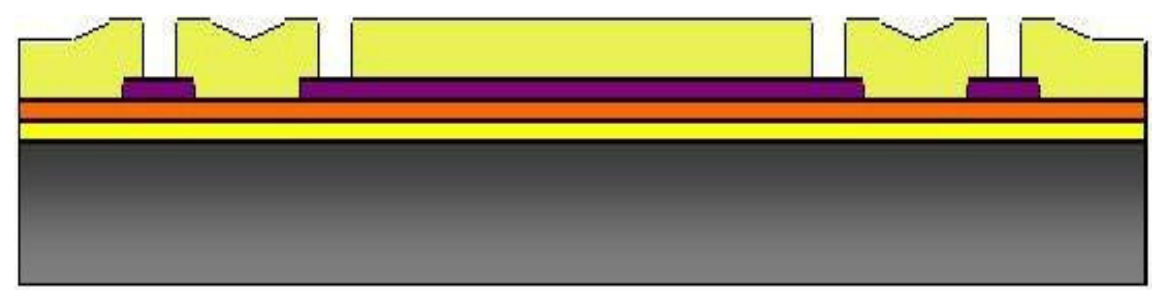

Figure 6.5(c): Device wafer following deposition and patterning of the poly0-poly1 dielectric. Vias provide locations for electrical signal transport as well as structural anchors.

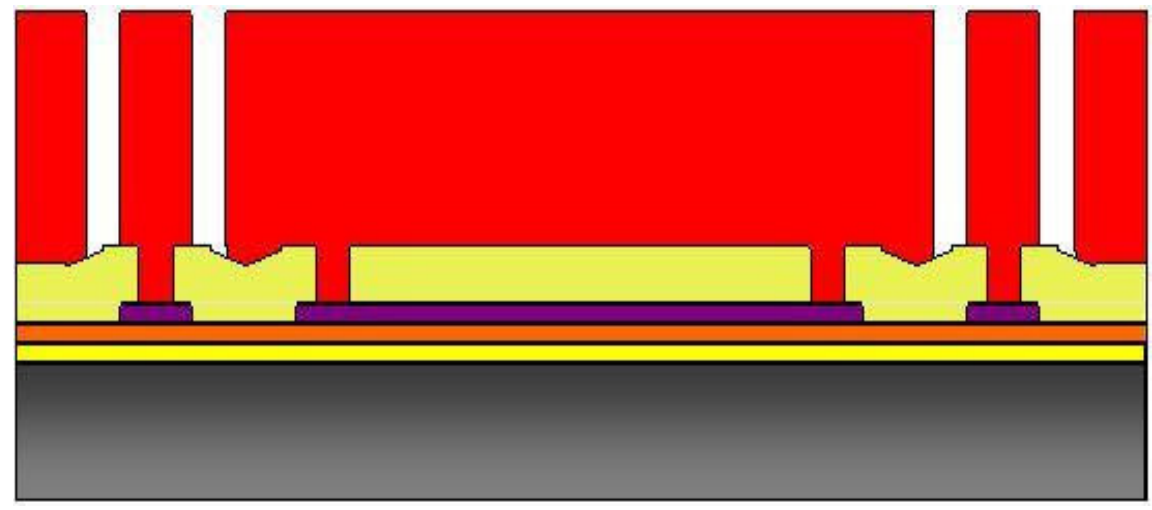

Figure 6.5(d): Device wafer following deposition and patterning of the poly1 structural layer. The center region shows the sensing structure while the surrounding studs reflect the use of the polyl layer to transfer electrical signals between the lower and upper interconnects. 


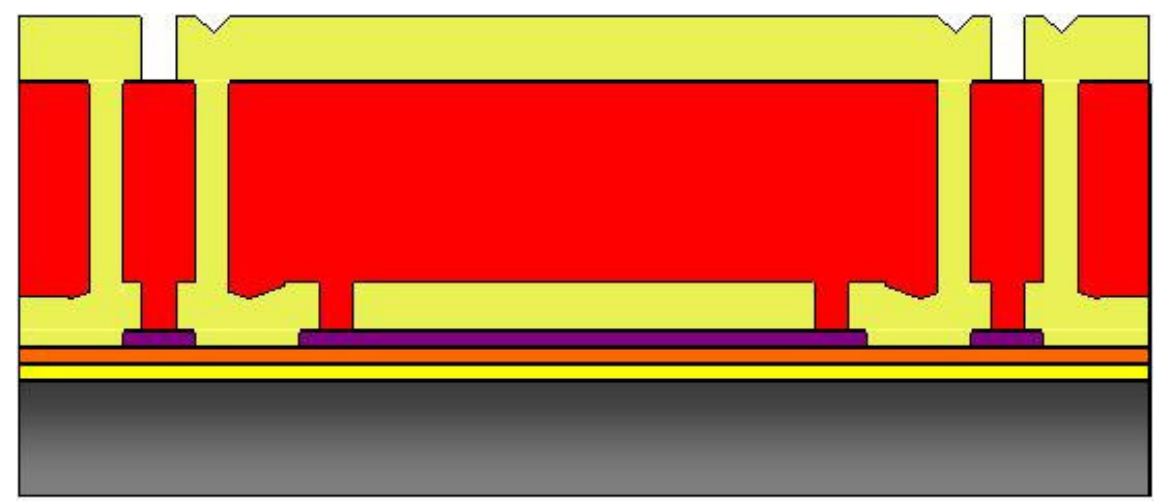

Figure 6.5(e): Device wafer following deposition and patterning of the polyl-metall dielectric. The silicon dioxide layer provides for insulation and vias between the two conducting layers as well as structural support for the metall bonding pads and thick ground traces.

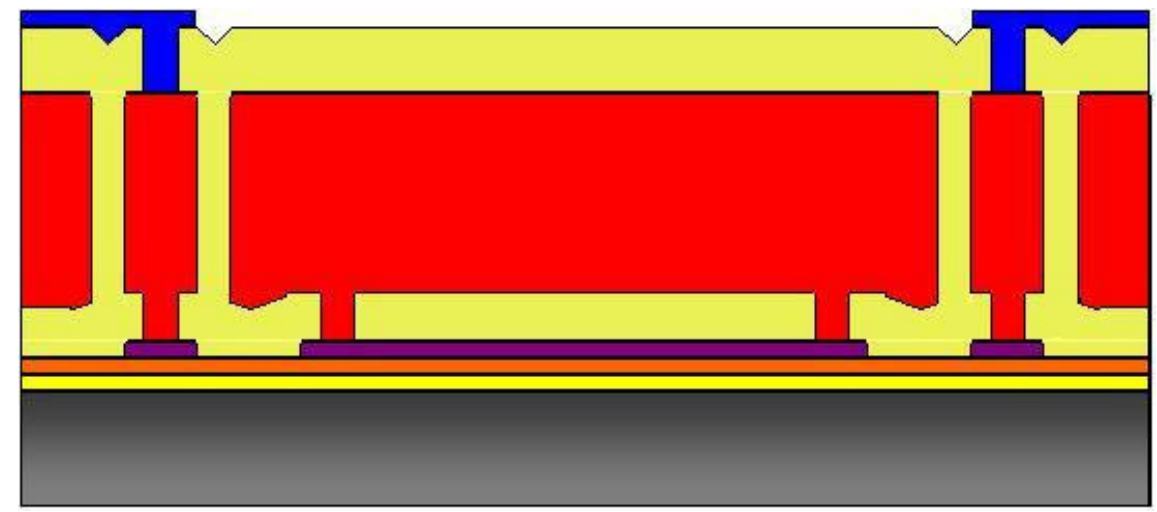

Figure 6.5(f): Device wafer following deposition and patterning of the metall1 upper interconnect layer. This layer provides bonding pads, thick ground traces, and airbridges between stationary electrodes.

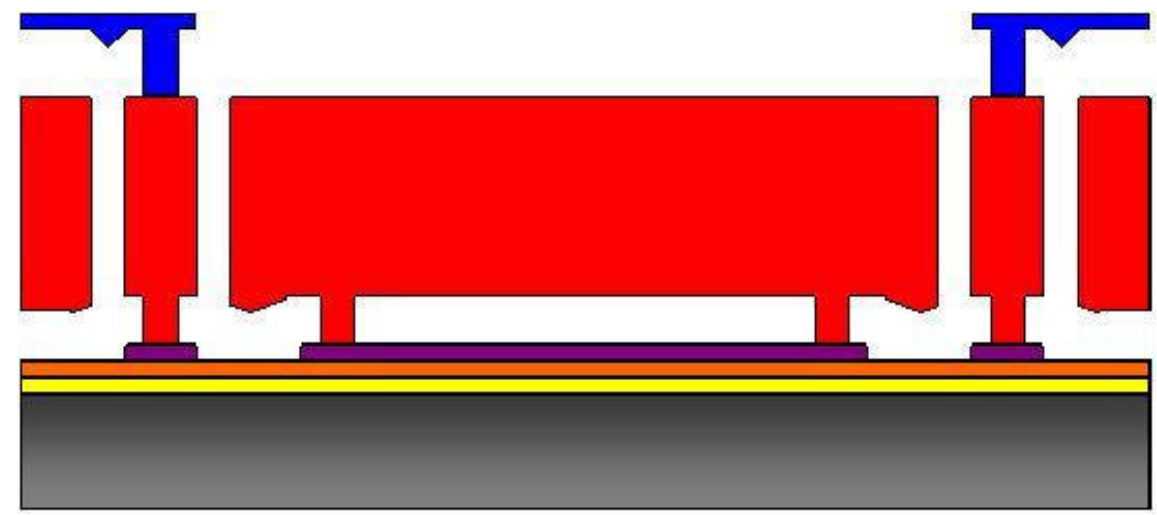

Figure 6.5(g): Device wafer following the sacrificial etch for structural release.

of the full device but not so long that significant undercut of the surroundings occurs. More specific details of the actual fabrication process, including film measurements, 
equipment used, and in-progress images are provided in Section 6.6. The detailed process flow is provided in Appendix C, both with (C.2) and without (C.1) the insertion of the inductive annealing procedure.

Device design was initiated in the Spring of 2003 as part of the EECS425 Semiconductor Fabrication course offered remotely through the University of Michigan, designated as EE5900 at MTU. The sensor was initially targeted to have a maximum acceleration of $+/-2 g$, where $g$ represents the earth's gravitational accelerating force, $9.8 \mathrm{~m} / \mathrm{s}^{2}$. The value of the gap spacing, $d$, between the capacitor electrodes was given by the minimum feature size for the fabrication process, $2 \mu \mathrm{m}$. Suggestions made by Dr. Bishnu Gogoi of Freescale Semiconductor included a nominal capacitance of around 1pF, a full-load capacitance change from $7.5-15 \%$ of the nominal value, an overall spring constant greater than $1.2 \mathrm{~N} / \mathrm{m}$ to minimize stiction-related problems, and self-test capability. Similar to the structures utilized in Freescale Semiconductor lateral accelerometers, the device would have four springs located internally to the proof mass. The final design had a proof mass with an interior region $994 \times 690 \mu \mathrm{m}$ in size and $2 \times 6 \mu \mathrm{m}$ etch holes staggered throughout the structure with $6 \mu \mathrm{m}$ spaced between them. The etch holes consumed approximately $12.5 \%$ of the structure, reducing the effective area from $0.686 \mathrm{~mm}^{2}$ to $0.600 \mathrm{~mm}^{2}$. For the electrodes, length and width values of $120 \mu \mathrm{m}$ and $6 \mu \mathrm{m}$, respectively, were assigned. Each side of the structure was given 36 sensing electrodes and three self-test electrodes for a total of 78 , adding $0.0562 \mathrm{~mm}^{2}$ to the structural area. Multiplying by the $20 \mu \mathrm{m}$ thickness dictated by the fabrication process gave an effective volume of $0.0131 \mathrm{~mm}^{3}$ and, using the typical polysilicon density value of $2331 \mathrm{~kg} / \mathrm{m}^{3}$ given in the Senturia textbook [Senturia, 2001 (p. 196)], the total mass of the structure 
calculated to $3.06 \mathrm{E}-8 \mathrm{~kg}$. Combining the electrode dimensions with the $2 \mu \mathrm{m}$ gap spacing resulted in a capacitance of $10.6 \mathrm{fF}$ per electrode pair for a total nominal capacitance of $0.765 \mathrm{pF}$, neglecting fringing effects. An estimate of the total capacitance, including fringing, can be obtained by adding the gap spacing to the length and height parameters as was suggested in Section 6.1, which predicted an actual value of $0.948 \mathrm{pF}$. Four different styles of springs were designed in order to examine some different ideas. The final designs yielded spring constant calculations that ranged from $1.19 \mathrm{~N} / \mathrm{m}$ to $4.42 \mathrm{~N} / \mathrm{m}$ per spring (this is multiplied by four for the overall spring constant), causing the theoretical sensing range of the four different accelerometers to vary from $2.38 g$ to $8.84 g$ in order to produce the necessary $7.5 \%$ minimum sensitivity. The resonant frequencies ranged from $1.99 \mathrm{kHz}$ to $3.83 \mathrm{kHz}$. Self-testing was designed such that a $5 \mathrm{~V}$ input would shift the structure by approximately $50 \%$ of the full sensing range for the nominal $2 \mathrm{~g}$ device. Substituting the design specifications into Equation (5.15) led to the selection of six self-test electrodes, or three on each side of the sensor. With the actual spring constant values, the shift would instead fall between $55.8 \%$ and $15.0 \%$, depending on the spring type, which meant that a larger voltage would likely be utilized in the testing of the stiffer devices. Thus the final sensor designs spanned a fairly broad scope, but device modeling gave a preliminary indication that each of the four would be functional. Simulation results and images obtained using Coventorware are presented in Sections 5.4 and 5.5 with detailed discussions of the specific aspects of each model.

Figures 6.6(a-c) present images from the final layout of the sensor. The first figure shows the full $2 \times 2 \mathrm{~mm}$ chip including the polysilicon device layer (red), bonding pads (dark blue), ground traces (dark blue), poly0 interconnects (white), and the overall 


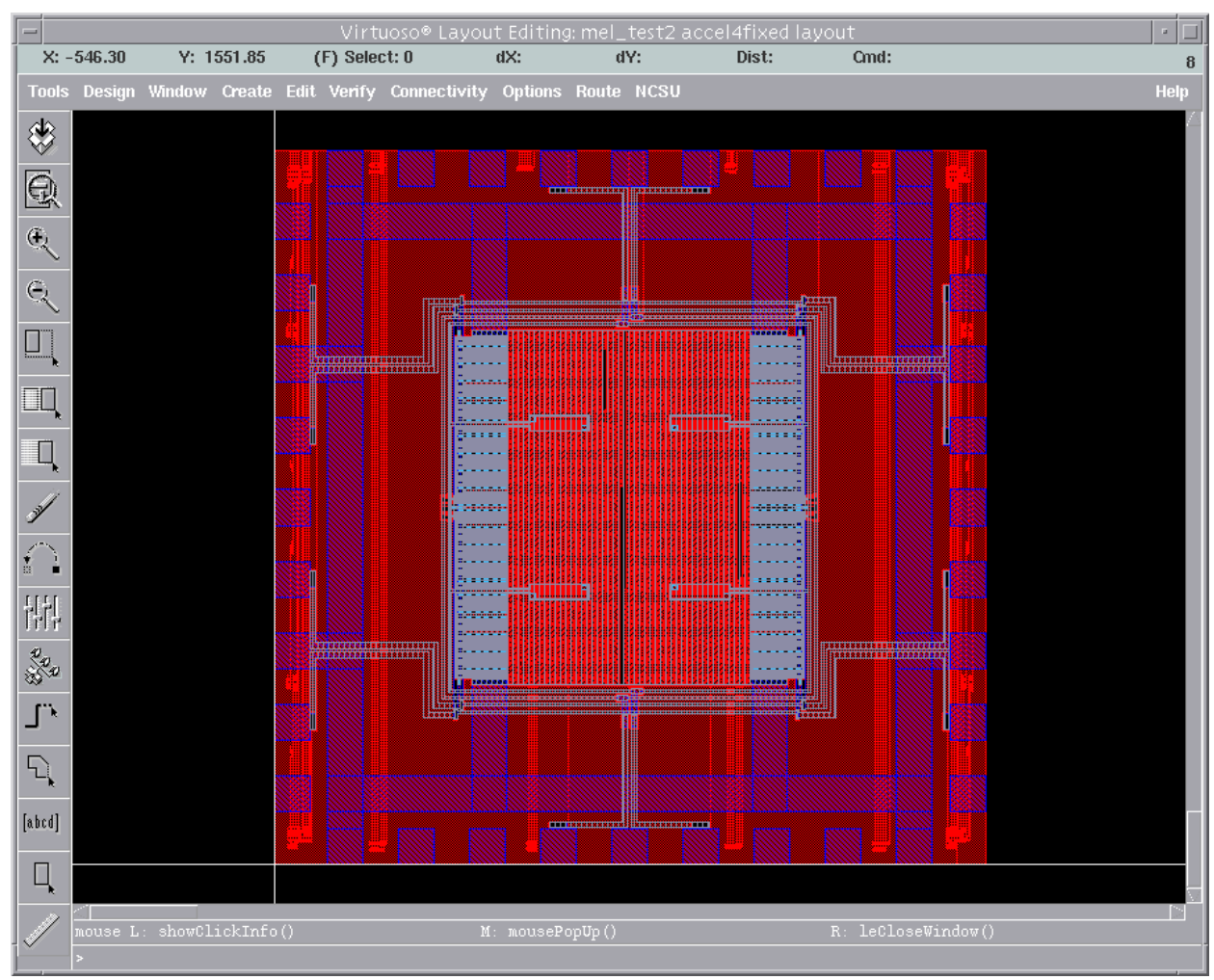

Figure 6.6(a): Full view of device layout on a $2 \times 2 \mathrm{~mm}$ die. Visible features include four internal springs, $36100 \times 100 \mu \mathrm{m}$ bonding pads along the edge of the die on the metall interconnect layer (blue), and $100 \mu \mathrm{m}$-wide ground trace (blue).

shape of the sensing structure including the placement of its four internal springs. Effort was made to maximize symmetry within the device, particularly when concerning the right and left capacitive sensing signals, so as not to introduce significant voltage offset. Bonding pad connections were made on all sides of the chip, with the right-hand and lefthand edges containing the sensing signals and the upper and lower edges containing the grounding and self-test signals. A close-up of the upper right-hand region of the structure is shown in Figure 6.6(b). Etch holes located throughout the interior to assist in release are visible as well as the design and anchoring (anchor region in light blue) of one of the springs. The electrodes spanned the entire length of both edges of the structure; shown here are movable electrodes attached to the proof mass, sense electrodes (light blue) 


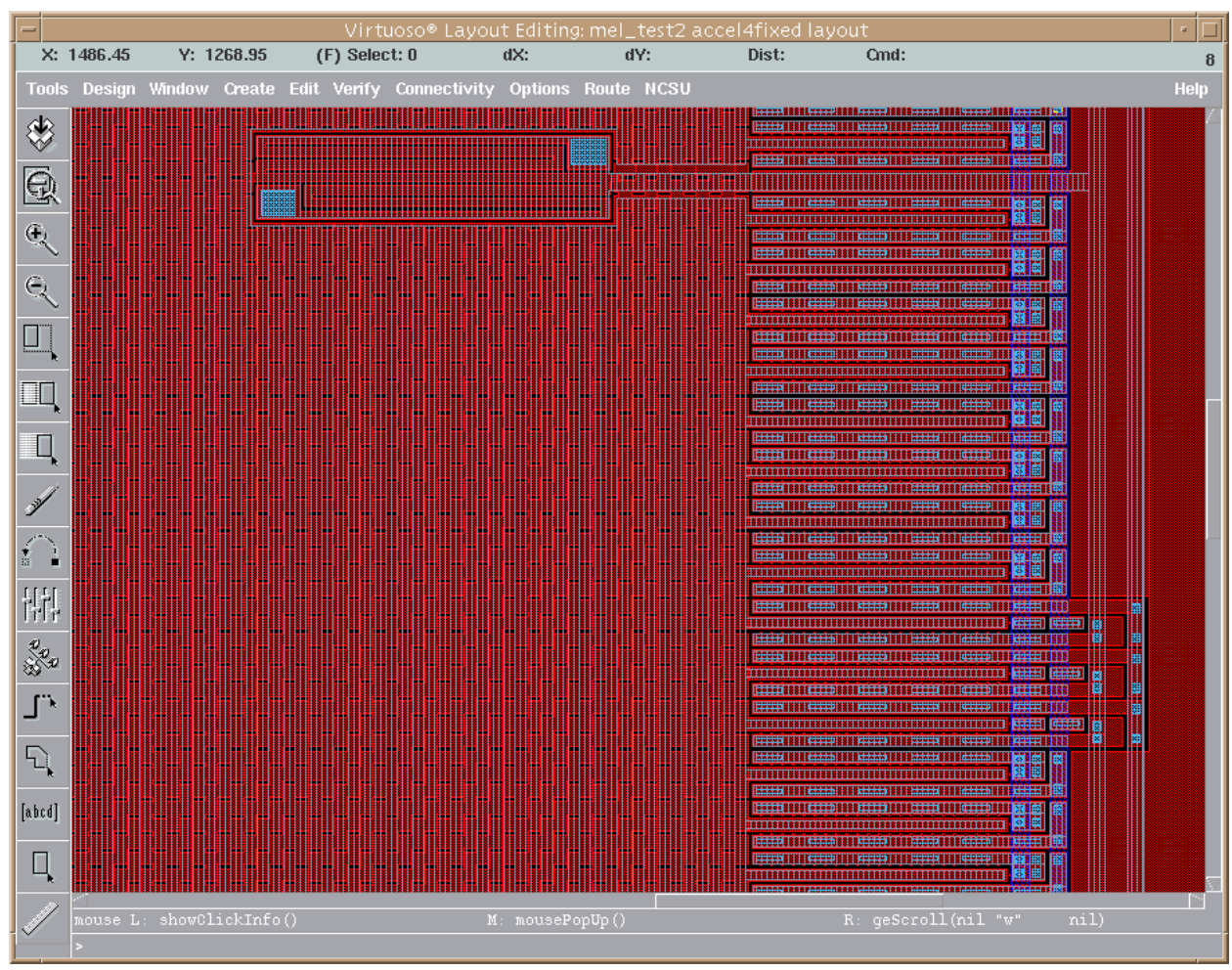

Figure 6.6(b): Right-hand side of the device showing connections to and anchoring of springs, structural electrodes and etch holes, stationary electrodes and anchors, self-test electrodes, ground plane (white), and upper (dark blue) and lower (white) interconnects.

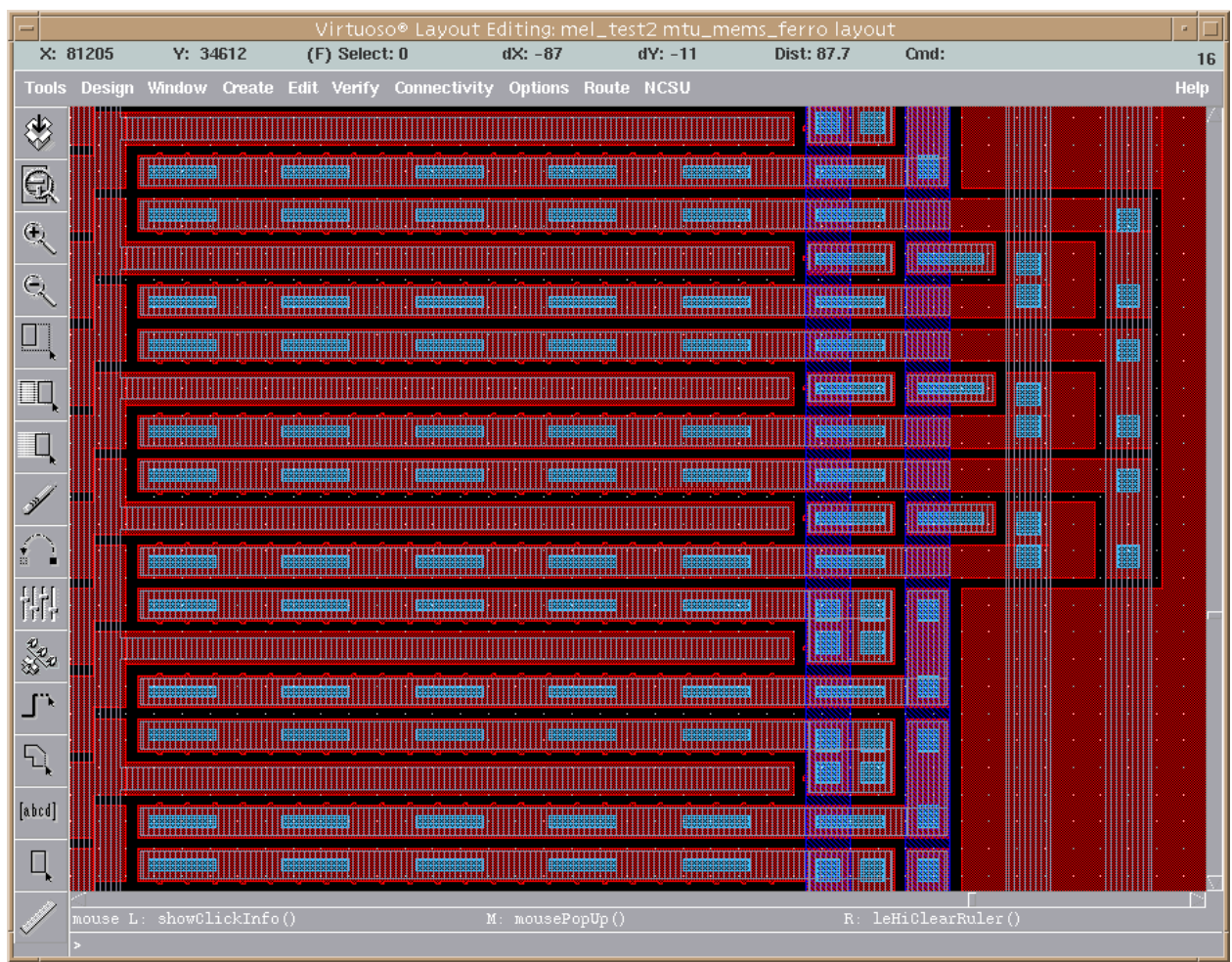

Figure 6.6(c): Close-up of sense electrode region showing dimensions of moving and stationary electrodes and surrounding gaps, vias, etch holes, and anti-stiction "bumps." 
anchored to the substrate, and the slightly larger self-test electrodes. Zooming in further, Figure 6.6(c) illustrates the finer details of the electrode shapes. The stationary electrodes were held in place using the same mask (light blue) that provided for poly0-poly1 signal transport and spring anchoring. The poly0 ground plane (white) located below the entire structural region also included the area below each of the moving electrodes. Antistiction "bumps" on the sides of the stationary electrodes gave a non-uniform texture in order to prevent adhesion to the structure during either release or operation. Details and dimensions of the spring designs are shown in Figure 6.7(a). The first was a singleanchor spring to which the inertial load was applied at two points. A more rigid beam perpendicular to the axis of motion transferred the force to a single beam down the

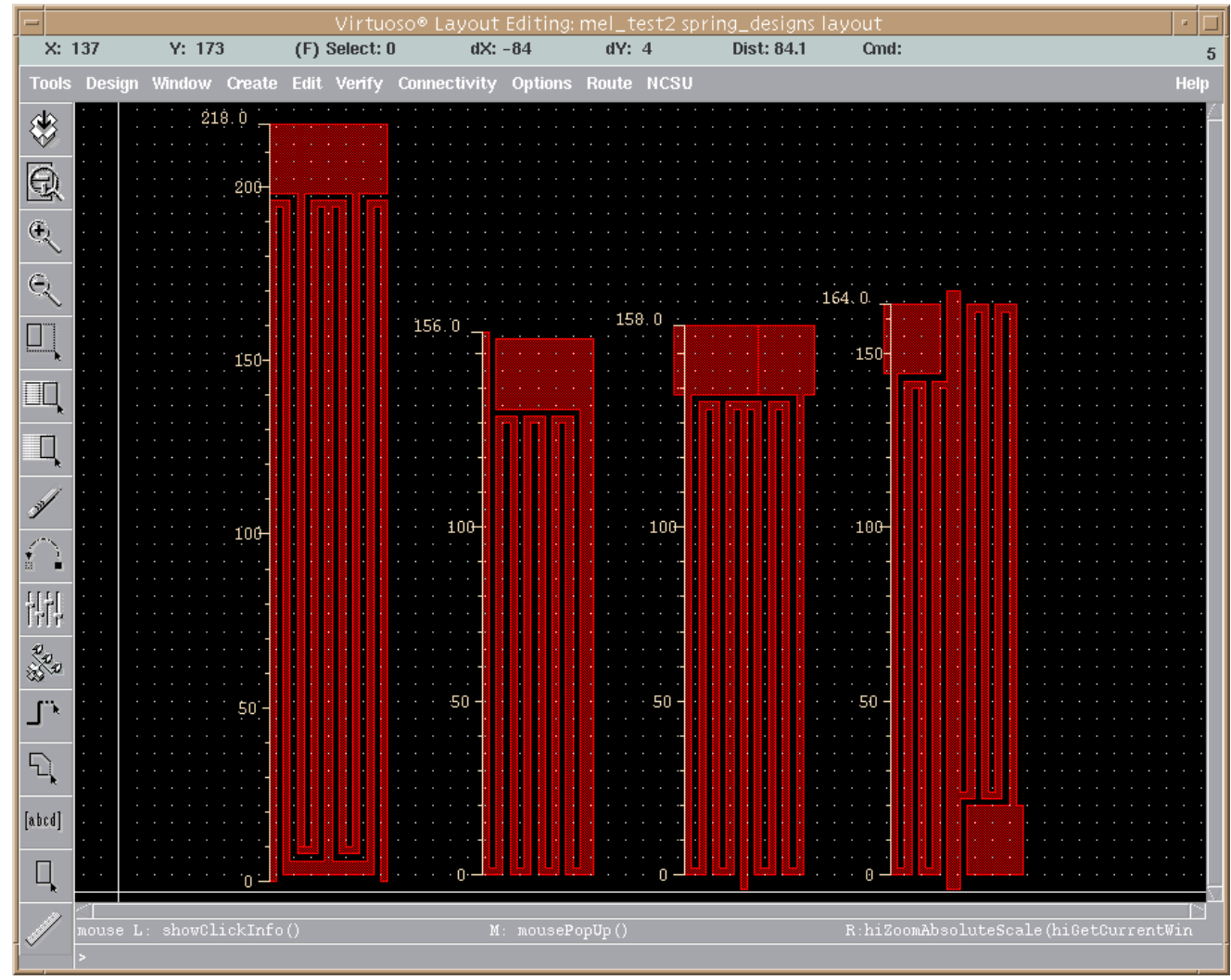

Figure 6.7(a): Styles and sizes of folded-beam springs for each of the four sensor designs. Each spring measured $40 \mu \mathrm{m}$ in overall width with individual segments either $2 \mu \mathrm{m}$ or $4 \mu \mathrm{m}$ wide in order to produce the desired spring constant. 
center, at which point the load was again divided before reaching the anchor region. The second spring, the least complex of the four, resembled many folded-beam springs encountered in similar industrial devices. The third design combined two springs of the second type, with fewer folds, into a more rigid single- anchor device. The fourth was perhaps the most unique, in which again two springs of the second type were combined with the difference that one of them was inverted, producing a double-anchor spring that was anticipated would minimize torsional elasticity. These springs were modeled as series and/or parallel combinations of individual beams as is illustrated in Figure 6.7(b), and approximate spring constant values were obtained from Equation (5.8b) using the typical polysilicon Young's Modulus of 160Gpa given by Senturia [Senturia, 2001 (p. 196)]. Figure 6.7(c) shows the details of the calculations, beginning with the values for the individual beams, combining them to model the full spring, and then multiplying by four to give the overall spring constant for the sensor since the four springs act in parallel. Simulation results presented in Section 6.5 confirm the accuracy of these calculations.

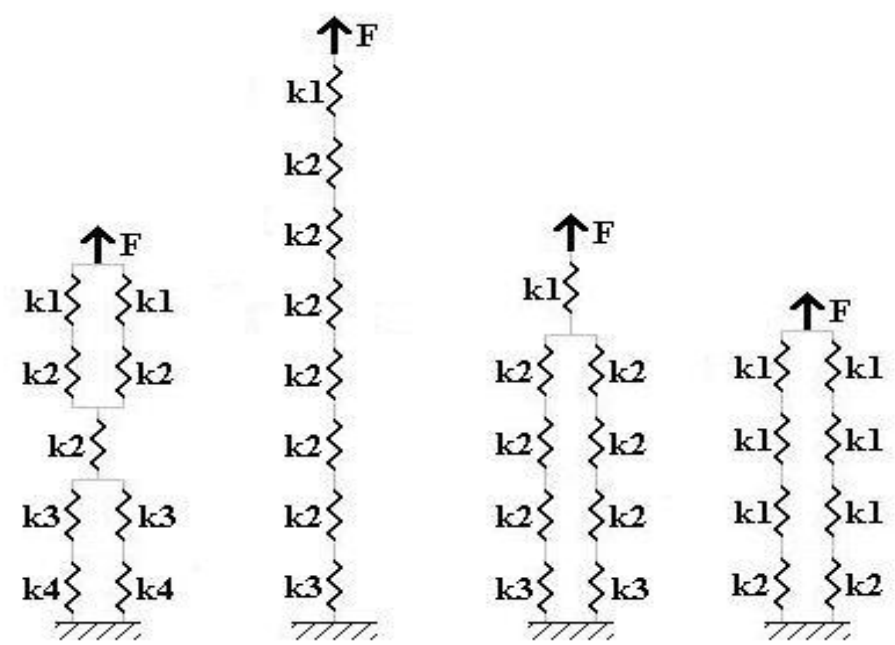

(1)

(2)

(3)

(4)

Figure 6.7(b): Models of each of the four spring styles shown in Figure 6.6(d) using series and/or parallel combinations of single-beam springs. Overall spring constants are additive for parallel springs and inverse additive for series springs. 


\begin{tabular}{|c|c|c|c|c|c|c|c|}
\hline Young's Modulus (Pa) & $1.60 \mathrm{E}+011$ & & & & & & \\
\hline \multirow[t]{4}{*}{ Thickness (Meters) } & 2.E-05 & & & & & & \\
\hline & & & & & & & \\
\hline & Spring 1 & & & & Spring 2 & & \\
\hline & k1 & $k 2$ & k3 & k4 & k1 & k2 & k3 \\
\hline Length (Meters) & $1.96 \mathrm{E}-04$ & 1.94E-04 & 1.88E-04 & 1.90E-04 & $1.56 \mathrm{E}-004$ & 1.32E-004 & 1.34E-004 \\
\hline Width (Meters) & 2.E-06 & 2.E-06 & 2.E-06 & 2.E-06 & 2.E-06 & 2.E-06 & 4.E-06 \\
\hline Individual Beam Constant (N/m) & 3.40 & 3.51 & 3.85 & 3.73 & 6.74 & 11.13 & 85.12 \\
\hline Individual Spring Constant ( $N / m)$ & 1.19243475 & & & & 1.43040837 & & \\
\hline \multirow[t]{5}{*}{ Total Spring Constant $(\mathrm{N} / \mathrm{m})$} & 4.76973899 & & & & 5.72163348 & & \\
\hline & & & & & & & \\
\hline & & & & & & & \\
\hline & Spring 3 & & & & Spring 4 & & \\
\hline & k1 & $k 2$ & k3 & & k1 & $k 2$ & \\
\hline Length (Meters) & 1.38E-04 & $1.36 \mathrm{E}-04$ & 1.38E-04 & & 1.42E-004 & 1.44E-004 & \\
\hline Width (Meters) & 2.E-06 & 2.E-06 & 2.E-06 & & 2.E-06 & 2.E-06 & \\
\hline Individual Beam Constant $(\mathrm{N} / \mathrm{m})$ & 9.74 & 10.18 & 9.74 & & 8.94 & 8.57 & \\
\hline Individual Spring Constant ( $\mathrm{N} / \mathrm{m})$ & 3.31808569 & & & & 4.4230021 & & \\
\hline Total Spring Constant $(\mathrm{N} / \mathrm{m})$ & 13.2723428 & & & & 17.6920084 & & \\
\hline
\end{tabular}

Figure 6.7(c): Microsoft Excel spreadsheet showing the numerical details of each of the spring constant calculations. The spring constants for the individual beams were determined and then combined to yield the overall value for the spring. Multiplication by four gave the total spring constant for the sensor.

\section{3: Solid Model Generation}

The Coventorware finite-element simulation software provides for the importation of layout files from other software packages such as Cadence. A process file is defined in which layers are deposited to the desired thicknesses with the layout masks applied to delineate their patterns, and the software utilizes this information to build a threedimensional solid model. The process file used to model the full sensor is shown in Figure 6.8. Two "imaginary" masks were created in order to separate each of the interlayer silicon dioxide films, labeled as phospho-silicate glass (PSG), into sacrificed and non-sacrificed portions. Otherwise, the software would completely remove the films during the final sacrificial etch, leaving no support for the upper layers. The model generation process therefore had to be adjusted. This was done by first defining the fraction of the deposited PSG layer intended to be permanent by an etch that utilized the "imaginary" mask. These holes were then refilled with "Oxide", creating two portions of 


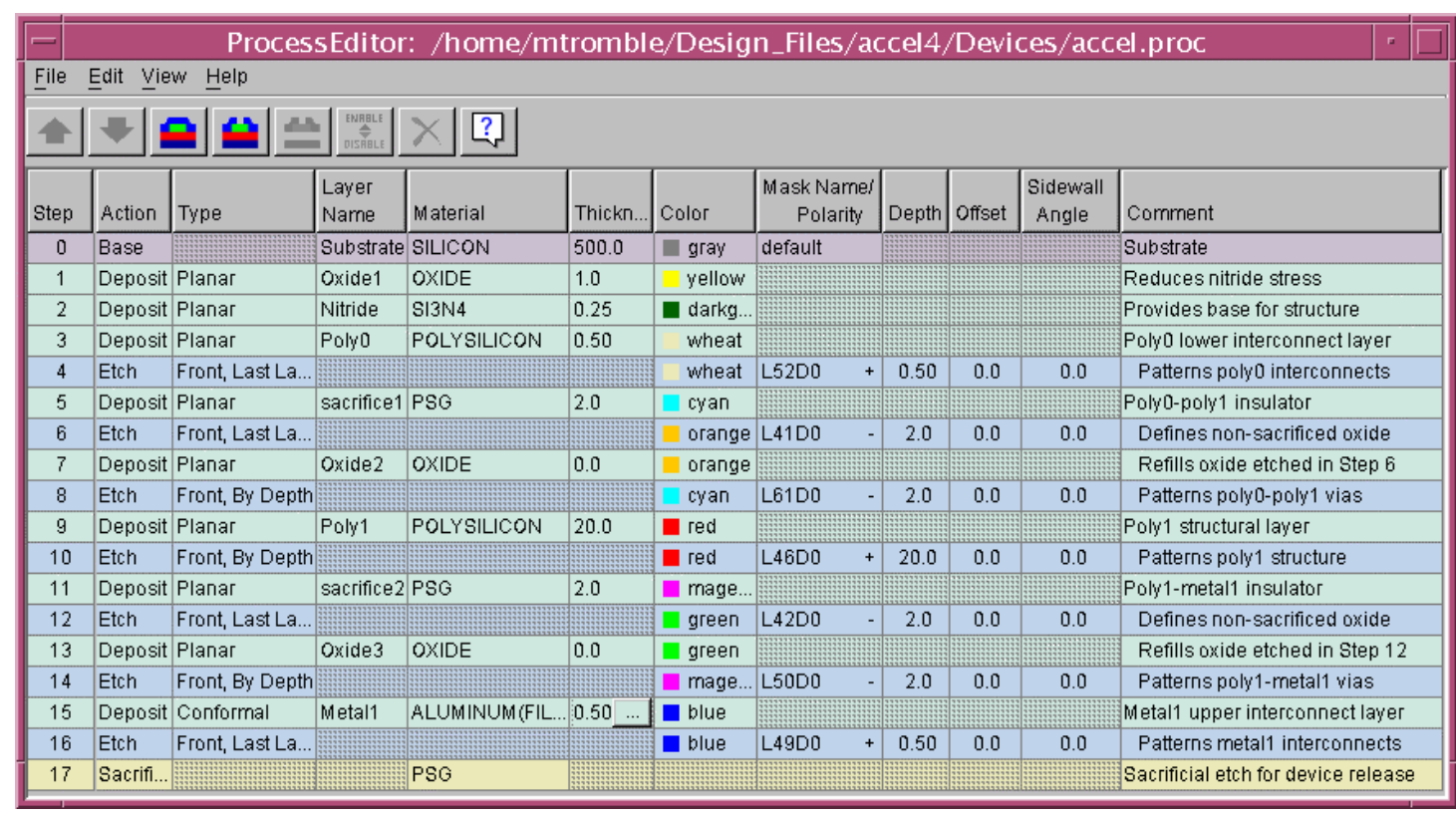

Figure 6.8: Fabrication process file utilized to construct the full solid model of the lateral accelerometer. Two additional masks were drawn to define non-sacrificed portions of the poly0-poly1 and poly1-metall oxide layers due to controlled timing of the sacrificial etch.

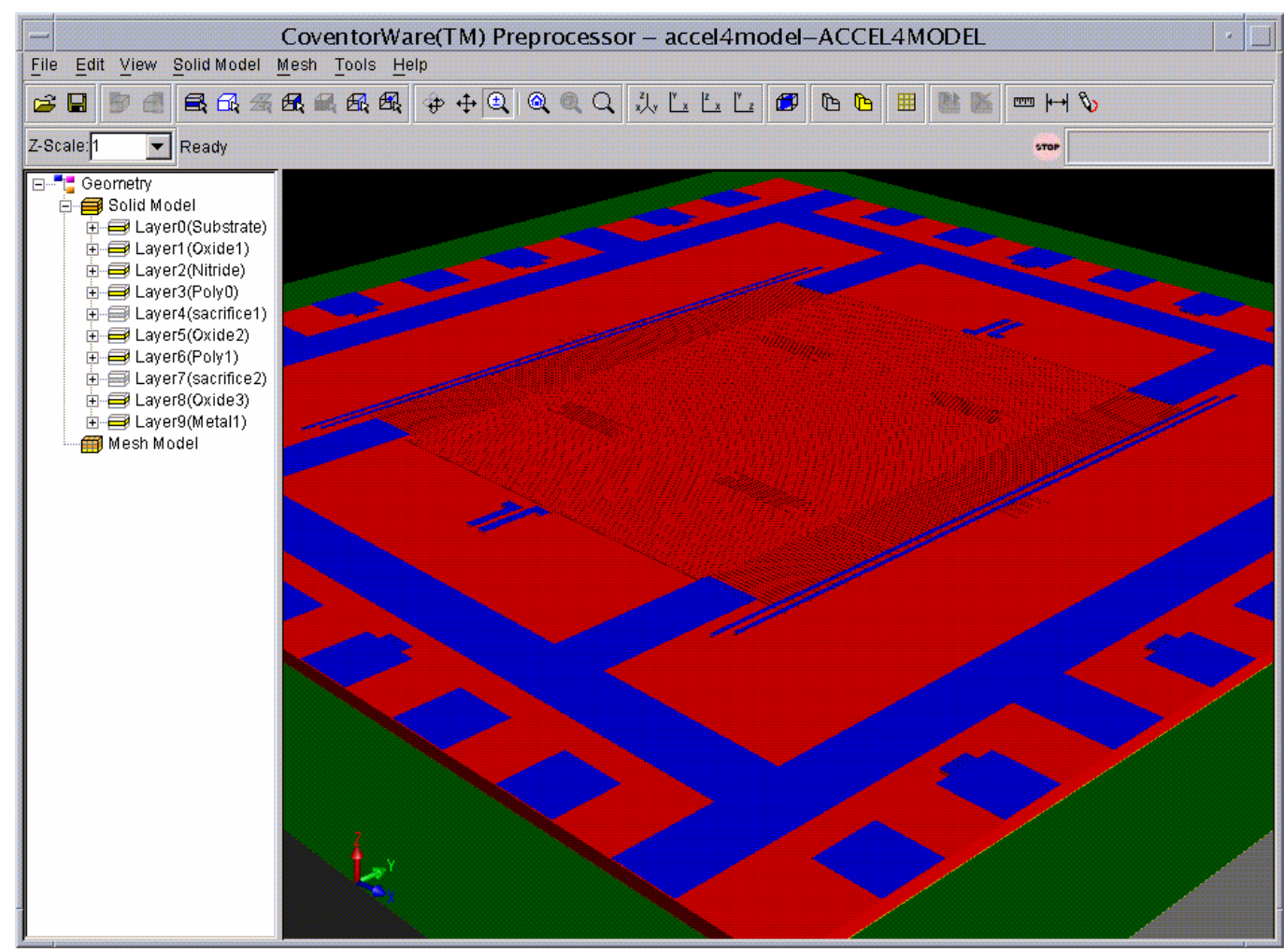

Figure 6.9(a): Solid model of the full accelerometer chip including the substrate (gray) and base dielectrics (nitride in green). This model was far too large for the software to simulate and was later divided into reduced electrical and mechanical portions so each simulation could be optimized as necessary. 
the same film distinguished by different names. A planarized refill was achieved by depositing the oxide film on top of the PSG with zero thickness, as the software defines film thicknesses based on the surface of the previous layer. Finally, the via locations were etched in the PSG using the original mask. At the end of the process, the sacrificial etch removed only the region of the film identified as PSG, leaving that identified as Oxide intact. Figures $6.9(\mathrm{a}-\mathrm{c})$ present images taken from the full solid model of the lateral accelerometer. The complete die is shown in Figure 6.9(a). For consistency, the colors of the patterned layers were chosen to match those from the Cadence layout masks. The Coventorware left-hand window enables the selection of individual layers for purposes such as adding to mesh regions, adjusting mesh configurations, or toggling visibility. Figure 6.9(b) shows a close-up of the right-hand portion of the sensor region, illustrating

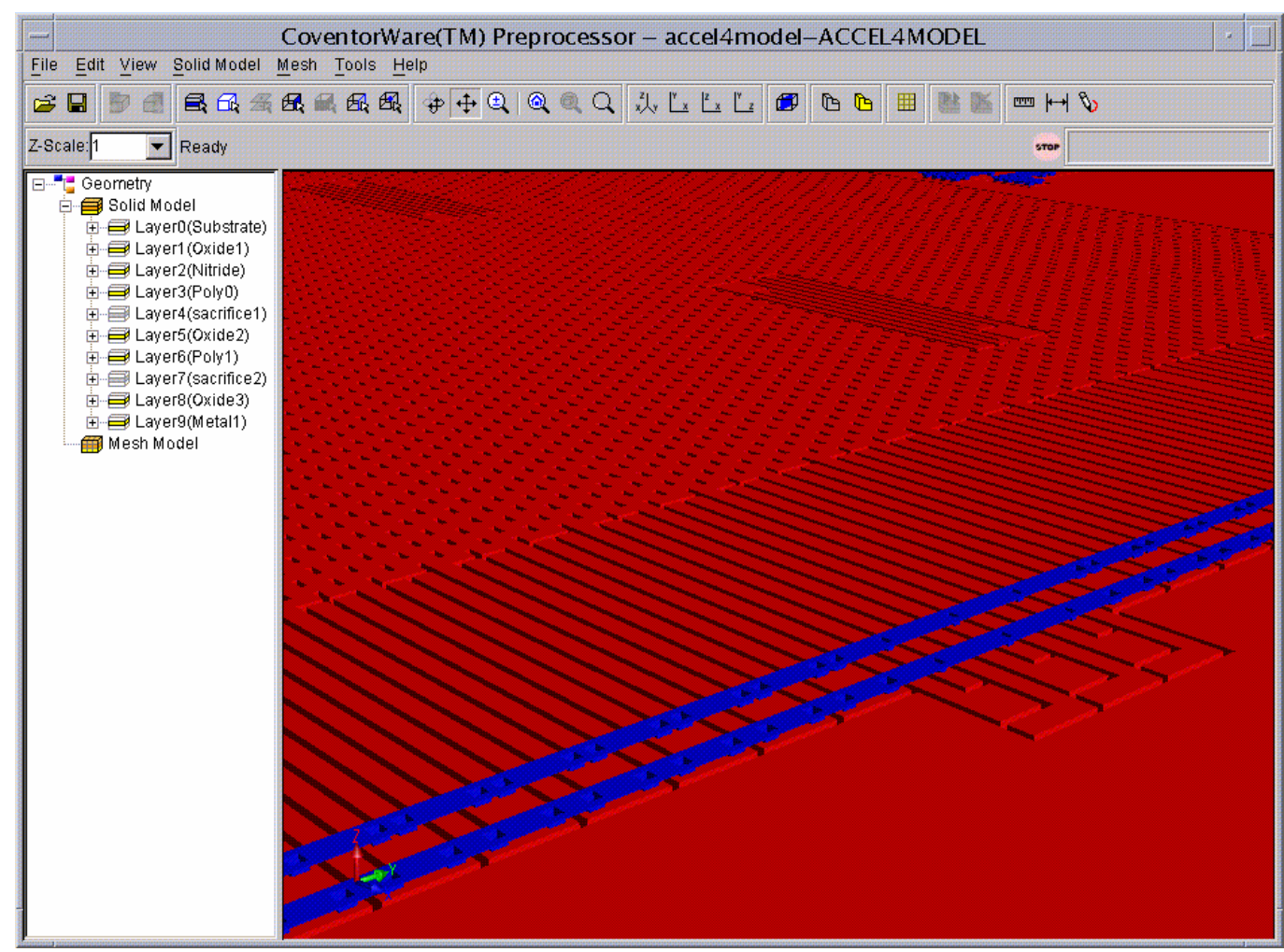

Figure 6.9(b): Close-up of the right-hand portion of the sensor surface showing details of the electrodes, both sensing and self-testing, as well as etch holes, a style- 4 spring, and the upper interconnect layer. 
in three dimensions the appearance of features such as etch holes and airbridges. Removing the structural and upper layers yields the image shown in Figure 6.9(c) in which the poly0 ground plane and interconnects are shown with the non-sacrificed portion of the poly0-poly1 oxide (yellow). Initially, an effort was made to simulate the full device in a coupled electro-mechanical mode, but numerous problems associated with the modeling package were encountered as a result of the large file sizes. In its present format, the Coventorware solvers have a $2 \mathrm{~GB}$ limit on memory addressing capability, and this was insufficient to simulate the full device. Based on recommendations by Coventorware technical support personnel, separate models were generated for simulating the electrical and mechanical portions of the sensor so that each

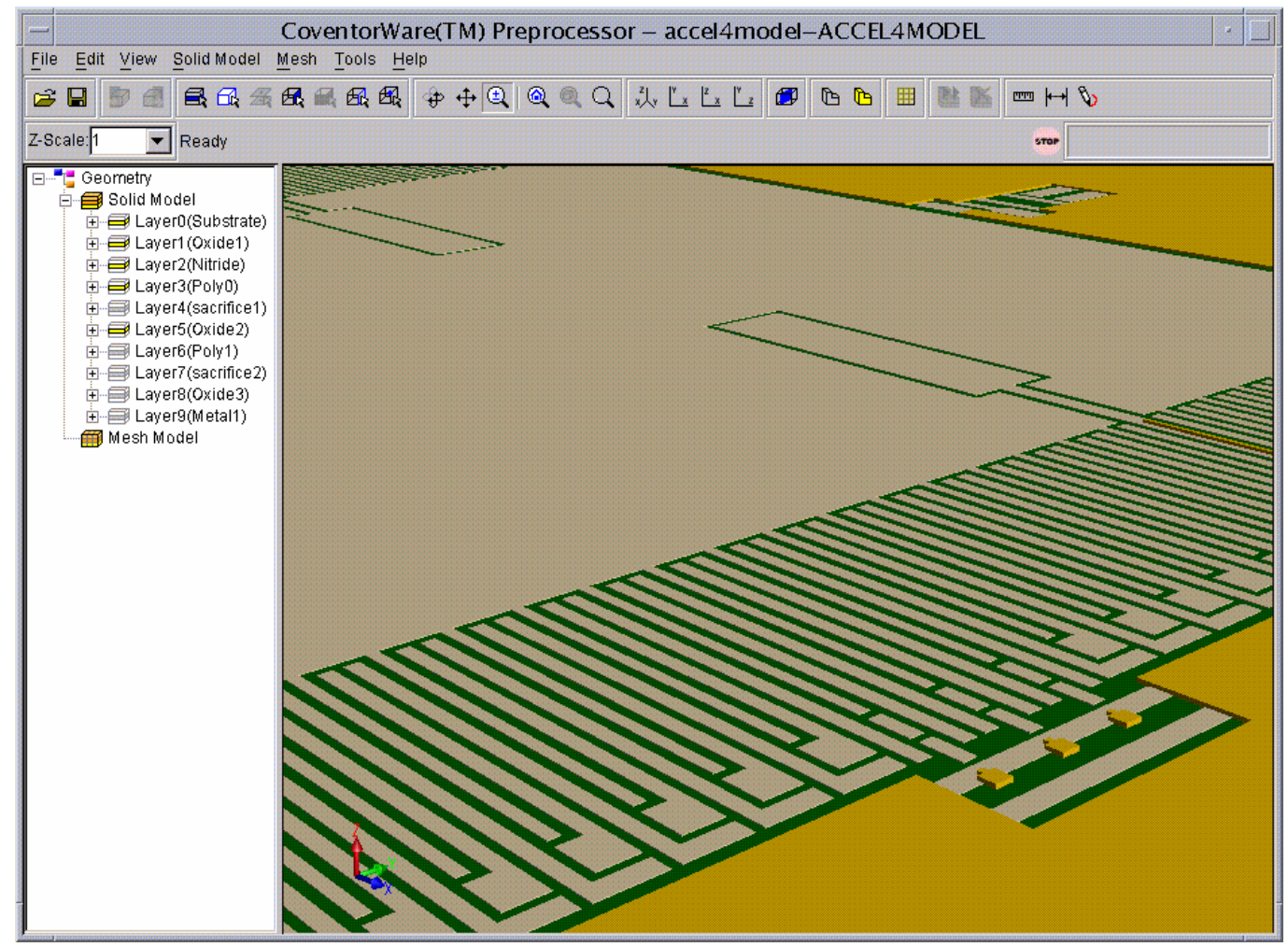

Figure 6.9(c): Close-up of the right-hand portion of the sensor with the poly1, metall, and poly1-metall oxide layers removed, illustrating the poly0 pattern as well as the nonsacrificed portion of the poly0-poly1 oxide layer 
could be reduced and optimized to their individual requirements. Details of the electrostatic model, in which capacitance chances are computed as the structure shifts laterally, are given in Section 6.4. The mechanical model predicts the shift of the structure for a particular applied acceleration; these results are presented in Section 6.5.

\section{4: Electrostatic Simulation}

The purpose of the electrostatic simulation of the lateral accelerometer was to determine the relationship between the structural offset and the resulting Center-Left and Center-Right capacitance values. Modeling the relevant portion of the sensor required only the regions containing the capacitive electrodes; specific details of the structure such as springs and etch holes were not necessary. The layout was reduced to encompass only the device region as shown in Figure 6.10. Etch holes and springs were filled in, and only the immediate interconnect traces were included. It was initially intended that the entire set of electrodes would be included in the simulation, but it was found that the model still exceeded the $2 \mathrm{~GB}$ addressing limit. Therefore, the model was cropped using an additional layer, shown in green, such that only half of the sensing electrodes were used. An electrode bank on each side of the structure was included in order to compensate for skew that would otherwise be introduced as a result of different stationary electrode lengths. The process file used to build the solid model is shown in Figure 6.11. This process differed from that used to build the full model in that the structural layer itself was split into two portions, one to be included in the meshed region and one to be left out. The means by which the layer was divided was the same as that used to split the inter-layer oxides, described previously. On the other hand, the poly1-metal1 oxide was no longer divided because the entire layer was sacrificed in this model. The three- 


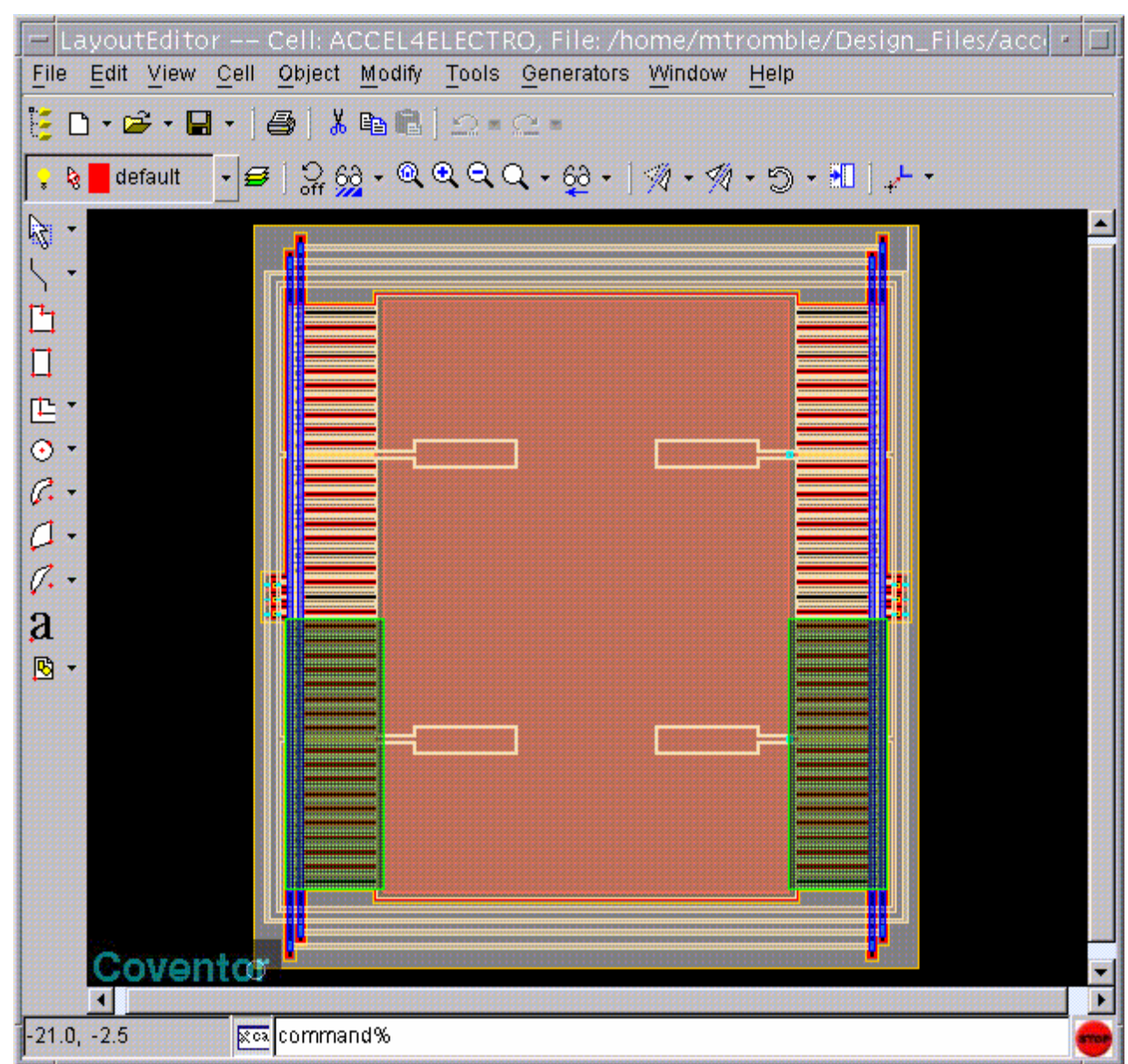

Figure 6.10: Imported layout of the modified device in which the two green areas defined the portion to be utilized for solid model generation. Half of each electrode bank was modeled, as the full set of electrodes were too large of a simulation to remain within the addressing limit.

dimensional meshed model is shown in Figure 6.12(a). Only the polyl and metall layers were included in the actual simulation, as the model was intended to simulate the relationships between polyl surfaces and thus required the metall connections in order to define the Left and Right capacitive signals in their entirety. The device was meshed using the Manhattan (brick) parabolic elements with size parameters of $20 \mu \mathrm{m}, 4 \mu \mathrm{m}$, and $10 \mu \mathrm{m}$ in the $\mathrm{x}, \mathrm{y}$, and $\mathrm{z}$ dimensions, respectively, and the additional stipulation was given that at least two elements must be defined on each surface. The left-hand window was utilized to define specific conductors, allowing the Center, Right, and Left signals from 


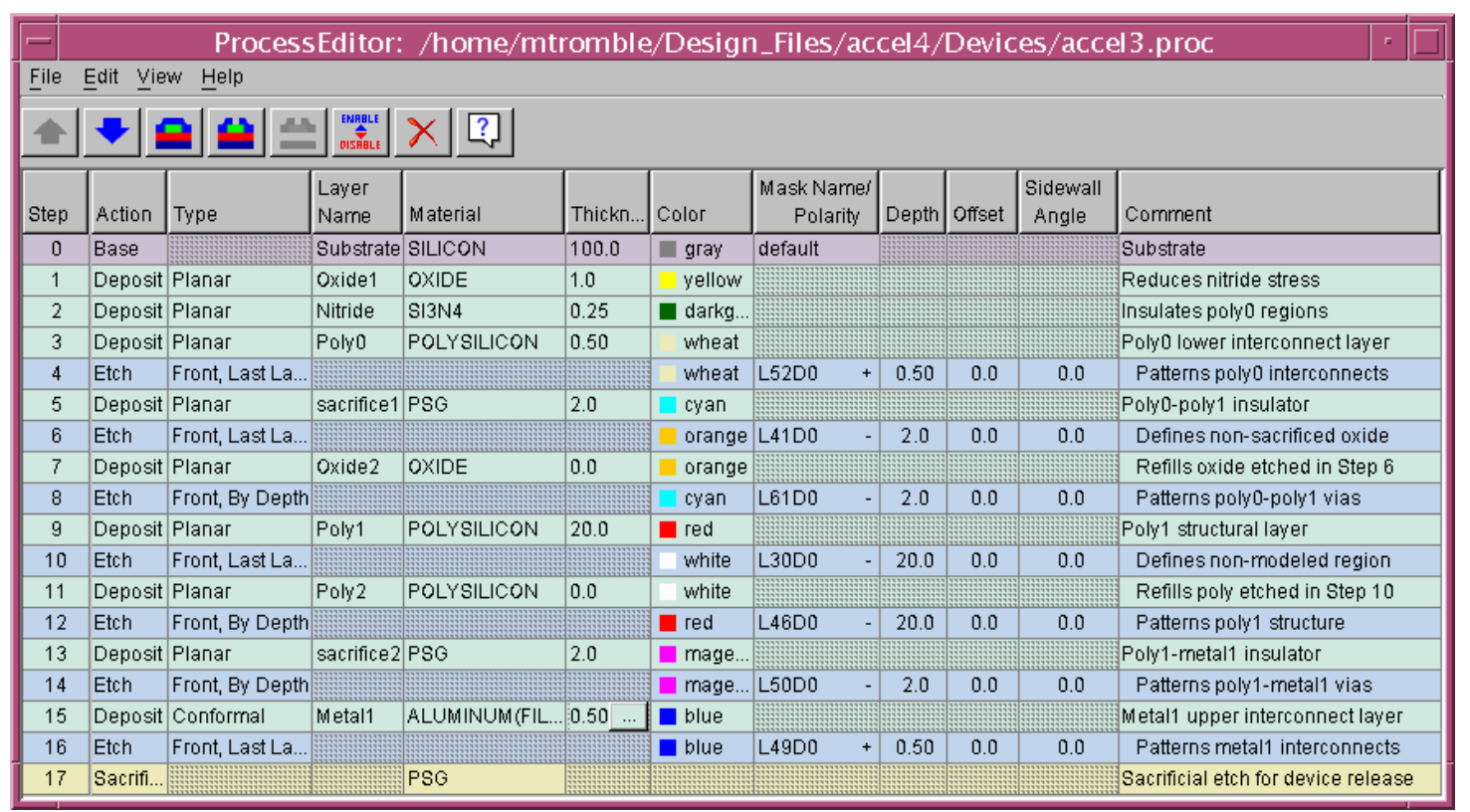

Figure 6.11: Modified fabrication process used to produce the electrostatic model. Two additional masks were required; the first defined non-sacrificed regions of the poly0poly1 oxide, and the second separated the modeled and non-modeled portions of poly1.

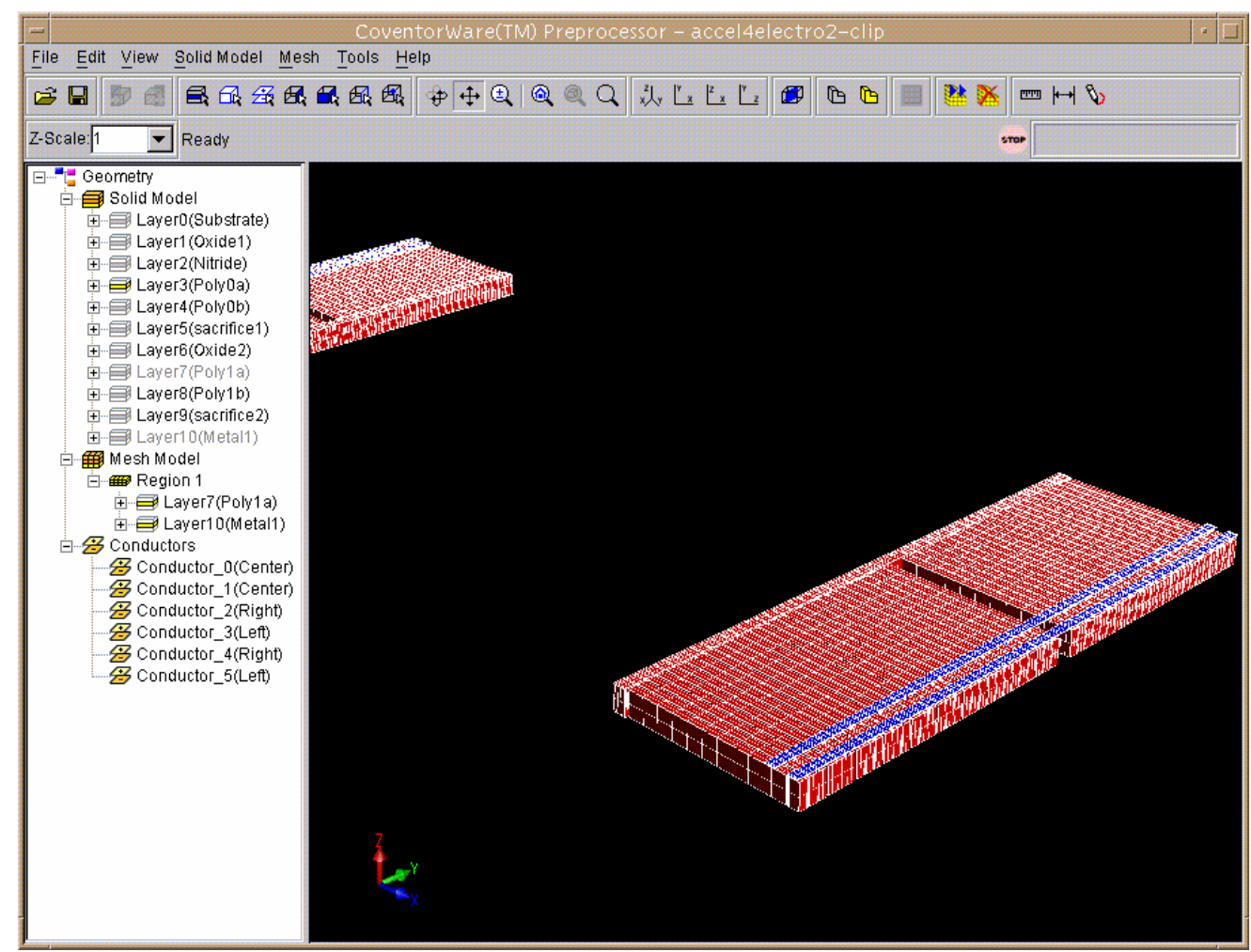

Figure 6.12(a): Meshed model of the capacitor banks utilized for the electrostatic model of the accelerometer. Only the polyl and metall layers were included in the simulation as the remaining layers did not contribute to the capacitance calculations. 


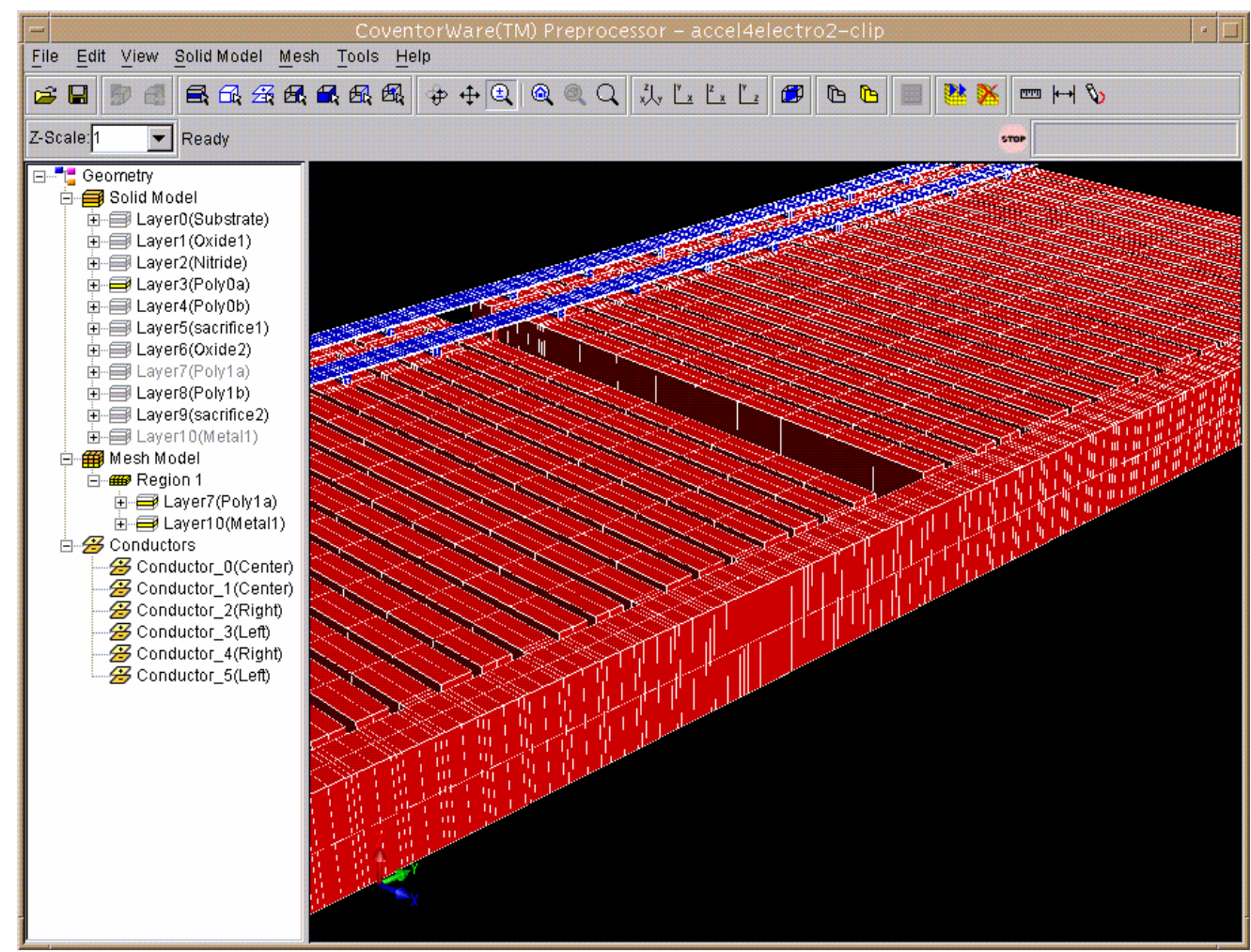

Figure 6.12(b): Close-up of the left-hand electrode bank of the electrostatic device model illustrating details of the capacitive sub-structure and its mesh configuration.

each of the two electrode banks to be linked for the simulation as they normally would if the remainder of the structure and interconnects were present. A close-up of the left-hand electrode bank is shown in Figure 6.12(b), illustrating the finer details of the substructure and its mesh. Once the features of the solid model were defined, the simulation parameters were set up as shown in Figures 6.12(a-d). Coventorware requires that voltages be assigned to all conductors, illustrated in the ConductorBCs window in Figure 6.13(a), though for this simulation the actual values were not relevant to the capacitance computation. Finally, a parametric sweep of offset values was applied to the structure in order to force it to shift laterally as desired. The offset trajectory was established as shown in Figure 6.13(b). Through the Edit tab, its direction was defined with an optional scaling factor, shown in Figure 6.13(c), and the components to receive the offset 


\begin{tabular}{|c|c|c|c|c|c|c|c|c|}
\hline \multicolumn{9}{|c|}{ ConductorBCs } \\
\hline ConductorBCs & \multicolumn{2}{|c|}{ Conductor } & \multicolumn{2}{|c|}{ BCType } & \multirow{2}{*}{$\begin{array}{c}\text { Voltage } \\
0.0\end{array}$} & \multirow{2}{*}{$\begin{array}{c}\text { Charge } \\
0.0\end{array}$} & \multicolumn{2}{|c|}{ Variable } \\
\hline Setting & Center & $\overline{7}$ & Fixed & $\nabla$ & & & none & $\overline{7}$ \\
\hline Setting & Left & 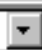 & Fixed & 7 & -5.0 & 0.0 & none & 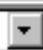 \\
\hline Setting & Right & \pm & Fixed & $\square$ & 5.0 & 0.0 & none & \pm \\
\hline Setting & none & $\nabla$ & Fixed & 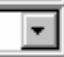 & 0.0 & 0.0 & none & \pm \\
\hline \multirow[t]{2}{*}{ Setting } & none & $\overline{-}$ & Fixed & $\overline{7}$ & 0.0 & 0.0 & none & $\overline{-}$ \\
\hline & & & & OK & Cancel & & & \\
\hline
\end{tabular}

Figure 6.13(a): Voltages applied to the Center, Left, and Right conductor signals. The software requires that values be assigned, though for this model the actual voltage magnitudes were not relevant to the simulation results.

\begin{tabular}{|c|c|c|c|c|c|c|}
\hline \multicolumn{7}{|c|}{ dimension } \\
\hline \multirow{2}{*}{\begin{tabular}{|l|} 
dimension \\
Transform1
\end{tabular}} & \multicolumn{2}{|c|}{ Trajectory } & \multicolumn{3}{|c|}{ Transform } & \multirow{2}{*}{$\frac{\text { Part }}{\text { List }}$} \\
\hline & $t 1$ & 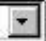 & Offset & $\mp$ & Edit & \\
\hline Transform2 & none & $\mp$ & none & $\nabla$ & Edit & List \\
\hline Transform3 & none & 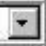 & none & \pm & Edit: & List \\
\hline Transform4 & none & 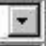 & none & \pm & Edit & List \\
\hline Transform5 & none & $\square$ & none & 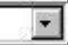 & Edit & List \\
\hline Transform6 & none & \pm & none & \pm & Edit: & List \\
\hline Transform? & none & $\nabla$ & none & $\nabla$ & Editt & List \\
\hline \multirow[t]{2}{*}{ Transform8 } & none & $\square$ & none & $\nabla$ & Edit: & List \\
\hline & & & & Cano & & \\
\hline
\end{tabular}

(b)

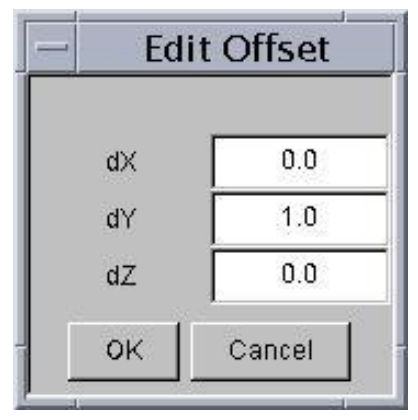

(c)

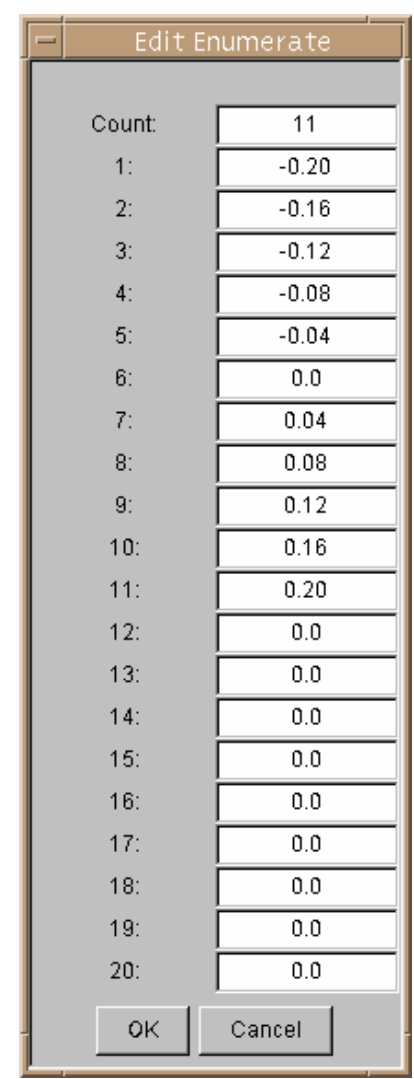

(d)

Figure 6.13(b-d): Definition of structural offset trajectory for the electrostatic simulation. The "dimension" window in (b) established the nature of the trajectory $t l$ as an offset which is applied to the structure. Selecting the "Edit" tab produced the window in (c) in which direction(s) and scaling factor(s) were applied to the trajectory values, which were enumerated as shown in (d). 
were selected; in this case the shift was applied only to the partial proof mass. The actual values that were used for the simulation are shown in Figure 6.13(d) and spanned the range of $+/-0.20 \mu \mathrm{m}$ in steps of $0.04 \mu \mathrm{m}$. The resulting values computed by the solver, multiplied by two, are plotted in Figure 6.14, in which the capacitances are shown in solid lines and the percent change relative to the nominal value are shown in dashed lines. The device was designed to have a $0.15 \mu \mathrm{m}$ shift under full load, and the plot shows that under normal operation the two capacitances ranged from approximately $0.92 \mathrm{pF}$ to $1.04 \mathrm{pF}$ with a nominal value of $0.975 \mathrm{pF}$, which is within $2.8 \%$ of the nominal value calculated using the fringe effect approximation. The relative capacitance change under full load fell within the 6-7\% range, depending on the amount of deflection, slightly lower than the $7.5 \%$ target but reasonably close. The results do not include capacitance

\section{Sense Capacitance vs Structural Shift}

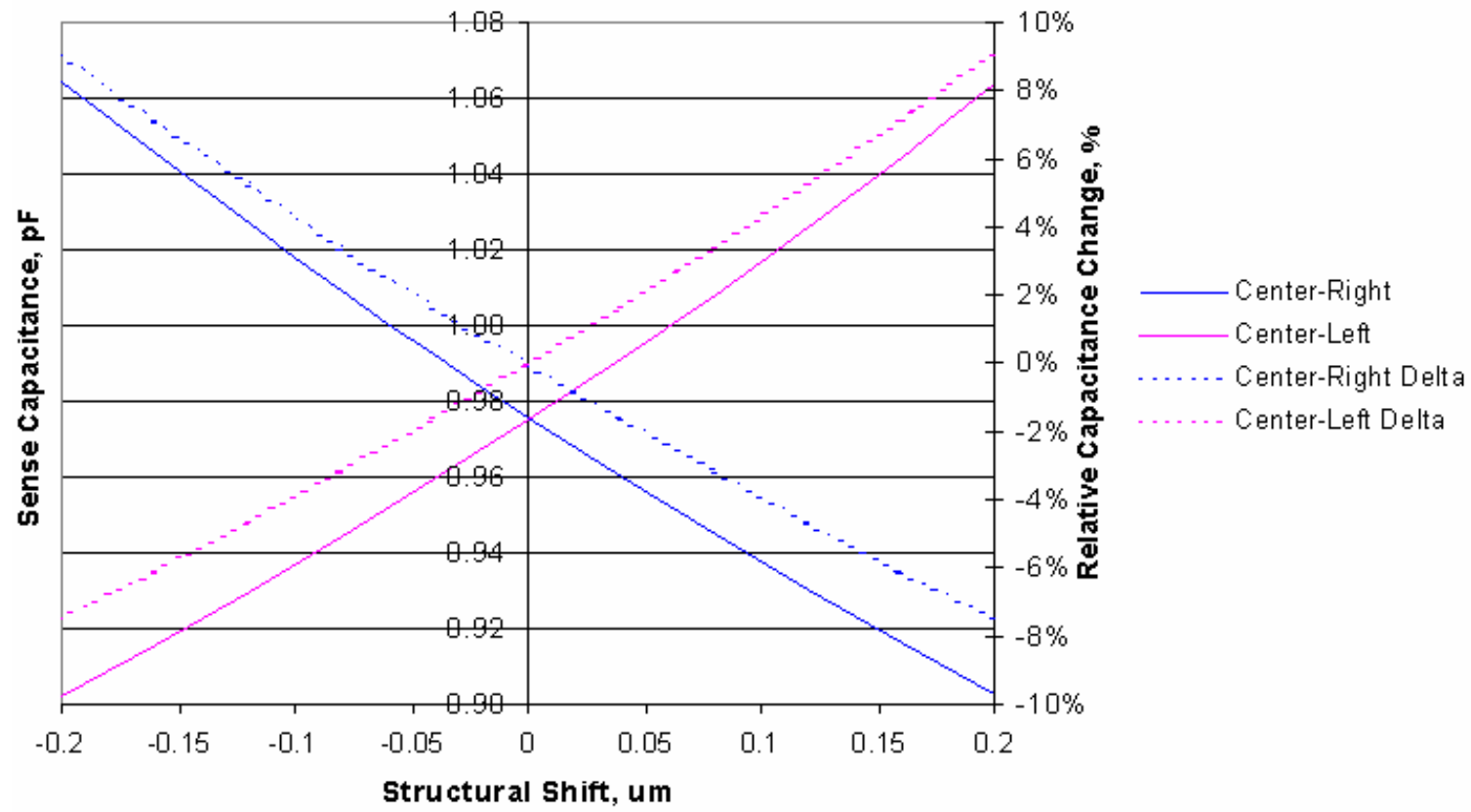

Figure 6.14: Results of lateral accelerometer electrostatic simulation. Capacitances vary from approximately $0.92 \mathrm{pF}$ to $1.04 \mathrm{pF}$ under a $0.15 \mu \mathrm{m}$ structural shift with a nominal value of around $0.975 \mathrm{pF}$. The relative capacitance change with respect to the nominal value is in the $6-7 \%$ range under full load. 
contributed by interconnect traces, which may have a small effect on the behavior of the fabricated device. Overall, however, the electrostatic model was expected to provide a reasonably accurate prediction of the sense capacitance values with respect to structural shift. The mechanical model was then used to translate this shift into the required input acceleration values.

\section{5: Mechanical Simulation}

An accurate mechanical simulation was of high importance due to the need to confirm the accuracy of the design values, particularly the spring constant calculations, as well as absence of unintentional electrical shorts. The mechanical model consisted only of the structure and springs as shown in Figure 6.15. The green-colored rectangles drawn at the spring regions were utilized by the software to separate the springs from the structure during the solid model generation, which enabled the independent meshing of the different regions for optimization purposes. For proper simulation, reattachment would later be required through the establishment of links between the adjacent surfaces. Meshing was also simplified through the consolidation of the etch holes into two large holes placed on opposite sides of the structure. This allowed for the use of a relatively coarse mesh through the interior of the structure while still maintaining the same inertial resistance to a change in motion. The process definition file used to generate the solid model is shown in Figure 6.16. This procedure was far more simple than that utilized for the electrostatic model, requiring only the base layers, a sacrificial layer patterned for spring anchoring, and the structural layer. Figure 6.17(a) shows the meshed solid model. The structural region was assigned mesh dimensions in the $\mathrm{x}-, \mathrm{y}-$, and $\mathrm{z}$-directions of $20 \mu \mathrm{m}, 12 \mu \mathrm{m}$, and $6 \mu \mathrm{m}$, respectively, and the spring regions had $2 \mu \mathrm{m}, 2 \mu \mathrm{m}$, and $4 \mu \mathrm{m}$ 


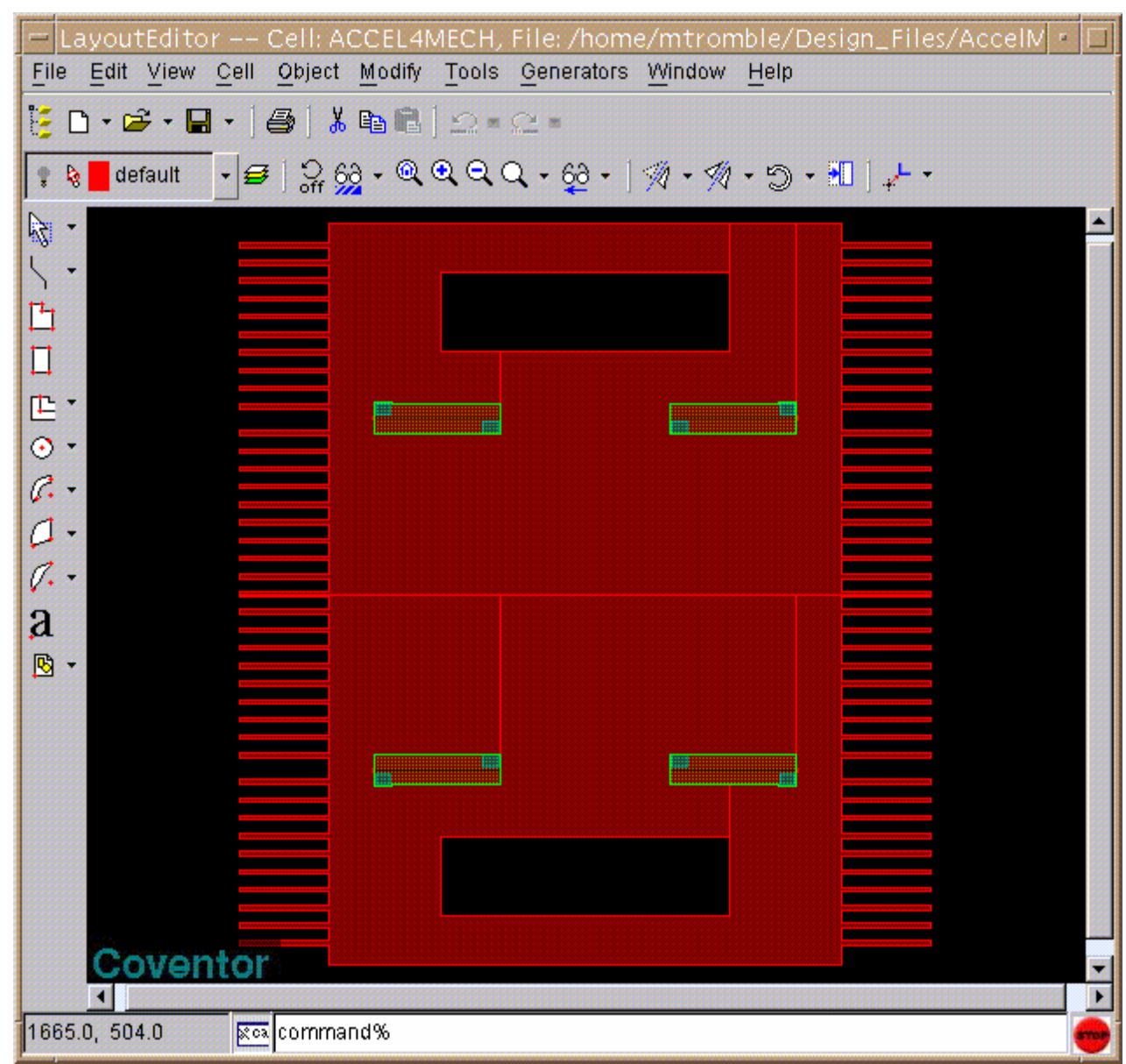

Figure 6.15: Imported layout of structural region consisting only of the proof mass, moving electrodes, and springs. Meshing was optimized through the consolidation of the etch holes and the separation of the springs from the structure.

\begin{tabular}{|c|c|c|c|c|c|c|c|c|c|c|c|c|}
\hline$=$ & \multicolumn{11}{|c|}{ ProcessEditor: /home/mtromble/Design_Files/AccelMech/Devices/AccelMech.proc } & r \\
\hline \multicolumn{13}{|c|}{ File Edit $\underline{\text { View }}$ Help } \\
\hline 삼 & 5 & nit & 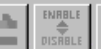 & $?$ & & & & & & & & \\
\hline Step & Action & Type & $\begin{array}{l}\text { Layer } \\
\text { Name }\end{array}$ & Material & Thickn... & Color & $\begin{array}{c}\text { Mask Name' } \\
\text { Polarity }\end{array}$ & Depth & Offset & $\begin{array}{l}\text { Sidewall } \\
\text { Angle }\end{array}$ & Comment & \\
\hline 0 & Base & & Substrate & SILICON & 100.0 & gray & default & & & & Substrate & \\
\hline 3 & Deposit & Planar & sacrifice1 & PSG & 2.0 & cyan & & & 䋖 & 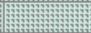 & Base-poly1 insul & rificed) \\
\hline 4 & Etch & Front, Last Le & & & & cyan & L61D0 & 2.00 & 0.0 & 0.0 & Patterns anchor & PSG \\
\hline 5 & Deposit & Planar & Poly1a & POLYSILICON & 20.0 & red & & & & 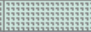 & Poly1 structural I & \\
\hline 6 & Etch & Front, Last Le & & & & red & L46D0 & 20.0 & 0.0 & 0.0 & Patterns poly1 & \\
\hline 7 & Sacrifi... & & & PSG & & & & & & & Sacrificial etch fo & ease \\
\hline
\end{tabular}

Figure 6.16: Modified fabrication process file utilizing only the base layers, sacrificial layer, and polyl structural layer. The interconnect layers were not required and thus the structure was anchored directly to silicon nitride. 


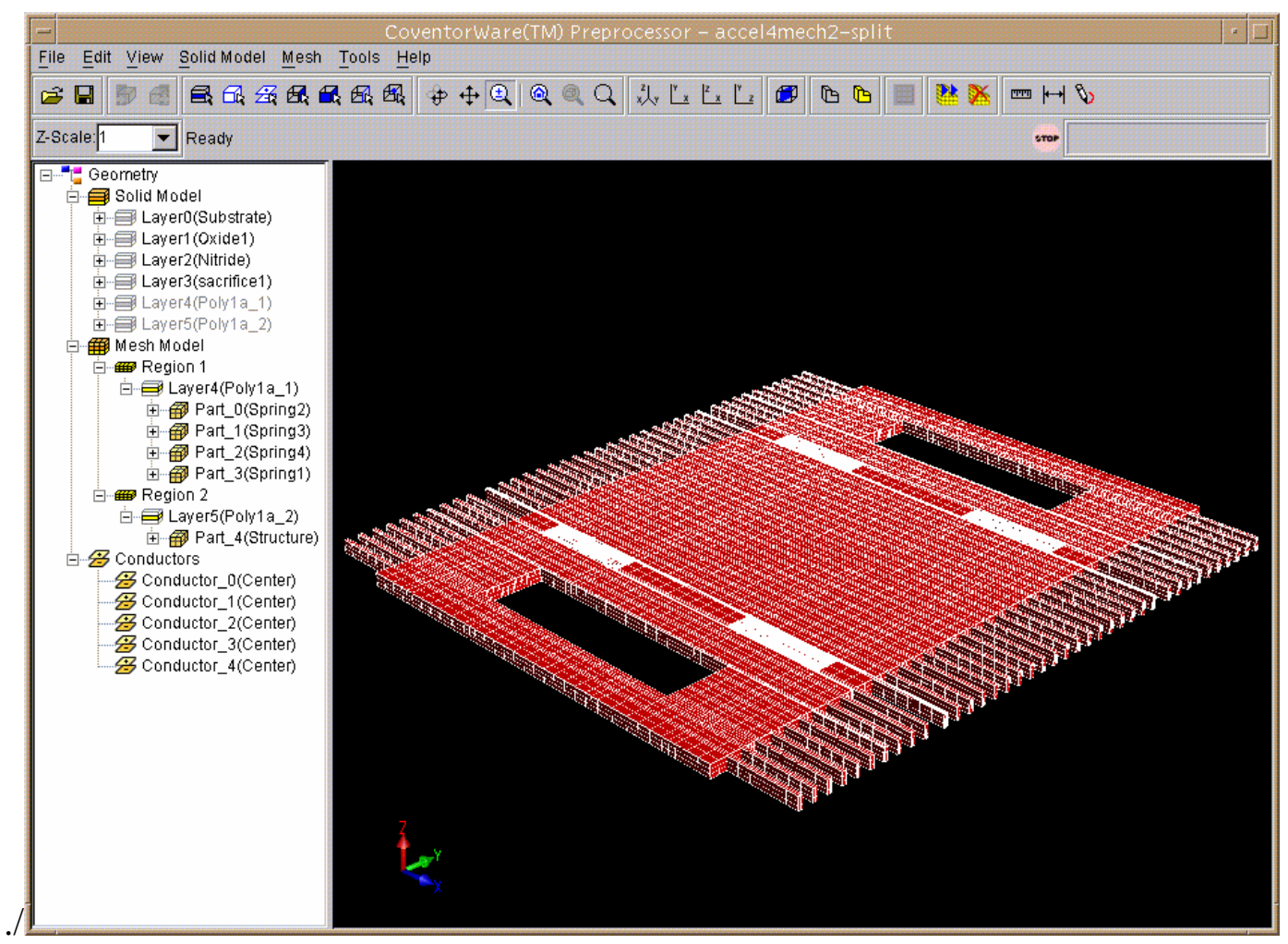

Figure 6.17(a): Meshed model utilized for mechanical simulation. Springs were modeled as separate regions and thus could be given a finer mesh, as is needed for accurate spring constant calculations, while the structural mesh remained coarse in order to minimize computational requirements.

nominal element sizes. The mesh generator was again instructed to create at least two elements for each surface, further reducing the element size in several locations. A closer look at one of the spring regions, given in Figure $6.17(\mathrm{~b})$, shows the relative difference between the two meshes. Had they not been separated, it would not have been possible to assign them different mesh parameters. For the software to recognize them as attached, each of the adjacent surfaces (or patches) had to be selected, assigned a name, and listed as a linkage boundary condition in the LinkageBCs window shown in Figure 6.18(a). Two types of links were available; rigid links place limits on the strain allowed in the attachment patches based upon the radius value assigned (integral values from 0.0 to 7.0 ), thus potentially reducing accuracy, whereas tied links allow for unrestricted deformation 


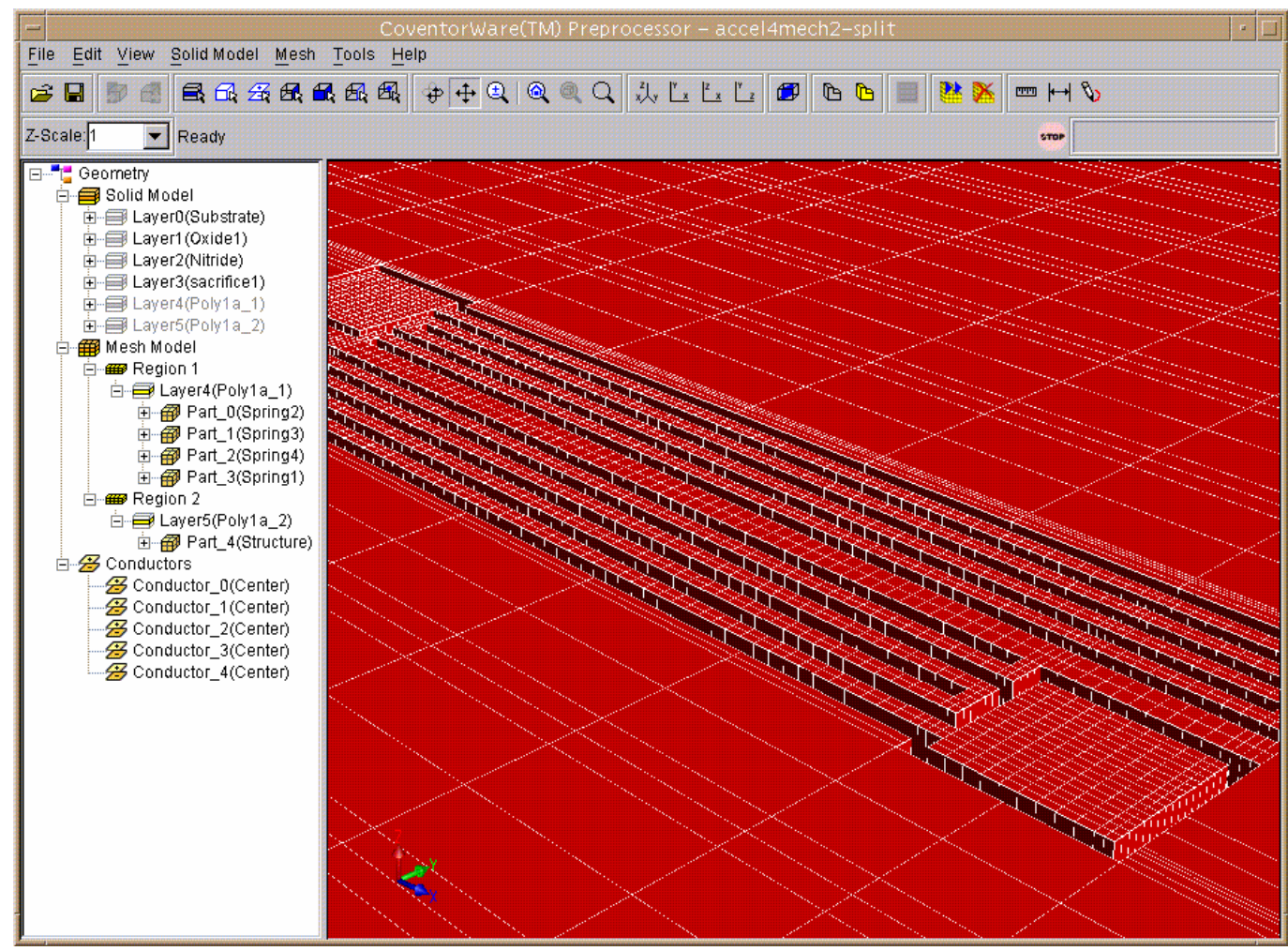

Figure 6.17(b): Close-up image of a spring region from the solid model showing the fine details of the mesh. The springs had five elements in the vertical direction while the remainder of the structure had three.

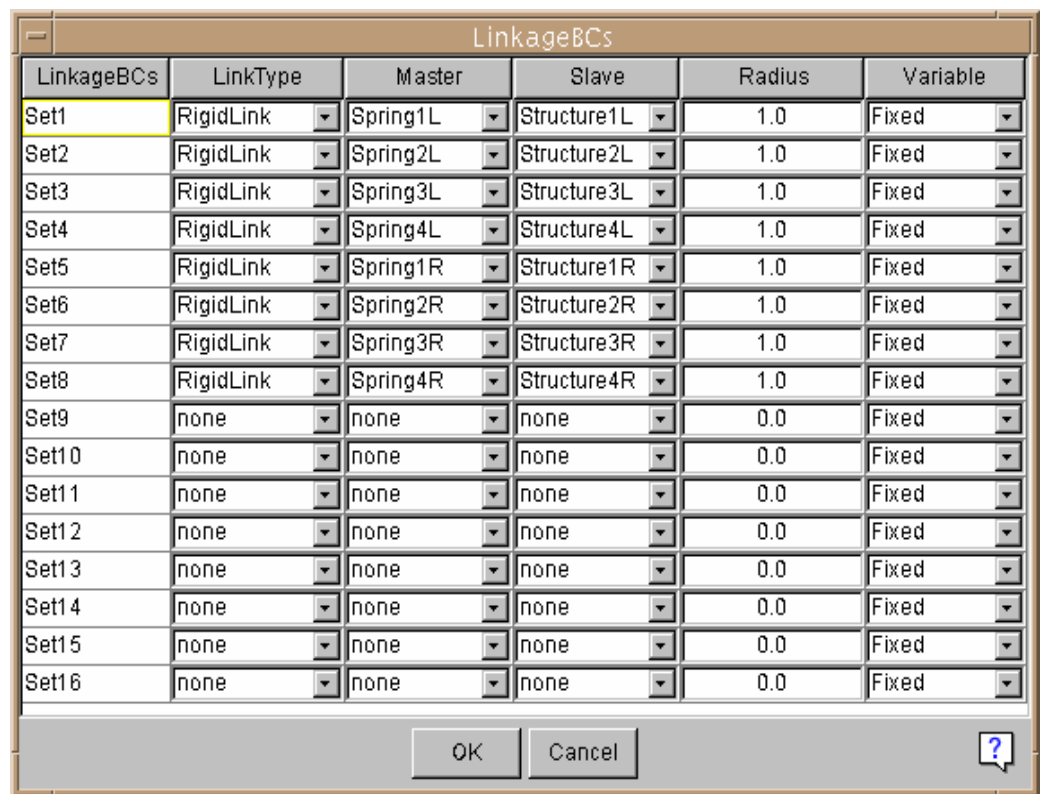

Figure 6.18(a): Re-attachment of the springs to the structure through linkage boundary conditions. Rigid links were used as opposed to the more complex tied links, which were found to increase computational requirements with a minimal effect on results. 
at the expense of computational resources. Experimentation with the two formats as well as different rigid link radii showed that the deformation in the patches was sufficiently minimal such that using rigid links with a radius value of 1.0 produced results very similar to those of tied links with far less computation time. Only a single surface boundary condition was needed, that being the restriction of motion in the anchor regions as shown in the SurfaceBCs window of Figure 6.18(b). Applying the loads was a multistep procedure that began with the VolumeBCs window of Figure 6.18(c). For this model, two separate accelerations were applied; the Set 1 load was a fixed $1 g$ gravitational force

\begin{tabular}{|c|c|c|c|c|c|c|c|c|c|c|c|c|c|c|c|c|c|}
\hline \multicolumn{18}{|c|}{ SurfaceBCs } \\
\hline SurfaceBCs & FixType & \multicolumn{2}{|c|}{ Patch1 } & \multicolumn{2}{|c|}{ and1 } & \multicolumn{2}{|c|}{ Patch2 } & \multicolumn{2}{|c|}{ and2 } & \multicolumn{2}{|c|}{ Patch3 } & \multicolumn{3}{|c|}{ LoadValue } & \multirow{2}{*}{\begin{tabular}{|l|} 
Variable \\
Fixed
\end{tabular}} & \multicolumn{2}{|c|}{ Transient } \\
\hline Set1 & fixAll & Anchors & $\nabla$ & and & \pm & none & $\nabla$ & and & $\nabla$ & none & $\nabla$ & Scalar & \pm & 0.0 & & Fixed & $\nabla$ \\
\hline Set2 & none & none & $\nabla$ & and & $\nabla$ & none & $\nabla$ & and & $\nabla$ & none & $\nabla$ & Scalar & $\nabla$ & 0.0 & Fixed $\square$ & |Fixed & $\nabla$ \\
\hline Set3 & none & none & $\nabla$ & and & 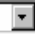 & none & $\nabla$ & and & $\nabla$ & |none & $\nabla$ & Scalar & $\nabla$ & 0.0 & Fixed $\square$ & Fixed & $\nabla$ \\
\hline Set4 & none & none & $\nabla$ & and & 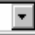 & none & 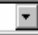 & and & $\nabla$ & |none & $\nabla$ & Scalar & 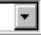 & 0.0 & Fixed $\square$ & Fixed & $\nabla$ \\
\hline Set5 & none & none & $\nabla$ & and & $\nabla$ & |rone & $\nabla$ & and & $\nabla$ & none & $\nabla$ & Scalar & $\nabla$ & 0.0 & Fixed & Fixed & $\nabla$ \\
\hline Set6 & none & none & $\nabla$ & and & $\nabla$ & none & $\nabla$ & and & $\nabla$ & none & $\nabla$ & Scalar & $\nabla$ & 0.0 & Fixed & Fixed & $\square$ \\
\hline Set? & none & none & 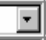 & and & $i$ & none & $I$ & and & 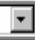 & none & 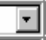 & Scalar & $\nabla$ & 0.0 & Fixed & Fixed & $\nabla$ \\
\hline Set8 & none & none & $\nabla$ & and & 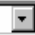 & | & $\nabla$ & and & $\nabla$ & none & $\nabla$ & Scalar & $\nabla$ & 0.0 & Fixed - & Fixed & $\nabla$ \\
\hline Set9 & none & none & 9 & and & 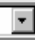 & none & $I$ & and & 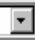 & none & 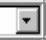 & Scalar & 7 & 0.0 & Fixed & Fixed & 7 \\
\hline Set10 & none & |none & $\nabla$ & and & $\nabla$ & none & $\nabla$ & and & $\nabla$ & |none & $\nabla$ & Scalar & $\nabla$ & 0.0 & Fixed $\square$ & Fixed & $\nabla$ \\
\hline Set11 & none & none & $\nabla$ & and & 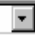 & none & $\nabla$ & and & $\nabla$ & none & $\nabla$ & $\sqrt{\text { Scalar }}$ & $\nabla$ & 0.0 & Fixed & Fixed & $\nabla$ \\
\hline Set12 & none & none & $\square$ & and & 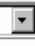 & none & \pm & and & $\square$ & none & 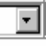 & Scalar & $\nabla$ & 0.0 & Fixed & Fixed & 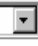 \\
\hline
\end{tabular}

Figure 6.18(b): Surface boundary condition preventing motion of the four anchor patches in any direction.

\begin{tabular}{|c|c|c|c|c|c|c|c|c|c|c|c|}
\hline- & & & & & Vo & neBCs & & & & & \\
\hline VolumeBCs & ВСТуре & & & & & Loadv & & Variable & & Tra & \\
\hline Set1 & Acceleration & $\nabla$ & ALL & $\nabla$ & Acceleration & $\nabla$ & Edit & Fixed & \pm & Fixed & $\nabla$ \\
\hline Set2 & Acceleration & $\nabla$ & ALL & $\nabla$ & Acceleration & 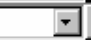 & Edit & MechBC1 & 7 & Fixed & 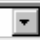 \\
\hline Set3 & none & $\nabla$ & none & $\nabla$ & Scalar & $\square$ & 0.0 & Fixed & 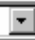 & Fixed & $\nabla$ \\
\hline Set4 & none & $\nabla$ & none & $\nabla$ & Scalar & $\nabla$ & 0.0 & Fixed & \pm & Fixed & $\nabla$ \\
\hline Set5 & none & $\nabla$ & none & $\nabla$ & Scalar & $\nabla$ & 0.0 & Fixed & $\nabla$ & Fixed & $\nabla$ \\
\hline Set6 & none & $\nabla$ & none & $\nabla$ & Scalar & $\nabla$ & 0.0 & Fixed & 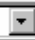 & Fixed & $\nabla$ \\
\hline Set? & none & $\nabla$ & none & $\nabla$ & Scalar & $\nabla$ & 0.0 & Fixed & $\nabla$ & Fixed & $\nabla$ \\
\hline Set8 & none & $\nabla$ & |none & $\nabla$ & Scalar & \pm & 0.0 & Fixed & 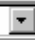 & Fixed & 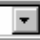 \\
\hline & & & & & OK & Cancel & & & & & ?] \\
\hline
\end{tabular}

Figure 6.18(c): Volumetric boundary conditions establishing two separate acceleration loads. The first was a fixed load defined as shown in Figure 6.17(d). The second was a trajectory by the name of MechBC1 defined as shown in Figures 6.17(e-h). 


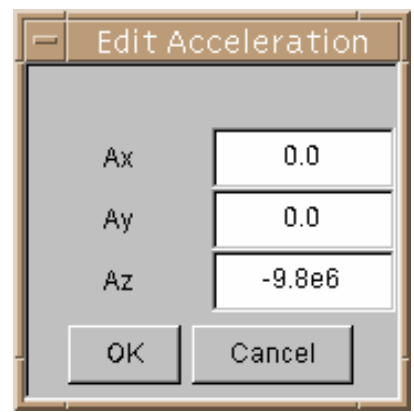

(d)

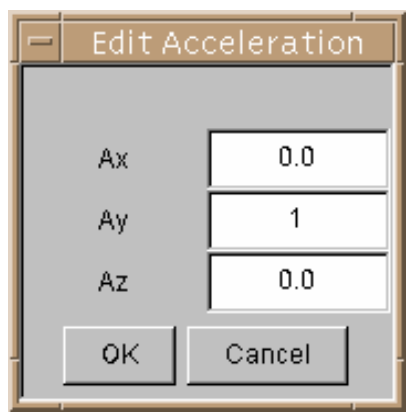

(e)

Figure 6.18(d,e): Acceleration loads applied separately but simultaneously to the structure under the two Edit tabs in Figure 6.17(c). The load in (d) referred to the downward gravitational force. The load in (e) was a scaling factor that was multiplied by the trajectory vector $t 1$, defined in Figures 6.17(f-h) to establish the load MechBCl.

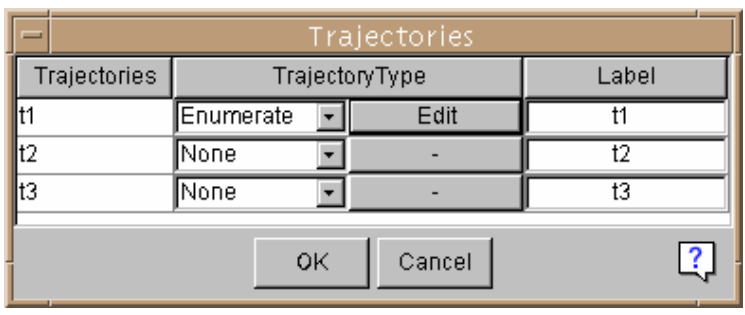

(f)

\begin{tabular}{|c|c|c|c|c|c|}
\hline \multicolumn{3}{|l|}{$=$} & \multicolumn{3}{|c|}{ mechBCs } \\
\hline mechBCs & \multicolumn{2}{|c|}{ Trajectory } & \multicolumn{2}{|c|}{ Type } & \multirow{2}{*}{$\frac{\text { ScaleFactor }}{1.0}$} \\
\hline MechBC1 & $t 1$ & 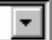 & Factor & 7 & \\
\hline MechBC2 & none & 7 & Factor & $\nabla$ & 1.0 \\
\hline WechBC3 & none & 7 & Factor & $\nabla$ & 1.0 \\
\hline MechBC4 & none & 7 & Factor & $\nabla$ & 1.0 \\
\hline MechBC5 & none & 7 & Factor & 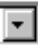 & 1.0 \\
\hline WechBC6 & none & 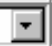 & Factor & $\square$ & 1.0 \\
\hline MechBC7 & none & 7 & Factor & 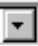 & 1.0 \\
\hline \multirow[t]{2}{*}{ WechBC8 } & none & 7 & Factor & 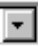 & 1.0 \\
\hline & & OK & \multicolumn{2}{|c|}{ Cancel } & \\
\hline
\end{tabular}

$(\mathrm{g})$

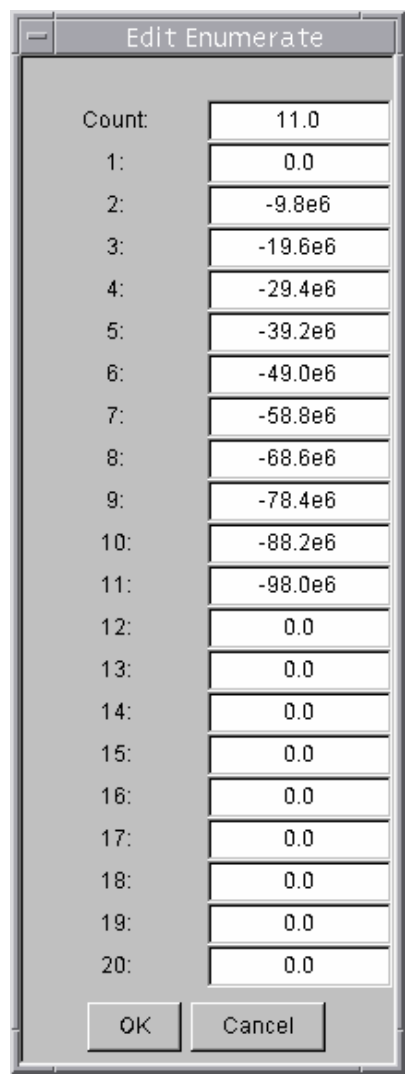

(h)

Figures 6.18(f-h): Definition of acceleration trajectory load $M e c h B C l$. The trajectory was established under the name $t l$ in (f) and assigned actual values through the Edit tab, shown in (h). It was then incorporated as a mechanical boundary condition in (g) at which point an additional scaling factor may be utilized. 
applied through the Edit tab as shown in Figure 6.18(d), and the Set2 load was a trajectory identified as $M e c h B C l$ defined elsewhere and assigned a direction and optional scaling factor through the Edit tab of Figure 6.18(e). Definition of MechBCl occurred through the series of windows shown in Figures 6.18(f-h). A trajectory was allocated under the name $t 1$ in Figure 6.18(f) that was assigned to the boundary condition load MechBCl through Figure 6.18(g) with another scaling opportunity. The actual values used in the simulation are shown in Figure 6.18(h) and correspond to applied accelerations ranging from $0 g-10 g$. Figure 6.19 shows the structural shift computed for each of the four different spring styles. The values verified the accuracy of the calculated spring constants, with results of $4.98 \mathrm{~N} / \mathrm{m}, 5.73 \mathrm{~N} / \mathrm{m}, 13.57 \mathrm{~N} / \mathrm{m}$, and $17.46 \mathrm{~N} / \mathrm{m}$ for Springs $1-4$, which were all within $5 \%$ of the calculated values of $4.77 \mathrm{~N} / \mathrm{m}, 5.72 \mathrm{~N} / \mathrm{m}, 13.27 \mathrm{~N} / \mathrm{m}$,

\section{Structural Shift vs Applied Acceleration}

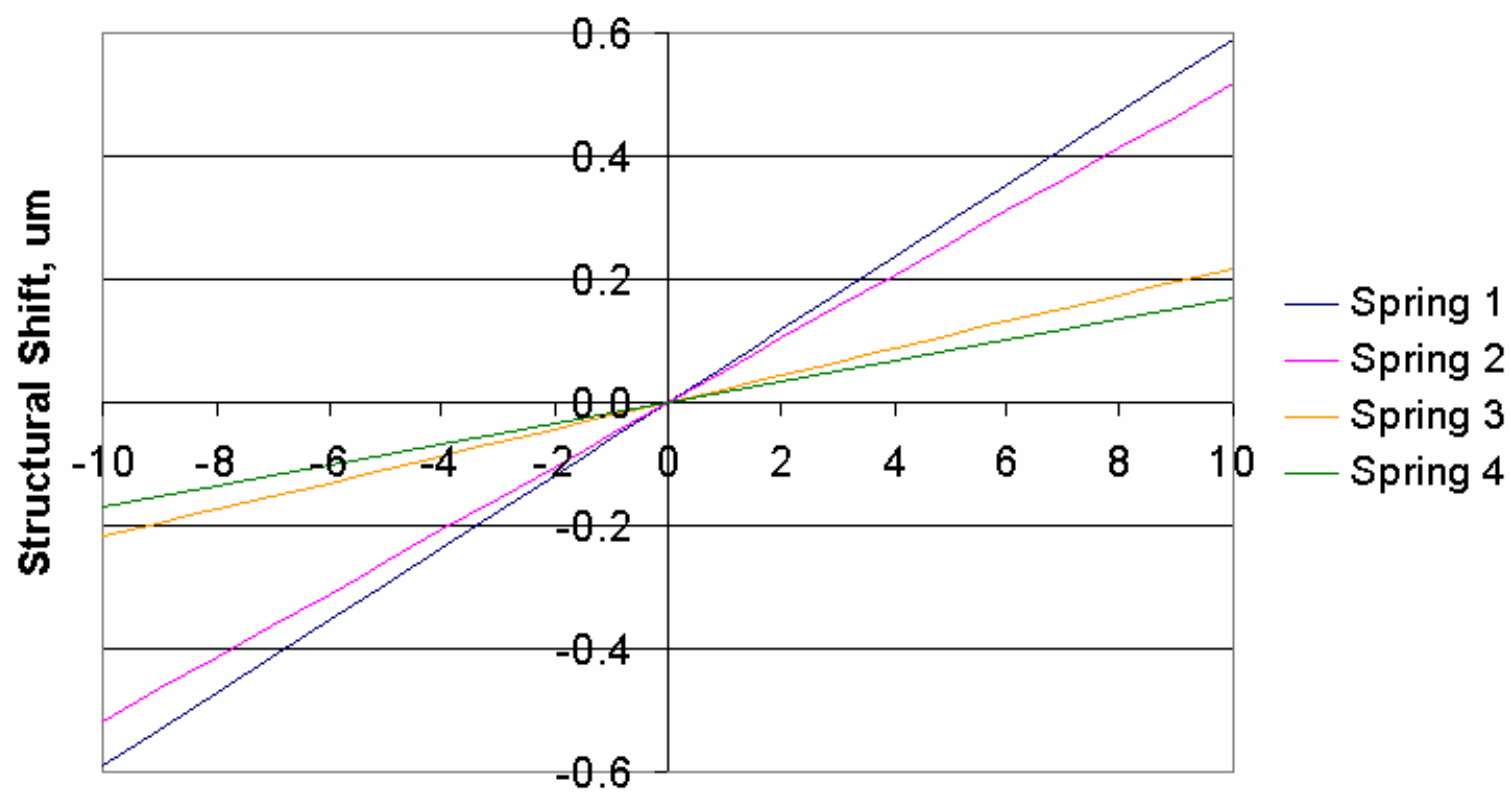

\section{Applied Acceleration, /g}

Figure 6.19: Mechanical simulation results for each of the four spring styles. Reaching the targeted $0.15 \mu \mathrm{m}$ structural shift under full load required accelerations of $2.54 \mathrm{~g}, 2.90 \mathrm{~g}$, $6.88 \mathrm{~g}$, and $8.86 \mathrm{~g}$ for Springs $1-4$, respectively, corresponding to spring constants of $4.98 \mathrm{~N} / \mathrm{m}, 5.73 \mathrm{~N} / \mathrm{m}, 13.57 \mathrm{~N} / \mathrm{m}$, and $17.46 \mathrm{~N} / \mathrm{m}$. 
and $17.69 \mathrm{~N} / \mathrm{m}$. Downward deflection in the z-direction due to the gravitational force was found to be in the range of $0.543-2.87 \mathrm{~nm}$, corresponding to vertical spring constants from $103.2-544.9 \mathrm{~N} / \mathrm{m}$. The accuracy in the results confirmed that the devices were ready to begin fabrication.

\section{6: Device Fabrication}

The accelerometer fabrication process was initiated with six $100 \mathrm{~mm}$ wafers purchased from University Wafer with $1 \mu \mathrm{m}$ of thermal silicon dioxide on the front and back sides. The wafers were of the $<100>$ orientation and boron-doped to the $10-20 \Omega-\mathrm{cm}$ range. The first three films were all deposited via low pressure chemical vapor deposition (LPCVD) in one of several TMX9K 4304 tube furnaces. Deposition of the base silicon nitride layer was done through the decomposition of $\mathrm{SiH}_{2} \mathrm{Cl}_{2}$ (40sccm) and $\mathrm{NH}_{3}$ $(160 \mathrm{sccm})$ at $150 \mathrm{mTorr}$ and $820^{\circ} \mathrm{C}$. Measurements based on spectroscopic reflectometry using a NanoSpec 6100 indicated an average actual thickness of $0.271 \mu \mathrm{m}$. This system was used for all film thickness measurements unless indicated otherwise. The poly0 lower interconnects were then formed from a polysilicon layer produced using $\mathrm{SiH}_{4}$ $(80 \mathrm{sccm})$ at $180 \mathrm{mTorr}$ and $625^{\circ} \mathrm{C}$. The measured thickness was found to be $0.316 \mu \mathrm{m}$. Following deposition, this film was doped with phosphorous, again in a TMX9K 4304 furnace, in order to enhance its conductivity. This was done at $1175^{\circ} \mathrm{C}$. Following doping, the sheet resistance was measured on a four-point probe and found to be $26.5 \Omega / \mathrm{square}$, or $8.38 \mathrm{E}-4 \Omega-\mathrm{cm}$. This layer was then patterned to form the interconnect traces and sub-structural ground plane to which the device is anchored. Etching was done in a LAM 9400 RIE system at 500W using $\mathrm{HBr}(100 \mathrm{sccm})$ with $\mathrm{He}(100 \mathrm{sccm})$ as a carrier gas. The first inter-layer dielectric, poly0-poly1, consisted of an undoped silicon 


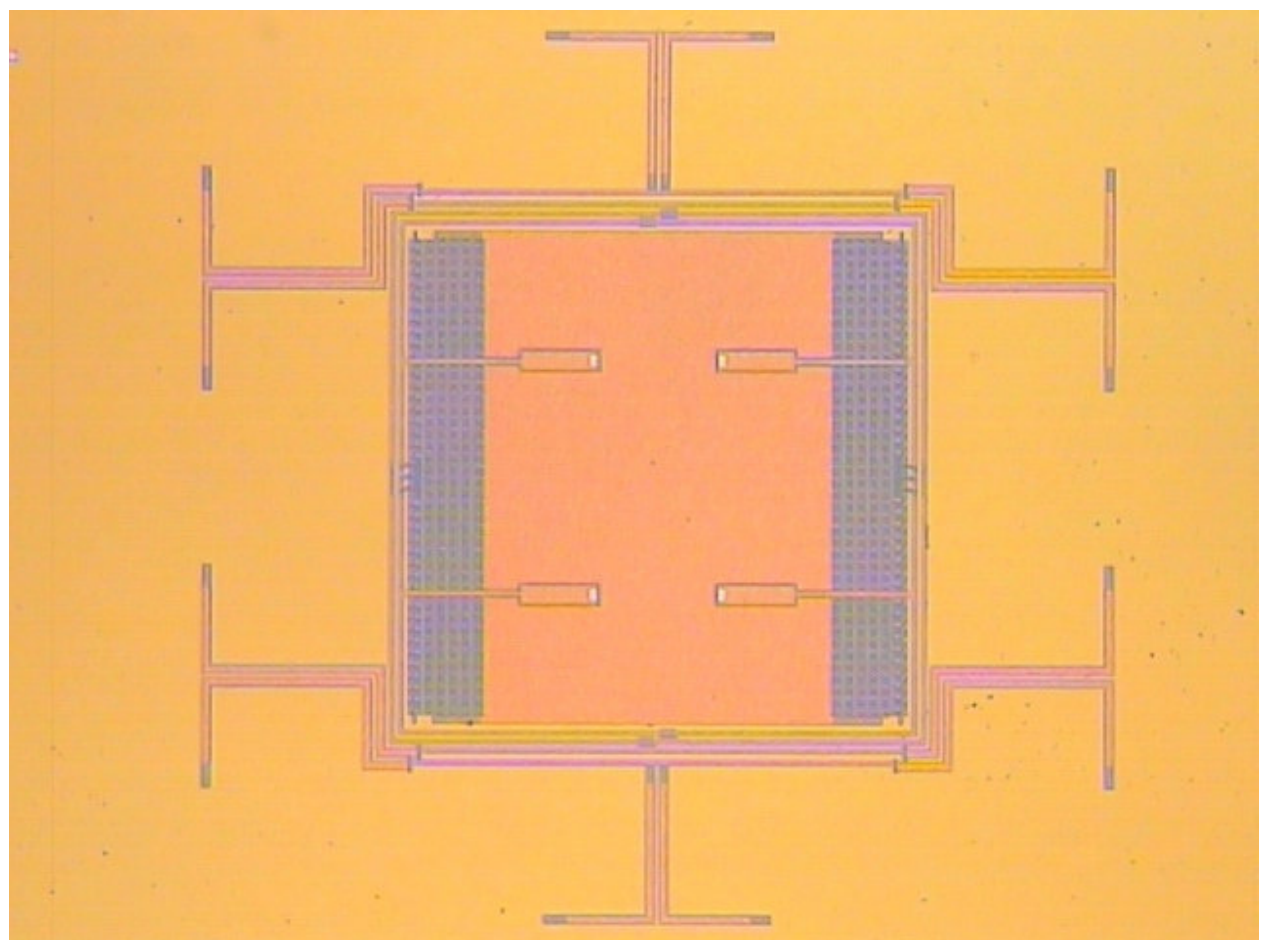

Figure 6.20(a): Image of full device following patterning of the poly0-polyl silicon dioxide layer, taken at $5 \mathrm{X}$ magnification.

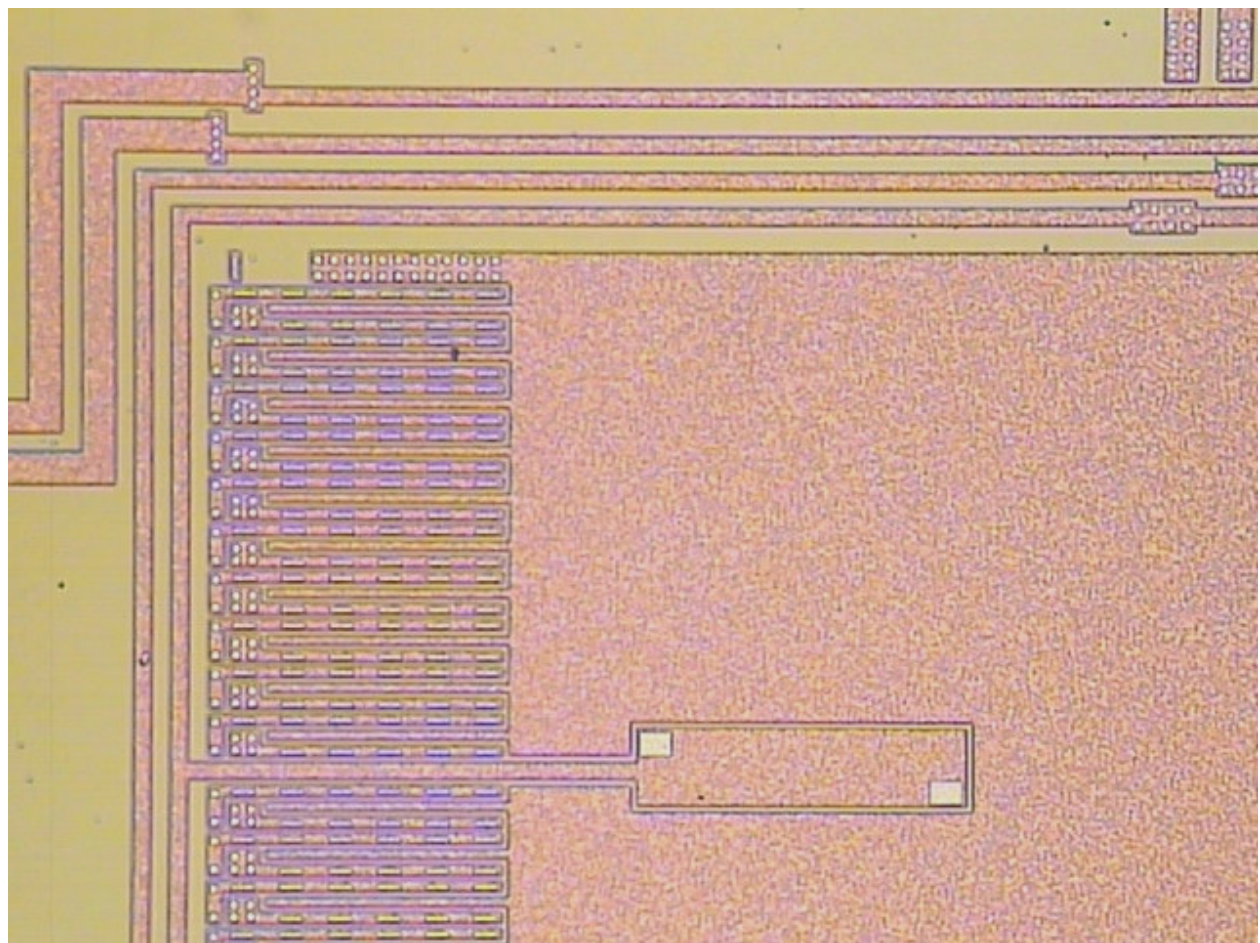

Figure 6.20(b): Close-up of upper left-hand corner of device following poly0-poly1 patterning, taken at 20X magnification. Vias and anchor regions were visible in white and appeared to be in good alignment. 
dioxide film deposited using $\mathrm{N}_{2} \mathrm{O}(120 \mathrm{sccm})$ and $\mathrm{SiH}_{2} \mathrm{Cl}_{2}(60 \mathrm{sccm})$ at $400 \mathrm{mTorr}$ and $920^{\circ} \mathrm{C}$. Measurement indicated an actual thickness of $1.19 \mu \mathrm{m}$, considerably lower than the $2 \mu \mathrm{m}$ target but nevertheless deemed to be sufficient as the device springs were highly rigid in the vertical direction. Patterning defined the electrical vias as well as the structural anchor points, and the film was etched using buffered hydrofluoric acid (BHF). The images in Figures $6.20(\mathrm{a}, \mathrm{b})$ illustrate the state of the device at this stage in fabrication. The poly1 structural layer was formed through one of two means; half of the six wafers underwent a $20 \mu \mathrm{m}$ epitaxial deposition at Lawrence Semiconductor and half received an $8 \mu \mathrm{m}$ LPCVD polysilicon layer at the University of Michigan (equipment usage policies dictated the limitation on the latter set). The former was done in an ASM 2000 epitaxial reactor at $1150^{\circ} \mathrm{C}$ under atmospheric pressure using $\mathrm{HSiCl}_{3}$, and the latter was done in a Tempress 6400 tube furnace at $600^{\circ} \mathrm{C}$ under $220 \mathrm{mTorr}$ with $60 \mathrm{sccm} \mathrm{SiH}_{4}$. The intent was for the epitaxial wafers to provide a low-stress baseline set of devices against which to compare the inductively annealed devices. This plan did not come to fruition, however, as will be discussed shortly. Following deposition, both sets of wafers were mechanically polished on a Logitech PM2A system in order to reduce the surface roughness. Figure 6.21 illustrates the non-uniform morphology of the epi-poly device layer. The silicon grown above the poly0-poly1 via sites appeared darker in color than the remainder, indicative of a transition boundary in which that formed above the vias was in direct contact with the poly0 polysilicon, which provided a seed layer, while the remainder was situated above silicon dioxide. The seed layer gave the epi-poly a base morphology upon which to form, resulting in material of better quality, whereas that grown rapidly above silicon dioxide exhibited a lower degree of crystallinity. Because the 


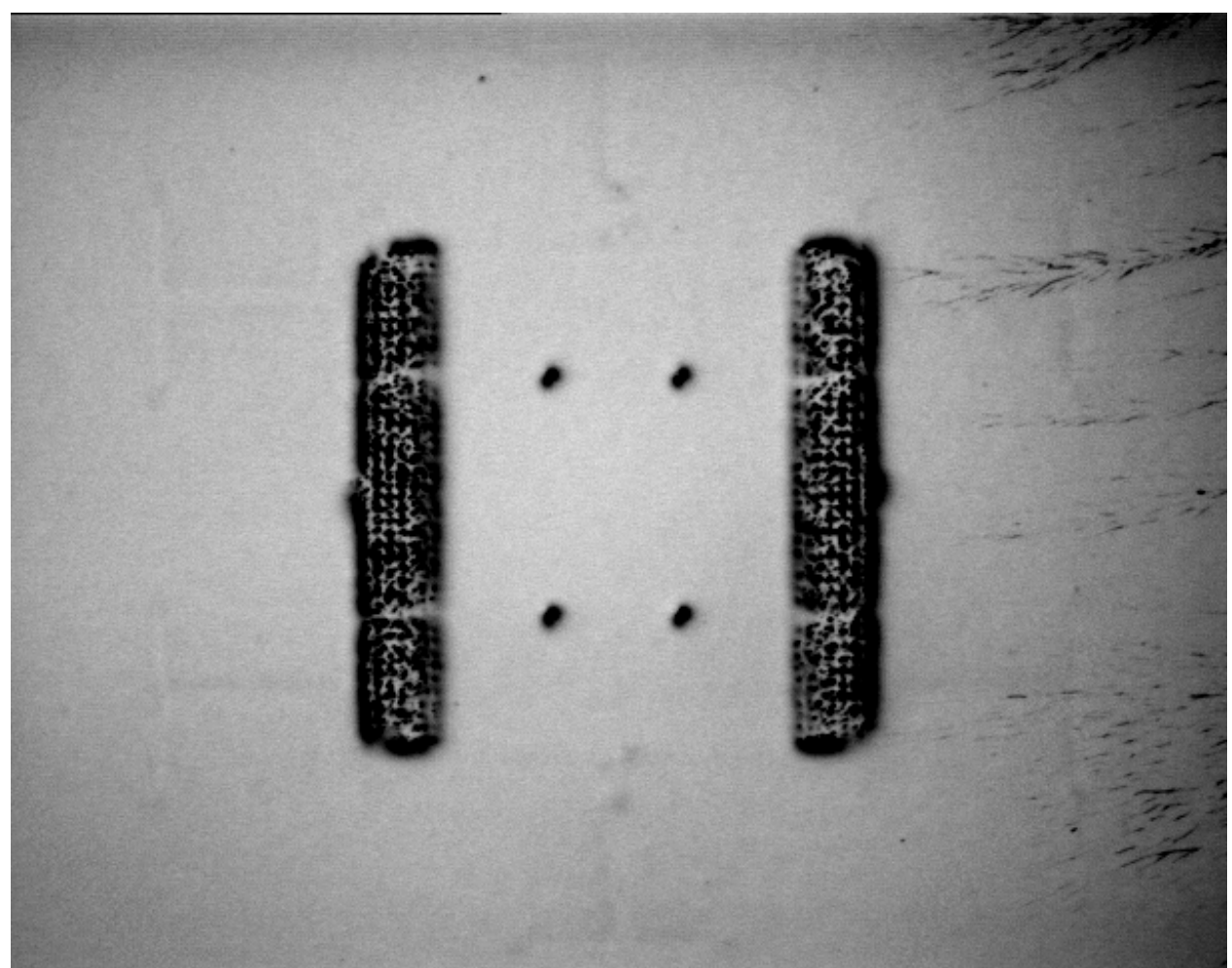

Figure 6.21: Image of epi-poly device layer following the polishing step and prior to patterning, taken at $5 \mathrm{X}$ magnification. Darker areas, which occurred above the poly0poly1 via locations, reflected differences in crystalline morphology as compared to that deposited on silicon dioxide.

polyl film was of such high thickness, any features formed in the layers below, such as alignment marks, were no longer visible. Thus the first pattern transferred to the device layer provided for the removal of the polysilicon in two large window-like sections on opposite sides of the wafer in order to reveal the alignment marks. A second mask was intended to provide thermal isolation trenches for the test wafers, as was discussed in Section 6.2 and illustrated in Figure 4.24, and thus was not utilized as the epitaxial wafers did not require annealing. The third and final pattern defined the actual shape of the sensors including the proof mass, moving and stationary electrodes, springs, and etch holes. All etching was done on a STS Multiplex ICP using a recipe formulated for deep trench etching: $100 \mathrm{~W}$ at the platen and $800 \mathrm{~W}$ at the coil generator with gas flow rates of 
$130 \mathrm{sccm} \mathrm{SF}_{6}$ and $13 \mathrm{sccm} \mathrm{O}_{2}$ for etching and $85 \mathrm{sccm} \mathrm{C}_{4} \mathrm{~F}_{8}$ for passivation. Future endeavors will likely combine the second and third masks into a single step, but for this initial attempt they were left separate in order to allow for independent changes to each as the process evolves. Figures 6.22(a,b) illustrate the appearance of the devices following DRIE etching. The "window" openings made alignment possible but nevertheless somewhat difficult due to the $20 \mu \mathrm{m}$ of vertical spacing between the alignment marks and the wafer surface; this approximately doubled the distance between the patterns to be aligned, which rendered simultaneous focusing more difficult. Following the DRIE steps, the second inter-layer dielectric was deposited via plasma enhanced chemical vapor deposition (PECVD) to a $2.1 \mu \mathrm{m}$ measured thickness in order to provide complete refill of

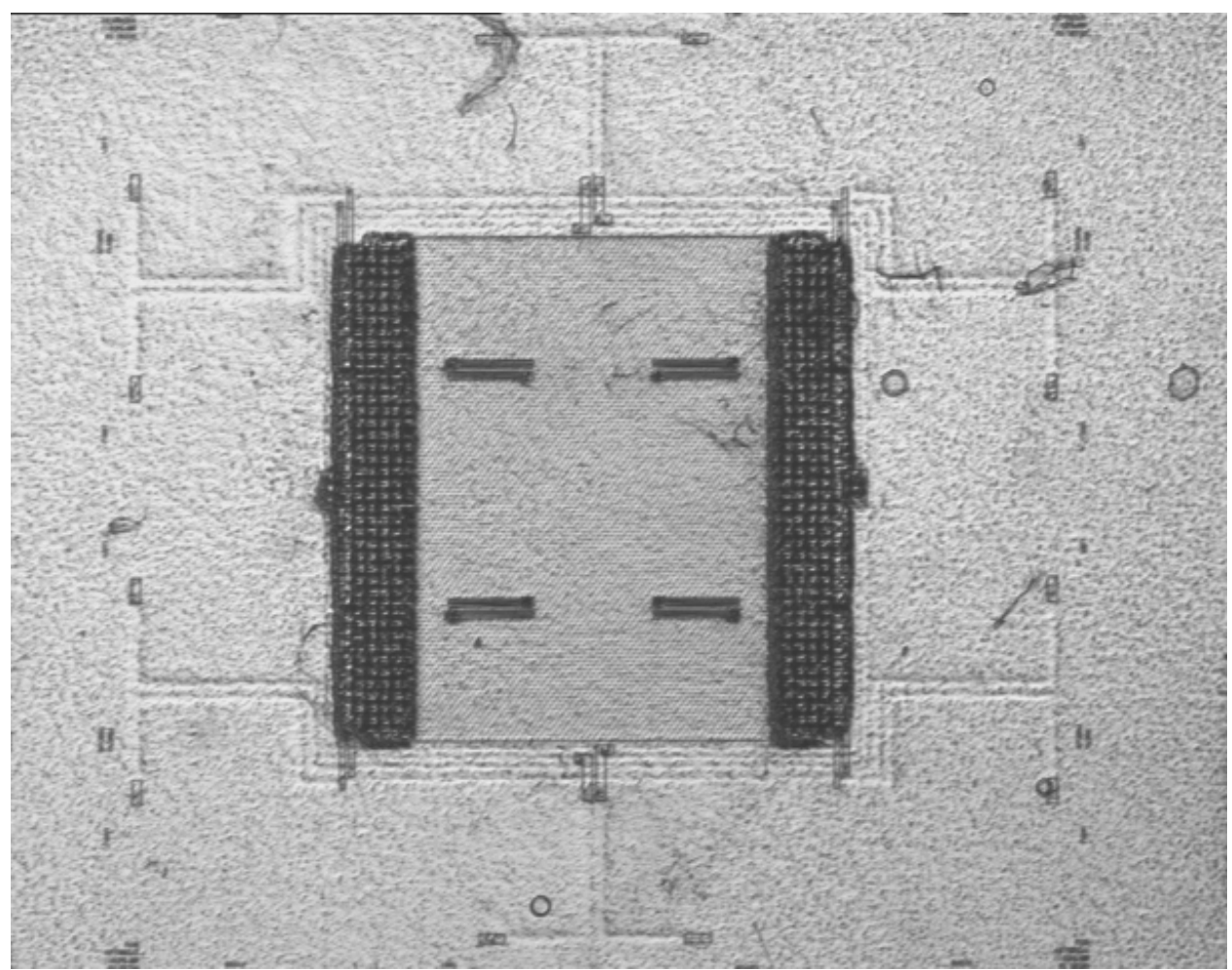

Figure 6.22(a): Image of sensor following patterning of the poly 1 device layer, taken at $5 \mathrm{X}$ magnification. The DRIE procedure first required the opening of two large "window" areas in order to reveal the alignment marks buried beneath. This made it possible to align to the previous layers. 


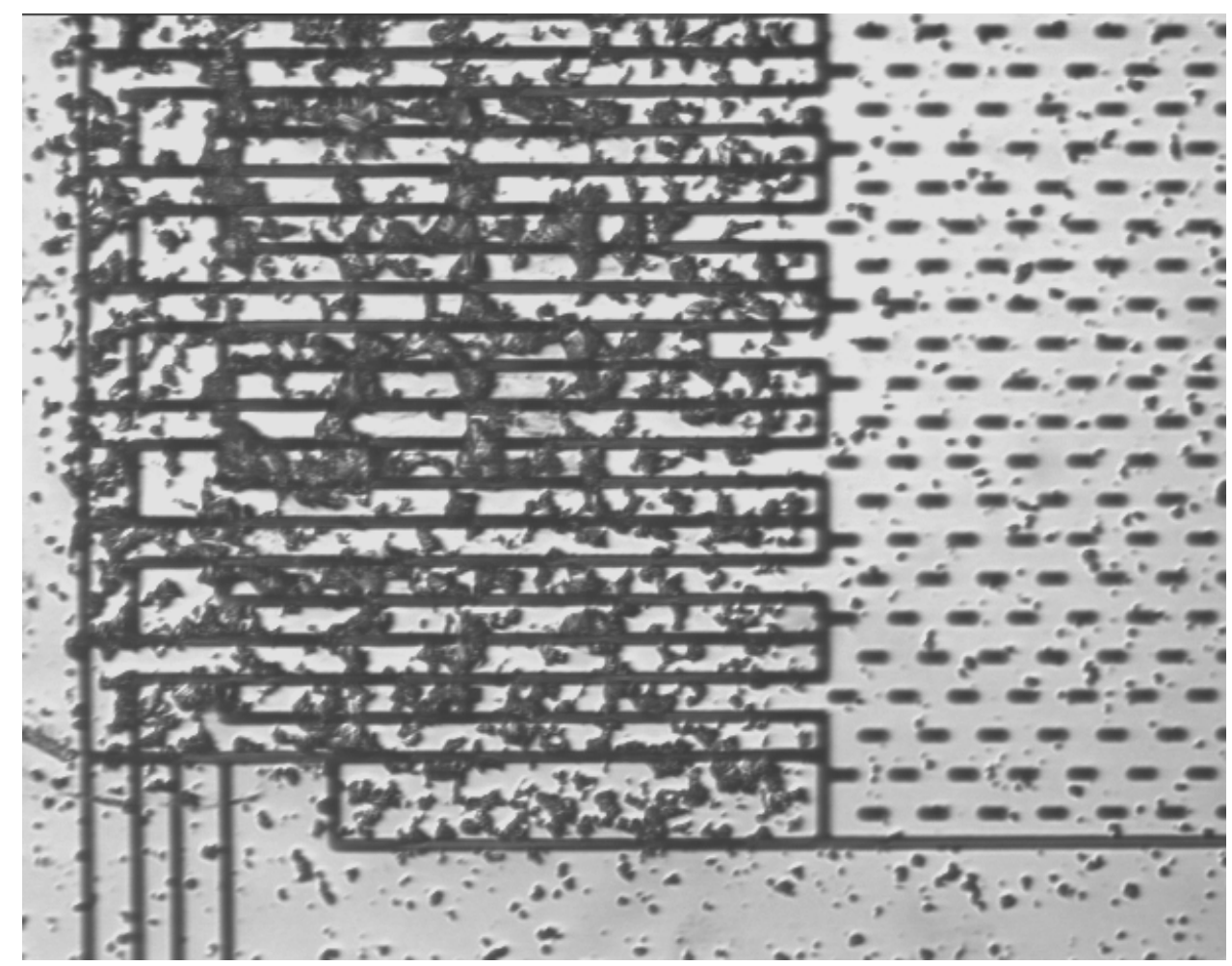

Figure 6.22(b): Close-up image showing the lower left-hand corner of the polyl structural layer following patterning, taken at 50X magnification. The $2 \times 6 \mu \mathrm{m}$ etch holes are visible, as are the seemingly random shapes of the regions of non-uniform morphology and their sizes relative to that of the electrodes.

the $2 \mu \mathrm{m}$-wide trenches in the structure. Deposition was done on a GSI Ultradep 2000 at $200^{\circ} \mathrm{C}$. Refill was far from complete, however, and it was largely the combination of the low quality of the poly1-metall silicon dioxide film and incomplete trench refill, as illustrated in Figure 6.23(a), that rendered the devices non-functional. It was at this stage that inductive annealing was intended to be performed on the three test wafers. Unfortunately, as was shown in Chapter 4, the technology had not yet advanced to a state in which the thorough annealing of an $8 \mu \mathrm{m}$ structure was achievable. Although temperatures in excess of $1000^{\circ} \mathrm{C}$ could be reached, limitations in ferromagnetic film quality limited the controllability range to below $900^{\circ} \mathrm{C}$, above which the substrates rapidly entered the thermal runaway condition and melted almost instantaneously. The 


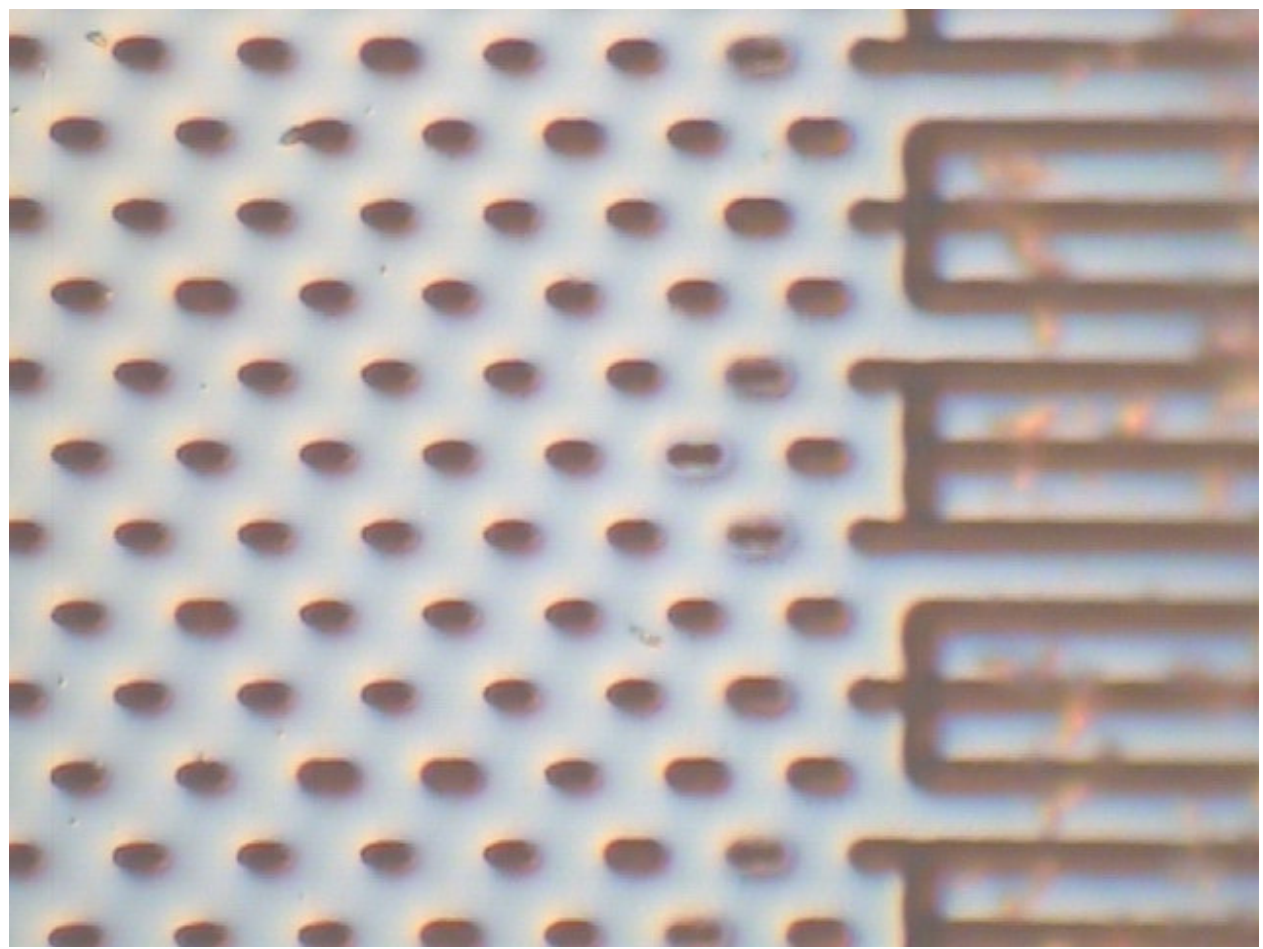

Figure 6.23(a): Image showing the partial but incomplete trench refill following deposition of the polyl-metall silicon dioxide layer, taken at 100X magnification. Etch holes appeared smaller and rounder than previously, with the new sizes exhibiting considerable variation as a result of non-uniformity in film thickness.

process had been designed around the ferromagnetic ring and thermal trench concept exactly as was illustrated in Figure 4.24, and would have followed the standard inductive annealing procedure: deposition of a chrome adhesion layer to a thickness of approximately $100-150 \mathrm{~nm}$, deposition of the ferromagnetic film, photolithography and etching, inductive heating on the BEAVIS test system, and lastly the removal of both the ferromagnetic and adhesion films. Instead, however, the test wafers were placed in storage for future use and the process continued with only the epitaxial wafers. The poly1-metal1 silicon dioxide layer was etched in a Semi Group RIE 1000 TP/CC system at $180 \mathrm{~W}$ and $20 \mathrm{mTorr}$ using $25 \mathrm{sccm}$ each of $\mathrm{CF}_{4}$ and $\mathrm{CHF}_{3}$. Figure 6.23(b) shows a portion of the device at this stage of the process. The vias appeared to have formed well, with minimal overetch. The final device layer, metall, turned out to present a rather 


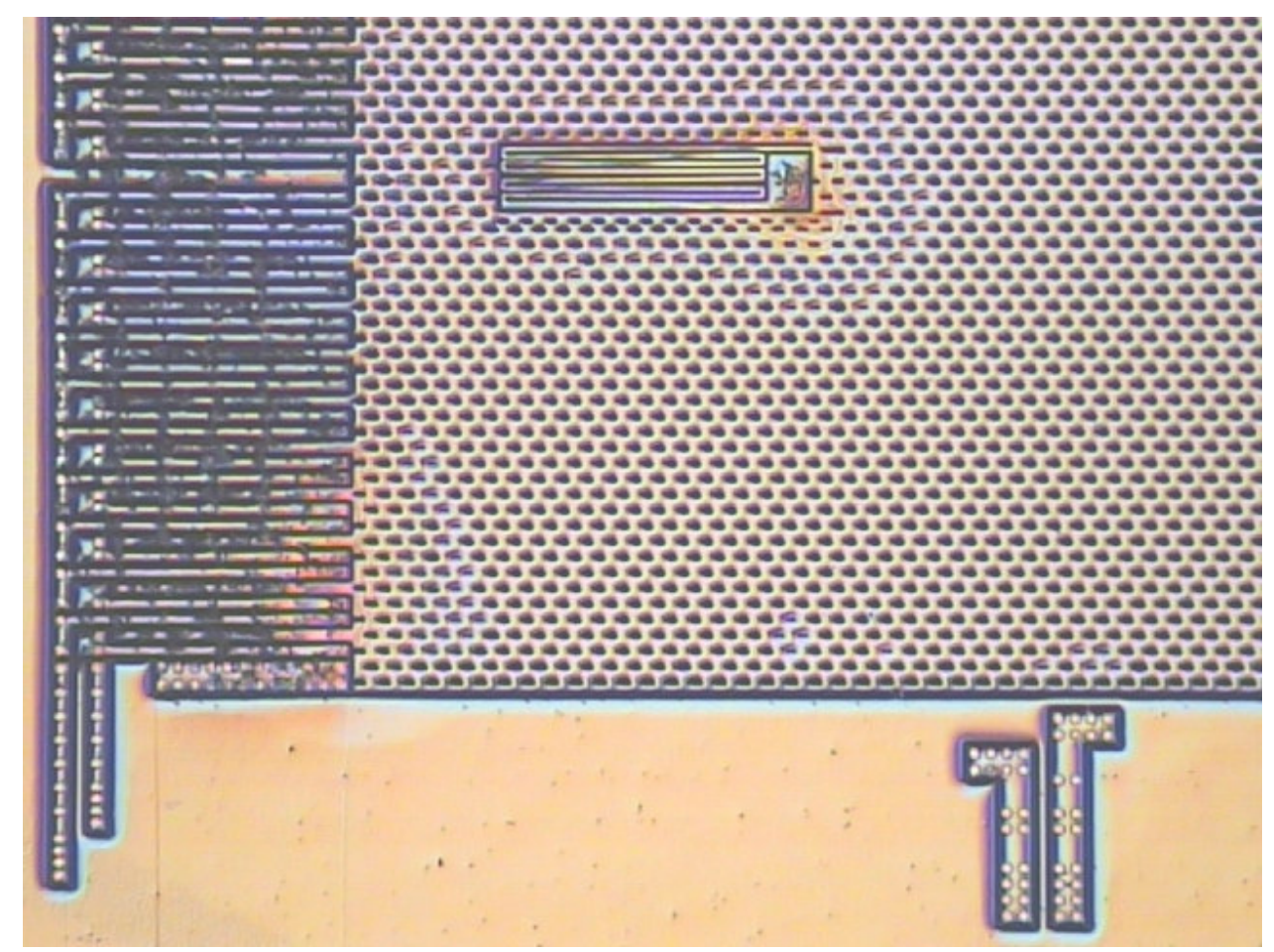

Figure 6.23(b): Close-up image of the lower left-hand corner of the device, taken at $20 \mathrm{X}$ magnification, showing polyl-metall vias along the outer portion of the stationary electrodes.

formidable challenge. With vias deeper than $2 \mu \mathrm{m}$ to fill, it was found that metallic films were not giving sufficient step coverage to overcome their relative softness, resulting in collapsed airbridges. For this reason, the decision was made to switch to polysilicon. Deposited via PECVD using a GSI Ultradep 2000, a $1.1 \mu \mathrm{m}$ polysilicon film gave much improved rigidity and step coverage. Following patterning, it was planned to sputter deposit a titanium layer for silicide formation at $1000^{\circ} \mathrm{C}$ via rapid thermal annealing (RTA) on a Heatpulse 410 RTA system. The unreacted titanium was to be removed during device release, as it is known to dissolve in BHF [Williams, et al., 1996]. Test samples showed, however, that the RTA chamber was substantially contaminated with metals such as lead. The result was an alloy that was not removable in standard etching solutions including BHF and thus would have prevented device release. Thus the 
silicidation procedure was abandoned for this first process iteration and the wafers were advanced to the release step. Wafers were cleaved into quarters and each portion was processed individually in order to maximize the chances of obtaining one or more functional devices. Release began with a 1-hour soak in BHF to dissolve the supporting silicon dioxide layers. This was followed with a ten-minute rinse in de-ionized water followed by immersion in isopropanol, methanol, and pentane for ten minutes each. The purpose of the three solvents was to reduce the likelihood of stiction; they exhibit increasing surface tension, helping to prevent released features from being pulled toward each other or to the substrate. Agitation was minimized as much as possible during each bath as well as when in transition, again for anti-stiction purposes. Thus concluded the first pass of the MTU high aspect ratio transducer process.

Despite the effort that was made, no functional devices were found after this first process iteration. Figure 6.24(a) shows a SEM image of a completed, unreleased sensor. From the multi-toned appearance of the surface layers, it was evident that both the polylmetall silicon dioxide and the metall silicon layers exhibited textural non-uniformity. The $2.1 \mu \mathrm{m}$ silicon dioxide failed to fully refill the poly 1 trenches as shown in Figure 6.24(b), indicating that the deposition reaction did not adequately permeate below the polysilicon surface. Increasing the dielectric's thickness would have the adverse effect of increasing the step height of the poly1-metall vias and further complicating airbridge formation, and thus a more elaborate refill procedure will likely be required. In the future, the use of a two-step PSG deposition with an intermediate heat treatment for reflow is suggested. Refinement of this portion of the process will be critical to device fabrication unless the airbridges are eliminated in favor of a second buried interconnect. 


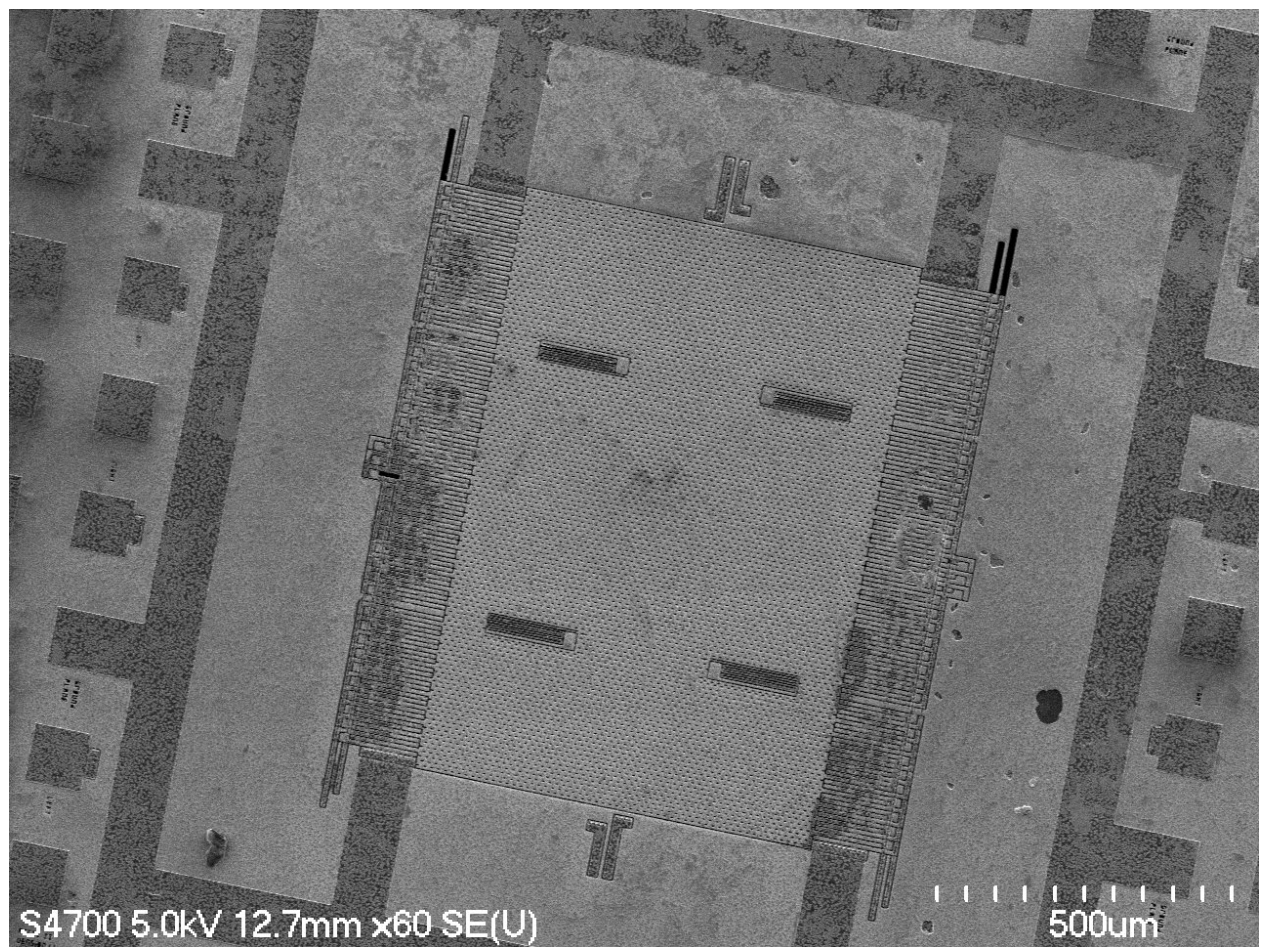

Figure 6.24(a): SEM image of a completed, unreleased accelerometer. Both of the two PECVD films, poly1-metall and metal1, exhibited significant non-uniformity in morphology, as is evidenced by the film discoloration.

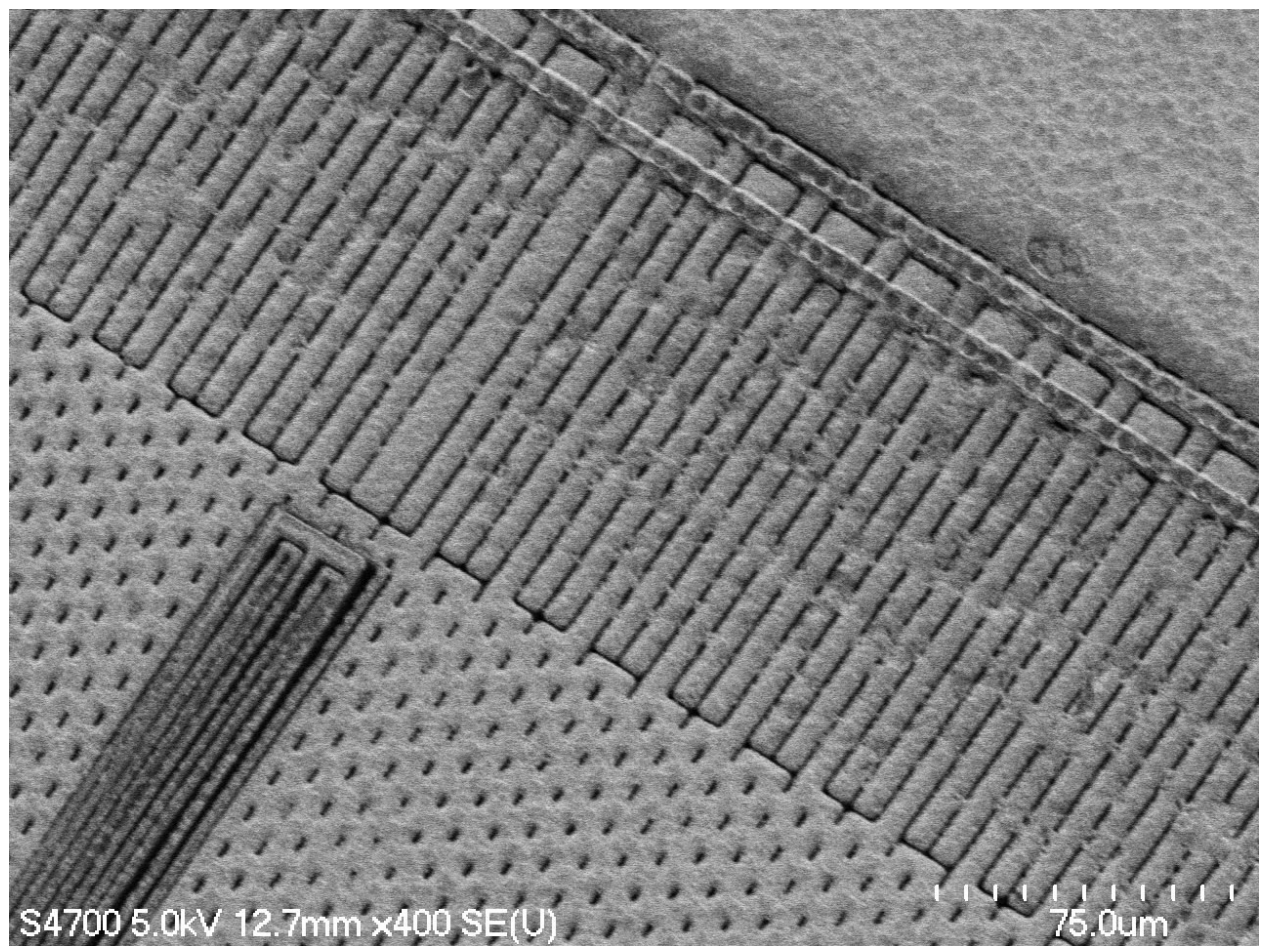

Figure 6.24(b): Close-up SEM image of the right-hand side of the device illustrating the incomplete refill of the structural trenches. While some portions of the electrode gaps had closed, the majority remained open and susceptible to trapping subsequent materials. 


\section{Conclusion}

This chapter presented the process by which a lateral accelerometer was designed and fabricated for the purpose of demonstrating the inductive annealing technology. The basic components of a device of this nature were defined, and the component values that resulted from the design choices were specified. Modeling through finite element simulation was utilized to confirm design accuracy as well as to help to verify the absence of basic layout errors, which are much easier to identify and correct in the software phase of development than in the later hardware phase. The combined results of the electrostatic and mechanical simulations produced a high degree of confidence that the devices should perform as expected. Unfortunately, the fabrication process was of a highly complex nature and will likely require several iterations before successful devices are produced. Nevertheless, the exercise served as a first iteration in the development of a high aspect ratio MEMS process. The goal of incorporating inductive annealing into a state of the art micromachining technology remains unfulfilled, but with continued effort will be realized in the near future. 


\section{Chapter 7: Conclusion and Recommendations for Future Work}

\section{1: Conclusion}

This dissertation proposed a novel semiconductor fabrication technology in which inductively heated ferromagnetic films were utilized to establish controlled temperature gradients within a semiconductor device. The primary research motivation was to address existing limitations to the monolithic integration of MEMS and CMOS due to conflicts in thermal budget as was described in Chapter 1. Development was concentrated on surface micromachined polysilicon structures in accordance with present industrial preferences. The inductive annealing technology utilized the superior magnetic properties of ferromagnetic materials to drive the efficient conversion of energy from magnetic to thermal. Selective patterning provided the ability to target a specific region of a structure or device for the purpose of localized polysilicon recrystallization.

Process characterization and refinement made use of theory, simulation, and experimentation. Correlation of these three counterparts was confirmed through analysis of output trends with respect to input parameters. The theoretical equations of Chapter 2 predicted the impact of film properties, dimensions, and the applied magnetic field on the resulting power dissipation and induced temperatures. Analysis of the simulated trends in Chapter 3 supported these relationships both in magnitude and in rate of change while also providing qualitative information on process behaviors. The experimental measurements in Chapter 4 reflected some deviation from theoretical assumptions but nevertheless were typically within an order of magnitude of expectation. Thus the underlying variables that defined process efficiency were well understood, as were the requirements for performance improvement. 
Many challenges were revealed during process development that limited the ability to achieve the research objectives as initially defined. Solutions were obtained through both innovation and directional changes, but the overall scope was nevertheless narrowed due in part to processing capabilities. The relative magnetic permeability of the heated film was perhaps the most important input variable to the process, but challenges in film quality limited that of the usable materials to only 15.8 , considerably short of the expectations discussed in Chapter 2. Other problems that rendered the technology in its present state undesirable for industrial application included thermal runaway above $800^{\circ} \mathrm{C}$, stress-induced delamination, non-uniformity across the heated wafer, and the overall footprint. Proposed solutions to these concerns are discussed in Section 7.2. Finally, the polysilicon annealing results failed to show a complete anneal on a functional micro-mechanical sensor as was originally intended. The accelerometer design presented in Chapter 6 was simulated successfully, but challenges encountered during fabrication rendered the final devices non-functional. Annealing was demonstrated on polysilicon cantilever beams and accelerometer structures in Chapter 5, but in both cases the recrystallization was incomplete due to trade-offs between induced temperature and process control. Thus thorough anneals were only demonstrated on the proof-of-concept cantilever beams, for which the early test configuration rendered essentially no process control, and on blanket polysilicon films. The realization of a functional, inductively annealed microsensor has been left for future research endeavors.

In conclusion, this initial phase of process development was successful in that the capability of the inductive annealing technology to resolve the CMOS-MEMS integration challenge was confirmed. Measures were taken to align with industrial fabrication 
processes and, although the work remains far from complete, the technology has the potential to resolve the drawbacks of existing methods and provide for maximum flexibility in device design and fabrication through system modularity, unrestricted structural materials, and precise heat treatment control.

\section{2: Recommendations for Future Work}

Continued research into the development of the technology should focus on the optimization of coupling efficiency between the magnetic field and the ferromagnetic films. One of the key avenues toward achieving this is the improvement of the properties of the magnetic films themselves, with studies recommended into alternate deposition methods, post-deposition magnetic annealing, optimum alloy composition for the research application, stress reduction for adhesion improvement and elimination of device tear-out. Enhanced thermal isolation methods are also of high importance, as was demonstrated through both simulation and experimentation. The utilization of a base layer extremely low in thermal conductivity so as to isolate the device region from the highly conductive substrate is of great importance, and a thermally resistive region of separation between the regions of high and low temperature is also recommended. Lastly, further development of the inductive heating test system must include a means by which the heated film can be controllably cooled back down so as to prevent stress-induced delamination, and methods to increase the strength of the applied magnetic field are also suggested. The latter could be achieved through increased power to the coil and/or improvements in coil design though increased turns of the solenoid or possibly even a different style altogether. The addition of a high-resolution thermal imaging camera 
would also be of great assistance, as it would provide a more direct, quantitative means by which the induced thermal gradients could be analyzed and optimized.

The high aspect ratio accelerometer technology presented in Chapter 6 also left many opportunities for improvement. The primary obstacle that prevented successful completion of the inertial sensors was the refill of the trenches within the polysilicon device layer. The development of a $\mathrm{SiO}_{2}$ reflow anneal would help to ensure a more thorough, uniform trench refill, but maintaining compatibility with the low-temperature nature of the post-CMOS integration approach presented a challenge. Other recommendations include improving the adhesion at the airbridge contacts, and developing a better means by which to perform alignments following the deposition of the thick device layer. This process must be optimized on its own before an attempt can be made to incorporate the inductive annealing technology. The successful integration would yield a true demonstration that would very likely gain the attention of the semiconductor industry. 


\section{References}

Advanced Energy Industries, "Sekidenko OR2000 Optical Fiber Thermometer," Application Notes, http://www.advanced-energy.com/upload/SL-2000-260-01.pdf, March 2002.

Allen, J.J., Kinney, R.D., Sarsfield, J., Daily, M.R., Ellis, J.R., Smith, J.H., Montague, S., Howe, R.T., Boser, B.E., Horowitz, R., Pisano, A.P., Lemkin, M.A., Clark, W.A., Juneau, T., "Integrated Micro-Electro-Mechanical Sensor Development for Inertial Applications," IEEE Aerospace and Electronic Systems Magazine, November 1998.

Allenspach, R., Bischof, A., Dürig, U., and Grütter, P., "Local modification of magnetic properties by an electron beam," Applied Physics Letters 73, December 1998.

Ameritherm, "Equipment Selection Guide," http://www.ameritherm.com/productcatalog_m.html, 2007.

ANSYS Basic Analysis Procedures Guide, Release 8.0, Copyright 2003 SAS IP, http://www1.ansys.com/customer/content/documentation/80/ansys/Hlp_G_BasTOC.html.

ANSYS Commands Reference, Release 8.0, Copyright 2003 SAS IP, http://www1.ansys.com/customer/content/documentation/80/ansys/Hlp_C_CmdTOC.htm $\underline{1 .}$

ANSYS Coupled-Field Analysis Guide, Release 8.0, Copyright 2003 SAS IP, http://www1.ansys.com/customer/content/documentation/80/ansys/Hlp_G_CouTOC.html

ANSYS Electromagnetic Field Analysis Guide, Release 8.0, Copyright 2003 SAS IP, http://www1.ansys.com/customer/content/documentation/80/ansys/Hlp_G_EleTOC.html.

ANSYS Element Reference, Release 8.0, Copyright 2003 SAS IP, http://www1.ansys.com/customer/content/documentation/80/ansys/Hlp_E_ElemTOC.htm $\underline{1 .}$

ANSYS Modeling and Meshing Guide, Release 8.0, Copyright 2003 SAS IP, http://www1.ansys.com/customer/content/documentation/80/ansys/Hlp_G_ModTOC.htm $\underline{1 .}$

Arajs, S., "Paramagnetic Behavior of Nickel just Above the Ferromagnetic Curie Temperature," Journal of Applied Physics 36, March 1965.

Ardesta, "Sandia, Ardesta join forces to commercialize MEMS and microsystems," Ardesta News Archives, http://www.ardesta.com/1about/abonea040401.asp, April 4, 2001. 
Bacher, W., Menz, W., and Mohr, J., "The LIGA Technique and Its Potential for Microsystems - A Survey," IEEE Transactions on Industrial Electronics 42, October 1995.

Balanis, C.A., Advanced Engineering Electromagnetics, Wiley: New York, 1989.

Barandiaran, J.M., Gorria, P., Gomez, J.C., Barquin, L.F., and Kaul, S.N., "Influence of Boron on the Magnetic and Transport Properties of FeZr Amorphous and Nanocrystalline Alloys," IEEE Transactions on Magnetics 30, November 1994.

Bergstrom, P.L. and Trombley, M.L., "Induction Heating of Thin Films", U.S. Patent 6,878,909, issued April 12, 2005.

Bergstrom, P.L. and Trombley, M.L., "Magnetic Annealing of Ferromagnetic Thin Films using Induction Heating,” U.S. Patent 7,193,193, issued March 20, 2007.

Bhave, S.A., Seeger, J.I., Jiang, X., Boser, B.E., Howe, R.T., and Yasaitis, J., "An Integrated, Vertical-Drive, In-Plane-Sense Microgyroscope," Proceedings of the International Conference on Solid State Sensors, Actuators, and Microsystems, 2003.

Bozorth, R.M., Ferromagnetism, IEEE Press: New York, 1993.

Budraa, N.K., Jackson, H.W., Barmatz, M., Pike, W.T., and Mai, J.D., "Low Pressure and Low Temperature Hermetic Wafer Bonding Using Microwave Heating," Proceedings of the IEEE International Conference on Microelectromechanical Systems, 1999, pp. 490-492.

Budraa, N., Ng, B., Wang, D., Ahsan, S., Zhang, Y., and Mai, J., "Microwave Techniques for High-Density Electronics Interconnect Bonding and Hybridization," IEEE Transactions on Nuclear Science 51, December 2004.

Bustillo, J.M., Howe, R.T., and Muller, R.S., "Surface Micromachining for Microelectromechanical Systems," Proceedings of the IEEE 86, August 1998.

Calvert, J.B., "Capacitors,” http://www.du.edu/ jcalvert/phys/caps.htm, April 2003.

Campo, E., Scheid, E., Bielle-Daspet, D., and Guillemet, J.-P., "Influence of Rapid Thermal and Low Temperature Processing on the Electrical Properties of Polysilicon Thin Film Transistors," IEEE Transactions on Semiconductor Manufacturing 8, August 1995, pp. 298-303.

Cao, A., Chio, M., Lin, L., "Selective and Localized Bonding using Induction Heating," Proceedings of the Solid-State Sensor, Actuator, and Microsystems Workshop, Hilton Head Island, SC, June 2002. 
Celem Passive Components, "A Quick Guide to Induction Heating," http://www.celem.com/support.html, 2005.

Chavan, A.V. and Wise, K.D., "A Monolithic Fully-Integrated Vacuum-Sealed CMOS Pressure Sensor," IEEE Transactions on Electron Devices 49, 2000.

Chae, J., Kulah, H., and Najafi, K., "A Hybrid Silicon-On-Glass (SOG) Lateral MicroAccelerometer with CMOS Readout Circuitry," Proceedings of the IEEE International Conference on Microelectromechanical Systems, 2002.

Chang, R., Chemistry, $5^{\text {th }}$ Ed., McGraw-Hill, 1994.

Chen, F.H. and Ng, V., "Effects of Temperature, Deposition Conditions, and Magnetic Field on $\mathrm{Ni}_{80} \mathrm{Fe}_{20} / \mathrm{Fe}_{50} \mathrm{Mn}_{50} / \mathrm{Ni}_{80} \mathrm{Fe}_{20} / \mathrm{Al}_{2} \mathrm{O}_{3} / \mathrm{Co}$ and $\mathrm{Ni}_{80} \mathrm{Fe}_{20} / \mathrm{Al}_{2} \mathrm{O}_{3} / \mathrm{Co}$ Magnetic Tunnel Junctions," IEEE Transactions on Magnetics 41, January 2005.

Chen, K.-S. and Ou, K.-S., "Modification of curvature-based thin-film residual stress measurement for MEMS applications," Journal of Micromechanics and Microengineering 12, 2002.

Cheng, Y.T., Lin, L, and Najafi, K., "Localized Silicon Fusion and Eutectic Bonding for MEMS Fabrication and Packaging," Journal of Microelectromechanical Systems 9, 2000.

Ciszek, T.F., "Some Applications of Cold Crucible Technology for Silicon Photovoltaic Material Preparation," Journal of the Electrochemical Society 132, April 1985.

Clendenin, J., Tung, S., Budraa, N., and Mai, J., "Microwave Bonding of Silicon Dies with Thin Metal Films for MEMS Applications," Proceedings of the Electronic Components and Technology Conference, 2003.

Coventor, "Microcosm Technologies and Sandia National Labs Team to Transfer MEMS Production Capability to Communications and Biotechnology Industries," In the News: Archive, January 8, 2001, http://www.coventor.com/news/more/010801.htm.

CRC, CRC Handbook of Chemistry and Physics, 70 ${ }^{\text {th }}$ Ed., CRC Press, 1989.

CRC, CRC Handbook of Chemistry and Physics, 80 ${ }^{\text {th }}$ Ed., CRC Press, 1999.

Cullity, B.D., Elements of X-Ray Diffraction, $1^{\text {st }}$ Ed., 1956.

Deferm, L., Decoutere, S., Claeys, C., and Declert, G., "Latch-up in a BiCMOS Technology," Proceedings of the IEEE International Electron Devices Meeting, 1988.

Detavernier, C., Van Meirhaeghe, R.L., Cardon, F., Maex, K., Bender, H., and Zhu, S., "CoSi $\mathrm{C}_{2}$ formation in the $\mathrm{Ti} / \mathrm{Co} / \mathrm{SiO}_{2} / \mathrm{Si}$ system," Journal of Applied Physics 88, 2000. 
Dobson, J., "Magnetism in Matter and Magnetic Biomaterials," Center for Science and Technology in Medicine, Keele University, http://www.keele.ac.uk/depts/stm/magmaterials.html, 2000.

Eibeck, P.A. and Muramatsu, B., "It Can Be Shown That ..." http://bits.me.berkeley.edu/beam/param_3z.html, 2000.

Eisberg, R. and Resnick, R., Quantum Physics of Atoms, Molecules, Solids, Nuclei, and Particles, Wiley: New York, 1985.

Elliott, S.R., The Physics and Chemistry of Solids, Wiley: Chichester, 1998.

Elwenspoek, M. and Wiegerink, R., Mechanical Microsensors, Springer-Verlag, 2001.

Feynman, R.P., Leighton, R.B., and Sands, M., The Feynman Lectures on Physics, Vol. 2, Addison-Wesley: Lebanon, IN, 1989.

Forsythe, W.E., "Smithsonian Physical Tables", $9^{\text {th }}$ Ed., Table 80: Computation of Total Emissivity Values for Various Glass Samples at Low Temperatures, Knovel, April 16, 2003.

Franca, D.R. and Blouin, A., "All-optical measurement of in-plane and out-of-plane Young's modulus and Poisson's ratio in silicon wafers by means of vibration modes," Measurement Science and Technology 15, 2004, pp. 859-868.

Franke, A.E., Heck, J.M., King, T.-J., and Howe, R.T., "Polycrystalline SiliconGermanium Films for Integrated Microsystems," Journal of Microelectromechanical Systems 12, April 2003.

French, P.J., "Polysilicon: a versatile material for microsystems," Sensors and Actuators $A$ 99, April 2002.

French, P.J., van Drieënhuizen, B.P., Poenar, D., Goosen, J.F.L., Mallée, R., Sarro, P.M., and Wolffenbuttel, R.F., "The Development of a Low-Stress Polysilicon Process Compatible with Standard Device Processing," Journal of Microelectromechanical Systems 5, September 1996, pp. 187-196.

Funk, K., Emmerich, H., Schilp, A., Offenberg, M., Neul, R., and Lärmer, F., "A Surface Micromachined Silicon Gyroscope using a Thick Polysilicon Layer," Proceedings of the IEEE International Conference on Microelectromechanical Systems, 1999.

Gad-el-Hak, M., Ed., “Inertial Sensors,” The MEMS Handbook, CRC Press: Boca Raton, 2002.

Galayko, D., Kaiser, A., Legrand, B., Collard, D., Buchaillot, L., and Combi, C., "HighFrequency High-Q Micro-Mechanical Resonators in Thick Epipoly Technology with 
Post-Process Gap Adjustment," Proceedings of the IEEE International Conference on Microelectromechanical Systems, 2002.

Garcia, F., Moritz, J., Ernult, F., Auffret, S., Rodmacq, B., Dieny, B., Camarero, J., Pennec, Y., Pizzini, S., and Vogel, J., "Exchange Bias With Perpendicular Anisotropy in (Pt-Co) $)_{\mathrm{n}}$-FeMn Multilayers," IEEE Transactions on Magnetics 38, September 2002.

Gianchandani, Y.B. and Najafi, K., "A Bulk Silicon Dissolved Wafer Process for Microelectromechanical Devices," Journal of Microelectromechanical Systems 1, June 1992.

Guckel, H., "High-Aspect-Ratio Micromachining Via Deep X-Ray Lithography," Proceedings of the IEEE, August 1998, pp. 1586-1593.

Guckel, H., Burns, D.W., Tilmans, H.A.C., Deroo, D., and Rutigliano, C.R., "Mechanical Properties of Fine Grained Polysilicon - The Repeatability Issue," Technical Digest IEEE Solid-State Sensor and Actuator Workshop, June 1988, pp. 96-99.

Guckel, H., Burns, D.W., Visser, C.C.G., Tilmans, H.A.C., and Deroo, D., "Fine-Grained Polysilicon Films with Built-In Tensile Strain," IEEE Transactions on Electron Devices 35, June 1988, pp. 800-801.

Hayzelden, C. and Batstone, J.L., "Silicide formation and silicide-mediated crystallization of nickel-implanted amorphous silicon thin films," Journal of Applied Physics 73, 1993.

Heuer, A.H., "LPCVD Polysilicon Films with Controlled Curvature for Optical MEMS: the MultiPoly Process," 2000 IEEE/LEOS International Conference on Optical MEMS, August 2000.

Holmes, A.S., "Laser fabrication and assembly processes for MEMS," Proceedings of LASE 2001 High Power Lasers and Applications, 2001.

Honda, H. and Kaya, S., "Magnetism of single crystals of iron," Science Reports of Tohoku University 15, pp. 721-753, 1926.

Ierardi, J.A., A Computer Model of Fire Spread from Engine to Passenger Compartments in Post-Collision Vehicles, http://users.wpi.edu/ ierardi/PDF/, Master's Thesis, Worcester Polytechnic Institute, May 1999.

Institute for Rock Magnetism (IRM), University of Minnesota, http://www.irm.umn.edu/hg2m/hg2m_c/hg2m_c.html.

Irwin, J. D., Basic Engineering Circuit Analysis, $5^{\text {th }}$ Ed., Prentice-Hall: NJ, 1996. 
Joachim, D. and Lin, L., "Characterization of Selective Polysilicon Deposition for MEMS Resonator Tuning," Journal of Microelectromechanical Systems 12, April 2003.

Juan, W.-H. and Pang. S.W., "Released Si microstructures fabricated by deep etching and shallow diffusion", Journal of Microelectromechanical Systems 5, March 1996.

Jun, K.-I., Lee, J.H., Shin, K.-H., Rhie, K., and Lee, B.C., "Rotation of the Pinned Direction in Artificial Antiferromagnetic Tunnel Junctions by Field Annealing," IEEE Transations on Magnetics 40, July 2004.

Kahn, H., Stemmer, S., Nandakumar, K., Heuer, A.H., Mullen, R.L., Ballarini, R., and Huff, M.A., "Mechanical Properties of Thick, Surface Micromachined Polysilicon Films," Proceedings of the IEEE International Conference on Microelectromechanical Systems, 1996.

Kawazu, Y., Kudo, H., Onari, S., and Arai, T., "Initial stage of the interfacial reaction between nickel and hydrogenated amorphous silicon," Japanese Journal of Applied Physics 29, 1990.

Kittel, C., "Introduction to Solid State Physics," $2^{\text {nd }}$ Ed., Wiley: New York, 1956.

Krygowski, T.W., Rodgers, M.S., Sniegowski, J.J., Miller, S.M., and Jakubczak, J., “A Low-Voltage Rotary Actuator Fabricated Using a Five-Level Polysilicon Surface Micromachining Technology," Proceedings of the IEEE International Electron Devices Meeting, 1999.

Kung, J.T., "Methods for planarization and encapsulation of micromechanical devices in semiconductor processes," U.S. Patent 5,504,026, 1996.

Kuriyama, H., "Excimer laser crystallization of silicon films for AMLCDs," AMLCDs, 1995.

Lange, P., Kirsten, M., Riethmuller, W., Wenk, B., Zwicker, G., Morante, J.R., Ericson, F., and Schweitz, J.A., "Thick polycrystalline silicon for surface-micromechanical applications: deposition, structuring, and mechanical characterization," Sensors and Actuators A 54, 1996.

Leung, T.C., Cheng, C.F., and Poon, M.C., "Poly Silicon Film Formation by NickelInduced-Lateral-Crystallization and Pulsed Rapid Thermal Annealing," Proceedings of the IEEE International Electron Devices Meeting, 2001.

Leus, V. and Elata, D., "Fringing field effect in electrostatic actuators," Technical Report ETR-2004-2， http://meeng.technion.ac.il/Research/TReports/2004/ETR-2004-02.html, May 2004. 
Lewis, S., Alie, S., Brosnihan, T., Core, C., Core, T., Howe, R., Geen, J., Hollocher, D., Judy, M., Memishian, J., Nunan, K., Paine, R., Sherman, S., Tsang, B., and Wachtmann, B., "Integrated Sensor and Electronics Processing for $>10^{\wedge} 8$ "iMEMS" Inertial Measurement Unit Components," Proceedings of the IEEE International Electron Devices Meeting, 2003.

Li, B., Xiong, B., Jiang, L., Zohar, Y., and Wong, M., "Germanium as a Versatile Material for Low-Temperature Micromachining," Journal of Microelectromechanical Systems 8, December 1999.

Li, G. and Tseng, A.A., "Low Stress Packaging of a Micromachined Accelerometer," IEEE Transactions on Electronics Packaging Manufacturing 24, January 2001.

Luo, H., Zhang, G., Carley, L.R., and Fedder, G.K., "A Post-CMOS Micromachined Lateral Accelerometer," Journal of Microelectromechanical Systems 11, June 2002.

Luxtron, "Simplifying Pyrometer Selection with Signal to Noise Ratio," http://www.luxtron.com/pdf/TP04_OFT01_PyrometerSNR(041504).pdf, April 2004.

MEMX, "Sandia National Laboratories Announces Private MEMS Spin-Off Company," http://www.memx.com/pr1.htm, 2003.

Mizutani, S., Yokoshima, T., Nam, H.-S., Nakanishi, T., Osaka, T., and Yamazaki, Y., "High-Frequency Permeability and Thermal Stability of Electrodeposited High-B CoNiFe Thin Films," IEEE Transactions on Magnetics 36, September 2000.

Moore, C.B., and Ast, D.G., "Instabilities in the resistivity of intrinsic polysilicon resistors during hydrogenation: Role of sodium and cooling kinetics," Journal of Applied Physics 76, September 1994.

Murley, D., Young, N., Trainor, M., and McCulloch, D., "An Investigation of Laser Annealed and Metal-Induced Crystallized Polycrystalline Silicon Thin-Film Transistors," IEEE Transactions on Electron Devices 48, June 2001.

National Bureau of Standards (NBS), JCPDS-ICDD powder diffraction file for polycrystalline silicon, 2001.

Nave, C.R., "Bragg's Law," http://hyperphysics.phy-astr.gsu.edu/hbase/quantum/bragg.html, 2005.

Nave, C.R., "Heat $\quad$ Conduction", $\quad$ http://hyperphysics.phyastr.gsu.edu/hbase/thermo/heatra.html," 2005.

Nave, C.R., "Magnetic Field of Current Loop," http://hyperphysics.phyastr.gsu.edu/hbase/magnetic/curloo.html, 2005. 
Nave, C.R., "Power”, http://hyperphysics.phy-astr.gsu.edu/hbase/pow.html,” 2005.

Nave, C.R., "Solenoid Field from Ampere's Law", http://hyperphysics.phy-astr.gsu.edu/hbase/magnetic/solenoid.html,", 2005.

Nave, C.R., "Specific Heat", http://hyperphysics.phy-astr.gsu.edu/hbase/thermo/spht.html," 2005.

Nunan, K., Ready, G., Garone, P., Sturdy, G., and Sledziewski, J., "Developing a Manufacturable Process for the Deposition of Thick Polysilicon Films for Micro Machined Devices," Proceedings of the IEEE/SEMI Advanced Semiconductor Manufacturing Conference, 2000.

Offenberg, M., Lärmer, F., Elsner, B., Münzel, H., and Riethmüller, W., "Novel Process for a Monolithic Integrated Accelerometer," Proceedings of the International Conference on Solid State Sensors and Actuators, 1995.

Okhotin, A.S., Pushkarskii, A.S., and Gorbachev, V.V., Thermophysical Properties of Semiconductors, “Atom” Publishing House: Moscow, 1972.

Palaniapan, M., Howe, R.T., and Yasaitis, J., "Performance Comparison of Integrated ZAxis Frame Microgyroscopes," Proceedings of the IEEE International Conference on Microelectromechanical Systems, 2003.

Painter, C.C. and Shkel, S.M., "Structural and thermal modeling of a z-axis rate integrating gyroscope," Journal of Micromechanics and Microengineering 13, January 2003.

Parr, A.A., Gill, K., Gardiner, D.J., Hoyland, J.D., Sands, D., Brunson, K., and Carline, R.T., "A comparison of laser- and furnace-annealed polysilicon structure," Semiconductor Science and Technology 17, 2002.

Partridge, A., Rice, A.E., Kenny, T.W., and Lutz, M., "New Thin Film Epitaxial Polysilicon Encapsulation for Piezoresistive Accelerometers," Proceedings of the IEEE International Conference on Microelectromechanical Systems, 2001.

Peng, D.-Z., Chang, T.-C., Chang, C.-Y., Tsai, M.-L., Tu, C.-H., and Liu, P.-T., "Characteristics and stress-induced polycrystalline silicon thin-film transistors," Journal of Applied Physics 93, February 2003.

Rafferty, N.R. and Tarbutton, G., "IEEE 844-2000: Recommended Practice for Electrical Impedance, Induction, and Skin Effect Heating of Pipelines and Vessels," IEEE Transactions on Industry Applications 38, July-August 2002.

Raeburn, S.J. and Aldridge, R.V., "The Hall effect, Resistivity and magnet moment of amorphous and polycrystalline iron films," Journal of Physics F: Metal Physics 8, 1978. 
Reed, R.P. and Clark, A.F., Ed., "Chapter 6 - Magnetic Properties," Materials at Low Temperatures, American Society for Metals: Metal Park, OH, 1983.

Reichenbach, R., Schubert, D., and Gerlach, G., "Micromechanical Triaxial Acceleration Sensor for Automotive Applications," Proceedings of the International Conference on Solid State Sensors, Actuators, and Microsystems, 2003.

Rudnev, V.I., Cook, R.L., Loveless, D.L., and Black, M.R., Induction Heat Treatment, Reprint of Chapters 11A and 11B of The Steel Heat Treatment Handbook, Marcel Dekker, 1997.

Samuels, H., "Single- and Dual-Axis Micromachined Accelerometers," Analog Dialogue 30, 1996, http://www.analog.com/library/analogDialogue/archives/30-4/acccel.html.

Sandia National Laboratories, "Technology Partnerships," Sandia LabNews, Vol. 54, http://www.sandia.gov/LabNews/LN02-22-02/LA2002/la02/partner_story.htm, February 2002.

Sato, T., "Spectral Emissivity of Silicon", Japanese Journal of Applied Physics 6, 1967, pp. 339-347.

Sedky, S., Howe, R.T., and King, T.-J., "Pulsed-Laser Annealing, a Low-ThermalBudget Technique for Eliminating Stress Gradient in Poly-SiGe MEMS Structures," Journal of Microelectromechanical Systems 13, August 2004.

Sedky, S., Witvrouw, A., Bender, H., and Baert, K., "Experimental Determination of the Maximum Post-Process Annealing Temperature for Standard CMOS Wafers," IEEE Transactions on Electron Devices 48, February 2001.

Senturia, S.D., Microsystem Design, Kluwer Academic Publishers: Norwell, 2001.

Shankar, R., Principles of Quantum Mechanics, $2^{\text {nd }}$ Ed., Plenum Press: New York, 1994.

Shaw, K.A., Zhang, Z.L., and MacDonald, N.C., "SCREAM I: A Single Mask, SingleCrystal Silicon Process for Microelectromechanical Structures," Proceedings of the Investigation of Micro Structures, Sensors, Actuators, Machines and Systems, 1993.

Shen, L.C., and Kong, J.A., Applied Electromagnetism, $3^{\text {rd }}$ Ed., PWS: Boston, 1995.

Solheid, P. and Oches, R., "Micro-VSM Maximizes Magnetic Measurements," The IRM Quarterly 5, Winter 1995-6.

Soohoo, R.F., Magnetic Thin Films, Harper: New York, 1985.

Spiegel, L. and Limbrunner, G.F., Applied Strength of Materials, Macmillan: New York, 1994. 
Sridhar, U., How, L.C., Jun, L.L., Bo, M.Y., Khen-Sang, T., Dow, F.P., Bergstrom, J., Sooriakumar, K., Hong, L.Y., San, L.H., and Kiat, T.C., "Trench oxide isolated Single crystal Silicon Micromachined Accelerometer," Proceedings of the IEEE International Electron Devices Meeting, 1998.

Srikar, V.T. and Senturia, S.D., "Thermoelastic Damping in Fine-Grained Polysilicon Flexural Beam Resonators," Journal of Microelectromechanical Systems 11, October 2002, pp. 499-504.

Suarez, J.E., Johnson, B.E., and El-Kareh, B., "Thermal Stability of Polysilicon Resistors," IEEE Transactions on Components, Hybrids, and Manufacturing Technology 15, June 1992, pp. 386-392.

Sze, S.M., "Properties of Ge, Si, GaAs at 300K," Physics of Semiconductor Devices, $2^{\text {nd }}$ Ed., Wiley, 1981.

Thompson, K, Gianchandani, Y.B., Booske, J., and Cooper, R.F., "Direct Silicon-Silicon Bonding by Electromagnetic Induction Heating," Journal of Microelectromechanical Systems 11, August 2002.

Thompson, K., Booske, J.H., Cooper, R.F., and Gianchandani, Y.B., "Electromagnetic Fast Firing for Ultrashallow Junction Formation," IEEE Transactions on Semiconductor Manufacturing 16, August 2003.

Thompson, K., Booske, J.H., Gianchandani, Y., Cooper, R., Bykov, Y., Eremeev, A., and Plotnikov, I., "Electromagnetic Induction Heating for Cold Wall Rapid Thermal Processing," Proceedings from the International Conference on Advanced Thermal Processing of Semiconductors-RTP, 2001.

Thermographic Measurements Ltd., "Irreversible Paints", http://www.t-m-c.com/PDF\%20Folder/PAINTSin.pdf, January 2005.

Tudbury, C.A., Basics of Induction Heating, Vol. 1, 1960.

Ueno, H., Hosaka, M., Zhang, Y., Tabata, O., Konishi, S., and Suriyama, S., "Study on Fabrication of High Aspect Ratio Microparts Using the LIGA Process," International Symposium on Micromechatronics and Human Science, 1997.

Van Kessel, P.F., Hornbeck, L.J., Meier, R.E., and Douglass, M.R., “A MEMS-Based Projection Display," Proceedings of the IEEE 86, August 1998.

Vas'ko, V.A., Inturi, V.R., Riemer, S.C., Morrone, A., Schouweiler, S., Knox, R.D., Kief, M.T., "High saturation magnetization films of FeCoCr," Journal of Applied Physics 91, May 2002. 
Wang, M., Meng, Z., Zohar, Y., and Wong, M., "A New Polycrystalline Silicon Technology for Integrated Sensor Applications," Proceedings of the IEEE International Conference on Microelectromechanical Systems, 2000.

Wang, M., Meng, Z., and Wong, M., "The Effects of High Temperature Annealing on Metal-Induced Laterally Crystallized Polycrystalline Silicon," IEEE Transactions on Electron Devices 47, November 2000.

Wang, M., Meng, Z., Zohar, Y., and Wong, M., "Metal-Induced Laterally Crystallized Polycrystalline Silicon for Integrated Sensor Applications," IEEE Transactions on Electron Devices 48, April 2001.

Weisstein, E.W., "Average Function," from MathWorld--A Wolfram Web Resource, http://mathworld.wolfram.com/Circle.html, 2006.

Weisstein, E.W., "Circle," from MathWorld--A Wolfram Web Resource, http://mathworld.wolfram.com/Circle.html, 2006.

Wikipedia, "Density of air," http://en.wikipedia.org/wiki/Density_of_air, 2007.

Williams, K.R. and Muller, R.S., "Etch rates for micromachining processes," Journal of Microelectromechanical Systems 5, 1996.

Winter, M., “WebElements Periodic Table,” http://www.webelements.com, 2006.

Wohlfarth, E.P., Ed., Ferromagnetic Materials, Vol. 1, North-Holland: New York, 1980.

Yasaitis, J., Judy, M., Brosnihan, T., Garone, P., Pokrovskiy, N., Sniderman, D., Limb, S., Howe, R., Boser, B., Palaniapan, M., Jiang, X., and Bhave, S., "A modular process for integrating thick polysilicon MEMS devices with sub-micron CMOS," Micromachining and Microfabrication Process Technology VIII 4979, 2003.

Zhang, G., Xie, H., de Rosset, L.E., and Fedder, G.K., "A Lateral Capacitive CMOS Accelerometer with Structural Curl Compensation," Proceedings of the IEEE International Conference on Microelectromechanical Systems, 1999.

Zhang, Z., Kang, K., and Suzuki, T., "Magnetic Properties of Granular-Type FePt-MgO Perpendicular Recording Media," IEEE Transactions on Magnetics 40, July 2004.

Zhang, Z.L. and MacDonald, N.C., "An RIE Process for Submicron, Silicon ElectroMechanical Structures," Proceedings of the International Conference on Solid State Sensors and Actuators, 1991. 


\section{Appendix A: ANSYS Code}

\section{A.1: Simulation Code for ANSYS Example Induction Heating Model}

Note: This simulation was obtained from the ANSYS Coupled-Field Analysis Guide [ANSYS Coupled-Field Analysis Guide, Sec. 2.10.2.9] and was re-printed with permission. An exclamation point signifies the beginning of a comment. For clarity in explanation, additional comments have been provided; these are indicated with double exclamation points.

/batch,list

/filenam,induc

/prep7

shpp,off ! Shape warning disabled

/title, induction heating of a solid cylinder billet

/com,

!!This model is a sequentially-coupled induction heating !!simulation. It a two-dimensional axisymmetric model, !!meaning that it's drawn in two dimensions but rotated !!about the y-axis.

!!ELEMENT TYPE DEFINITIONS FOR ELECTROMAGNETIC MODEL

!!Sets up two Plane13 planar element types to distinguish

!!between two different portions of the model, and a Surf151

!!surface element type utilized for simulating thermal

!!radiation. Element1 (Plane13) is used for the structure

!!and is converted to Plane55 in the thermal model. Element2

!!(Plane13) is used for the coil and surrounding air, and is

!!converted to the Nullo type (not simulated) in the thermal

!!model. Element3 (Surf151) overlays onto the surface of the

!!heated structure and is used only in the thermal model.

et, 1,13,, ! ! PLANE13, axisymmetric, AZ dof

et, $2,13,, 1$

et, $3,151,, 1,1,1 \quad$ ! SURF151, thermal, radiation

!!VARIABLE DEFINITIONS FOR BOTH MODELS

!! These variables set the size of the structure, the

!!frequency and time durations of the simulations, and the

!!skin depth which determines the minimum element size.
$\mathrm{r}, 3,0$
! Real constant set for SURF151
row $=.015$
! outer radius of workpiece
ric $=.0175$
! inner radius of coil
$\mathrm{roc}=.0200$
! outer radius of coil 


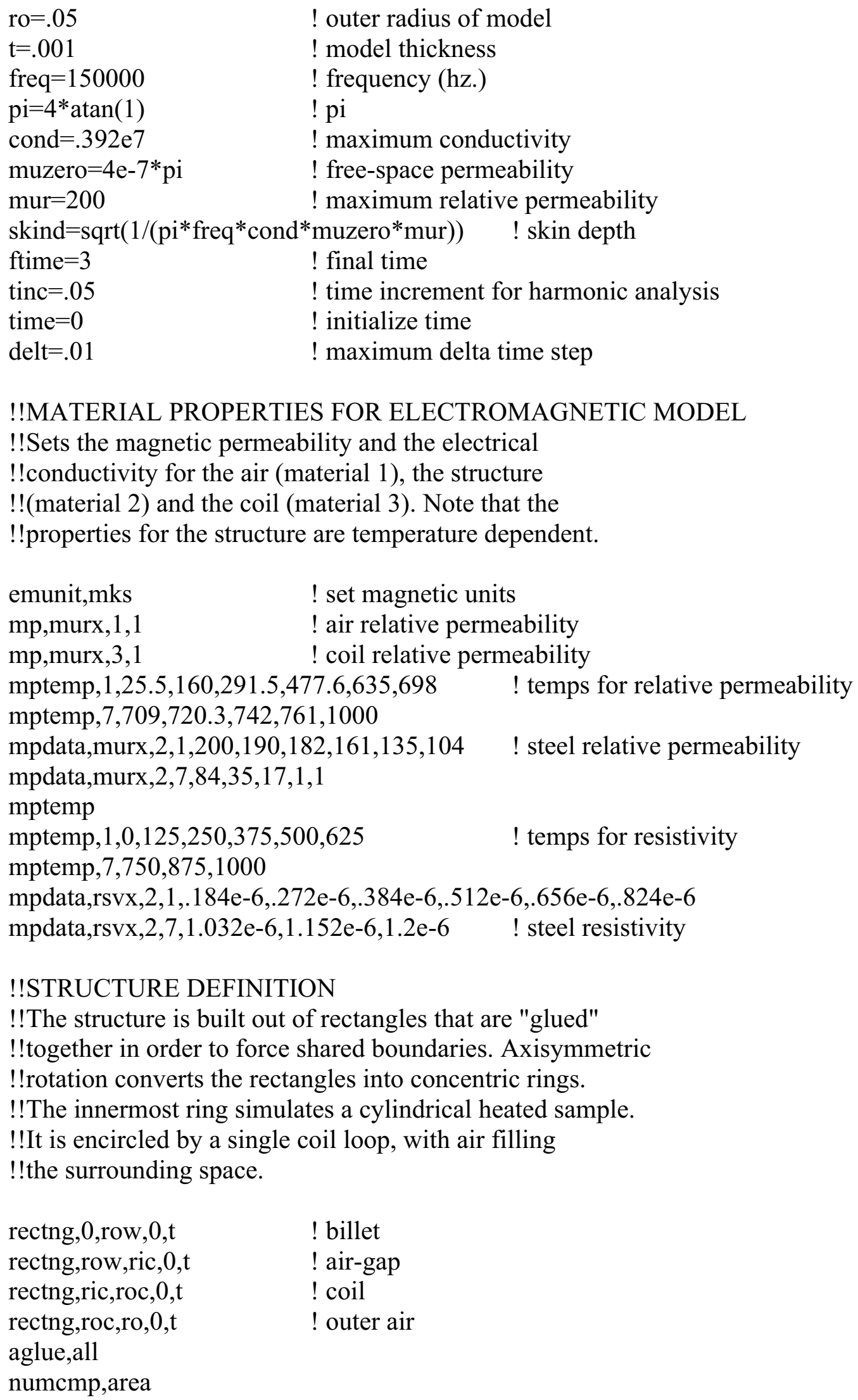

!!MATERIAL PROPERTIES FOR ELECTROMAGNETIC MODEL

!!Sets the magnetic permeability and the electrical

!!conductivity for the air (material 1), the structure

!!(material 2) and the coil (material 3). Note that the

!!properties for the structure are temperature dependent. 


\section{!!MESHING}

!!The mesh sizes are selected via keypoints. The mesh size

!! in the heated structure is modulated from $1 / 2$ of the

!!minimum skin depth, at the outer edge, to 40 skin depths

!!at the inner edge. The other areas (air and coil) are

!!divided into $1 \mathrm{~mm}$ regions. Lastly, a single node, called a

!!"space node", is created for the purpose of modeling

!!thermal radiation. The space node provides a site at which

!!the radiated energy is collected.

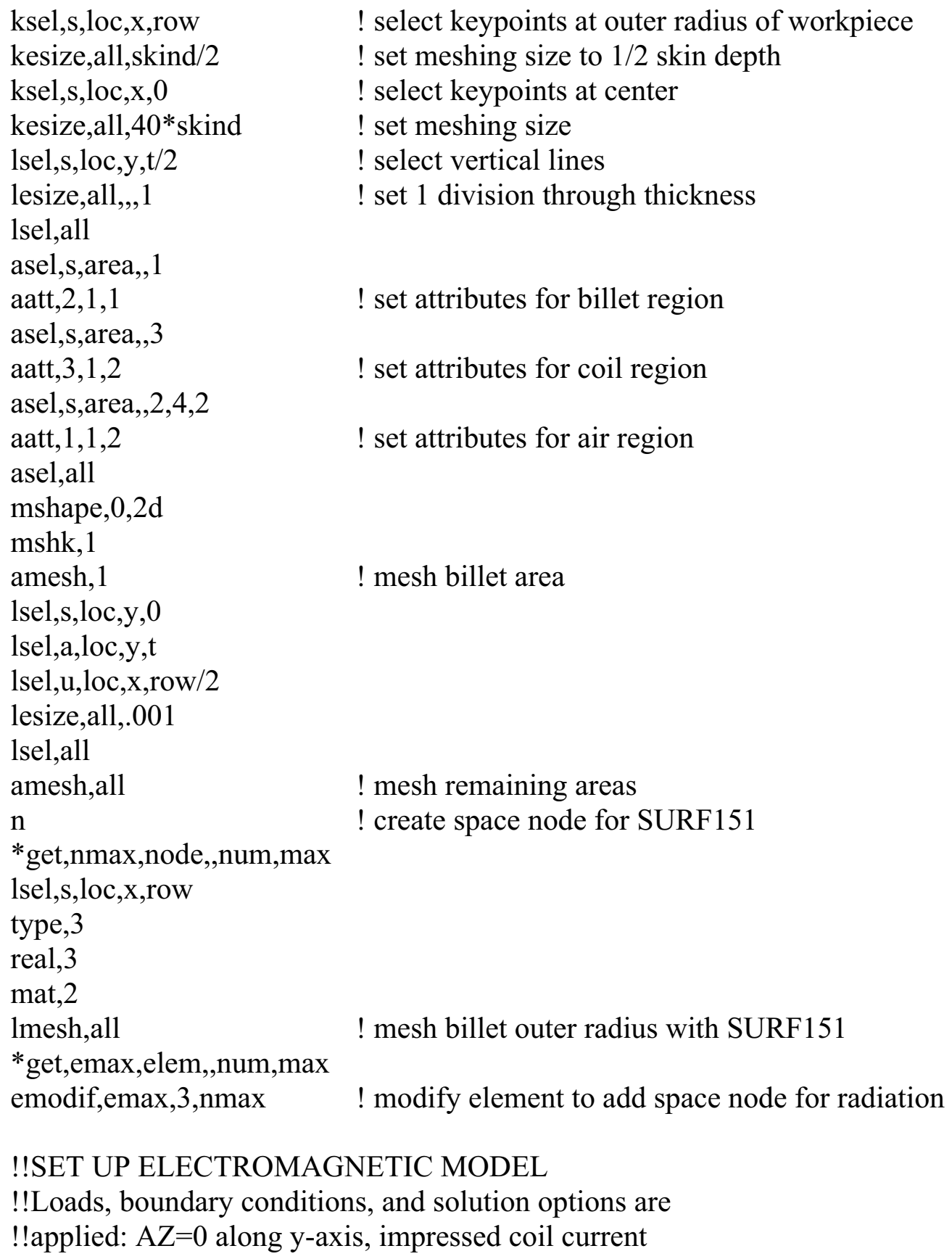


!!density, harmonic frequency, and analysis type (harmonic).

!! The elements are set to their required configuration for !! the electromagnetic model (Plane13, Plane13, Null0). !!Lastly, the physics environment is stored.

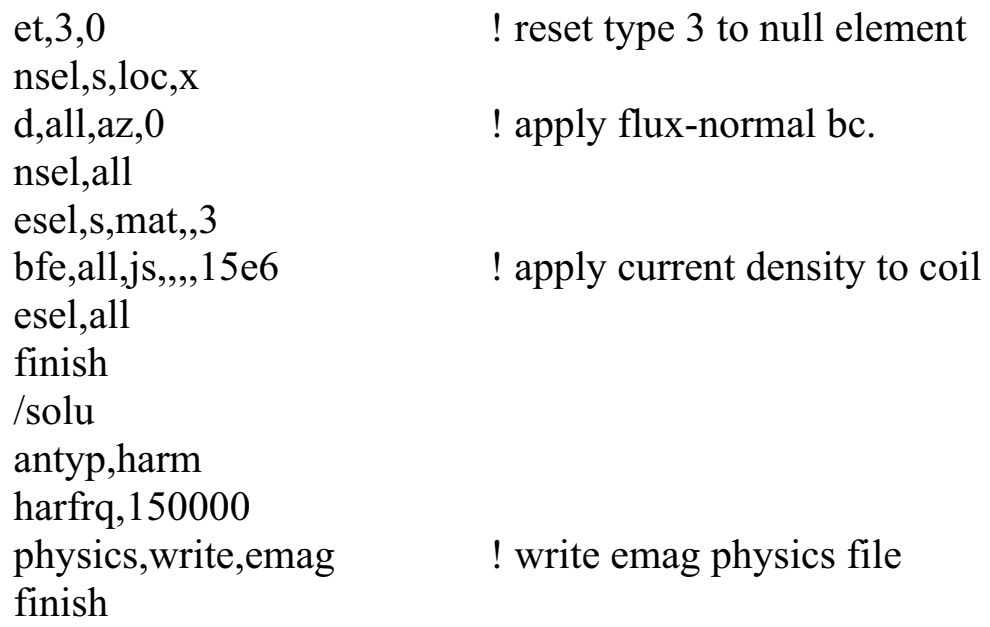

!!MATERIAL PROPERTIES FOR THERMAL MODEL

!!Sets the thermal conductivity, enthalpy (or internal !!energy), and emissivity for the heated structure. Note !! that the thermal conductivity and enthalpy are both !!temperature dependent.

keyopt,3,9,1

r, 3,1,5.67e-8 ! form factor, Stefan-Boltzman constant

mptemp

mptemp,1,0,730,930,1000 ! temps for conductivity

mpdata,kxx,2,1,60.64,29.5,28,28

mptemp ! temps for enthalpy

mptemp,1,0,27,127,327,527,727

mptemp,7,765,765.001,927

mpdata,enth,2,1,0,91609056,453285756,1.2748e9,2.2519e9,3.3396e9 
mpdata,enth, 2,7,3.548547e $9,3.548556 \mathrm{e} 9,4.3520 \mathrm{e} 9$

mp,emis, $2, .68$ ! emissivity

finish

!!SET UP THERMAL MODEL

!!Initial conditions and model parameters are applied:

!!Celsius temperature scale, $100 \mathrm{C}$ initial temperature, $25 \mathrm{C}$

!!far-field temperature, transient analysis type, and

!!computer-chosen time steps in the range of 1 us- $10 \mathrm{~ms}$

!!(delt $=10 \mathrm{~ms}$ from variable definitions). Lastly, the physics

!!environment is stored.

/solu

antype,trans

toffst, 273

tunif, 100

! initial uniform temperature

d,nmax,temp, 25

! ambient temperature

cnvtol,heat, 1

! convergence tolerance

$\mathrm{kbc}, 1$

! step loads

trnopt,full

autos,on

! auto time-stepping

deltim,1e-5,1e-6,delt,on

! time step control

outres,basic,all

! save all load step information

physics, write, thermal ! write thermal physics file

finish

!!EXECUTE MODEL

!!This do-loop runs the simulation. The number of loops

!!depends on the user-specified final time (ftime) and time

!!duration between re-evaluation of electromagnetic fields

!!(tinc). Each iteration of the loop begins with the

!!electromagnetic model. It assigns a uniform temperature of

!!100C for the first pass and reads in the results of the

!!thermal model for each subsequent pass. After reaching a

!!solution, the code switches to the thermal model. The

!!thermal simulation is restarted with the updated Joule

!!heat data, and evolution of the transient thermal behavior

!!continues until the time duration specified (time) is reached.

*do,i,1,ftime/tinc $\quad$ ! solution *do loop

time $=$ time + tinc $\quad$ ! increment time

physics,read,emag ! read emag physics file

/solu

*if,i,eq, 1 ,then

tunif, 100 ! initial temperature

*else 


\begin{tabular}{|c|c|}
\hline $\begin{array}{l}\text { ldread,temp,last,,,,,rth } \\
\text { *endif }\end{array}$ & ! read thermal analysis temperatures \\
\hline solve & ! solve harmonic analysis \\
\hline finish & \\
\hline physics,read,thermal & ! read thermal physics file \\
\hline /assign,esav,therm,esav & ! redirect files for use in thermal restart \\
\hline $\begin{array}{l}\text { /assign,emat,therm,emat } \\
\text { /solu }\end{array}$ & \\
\hline$*_{\text {if,i,gt, } 1, \text { then }}$ & \\
\hline $\begin{array}{l}\text { antype,trans,rest } \\
\text { *endif }\end{array}$ & ! thermal restart \\
\hline time,time & ! time at end of thermal run \\
\hline esel,s,mat,,2 & ! select billet region \\
\hline $\begin{array}{l}\text { ldread,hgen,,,2,,rmg } \\
\text { esel,all } \\
\text { solve } \\
\text { finish }\end{array}$ & ! apply coupled joule heating load from emag \\
\hline $\begin{array}{l}\text { /assign,esav } \\
\text { /assign.emat }\end{array}$ & ! reassign files to default \\
\hline *enddo & ! end of solution looping \\
\hline finish & \\
\hline save & ! save database \\
\hline !!PLOT RESULTS & \\
\hline $\begin{array}{l}\text { !!The center and outer ed } \\
\text { !'selected for evaluation. } \\
\text { !!are given over time botl }\end{array}$ & $\begin{array}{l}\text { of the heated structure are } \\
\text { e temperatures at each location } \\
\text { raphically and numerically. }\end{array}$ \\
\hline /post26 & ! time-history postprocessor \\
\hline /show & \\
\hline nsol,2,1,temp,,tempcl & ! store temperature at billet centerline \\
\hline nsol,3,2,temp,tempsurf & ! store temperature at billet outer diameter \\
\hline plvar, 2,3 & ! plot temperature rise over time \\
\hline prvar, 2,3 & ! print temperature rise over time \\
\hline finish & \\
\hline
\end{tabular}

\section{A.2: Material Properties for Thin Film Simulations}

\section{Silicon Substrate - 1E15 Boron Doped}

Density: Senturia, 2001 (p. 196).

Thermal Conductivity: CRC, 1989 (pp. E-14 - E-16).

Resistivity: Sze, 1981 (pp. 32-33).

Specific Heat: Okhotin, et al., 1972.

Emissivity: Sato, 1967.

Relative Permeability: CRC, 1999 (pp. 4-131 -4-138). 


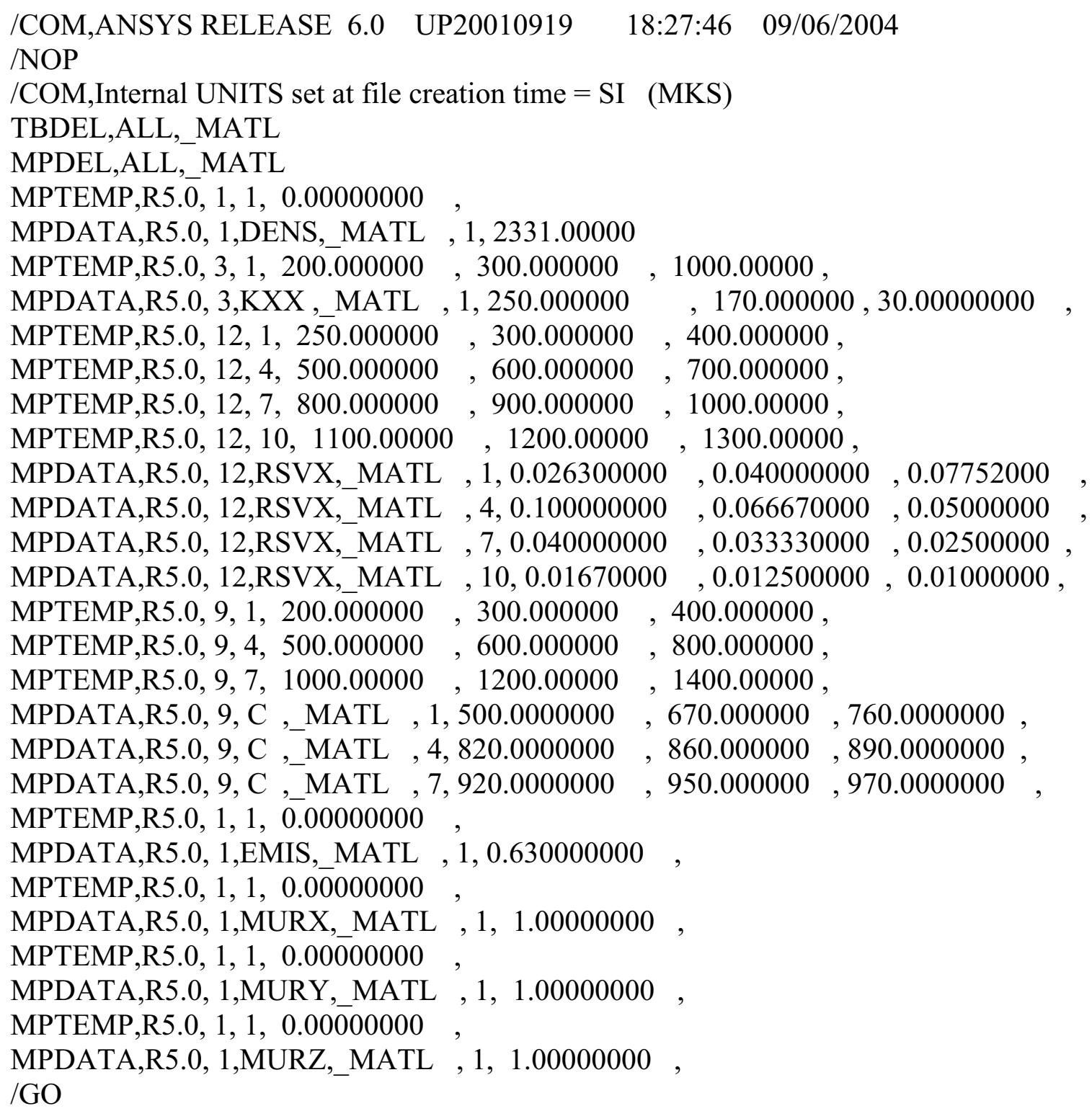

\section{Silicon Dioxide}

Density: Senturia, 2001 (p. 196).

Thermal Conductivity: CRC, 1989 (pp. E-14 - E-16).

Resistivity: Sze, 1981 (pp. 850-851).

Specific Heat: Grove, 1967.

Emissivity: Forsythe, 2003.

Relative Permeability: CRC, 1999 (pp. 4-131 -4-138).

/COM,ANSYS RELEASE 6.0 UP20010919 17:45:10 07/29/2004

NOP

$/ \mathrm{COM}$,Internal UNITS set at file creation time $=\mathrm{SI} \quad(\mathrm{MKS})$

TBDEL,ALL, MATL

MPDEL,ALL,_MATL 


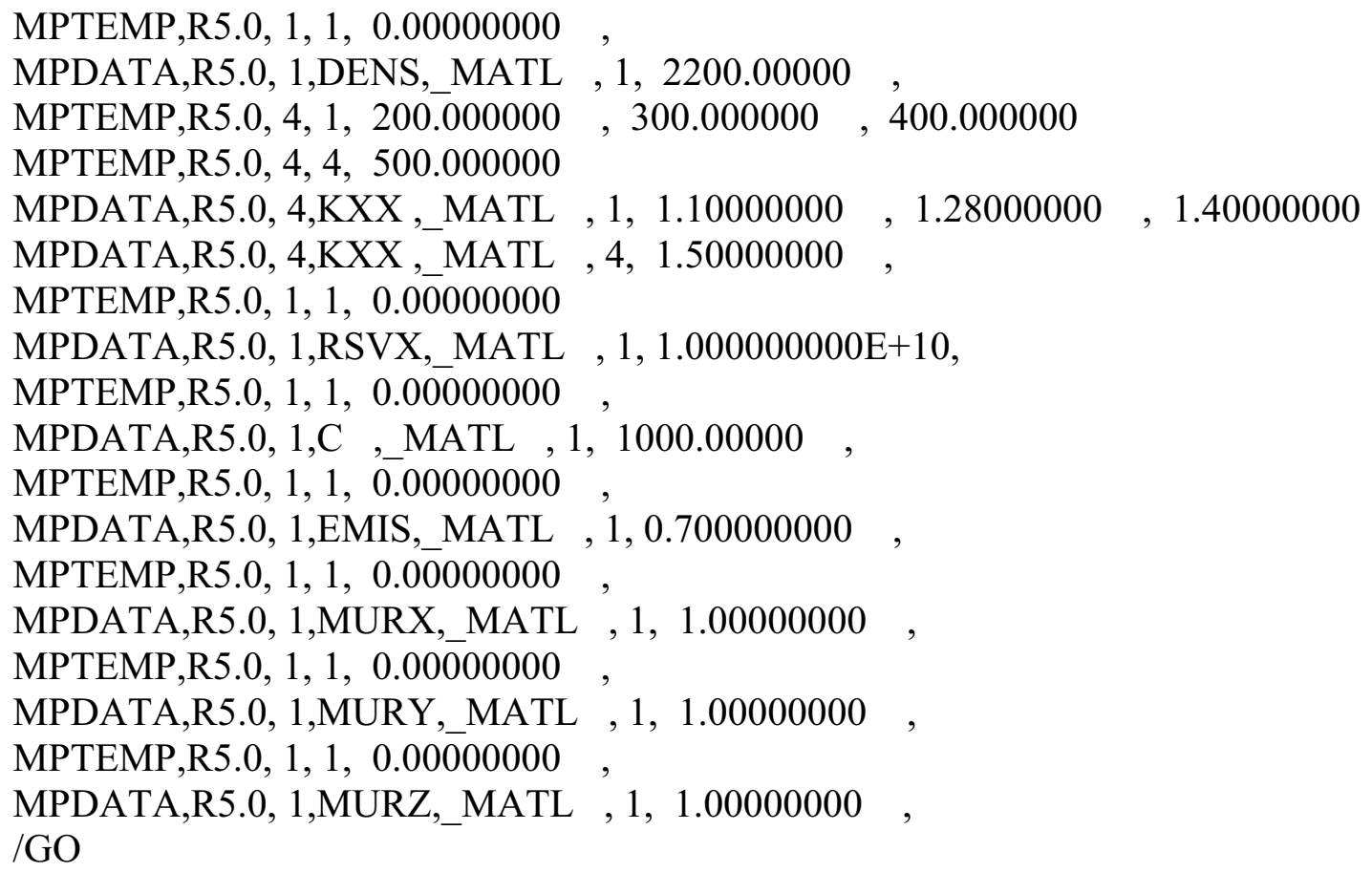

\section{Polysilicon - Undoped}

Density: Senturia, 2001 (p. 196).

Thermal Conductivity: CRC, 1989 (pp. E-14 - E-16); assumed the same as for silicon.

Resistivity: Sze, 1981 (pp. 32-33); Moore, et al., 1994.

Specific Heat: Okhotin, et al., 1972; assumed the same as for silicon.

Emissivity: Sato, 1967; assumed the same as for silicon.

Relative Permeability: CRC, 1999 (pp. 4-131 - 4-138).

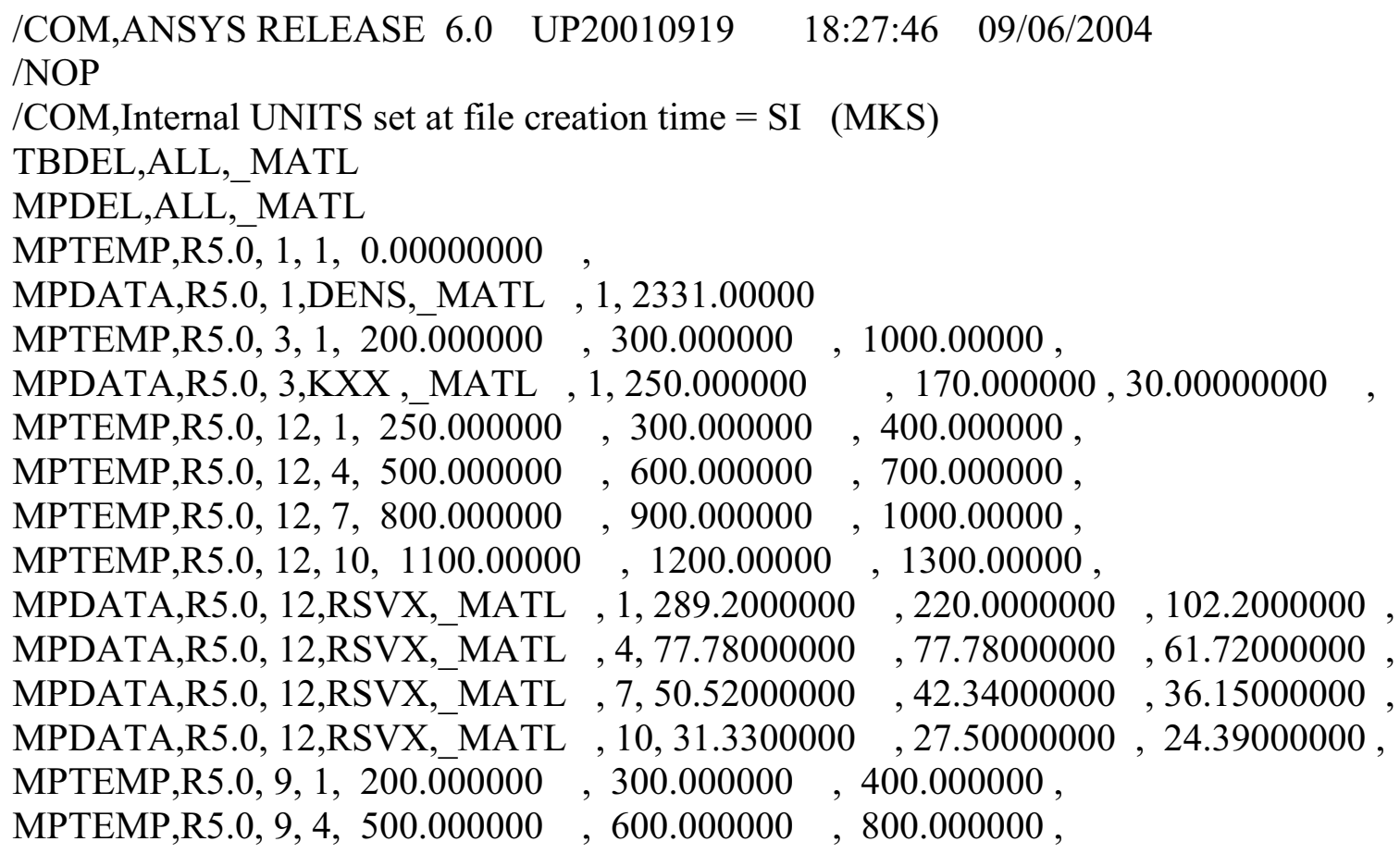




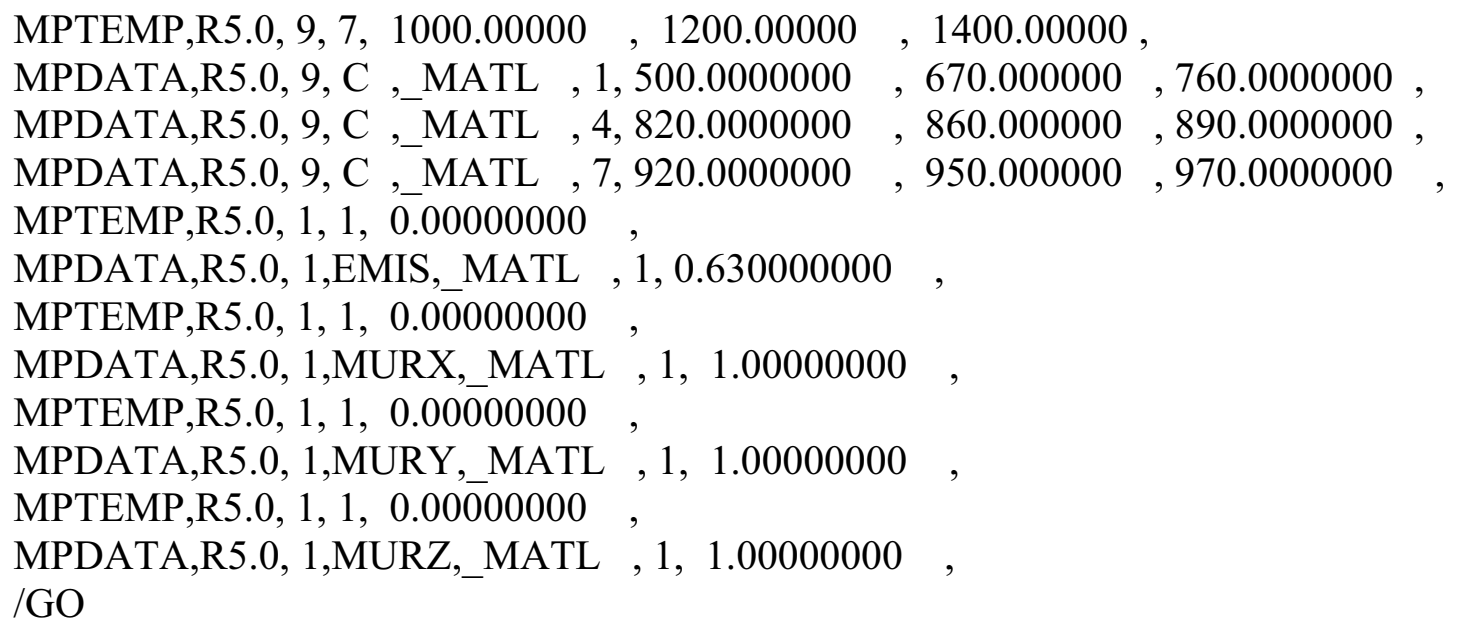

\section{NiFe19}

Density: CRC, 1989 (pp. B68-B146); interpolated between nickel and iron.

Thermal Conductivity: CRC, 1989 (pp. E14-E16); interpolated between nickel and iron.

Resistivity: Measured via Four-Point Probe.

Specific Heat: CRC, 1989 (pp. D180-D181); interpolated between nickel and iron.

Emissivity: CRC, 1989 (p. E-406).

Relative Permeability: Measured via Vibrating Sample Magnetrometry.

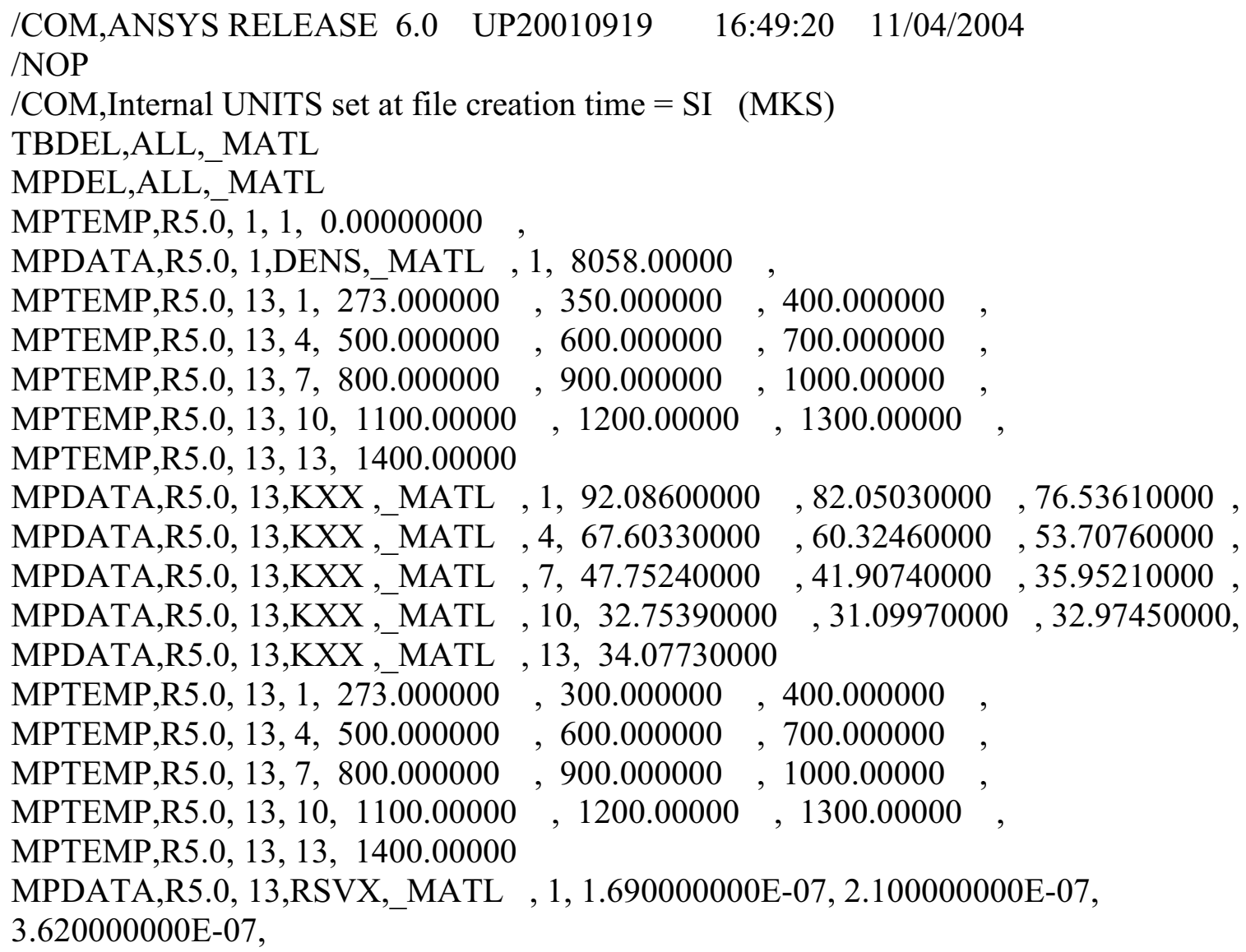


MPDATA,R5.0, 13,RSVX,_MATL , 4, 5.130000000E-07, 6.650000000E-07, 8.170000000E-07,

MPDATA,R5.0, 13,RSVX,_MATL , 7, 9.680000000E-07, 1.120000000E-06, $1.270000000 \mathrm{E}-06$,

MPDATA,R5.0, 13,RSVX,_MATL , 10, 1.420000000E-06, 1.580000000E-06, $1.730000000 \mathrm{E}-06$,

MPDATA,R5.0, 13,RSVX,_MATL , 13, 1.880000000E-06

MPTEMP,R5.0, 13, 1, $273.000000 ， 300.000000 \quad, 400.000000$ ，

MPTEMP,R5.0, 13, 4, 500.000000 , 600.000000 , 700.000000 ,

MPTEMP,R5.0, 13, 7, 800.000000 , $900.000000 ， 1000.00000$,

MPTEMP,R5.0, 13, 10, $1100.00000,1200.00000,1300.00000$ ，

MPTEMP,R5.0, 13, 13, 1400.00000

MPDATA,R5.0, 13,RSVY,_MATL , 1, 1.690000000E-07, 2.100000000E-07, 3.620000000E-07,

MPDATA,R5.0, 13,RSVY,_MATL , 4, 5.130000000E-07, 6.650000000E-07, 8.170000000E-07,

MPDATA,R5.0, 13,RSVY,_MATL , 7, 9.680000000E-07, 1.120000000E-06, $1.270000000 \mathrm{E}-06$,

MPDATA,R5.0, 13,RSVY,_MATL , 10, 1.420000000E-06, 1.580000000E-06, $1.730000000 \mathrm{E}-06$,

MPDATA,R5.0, 13,RSVY, MATL , 13, 1.880000000E-06

MPTEMP,R5.0, 13, 1, 273.000000 ，300.000000 ，400.000000 ，

MPTEMP,R5.0, 13, 4, 500.000000 ，600.000000 ，700.000000 ，

MPTEMP,R5.0, 13, 7, 800.000000 , $900.000000 ， 1000.00000$,

MPTEMP,R5.0, 13, 10, $1100.00000 ， 1200.00000 ， 1300.00000$,

MPTEMP,R5.0, 13, 13, 1400.00000

MPDATA,R5.0, 13,RSVZ,_MATL , 1, 1.690000000E-07, 2.100000000E-07, 3.620000000E-07,

MPDATA,R5.0, 13,RSVZ,_MATL , 4, 5.130000000E-07, 6.650000000E-07, 8.170000000E-07,

MPDATA,R5.0, 13,RSVZ,_MATL , 7, 9.680000000E-07, 1.120000000E-06, $1.270000000 \mathrm{E}-06$,

MPDATA,R5.0, 13,RSVZ,_MATL , 10, 1.420000000E-06, 1.580000000E-06, $1.730000000 \mathrm{E}-06$,

MPDATA,R5.0, 13,RSVZ,_MATL , 13, 1.880000000E-06

MPTEMP,R5.0, 5, 1, $200.000000 \quad, 300.000000 \quad, 400.000000$

MPTEMP,R5.0, 5, 4, 700.000000 , 1400.00000

MPDATA,R5.0, 5,C ,_MATL , 1, $398.000000,461.000000 \quad, 502.000000$ ，

MPDATA,R5.0, 5,C ,_MATL , 4, $628.000000 \quad, 921.000000$,

MPTEMP,R5.0, 1, 1, 0.00000000 ,

MPDATA,R5.0, 1,EMIS, MATL , 1, 0.09000000 ,

MPTEMP,R5.0, 10, 1, 273.000000 ，298.000000 ，373.000000 ，

MPTEMP,R5.0, 10, 4, $473.000000 ， 573.000000 ， 673.000000$,

MPTEMP,R5.0, 10, 7, $773.000000 ， 873.000000 ， 973.000000$,

MPTEMP,R5.0, 10, 10, 1400.00000

MPDATA,R5.0, 10,MURX,_MATL , 1, $48.30400000 \quad, 48.30400000 \quad, 31.19400000$ ， 
MPDATA,R5.0, 10,MURX,_MATL , 4, $32.22900000 \quad, 30.63700000 \quad, 22.60000000$, MPDATA,R5.0, 10,MURX,_MATL , 7, $6.461700000,1.010600000 \quad, 0.970850000$, MPDATA,R5.0, 10,MURX,_MATL , 10, 1.000000000

MPTEMP,R5.0, 10, 1, 273.000000 ，298.000000 , 373.000000 ,

MPTEMP,R5.0, 10, 4, 473.000000 , 573.000000 , 673.000000 ,

MPTEMP,R5.0, 10, 7, $773.000000 ， 873.000000 ， 973.000000$,

MPTEMP,R5.0, 10, 10, 1400.00000

MPDATA,R5.0, 10,MURZ,_MATL , 1, $48.30400000 \quad, 48.30400000 \quad, 31.19400000$, MPDATA,R5.0, 10,MURZ,_MATL , 4, $32.22900000 \quad, 30.63700000 \quad, 22.60000000$, MPDATA,R5.0, 10,MURZ,_MATL , 7, $6.461700000 \quad, 1.010600000 \quad, 0.970850000$, MPDATA,R5.0, 10,MURZ,_MATL , 10, 1.000000000

MPTEMP,R5.0, 10, 1, 273.000000 ，298.000000 , 373.000000 ,

MPTEMP,R5.0, 10, 4, 473.000000 ，573.000000 , 673.000000 ,

MPTEMP,R5.0, 10, 7, 773.000000 , $873.000000 ， 973.000000$,

MPTEMP,R5.0, 10, 10, 1400.00000

MPDATA,R5.0, 10,MURY,_MATL , 1, $15.75600000 ， 15.75600000,15.19900000$, MPDATA,R5.0, 10,MURY,_MATL , 4, $14.80100000 \quad, 13.84600000 \quad, 12.57300000$, MPDATA,R5.0, 10,MURY,_MATL , 7, 9.310600000 , $1.106100000,1.082260000$, MPDATA,R5.0, 10,MURY,_MATL , 10, 1.000000000 /GO

\section{Vacuum - 50mTorr}

Density: Wikipedia, 2007.

Thermal Conductivity: Ierardi, 1999

Resistivity: CRC, 1999 (pp. 12-45 - 12-47).

Specific Heat: Ierardi, 1999.

Relative Permeability: Balanis, 1989 (p. 55).

/COM,ANSYS RELEASE $6.0 \quad$ UP20010919 20:31:24 12/03/2003

/NOP

$/$ COM,Internal UNITS set at file creation time $=$ SI $(\mathrm{MKS})$

TBDEL,ALL,_MATL

MPDEL,ALL,_MATL

MPTEMP,R5.0,11, 1, $300.000000 ， 400.000000 ， 500.000000$

MPTEMP,R5.0,11, 4, 600.000000 , $700.000000,800.000000$

MPTEMP,R5.0,11, 7, 900.000000 , $1000.00000,1100.00000$

MPTEMP,R5.0,11,10, $1200.00000 ， 1300.00000$,

MPDATA,R5.0,11,DENS,_MATL , 1, 7.740800000E-05, 5.810600000E-05, 4.644500000E-05

MPDATA,R5.0,11,DENS,_MATL , 4, 3.870400000E-05, 3.317500000E-05, $2.902800000 \mathrm{E}-05$

MPDATA,R5.0,11,DENS,_MATL , 7, 2.580300000E-05, 2.322200000E-05, $2.111100000 \mathrm{E}-05$

MPDATA,R5.0,11,DENS,_MATL , 10, 1.935200000E-05, 1.786300000E-05, MPTEMP,R5.0,11, 1, 300.000000 ，400.000000 ，500.000000

MPTEMP,R5.0,11, 4, 600.000000 ，700.000000 , 800.000000 
MPTEMP,R5.0,11, 7, 900.000000 , $1000.00000,1100.00000$

MPTEMP,R5.0,11,10, $1200.00000 \quad, 1300.00000$,

MPDATA,R5.0,11,KXX,_MATL , 1, 2.600000000E-02, 3.500000000E-02, 4.000000000E-02

MPDATA,R5.0,11,KXX,_MATL , 4, 4.700000000E-02, 5.300000000E-02, 5.800000000E-02

MPDATA,R5.0,11,KXX ,_MATL , 7, 6.200000000E-02, 6.800000000E-02, 7.200000000E-02

MPDATA,R5.0,11,KXX,_MATL , 10, 7.800000000E-02, 8.300000000E-02, MPTEMP,R5.0, 1, 1, 0.00000000 ,

MPDATA,R5.0, 1,RSVX,_MATL , 1, 2.00000000E13. ,

MPTEMP,R5.0,12, 1, 300.000000 , $400.000000 \quad, 500.000000$

MPTEMP,R5.0,12, 4, 600.000000 , 700.000000 , 800.000000

MPTEMP,R5.0,12, 7, 900.000000 , $1000.00000,1100.00000$

MPTEMP,R5.0,12,10, $1200.00000 ， 1300.00000 ， 1400.00000$

MPDATA,R5.0,12,C ,_MATL , 1, $1005.00000,1015.00000,1030.00000$

MPDATA,R5.0,12,C ,_MATL , 4, $1050.00000,1075.00000,1100.00000$

MPDATA,R5.0,12,C ,_MATL , 7, $1125.00000,1150.00000,1165.00000$

MPDATA,R5.0,12,C ,_MATL ,10, $1180.00000 ， 1200.00000 \quad, 1220.00000$

MPTEMP,R5.0, 1, 1, 0.00000000 ,

MPDATA,R5.0, 1,MURX,_MATL , 1, 1.00000000 ,

MPTEMP,R5.0, 1, 1, 0.00000000,

MPDATA,R5.0, 1,MURY,_MATL , 1, 1.00000000 ,

MPTEMP,R5.0, 1, 1, 0.00000000 ,

MPDATA,R5.0, 1,MURZ,_MATL , 1, 1.00000000 ，

/GO

\section{Copper (Magnetic Coil)}

Density: CRC, 1989 (pp. B68-B146).

Thermal Conductivity: CRC, 1989 (pp. E-14 - E-16).

Resistivity: CRC, 1999 (pp. 12-45 - 12-47).

Specific Heat: CRC, 1989 (pp. D-180 - D-181).

Emissivity: CRC, 1989 (p. E-406).

Relative Permeability: CRC, 1999 (pp. 4-131 - 4-138).

/COM,ANSYS RELEASE $6.0 \quad$ UP20010919 $\quad 16: 54: 29 \quad 06 / 02 / 2003$

/NOP

$/$ COM,Internal UNITS set at file creation time $=$ SI $\quad($ MKS $)$

TBDEL,ALL,_MATL

MPDEL,ALL, MATL

MPTEMP,R5.0, 1, 1, 0.00000000 ,

MPDATA,R5.0, 1,DENS,_MATL , 1, 8920.00000 ，

MPTEMP,R5.0, 12, 1, 273.000000 , $350.000000 \quad, 400.000000$,

MPTEMP,R5.0, 12, 4, 500.000000 , 600.000000 , 700.000000 ,

MPTEMP,R5.0, 12, 7, 800.000000 ，900.000000 ，1000.00000 ，

MPTEMP,R5.0, 12, 10, $1100.00000,1200.00000,1300.00000$, 


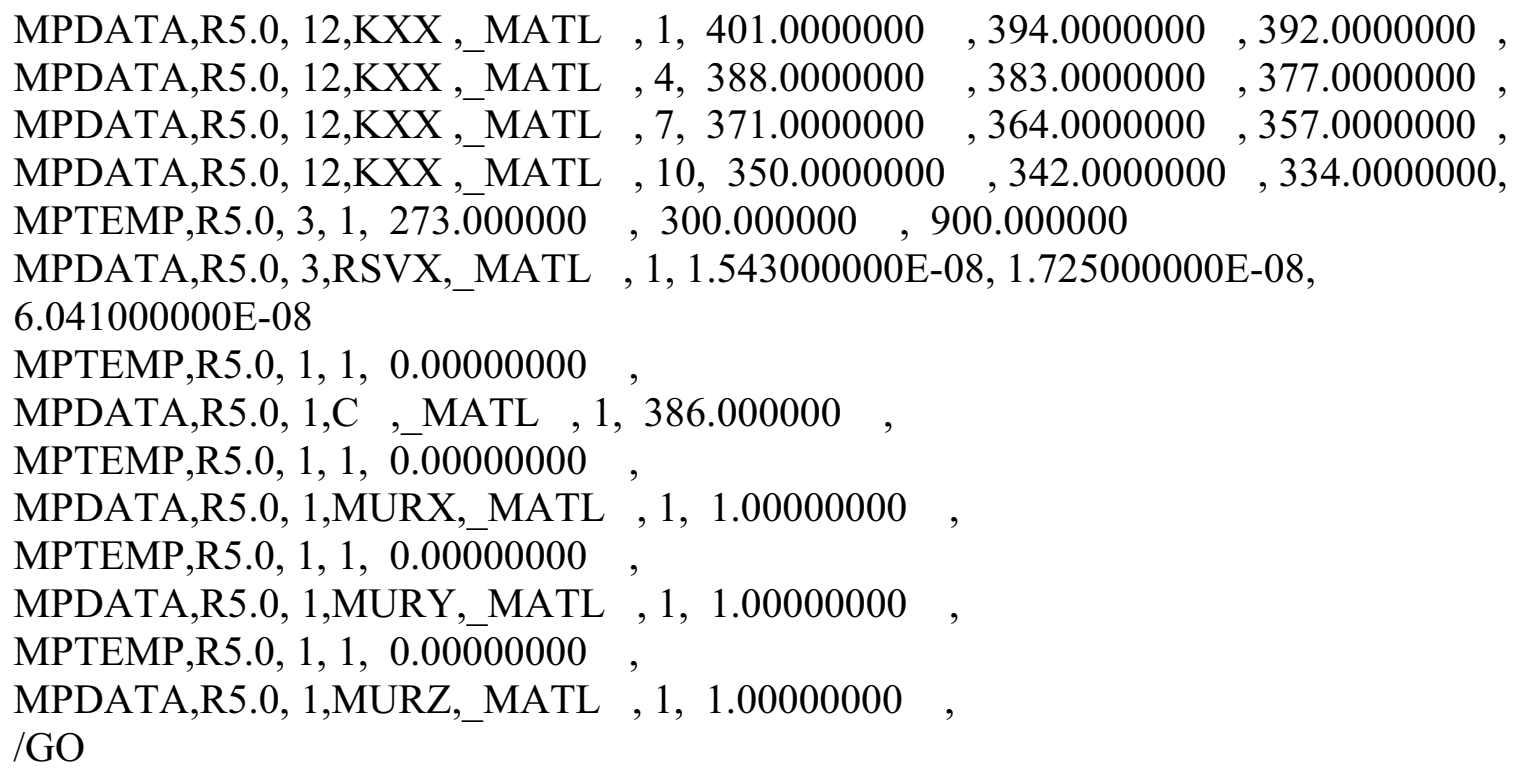

\section{A.3: Simulation Code for Thin Film Induction Heating Model}

! Thin film simulation with hollow ferromagnetic ring

$! 1.2 \mathrm{~cm}$ nife 19 outer radius, $0.6 \mathrm{~cm}$ inner radius, $7.3 \mathrm{um}$ thickness

$! 4.5 \mathrm{~mm}$ poly radius, $3 \mathrm{um}$ thickness, $4.5 \mathrm{~mm}$-wide outer poly

! 1 coil turn (multiply current by 3 )

! This model is an axisymmetric induction heating simulation ! that models a thin film stack. The energy from the heated

! NiFe19 film ring spreads to polysilicon films both inside

! and outside, simulating polysilicon devices, to examine the.

! heat transfer behaviors.

\section{! DEFINE MODEL DIMENSIONS}

! The model dimensions are defined via connected ! keypoints. Keypoints are points defined at specific ! locations (eg, $\mathrm{x}, \mathrm{y}, \mathrm{z}$ coordinates). Area definitions ! include all keypoints through which they

! intersect in order to avoid mesh discontinuities.

/prep7

! Y-coordinates

SubstrateY $=-0.5 \mathrm{E}-3$

OxideY=1E-6

PolyY $=4 \mathrm{E}-6$

! 1um lower oxide

FerroY $=8.3 \mathrm{E}-6$

! 3um poly

! 7.3um nife19 
! X-coordinates

SubstrateXMax $=1.8 \mathrm{E}-2 \quad ! 1.8 \mathrm{~cm}$ model width

PolyXMax $=4.5 \mathrm{E}-3 \quad$ ! Inner poly region

FerroXMax $=12 \mathrm{E}-3$

FerroXMin $=6 \mathrm{E}-3$

Poly2XMin=13.5E-3 ! Outer poly region

! Finite and Inifinite Space Dimensions

$\begin{array}{ll}\text { AirMinY }=-0.25 \mathrm{E}-2 & \text { ! Air range is }-.25 \mathrm{~cm} \text { to } 1 \mathrm{~cm} \\ \text { AirMaxY }=1 \mathrm{E}-2 & \\ \text { AirMaxX }=2.25 \mathrm{E}-2 & \\ \text { InfMin } Y=-0.35 \mathrm{E}-2 & \\ \text { InfMaxY }=1.1 \mathrm{E}-2 & \\ \text { InfMaxX }=2.35 \mathrm{E}-2 & \end{array}$

! Define Areas via Keypoints

$\mathrm{k}, 1,0$, SubstrateY,0

k,2,SubstrateXMax,SubstrateY,0

$\mathrm{k}, 3,0,0,0$

k,4,SubstrateXMax,0,0

a, $1,2,4,3 \quad$ ! Substrate (area 1)

k,5,0,OxideY,0

k,6,PolyXMax,OxideY,0

k,7,FerroXMin,OxideY,0

k,8,FerroXMax,OxideY,0

k,9,Poly2Xmin,OxideY,0

k,10,SubstrateXMax,OxideY,0

a,3,4,10,9,8,7,6,5 ! Oxide (area 2)

k, 11,0,PolyY,0

k, 12,PolyXMax,PolyY,0

k, 13,Poly2XMin,PolyY,0

k, 14,SubstrateXMax,PolyY,0

a, 5,6,12,11 ! Inner Poly (area 3)

a, $9,10,14,13 \quad$ ! Outer Poly (area 4)

k, 15,FerroXMin,FerroY,0

k,16,FerroXMax,FerroY,0 


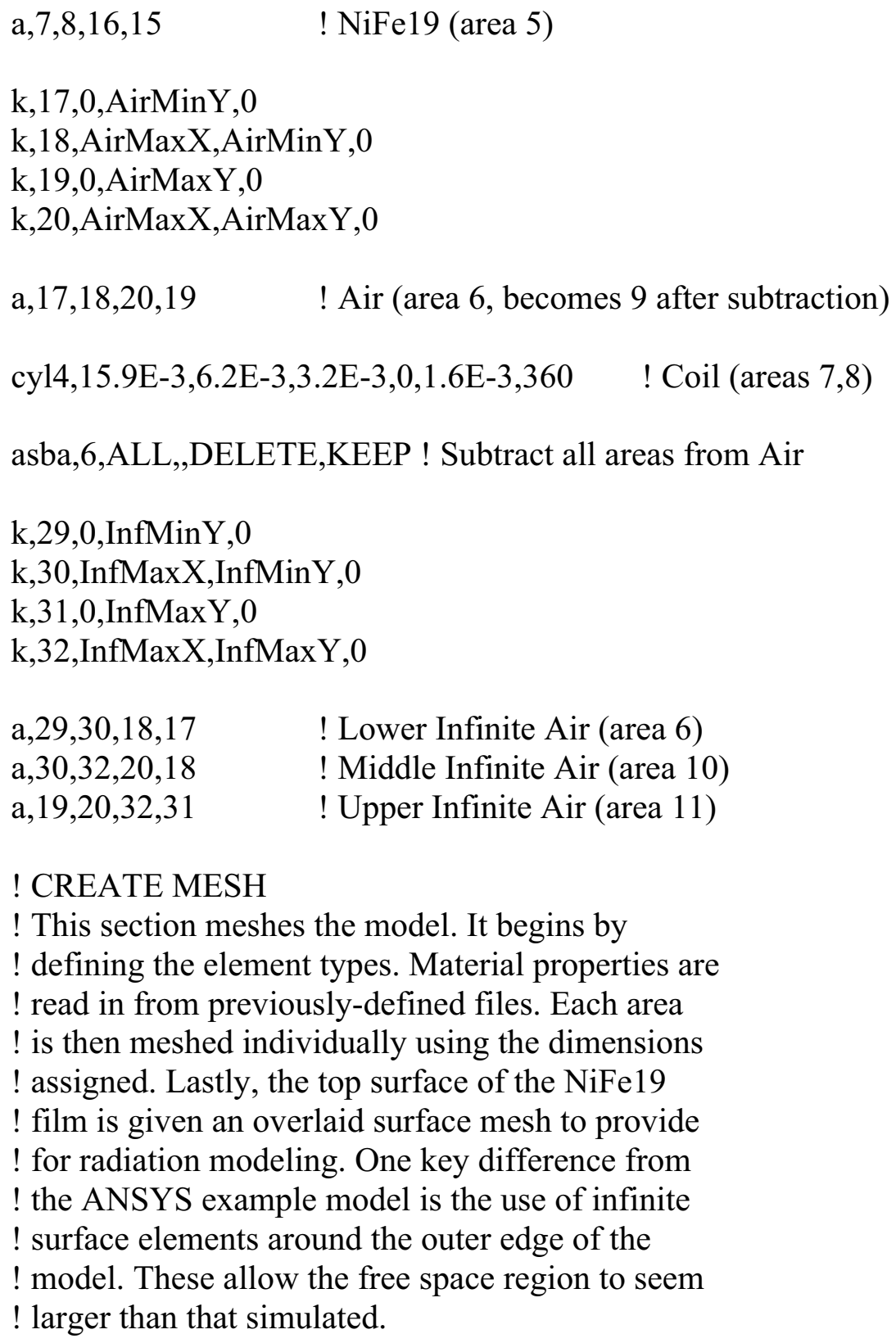

! Define element types
et, $1,53,, 1$
! PLANE53, AZ DOF, included in thermal sim
et, $2,53,, 1$ ! PLANE53, AZ DOF, not in thermal sim
et, $3,110,, 1,1$
! INFIN110, for infinite area
et, $4,151,, 1,0,1$
! SURF151, for including radiation
$\mathrm{r}, 4,0$

! Define material properties (from files)

emunit,mks 


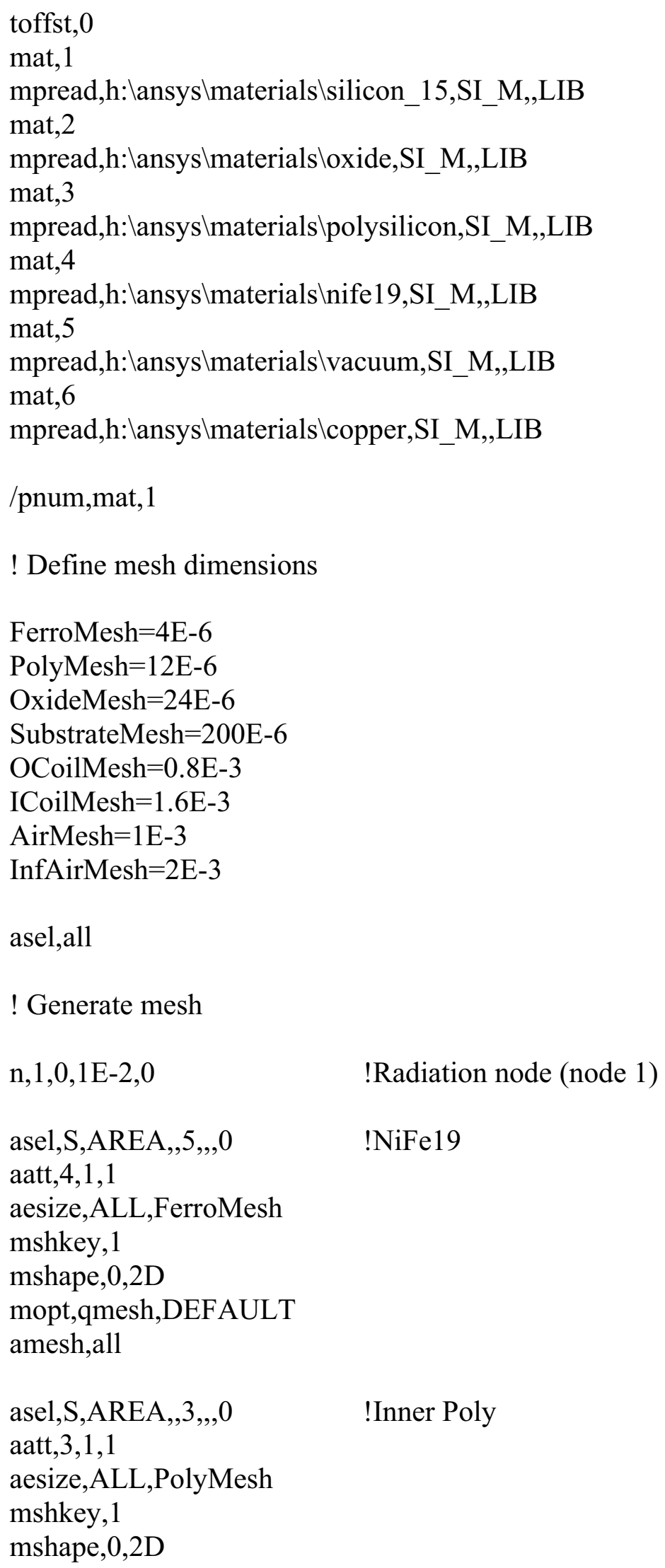




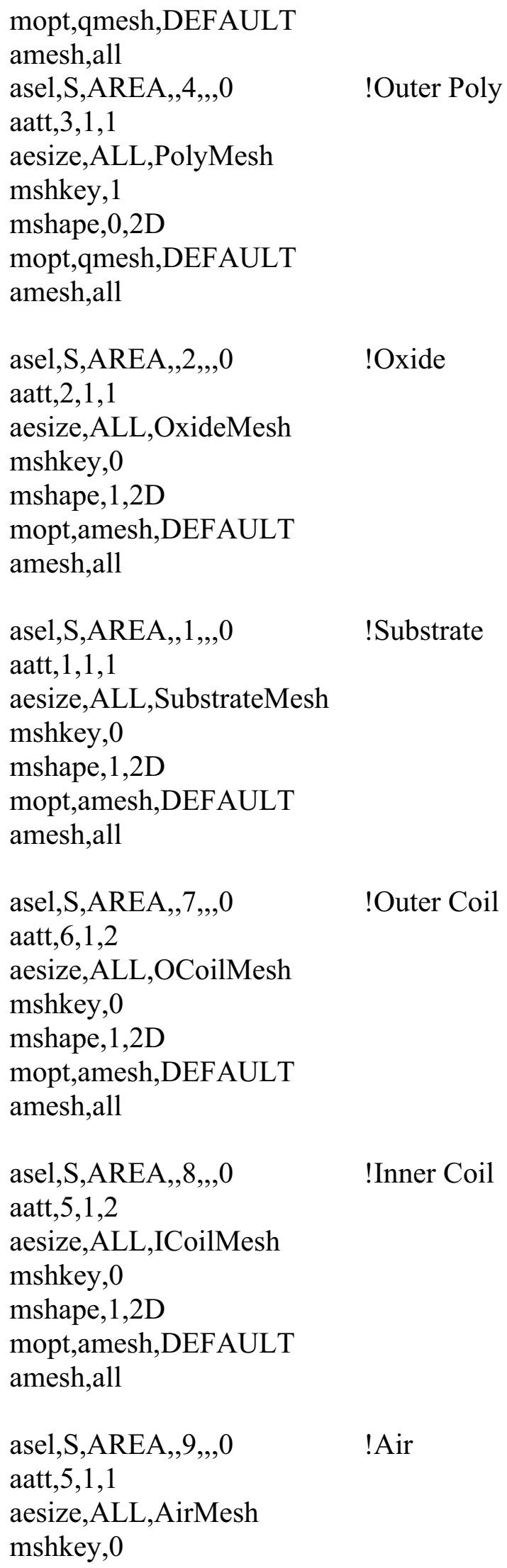


mshape, $0,2 \mathrm{D}$

mopt,qmesh,DEFAULT

amesh,all

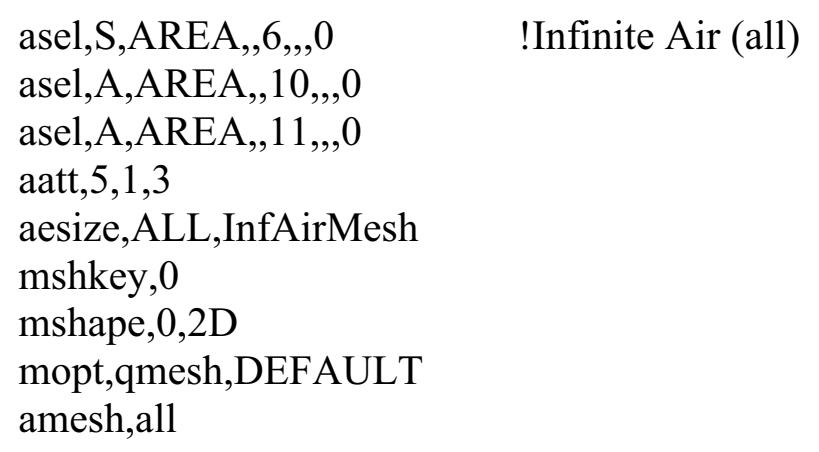

! Mesh top line of ferromagnetic film as Surf151

allsel

lsel,S,LINE, ,19,„,0

nsll,S,1

esln, 0

esel,R,MAT, ,4

type, 4

real, 4

mat, 4

esurf, 1

allsel

! Generate mesh and assign space node (node 1)

finish

\section{! WRITE ELECTROMAGNETIC PHYSICS ENVIRONMENT}

! The electromagnetic physics environment requires

! only one load, that being the applied current

! density to the magnetic coil. The boundary

! conditions required are the infinite surface on the

! outer lines and the flux-normal condition for $\mathrm{AZ}$

! along the y-axis. An additional $\mathrm{BC}$ is optionally

! utilized to artificially control the substrate

! temperature. The harmonic frequency is also

! provided.

/prep7

allsel

toffst, 0

et, 4,0

nsel, S,loc, $\mathrm{x}, 0$

d,ALL,az,0

! Apply flux-normal on y-axis

nsel,ALL 


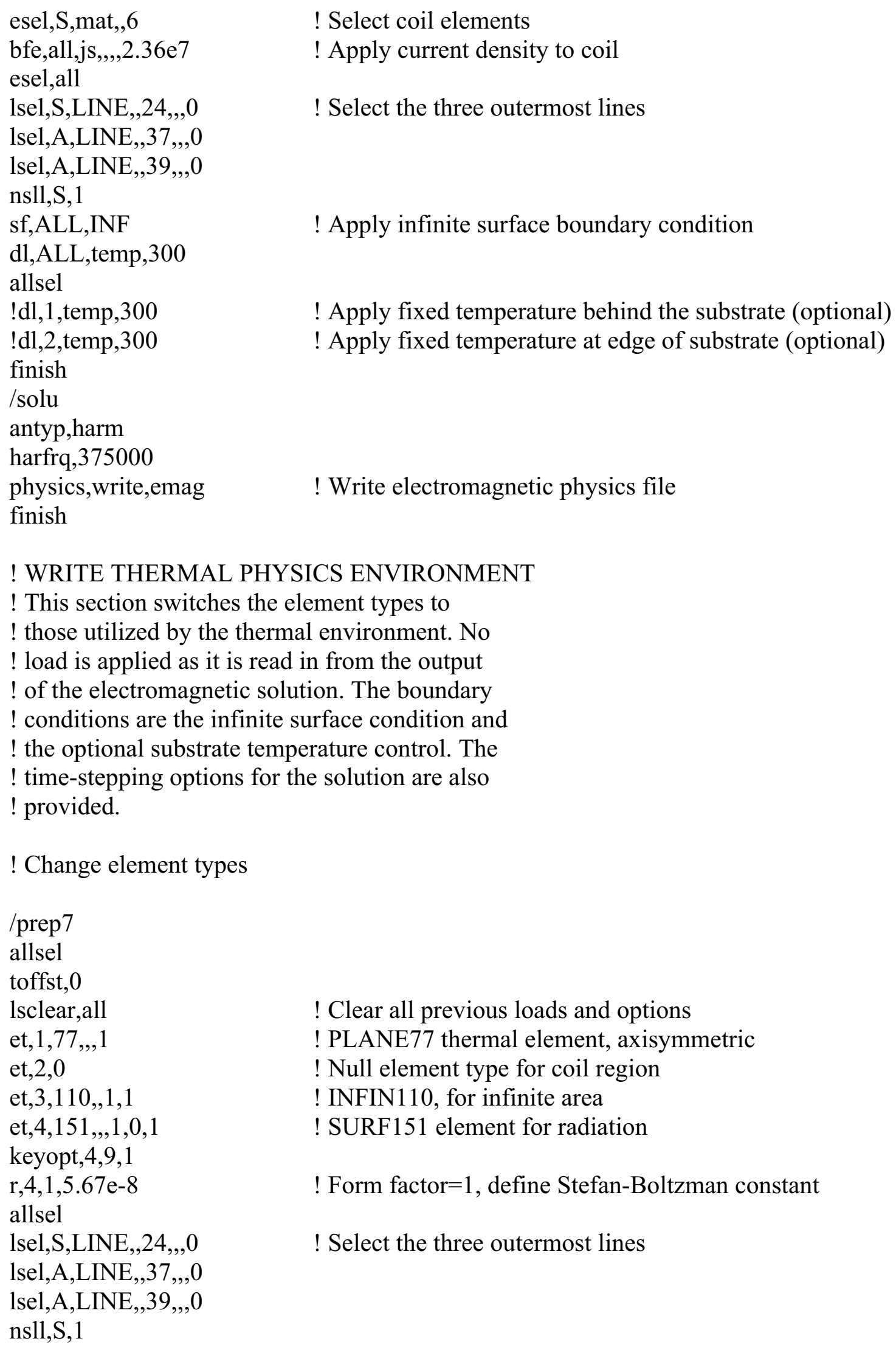




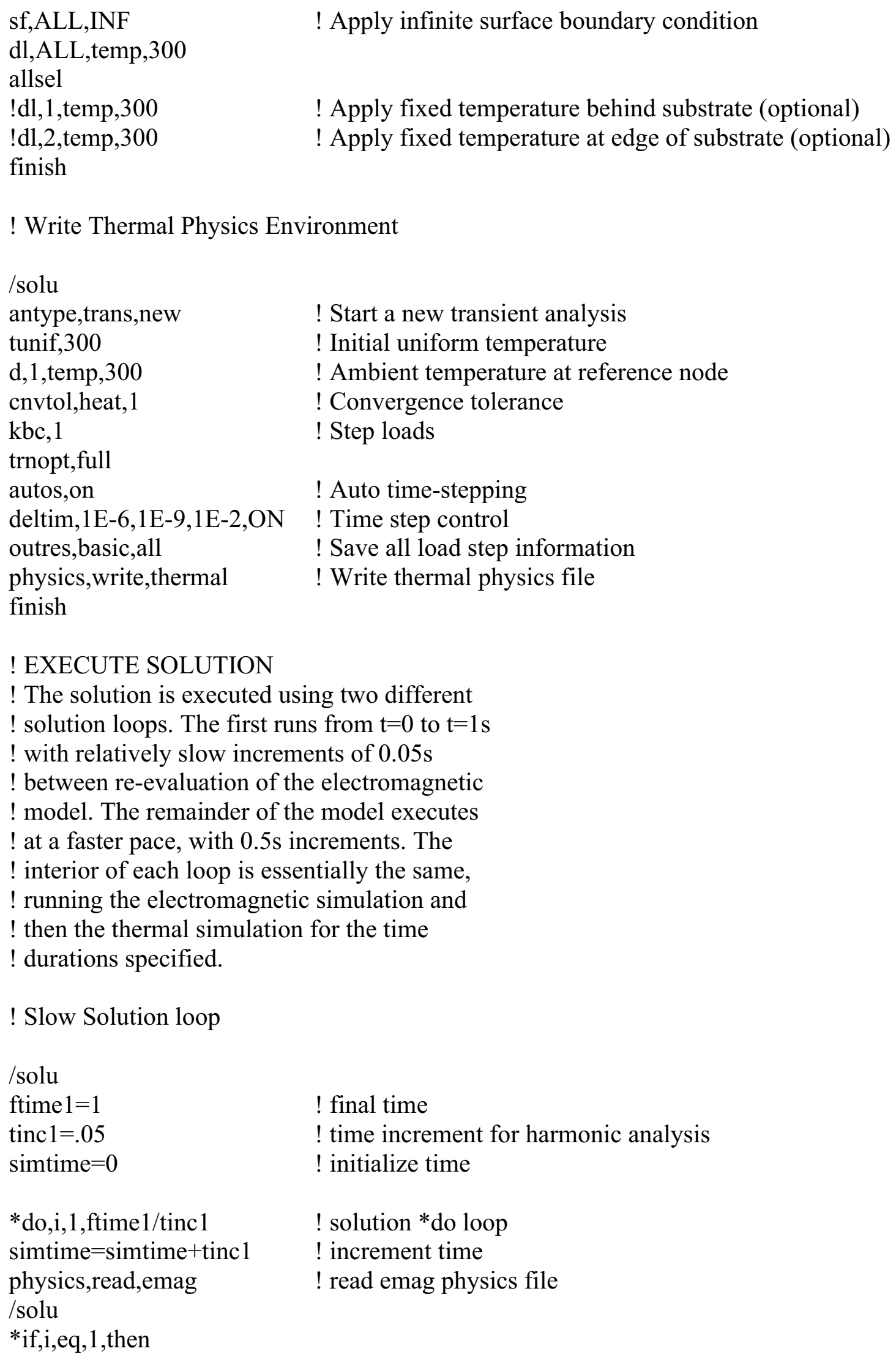

! Slow Solution loop

/solu

$\begin{array}{ll}\text { ftime } 1=1 & \text { ! final time } \\ \text { tinc } 1=.05 & \text { ! time increment for harmonic analysis } \\ \text { simtime }=0 & \text { ! initialize time } \\ \text { *do,i,1,ftime1/tinc1 } & \text { ! solution *do loop } \\ \begin{array}{l}\text { simtime=simtime+tinc1 } \\ \text { physics,read,emag }\end{array} & \text { ! increment time } \\ \text { /solu } & \text { read emag physics file } \\ * \text { if,i,eq, }, \text { then } & \end{array}$




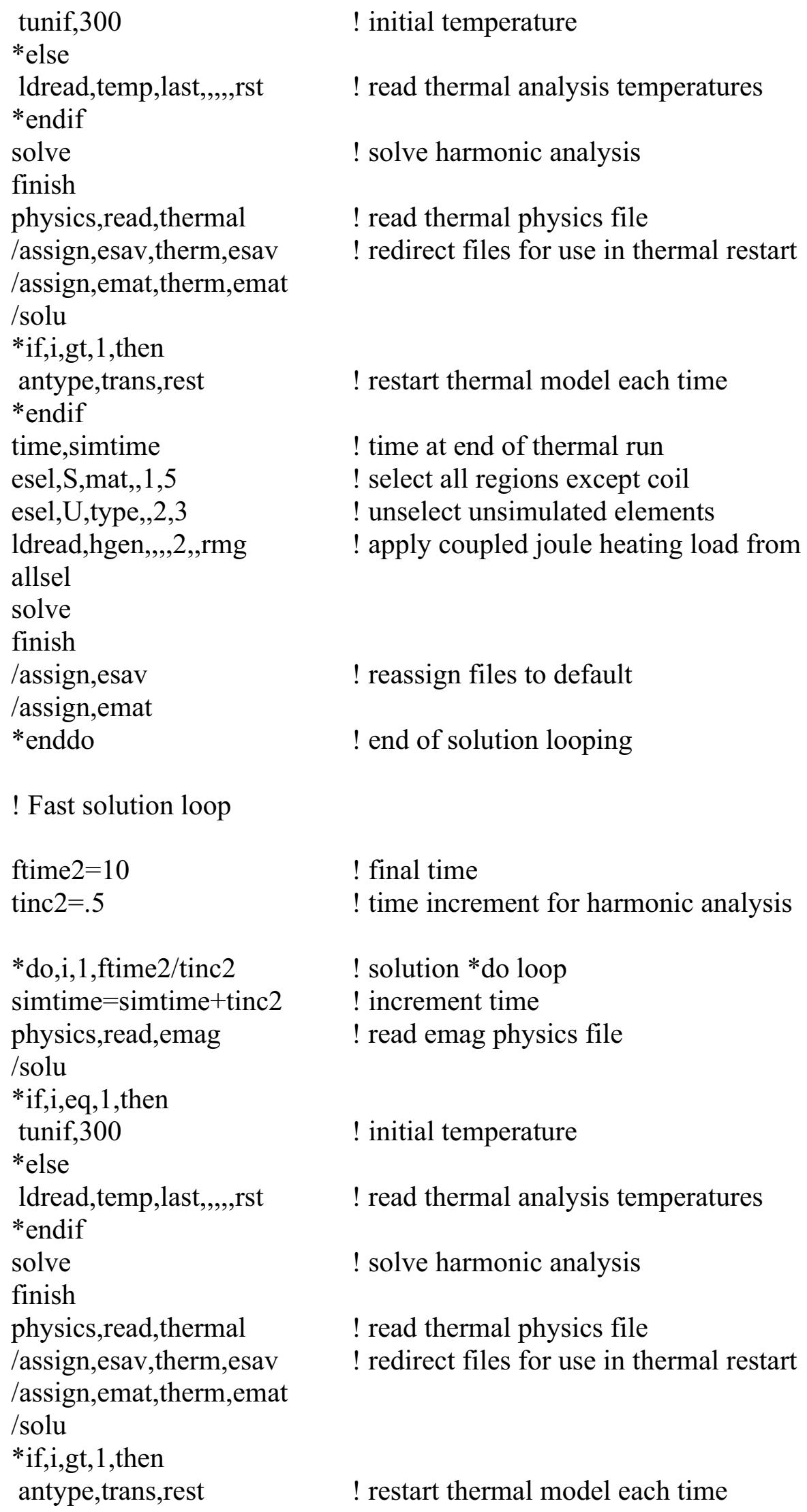




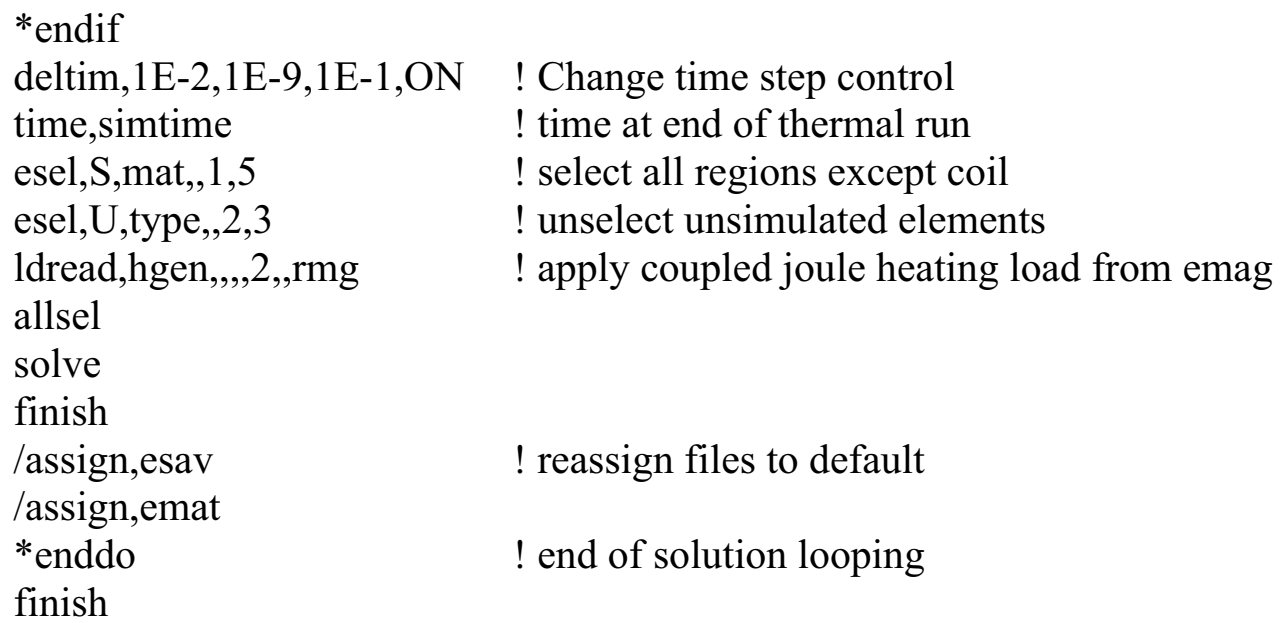




\section{A.4: Simulation Code for Thin Film Induction Heating Model with Thermal Isolation}

! Thin film simulation with hollow ferromagnetic ring

! $1.2 \mathrm{~cm}$ nife 19 outer radius, $0.6 \mathrm{~cm}$ inner radius, $7.1 \mathrm{um}$ thickness

! 3um poly layer with two sets of three thermal isolation trenches

! 1 coil turn (multiply current by 3 )

! This model is an axisymmetric induction heating simulation

! that models a thin film stack. The energy from the heated

! NiFe19 film ring spreads to polysilicon film below, and an

! attempt is made to contain the thermal energy using

! various isolation methods.

\section{! DEFINE MODEL DIMENSIONS}

! The model dimensions are defined via connected

! keypoints. Keypoints are points defined at specific

! locations (eg, x,y,z coordinates). Area definitions

! include all keypoints through which they

! intersect in order to avoid mesh discontinuities.

/prep7

! Y-coordinates

Substrate $\mathrm{Y}=-0.5 \mathrm{E}-3$

$\begin{array}{ll}\text { OxideY }=1 \mathrm{E}-6 & \text { ! 1 um lower oxide } \\ \text { PolyY=4E-6 } & \text { ! 3um poly } \\ \text { FerroY }=11.1 \mathrm{E}-6 & ! \text { 7.1um nife19 }\end{array}$

! X-coordinates

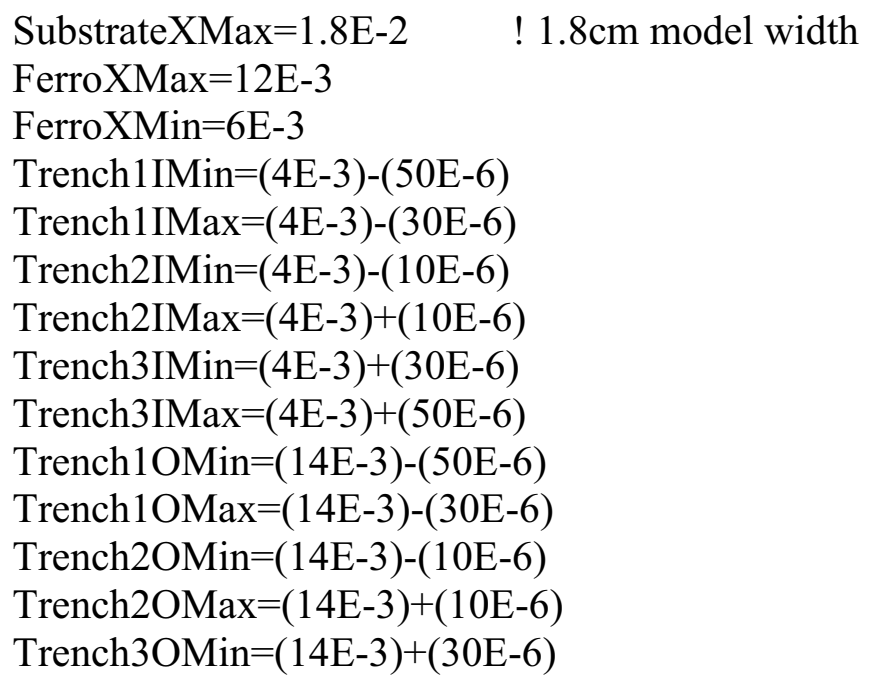


Trench3OMax $=(14 \mathrm{E}-3)+(50 \mathrm{E}-6)$

! Finite and Inifinite Space Dimensions

AirMinY $=-0.25 \mathrm{E}-2 \quad$ ! Air range is $-.25 \mathrm{~cm}$ to $1 \mathrm{~cm}$

AirMaxY=1E-2

AirMaxX $=2.25 \mathrm{E}-2$

InfMinY $=-0.35 \mathrm{E}-2$ ! Infinite space range is $-.35 \mathrm{~cm}$ to $1.1 \mathrm{~cm}$

$\operatorname{InfMaxY}=1.1 \mathrm{E}-2$

$\operatorname{InfMaxX}=2.35 \mathrm{E}-2$

! Define Areas via Keypoints

$\mathrm{k}, 1,0$, SubstrateY, 0

k,2,SubstrateXMax,SubstrateY,0

$\mathrm{k}, 3,0,0,0$

$\mathrm{k}, 4$, SubstrateXMax, 0,0
a, $1,2,4,3$
! Substrate (area 1)

$\mathrm{k}, 5,0$, OxideY, 0

k,6,Trench1IMin,OxideY,0

k,7,Trench1IMax,OxideY,0

k,8,Trench2IMin,OxideY,0

k,9,Trench2IMax,OxideY,0

$\mathrm{k}, 10$,Trench3IMin,OxideY, 0

$\mathrm{k}, 11$,Trench3IMax,OxideY, 0

$\mathrm{k}, 12$,Trench1OMin,OxideY, 0

$\mathrm{k}, 13$,Trench1OMax,OxideY, 0

k,14,Trench2OMin,OxideY, 0

$\mathrm{k}, 15$,Trench2OMax,OxideY,0

k,16,Trench3OMin,OxideY,0

k,17,Trench3OMax,OxideY,0

k,18,SubstrateXMax,OxideY,0

a,3,4,18,17,16,15,14,13,12,11,10,9,8,7,6,5 ! Oxide (area 2)

k, 19,0,PolyY,0

k,20,Trench1IMin,PolyY,0

k,21,Trench1IMax,PolyY,0

k,22,Trench2IMin,PolyY,0

k,23,Trench2IMax,PolyY,0

k,24,Trench3IMin,PolyY,0

k,25,Trench3IMax,PolyY,0

k,26,FerroXMin,PolyY,0 
k,27,FerroXMax,PolyY,0
k,28,Trench1OMin,PolyY,0
k,29,Trench1OMax,PolyY,0
k,30,Trench2OMin,PolyY,0
k,31,Trench2OMax,PolyY,0
k,32,Trench3OMin,PolyY,0
k,33,Trench3OMax,PolyY,0
k,34,SubstrateXMax,PolyY,0

a,5,6,20,19

a, $6,7,21,20$

a, $7,8,22,21$

a, 8,9,23, 22

a,9, 10,24,23

a, 10,11,25,24

a,11,12,28,27,26,25

a, 12,13,29,28

a, $13,14,30,29$

a, $14,15,31,30$

a, 15,16,32,31

a, 16,17,33,32

a, 17, 18,34,33

k,35,FerroXMin,FerroY,0

k,36,FerroXMax,FerroY,0

a,26,27,36,35

k,37,0,AirMinY,0

k,38,AirMaxX,AirMinY,0

k,39,0,AirMaxY,0

k,40,AirMaxX,AirMaxY,0

a,37,38,40,39

! Air (area 17, becomes 20 after subtraction)

cyl4,15.9E-3,6.2E-3,3.2E-3,0,1.6E-3,360 ! Coil (areas 18,19)

asba,17,ALL,DELETE,KEEP ! ! Subtract all areas from Air

$\mathrm{k}, 49,0, \operatorname{InfMin} Y, 0$

k,50,InfMaxX,InfMinY,0

$\mathrm{k}, 51,0$,InfMaxY,0

k,52,InfMaxX,InfMaxY,0

a, $49,50,38,37$

a, $50,52,40,38$
! Inner Poly Structure (area 3)

! Inner Trench 1 (area 4)

! Poly between T1 and T2 (area 5)

! Inner Trench 2 (area 6)

! Poly between T2 and T3 (area 7)

! Inner Trench 3 (area 8)

! Poly below NiFe19 (area 9)

! Outer Trench 1 (area 10)

! Poly between T1 and T2 (area 11)

! Outer Trench 2 (area 12)

! Poly between T2 and T3 (area 13)

! Outer Trench 3 (area 14)

! Outer Poly Structure (area 15)
! NiFe19 (area 16) 
a,39,40,52,51 ! Upper Infinite Air (area 22)

\section{! CREATE MESH}

! This section meshes the model. It begins by

! defining the element types. Material properties are

! read in from previously-defined files. Each area

! is then meshed individually using the dimensions

! assigned. Lastly, the top surface of the NiFe19

! film is given an overlaid surface mesh to provide

! for radiation modeling. One key difference from

! the ANSYS example model is the use of infinite

! surface elements around the outer edge of the

! model. These allow the free space region to seem

! larger than that simulated.

! Define element types
et, $1,53,, 1$
! PLANE53, AZ DOF, included in thermal sim
et, $2,53,,, 1$
! PLANE53, AZ DOF, not in thermal sim
et, $3,110,1,1$
! INFIN110, for infinite area
et, $4,151,, 1,0,1$
! SURF151, for including radiation
$\mathrm{r}, 4,0$

! Define material properties (from files)

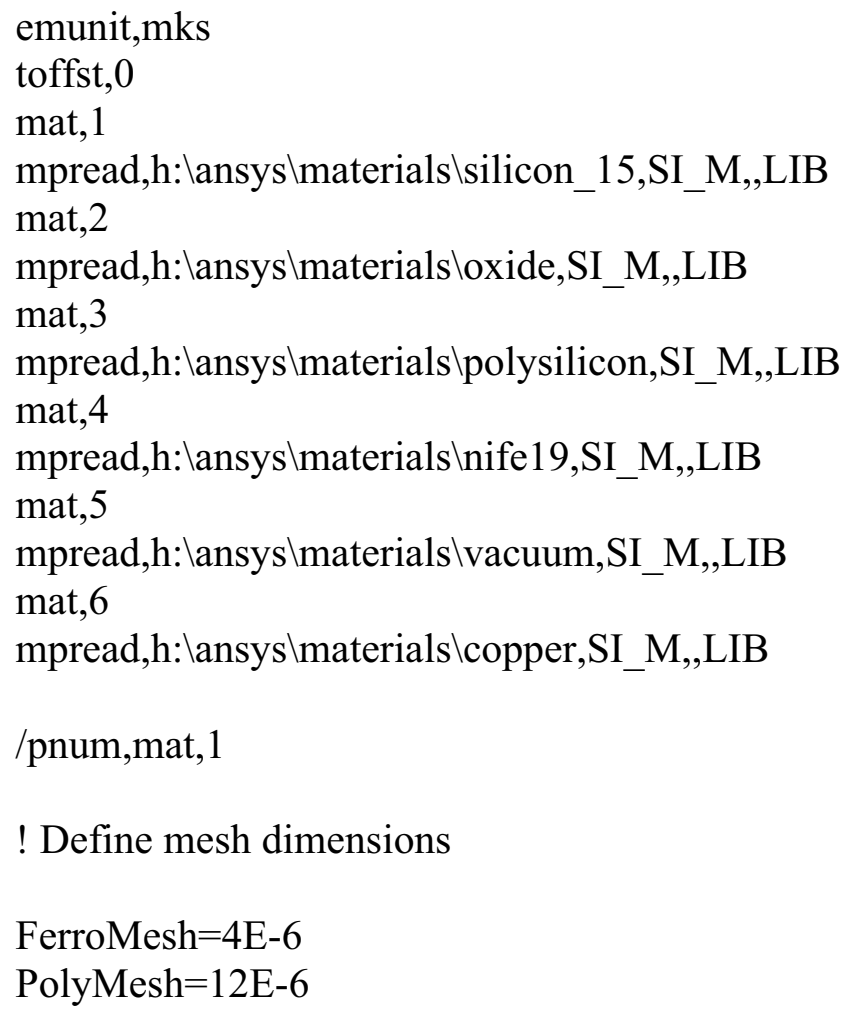




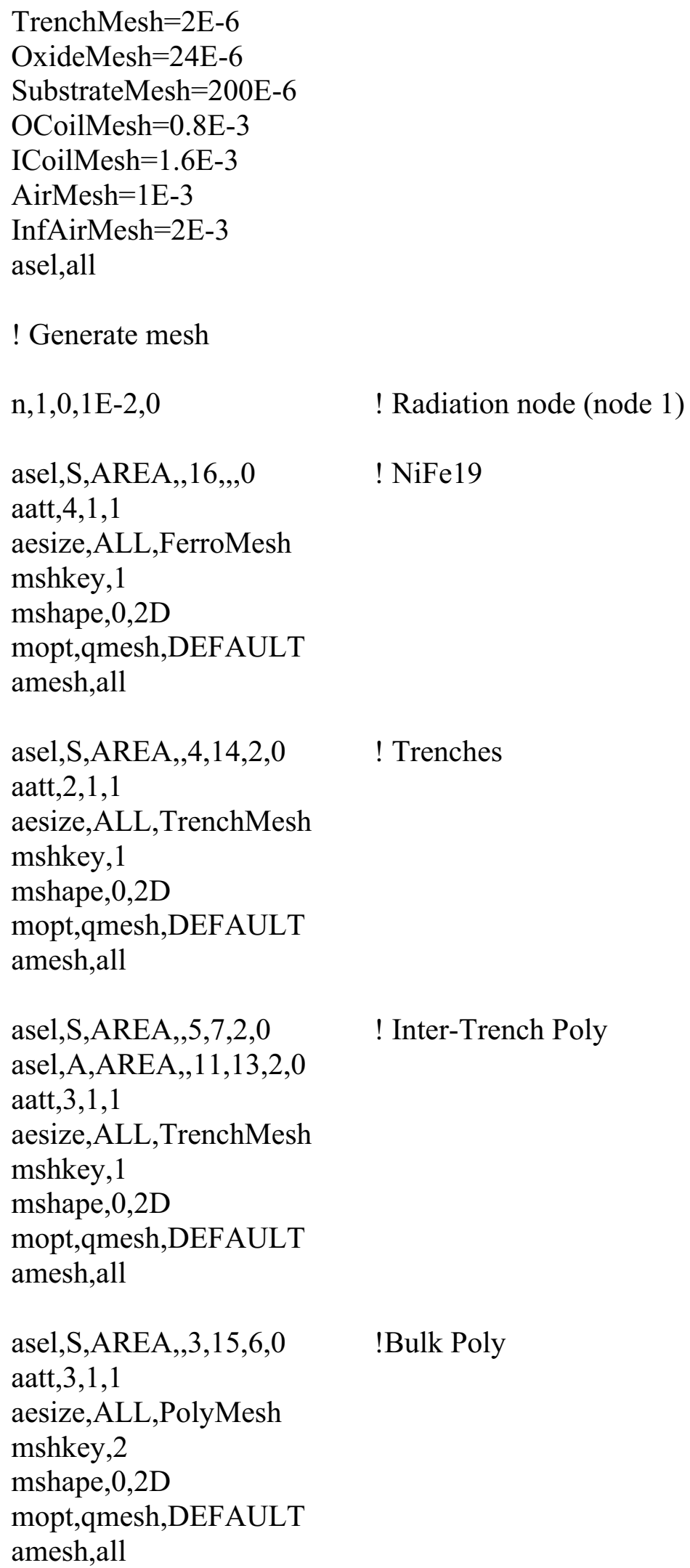

asel,S,AREA,,16,,0 $\quad$ ! NiFe19

asel,S,AREA,,4,14,2,0 ! Trenches

! Radiation node (node 1) 


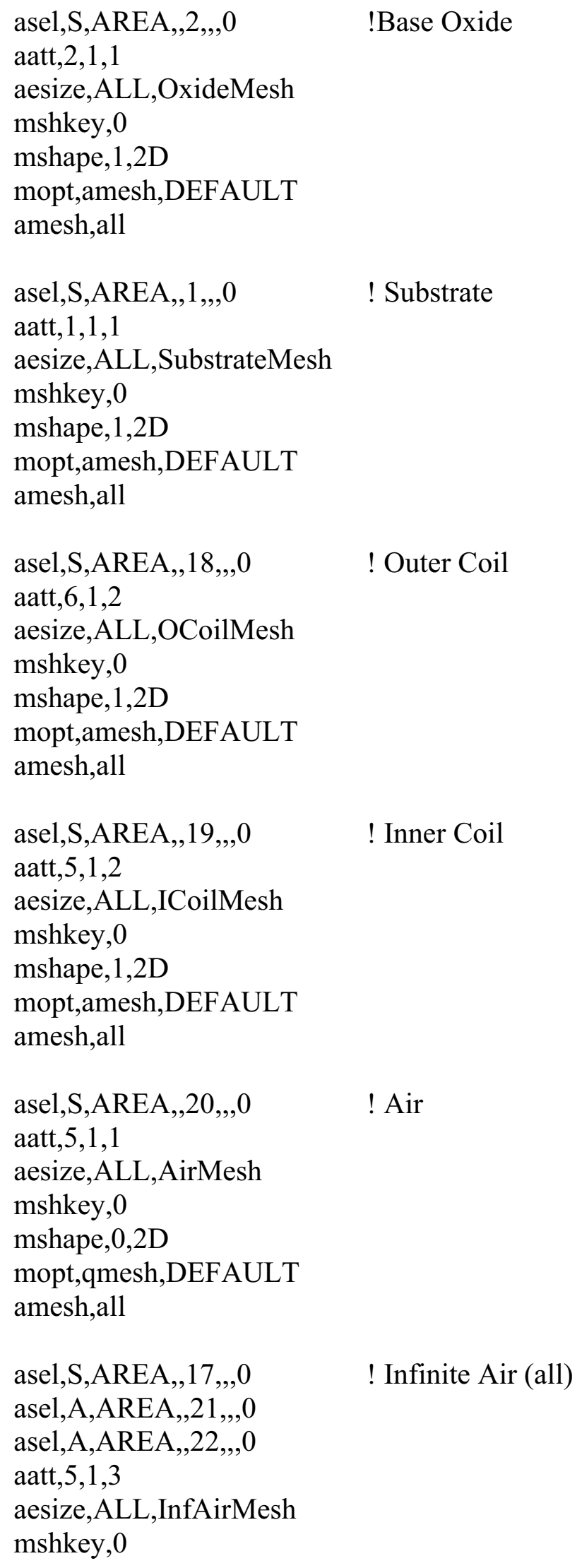


mshape, $0,2 \mathrm{D}$

mopt,qmesh,DEFAULT

amesh,all

allsel

! Mesh top line of ferromagnetic film as Surf151

* get,emin,elem,,num,max

lsel,S,LINE, 50,,0

nsll,S, 1

esln, 0

esel,R,MAT,„4

type, 4

real, 4

mat, 4

esurf, 1

*get,emax,elem,,num,max

allsel

! WRITE ELECTROMAGNETIC PHYSICS ENVIRONMENT

! The electromagnetic physics environment requires

! only one load, that being the applied current

! density to the magnetic coil. The boundary

! conditions required are the infinite surface on the

! outer lines and the flux-normal condition for $\mathrm{AZ}$

! along the y-axis. An additional BC is optionally

! utilized to artificially control the substrate

! temperature. The harmonic frequency is also

! provided.

/prep7

allsel

toffst, 0

et, 4,0

nsel,S,loc, $\mathrm{x}, 0$

$\mathrm{d}, \mathrm{ALL}, \mathrm{az}, 0$

nsel,ALL

esel,S,mat,,6

! Apply flux-normal on y-axis

bfe,all,js, ,,,2.36e 7

esel,all

1sel,S,LINE, 55, ,0

1sel,A,LINE,,68,,0

1sel,A,LINE, ,70,,0

nsll,S,1

sf,ALL,INF

dl,ALL,,temp,300

! Select coil elements

! Apply current density to coil

! Select the three outermost lines

! Apply infinite surface boundary condition 


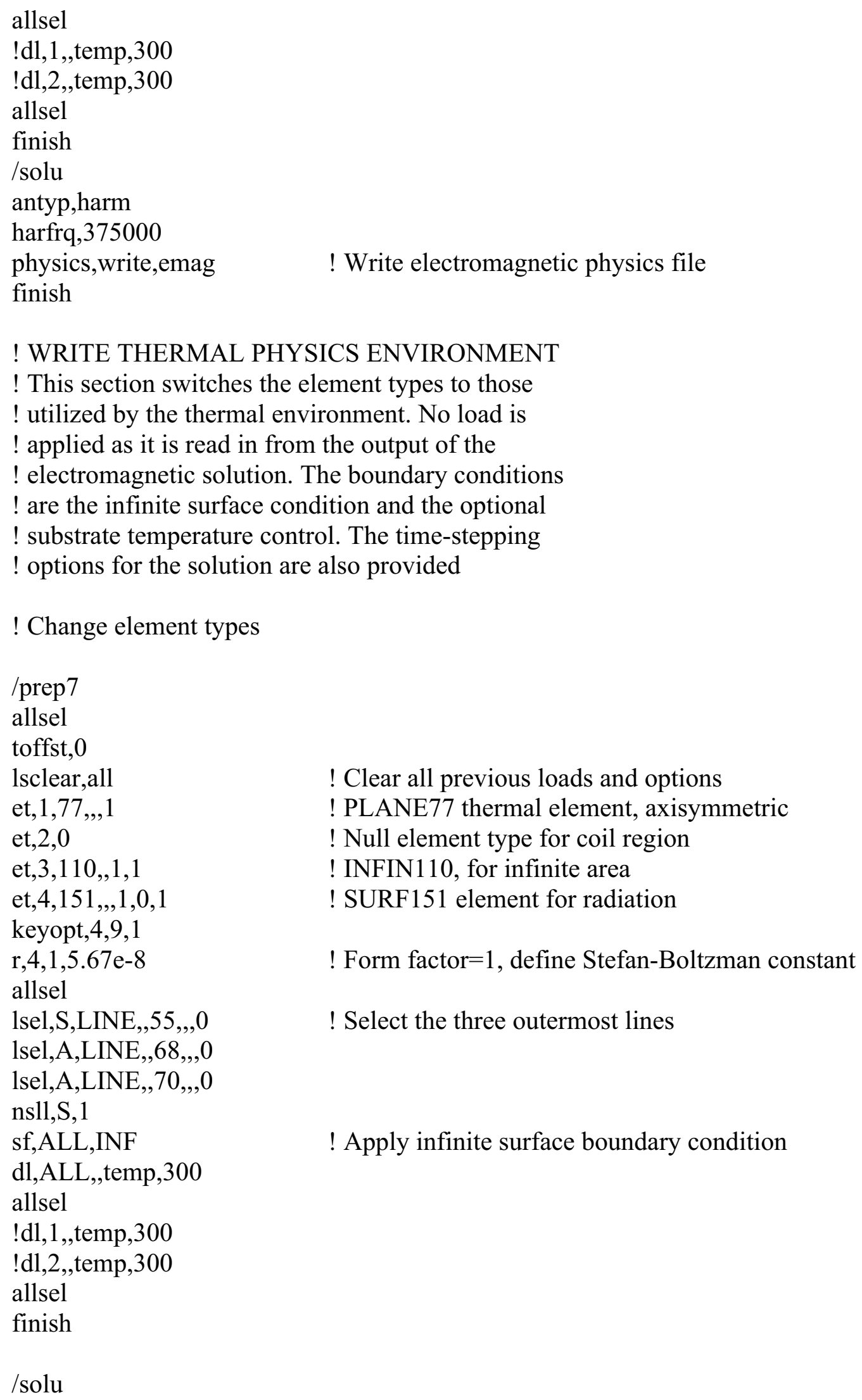




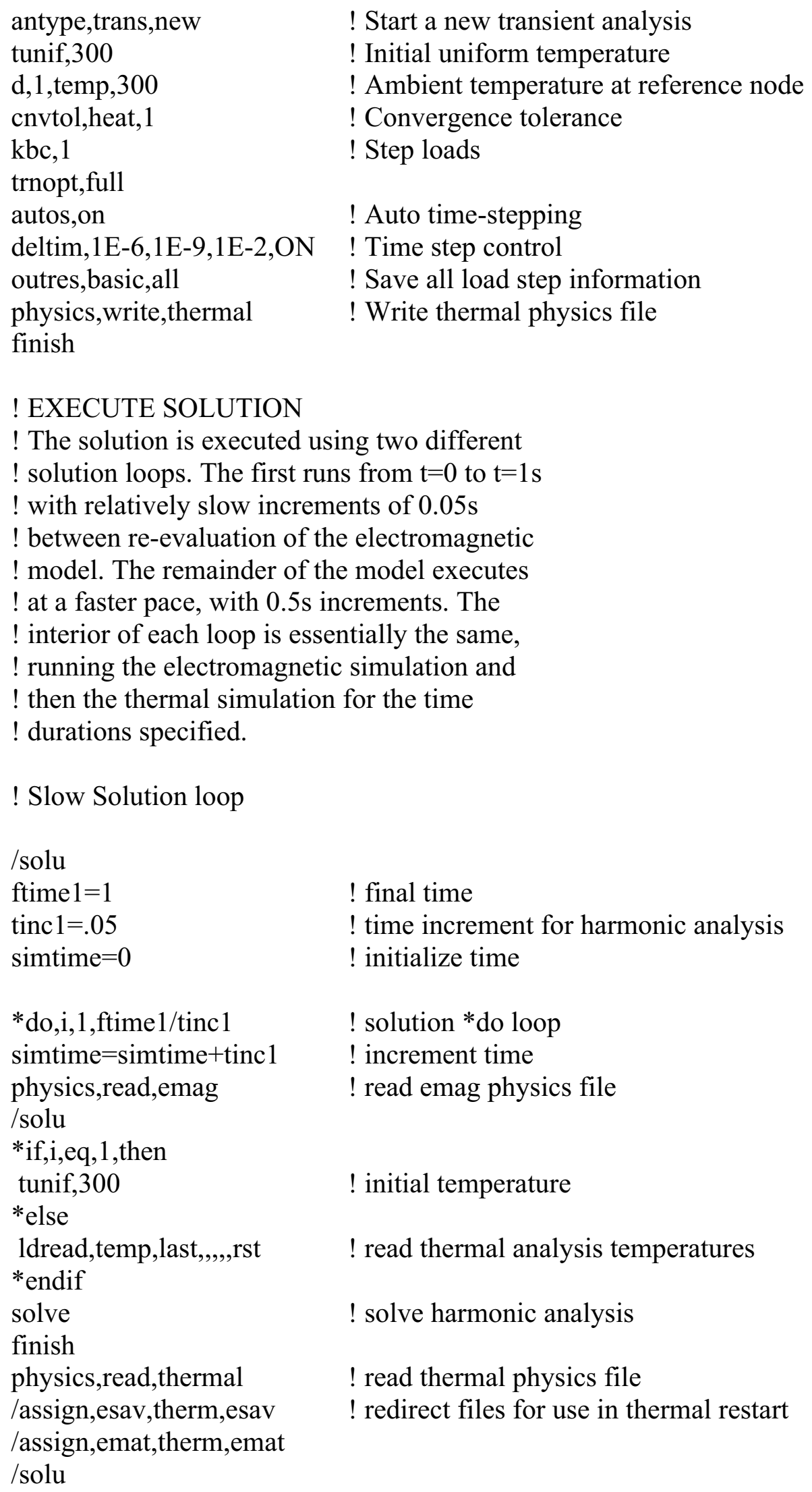

! Slow Solution loop

/solu

ftime $1=1$

! final time

tinc $1=.05$

! time increment for harmonic analysis

simtime $=0$

! initialize time

*do,i,1,ftime1/tinc1 ! solution *do loop

simtime $=$ simtime + tinc $1 \quad$ ! increment time

physics,read,emag ! read emag physics file

/solu

*if,i,eq, 1 ,then

tunif,300 ! initial temperature

*else

ldread,temp,last,,,,,rst $\quad$ ! read thermal analysis temperatures

*endif

solve

! solve harmonic analysis

finish

physics,read,thermal ! read thermal physics file

/assign,esav,therm,esav ! redirect files for use in thermal restart

/assign,emat,therm,emat

/solu 
*if,i,gt, 1 ,then

antype,trans,rest

! restart thermal model each time

*endif

time,simtime

! time at end of thermal run

esel,S,mat, ,1,5

esel,U,type, 2,3

! select all regions except coil

! unselect unsimulated elements

ldread,hgen,,,2,,rmg

! apply coupled joule heating load from emag

allsel

solve

finish

/assign,esav

! reassign files to default

/assign,emat

*enddo

! end of solution looping

! Fast solution loop

ftime $2=10$

! final time

tinc $2=.5$

! time increment for harmonic analysis

*do,i,1,(ftime2-simtime)/tinc2 ! solution *do loop

simtime $=$ simtime + tinc $2 \quad$ ! increment time

physics,read,emag ! read emag physics file

/solu

*if,i,eq, 1 ,then

tunif,300

! initial temperature

*else

ldread,temp,last,,,,,rst ! read thermal analysis temperatures

*endif

solve

! solve harmonic analysis

finish

! read thermal physics file

physics,read,thermal

! redirect files for use in thermal restart

/assign,emat,therm,emat

/solu

*if,i,gt, 1 , then

antype,trans, rest

*endif

time,simtime

! restart thermal model each time

deltim,1E-2,1E-9,1E-1,ON

esel,S,mat, ,1,5

esel,U,type, 2,3

ldread,hgen,,,2,,rmg

allsel

solve

! time at end of thermal run

! Change time step control

! select all regions except coil

! unselect unsimulated elements

! apply coupled joule heating load from emag

finish 


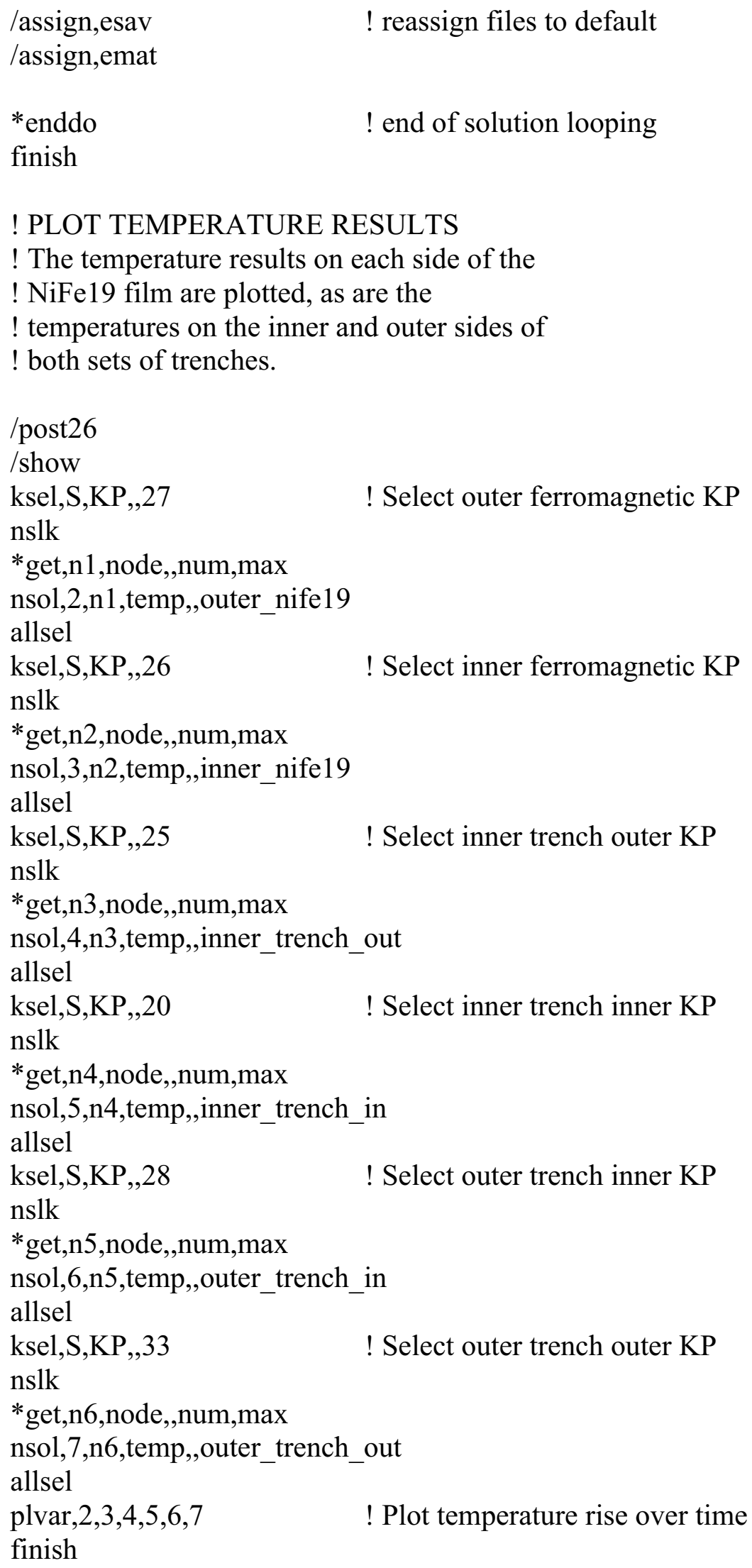




\section{Appendix B: Ferromagnetic Film Data}

\section{B.1: Nickel and Cobalt}

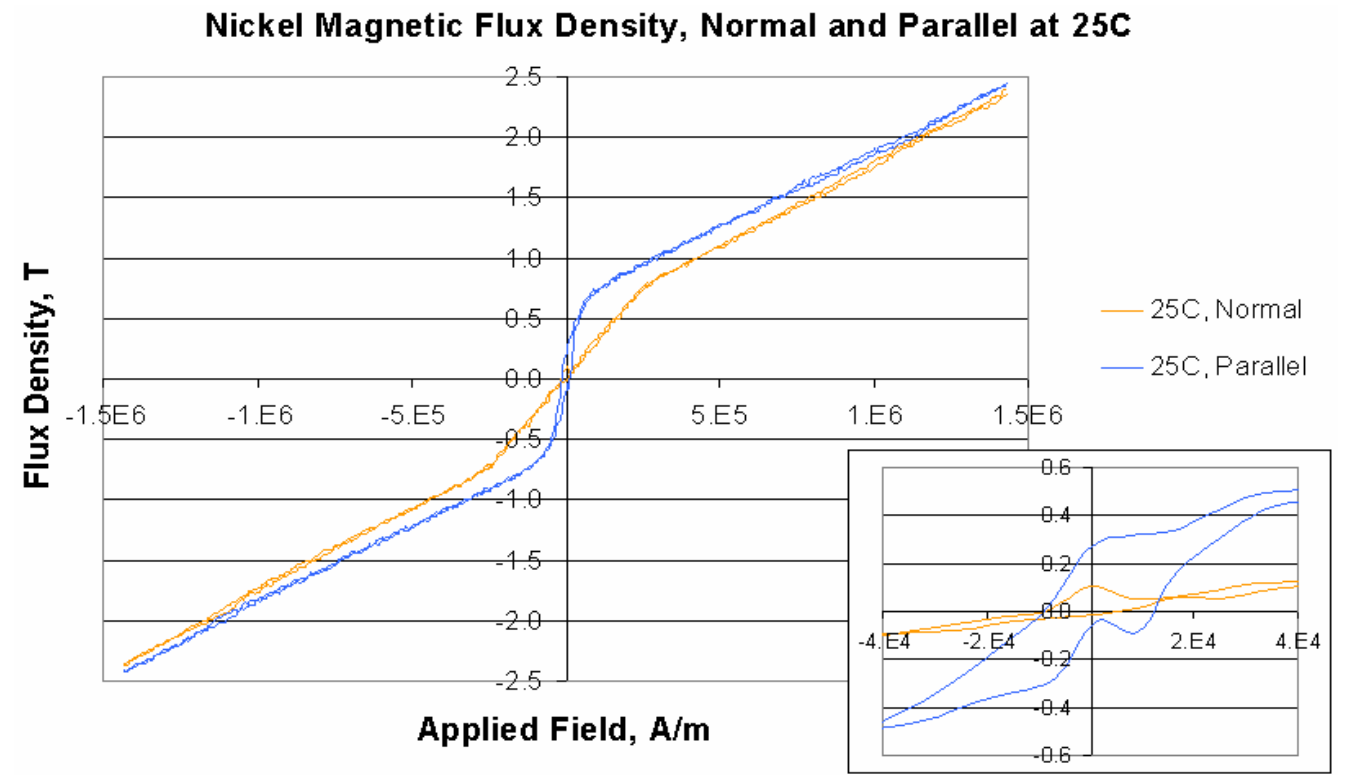

Figure B.1: Magnetic flux density plots for a RF sputtered nickel film illustrating properties both parallel and normal to the substrate at room temperature. Deposition was done at $700 \mathrm{~W}$ with no applied magnetic field, resulting in a $0.19 \mu \mathrm{m}$ film. Hysteresis was evident in the parallel direction but is difficult to distinguish over noise interference.

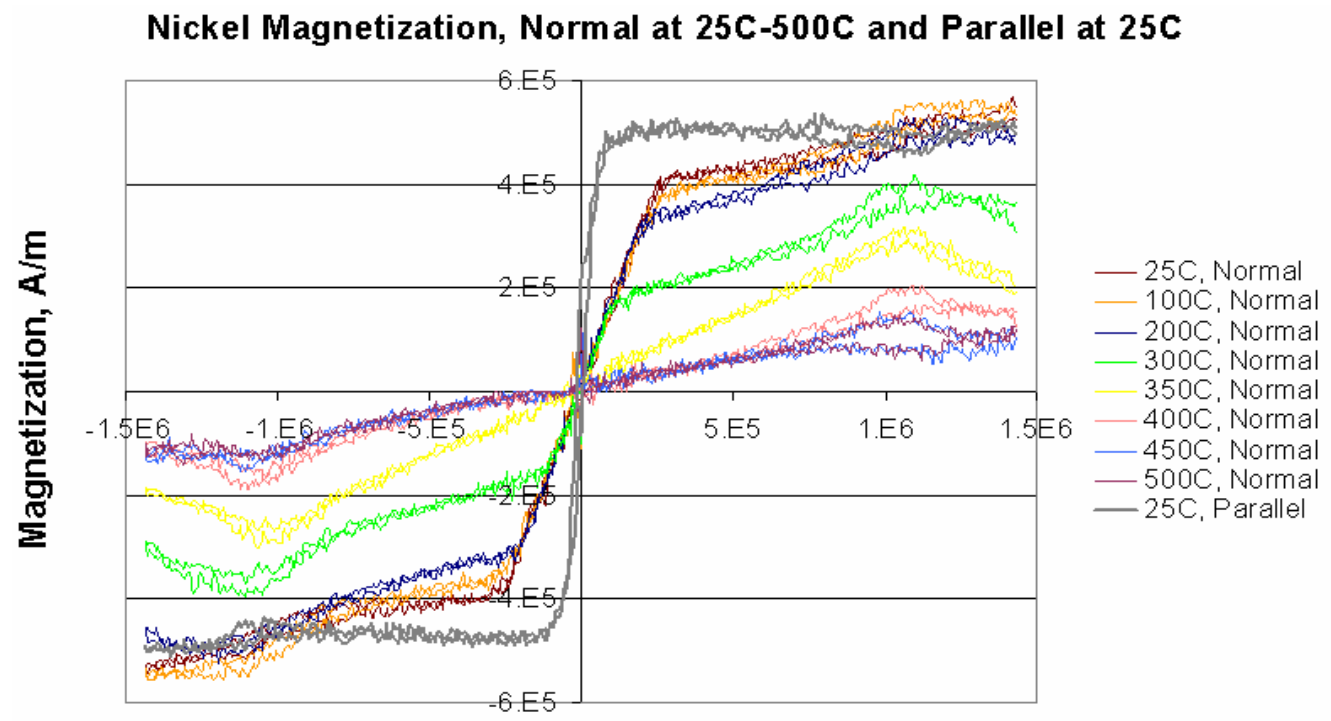

\section{Applied Field, A/m}

Figure B.2: Magnetization plots for the RF sputtered nickel film showing the effect of elevated temperature on its magnetic behavior in the direction normal to the substrate relative to the $25^{\circ} \mathrm{C}$ data. The parallel curve at $25^{\circ} \mathrm{C}$ is also included for reference. Noise in the data made curve shapes unusual. Ferromagnetic behavior was minimal at and above $400^{\circ} \mathrm{C}$. The nickel Curie temperature is $358^{\circ} \mathrm{C}$ [Eisberg, et al., 1985 (p 500)]. 


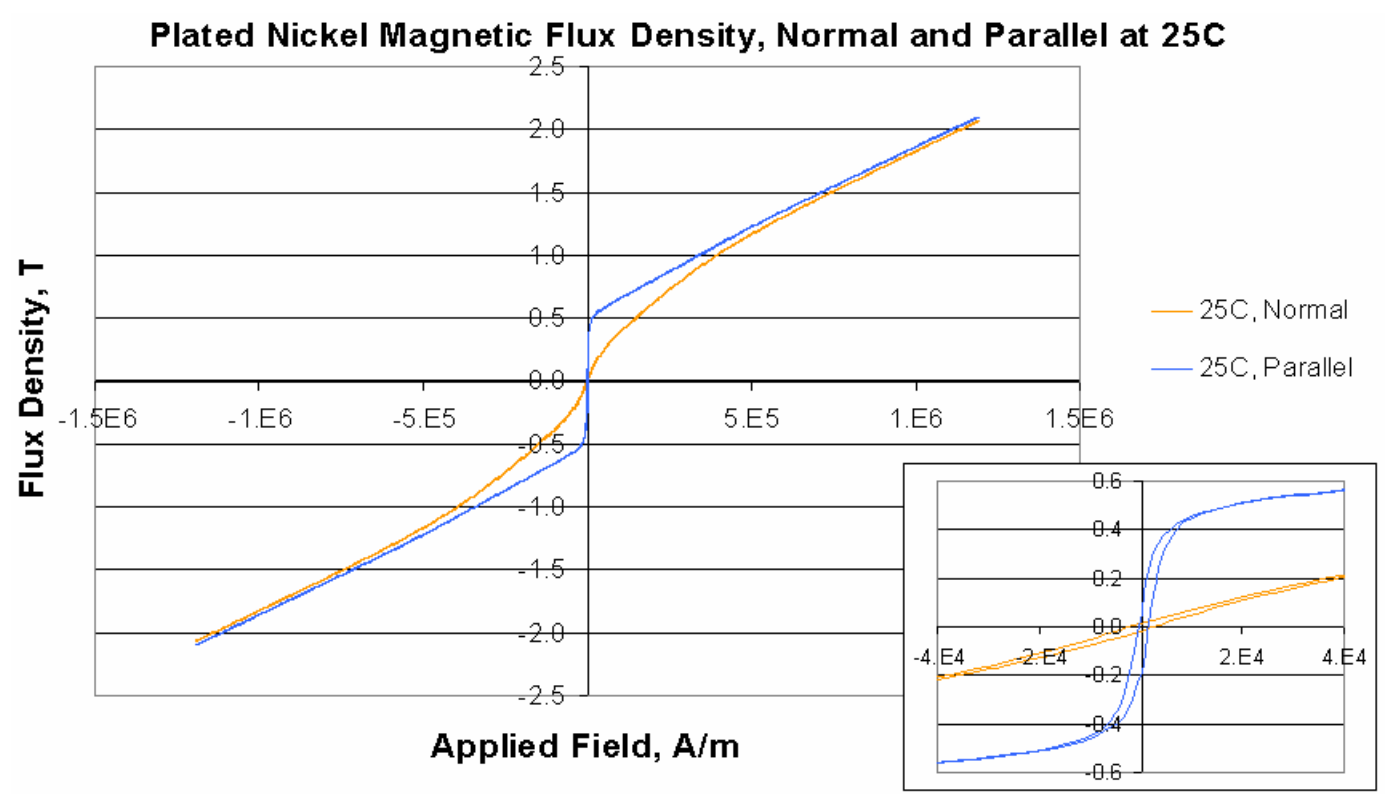

Figure B.3: Magnetic flux density plots for an electroplated nickel film illustrating properties both parallel and normal to the substrate at room temperature. The film was formed using a $\mathrm{NiSO}_{4}$-based solution (see Section 4.3) with a ??mA current under no applied magnetic field, resulting in a $11 \mu \mathrm{m}$ film. Hysteresis loops were more clearly defined than for the sputtered nickel film shown previously. Their lack of appreciable width is likely due to the more amorphous film structure.

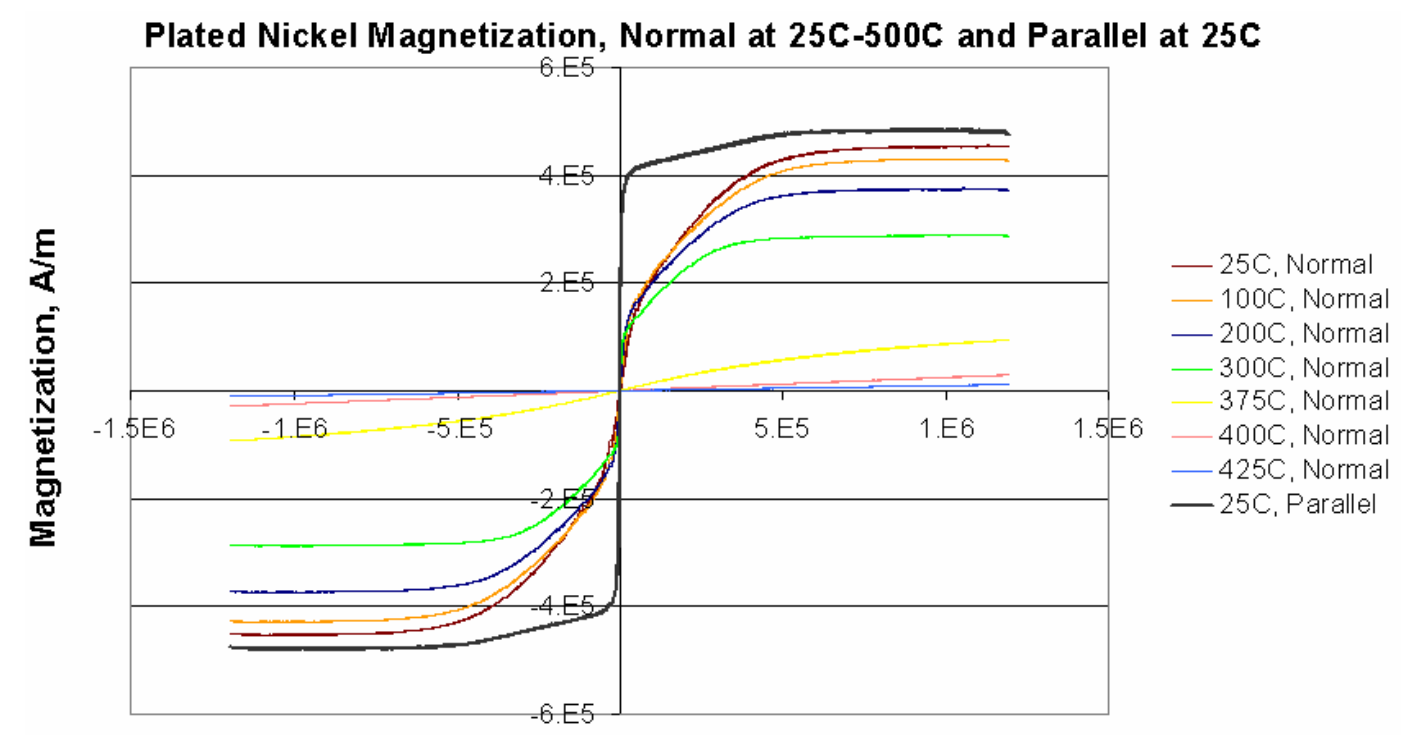

Applied Field, A/m

Figure B.4: Magnetization plots for the electroplated nickel film showing the effect of elevated temperature on its magnetic behavior in the direction normal to the substrate relative to the $25^{\circ} \mathrm{C}$ data. The parallel curve at $25^{\circ} \mathrm{C}$ is also included for reference. Ferromagnetic behavior was minimal at and above $375^{\circ} \mathrm{C}$, as anticipated. 


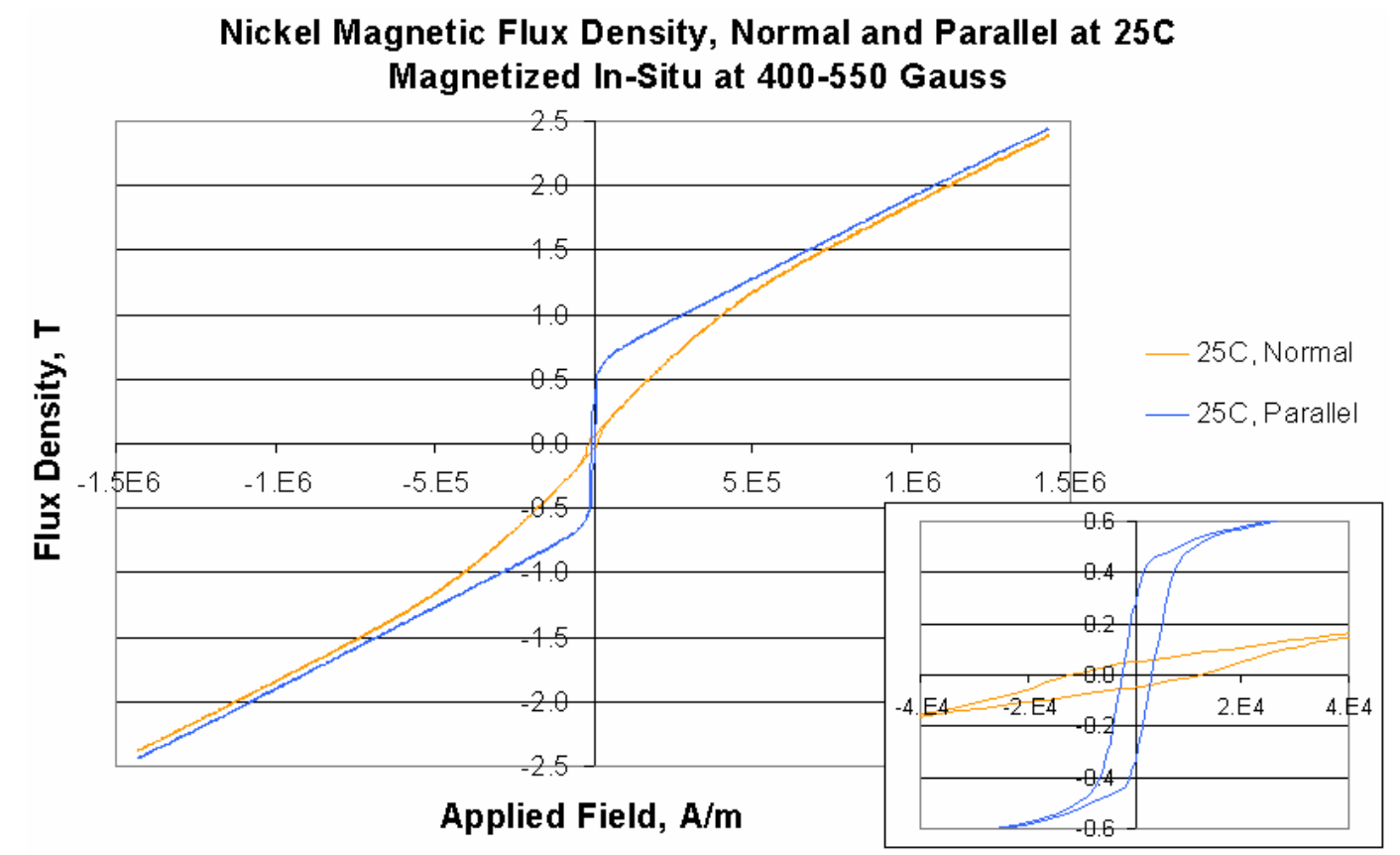

Figure B.5: Magnetic flux density plots for a RF sputtered nickel film illustrating properties both parallel and normal to the substrate at room temperature. Deposition was done at $700 \mathrm{~W}$ with a $400-550$ Gauss applied magnetic field, resulting in a $2.8 \mu \mathrm{m}$ film. Hysteresis in both directions was wider than that of the non-magnetized films.

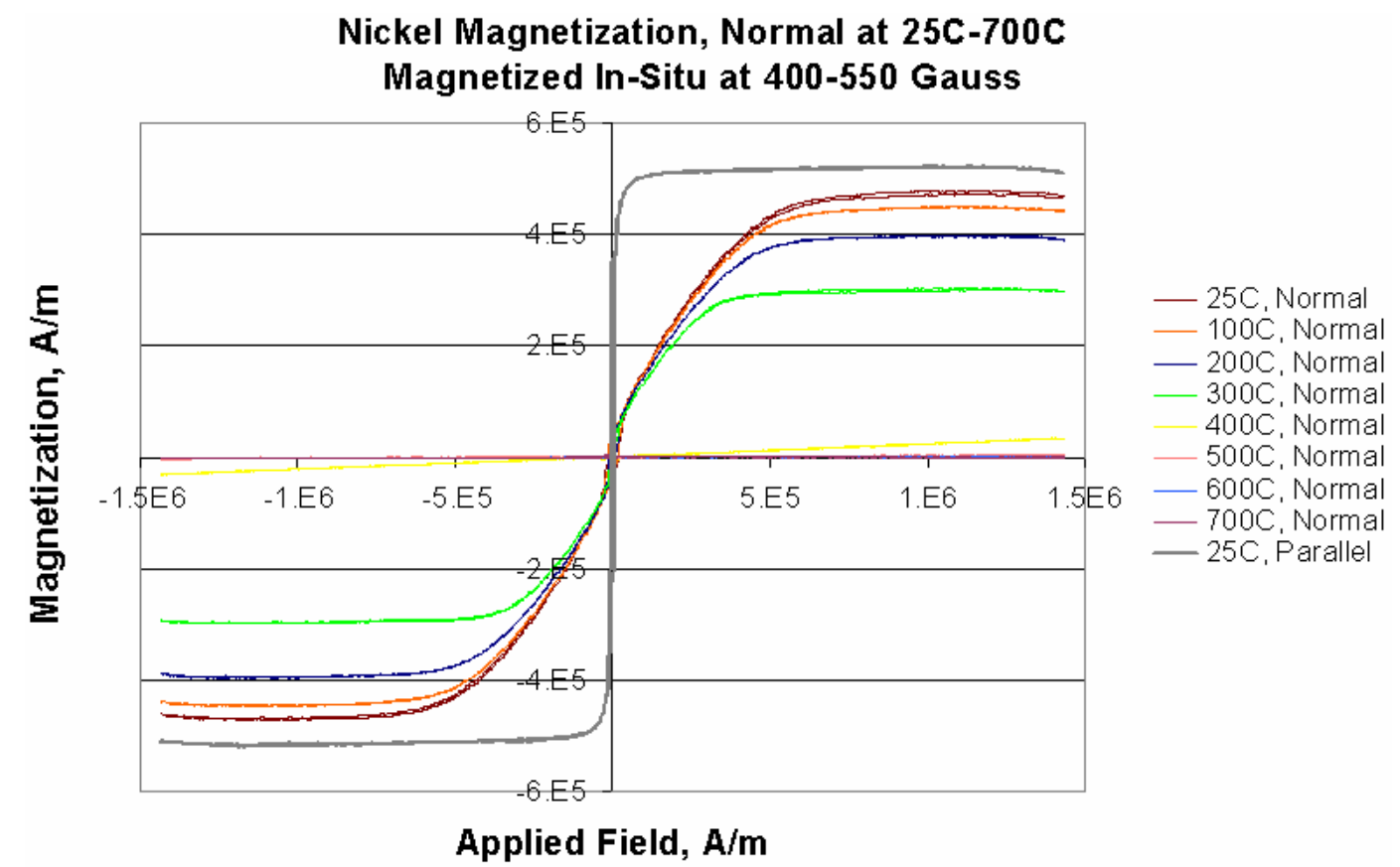

Figure B.6: Magnetization plots for the in-situ magnetized RF sputtered nickel film showing the effect of elevated temperature on its magnetic behavior in the direction normal to the substrate relative to the $25^{\circ} \mathrm{C}$ data. The parallel curve at $25^{\circ} \mathrm{C}$ is also included for reference. Ferromagnetic behavior was minimal at and above $400^{\circ} \mathrm{C}$. 
Cobalt Magnetic Flux Density, Normal and Parallel at 25C

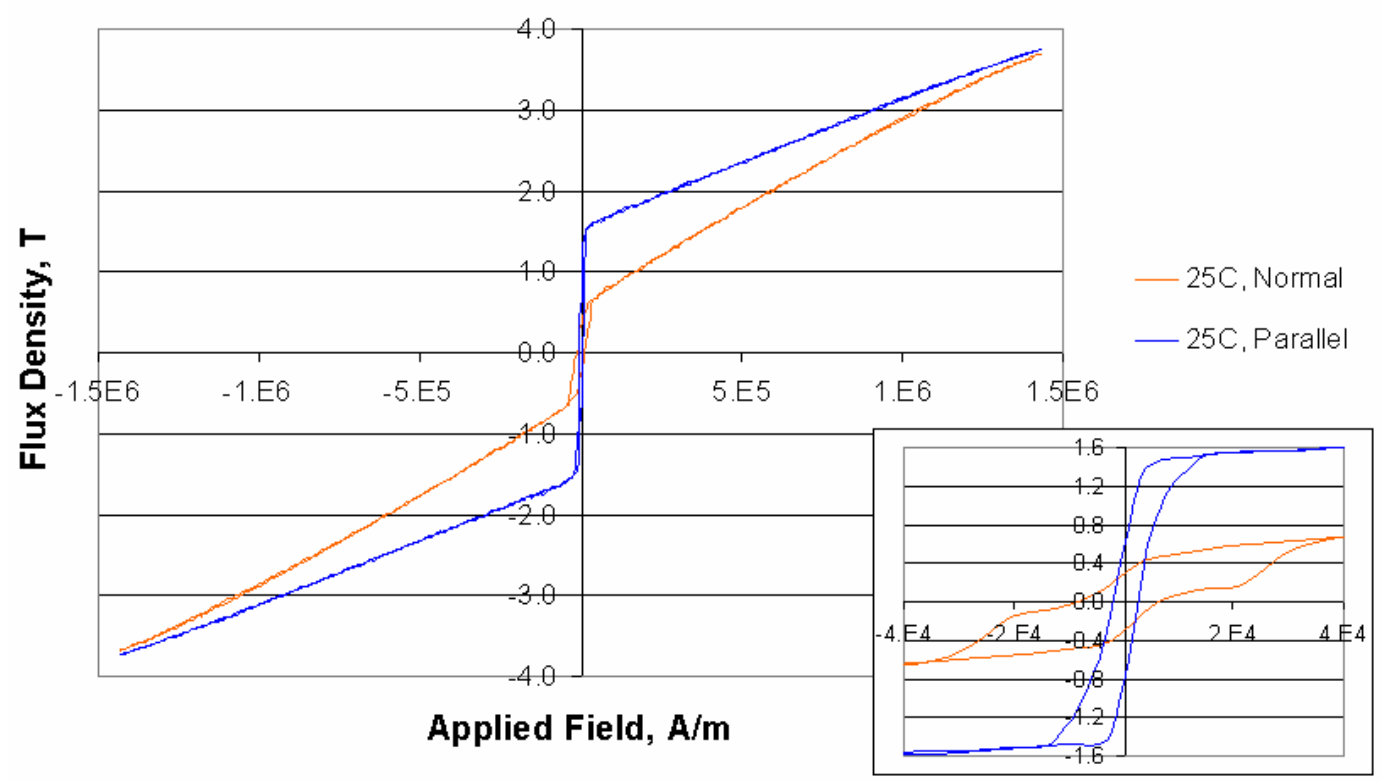

Figure B.7: Magnetic flux density plots for a RF sputtered cobalt film illustrating properties both parallel and normal to the substrate at room temperature. Deposition was done at $800 \mathrm{~W}$ with no applied magnetic field, resulting in a $0.20 \mu \mathrm{m}$ film. Both directions exhibited measurable hysteresis, taller than that of the nickel films due to cobalt's higher saturation level.

Cobalt Magnetization, Normal at 25C-700C and Parallel at 25C

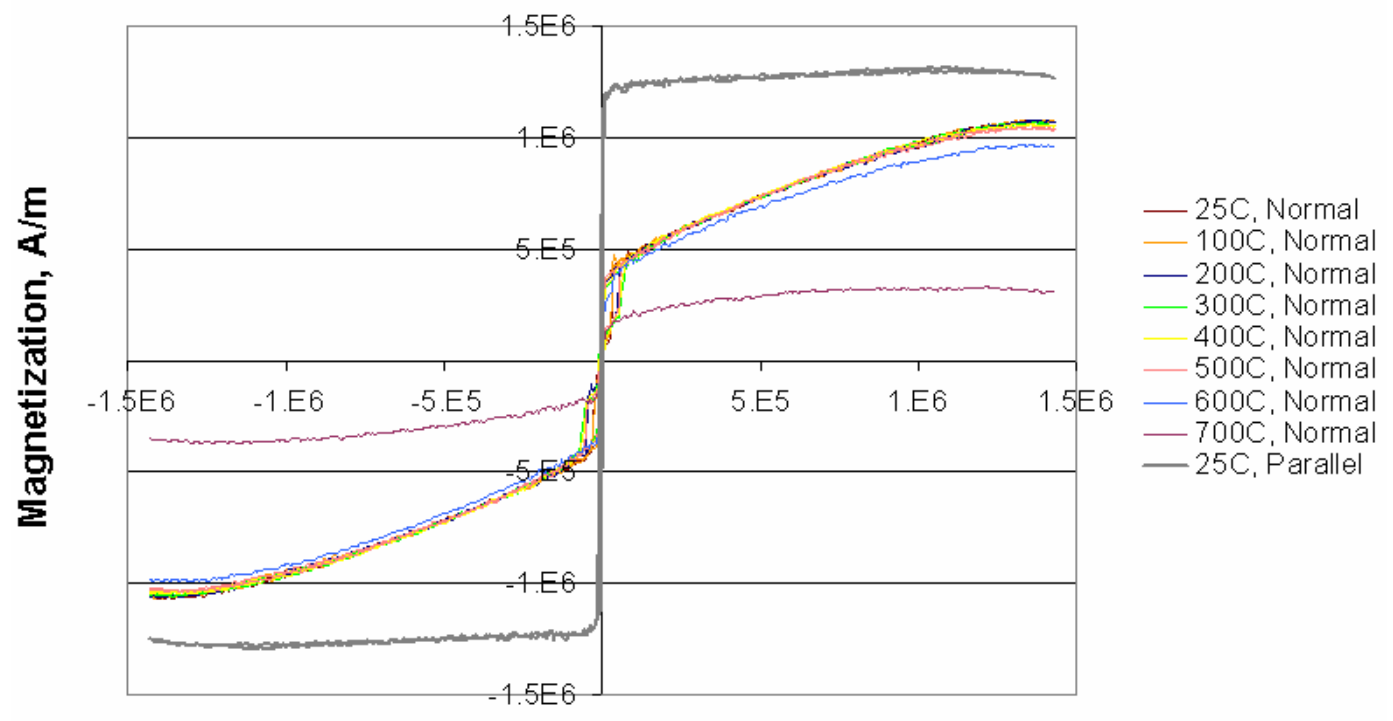

Applied Field, A/m

Figure B.8: Magnetization plots for the RF sputtered cobalt film showing the effect of elevated temperature on its magnetic behavior in the direction normal to the substrate relative to the $25^{\circ} \mathrm{C}$ data. The parallel curve at $25^{\circ} \mathrm{C}$ is also included for reference. Ferromagnetic properties showed minimal degradation up to $600^{\circ} \mathrm{C}$ but exhibited a sharp, unexpected decrease at $700^{\circ} \mathrm{C}$. The cobalt Curie temperature is $1127^{\circ} \mathrm{C}$ [Eisberg, et al., 1985 (p 500)]. 


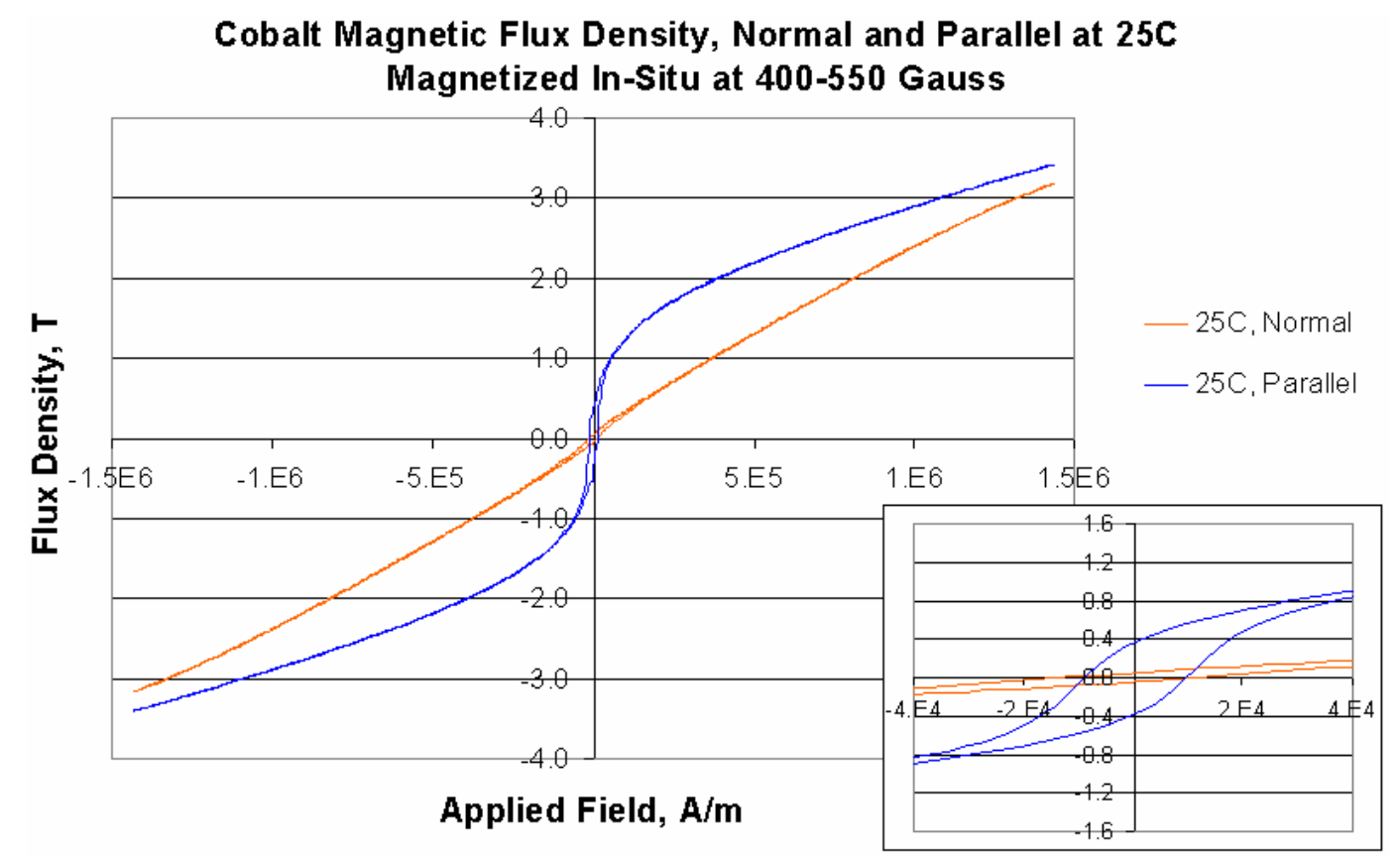

Figure B.9: Magnetic flux density plots for a RF sputtered cobalt film illustrating properties both parallel and normal to the substrate at room temperature. Deposition was done at $700 \mathrm{~W}$ with a $400-550$ Gauss applied magnetic field, resulting in a $2.4 \mu \mathrm{m}$ film. Both hysteresis loops decreased in height but increased in width relative to the nonmagnetized films. This was a result of the more rounded magnetization curves as shown below.

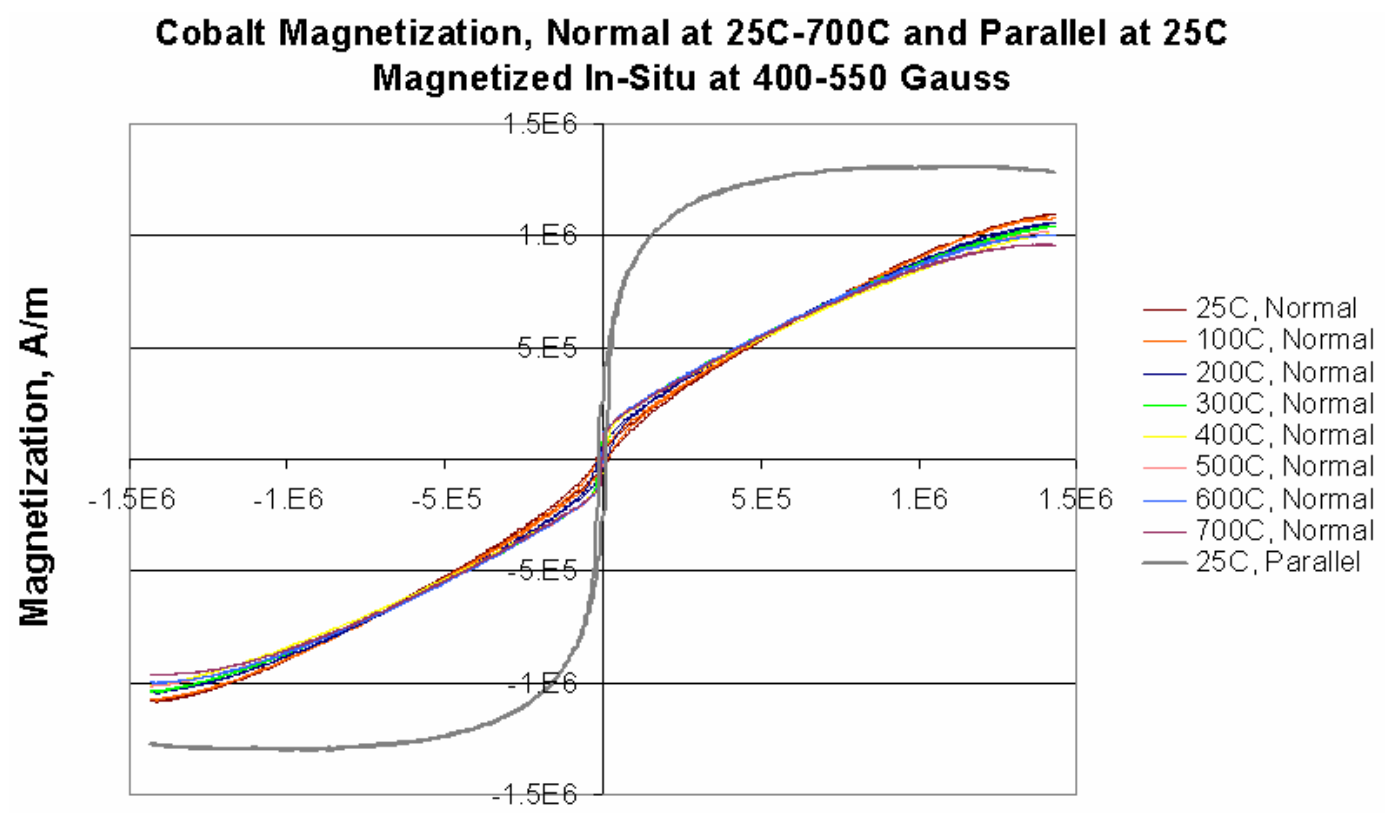

Applied Field, A/m

Figure B.10: Magnetization plots for the in-situ magnetized RF sputtered cobalt film showing the effect of elevated temperature on its magnetic behavior in the direction normal to the substrate relative to the $25^{\circ} \mathrm{C}$ data. The parallel curve at $25^{\circ} \mathrm{C}$ is also included for reference. The magnetic properties show minimal degradation up to $700^{\circ} \mathrm{C}$. 


\section{B.2: Nickel-Iron Alloys}

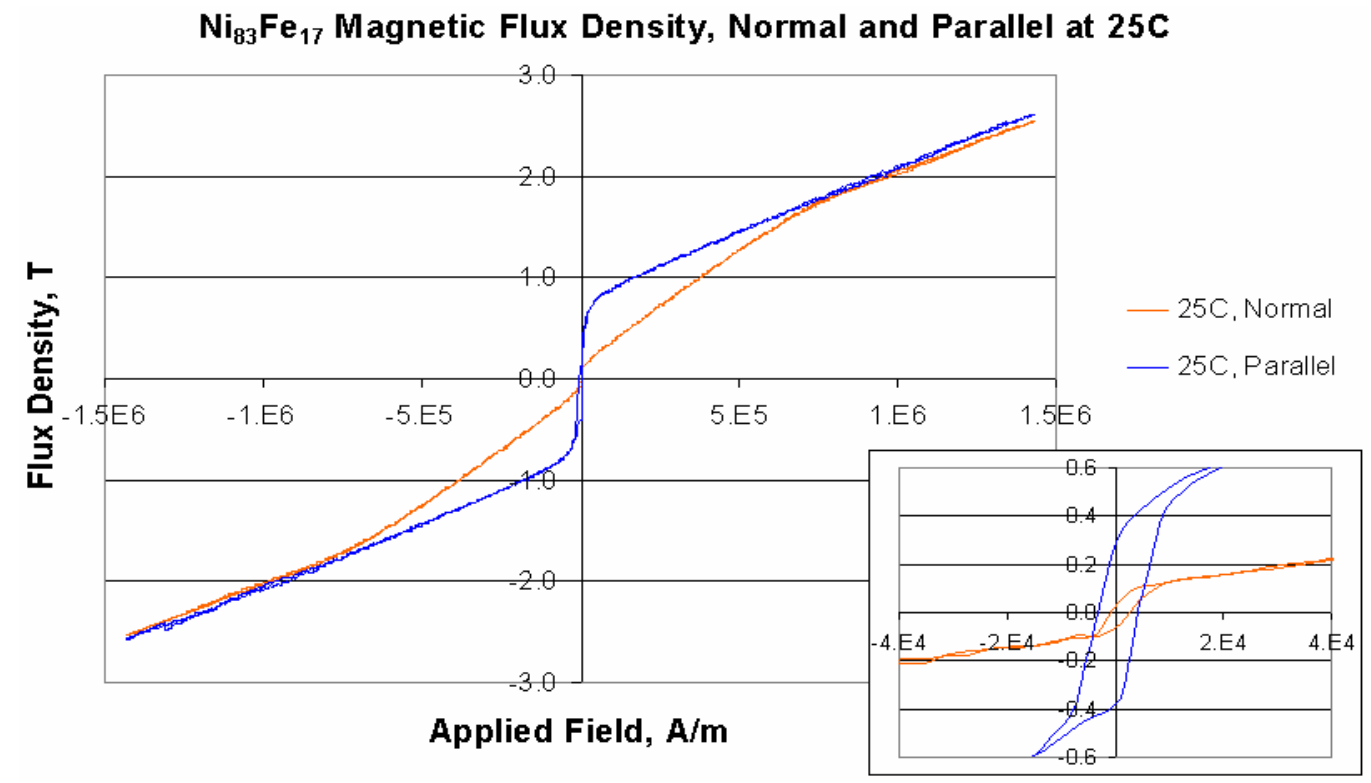

Figure B.11: Magnetic flux density plots for a $\mathrm{RF}$ sputtered $\mathrm{Ni}_{83} \mathrm{Fe}_{17}$ alloy illustrating properties both parallel and normal to the substrate at room temperature. Deposition was done at $800 \mathrm{~W}$ with no applied magnetic field, resulting in a $0.18 \mu \mathrm{m}$ film. Hysteresis resembled that of in-situ magnetized nickel and likely would have resembled the nonmagnetized nickel had that data been less noisy.

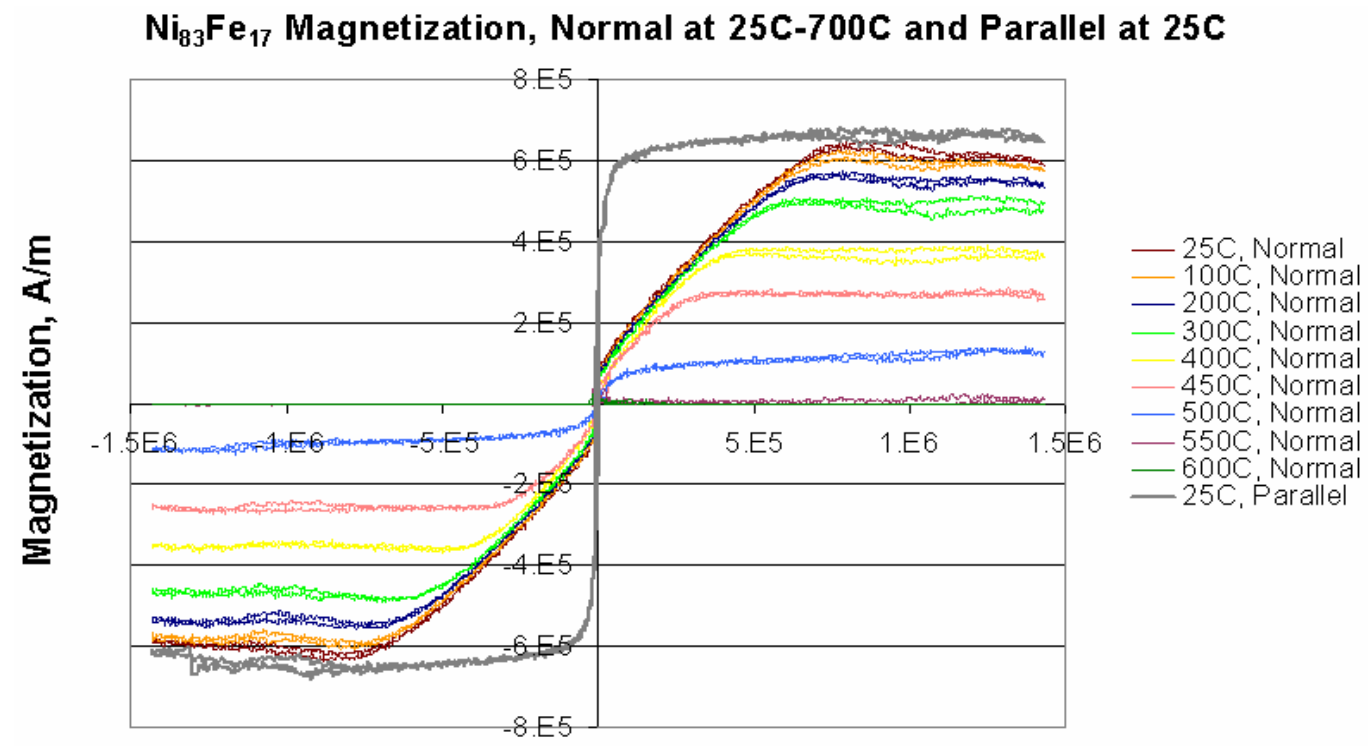

Applied Field, A/m

Figure B.12: Magnetization plots for the RF sputtered $\mathrm{Ni}_{83} \mathrm{Fe}_{17}$ alloy showing the effect of elevated temperature on its magnetic behavior in the direction normal to the substrate relative to that at $25^{\circ} \mathrm{C}$. The parallel curve at $25^{\circ} \mathrm{C}$ is also included for reference. The nickel and iron Curie temperatures are $358^{\circ} \mathrm{C}$ and $761^{\circ} \mathrm{C}$, respectively [Eisberg, et al., 1985 (p 500)], and thus the iron content improved the high-temperature stability of the film's properties over that of pure nickel. 


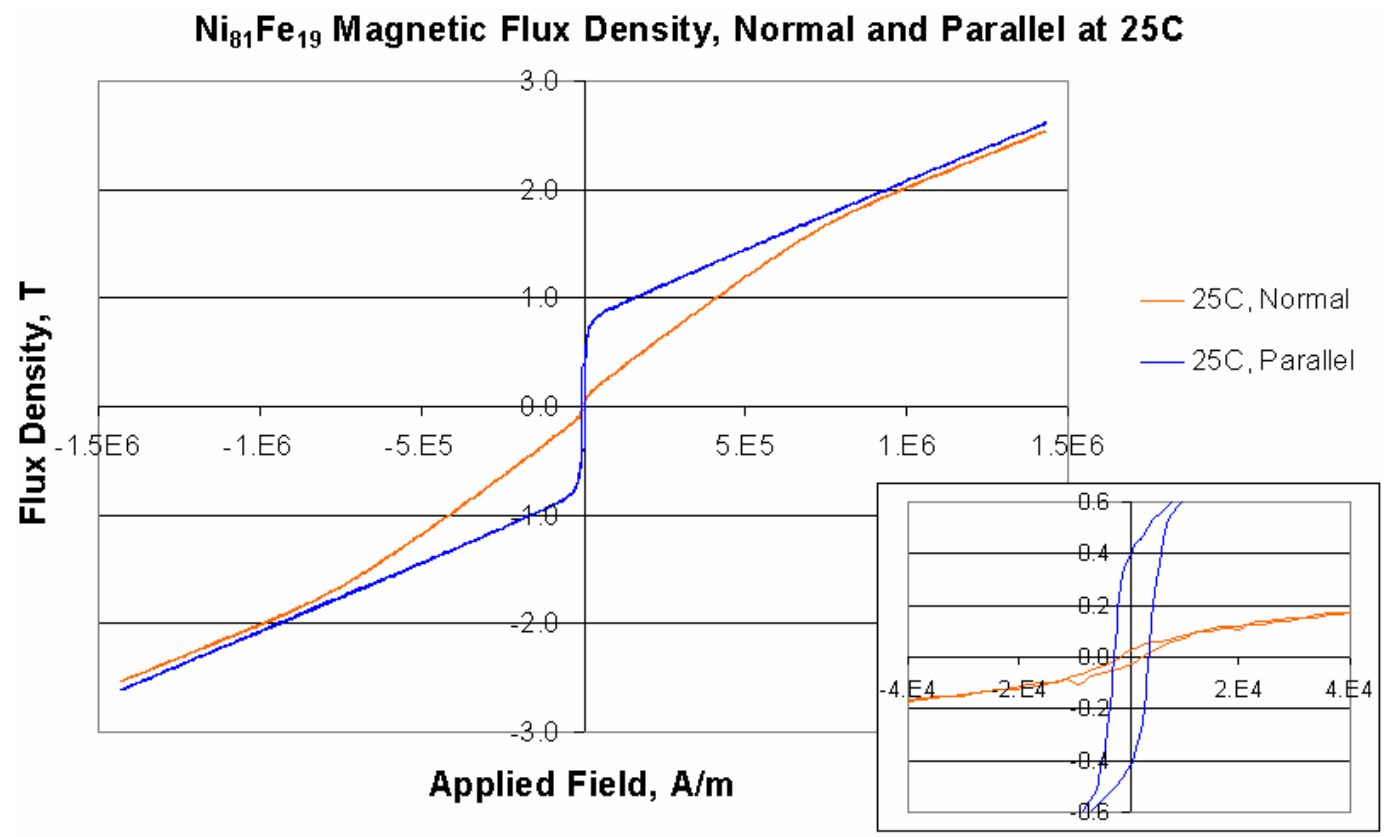

Figure B.13: Magnetic flux density plots for a $\mathrm{RF}$ sputtered $\mathrm{Ni}_{81} \mathrm{Fe}_{19}$ alloy illustrating properties both parallel and normal to the substrate at room temperature. Deposition was done at $800 \mathrm{~W}$ with no applied magnetic field, resulting in a $0.28 \mu \mathrm{m}$ film. Hysteresis and other properties were very similar to that of $\mathrm{Ni}_{83} \mathrm{Fe}_{17}$.

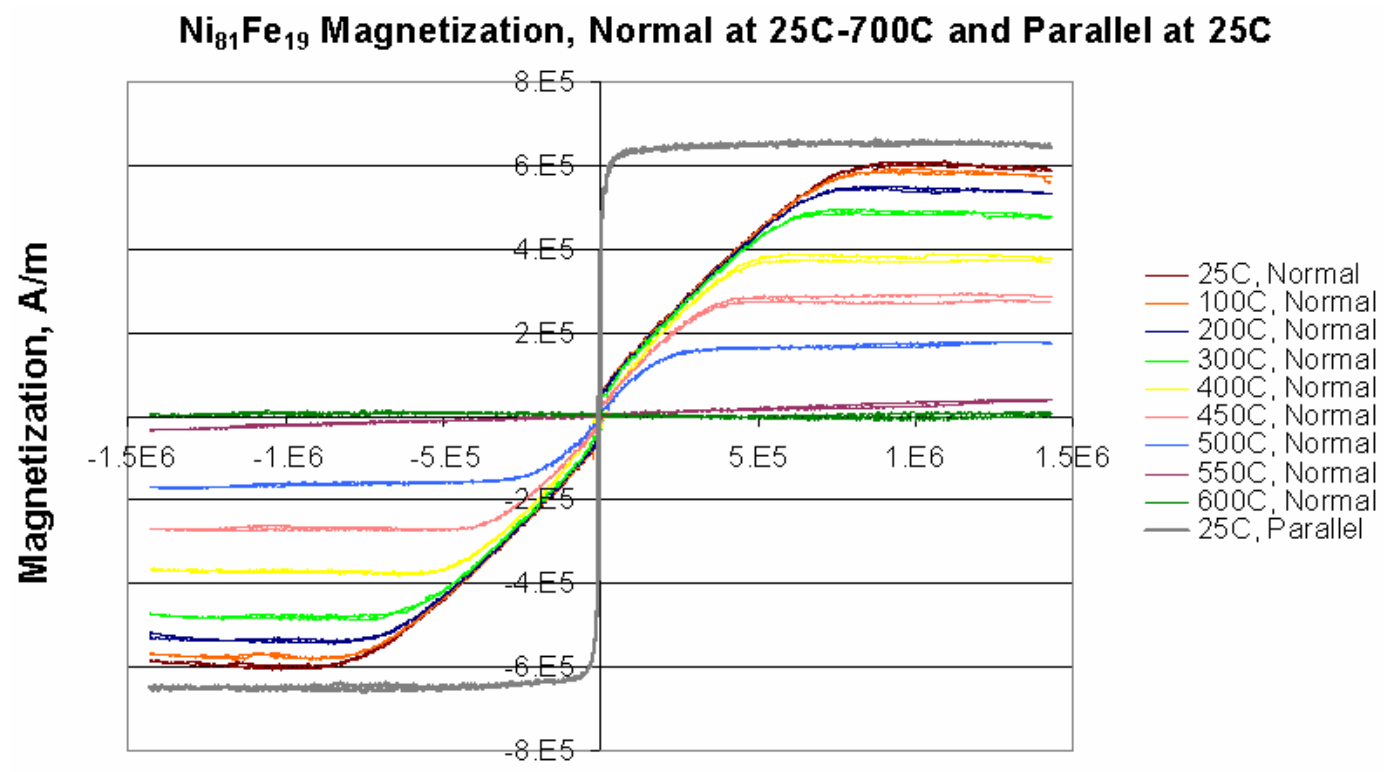

Applied Field, A/m

Figure B.14: Magnetization plots for the RF sputtered $\mathrm{Ni}_{81} \mathrm{Fe}_{19}$ alloy showing the effect of elevated temperature on its magnetic behavior in the direction normal to the substrate relative to that at $25^{\circ} \mathrm{C}$. The parallel curve at $25^{\circ} \mathrm{C}$ is also included for reference. Hightemperature ferromagnetic properties were similar to that for the $\mathrm{Ni}_{83} \mathrm{Fe}_{17}$ film. 


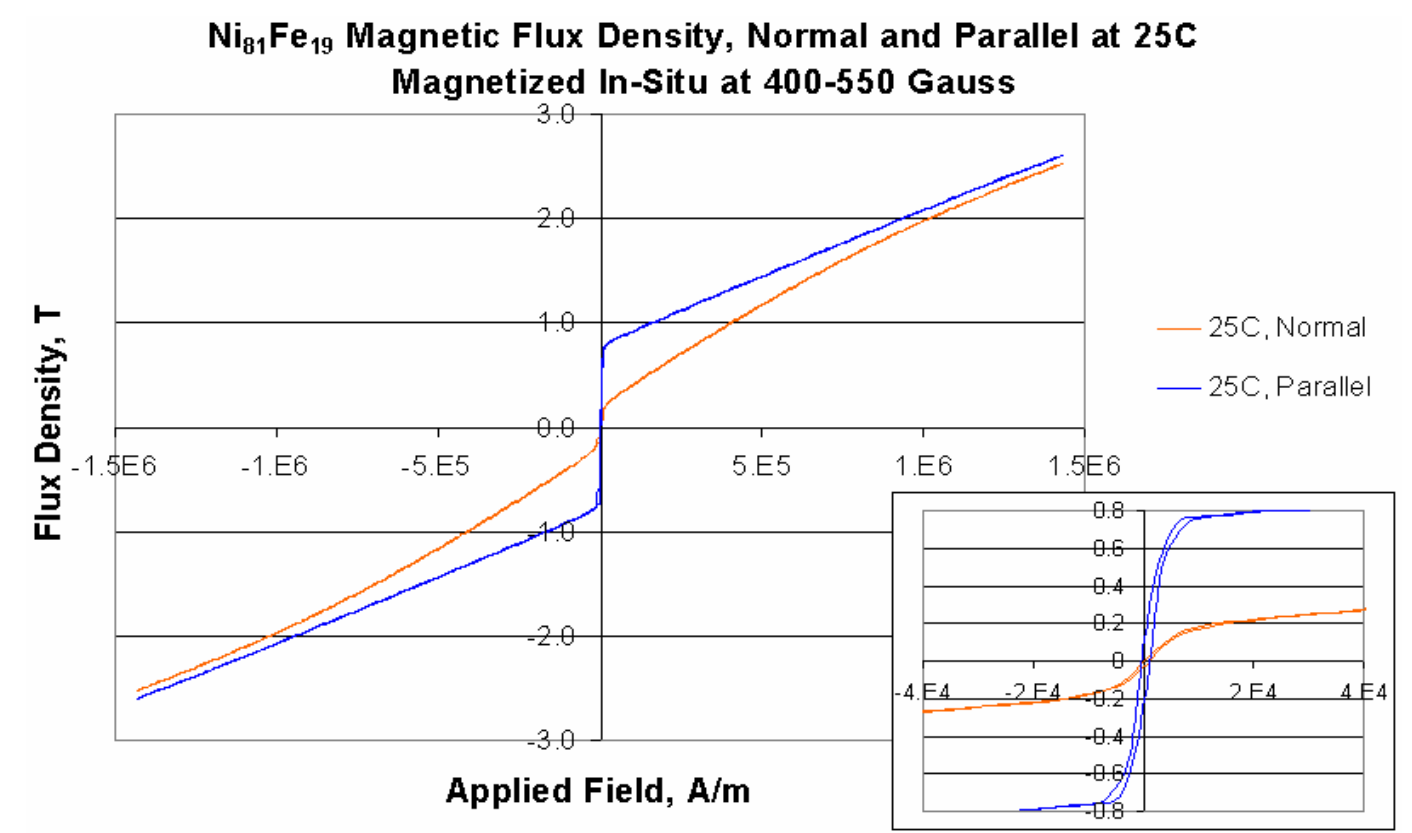

Figure B.15: Magnetic flux density plots for a $\mathrm{RF}$ sputtered $\mathrm{Ni}_{81} \mathrm{Fe}_{19}$ alloy illustrating properties both parallel and normal to the substrate at room temperature. Deposition was done at $700 \mathrm{~W}$ with a $400-550$ Gauss applied magnetic field, resulting in a $2.1 \mu \mathrm{m}$ film. Hysteresis loops appeared to have narrowed in width as compared to the non-magnetized $\mathrm{Ni}_{81} \mathrm{Fe}_{19}$ film, an unexpected result.

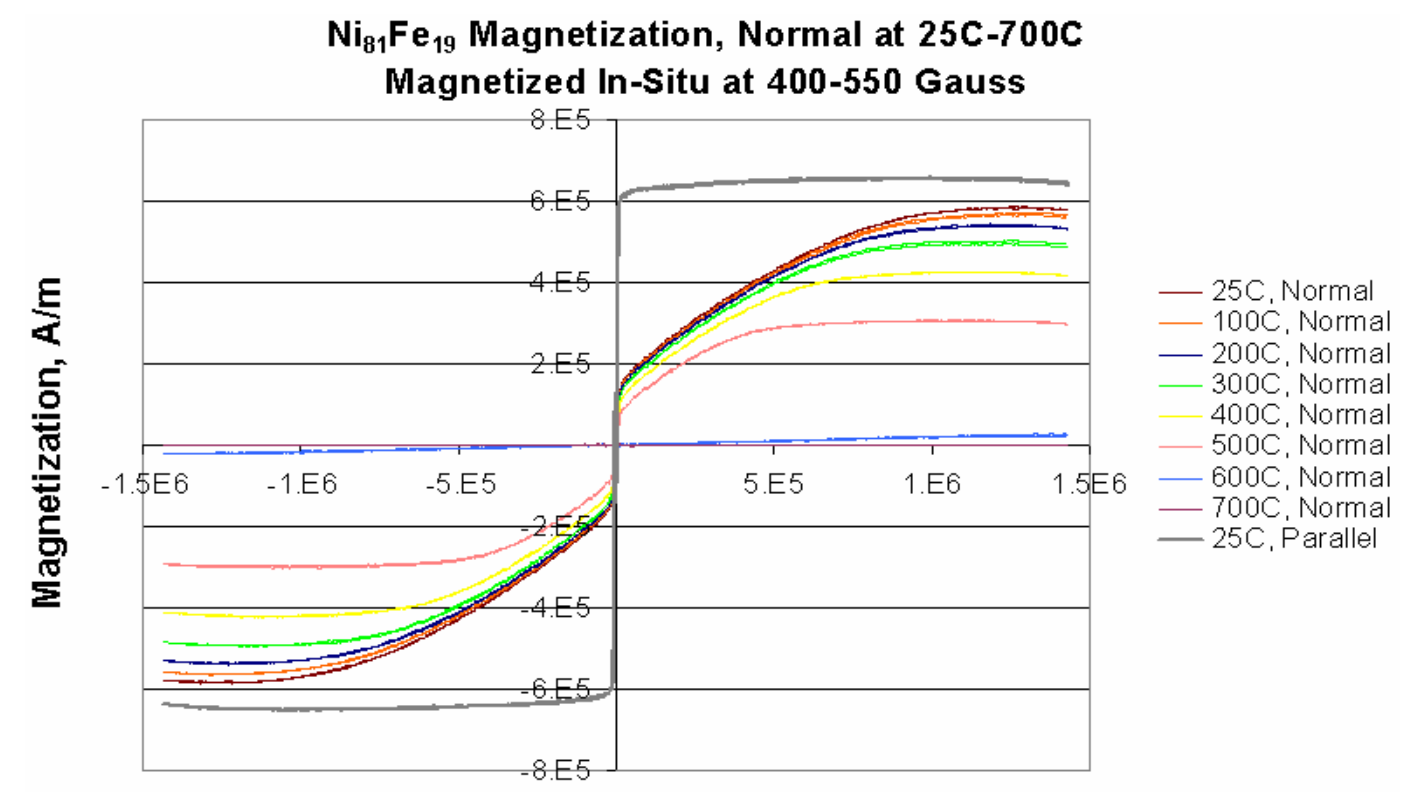

\section{Applied Field, A/m}

Figure B.16: Magnetization plots for the in-situ magnetized RF sputtered $\mathrm{Ni}_{81} \mathrm{Fe}_{19}$ alloy showing the effect of elevated temperature on its magnetic behavior in the direction normal to the substrate relative to that at $25^{\circ} \mathrm{C}$. The parallel curve at $25^{\circ} \mathrm{C}$ is also included for reference. High-temperature performance was similar to that of the non-magnetized $\mathrm{Ni}_{81} \mathrm{Fe}_{19}$ and $\mathrm{Ni}_{83} \mathrm{Fe}_{17}$ alloys, though once again the field caused the curves to become somewhat rounded. 
$\mathrm{Ni}_{45} \mathrm{Fe}_{55}$ Magnetic Flux Density, Normal and Parallel at 25C

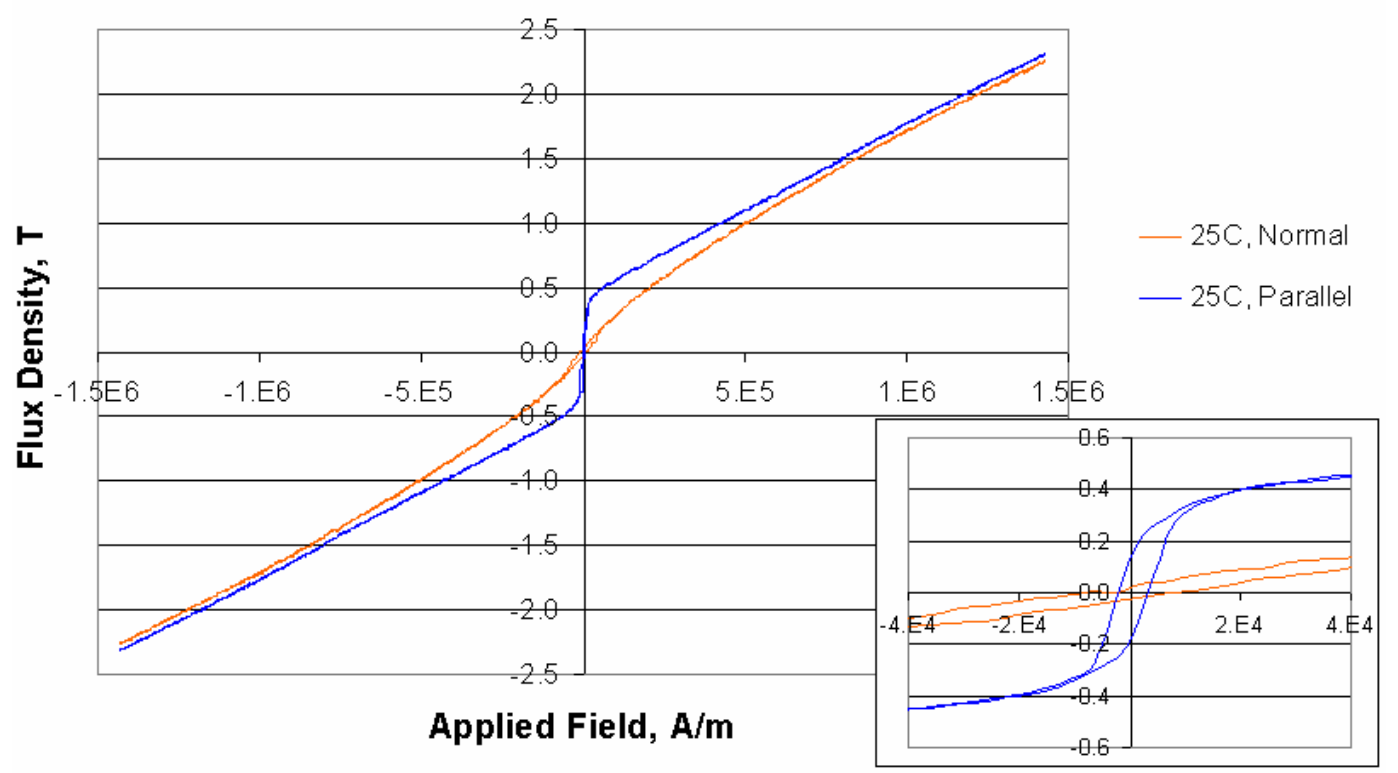

Figure B.17: Magnetic flux density plots for a $\mathrm{RF}$ sputtered $\mathrm{Ni}_{45} \mathrm{Fe}_{55}$ alloy illustrating properties both parallel and normal to the substrate at room temperature. Deposition was done at $1000 \mathrm{~W}$ with no applied magnetic field, resulting in a $0.67 \mu \mathrm{m}$ film. Saturation levels were reduced in magnitude from those of the alloys with lower iron content, an unexpected result, though normal hysteresis showed increased width.

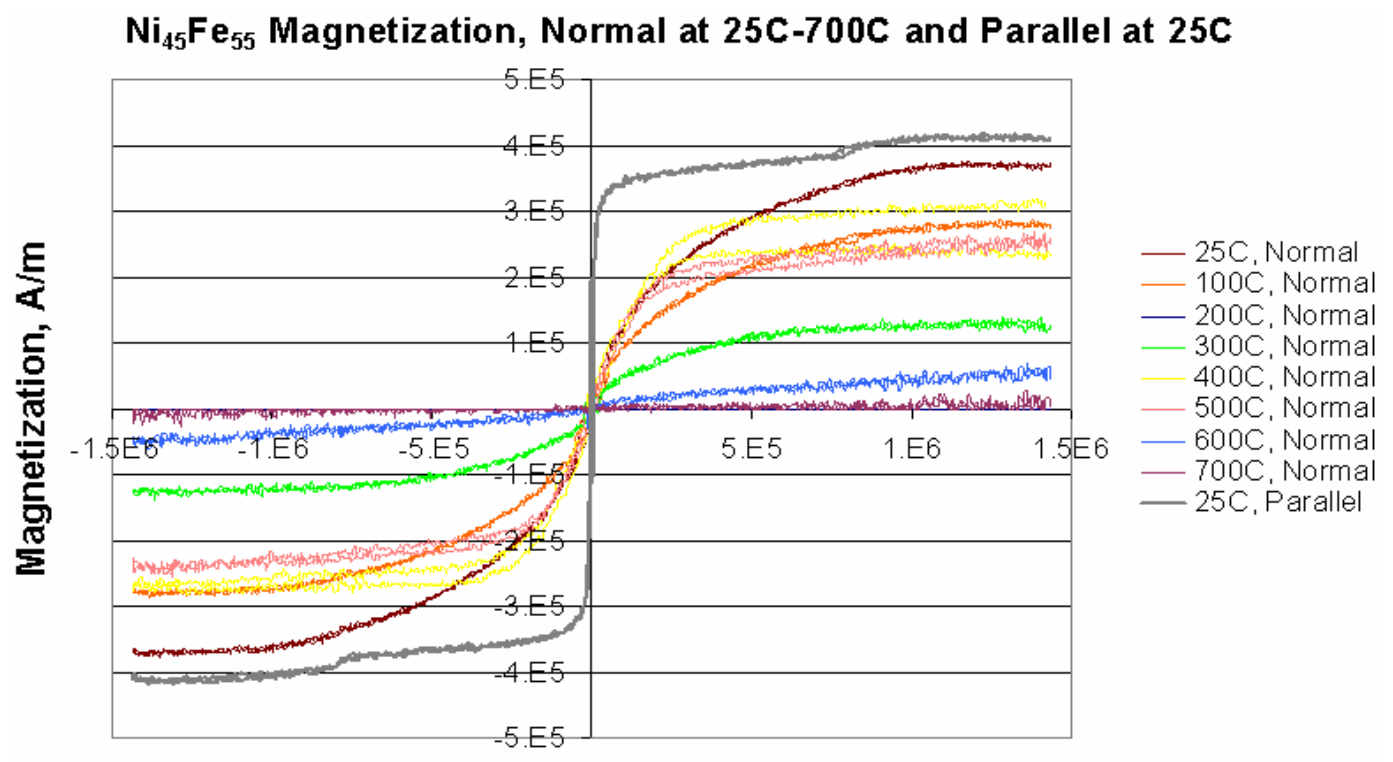

Applied Field, A/m

Figure B.18: Magnetization plots for the RF sputtered $\mathrm{Ni}_{45} \mathrm{Fe}_{55}$ alloy showing the effect of elevated temperature on its magnetic behavior in the direction normal to the substrate relative to that at $25^{\circ} \mathrm{C}$. The parallel curve at $25^{\circ} \mathrm{C}$ is also included for reference. The data indicates that the magnetization fell rapidly with increasing temperature until between $300-400^{\circ} \mathrm{C}$, at which point it recovered to nearly the $25^{\circ} \mathrm{C}$ level. This suggests that a phase transformation may have taken place. Similar to the other two iron-nickel alloys, ferromagnetic quality was negligible at $600^{\circ} \mathrm{C}$ and above. 


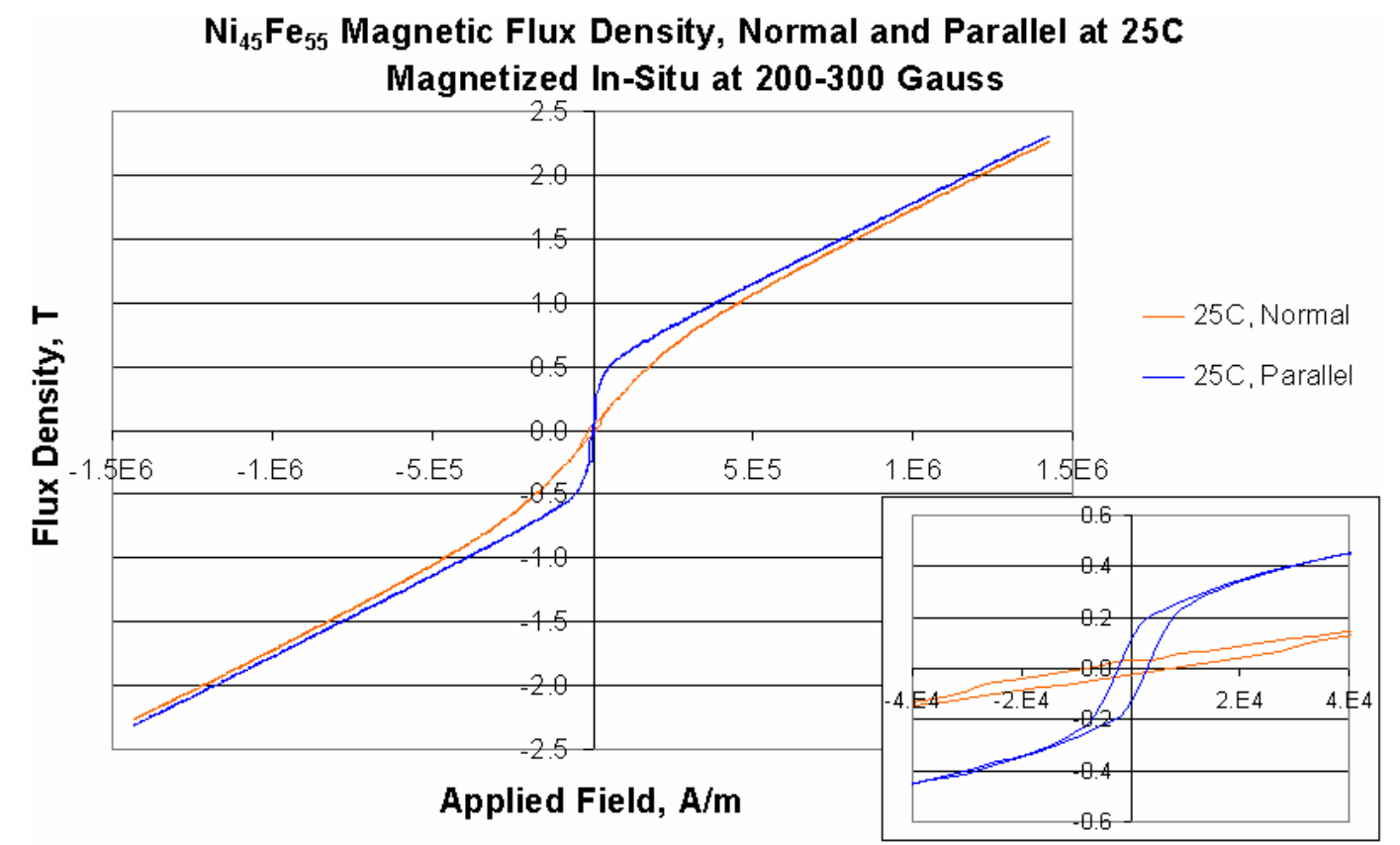

Figure B.19: Magnetic flux density plots for a $\mathrm{RF}$ sputtered $\mathrm{Ni}_{45} \mathrm{Fe}_{55}$ alloy illustrating properties both parallel and normal to the substrate at room temperature. Deposition was done at 500W with a 200-300 Gauss applied magnetic field, resulting in a $1.6 \mu \mathrm{m}$ film. Hysteresis loops were very similar to those of the non-magnetized film.

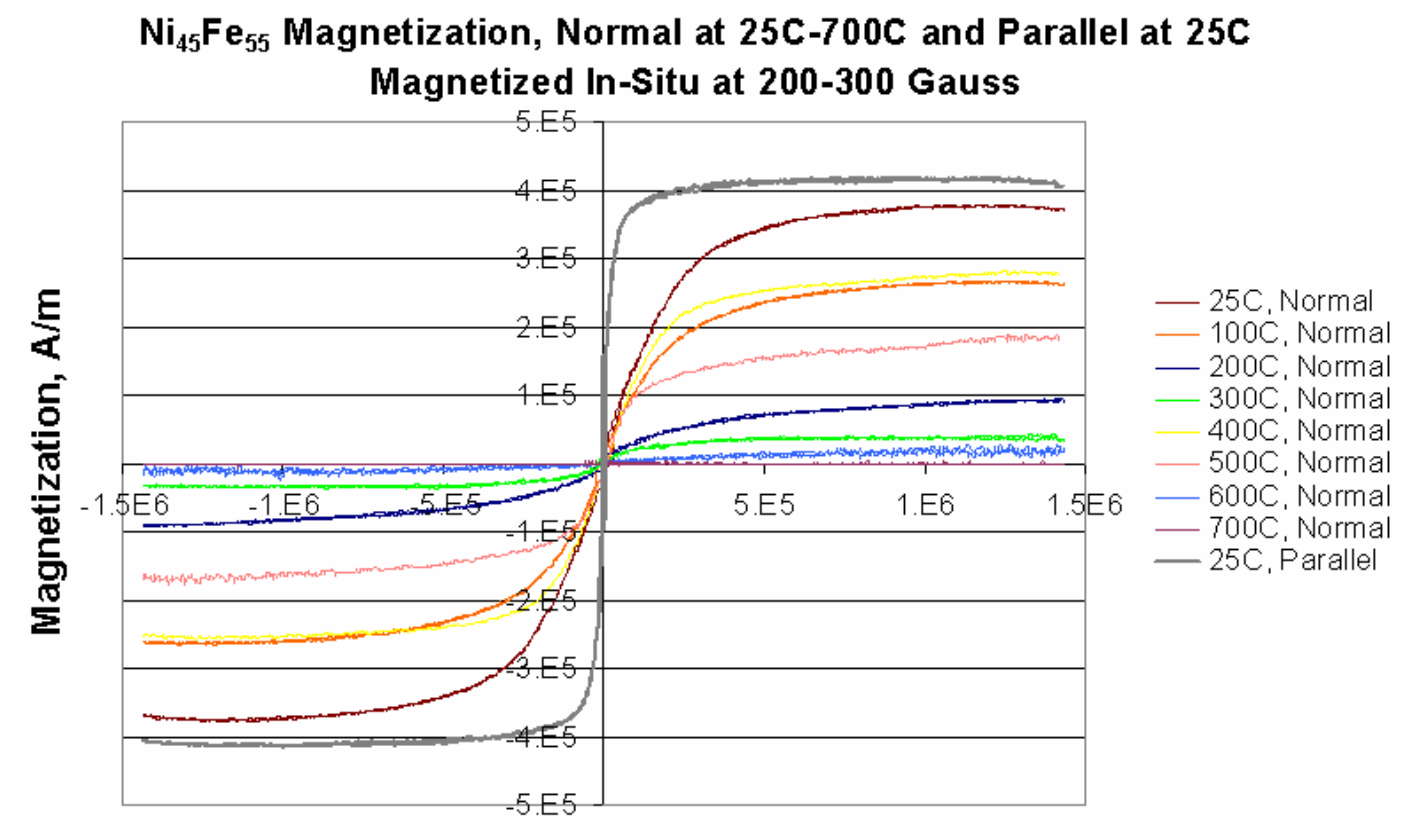

\section{Applied Field, A/m}

Figure B.20: Magnetization plots for the in-situ magnetized RF sputtered $\mathrm{Ni}_{45} \mathrm{Fe}_{55}$ alloy showing the effect of elevated temperature on its magnetic behavior in the direction normal to the substrate relative to that at $25^{\circ} \mathrm{C}$. The parallel curve at $25^{\circ} \mathrm{C}$ is also included for reference. The data was very similar to that of the non-magnetized film, again suggesting a phase transformation between $300-400^{\circ} \mathrm{C}$. 


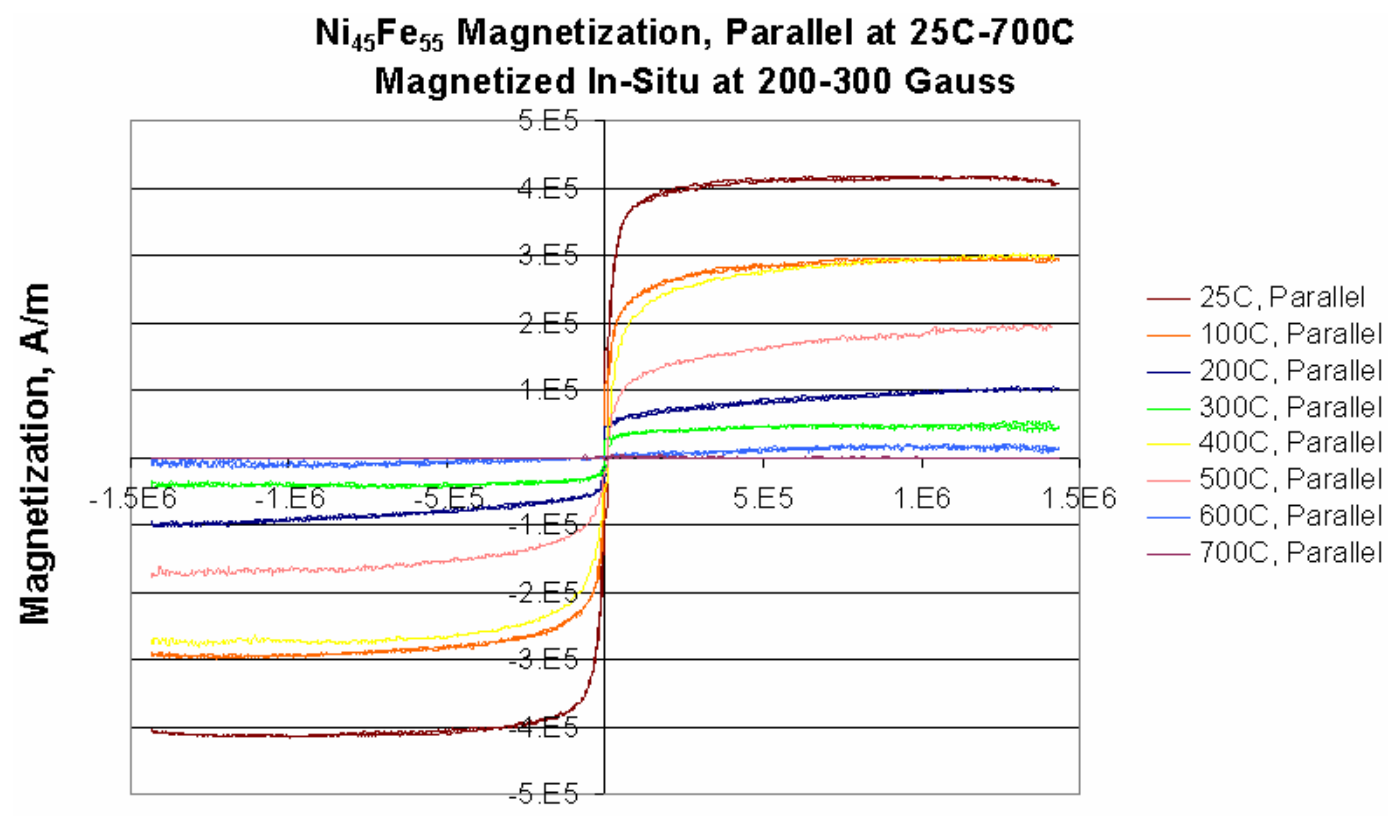

Applied Field, A/m

Figure B.21: Magnetization plots for the in-situ magnetized RF sputtered $\mathrm{Ni}_{45} \mathrm{Fe}_{55}$ alloy showing the effect of elevated temperature on its magnetic behavior in the direction parallel to the substrate relative to that at $25^{\circ} \mathrm{C}$. The high-temperature quality in the parallel direction agreed with that in the normal direction, further supporting the assumption of a phase transformation in the $300-400^{\circ} \mathrm{C}$ range. 


\section{B.3: Iron-Cobalt Alloys}

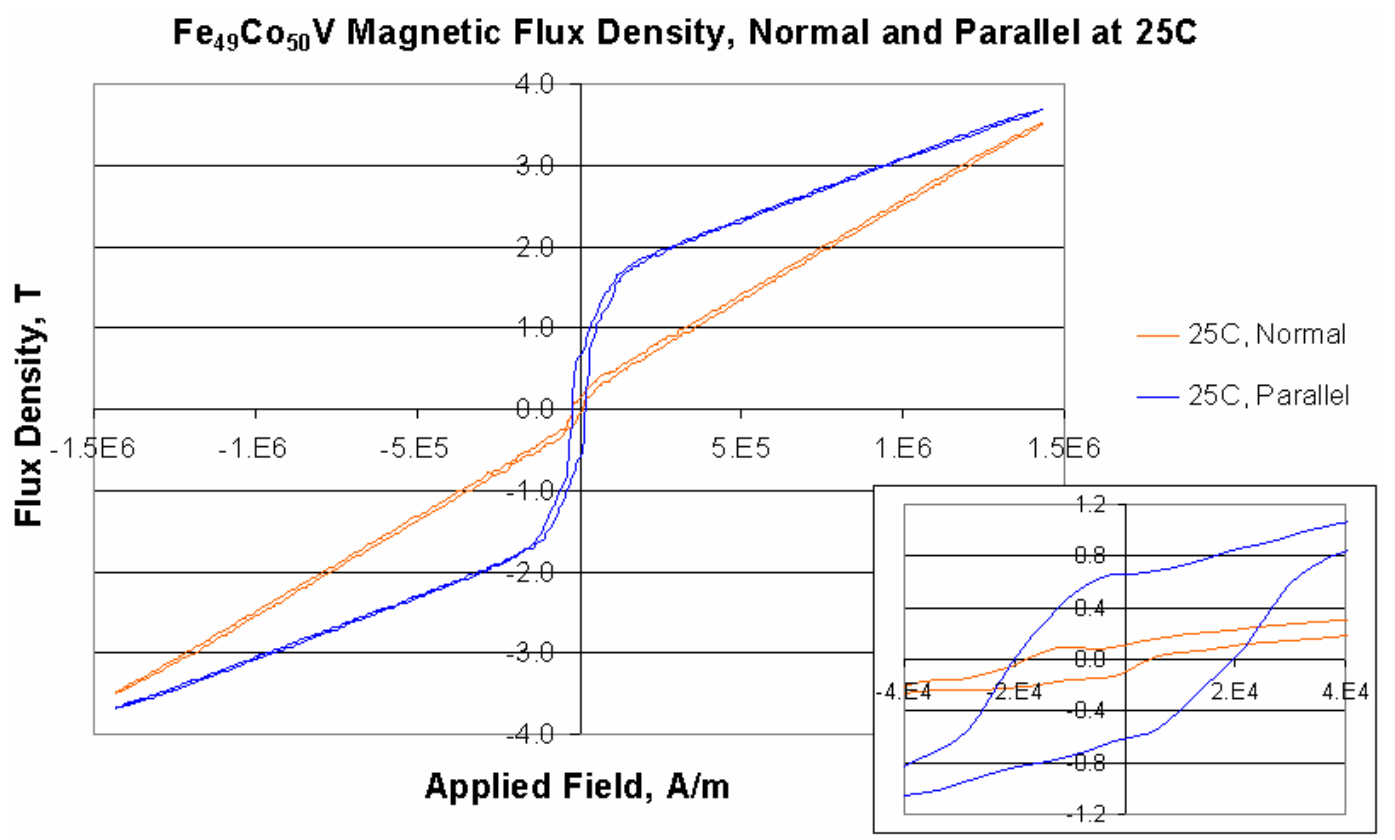

Figure B.22: Magnetic flux density plots for a RF sputtered $\mathrm{Fe}_{49} \mathrm{Co}_{50} \mathrm{~V}$ alloy illustrating properties both parallel and normal to the substrate at room temperature. Deposition was done at $850 \mathrm{~W}$ with no applied magnetic field, resulting in a $0.20 \mu \mathrm{m}$ film. Both hysteresis loops were relatively wide and exhibited somewhat of a rounded shape.

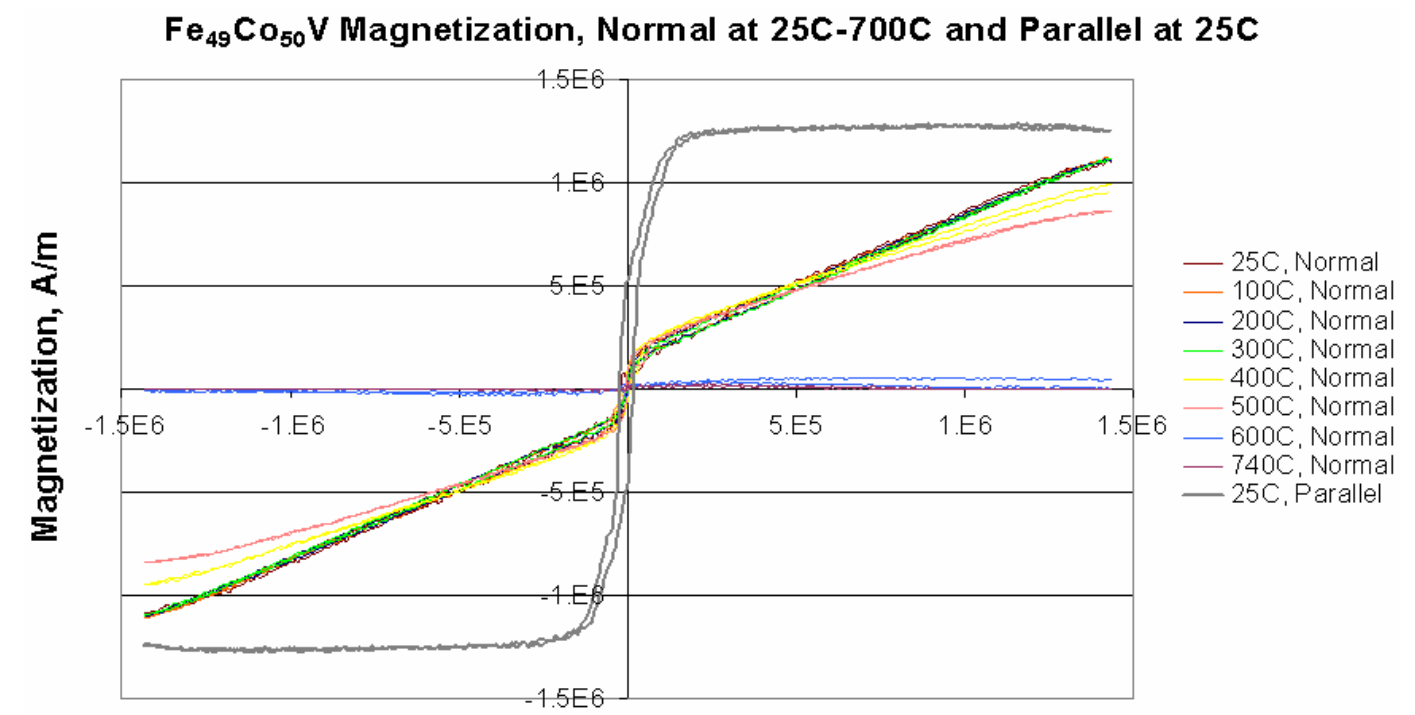

Applied Field, A/m

Figure B.23: Magnetization plots for the RF sputtered $\mathrm{Fe}_{49} \mathrm{Co}_{50} \mathrm{~V}$ alloy showing the effect of elevated temperature on its magnetic behavior in the direction normal to the substrate relative to that at $25^{\circ} \mathrm{C}$. The parallel curve at $25^{\circ} \mathrm{C}$ is also included for reference. The alloy remained ferromagnetic only to approximately $500^{\circ} \mathrm{C}$, considerably lower than expected, though its magnetized counterparts performed better. The iron and cobalt Curie temperatures are $761^{\circ} \mathrm{C}$ and $1127^{\circ} \mathrm{C}$, respectively [Eisberg, et al., 1985 (p 500)]. 


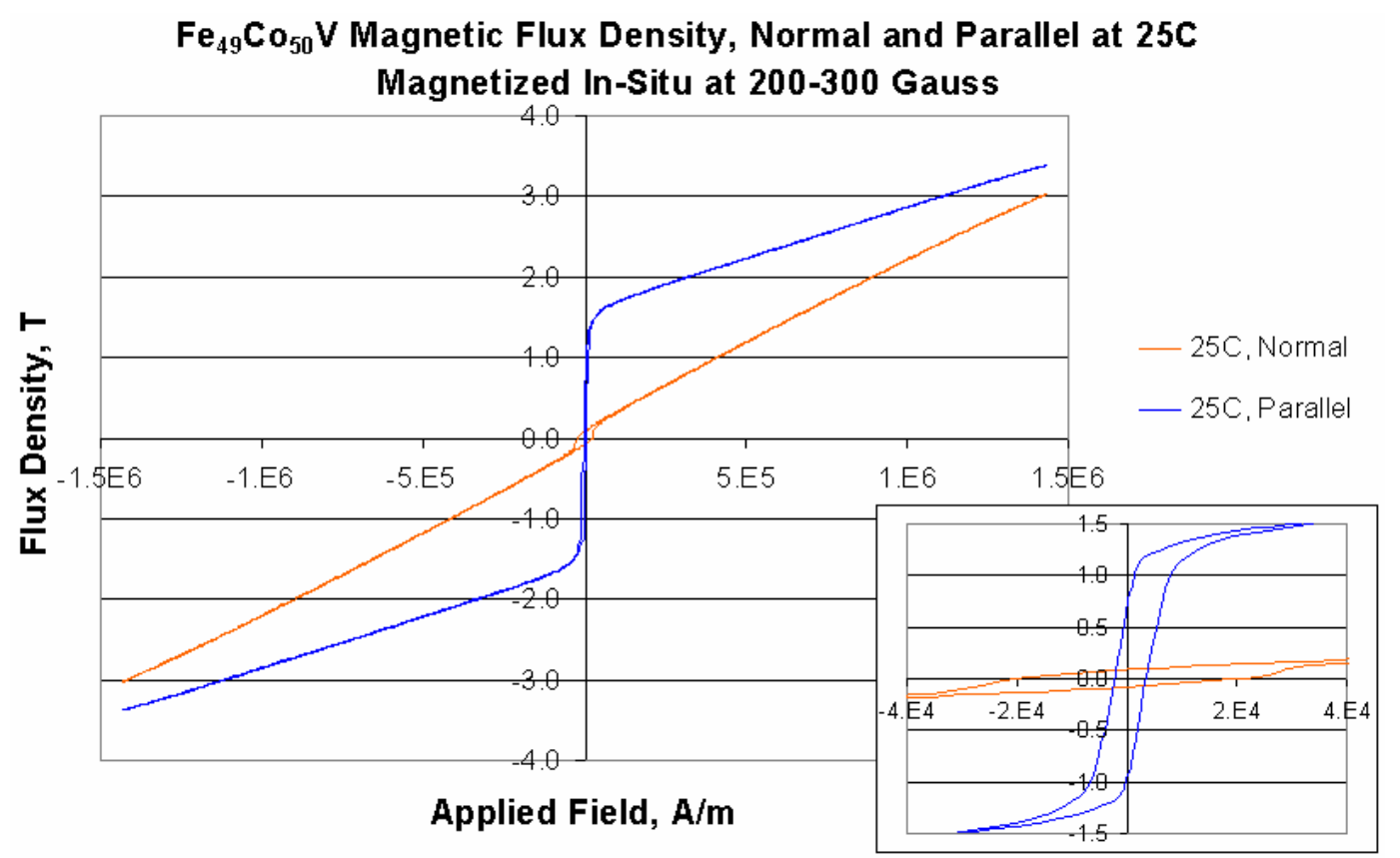

Figure B.24: Magnetic flux density plots for a $\mathrm{RF}$ sputtered $\mathrm{Fe}_{49} \mathrm{Co}_{50} \mathrm{~V}$ alloy illustrating properties both parallel and normal to the substrate at room temperature. Deposition was done at $700 \mathrm{~W}$ with a $200-300$ Gauss applied magnetic field, resulting in a $1.7 \mu \mathrm{m}$ film. The parallel hysteresis loop reflected increased permeability with respect to the unmagnetized film, whereas the normal hysteresis loop showed less significant changes.

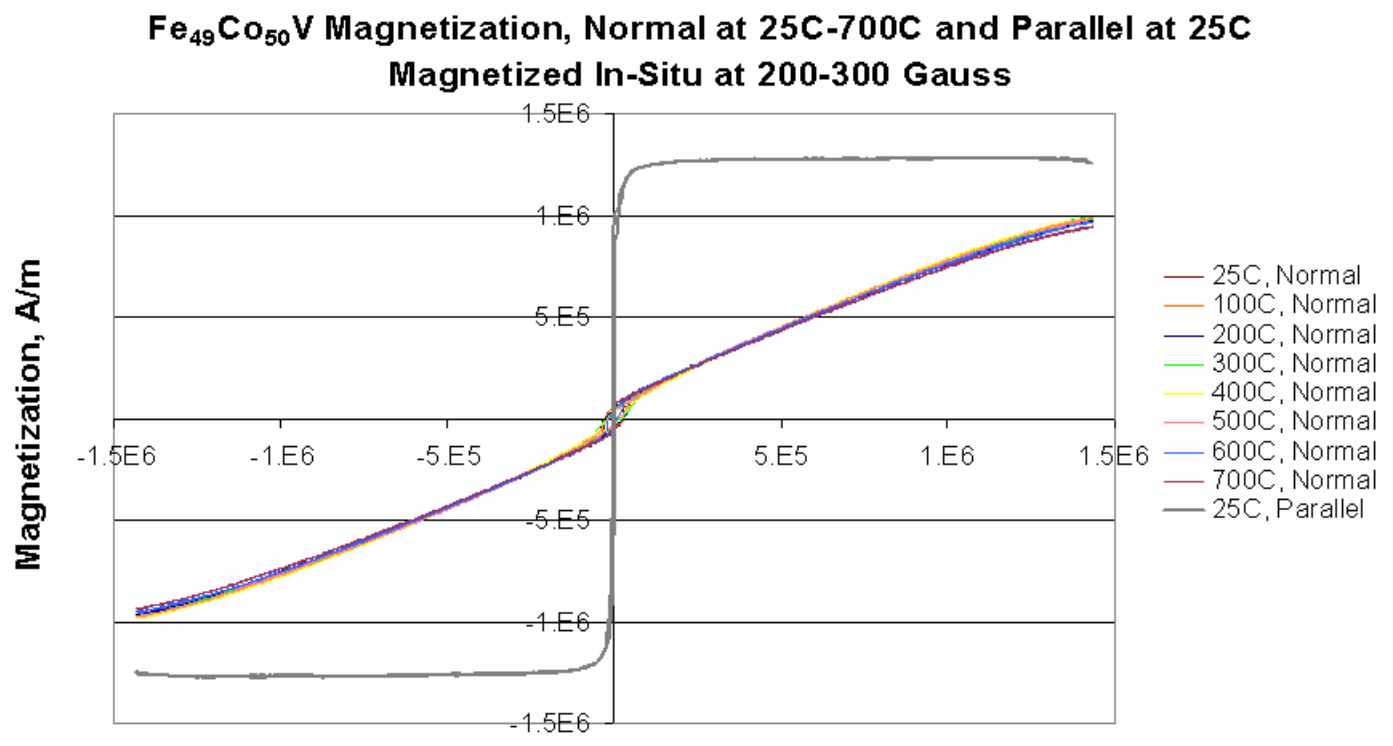

\section{Applied Field, A/m}

Figure B.25: Magnetization plots for the magnetized RF sputtered $\mathrm{Fe}_{49} \mathrm{Co}_{50} \mathrm{~V}$ alloy showing the effect of elevated temperature on its magnetic behavior in the direction normal to the substrate relative to that at $25^{\circ} \mathrm{C}$. The parallel curve at $25^{\circ} \mathrm{C}$ is also included for reference. The alloy remained ferromagnetic over the full $700^{\circ} \mathrm{C}$ range, showing almost no degradation in quality. 


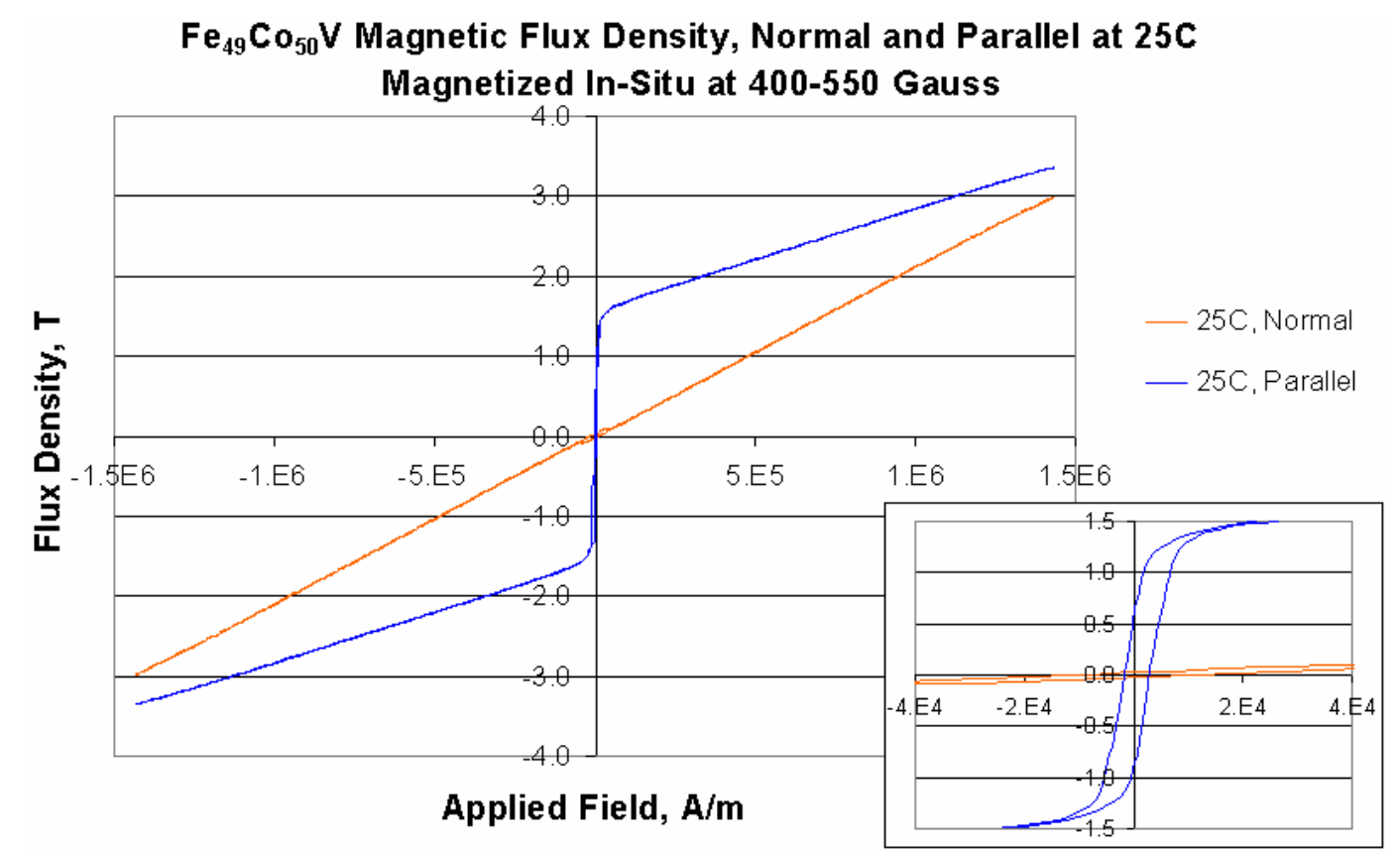

Figure B.26: Magnetic flux density plots for a $\mathrm{RF}$ sputtered $\mathrm{Fe}_{49} \mathrm{Co}_{50} \mathrm{~V}$ alloy illustrating properties both parallel and normal to the substrate at room temperature. Deposition was done at $700 \mathrm{~W}$ with a $400-550$ Gauss applied magnetic field, resulting in a $3.0 \mu \mathrm{m}$ film. Hysteresis is similar to that of the same alloy deposited under a 200-300 Gauss field.

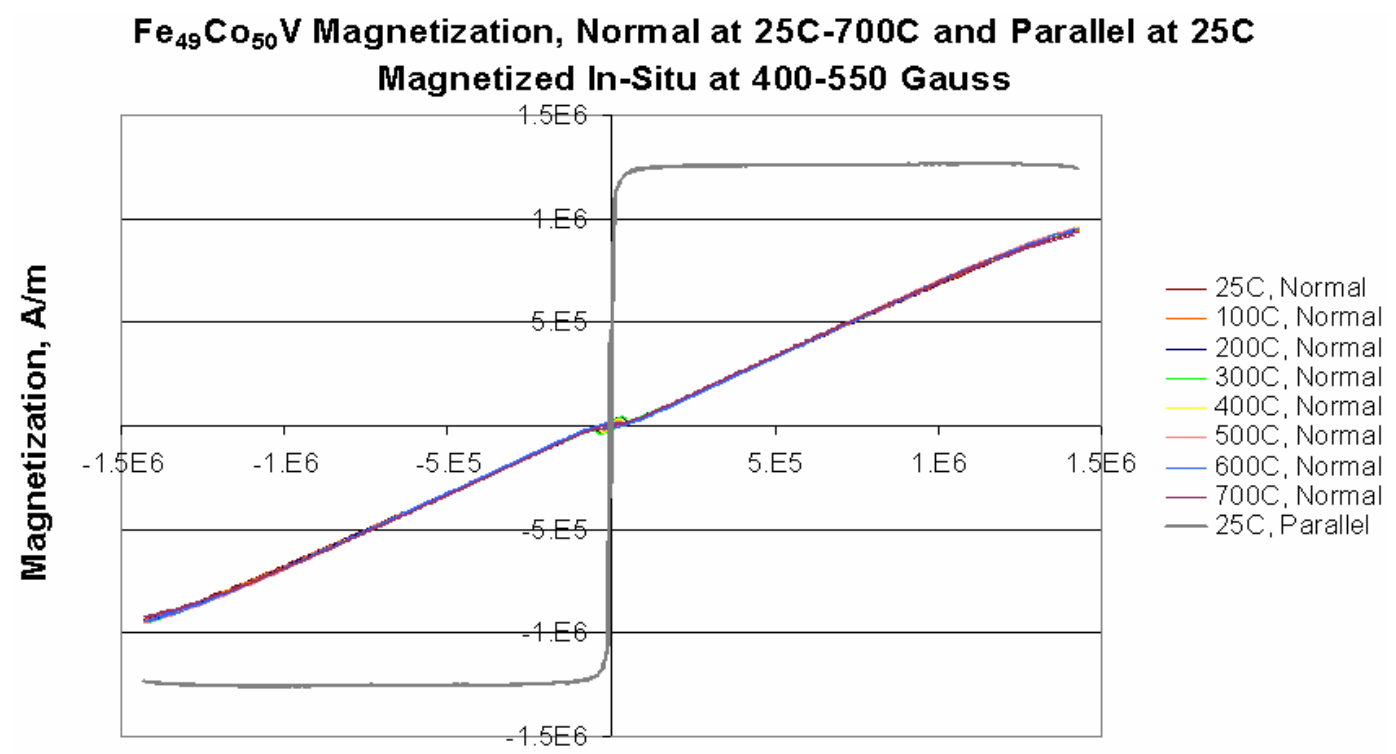

Applied Field, A/m

Figure B.27: Magnetization plots for the magnetized RF sputtered $\mathrm{Fe}_{49} \mathrm{Co}_{50} \mathrm{~V}$ alloy showing the effect of elevated temperature on its magnetic behavior in the direction normal to the substrate relative to that at $25^{\circ} \mathrm{C}$. The parallel curve at $25^{\circ} \mathrm{C}$ is also included for reference. Once again, the film remained fully ferromagnetic over the entire $700^{\circ} \mathrm{C}$ range. The data suggests that the effect of doubling the applied field during deposition is negligible. 


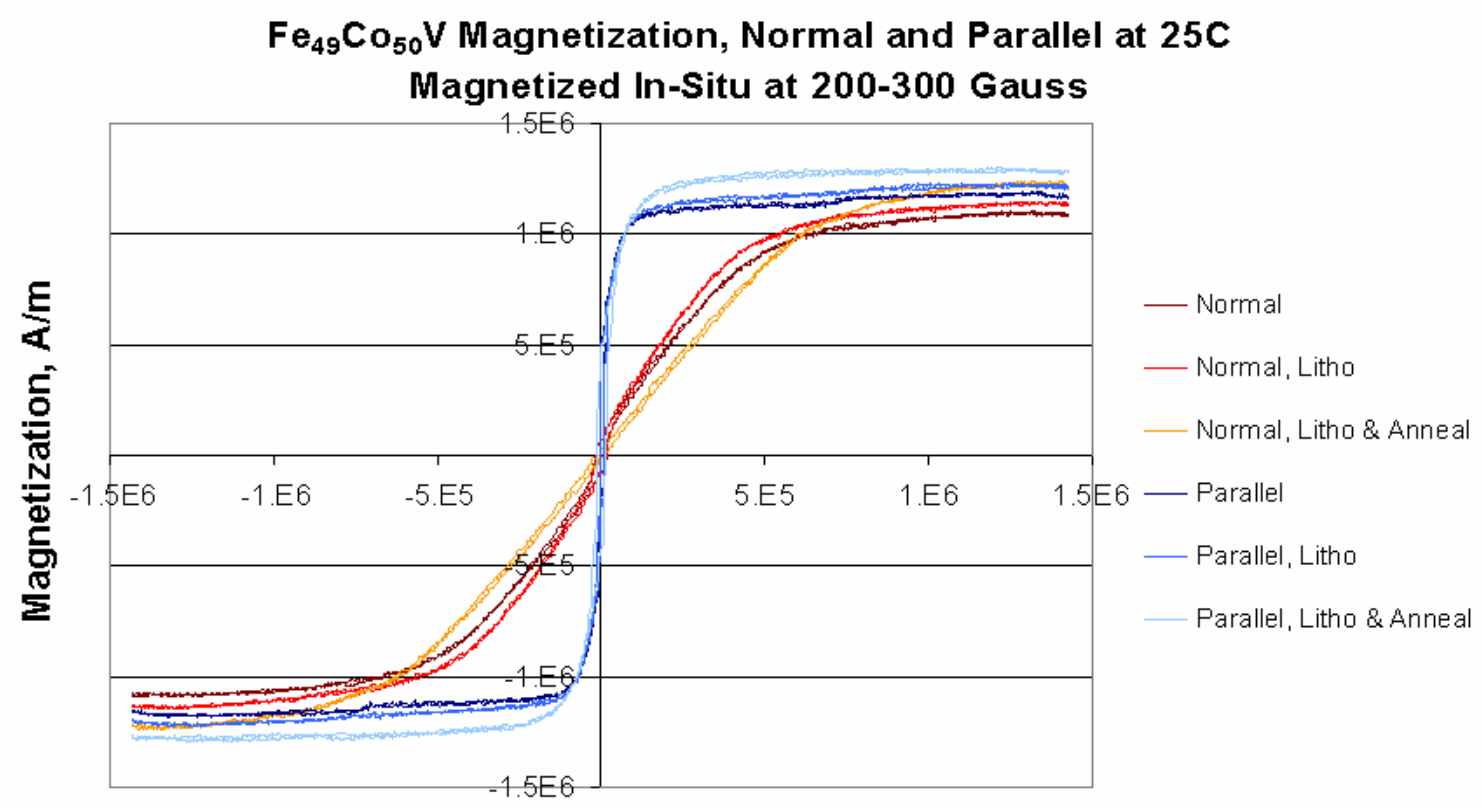

Applied Field, A/m

Figure B.28: Magnetization plots for a RF sputtered $\mathrm{Fe}_{49} \mathrm{Co}_{50} \mathrm{~V}$ alloy parallel and normal to the substrate. Deposition was done at $300 \mathrm{~W}$ with a 200-300 Gauss applied magnetic field, resulting in a $0.40 \mu \mathrm{m}$ film. Some samples underwent a photolithography process to simulate the stresses to which the magnetic films are exposed during patterning. Of those, half were then magnetically annealed at $300^{\circ} \mathrm{C}$ in a nitrogen atmosphere. The data showed that both the lithography process and the magnetic anneal had a minimal impact on the $\mathrm{Fe}_{49} \mathrm{Co}_{50} \mathrm{~V}$ magnetic properties. Furthermore, the magnetization normal to the substrate reached saturation far more quickly than that for the other in-situ magnetized $\mathrm{Fe}_{49} \mathrm{Co}_{50} \mathrm{~V}$ films, suggesting that the lower deposition power (and thus lower deposition rate) allowed the film to magnetize more strongly as it formed. 


\section{B.4: Iron-Nickel-Cobalt Alloys}

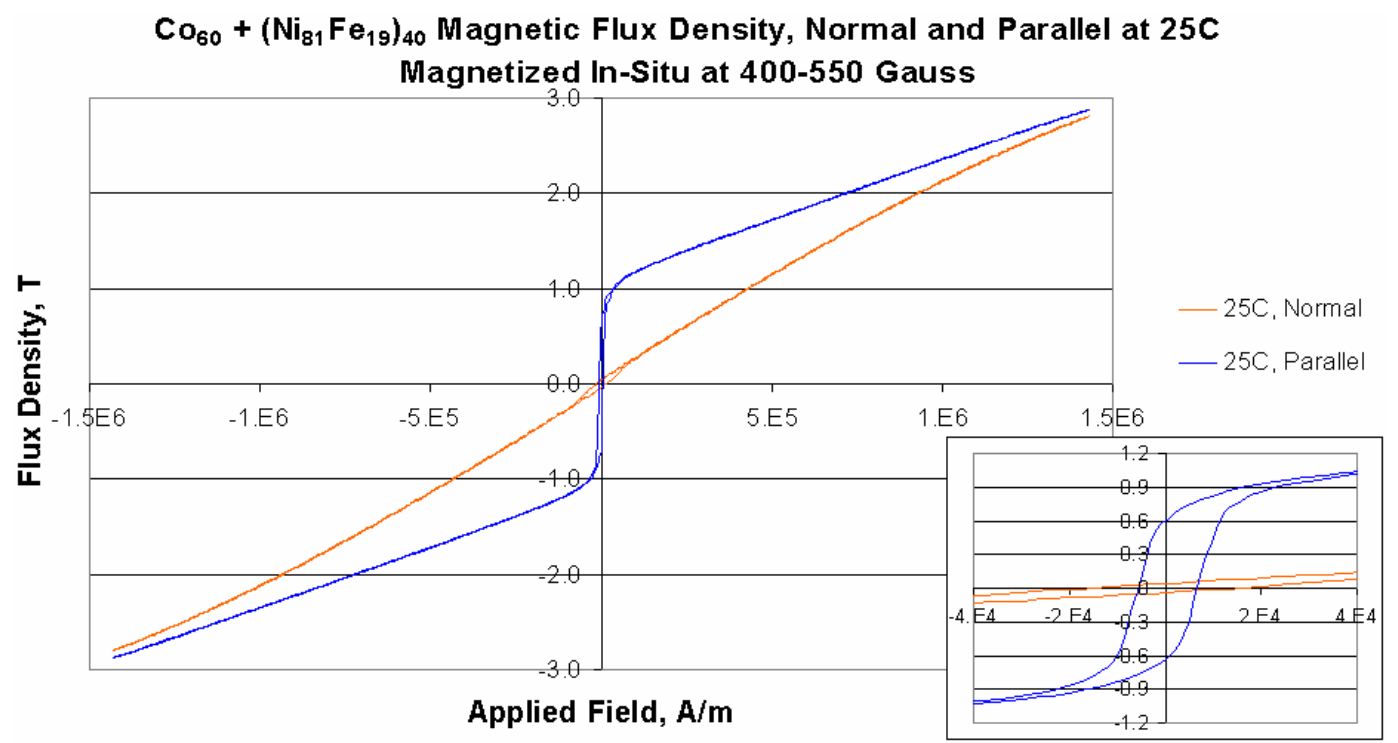

Figure B.29: Magnetic flux density plots for a RF sputtered $\mathrm{Co}_{60}\left(\mathrm{Ni}_{81} \mathrm{Fe}_{19}\right)_{40}$ alloy illustrating properties both parallel and normal to the substrate at room temperature. Deposition was done by alternating between the $\mathrm{Co}$ and $\mathrm{Ni}_{81} \mathrm{Fe}_{19}$ targets for 3 and 2 minutes, respectively, at $700 \mathrm{~W}$ each with a 400-550 Gauss applied magnetic field, resulting in a $3.1 \mu \mathrm{m}$ film. Hysteresis is fairly wide like that of cobalt, but the parallel slope is more like that for $\mathrm{Ni}_{81} \mathrm{Fe}_{19}$ (with almost identical maximum permeability).

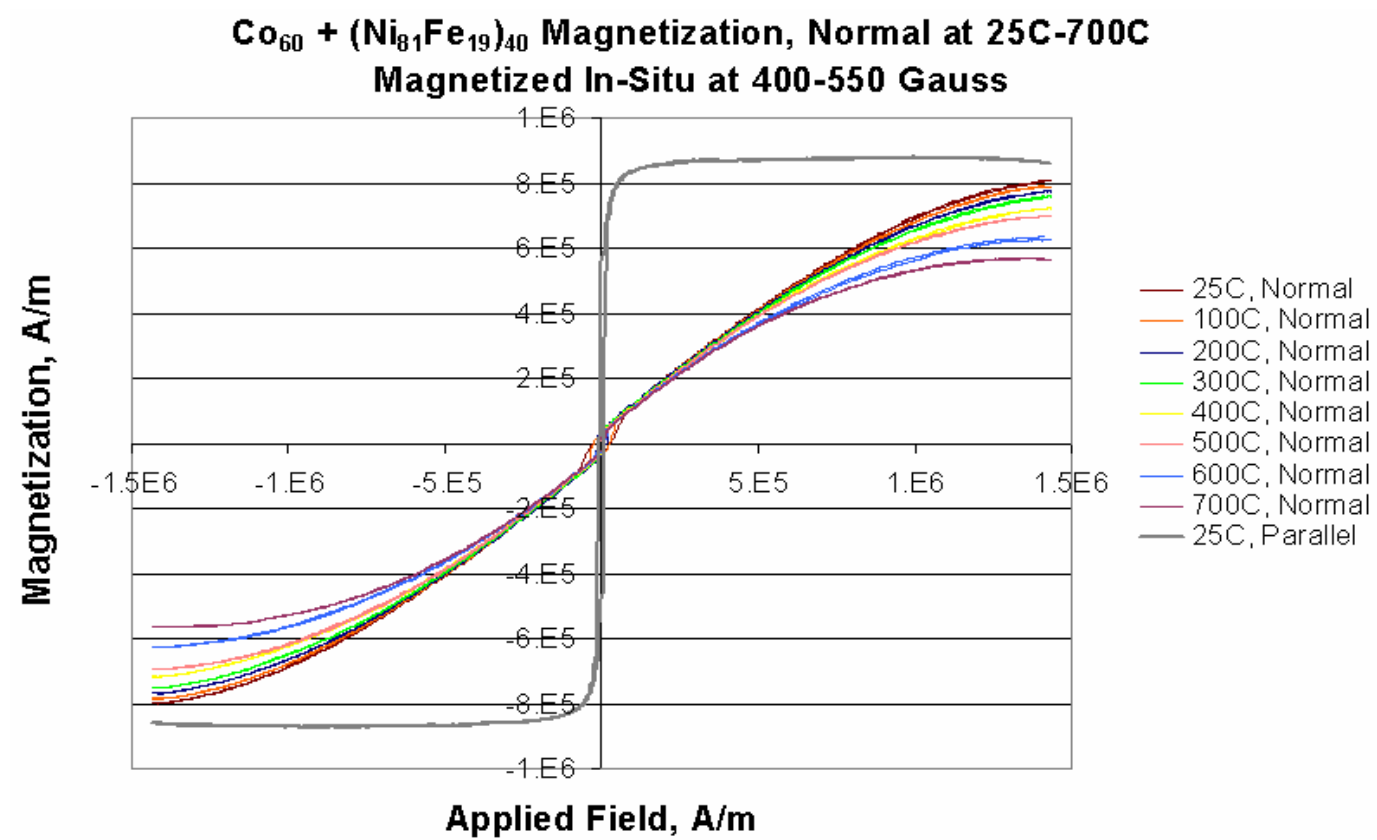

Figure B.30: Magnetization plots for the magnetized RF sputtered $\mathrm{Co}_{60}\left(\mathrm{Ni}_{81} \mathrm{Fe}_{19}\right)_{40}$ alloy showing the effect of elevated temperature on its magnetic behavior in the direction normal to the substrate relative to that at $25^{\circ} \mathrm{C}$. The parallel curve at $25^{\circ} \mathrm{C}$ is also included for reference. The film retained approximately $70 \%$ of its room temperature magnetization at $700^{\circ} \mathrm{C}$, the best high-temperature performance of the ternary alloys. 
$\left(\mathrm{Ni}_{81} \mathrm{Fe}_{19}\right)_{70}+\mathrm{Co}_{30}$ Magnetic Flux Density, Normal and Parallel at 25C Magnetized In-Situ at 400-550 Gauss

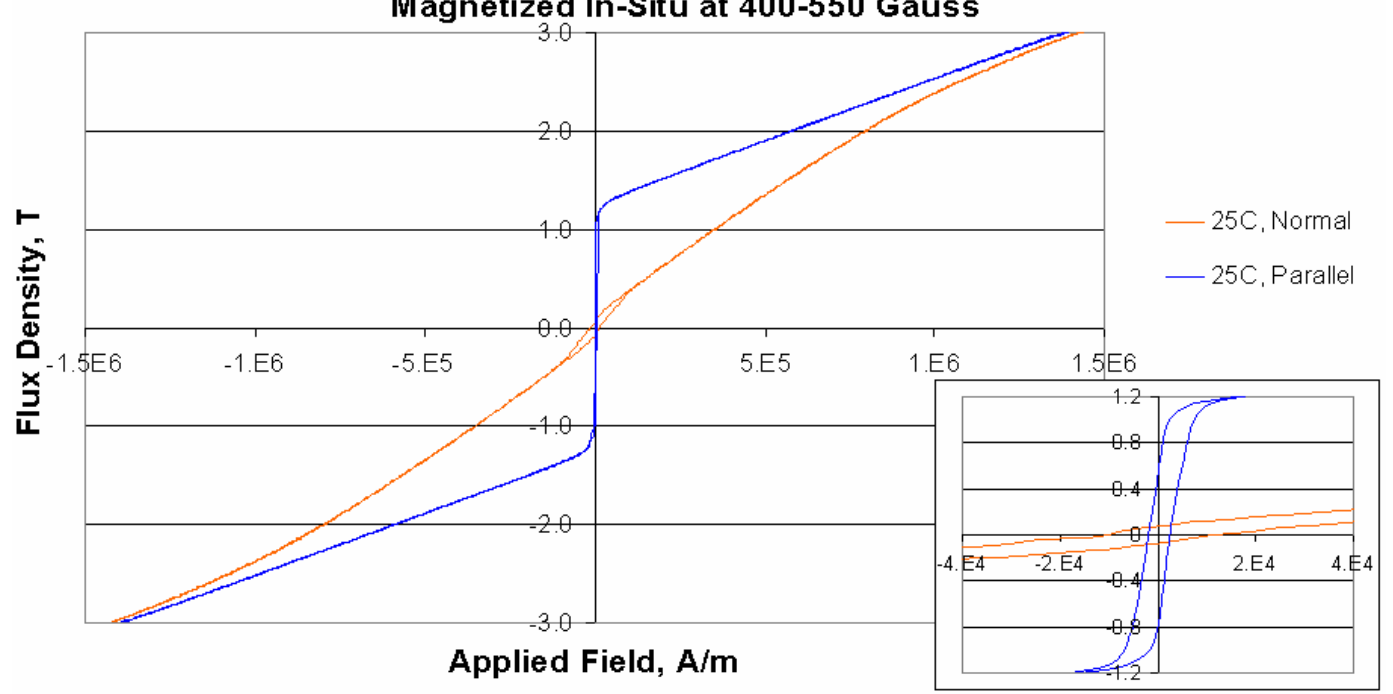

Figure B.31: Magnetic flux density plots for a $\mathrm{RF}$ sputtered $\left(\mathrm{Ni}_{81} \mathrm{Fe}_{19}\right)_{70} \mathrm{Co}_{30}$ alloy illustrating properties both parallel and normal to the substrate at room temperature. Deposition was done by alternating between the $\mathrm{Co}$ and $\mathrm{Ni}_{81} \mathrm{Fe}_{19}$ targets for 1.5 and 3.5 minutes, respectively, at $700 \mathrm{~W}$ each with a $400-550$ Gauss applied magnetic field, resulting in a $2.8 \mu \mathrm{m}$ film. Normal hysteresis is similar to that of cobalt, whereas parallel hysteresis yields a higher permeability value than either of the two constituent materials.

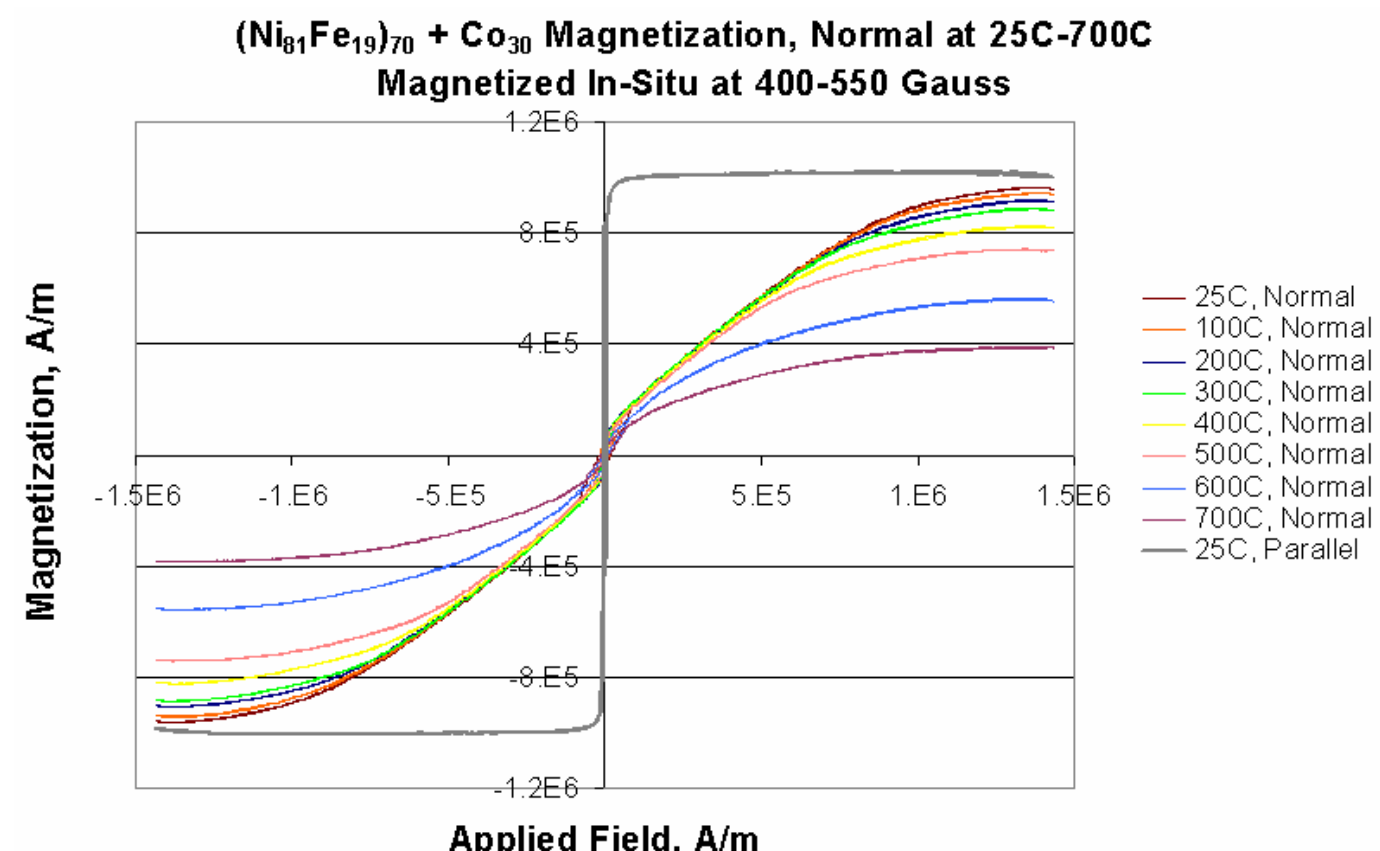

Figure B.32: Magnetization plots for the magnetized RF sputtered $\left(\mathrm{Ni}_{81} \mathrm{Fe}_{19}\right)_{70} \mathrm{Co}_{30}$ alloy showing the effect of elevated temperature on its magnetic behavior in the direction normal to the substrate relative to that at $25^{\circ} \mathrm{C}$. The parallel curve at $25^{\circ} \mathrm{C}$ is also included for reference. The addition of cobalt improved the thermal performance by a factor of 70 over that of pure $\mathrm{Ni}_{81} \mathrm{Fe}_{19}$, though the normal permeability values were approximately $2 / 3$ lower. 


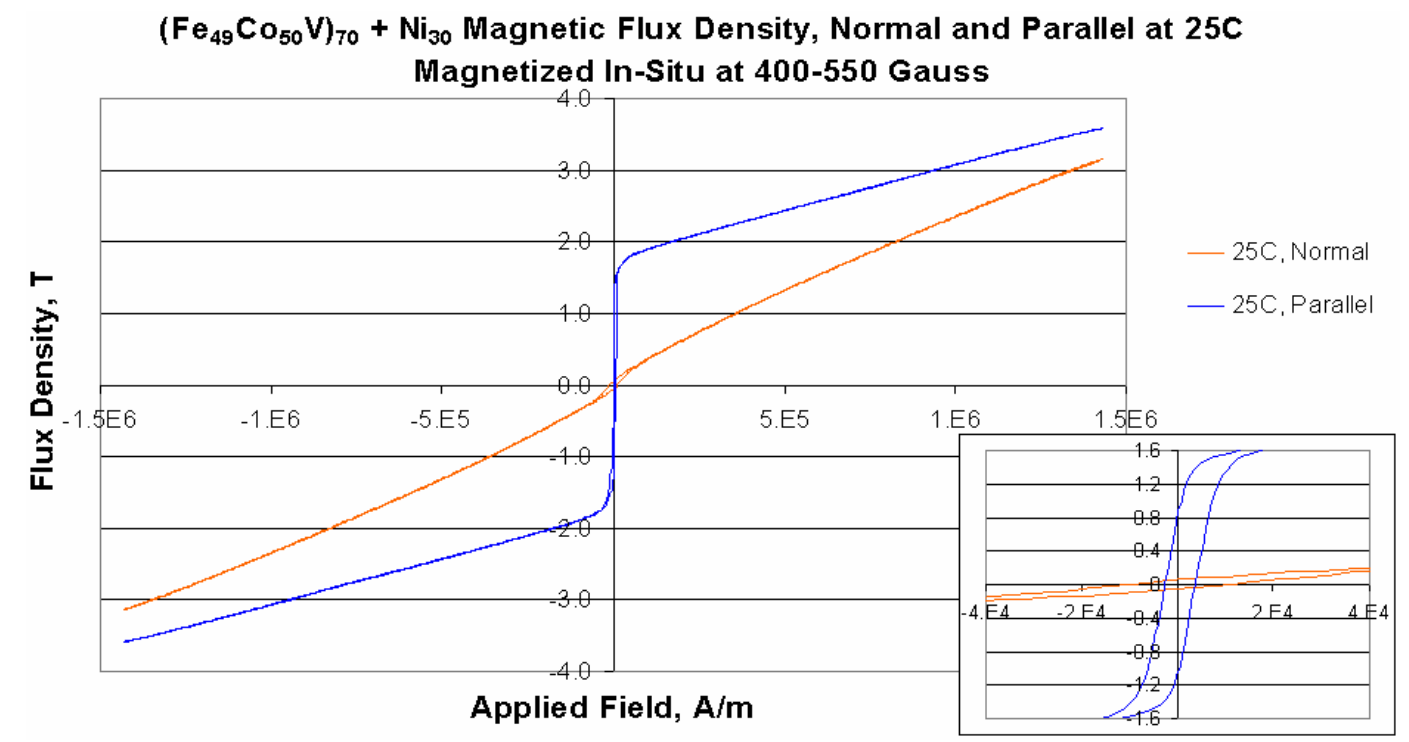

Figure B.33: Magnetic flux density plots for a RF sputtered $\left(\mathrm{Fe}_{49} \mathrm{Co}_{50} \mathrm{~V}\right)_{70} \mathrm{Ni}_{30}$ alloy illustrating properties both parallel and normal to the substrate at room temperature. Deposition was done by alternating between the $\mathrm{Fe}_{49} \mathrm{Co}_{50} \mathrm{~V}$ and $\mathrm{Ni}$ targets for 3.5 and 1.5 minutes, respectively, at $700 \mathrm{~W}$ each with a 400-550 Gauss applied magnetic field, resulting in a $3.0 \mu \mathrm{m}$ film. Hysteresis loops were similar to those of $\mathrm{Fe}_{49} \mathrm{Co}_{50} \mathrm{~V}$.

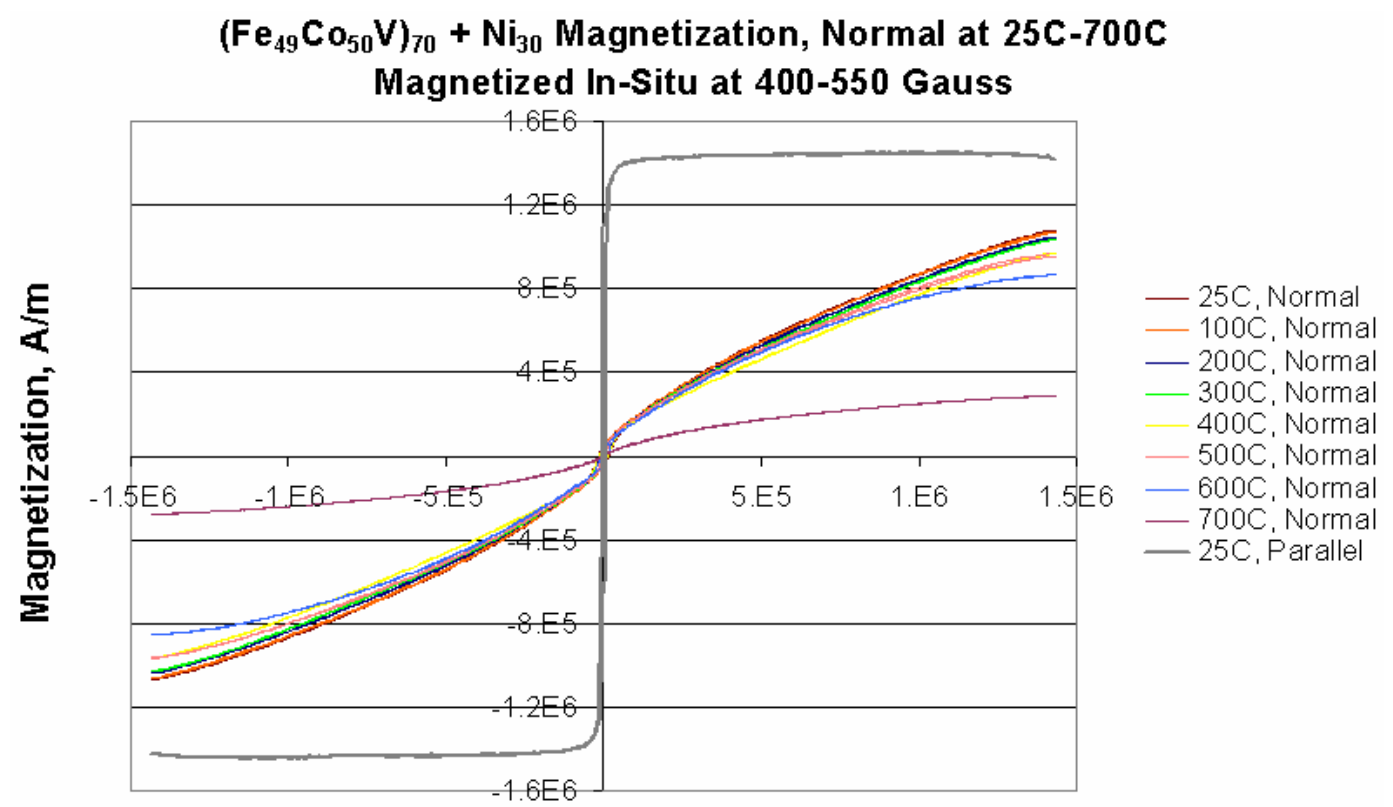

Applied Field, A/m

Figure B.34: Magnetization plots for the magnetized RF sputtered $\left(\mathrm{Fe}_{49} \mathrm{Co}_{50} \mathrm{~V}\right)_{70} \mathrm{Ni}_{30}$ alloy showing the effect of elevated temperature on its magnetic behavior in the direction normal to the substrate relative to that at $25^{\circ} \mathrm{C}$. The parallel curve at $25^{\circ} \mathrm{C}$ is also included for reference. The addition of nickel reduced the high-temperature performance from that of pure $\mathrm{Fe}_{49} \mathrm{Co}_{50} \mathrm{~V}$, and furthermore the normal permeabilities were approximately $2 / 3$ lower. Permeability values in the parallel direction, however, were the highest among all samples tested, reaching 124 times that of free space at $25^{\circ} \mathrm{C}$. 


\section{Appendix C: MTU High Aspect Ratio Polysilicon Sensor Process}

\section{C.1: Low Temperature Deposition with Annealing}

1) Wafer Procurement

Vendor: University Wafer

Orientation: $<100>$

Dopant: Boron

Resistivity: $10-20 \Omega-\mathrm{cm}$

Films present: $1 \mu \mathrm{m}$ thermal oxide

Comments: Dopant species not critical, Resistivity critical for inductively annealed wafers only

2) LPCVD Silicon Nitride Deposition

System: TMX9K 4304

Gas flow: $40 \mathrm{sccm} \mathrm{SiH}_{2} \mathrm{Cl}_{2}, 160 \mathrm{sccm} \mathrm{NH}_{3}$

Temperature: $820^{\circ} \mathrm{C}$

Pressure: $150 \mathrm{mTorr}$

Target thickness: $0.25 \mu \mathrm{m}$

3) LPCVD Polysilicon Deposition

System: TMX9K 4304

Gas flow: $80 \mathrm{sccm} \mathrm{SiH}_{4}$

Temperature: $625^{\circ} \mathrm{C}$

Pressure: $180 \mathrm{mTorr}$

Target thickness: $0.5 \mu \mathrm{m}$

Comment: Poly0 lower interconnect layer

4) Phosphorous Doping of Polysilicon

System: TMX9K 4304

Temperature: $1175^{\circ} \mathrm{C}$

Pressure: Atmospheric

Measured resistivity: $26.5 \Omega /$ square

Comment: Doping intended to enhance conductivity of poly0 layer

5) Photolithography

Mask: Poly0

Resist polarity: Negative

6) Polysilicon RIE Etch

System: LAM 9400

Power: 500W

Gas flow: $100 \mathrm{sccm} \mathrm{HBr}, 100 \mathrm{sccm} \mathrm{He}$

Pressure: $120 \mathrm{mTorr}$

7) LPCVD Silicon Dioxide Deposition 
System: TMX9K 4304

Gas flow: $120 \mathrm{sccm} \mathrm{N} \mathrm{N}_{2} \mathrm{O}, 60 \mathrm{sccm} \mathrm{SiH}_{2} \mathrm{Cl}_{2}$

Temperature: $920^{\circ} \mathrm{C}$

Pressure: $400 \mathrm{mT}$ Torr

Target Thickness: $2 \mu \mathrm{m}$

Comment: Poly0-poly1 sacrificial layer

8) Photolithography

Mask: Poly0-poly1

Resist polarity: Negative

9) Silicon Dioxide Chemical Etch

Chemical: Buffered hydrofluoric acid (BHF)

10) LPCVD Silicon Deposition

System: Tempress 6400

Gas flow: $60 \mathrm{sccm} \mathrm{SiH}_{4}$

Temperature: $600^{\circ} \mathrm{C}$

Pressure: $220 \mathrm{mTorr}$

Target thickness: $6 \mu \mathrm{m}$

Comment: Alternate silicon deposition methods with temperatures below $400^{\circ} \mathrm{C}$ were preferred but were not available at the time of device fabrication

11) Wafer Polishing

System: Logitech PM2A

Rotation rate: 60 RPM

Slurry: Logitech Polishing Suspension Type SF1

12) Photolithography

Mask: Windows

Resist polarity: Positive

Comment: Removes thick polysilicon from two areas on opposite sides of the wafer approximately $1 \times 1 \mathrm{~cm}^{2}$ in order to reveal the alignment marks

13) Polysilicon DRIE Etch

System: STS Multiplex ICP

Power: $100 \mathrm{~W}$ platen, $800 \mathrm{~W}$ coil generator (etching) / $800 \mathrm{~W}$ coil generator (passivation)

Gas flow: $130 \mathrm{sccm} \mathrm{SF}_{6}, 13 \mathrm{sccm} \mathrm{O} \mathrm{O}_{2}$ (etching) / 85 $\mathrm{sccm} \mathrm{C}_{4} \mathrm{~F}_{8}$ (passivation)

Pressure: 10mTorr

14) Photolithography

Mask: Thermal trenches

Resist polarity: Positive

Comment: Defines thermal isolation trenches in order to enhance the temperature

differential during localized annealing 
15) Polysilicon DRIE Etch

System: STS Multiplex ICP

Power: $100 \mathrm{~W}$ platen, $800 \mathrm{~W}$ coil generator (etching) / $800 \mathrm{~W}$ coil generator (passivation)

Gas flow: $130 \mathrm{sccm} \mathrm{SF}_{6}, 13 \mathrm{sccm} \mathrm{O} \mathrm{O}_{2}$ (etching) / 85 $\mathrm{sccm} \mathrm{C}_{4} \mathrm{~F}_{8}$ (passivation)

Pressure: 10mTorr

16) Photolithography

Mask: Poly1

Resist polarity: Positive

17) Polysilicon DRIE Etch

System: STS Multiplex ICP

Power: $100 \mathrm{~W}$ platen, $800 \mathrm{~W}$ coil generator (etching) / $800 \mathrm{~W}$ coil generator (passivation)

Gas flow: $130 \mathrm{sccm} \mathrm{SF}_{6}, 13 \mathrm{sccm} \mathrm{O}$ (etching) / 85 $\mathrm{sccm} \mathrm{C}_{4} \mathrm{~F}_{8}$ (passivation)

Pressure: 10mTorr

18) PECVD Silicon Dioxide Deposition

System: GSI Ultradep 2000

Power: $30 \mathrm{~W}$ platen, $90 \mathrm{~W}$ coil generator

Gas flow: $15 \% \mathrm{SiH}_{4}, 99 \% \mathrm{~N}_{2} \mathrm{O}, 50 \% \mathrm{He}$

Temperature: $200^{\circ} \mathrm{C}$

Pressure:

Target thickness: $2 \mu \mathrm{m}$

Comment: Low temperature required to minimize effect on polysilicon properties

19) Sputtered Chromium Deposition

System: Perkin-Elmer 2400-8J

Power: $700 \mathrm{~W}$

Gas flow: 20scem Ar

Pressure: $10 \mathrm{mT}$ Torr

Target thickness: $120 \mathrm{~nm}$

Comment: Provides adhesion assistance for ferromagnetic films

20) Sputtered $\mathrm{Ni}_{81} \mathrm{Fe}_{19}$ Deposition

System: Perkin-Elmer 2400-8J

Power: $500 \mathrm{~W}$

Gas flow: $15 \mathrm{sccm} \mathrm{Ar}$

Pressure: $8 \mathrm{mT}$ Torr

Target thickness: To be determined

21) Photolithography

Mask: Ferromagnetic Grid

Resist polarity: Positive

22) Ferromagnetic Chemical Etch

Chemical: Transene Nickel Etchant Type I 
Comment: Etchant removes $\mathrm{NiFe}, \mathrm{FeCo}$, and $\mathrm{Cr}$

23) Inductive Anneal

System: MTU BEAVIS

Power: Full

Pressure: $5 \mathrm{mTorr}$

Time: 5 minutes

Temperature: $1000^{\circ} \mathrm{C}$

24) Ferromagnetic Film Removal

Chemical: Transene Nickel Etchant Type I

25) Photolithography

Mask: Poly1-Metal1

Resist polarity: Positive

26) Silicon Dioxide RIE Etch

System: Semi Group RIE System 1000 TP/CC

Power: $180 \mathrm{~W}$

Gas flow: $25 \mathrm{sccm} \mathrm{CF}_{4}, 25 \mathrm{sccm} \mathrm{CHF}_{3}$

Pressure: 20mTorr

27) PECVD Silicon Deposition

System: GSI Ultradep 2000

Target thickness: $1.5 \mu \mathrm{m}$

Comment: Substitution material for Metall interconnect layer

28) Photolithography

Mask: Metall

Resist polarity: Negative

29) Silicon RIE etch

System: March Instruments Jupiter II

Power: $30 \mathrm{~W}$

Gas flow: $80 \mathrm{sccm} \mathrm{SF}_{4}$

Pressure: $100 \mathrm{mTorr}$

30) Device Release

Chemical: Buffered hydrofluoric acid (BHF)

Comment: Etchant removes unreacted titanium also 


\section{C.2: High Temperature Deposition without Annealing}

1) Wafer Procurement

Vendor: University Wafer

Orientation: $<100>$

Dopant: Boron

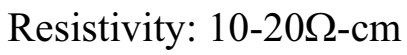

Films present: $1 \mu \mathrm{m}$ thermal oxide

Comments: Dopant species not critical, Resistivity critical for inductively annealed wafers only

2) LPCVD Silicon Nitride Deposition

System: TMX9K 4304

Gas flow: 40sccm $\mathrm{SiH}_{2} \mathrm{Cl}_{2}, 160 \mathrm{sccm} \mathrm{NH}_{3}$

Temperature: $820^{\circ} \mathrm{C}$

Pressure: $150 \mathrm{mTorr}$

Target thickness: $0.25 \mu \mathrm{m}$

3) LPCVD Polysilicon Deposition

System: TMX9K 4304

Gas flow: $80 \mathrm{sccm} \mathrm{SiH}_{4}$

Temperature: $625^{\circ} \mathrm{C}$

Pressure: $180 \mathrm{~m}$ Torr

Target thickness: $0.5 \mu \mathrm{m}$

Comment: Poly0 lower interconnect layer

4) Phosphorous Doping of Polysilicon

System: TMX9K 4304

Temperature: $1175^{\circ} \mathrm{C}$

Pressure: Atmospheric

Measured resistivity: $26.5 \Omega /$ square

Comment: Doping intended to enhance conductivity of poly0 layer

5) Photolithography

Mask: Poly0

Resist polarity: Negative

6) Polysilicon RIE Etch

System: LAM 9400

Power: 500W

Gas flow: 100sccm HBr, 100sccm He

Pressure: $120 \mathrm{~m}$ Torr

7) LPCVD Silicon Dioxide Deposition

System: TMX9K 4304

Gas flow: $120 \mathrm{sccm} \mathrm{N}_{2} \mathrm{O}, 60 \mathrm{sccm} \mathrm{SiH}_{2} \mathrm{Cl}_{2}$ 
Temperature: $920^{\circ} \mathrm{C}$

Pressure: $400 \mathrm{mTorr}$

Target Thickness: $2 \mu \mathrm{m}$

Comment: Poly0-polyl sacrificial layer

8) Photolithography

Mask: Poly0-poly1

Resist polarity: Negative

9) Silicon Dioxide Chemical Etch

Chemical: Buffered hydrofluoric acid (BHF)

10) Epitaxial Silicon Deposition

System: ASM 2000

Gas flow: $\mathrm{HSiCl}_{3}$ with $\mathrm{H}_{2}$ carrier gas

Temperature: $1150^{\circ} \mathrm{C}$

Pressure: Atmospheric

Target thickness: $20 \mu \mathrm{m}$

Comment: Film deposited at high temperature, no annealing required.

11) Wafer Polishing

System: Logitech PM2A

Rotation rate: 60 RPM

Slurry: Logitech Polishing Suspension Type SF1

12) Photolithography

Mask: Windows

Resist polarity: Positive

Comment: Removes thick polysilicon from two areas on opposite sides of the wafer

approximately $1 \times 1 \mathrm{~cm}^{2}$ in order to reveal the alignment marks

13) Polysilicon DRIE Etch

System: STS Multiplex ICP

Power: $100 \mathrm{~W}$ platen, $800 \mathrm{~W}$ coil generator (etching) / 800W coil generator (passivation)

Gas flow: $130 \mathrm{sccm} \mathrm{SF} 6,13 \mathrm{sccm} \mathrm{O}_{2}$ (etching) / $85 \mathrm{sccm} \mathrm{C}_{4} \mathrm{~F}_{8}$ (passivation)

Pressure: $10 \mathrm{mTorr}$

14) Photolithography

Mask: Poly1

Resist polarity: Positive

15) Polysilicon DRIE Etch

System: STS Multiplex ICP

Power: $100 \mathrm{~W}$ platen, $800 \mathrm{~W}$ coil generator (etching) / 800W coil generator (passivation)

Gas flow: $130 \mathrm{sccm} \mathrm{SF} 6,13 \mathrm{sccm} \mathrm{O} \mathrm{O}_{2}$ (etching) / $85 \mathrm{sccm} \mathrm{C}_{4} \mathrm{~F}_{8}$ (passivation)

Pressure: $10 \mathrm{mTorr}$ 
16) PECVD Silicon Dioxide Deposition

System: GSI Ultradep 2000

Gas flow:

Temperature: $200^{\circ} \mathrm{C}$

Pressure:

Target thickness: $2 \mu \mathrm{m}$

Comment: Poly1-Metall insulating layer, sacrificial in structural region

17) Photolithography

Mask: Poly1-Metal1

Resist polarity: Positive

18) Silicon Dioxide RIE Etch

System: Semi Group RIE System 1000 TP/CC

Power: $180 \mathrm{~W}$

Gas flow: $25 \mathrm{sccm} \mathrm{CF}_{4}, 25 \mathrm{sccm} \mathrm{CHF}_{3}$

Pressure: 20mTorr

19) PECVD Silicon Deposition

System: GSI Ultradep 2000

Power:

Gas flow:

Pressure:

Target thickness: $1.5 \mu \mathrm{m}$

Comment: Substitution material for Metall interconnect layer

20) Photolithography

Mask: Metall

Resist polarity: Negative

21) Silicon RIE etch

System: March Instruments Jupiter II

Power: $30 \mathrm{~W}$

Gas flow: $80 \mathrm{sccm} \mathrm{SF}_{4}$

Pressure: $100 \mathrm{mTorr}$

22) Device Release

Chemical: Buffered hydrofluoric acid (BHF) 